\title{
Unified Synthesis of Polycyclic Alkaloids via Complementary Carbonyl Activation
}

\author{
Guoli He${ }^{[a]}$, Benjamin List ${ }^{*[b]}$ and Mathias Christmann ${ }^{*[a]}$
}

\begin{abstract}
A complementary dual carbonyl activation strategy for the synthesis of polycyclic alkaloids has been developed. Successful applications include the synthesis of tetracyclic alkaloids harmalanine, harmalacinine, pentacyclic indoloquinolizidine alkaloid nortetoyobyrine, and octacyclic $\beta$-carboline alkaloid peganumine $A$. The latter synthesis features a protecting-group-free assembly and an asymmetric disulfonimide catalyzed cyclization. Furthermore, formal syntheses of hirsutine, deplancheine, 10-desbromoarborescidine $A$, and oxindole alkaloids rhynchophylline and isorhynchophylline have been achieved. Finally, a concise synthesis of berberine alkaloid ilicifoline $B$ was completed.
\end{abstract}

Despite the advancement of combinatorial strategies, natural products remain an indispensable source for the discovery of new molecular entities. ${ }^{[1]}$ Their diverse scaffolds with hydrogen bond donor and acceptor groups positioned in a well-defined spatial arrangement make them attractive starting points and inspiration for drug development. ${ }^{[2]}$ Bioactive polycyclic alkaloids, such as yohimbine (1), hirsutine (2), deplancheine (3), eburnamonine (4), ilicifoline B (5), peganumine A (6), and reserpine (7), contain the common quinolizidine core I fused to different heterocyclic rings (Figure 1). We reasoned that developing a straightforward annulation method for efficient construction of these scaffolds is beneficial for the total synthesis of polycyclic natural products and their analogs. Since the indole substructure is a privileged ${ }^{[3]}$ and very common motif in these polycyclic natural products, we started our synthetic journey with the quinolizidine-fused indole core. We strategized that incorporating an enamide motif into the A ring would provide a flexible handle for subsequent transformations. Therefore, intermediate III was considered the central linchpin for a divergent synthesis of polycyclic alkaloids. It was envisioned to be derived from IV by an annulation sequence involving an electrophilic cyclization followed by lactamization. Toward this goal we identified two major challenges: 1) selective activation of the amide carbonyl group to participate in the electrophilic cyclization; ${ }^{[4]}$ 2) subsequent selective activation of the second carbonyl group to achieve lactamization.

[a] G. He, Prof. Dr. M. Christmann

Freie Universität Berlin, Institute of Chemistry and Biochemistry

Takustr.3,14195 Berlin (Germany)

E-Mail: mathias.christmann@fu-berlin.de

[b] Prof. Dr. B. List

Max-Planck-Institut für Kohlenforschung

Kaiser-Wilhelm-Platz 1, 45470 Mülheim an der Ruhr (Germany)

E-Mail: list@kofo.mpg.de

Supporting information for this article is given via a link at the end of the document.

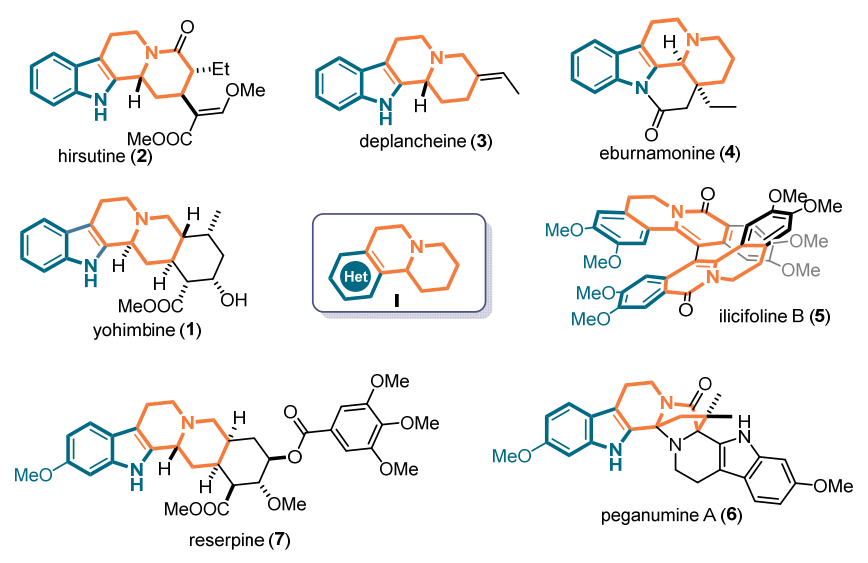

Figure 1. Common alkaloids with a tetracyclic substructure.

We conceived a one-pot approach in which IV can be prepared by two-fold condensation of $\mathbf{V}, \mathbf{V} \mathbf{l}$ and active ester component. By permutation of the substrates $\mathbf{V}$ and $\mathbf{V I}$, a variety of polycyclic ring system with diverse substitution patterns could be accessed (Scheme 1).

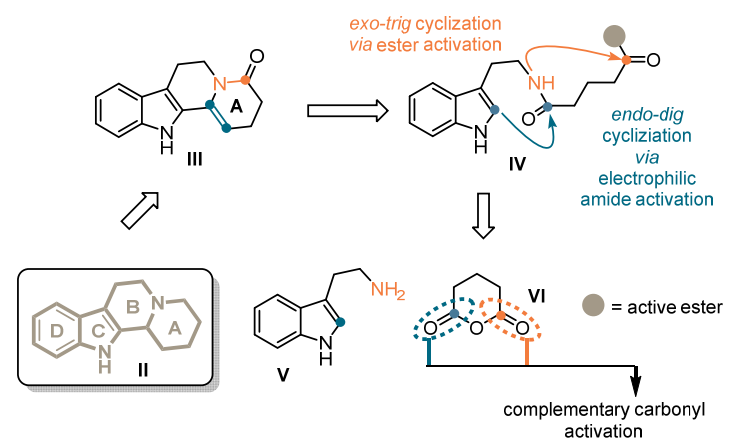

Scheme 1. Synthetic strategy.

In order to orchestrate the subsequent activations, we initially investigated the reaction using the amide $\mathbf{8 a}$ as the model substrate (for its preparation see the Supporting Information). We tested different conditions to achieve selective amide activation via reactive nitrilium ions, ${ }^{[3]}$ including a variety of phosphorus $(\mathrm{V})$ reagents frequently applied in the BischlerNapieralski reaction ${ }^{[5]}$ and the von Braun amide degradation (Table 1, entries 1-4). ${ }^{[6]}$ We also screened strong electrophiles, such as $\mathrm{Me}_{3} \mathrm{SiCl}$ and $(\mathrm{COCl})_{2}{ }^{[7]}$ and the $\mathrm{Tf}_{2} \mathrm{O} / 2$ 2-chloropyridine system $^{[8]}$ which have been used successfully in electrophilic amide activation recently (Table 1 , entries $5-7$ ). Most of these 
conditions afforded tricyclic imine 9 as the major product. Unfortunately, the subsequent imine acylation to give tetracyclic product turned out to be challenging. With $\mathrm{POCl}_{3}, 10$ was isolated in $6 \%$ yield along with $84 \%$ of imine 9 (Table 1, entry 1 ). This result indicated that the reaction had stopped after the first cyclization. We hypothesized that imine-enamine tautomerization during second cyclization could also be a critical prerequisite for the second cyclization. ${ }^{[9]}$ After a screening of bases (see the $\mathrm{SI}$ ), we achieved a slight improvement to $10 \%$ yield of 10 using $\mathrm{K}_{2} \mathrm{CO}_{3}$ (Table 1, entry 8). With $n \mathrm{Bu}_{4} \mathrm{NBr}$ as phase transfer catalyst and methanol, the yield of $\mathbf{1 0}$ was further increased to $18 \%$ (Table 1 , entry 9 ). Inspired by active ester activation strategies used in peptide synthesis, ${ }^{[10]}$ we tested a variety of ester derivatives (see the SI). Satisfyingly, with 1,1,1,3,3,3-hexafluoro-2-propoxy ester $\mathbf{8 d}$, we achieved a $90 \%$ yield of $\mathbf{1 0}$ (Table 1 , entry 12 ).

Table 1. Optimization of reaction condition.

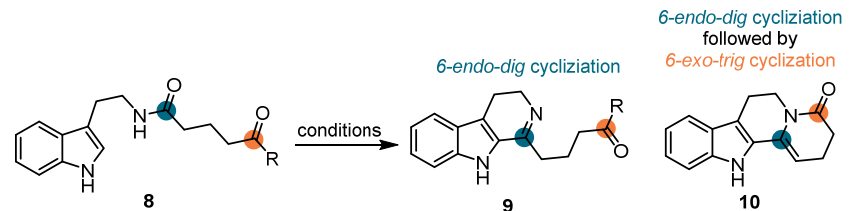

8a: $R=$ methoxy 8b: $R=4$-nitrophenoxy $\mathbf{8 c}: \mathbf{R}=2$-propoxy $\mathbf{8 d :} R=1,1,1,3,3,3$-hexafluoro-2-propoxy

\begin{tabular}{|c|c|c|c|c|c|}
\hline Entry ${ }^{[a]}$ & Substrate & $\begin{array}{c}\text { Amide } \\
\text { activation } \\
\text { reagent }\end{array}$ & Solvent & $\left.\pi^{\circ} \mathrm{C}\right]$ & Yield[\%] $]^{[b]}$ \\
\hline 1 & $8 a$ & $\mathrm{POCl}_{3}$ & toluene & 110 & $9(84) 10(6)$ \\
\hline 2 & $8 a$ & $\mathrm{P}_{2} \mathrm{O}_{5}$ & toluene & 110 & $9(8) 10(4)$ \\
\hline 3 & $8 a$ & $\mathrm{~T}_{3} \mathrm{P}$ & toluene & 110 & $9(3)$ \\
\hline 4 & $8 \mathbf{a}$ & $\mathrm{PCl}_{5}$ & toluene & 110 & $9(12) 10(7)$ \\
\hline 5 & $8 a$ & TMSCI & THF & 60 & N.R. \\
\hline 6 & $8 \mathbf{a}$ & $(\mathrm{COCl})_{2}$ & DCM & 23 & N.D. \\
\hline $7^{[c]}$ & $8 a$ & $\mathrm{Tf}_{2} \mathrm{O}$ & DCM & 23 & $9(53)$ \\
\hline $8^{[\mathrm{d}]}$ & $8 a$ & $\mathrm{POCl}_{3}$ & toluene & 110 & $10(10)$ \\
\hline $9^{[e]}$ & $8 a$ & $\mathrm{POCl}_{3}$ & toluene/MeOH & 110 to 80 & $10(18)$ \\
\hline $10^{[e]}$ & $8 b$ & $\mathrm{POCl}_{3}$ & toluene/MeOH & 110 to 80 & $10(62)$ \\
\hline $11^{[\mathrm{e}]}$ & $8 c$ & $\mathrm{POCl}_{3}$ & toluene/MeOH & 110 to 80 & $10(18)$ \\
\hline $12^{[\mathrm{e}]}$ & $8 d$ & $\mathrm{POCl}_{3}$ & toluene/MeOH & 110 to 80 & $10(90)$ \\
\hline
\end{tabular}

[a] Reactions were performed with substrate $(0.15 \mathrm{mmol})$, amide activation reagent $(0.15 \mathrm{mmol})$ in solvent $(2.0 \mathrm{~mL})$ as stated. [b] isolated yield. [c] 2-Clpyridine was used. [d] $\mathrm{K}_{2} \mathrm{CO}_{3}$ was used. [e] mixture of $\mathrm{K}_{2} \mathrm{CO}_{3}$ and $n \mathrm{Bu} \mathrm{u}_{4} \mathrm{NBr}$ in $\mathrm{MeOH}$ was added and temperature was decreased to $80^{\circ} \mathrm{C}$ after the addition.

With optimized conditions in hand, we explored the scope of the reaction for the synthesis of diverse polycyclic scaffolds (Table 2). Substitutions at the indole ring with electron donating groups (12a and 12b) and electron withdrawing groups (12c and 12d) were well tolerated, providing the corresponding tetracyclic scaffolds in good yields (76-86\%).

Table 2. Substrate scope. ${ }^{[a]}$
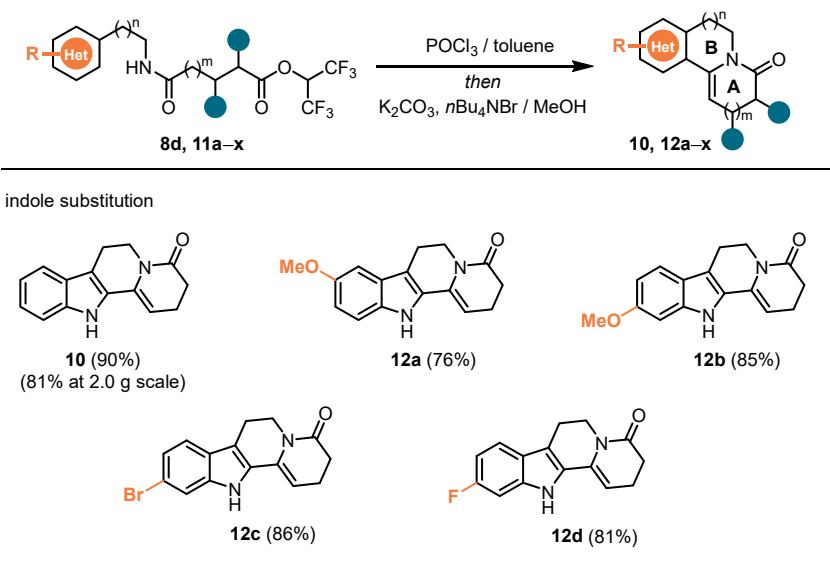

quinolizidine substitution

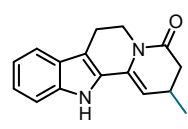

$12 \mathrm{e}(75 \%)$

(70\% at $1.0 \mathrm{~g}$ scale)

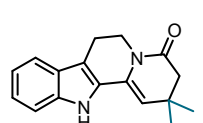

$12 \mathrm{~h}(83 \%)$

alternate ring systems

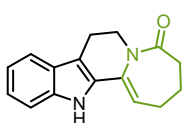

$12 k(26 \%)$

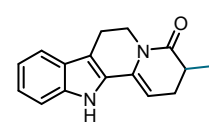

$12 f(72 \%)$

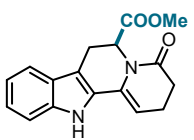

$12 \mathbf{i}(71 \%)$
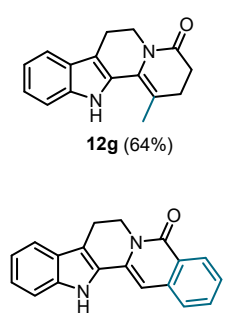

12j nortetoyobyrine (83\%)

(n)<smiles>CC1(C)C=C2c3ccccc3CCN2C(=O)C1</smiles>

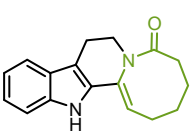

12I (39\%)

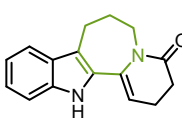

$12 \mathrm{~m}(52 \%)$

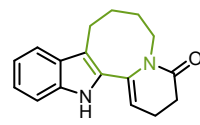

12n $(43 \%)$

substitution of the indole moiety

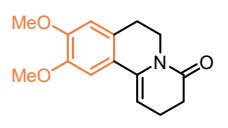

$12 \mathrm{r}(76 \%)$

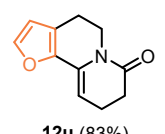

$12 u(83 \%)$
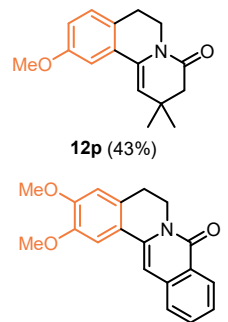

$12 \mathrm{~s}(83 \%)$

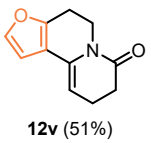

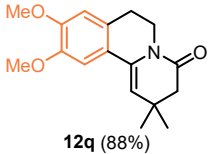

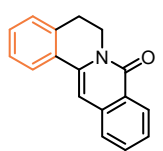

$12 t(48 \%)$

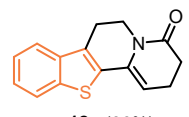

$12 \times(86 \%)$ [a] Reactions were performed with substrate $(0.10-4.7 \mathrm{mmol})$ using the standard procedure, isolated yields. See the SI for details.

Encouraged by these results, we investigated additional substitution patterns and ring systems. Substituting the quinolizidine core afforded the tetracycles 12e-12j in good yields $(64-83 \%)$ thus providing access to the indoloquinolizidinetype alkaloid nortetoyobyrine $(\mathbf{1 2 j})^{[11]}$ in an additional step. The $7 / 6,8 / 6,6 / 7$ and $6 / 8$ fused ring systems were obtained in moderate yield (12k-12n, 26\%-52\%). Finally, we successfully expanded our strategy to benzene derivatives and hetero- 
aromatic compounds, such as furan, thiophene, and benzothiophene (12o-12x, 33\%-91\%)

We next turned our attention to manipulations in the A ring in order to fully exploit our scaffold for natural product synthesis. Through oxidation, a second double bond could be easily introduced to the 3,4-position (VII). Reduction of the double bond in the 1,2-position (VIII) could be achieved with or without concomitant reduction of the lactam. Moreover, introduction of a carbonyl group in 4-position (IX) was key to the synthesis of more complex natural product.

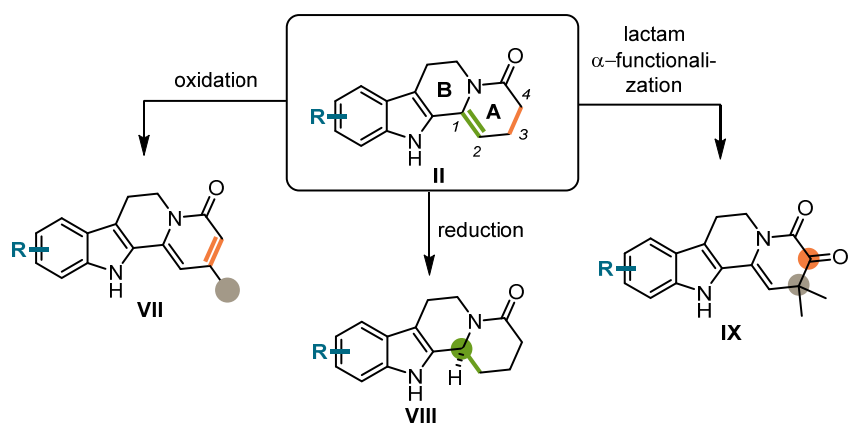

Scheme 2. Diversification strategy for the tetracyclic scaffold.

Starting with the dehydrogenation, we tested selenium- and sulfur-based reagents, such as $\mathrm{PhSeCl}, \mathrm{PhSeBr}, \mathrm{PhSSPh}$ and $N$-tert-butyl phenylsulfinimidoyl chloride (see the SI). ${ }^{[12]}$ Among standard protocols, only $\mathrm{N}$-tert-butyl phenylsulfinimidoyl chloride afforded traces of the desired product. Gratifyingly, using the palladium-catalyzed amide dehydrogenation protocol developed by Newhouse, ${ }^{[13]}$ demethoxyharmalanine (14a), harmalanine (14b), demethoxyharmalacinine (14c) and harmalacinine $(\mathbf{1 4 d}),{ }^{[14]}$ were successfully obtained in an excellent yield $(60$ $77 \%)$.

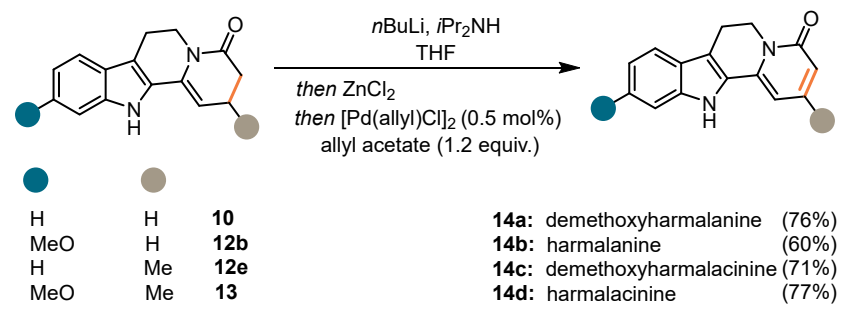

Scheme 3. Oxidative diversification.

Racemic 15 can be obtained through selective catalytic hydrogenation of 10 using palladium on carbon. From this intermediate, selenoxide elimination affords 16, a key intermediate in the total synthesis of hirsutine (2), rhynchophylline (17) and isorhynchophylline (18). ${ }^{[15]}$ An asymmetric reduction of the $\mathrm{C}-\mathrm{C}$ double bond was realized using chiral phosphoric acid (CPA) 19 and Hantzsch ester (20) system $^{[16]}$ to give 15 in $80 \%$ ee and $61 \%$ yield. This material can be converted into (S)-deplancheine (3) and (S)-10desbromoarborescidine $A(\mathbf{2 1})$ as previously reported. ${ }^{[17]}$

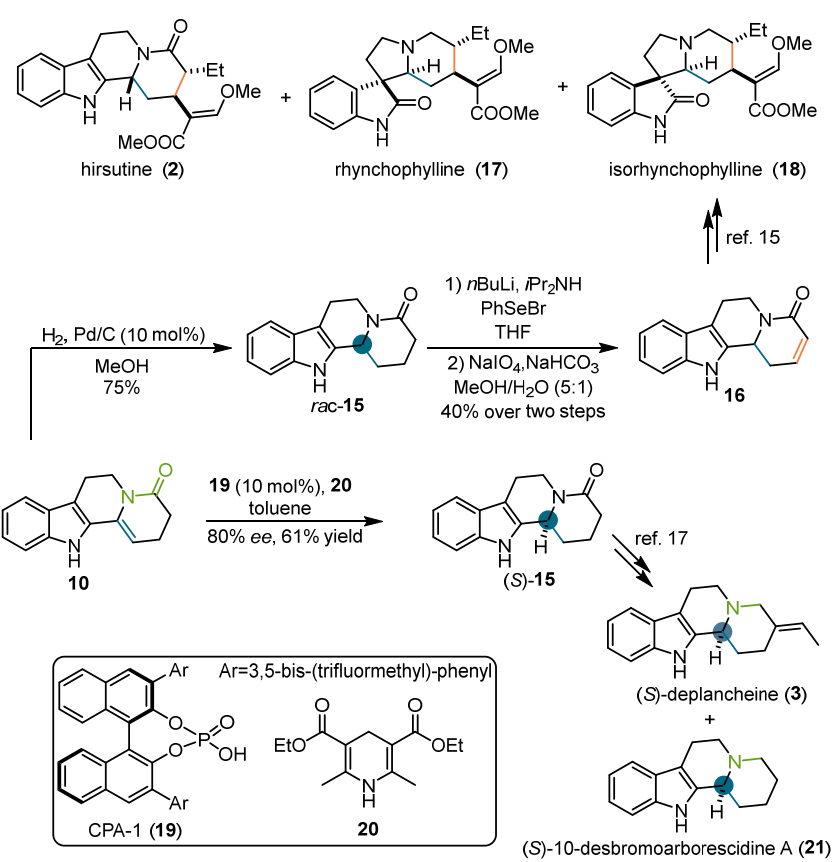

Scheme 4. Reductive diversification.

To further demonstrate the synthetic potential of this method, we envisioned to use our annulation in a protecting-group- and transition-metal-free asymmetric total synthesis of peganumine A (6). ${ }^{[18]}$

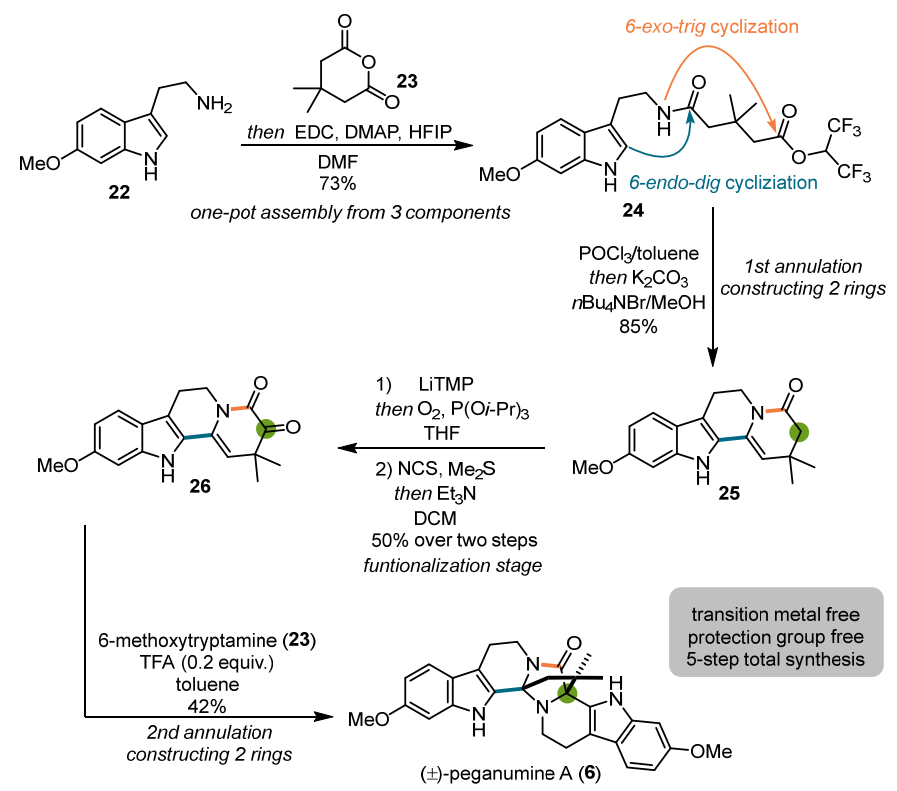

Scheme 5. Protecting-group-free synthesis of $( \pm)$-peganumine A.

Following the established protocol, we successfully prepared the tetracyclic intermediate $\mathbf{2 5}$ in $85 \%$ yield. Subsequently, ketoenamide $\mathbf{2 6}$ was obtained in $50 \%$ yield through a two-step $\alpha$-oxidation sequence. The Boc-derivative of $\mathbf{2 6}$ constitutes an intermediate in Zhu's elegant total synthesis of peganumine $A$ (6). ${ }^{[19]}$ At this point, we contemplated the possibility of a 
protecting-group-free synthesis. The key cascade cyclization was achieved using 0.2 equiv. of TFA in toluene to complete a protecting-group-free synthesis of ( \pm )- 6 in $42 \%$ yield.

Encouraged by the success of the previous cascade cyclization, we initiated investigations toward an asymmetric total synthesis. First, we tested the chiral thiourea (CTU) and $\mathrm{PhCO}_{2} \mathrm{H}$ system developed by Jacobsen, ${ }^{[20]}$ which afforded $92 \%$ ee in Zhu's synthesis ${ }^{[19]}$ for the Boc-protected substrate. In our protectinggroup-free substrate, with 27 and $\mathrm{PhCO}_{2} \mathrm{H}$, the enantioselectivity was $9 \%$ ee. We speculated that the remarkable difference in enantioselectivity could be attributed to an impaired recognition between substrate and catalyst. It is possible that either the Boc group is crucial for the recognition, or that the free $\alpha$ ketoenamide 26 interrupted the substrate binding. Based on these considerations, we proposed to either apply a multibinding-site catalyst to rigidify the transition state, or to use asymmetric counteranion directed catalysis $(A C D C)^{[21]}$ as stronger chiral acid to activate the imine more efficiently.

First, we tested the conjugate-base-stabilized Brønsted acid (CBSBA) 28 developed by Seidel, ${ }^{[22]}$ and 1,2,3,4,5pentacarboxycyclopentadiene (PCCP) derived pentamenthyl ester 29, a novel $\mathrm{C}-\mathrm{H}$ acid discovered by Lambert, ${ }^{[23]}$ which are all multi-binding-site catalysts. However, no improvement of the enantioselectivity could be achieved with our substrate. Moving to the ACDC using CPA-2 (30), a significant improvement of the enantioselectivity $(31 \%$ ee $)$ was observed. Expanding on this idea, we applied the stronger chiral Brønsted acid disulfonimide $(\mathrm{DSI})^{[24]}$ to the reaction and obtained $79 \%$ ee with DSI-1 (31). Encouraged by this promising result, and after intensive screening of DSIs (see the SI), we finally discovered that using DSI-2 (32) could achieve $97 \%$ ee and $81 \%$ yield.<smiles>CC(C)(C)C(C(=O)N(I)Cc1ccccc1)C(NC(=S)Nc1cc(C(F)(F)F)cc(C(F)(F)F)c1)C(=O)O</smiles><smiles>O=C(NC(=S)Nc1cc(C(F)(F)F)cc(C(F)(F)F)c1)C(=O)NC1CCCCC1</smiles>

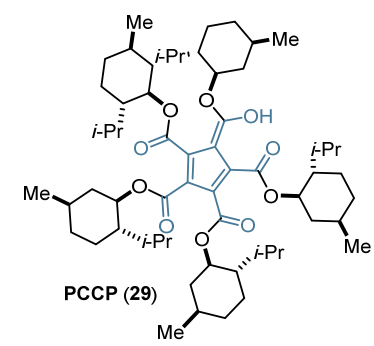

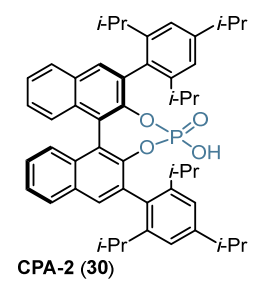<smiles></smiles><smiles></smiles>

Figure 2. Representative chiral Brønsted acids.
Table 3. Optimization of the asymmetric Pictet-Spengler reaction cascade.

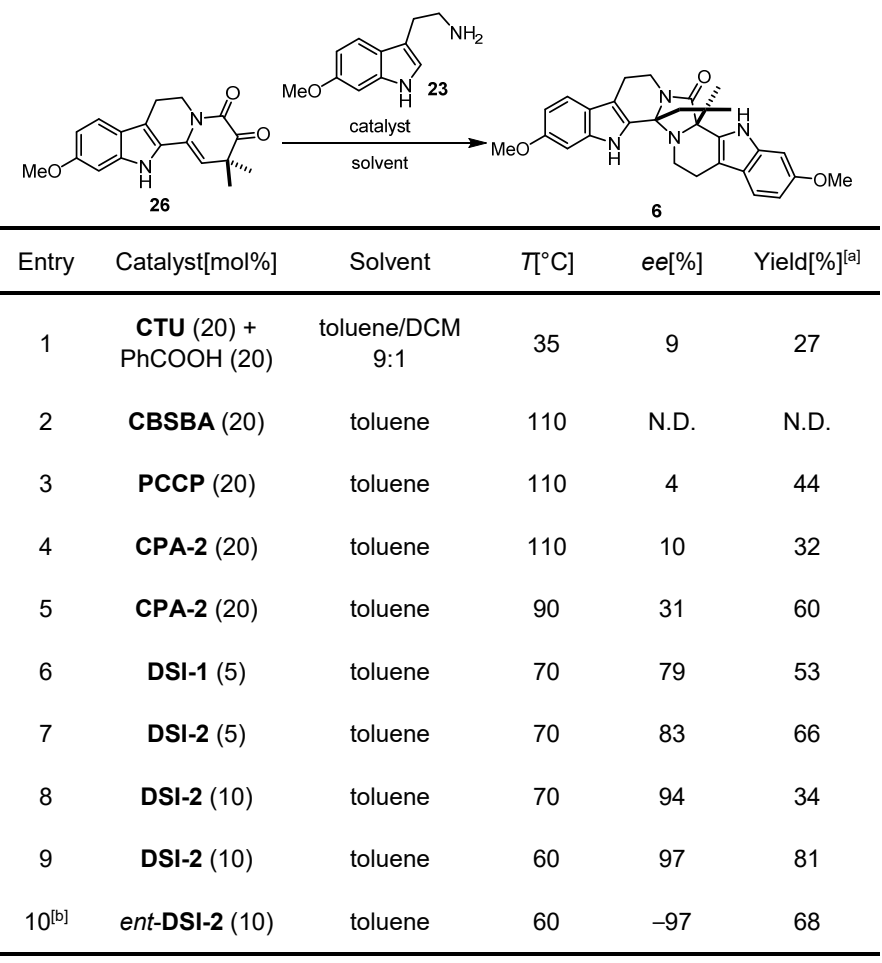

[a] isolated yields, see SI for detailed screenings. [b] using the enantiomer of the DSI-2 to obtain the enantiomer of pegamunine A.

Inspired by the great potential of total synthesis of indole alkaloids, this annulation was further extended to the synthesis of dimeric berberine alkaloid ilicifoline B (5). ${ }^{[25]}$ Using our standard reaction sequence, 8-oxopseudopalmatine (36) was obtained in 95\% yield for the annulation. Using Opatz's dimerization procedure ${ }^{[26]}$ racemic ilicifoline B was synthesized. Moreover, 8-oxopseudopalmatine (36) can be transformed into the tetracyclic protoberberine alkaloid xylopinine (37) according to the reported method. ${ }^{[27]}$

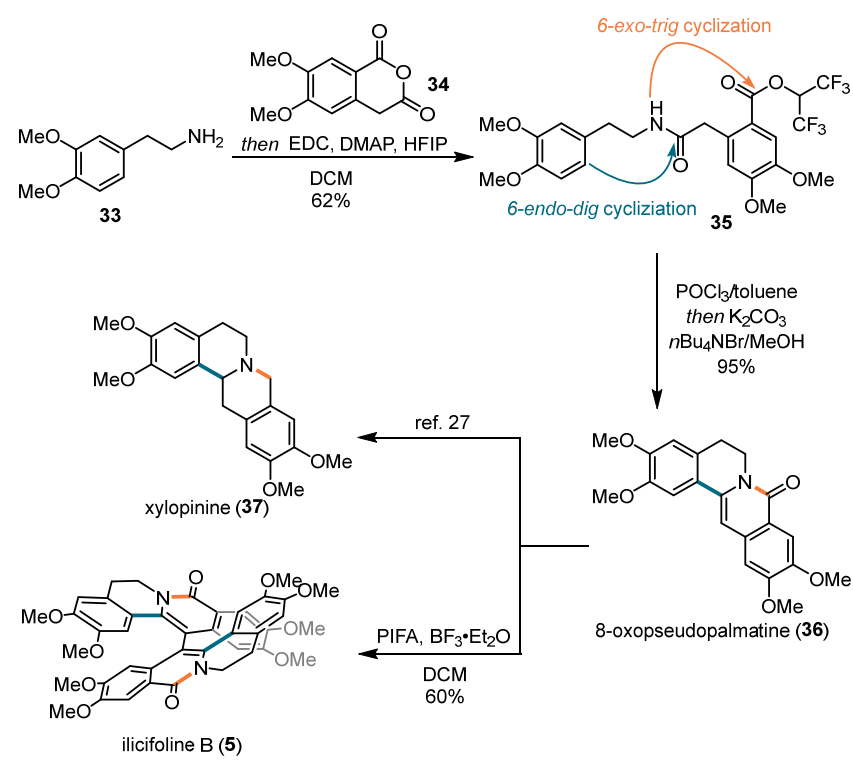

Scheme 6. Synthesis of berberine alkaloids 
In summary, we have developed an efficient method that is enabling to the rapid assembly of polycyclic scaffolds of bioactive alkaloids, through a straightforward annulation reaction featuring a complementary carbonyl activation strategy. Diverse polycyclic ring systems were accessed in good yields, enabling the total synthesis of different types of alkaloids and their analogs. Through diverging pathways, the total synthesis of five alkaloids and formal total synthesis of six alkaloids were completed. Among them, a synthesis of (+)- and (-)-peganumine $A(6)$ was achieved in a protecting-group-free sequence using a DSI catalyzed Pictet-Spengler reaction as the key step. Finally, we also applied this method to a synthesis of dimeric berberine alkaloid ilicifoline B (5).

\section{Acknowledgements}

G. He thanks the China Scholarship Council and Dahlem Research School for doctoral scholarships. We thank members and technician team in AK List (Max-Planck-Institut für Kohlenforschung) for the support of the chiral catalysts. Christiane Groneberg, Anja Peukert and Gregor Drendel (Freie Universität Berlin) are acknowledged for analytical support.

Keywords: polycyclic alkaloids $\bullet$ total synthesis $\cdot$ disulfonimide $\bullet$ organocatalysis $\cdot$ peganumine $\mathrm{A}$

[1] D. J. Newman, G. M. Cragg, J. Nat. Prod. 2020, 83, 770.

[2] a) J. Kim, H. Kim, S. B. Park, J. Am. Chem. Soc. 2014, 136 14629; b) M. Garcia-Castro, S. Zimmermann, M. G. Sankar, K. Kumar, Angew. Chem. Int. Ed. 2016, 55, 7586.

[3] a) P. Pérez-Galán, H. Waldmann, K. Kumar, Tetrahedron 2016, 72, 3647; b) Y.-C. Lee, S. Patil, C. Golz, C. Strohmann, S. Ziegler, K. Kumar, H. Waldmann, Nat. Commun. 2017, 8 , 14043 ; c) V. Němec, M. Hylsová, L. Maier, J. Flegel, S. Sievers, S. Ziegler, M. Schröder, B.-T. Berger, A. Chaikuad, B. Valčíková, S. Uldrijan, S. Drápela, K. Souček, H. Waldmann, S. Knapp, K. Paruch, Angew. Chem. Int. Ed. 2019, 58, 1062; d) A Fernando Rodrigues de Sa, J. B. Eliezer, F. Carlos Alberto Manssour, Mini-Rev. Med. Chem. 2009, 9, 782; e) T. V. Sravanthi, S. L. Manju, Eur. J. Pharm. Sci. 2016, 91, 1.

[4] T. van Dijk, J. Chris Slootweg, K. Lammertsma, Org. Biomol. Chem. 2017, 15, 10134

[5] a) A. Bischler, B. Napieralski, Ber. Dtsch. Chem. Ges. 1893 26, 1903; b) G. Fodor, J. Gal, B. A. Phillips, Angew. Chem. Int. Ed. 1972, 11, 919; Angew. Chem. 1972, 84, 947.

[6] J. V. Braun, Ber. Dtsch. Chem. Ges. 1904, 37, 3210.

[7] a) B. Ravinder, S. Rajeswar Reddy, A. Panasa Reddy, R. Bandichhor, Tetrahedron Lett. 2013, 54, 4908; b) A. J. Speziale, L. R. Smith, J. Org. Chem. 1963, 28, 1805.

[8] a) D. Kaiser, A. Bauer, M. Lemmerer, N. Maulide, Chem. Soc. Rev. 2018, 47, 7899; b) D. Kaiser, N. Maulide, J. Org. Chem. 2016, 81, 4421; c) H. Zhang, M. Riomet, A. Roller, N. Maulide, Org. Lett. 2020, 22, 2376.

[9] a) K. Lammertsma, P. V. Bharatam, J. Org. Chem. 2000, 65, 4662; b) K. Lammertsma, B. V. Prasad, J. Am. Chem. Soc. 1994, 116, 642; c) P. Pérez, A. Toro-Labbé, Theor. Chem. Acc. 2001, 105, 422

[10] a) C. A. G. N. Montalbetti, V. Falque, Tetrahedron 2005, 61, 10827; b) A. El-Faham, F. Albericio, Chem. Rev. 2011, 111 6557.

[11] K. Li, J. Ou, S. Gao, Angew. Chem. Int. Ed. 2016, 55, 14778.

[12] a) C. A. Kingsbury, D. J. Cram, J. Am. Chem. Soc. 1960, 82, 1810; b) H. J. Reich, I. L. Reich, J. M. Renga, J. Am. Chem.
Soc. 1973, 95, 5813; c) H. J. Reich, J. M. Renga, I. L. Reich, J. Am. Chem. Soc. 1975, 97, 5434; d) M. Teruaki, M. Jun-ichi, K. Hideo, Chem. Lett. 2000, 29, 1250.

[13] a) Y. Chen, A. Turlik, T. R. Newhouse, J. Am. Chem. Soc. 2016, 138, 1166; b) A. Turlik, Y. Chen, T. R. Newhouse, Synlett 2016, 27, 331

[14] a) S. Siddiqui, O. Y. Khan, S. Faizi, B. S. Siddiqui, Heterocycles 1988, 27, 1401; b) S. Siddiqui, O. Y. Khan, S Faizi, B. S. Siddiqui, Heterocycles 1989, 29, 521; c) K.-B. Wang, D.-H. Li, Y. Bao, F. Cao, W.-J. Wang, C. Lin, W. Bin, J. Bai, Y.-H. Pei, Y.-K. Jing, D. Yang, Z.-L. Li, H.-M. Hua, J. Nat. Prod. 2017, 80, 551

[15] A. Deiters, M. Pettersson, S. F. Martin, J. Org. Chem. 2006, 71, 6547.

[16] M. Rueping, C. Brinkmann, A. P. Antonchick, I. Atodiresei, Org Lett. 2010, 12, 4604

[17] W. A. da Silva, M. T. Rodrigues, N. Shankaraiah, R. B. Ferreira, C. K. Z. Andrade, R. A. Pilli, L. S. Santos, Org. Lett. 2009, 11, 3238

[18] K.-B. Wang, Y.-T. Di, Y. Bao, C.-M. Yuan, G. Chen, D.-H. Li, J. Bai, H.-P. He, X.-J. Hao, Y.-H. Pei, Y.-K. Jing, Z.-L. Li, H.-M. Hua, Org. Lett. 2014, 16, 4028.

[19] C. Piemontesi, Q. Wang, J. Zhu, J. Am. Chem. Soc. 2016, 138, 11148

[20] a) M. S. Taylor, E. N. Jacobsen, J. Am. Chem. Soc. 2004, 126, 10558; b) I. T. Raheem, P. S. Thiara, E. A. Peterson, E. N. Jacobsen, J. Am. Chem. Soc. 2007, 129, 13404; c) R. S. Klausen, E. N. Jacobsen, Org. Lett. 2009, 11, 887.

[21] a) N. J. A. Martin, B. List, J. Am. Chem. Soc. 2006, 128 13368; b) S. Mayer, B. List, Angew. Chem. Int. Ed. 2006, 45 4193; c) M. Mahlau, P. García-García, B. List, Chem. Eur. J. 2012, 18, 16283; d) M. Mahlau, B. List, Isr. J. Chem. 2012, 52, 630; e) M. Mahlau, B. List, Angew. Chem. Int. Ed. 2013, 52, 518; f) Z. Zhang, H. Y. Bae, J. Guin, C. Rabalakos, M. van Gemmeren, M. Leutzsch, M. Klussmann, B. List, Nat Commun. 2016, 7, 12478.

[22] N. Mittal, D. X. Sun, D. Seidel, Org. Lett. 2014, 16, 1012.

[23] C. D. Gheewala, B. E. Collins, T. H. Lambert, Science 2016, 351, 961.

[24] a) L. Ratjen, P. García-García, F. Lay, M. E. Beck, B. List, Angew. Chem. Int. Ed. 2011, 50, 754; b) J. Guin, C Rabalakos, B. List, Angew. Chem. Int. Ed. 2012, 51, 8859; c) Q. Wang, M. Leutzsch, M. van Gemmeren, B. List, J. Am. Chem. Soc. 2013, 135, 15334; d) L. Ratjen, M. van Gemmeren, F. Pesciaioli, B. List, Angew. Chem. Int. Ed. 2014, 53, 8765; e) Q. Wang, M. van Gemmeren, B. List, Angew. Chem. Int. Ed. 2014, 53, 13592; f) T. James, M. van Gemmeren, B. List, Chem. Rev. 2015, 115, 9388; g) V. N. Wakchaure, P. S. J. Kaib, M. Leutzsch, B. List, Angew. Chem. Int. Ed. 2015, 54, 11852; h) V. N. Wakchaure, B. List, Angew. Chem. Int. Ed. 2016, 55, 15775; i) F. Mandrelli, A. Blond, T. James, H. Kim, B. List, Angew. Chem. Int. Ed. 2019, 58, 11479 ; j) V. N. Wakchaure, C. Obradors, B. List, Synlett 2020, 31,1707

[25] V. Fajardo, C. Carcamo, B. Moreno, Heterocycles 1996, 43, 949.

[26] D. Stubba, G. Lahm, M. Geffe, J. W. Runyon, A. J. Arduengo III, T. Opatz, Angew. Chem. Int. Ed. 2015, 54, 14187

[27] J. Yu, Z. Zhang, S. Zhou, W. Zhang, R. Tong, Org. Chem. Front. 2018, 5, 242. 


\section{Entry for the Table of Contents}

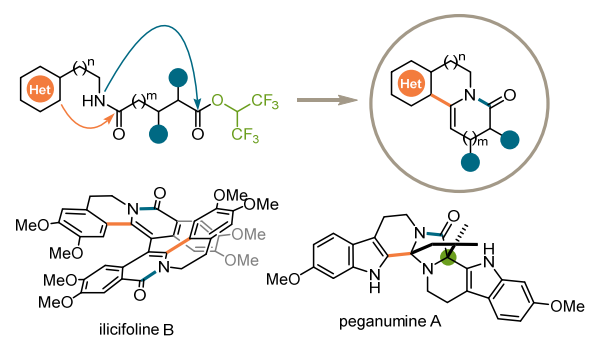

An efficient construction of polycyclic scaffolds of bioactive alkaloids via a novel annulation strategy has been developed. Among several natural products prepared, a synthesis of the dimeric berberine alkaloid ilicifoline $B$ and a protecting-group-free synthesis of $(+)$ - and (-)-peganumine A have been achieved. The latter features an asymmetric Brønsted acid catalyzed cyclization cascade.

Institute and/or researcher Twitter usernames: @ChristmannGroup @FU_Berlin @ListLaboratory @maxplanckpress 
Supporting Information for

Unified Synthesis of Polycyclic Alkaloids via Complementary Carbonyl Activation

Guoli He, Benjamin List* and Mathias Christmann* 


\section{Table of Content}

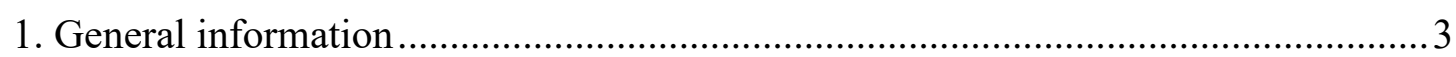

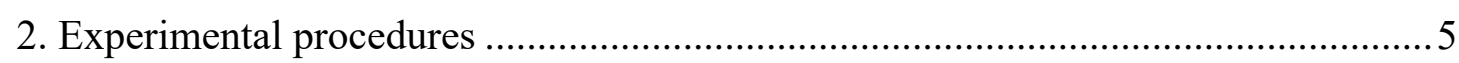

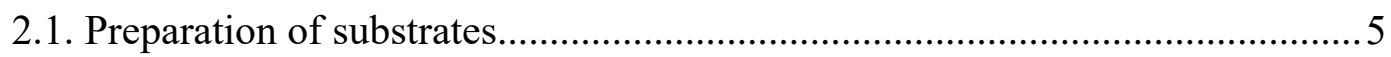

2.1.1. Substrates for the reaction optimization ........................................... 6

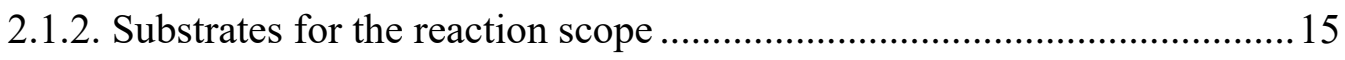

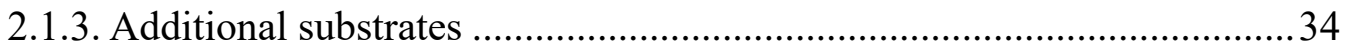

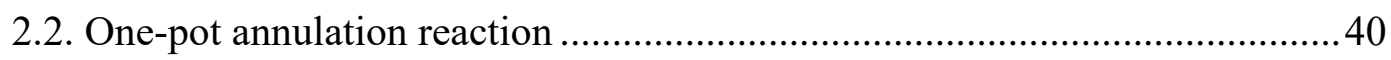

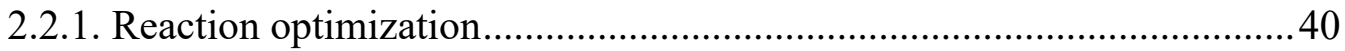

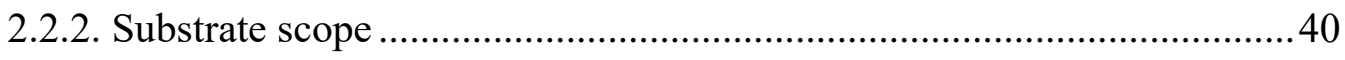

2.2.3. Detail and consideration of the annulation of the additional substrates...59

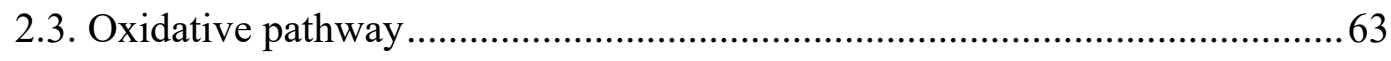

2.3.1. Natural products and analogues synthesis ...........................................63

2.3.2. Optimization of selective dehydrogenation.........................................67

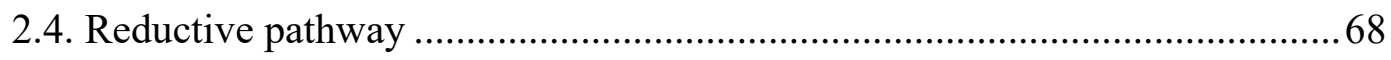

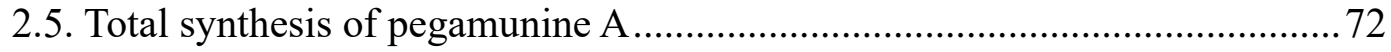

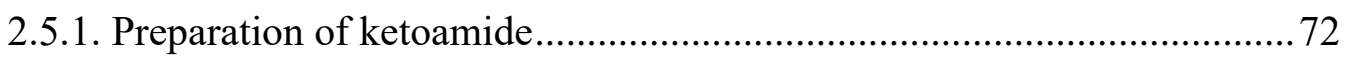

2.5.2. Racemic protection group free annulation reaction............................... 75

2.5.3. Enantioselective protection group free annulation reaction ....................76

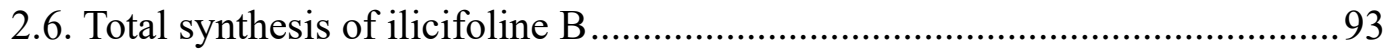

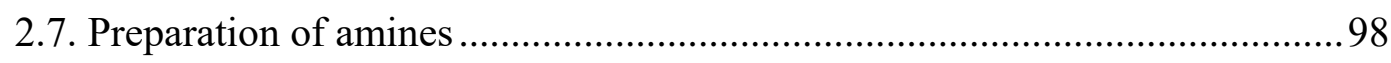

2.7.1. Commercially available amines ........................................................ 98

2.7.2. Noncommercially available amines synthesis by reported methods .......98

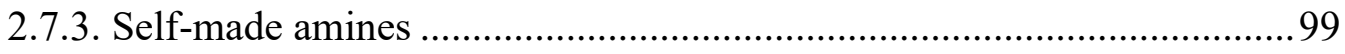

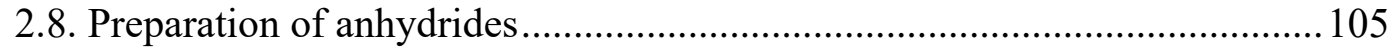

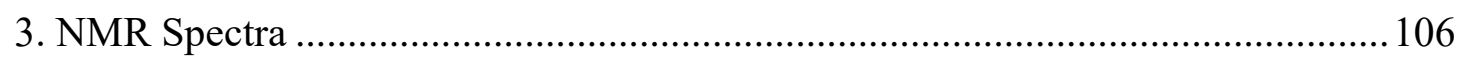




\section{General information}

Unless otherwise stated, all reactions were magnetically stirred and conducted in anhydrous solvents under argon, applying standard Schlenk techniques. Solvents and liquid reagents, as well as solutions of solid or liquid reagents were added via syringes, stainless steel cannulas through rubber septa or micropipettes in a weak stream of argon. Solid reagents were added through a weak argon counter-flow. Cooling baths were prepared in Dewar vessels, filled with ice/water $\left(0^{\circ} \mathrm{C}\right)$, or dry ice/acetone $\left(-78^{\circ} \mathrm{C}\right)$. Heated oil baths were used for reactions requiring elevated temperatures. Solvents were removed under reduced pressure at $40{ }^{\circ} \mathrm{C}$ using a rotary evaporator. All given yields are isolated yields of NMR spectroscopically pure materials, unless otherwise stated.

\section{Chemicals}

Chemicals (Abcr, Alfa Aesar, Apollo Scientific, Fisher Scientific, Fluorochem, SigmaAldrich, TCI) were purchased as reagent grade and used without further purification unless otherwise stated. Molecular sieves (MS) $4 \AA$ were dried at $150{ }^{\circ} \mathrm{C}$ under vacuum and stored in Schlenk flask.

\section{Solvents}

Solvents (toluene, tetrahydrofuran (THF), dichloromethane (DCM)) were purified with the solvent purification system Braun MB-SPS-800 (Institut für Chemie und Biochemie, Freie Universität Berlin) or dried by distillation from an appropriate drying agent and stored in Schlenk flasks under argon (Max-Planck-Institut für Kohlenforschung). Additional solvents (dimethylformamide (DMF) and methanol) were purchased from Fisher Scientific in AcroSeal $^{\odot}$-bottles under argon atmosphere with MS $4 \AA$.

\section{Thin-layer chromatography (TLC)}

TLC was performed using silica gel coated aluminum plates (ALUGRAM® Xtra SIL G/UV254, Macherey-Nagel) or pre-coated silica gel plastic sheets (Polygram SIL G/UV254, $0.20 \mathrm{~mm}$, with fluorescent indicator; Macherey-Nagel) which were visualized under irradiation with UV light $(\lambda=254)$ or vanillin stain $(500 \mathrm{mg}$ vanillin, $5 \mathrm{~mL} \mathrm{H}_{2} \mathrm{SO}_{4}$ (conc.), $10 \mathrm{~mL} \mathrm{HOAc,} 100 \mathrm{~mL} \mathrm{MeOH}$ ). 


\section{Column Chromatography}

Column chromatography was carried out using silica gel from Merck or Macherey \&

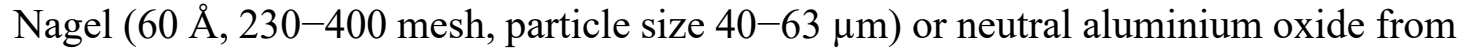
Fisher Scientific (Brockmann I, $60 \AA$, 50-200 $\mu \mathrm{m}$ ) using technical grade solvents. Fractions containing a desired substance were combined and concentrated in vacuo. Solvent mixtures (mobile phase) are reported in terms of volume ratios (v/v).

\section{Nomenclature}

Nomenclature follows the suggestions proposed by the computer program ChemDraw Professional (17.1.0.105) of PerkinElmer.

\section{Nuclear Magnetic Resonance (NMR) Spectroscopy}

${ }^{1} \mathrm{H}$ and ${ }^{13} \mathrm{C}$ NMR spectra were recorded on a JEOL ECX 400 (400 MHz), JEOL ECP $500(500 \mathrm{MHz})$, Bruker Avance 500 (500 MHz), JEOL ECZ600 S (600 MHz) and Bruker Avance $700(700 \mathrm{MHz})$ spectrometers in reported deuterated solvent. All spectra were processed with MestReNova 12.0.3. The residual deuterated solvent signal relative to tetramethylsilane (TMS) was used as the internal reference in ${ }^{1} \mathrm{H}$ NMR $\operatorname{spectra}\left(\mathrm{DMSO} \delta[\mathrm{ppm}]=2.50 ; \mathrm{CD}_{2} \mathrm{Cl}_{2} \delta[\mathrm{ppm}]=5.32 ; \mathrm{CD}_{3} \mathrm{OD} \delta[\mathrm{ppm}]=3.31 ; \mathrm{C}_{6} \mathrm{D}_{6}\right.$ $\left.\delta[\mathrm{ppm}]=7.16 ; \mathrm{CDCl}_{3} \delta[\mathrm{ppm}]=7.26\right)$. Data are reported as follows: chemical shift, multiplicity $(\mathrm{s}=$ singlet, $\mathrm{bs}=$ broad singlet, $\mathrm{d}=$ doublet, $\mathrm{t}=$ triplet, $\mathrm{q}=$ quartet, $\mathrm{m}=$ multiplet), coupling constants $(\mathrm{Hz})$ and integration. ${ }^{13} \mathrm{C}$ NMR spectra reported in ppm from tetramethylsilane (TMS) with the solvent resonance as the internal standard $\left(\mathrm{DMSO} \delta[\mathrm{ppm}]=39.5 ; \mathrm{CD}_{2} \mathrm{Cl}_{2} \delta[\mathrm{ppm}]=53.8 ; \mathrm{CD}_{3} \mathrm{OD} \delta[\mathrm{ppm}]=49.0, \mathrm{CDCl}_{3} \delta[\mathrm{ppm}]\right.$ $\left.=72.16 ; \mathrm{C}_{6} \mathrm{D}_{6} \delta[\mathrm{ppm}]=128.0\right)$.

\section{Mass Spectrometry}

High resolution mass spectrometry (HRMS) was performed on a Finnigan MAT 95 (EI), Bruker APEX III FTMS (ESI) or Agilent 6210 (ESI). The ionization method and mode of detection employed is indicated for the respective experiment and all masses are reported in atomic units per elementary charge $(\mathrm{m} / \mathrm{z})$. 


\section{IR Spectroscopy}

IR Spectra were recorded on a JASCO FT/IR-4100 spectrometer. Characteristic absorption bands are reported in wavelengths $v$ in $\mathrm{cm}^{-1}$ and were analyzed with the software Spectral Manager from JASCO.

\section{Specific Rotations}

Specific rotations $[\alpha]_{\mathrm{D}}^{T}$ were measured on a Rudolph RA Autopol IV Automatic Polarimeter or JASCO P-2000 Polarimeter at the indicated temperature with a sodium lamp (sodium D line, $\lambda=589 \mathrm{~nm}$ ). Measurements were performed in an acid resistant $50 \mathrm{~mm}$ cell (Rudolph) or $100 \mathrm{~mm}$ (JASCO) with concentrations $(\mathrm{g} / 100 \mathrm{~mL})$ reported in the corresponding solvent.

\section{High Performance Liquid Chromatography (HPLC)}

HPLC was performed on Shimadzu LC-20AD liquid chromatograph (SIL-20AC auto sampler, CMB-20A communication bus module, DGU-20A5 degasser, CTO-20AC column oven, SPD-M20A diode array detector), Shimadzu LC-20AB liquid chromatograph (SIL-20ACHT auto sampler, DGU-20A5 degasser, CTO-20AC column oven, SPD-M20A diode array detector), or Agilent Technologies 1200 series with CHIRALPAK $^{\circledR}$ IA. All solvents used were HPLC-grade solvents purchased from Sigma-Aldrich or VWR. The column employed and respective solvent mixture are indicated for each experiment.

\section{Experimental procedures}

\subsection{Preparation of substrates}

\section{General procedure A}

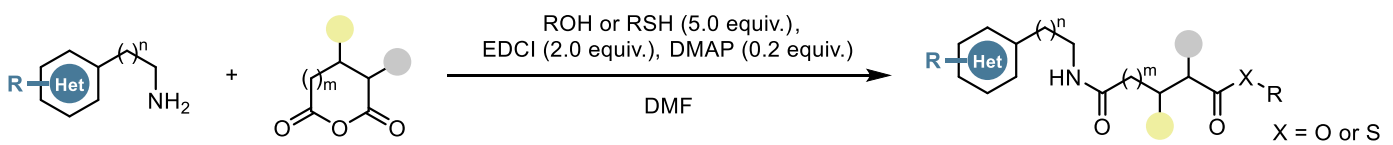

A mixture of the amine (1.0 equiv.) and the anhydride (1.0 equiv.) in DMF, was stirred overnight at room temperature. Then $N$-(3-dimethylaminopropyl)- $N$ 'ethylcarbodiimide hydrochloride (EDCI) (2.0 equiv.), 4-dimethylaminopyridine (DMAP) ( 0.2 equiv.) and corresponding alcohol (5.0 equiv.) were added to the reaction 
mixture subsequently. The resulting mixture was stirred for $24 \mathrm{~h}$ at room temperature, before the reaction mixture was diluted with $\mathrm{H}_{2} \mathrm{O}$ and EtOAc. After washing by brine for three times, the organic phase was dried over $\mathrm{MgSO}_{4}$, and concentrated under reduced pressure. The crude product was purified by silica gel column chromatography. (Note: It is not necessary to perform the reaction under argon.)

\section{General procedure B}

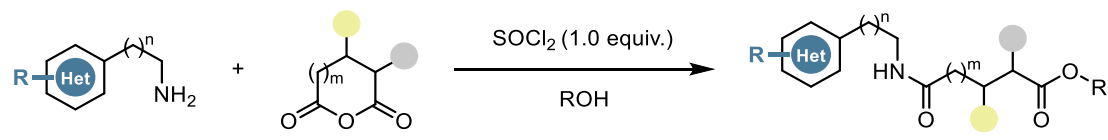

A mixture of the amine (1.0 equiv.) and the anhydride (1.0 equiv.) in corresponding alcohol was stirred overnight at room temperature. The mixture was then cooled to $0{ }^{\circ} \mathrm{C}$ and $\mathrm{SOCl}_{2}$ (1.0 equiv.) was added dropwise. Immediately after the addition, the reaction mixture was allowed to warm to room temperature and stirred for $2 \mathrm{~h}$. The reaction was quenched with $\mathrm{NaHCO}_{3}$ (sat. aq.) and extracted with DCM. The combined organic phases were washed with brine, dried over $\mathrm{MgSO}_{4}$ and concentrated under reduced pressure. The crude product was purified by silica gel column chromatography.

(Note: It is not necessary to perform the reaction under argon.)

\subsubsection{Substrates for the reaction optimization}

Methyl 5-((2-(1H-indol-3-yl)ethyl)amino)-5-oxopentanoate (8a).

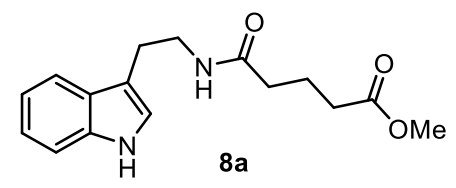

8a was prepared according to General procedure B, starting from tryptamine (1.50 g, $9.38 \mathrm{mmol}, 1.0$ equiv.) and glutaric anhydride (1.07 g, $9.38 \mathrm{mmol}, 1.0$ equiv.) in $\mathrm{MeOH}$ (30 mL). The reaction mixture was subsequently treated with $\mathrm{SOCl}_{2}(680 \mu \mathrm{L}$, $9.38 \mathrm{mmol}, 1.0$ equiv.). Purification by silica gel column chromatography (pentane/EtOAc $=3: 2)$ afforded $8 \mathbf{a}(2.39 \mathrm{~g}, 8.33 \mathrm{mmol}, 88 \%)$ as a white solid.

${ }^{1} \mathbf{H}$ NMR $\left(500 \mathrm{MHz}, \mathrm{CDCl}_{3}\right) \delta[\mathrm{ppm}]=8.51(\mathrm{~s}, 1 \mathrm{H}), 7.36(\mathrm{dt}, J=8.1,1.0 \mathrm{~Hz}, 1 \mathrm{H}), 7.36$ 
(dt, $J=8.1,1.0 \mathrm{~Hz}, 1 \mathrm{H}), 7.19$ (ddd, $J=8.2,7.0,1.2 \mathrm{~Hz}, 1 \mathrm{H}), 7.11$ (ddd, $J=8.0,7.0$, $1.0 \mathrm{~Hz}, 1 \mathrm{H}), 6.99$ (d, $J=2.3 \mathrm{~Hz}, 1 \mathrm{H}), 5.72(\mathrm{~s}, 1 \mathrm{H}), 3.64(\mathrm{~s}, 3 \mathrm{H}), 3.58(\mathrm{td}, J=6.8,5.7$ $\mathrm{Hz}, 2 \mathrm{H}), 2.95(\mathrm{td}, J=6.8,0.8 \mathrm{~Hz}, 2 \mathrm{H}), 2.33(\mathrm{t}, J=7.3 \mathrm{~Hz}, 2 \mathrm{H}), 2.14(\mathrm{t}, J=7.4 \mathrm{~Hz}, 2 \mathrm{H})$, $1.94-1.89(\mathrm{~m}, 2 \mathrm{H})$.

${ }^{13} \mathbf{C ~ N M R}\left(126 \mathrm{MHz}, \mathrm{CDCl}_{3}\right) \delta[\mathrm{ppm}]=173.8,172.3,136.6,127.4,122.3,122.2,119.4$, $118.7,112.8,111.4,51.7,39.8,35.6,33.2,25.4,20.9$.

HRMS-ESI: calcd. for $\mathrm{C}_{16} \mathrm{H}_{20} \mathrm{~N}_{2} \mathrm{O}_{3} \mathrm{Na}[\mathrm{M}+\mathrm{Na}]^{+}: 311.1366$; found: 311.1371 .

FT-IR: $v\left[\mathrm{~cm}^{-1}\right]=3394,3297,2949,1731,1647,1533,1457,1436,1226,1152,743$.

\section{4-Nitrophenyl 5-((2-(1H-indol-3-yl)ethyl)amino)-5-oxopentanoate (8b).}

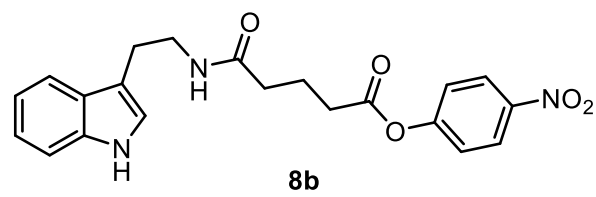

$\mathbf{8 b}$ was prepared according to General procedure A, starting from tryptamine (300 $\mathrm{mg}$, $1.87 \mathrm{mmol}, 1.0$ equiv.) and glutaric anhydride (214 mg, $1.87 \mathrm{mmol}, 1.0$ equiv.) in DMF $(10.0 \mathrm{~mL})$. The reaction mixture was subsequently treated with EDCI $(718 \mathrm{mg}$, $3.74 \mathrm{mmol}, 2.0$ equiv.), DMAP (45.8 mg, $0.374 \mathrm{mmol}, 0.2$ equiv.) and $p$-nitrophenol (1.30 g, 9.36 mmol, 5.0 equiv.). Purification by silica gel column chromatography $(\mathrm{DCM} / \mathrm{MeOH}=10: 1)$ afforded $\mathbf{8 b}(318 \mathrm{mg}, 0.806 \mathrm{mmol}, 43 \%)$ as a yellow solid.

${ }^{1} \mathbf{H}$ NMR $\left(500 \mathrm{MHz}, \mathrm{CD}_{3} \mathrm{OD}: \mathrm{CDCl}_{3}(\mathrm{v} / \mathrm{v}=1: 1)\right) \delta[\mathrm{ppm}]=8.27-8.21(\mathrm{~m}, 2 \mathrm{H}), 7.57-$ 7.53 (m, 1H), 7.35-7.31 (m, 1H), 7.29-7.25 (m, 2H), 7.09 (ddd, $J=8.2,6.9,1.2 \mathrm{~Hz}$, 1H), 7.05-6.98 (m, 2H), $3.51(\mathrm{t}, J=7.2 \mathrm{~Hz}, 2 \mathrm{H}), 2.95(\mathrm{t}, J=7.3 \mathrm{~Hz}, 2 \mathrm{H}), 2.60(\mathrm{t}, J=$ $7.4 \mathrm{~Hz}, 2 \mathrm{H}), 2.26(\mathrm{t}, J=7.3 \mathrm{~Hz}, 2 \mathrm{H}), 2.03-1.97(\mathrm{~m}, 2 \mathrm{H})$.

${ }^{13} \mathrm{C} \mathrm{NMR}\left(176 \mathrm{MHz}, \mathrm{CD}_{3} \mathrm{OD}: \mathrm{CDCl}_{3}(\mathrm{v} / \mathrm{v}=1: 1)\right) \delta[\mathrm{ppm}]=173.0,170.7,155.2,144.9$, $136.3,127.0,124.7,124.6,122.1,121.9,121.0,118.2,117.8,117.8,111.6,110.9,39.7$, $34.4,32.7,24.7,20.2$.

(Due the poor solubility of this compound, the mixture of $\mathrm{CD}_{3} \mathrm{OD}: \mathrm{CDCl}_{3}(\mathrm{v} / \mathrm{v}=1: 1$ ) was used for ${ }^{1} \mathrm{H}$ NMR and ${ }^{13} \mathrm{C}$ NMR spectroscopies.)

HRMS-ESI: calcd. for $\mathrm{C}_{21} \mathrm{H}_{21} \mathrm{~N}_{3} \mathrm{O}_{5} \mathrm{~K}[\mathrm{M}+\mathrm{K}]^{+}$: 434.1113; found: 434.1125.

FT-IR: $v\left[\mathrm{~cm}^{-1}\right]=2925,1591,1522,1336,1290,1208,1109$. 
Isopropyl 5-((2-(1H-indol-3-yl)ethyl)amino)-5-oxopentanoate (8c).

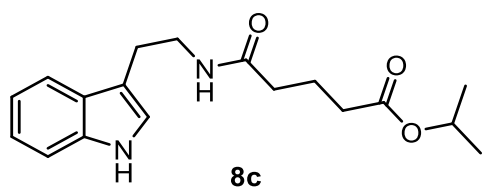

8c was prepared according to General procedure B, starting from tryptamine (500 $\mathrm{mg}$, $3.12 \mathrm{mmol}, 1.0$ equiv.) and glutaric anhydride (356 mg, $3.12 \mathrm{mmol}, 1.0$ equiv.) in $i \mathrm{PrOH}$ $(10.0 \mathrm{~mL})$. The reaction mixture was subsequently treated with $\mathrm{SOCl}_{2}(221 \mu \mathrm{L}$, $3.12 \mathrm{mmol}, 1.0$ equiv.). Purification by silica gel column chromatography (pentane/EtOAc $=3: 2)$ afforded $8 \mathbf{c}(524 \mathrm{mg}, 1.66 \mathrm{mmol}, 53 \%)$ as a white solid.

${ }^{1} \mathbf{H}$ NMR $\left(500 \mathrm{MHz}, \mathrm{CDCl}_{3}\right) \delta[\mathrm{ppm}]=8.44(\mathrm{~s}, 1 \mathrm{H}), 7.59(\mathrm{~d}, J=7.9 \mathrm{~Hz}, 1 \mathrm{H}), 7.36(\mathrm{~d}$, $J=8.1 \mathrm{~Hz}, 1 \mathrm{H}), 7.19(\mathrm{t}, J=7.6 \mathrm{~Hz}, 1 \mathrm{H}), 7.11(\mathrm{t}, J=7.5 \mathrm{~Hz}, 1 \mathrm{H}), 7.00(\mathrm{~s}, 1 \mathrm{H}), 5.69(\mathrm{~s}$, 1H), 5.06-4.90 (m, 1H), 3.59 (q, $J=6.4 \mathrm{~Hz}, 2 \mathrm{H}), 2.96(\mathrm{t}, J=6.8 \mathrm{~Hz}, 2 \mathrm{H}), 2.28(\mathrm{t}, J=$ $7.2 \mathrm{~Hz}, 2 \mathrm{H}), 2.14$ (t, $J=7.4 \mathrm{~Hz}, 2 \mathrm{H}), 1.91$ (t, $J=7.3 \mathrm{~Hz}, 2 \mathrm{H}), 1.22(\mathrm{~d}, J=1.9 \mathrm{~Hz}, 3 \mathrm{H})$, $1.20(\mathrm{~d}, J=1.8 \mathrm{~Hz}, 3 \mathrm{H})$.

${ }^{13} \mathbf{C ~ N M R}\left(126 \mathrm{MHz} \mathrm{CDCl}_{3}\right) \delta[\mathrm{ppm}]=172.9,172.4,136.6,127.4,122.3,122.2,119.5$, $118.8,112.9,111.4,67.8,39.8,35.7,33.8,25.4,21.9,21.1$.

HRMS-ESI: calcd. for $\mathrm{C}_{18} \mathrm{H}_{24} \mathrm{~N}_{2} \mathrm{O}_{3} \mathrm{Na}[\mathrm{M}+\mathrm{Na}]^{+}$: 339.1679; found: 339.1692 .

FT-IR: $v\left[\mathrm{~cm}^{-1}\right]=3287,2978,1724,1646,1531,1456,1373,1226,1105,740$.

\section{1,1,1,3,3,3-Hexafluoropropan-2-yl 5-((2-(1H-indol-3-yl)ethyl)amino)-5-oxopenta- noate (8d).}

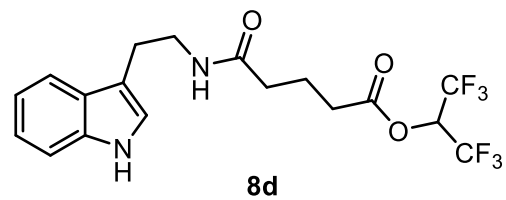

8d was prepared according to General procedure A, starting from tryptamine (3.60 g, 22.5 mmol, 1.0 equiv.) and glutaric anhydride (2.56 g, $22.5 \mathrm{mmol}, 1.0$ equiv.) in DMF $(80 \mathrm{~mL})$. The reaction mixture was subsequently treated with EDCI ( $8.59 \mathrm{~g}, 45.0 \mathrm{mmol}$, 2.0 equiv.), DMAP (550 mg, $4.50 \mathrm{mmol}, 0.2$ equiv.) and hexafluoroisopropano (HFIP) (11.9 mL, 112 mmol, 5.0 equiv.). Purification by silica gel column chromatography (pentane/EtOAc $=3: 2)$ afforded $\mathbf{8 d}(4.80 \mathrm{~g}, 11.3 \mathrm{mmol}, 50 \%)$ as a white solid. 
${ }^{1} \mathbf{H}$ NMR $\left(600 \mathrm{MHz}, \mathrm{CDCl}_{3}\right) \delta[\mathrm{ppm}]=8.21(\mathrm{~s}, 1 \mathrm{H}), 7.60(\mathrm{~d}, J=7.9 \mathrm{~Hz}, 1 \mathrm{H}), 7.37(\mathrm{dd}$, $J=8.1,1.0 \mathrm{~Hz}, 1 \mathrm{H}), 7.24-7.18(\mathrm{~m}, 1 \mathrm{H}), 7.13(\mathrm{ddd}, J=8.0,7.0,1.1 \mathrm{~Hz}, 1 \mathrm{H}), 7.01(\mathrm{~d}, J$ $=2.3 \mathrm{~Hz}, 1 \mathrm{H}), 5.79-5.73(\mathrm{~m}, 1 \mathrm{H}), 5.56(\mathrm{t}, J=5.8 \mathrm{~Hz}, 1 \mathrm{H}), 3.62-3.59(\mathrm{~m}, 2 \mathrm{H}), 2.98(\mathrm{t}$, $J=6.8 \mathrm{~Hz}, 2 \mathrm{H}), 2.54(\mathrm{t}, J=7.3 \mathrm{~Hz}, 2 \mathrm{H}), 2.15(\mathrm{t}, J=7.2 \mathrm{~Hz}, 2 \mathrm{H}), 2.01-1.97(\mathrm{~m}, 2 \mathrm{H})$. ${ }^{13} \mathbf{C}$ NMR $\left(151 \mathrm{MHz}, \mathrm{CDCl}_{3}\right) \delta[\mathrm{ppm}]=171.5,170.1,136.6,127.5,122.4,122.2,120.5$ (q, $J=282.2 \mathrm{~Hz}), 119.7,118.8,113.0,111.4,67.0-66.1(\mathrm{~m}), 39.9,34.9,32.4,25.4,20.4$. ${ }^{19}$ F NMR $\left(565 \mathrm{MHz}, \mathrm{CDCl}_{3}\right) \delta[\mathrm{ppm}]=-73.19(\mathrm{~d}, J=5.9 \mathrm{~Hz})$.

HRMS-ESI: calcd. for $\mathrm{C}_{18} \mathrm{H}_{18} \mathrm{~F}_{6} \mathrm{~N}_{2} \mathrm{O}_{3} \mathrm{Na}[\mathrm{M}+\mathrm{Na}]^{+}$: 447.1114; found: 447.1113 .

FT-IR: $v\left[\mathrm{~cm}^{-1}\right]=3418,3328,1777,1753,1633,1540,1384,1359,1286,1228,1201$, $1108,740$.

\section{2,2,2-Trifluoroethyl 5-((2-(1H-indol-3-yl)ethyl)amino)-5-oxopentanoate (8e).}

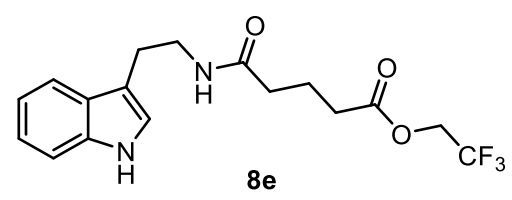

8e was prepared according to General procedure A, starting from tryptamine (285 $\mathrm{mg}$, $1.78 \mathrm{mmol}, 1.0$ equiv.) and glutaric anhydride (203 mg, $1.78 \mathrm{mmol}, 1.0$ equiv.) in DMF $(10.0 \mathrm{~mL})$. The reaction mixture was subsequently treated with EDCI $(680 \mathrm{mg}$, $3.56 \mathrm{mmol}, 2.0$ equiv.), DMAP (43.5 mg, $0.356 \mathrm{mmol}, 0.2$ equiv.) and 2,2,2trifluoroethanol (675 $\mu \mathrm{L}, 8.91 \mathrm{mmol}, 5.0$ equiv.). Purification by silica gel column chromatography (pentane/EtOAc $=3: 2)$ afforded $\mathbf{8 e}(275 \mathrm{mg}, 0.772 \mathrm{mmol}, 43 \%)$ as a white solid.

${ }^{1} \mathbf{H}$ NMR $\left(500 \mathrm{MHz}, \mathrm{CDCl}_{3}\right) \delta[\mathrm{ppm}]=8.13(\mathrm{~s}, 1 \mathrm{H}), 7.60(\mathrm{dd}, J=7.9,1.1 \mathrm{~Hz}, 1 \mathrm{H})$, $7.38(\mathrm{dt}, J=8.1,1.0 \mathrm{~Hz}, 1 \mathrm{H}), 7.21$ (ddd, $J=8.2,7.0,1.2 \mathrm{~Hz}, 1 \mathrm{H}), 7.13$ (ddd, $J=7.9$, 7.0, 1.1 Hz, 1H), 7.04 (d, $J=2.3 \mathrm{~Hz}, 1 \mathrm{H}), 5.54(\mathrm{~s}, 1 \mathrm{H}), 4.44$ (q, $J=8.5 \mathrm{~Hz}, 2 \mathrm{H}), 3.61$ (q, $J=6.5 \mathrm{~Hz}, 2 \mathrm{H}), 2.98(\mathrm{td}, J=6.7,0.8 \mathrm{~Hz}, 2 \mathrm{H}), 2.44(\mathrm{t}, J=7.2 \mathrm{~Hz}, 2 \mathrm{H}), 2.16(\mathrm{t}, J=$ $7.3 \mathrm{~Hz}, 2 \mathrm{H}), 1.99-1.93(\mathrm{~m}, 2 \mathrm{H})$.

${ }^{13} \mathbf{C ~ N M R}\left(126 \mathrm{MHz}, \mathrm{CDCl}_{3}\right) \delta[\mathrm{ppm}]=171.8,171.7,136.6,127.5,123.1$ (q, $J=277.0$ Hz), 122.4, 122.2, 119.7, 118.8, 113.1, 111.4, 60.7-59.9 (m), 39.8, 35.3, 32.8, 25.4, 20.6 . 
${ }^{19}$ F NMR $\left(471 \mathrm{MHz}, \mathrm{CDCl}_{3}\right) \delta[\mathrm{ppm}]=-73.65(\mathrm{t}, J=8.6 \mathrm{~Hz})$.

HRMS-ESI: calcd. for $\mathrm{C}_{17} \mathrm{H}_{19} \mathrm{~F}_{3} \mathrm{~N}_{2} \mathrm{O}_{3} \mathrm{Na}[\mathrm{M}+\mathrm{Na}]^{+}$: 379.1240; found: 379.1244 .

FT-IR: $v\left[\mathrm{~cm}^{-1}\right]=3388,3322,2927,1736,1630,1545,1411,1275,1261,1179,740$.

\section{1,1,1-Trifluoropropan-2-yl 5-((2-(1H-indol-3-yl)ethyl)amino)-5-oxopentanoate(8f).}

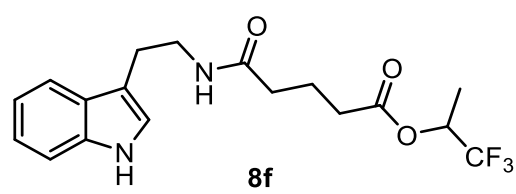

8f was prepared according to General procedure A, starting from tryptamine (150 mg, $0.938 \mathrm{mmol}, 1.0$ equiv.) and glutaric anhydride (107 mg, $0.938 \mathrm{mmol}, 1.0$ equiv.) in DMF $(5.0 \mathrm{~mL})$. The reaction mixture was subsequently treated with EDCI (358 mg, $1.88 \mathrm{mmol}, 2.0$ equiv.), DMAP (22.9 mg, $0.188 \mathrm{mmol}, 0.2$ equiv.) and 1,1,1-trifluoro2-propanol (424 $\mu \mathrm{L}, 4.69 \mathrm{mmol}, 5.0$ equiv.). Purification by silica gel column chromatography (pentane/EtOAc $=3: 2)$ afforded $\mathbf{8 f}(70.0 \mathrm{mg}, 0.189 \mathrm{mmol}, 20 \%)$ as a white solid.

${ }^{1} \mathbf{H}$ NMR $\left(500 \mathrm{MHz}, \mathrm{CD}_{3} \mathrm{OD}\right) \delta[\mathrm{ppm}]=7.55(\mathrm{dt}, J=7.9,1.0 \mathrm{~Hz}, 1 \mathrm{H}), 7.32(\mathrm{dt}, J=$ 8.2, $0.9 \mathrm{~Hz}, 1 \mathrm{H}), 7.08$ (ddd, $J=8.2,6.9,1.2 \mathrm{~Hz}, 1 \mathrm{H}), 7.04$ (s, 1H), 7.00 (ddd, $J=7.9$, 7.0, 1.0 Hz, 1H), 5.41-5.33 (m, 1H), 3.47 (t, $J=7.3 \mathrm{~Hz}, 2 \mathrm{H}), 2.98-2.88$ (m, 2H), 2.35 (t, $J=7.5 \mathrm{~Hz}, 2 \mathrm{H}), 2.18$ (t, $J=7.4 \mathrm{~Hz}, 2 \mathrm{H}), 1.90-1.84(\mathrm{~m}, 2 \mathrm{H}), 1.36$ (d, $J=6.6 \mathrm{~Hz}$, $3 \mathrm{H})$.

${ }^{13} \mathrm{C}$ NMR $\left(126 \mathrm{MHz}, \mathrm{CD}_{3} \mathrm{OD}\right) \delta[\mathrm{ppm}]=174.9,172.6,138.1,128.8,125.6(\mathrm{q}, J=$ 279.1 Hz), 123.4, 119.6, 119.3, 113.2, 112.2, 68.2-67.4 (m), 41.4, 35.8, 33.7, 26.2, 21.9, $13.7,13.7$.

${ }^{19}$ F NMR (471 MHz, $\left.\mathrm{CD}_{3} \mathrm{OD}\right) \delta[\mathrm{ppm}]=-80.2$. (Due to the resolution, the coupling was not observed.)

HRMS-ESI: calcd. for $\mathrm{C}_{18} \mathrm{H}_{21} \mathrm{~F}_{3} \mathrm{~N}_{2} \mathrm{O}_{3} \mathrm{Na}[\mathrm{M}+\mathrm{Na}]^{+}$: 393.1396; found: 393.1410 .

FT-IR: $v\left[\mathrm{~cm}^{-1}\right]=3396,3324,2932,1749,1730,1631,1542,1458,1386,1278,1187$, $1165,1118,1092,1017,741$. 
Perfluorophenyl 5-((2-(1H-indol-3-yl)ethyl)amino)-5-oxopentanoate (8g).

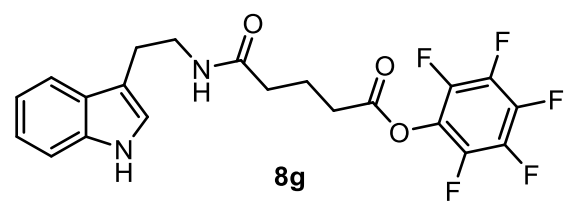

$\mathbf{8 g}$ was prepared according to General procedure A, starting from tryptamine (300 mg, $1.87 \mathrm{mmol}, 1.0$ equiv.) and glutaric anhydride (214 mg, $1.87 \mathrm{mmol}, 1.0$ equiv.) in DMF $(10.0 \mathrm{~mL})$. The reaction mixture was subsequently treated with EDCI $(715 \mathrm{mg}$, $3.74 \mathrm{mmol}, 2.0$ equiv.), DMAP (45.8 $\mathrm{mg}, 0.374 \mathrm{mmol}, 0.2$ equiv.) and pentafluorophenol (1.71 g, $9.36 \mathrm{mmol}, 5.0$ equiv.). Purification by silica gel column chromatography $(\mathrm{DCM} / \mathrm{MeOH}=10: 1)$ afforded $8 \mathrm{~g}(382 \mathrm{mg}, 0.867 \mathrm{mmol}, 46 \%)$ as a yellow solid.

${ }^{1} \mathbf{H}$ NMR $\left(500 \mathrm{MHz}, \mathrm{CD}_{3} \mathrm{OD}\right) \delta[\mathrm{ppm}]=7.54(\mathrm{dd}, J=7.9,1.0 \mathrm{~Hz}, 1 \mathrm{H}), 7.30(\mathrm{~d}, J=8.1$ Hz, 1H), 7.10-7.01 (m, 2H), 7.01-6.93 (m, 1H), 3.49 (t, $J=7.3 \mathrm{~Hz}, 2 \mathrm{H}), 2.94$ (t, $J=$ $7.3 \mathrm{~Hz}, 2 \mathrm{H}), 2.66(\mathrm{t}, J=7.4 \mathrm{~Hz}, 2 \mathrm{H}), 2.26(\mathrm{t}, J=7.4 \mathrm{~Hz}, 2 \mathrm{H}), 2.00-1.94(\mathrm{~m}, 2 \mathrm{H})$.

${ }^{13} \mathrm{C}$ NMR (126 MHz, CD $\left.3 \mathrm{OD}\right) \delta[\mathrm{ppm}]=174.7,170.4,143.4,141.5,140.2,139.6$, $138.1,128.8,126.4,123.6,123.4,122.3,119.6,119.5,119.3,113.2,112.2,41.3,35.6$, 33.0, 26.2, 21.9. (Due to the complexity of the F-C coupling, the coupling constants were not calculated.)

${ }^{19}$ F NMR (471 MHz, CD 3 OD) $\delta[\mathrm{ppm}]=-155.3(\mathrm{~d}, J=17.7 \mathrm{~Hz}),-161.3(\mathrm{t}, J=20.7$ $\mathrm{Hz}),-165.5(\mathrm{~m})$.

HRMS-ESI: calcd. for $\mathrm{C}_{21} \mathrm{H}_{17} \mathrm{~F}_{5} \mathrm{~N}_{2} \mathrm{O}_{3} \mathrm{Na}[\mathrm{M}+\mathrm{Na}]^{+}$: 463.1051 ; found: 463.1067 .

FT-IR: $v\left[\mathrm{~cm}^{-1}\right]=3289,2933,1787,1652,1457,1107,1101,743$.

3-Methylbut-2-en-1-yl 5-((2-(1H-indol-3-yl)ethyl)amino)-5-oxopentanoate $(8 \mathrm{~h})$.

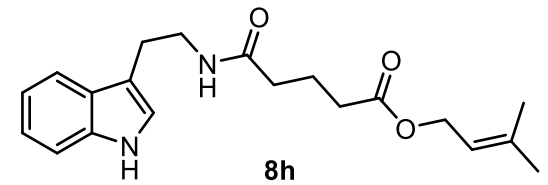

8h was prepared according to General procedure A, starting from tryptamine (509 $\mathrm{mg}$, $3.18 \mathrm{mmol}, 1.0$ equiv.) and glutaric anhydride (363 mg, $3.18 \mathrm{mmol}, 1.0$ equiv.) in DMF $(15.0 \mathrm{~mL})$. The reaction mixture was subsequently treated with EDCI (1.21 g, 
$6.36 \mathrm{mmol}, 2.0$ equiv.), DMAP (77.7 $\mathrm{mg}, 0.636 \mathrm{mmol}, 0.2$ equiv.) and 3-methyl-2buten-1-ol (1.61 mL, $15.9 \mathrm{mmol}, 5.0$ equiv.). Purification by silica gel column chromatography (pentane/EtOAc $=5: 2)$ afforded $\mathbf{8 h}(468 \mathrm{mg}, 1.37 \mathrm{mmol}, 43 \%)$ as a light yellow solid.

${ }^{1} \mathbf{H}$ NMR $\left(500 \mathrm{MHz}, \mathrm{CDCl}_{3}\right) \delta[\mathrm{ppm}]=8.20(\mathrm{~s}, 1 \mathrm{H}), 7.63-7.57(\mathrm{~m}, 1 \mathrm{H}), 7.37(\mathrm{dt}, J=$ 8.2, $0.9 \mathrm{~Hz}, 1 \mathrm{H}), 7.21(\mathrm{ddd}, J=8.2,7.0,1.2 \mathrm{~Hz}, 1 \mathrm{H}), 7.13(\mathrm{ddd}, J=8.0,7.0,1.0 \mathrm{~Hz}$, $1 \mathrm{H}), 7.03(\mathrm{~d}, J=2.4 \mathrm{~Hz}, 1 \mathrm{H}), 5.59(\mathrm{~s}, 1 \mathrm{H}), 5.34-5.30(\mathrm{~m}, 1 \mathrm{H}), 4.55(\mathrm{~d}, J=7.3 \mathrm{~Hz}, 2 \mathrm{H})$, $3.60(\mathrm{td}, J=6.7,5.7 \mathrm{~Hz}, 2 \mathrm{H}), 2.97(\mathrm{td}, J=6.8,0.9 \mathrm{~Hz}, 2 \mathrm{H}), 2.33(\mathrm{t}, J=7.2 \mathrm{~Hz}, 2 \mathrm{H})$, $2.16(\mathrm{t}, J=7.4 \mathrm{~Hz}, 2 \mathrm{H}), 1.95-1.89(\mathrm{~m}, 2 \mathrm{H}), 1.75(\mathrm{~d}, J=1.4 \mathrm{~Hz}, 3 \mathrm{H}), 1.70(\mathrm{~d}, J=1.4$ $\mathrm{Hz}, 3 \mathrm{H})$.

${ }^{13} \mathrm{C}$ NMR $\left(126 \mathrm{MHz}, \mathrm{CDCl}_{3}\right) \delta[\mathrm{ppm}]=173.4,172.3,139.2,136.6,127.4,122.24$, 122.22, 119.5, 118.8, 118.6, 112.9, 111.4, 61.5, 39.8, 35.7, 33.5, 25.8, 25.4, 21.0, 18.1.

HRMS-ESI: calcd. for $\mathrm{C}_{20} \mathrm{H}_{26} \mathrm{~N}_{2} \mathrm{O}_{3} \mathrm{Na}[\mathrm{M}+\mathrm{Na}]^{+}$: 365.1835 ; found: 365.1846 .

FT-IR: $v\left[\mathrm{~cm}^{-1}\right]=3398,3293,2969,2932,1728,1650,1537,1457,1378,1339,1229$, $1151,956,742$.

\section{Benzhydryl 5-((2-(1H-indol-3-yl)ethyl)amino)-5-oxopentanoate (8i).}

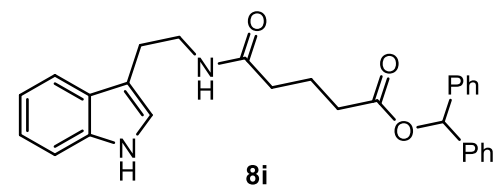

$\mathbf{8 i}$ was prepared according to General procedure A, starting from tryptamine (513 $\mathrm{mg}$, $3.20 \mathrm{mmol}, 1.0$ equiv.) and glutaric anhydride (366 mg, $3.20 \mathrm{mmol}, 1.0$ equiv.) in DMF $(18.0 \mathrm{~mL})$. The reaction mixture was subsequently treated with EDCI (1.22 g, $6.41 \mathrm{mmol}, 2.0$ equiv.), DMAP (78.3 mg, $0.641 \mathrm{mmol}, 0.2$ equiv.) and diphenylmethanol (2.95 g, $16.0 \mathrm{mmol}, 5.0$ equiv.). Purification by silica gel column chromatography (pentane/EtOAc $=5: 2)$ afforded $8 \mathbf{i}(772 \mathrm{mg}, 1.75 \mathrm{mmol}, 55 \%)$ as a white solid.

${ }^{1} \mathbf{H}$ NMR $\left(500 \mathrm{MHz}, \mathrm{CD}_{2} \mathrm{Cl}_{2}\right) \delta[\mathrm{ppm}]=8.30(\mathrm{~s}, 1 \mathrm{H}), 7.55(\mathrm{dd}, J=7.9,2.7 \mathrm{~Hz}, 1 \mathrm{H})$, 7.36-7.28 (m, 9H), 7.27-7.23 (m, 2H), 7.18-7.11 (m, 1H), $7.06(\mathrm{dd}, J=8.6,6.0 \mathrm{~Hz}$, $1 \mathrm{H}), 6.97(\mathrm{~d}, J=2.7 \mathrm{~Hz}, 1 \mathrm{H}), 6.80(\mathrm{~d}, J=3.0 \mathrm{~Hz}, 1 \mathrm{H}), 5.55(\mathrm{~s}, 1 \mathrm{H}), 3.52-3.48(\mathrm{~m}, 2 \mathrm{H})$, 
$2.89(\mathrm{td}, J=7.0,2.8 \mathrm{~Hz}, 2 \mathrm{H}), 2.47-2.37(\mathrm{~m}, 2 \mathrm{H}), 2.06(\mathrm{td}, J=7.5,2.8 \mathrm{~Hz}, 2 \mathrm{H}), 1.95-$ $1.84(\mathrm{~m}, 2 \mathrm{H})$.

${ }^{13} \mathrm{C}$ NMR $\left(126 \mathrm{MHz}, \mathrm{CD}_{2} \mathrm{Cl}_{2}\right) \delta[\mathrm{ppm}]=172.2,171.8,140.6,136.5,128.6,127.9,127.4$, 126.9, 122.2, 122.0, 119.3, 118.6, 112.9, 111.3, 76.9, 39.7, 35.4, 33.6, 25.4, 21.0.

HRMS-ESI: calcd. for $\mathrm{C}_{28} \mathrm{H}_{28} \mathrm{~N}_{2} \mathrm{O}_{3} \mathrm{Na}[\mathrm{M}+\mathrm{Na}]^{+}$: 463.1992; found: 463.2008.

FT-IR: $v\left[\mathrm{~cm}^{-1}\right]=3407,3297,2930,1733,1651,1529,1455,1231,1148,743$.

\section{Cyclohexyl 5-((2-(1H-indol-3-yl)ethyl)amino)-5-oxopentanoate (8j).}

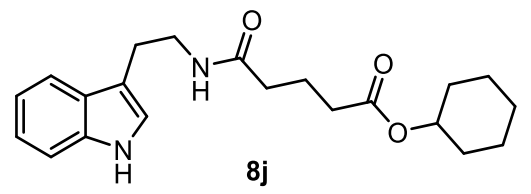

$\mathbf{8 j}$ was prepared according to General procedure A, starting from tryptamine (509 $\mathrm{mg}$, $3.18 \mathrm{mmol}, 1.0$ equiv.) and glutaric anhydride (363 mg, $3.18 \mathrm{mmol}, 1.0$ equiv.) in DMF $(18.0 \mathrm{~mL})$. The reaction mixture was subsequently treated with EDCI (1.21 g, $6.36 \mathrm{mmol}, 2.0$ equiv.), DMAP (77.7 mg, $0.636 \mathrm{mmol}, 0.2$ equiv.) and cyclohexanol (1.65 mL, $15.9 \mathrm{mmol}, 5.0$ equiv.). Purification by silica gel column chromatography (pentane/EtOAc = 3:2) afforded 8j (362 mg, $1.02 \mathrm{mmol}, 32 \%$ ) as a white solid.

${ }^{1} \mathbf{H}$ NMR $\left(700 \mathrm{MHz}, \mathrm{CDCl}_{3}\right) \delta[\mathrm{ppm}]=8.28(\mathrm{~s}, 1 \mathrm{H}), 7.59(\mathrm{dt}, J=7.8,1.0 \mathrm{~Hz}, 1 \mathrm{H}), 7.37$ $(\mathrm{dt}, J=8.1,0.9 \mathrm{~Hz}, 1 \mathrm{H}), 7.20(\mathrm{ddd}, J=8.2,7.0,1.2 \mathrm{~Hz}, 1 \mathrm{H}), 7.12(\mathrm{ddd}, J=8.0,7.0$, $1.0 \mathrm{~Hz}, 1 \mathrm{H}), 7.03-7.00(\mathrm{~m}, 1 \mathrm{H}), 5.65(\mathrm{~s}, 1 \mathrm{H}), 3.60(\mathrm{td}, J=6.8,5.8 \mathrm{~Hz}, 2 \mathrm{H}), 3.49(\mathrm{td}, J$ $=10.3,4.0 \mathrm{~Hz}, 1 \mathrm{H}), 2.97(\mathrm{td}, J=6.8,0.9 \mathrm{~Hz}, 2 \mathrm{H}), 2.52(\mathrm{t}, J=7.2 \mathrm{~Hz}, 2 \mathrm{H}), 2.14(\mathrm{t}, J=$ $7.4 \mathrm{~Hz}, 2 \mathrm{H}), 1.96-1.92(\mathrm{~m}, 2 \mathrm{H}), 1.91-1.85(\mathrm{~m}, 2 \mathrm{H}), 1.72-1.65(\mathrm{~m}, 2 \mathrm{H}), 1.62-1.55(\mathrm{~m}$, 1H), 1.45-1.35 (m, 4H), 1.29-1.23 (m, 1H).

${ }^{13} \mathrm{C}$ NMR $\left(126 \mathrm{MHz}, \mathrm{CDCl}_{3}\right) \delta[\mathrm{ppm}]=172.8,172.4,136.6,127.4,122.24,122.17$, $119.5,118.7,112.8,111.4,72.8,39.9,35.7,33.9,31.7,25.4,23.8,21.2$. (One $\mathrm{sp}^{3}$ carbon is missing, probably due to the resonance overlap.)

HRMS-ESI: calcd. for $\mathrm{C}_{21} \mathrm{H}_{28} \mathrm{~N}_{2} \mathrm{O}_{3} \mathrm{Na}[\mathrm{M}+\mathrm{Na}]^{+}$: 379.1992; found: 379.2006.

FT-IR: $v\left[\mathrm{~cm}^{-1}\right]=3357,3325,2948,2920,2853,1707,1635,1539,1386,1189,1011$, $748,727$. 
Cyclohexyl 5-((2-(1H-indol-3-yl)ethyl)amino)-5-oxopentanethioate (8k).

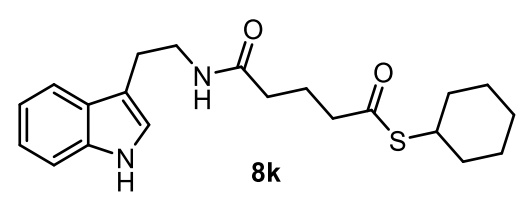

8k was prepared according to General procedure A, starting from tryptamine (506 mg, $3.16 \mathrm{mmol}, 1.0$ equiv.) and glutaric anhydride (360 mg, $3.16 \mathrm{mmol}, 1.0$ equiv.) in DMF $(18.0 \mathrm{~mL})$. The reaction mixture was subsequently treated with EDCI (1.21 g, $6.36 \mathrm{mmol}, 2.0$ equiv.), DMAP (77.2 mg, $0.632 \mathrm{mmol}, 0.2$ equiv.) and cyclohexanethiol (1.93 mL, $15.8 \mathrm{mmol}, 5.0$ equiv.). Purification by silica gel column chromatography (pentane/EtOAc $=3: 2)$ afforded $\mathbf{8 k}(401 \mathrm{mg}, 1.08 \mathrm{mmol}, 34 \%)$ as a white solid.

${ }^{1} \mathbf{H}$ NMR $\left(500 \mathrm{MHz}, \mathrm{CDCl}_{3}\right) \delta[\mathrm{ppm}]=8.23(\mathrm{~s}, 1 \mathrm{H}), 7.62(\mathrm{dd}, J=7.9,1.0 \mathrm{~Hz}, 1 \mathrm{H})$, 7.39 (dt, $J=8.1,1.0 \mathrm{~Hz}, 1 \mathrm{H}), 7.23$ (ddd, $J=8.2,7.0,1.2 \mathrm{~Hz}, 1 \mathrm{H}), 7.15$ (ddd, $J=8.0$, 7.0, 1.1 Hz, 1H), $7.05(\mathrm{~d}, J=2.3 \mathrm{~Hz}, 1 \mathrm{H}), 5.62(\mathrm{~s}, 1 \mathrm{H}), 3.62(\mathrm{td}, J=6.8,5.7 \mathrm{~Hz}, 2 \mathrm{H})$, 3.51 (ddt, $J=10.3,6.2,3.6 \mathrm{~Hz}, 1 \mathrm{H}), 2.99(\mathrm{td}, J=6.8,0.8 \mathrm{~Hz}, 2 \mathrm{H}), 2.55(\mathrm{t}, J=7.2 \mathrm{~Hz}$, 2H), $2.16(\mathrm{t}, J=7.4 \mathrm{~Hz}, 2 \mathrm{H}), 1.99-1.94(\mathrm{~m}, 2 \mathrm{H}), 1.93-1.87(\mathrm{~m}, 2 \mathrm{H}), 1.75-1.68(\mathrm{~m}, 2 \mathrm{H})$, 1.65-1.56 (m, 1H), 1.49-1.36 (m, 4H), 1.34-1.23 (m, 1H).

${ }^{13} \mathbf{C ~ N M R}\left(176 \mathrm{MHz}, \mathrm{CDCl}_{3}\right) \delta[\mathrm{ppm}]=199.2,172.1,136.6,127.4,122.3,122.2,119.6$, 118.8, 113.0, 111.4, 43.1, 42.4, 39.8, 35.4, 33.1, 26.0, 25.6, 25.5, 21.7.

HRMS-ESI: calcd. for $\mathrm{C}_{21} \mathrm{H}_{28} \mathrm{~N}_{2} \mathrm{O}_{2} \mathrm{SNa}[\mathrm{M}+\mathrm{Na}]^{+}: 395.1763$; found: 395.1782 .

FT-IR: $v\left[\mathrm{~cm}^{-1}\right]=3399,3285,2929,2852,1652,1530,1448,1341,998,741$.

\section{Tert-butyl 5-((2-(1H-indol-3-yl)ethyl)amino)-5-oxopentanoate (8I).}

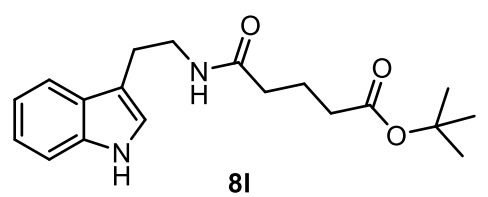

$\mathbf{8 1}$ was prepared according to General procedure A, starting from tryptamine (150 mg, $0.938 \mathrm{mmol}, 1.0$ equiv.) and glutaric anhydride (107 mg, $0.938 \mathrm{mmol}, 1.0$ equiv.) in DMF $(5.0 \mathrm{~mL})$. The reaction mixture was subsequently treated with EDCI (358 mg, $1.88 \mathrm{mmol}, 2.0$ equiv.), DMAP (22.9 mg, $0.188 \mathrm{mmol}, 0.2$ equiv.) and $t \mathrm{BuOH}$ (445 $\mu \mathrm{L}$, 4.69 mmol, 5.0 equiv.). Purification by silica gel column chromatography (pentane/EtOAc $=3: 2)$ afforded $\mathbf{8 1}(21.0 \mathrm{mg}, 636 \mu \mathrm{mol}, 7 \%)$ as a white solid. 
${ }^{1} \mathbf{H}$ NMR $\left(600 \mathrm{MHz} \mathrm{CDCl}_{3}\right) \delta[\mathrm{ppm}]=8.28(\mathrm{~s}, 1 \mathrm{H}), 7.63-7.58(\mathrm{~m}, 1 \mathrm{H}), 7.37(\mathrm{dd}, J=$ 8.1, $1.0 \mathrm{~Hz}, 1 \mathrm{H}), 7.20(\mathrm{ddd}, J=8.1,7.1,1.2 \mathrm{~Hz}, 1 \mathrm{H}), 7.12(\mathrm{ddd}, J=8.0,7.0,1.0 \mathrm{~Hz}$, 1H), 7.04-7.00 (m, 1H), $5.65(\mathrm{~s}, 1 \mathrm{H}), 3.64-3.56(\mathrm{~m}, 2 \mathrm{H}), 2.97(\mathrm{t}, J=6.8 \mathrm{~Hz}, 2 \mathrm{H}), 2.23$ (t, $J=7.2 \mathrm{~Hz}, 2 \mathrm{H}), 2.14$ (t, $J=7.4 \mathrm{~Hz}, 2 \mathrm{H}), 1.90-1.86(\mathrm{~m}, 2 \mathrm{H}), 1.43(\mathrm{~s}, 9 \mathrm{H})$.

${ }^{13} \mathbf{C ~ N M R}\left(151 \mathrm{MHz}, \mathrm{CDCl}_{3}\right) \delta[\mathrm{ppm}]=172.8,172.4,136.6,127.4,122.3,122.2,119.6$, $118.8,113.0,111.4,80.5,39.8,35.8,34.8,28.2,25.5,21.2$.

HRMS-ESI: calcd. for $\mathrm{C}_{19} \mathrm{H}_{26} \mathrm{~N}_{2} \mathrm{O}_{3} \mathrm{Na}[\mathrm{M}+\mathrm{Na}]^{+}: 353.1835$; found: 353.1852 .

FT-IR: $v\left[\mathrm{~cm}^{-1}\right]=3398,3296,2965,2930,1702,1649,1531,1457,1367,1236,1164$, 1143,741 .

\subsubsection{Substrates for the reaction scope}

\section{1,1,1,3,3,3-Hexafluoropropan-2-yl 5-((2-(5-methoxy-1H-indol-3-yl)ethyl)amino)-} 5-oxopentanoate (11a).

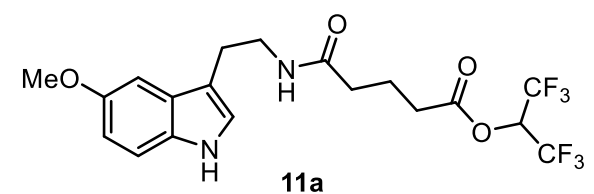

11a was prepared according to General procedure A, starting from 5methoxytryptamine (200 mg, $1.05 \mathrm{mmol}, 1.0$ equiv.) and glutaric anhydride (120 mg, $1.05 \mathrm{mmol}, 1.0$ equiv.) in DMF $(5.0 \mathrm{~mL})$. The reaction mixture was subsequently treated with EDCI (402 mg, 2.10 mmol, 2.0 equiv.), DMAP (25.7 mg, $0.211 \mathrm{mmol}$, 0.2 equiv.) and HFIP (556 $\mu \mathrm{L}, 5.26 \mathrm{mmol}, 5.0$ equiv.). Purification by silica gel column chromatography (pentane/EtOAc $=3: 2)$ afforded $11 \mathrm{a}(270 \mathrm{mg}, 0.595 \mathrm{mmol}, 56 \%)$ as a white solid.

${ }^{1} \mathbf{H}$ NMR $\left(500 \mathrm{MHz}, \mathrm{CDCl}_{3}\right) \delta[\mathrm{ppm}]=8.15(\mathrm{~s}, 1 \mathrm{H}), 7.28-7.24(\mathrm{~m}, 1 \mathrm{H}), 7.03(\mathrm{~d}, J=$ $2.4 \mathrm{~Hz}, 1 \mathrm{H}), 7.00$ (t, $J=2.4 \mathrm{~Hz}, 1 \mathrm{H}), 6.88(\mathrm{dd}, J=8.8,2.4 \mathrm{~Hz}, 1 \mathrm{H}), 5.82-5.71(\mathrm{~m}, 1 \mathrm{H})$, $5.60(\mathrm{~s}, 1 \mathrm{H}), 3.86(\mathrm{~s}, 3 \mathrm{H}), 3.65-3.57(\mathrm{~m}, 2 \mathrm{H}), 2.97-2.91(\mathrm{~m}, 2 \mathrm{H}), 2.56(\mathrm{td}, J=7.3,1.0$ $\mathrm{Hz}, 2 \mathrm{H}), 2.17$ (t, $J=7.2 \mathrm{~Hz}, 2 \mathrm{H}), 2.03-1.97(\mathrm{~m}, 2 \mathrm{H})$.

${ }^{13} \mathbf{C ~ N M R}\left(126 \mathrm{MHz}, \mathrm{CDCl}_{3}\right) \delta[\mathrm{ppm}]=171.6,170.1,154.2,131.7,127.8,123.0,120.5$ $(\mathrm{q}, J=285.6 \mathrm{~Hz}), 112.7,112.5,112.2,100.7,66.8-66.2(\mathrm{~m}), 56.1,39.7,34.9,32.4,25.4$, 20.4 . 
${ }^{19}$ F NMR $\left(565 \mathrm{MHz}, \mathrm{CDCl}_{3}\right) \delta[\mathrm{ppm}]=-73.18(\mathrm{~d}, J=5.8 \mathrm{~Hz})$.

HRMS-ESI: calcd. for $\mathrm{C}_{19} \mathrm{H}_{21} \mathrm{~F}_{6} \mathrm{~N}_{2} \mathrm{O}_{4}[\mathrm{M}+\mathrm{H}]^{+}$: 455.1400; found: 455.1408 .

FT-IR: $v\left[\mathrm{~cm}^{-1}\right]=3432,3325,2928,1779,1759,1635,1541,1487,1218,1195,1108$, $927,794$.

\section{1,1,1,3,3,3-Hexafluoropropan-2-yl 5-((2-(6-methoxy-1H-indol-3-yl)ethyl)amino)-} 5-oxopentanoate (11b).

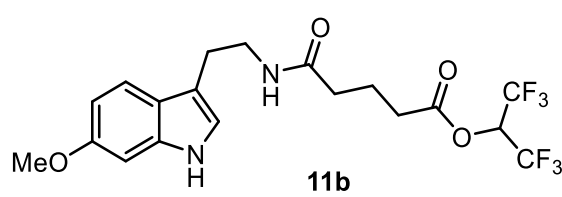

11b was prepared according to General procedure A, starting from 6methoxytryptamine (100 mg, $0.526 \mathrm{mmol}, 1.0$ equiv.) and glutaric anhydride (60.0 mg, $0.526 \mathrm{mmol}, 1.0$ equiv.) in DMF (3.0 mL). The reaction mixture was subsequently treated with EDCI (201 mg, $1.05 \mathrm{mmol}, 2.0$ equiv.), DMAP (12.9 mg, $0.105 \mathrm{mmol}$, 0.2 equiv.) and HFIP (278 $\mu \mathrm{L}, 2.63 \mathrm{mmol}, 5.0$ equiv.). Purification by silica gel column chromatography (pentane/EtOAc $=3: 2)$ afforded $11 \mathbf{b}(100 \mathrm{mg}, 0.220 \mathrm{mmol}, 42 \%)$ as a white solid.

${ }^{1} \mathbf{H}$ NMR $\left(500 \mathrm{MHz}, \mathrm{CD}_{2} \mathrm{Cl}_{2}\right) \delta[\mathrm{ppm}]=8.14(\mathrm{~s}, 1 \mathrm{H}), 7.44(\mathrm{~d}, J=8.6 \mathrm{~Hz}, 1 \mathrm{H}), 6.93$ $(\mathrm{dd}, J=2.2,1.1 \mathrm{~Hz}, 1 \mathrm{H}), 6.87(\mathrm{~d}, J=2.3 \mathrm{~Hz}, 1 \mathrm{H}), 6.75(\mathrm{dd}, J=8.7,2.3 \mathrm{~Hz}, 1 \mathrm{H}), 5.86-$ $5.79(\mathrm{~m}, 1 \mathrm{H}), 5.59(\mathrm{~s}, 1 \mathrm{H}), 3.82(\mathrm{~s}, 3 \mathrm{H}), 3.54(\mathrm{td}, J=6.9,5.8 \mathrm{~Hz}, 2 \mathrm{H}), 2.90(\mathrm{td}, J=6.9$, $0.9 \mathrm{~Hz}, 2 \mathrm{H}), 2.56$ (t, $J=7.4 \mathrm{~Hz}, 2 \mathrm{H}), 2.15$ (t, $J=7.3 \mathrm{~Hz}, 2 \mathrm{H}), 2.00-1.90(\mathrm{~m}, 2 \mathrm{H})$.

${ }^{13} \mathbf{C ~ N M R}\left(126 \mathrm{MHz}, \mathrm{CD}_{2} \mathrm{Cl}_{2}\right) \delta[\mathrm{ppm}]=171.5,170.5,157.1,137.6,122.2,121.2,120.9$ (q, $J=282.4 \mathrm{~Hz}), 119.6,113.3,109.8,94.9,67.3-66.2(\mathrm{~m}), 55.9,40.1,35.1,32.7,25.8$, 20.7 .

${ }^{19}$ F NMR $\left(471 \mathrm{MHz}, \mathrm{CD}_{2} \mathrm{Cl}_{2}\right) \delta[\mathrm{ppm}]=-73.66$. (Due to the resolution, the coupling was not observed.)

HRMS-ESI: calcd. for $\mathrm{C}_{19} \mathrm{H}_{20} \mathrm{~F}_{6} \mathrm{~N}_{2} \mathrm{O}_{4} \mathrm{Na}[\mathrm{M}+\mathrm{Na}]^{+}: 477.1219$ found: 477.1222 .

FT-IR: $v\left[\mathrm{~cm}^{-1}\right]=3302,2965,2937,1764,1635,1384,1281,1257,1107,824$. 


\section{1,1,1,3,3,3-Hexafluoropropan-2-yl $\quad 5-((2-(6-b r o m o-1 H$-indol-3-yl)ethyl)amino)-5-}

oxopentanoate (11c).

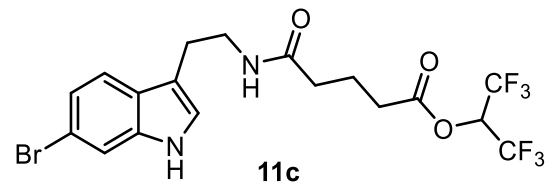

11c was prepared according to General procedure A, starting from 6-bromotryptamine (100 mg, $0.420 \mathrm{mmol}, 1.0$ equiv.) and glutaric anhydride (47.9 mg, $0.420 \mathrm{mmol}$, 1.0 equiv.) in DMF (3.0 mL). The reaction mixture was subsequently treated with EDCI (160 mg, 0.840 mmol, 2.0 equiv.), DMAP (10.3 mg, $84.0 \mu$ mol, 0.2 equiv.) and HFIP (222 $\mu \mathrm{L}, 2.10 \mathrm{mmol}, 5.0$ equiv.). Purification by silica gel column chromatography (pentane/EtOAc $=3: 2)$ afforded $11 \mathrm{c}(120 \mathrm{mg}, 0.239 \mathrm{mmol}, 57 \%)$ as a white solid.

${ }^{1} \mathbf{H}$ NMR $\left(700 \mathrm{MHz}, \mathrm{CDCl}_{3}\right) \delta[\mathrm{ppm}]=8.23(\mathrm{~s}, 1 \mathrm{H}), 7.52(\mathrm{dd}, J=1.7,0.6 \mathrm{~Hz}, 1 \mathrm{H})$, $7.44(\mathrm{dd}, J=8.4,0.7 \mathrm{~Hz}, 1 \mathrm{H}), 7.22(\mathrm{dd}, J=8.4,1.7 \mathrm{~Hz}, 1 \mathrm{H}), 7.00(\mathrm{dd}, J=2.3,0.9 \mathrm{~Hz}$, 1H), 5.77-5.74 (m, 1H), $5.53(\mathrm{~d}, J=6.2 \mathrm{~Hz}, 1 \mathrm{H}), 3.58(\mathrm{td}, J=6.9,5.8 \mathrm{~Hz}, 2 \mathrm{H}), 2.94$ (td, $J=6.9,0.9 \mathrm{~Hz}, 2 \mathrm{H}), 2.56(\mathrm{t}, J=7.3 \mathrm{~Hz}, 2 \mathrm{H}), 2.18(\mathrm{t}, J=7.3 \mathrm{~Hz}, 2 \mathrm{H}), 2.02-1.98$ $(\mathrm{m}, 2 \mathrm{H})$.

${ }^{13} \mathbf{C ~ N M R}\left(176 \mathrm{MHz}, \mathrm{CDCl}_{3}\right) \delta[\mathrm{ppm}]=171.6,170.0,137.3,126.4,123.0,122.7,121.3$, $120.5(\mathrm{q}, J=281.7 \mathrm{~Hz}), 120.0,116.0,114.4,113.3,66.9-66.1(\mathrm{~m}), 39.9,34.9,32.4$, $25.4,20.4$

${ }^{19}$ F NMR $\left(471 \mathrm{MHz}, \mathrm{CDCl}_{3}\right) \delta[\mathrm{ppm}]=-73.17(\mathrm{~d}, J=6.4 \mathrm{~Hz})$.

HRMS-ESI: calcd. for $\mathrm{C}_{18} \mathrm{H}_{17} \mathrm{BrF}_{6} \mathrm{~N}_{2} \mathrm{O}_{3} \mathrm{Na}$ [M + Na] $]^{+}$: 525.0219; found: 525.0244.

FT-IR: $v\left[\mathrm{~cm}^{-1}\right]$ 3396, 3326, 2930, 1779, 1749, 1638, 1385, 1287, 1231, 1201, 1110 , $803,746$.

\section{1,1,1,3,3,3-Hexafluoropropan-2-yl 5-((2-(6-fluoro- $1 H$-indol-3-yl)ethyl)amino)-5- oxopentanoate (11d).}

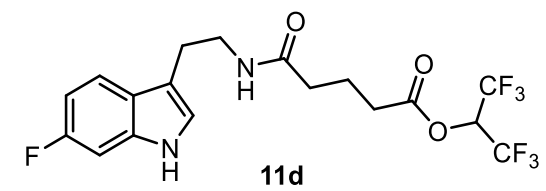

11d was prepared according to General procedure A, starting from 6-fluorotryptamine 
(260 mg, $1.46 \mathrm{mmol}, 1.0$ equiv.) and glutaric anhydride (166 mg, $1.46 \mathrm{mmol}, 1.0$ equiv.) in DMF (6.0 mL). The reaction mixture was subsequently treated with EDCI (558 mg, $2.92 \mathrm{mmol}, 2.0$ equiv.), DMAP (35.7 mg, $0.292 \mathrm{mmol}, 0.2$ equiv.) and HFIP (772 $\mu \mathrm{L}$, 7.30 mmol, 5.0 equiv.). Purification by silica gel column chromatography (pentane/EtOAc $=3: 2)$ afforded $11 \mathrm{~d}(350 \mathrm{mg}, 0.792 \mathrm{mmol}, 54 \%)$ as a white solid.

${ }^{1} \mathbf{H}$ NMR $\left(400 \mathrm{MHz}, \mathrm{CDCl}_{3}\right) \delta[\mathrm{ppm}]=8.07(\mathrm{~s}, 1 \mathrm{H}), 7.50(\mathrm{dd}, J=8.7,5.3 \mathrm{~Hz}, 1 \mathrm{H})$, $7.05(\mathrm{dd}, J=9.7,2.3 \mathrm{~Hz}, 1 \mathrm{H}), 7.01(\mathrm{~s}, 1 \mathrm{H}), 6.90(\mathrm{td}, J=9.1,2.3 \mathrm{~Hz}, 1 \mathrm{H}), 5.78-5.72$ $(\mathrm{m}, 1 \mathrm{H}), 5.51(\mathrm{~s}, 1 \mathrm{H}), 3.59(\mathrm{q}, J=6.5 \mathrm{~Hz}, 2 \mathrm{H}), 2.95(\mathrm{t}, J=6.8 \mathrm{~Hz}, 2 \mathrm{H}), 2.56(\mathrm{t}, J=7.2$ $\mathrm{Hz}, 2 \mathrm{H}), 2.18(\mathrm{t}, J=7.2 \mathrm{~Hz}, 2 \mathrm{H}), 2.04-1.97(\mathrm{~m}, 2 \mathrm{H})$.

${ }^{13} \mathrm{C}$ NMR $\left(176 \mathrm{MHz}, \mathrm{CDCl}_{3}\right) \delta[\mathrm{ppm}]=171.5,170.1,160.3(\mathrm{~d}, J=238.1 \mathrm{~Hz}), 136.5$ $(\mathrm{d}, J=12.6 \mathrm{~Hz}), 124.1,122.3(\mathrm{~d}, J=2.9 \mathrm{~Hz}), 120.5$ (d, $J=278.8 \mathrm{~Hz}), 119.6$ (d, $J=$ $10.1 \mathrm{~Hz}), 113.3,108.6(\mathrm{~d}, J=24.5 \mathrm{~Hz}), 97.7$ (d, $J=25.6 \mathrm{~Hz}), 66.9-66.1$ (m), 39.8, 34.9, $32.4,25.5,20.4$.

${ }^{19}$ F NMR $\left(376 \mathrm{MHz}, \mathrm{CDCl}_{3}\right) \delta[\mathrm{ppm}]=-73.17(\mathrm{~d}, J=10.2 \mathrm{~Hz}),(-120.71)-(-120.78)$ (m).

HRMS-ESI: calcd. for $\mathrm{C}_{18} \mathrm{H}_{18} \mathrm{~F}_{7} \mathrm{~N}_{2} \mathrm{O}_{3}\left[\mathrm{M}+\mathrm{H}^{+}\right.$: 443.1200 ; found: 443.1217 .

FT-IR: $v\left[\mathrm{~cm}^{-1}\right]=3411,3397,3277,1778,1653,1543,1522,1290,1239,1199,1110$.

\section{1,1,1,3,3,3-Hexafluoropropan-2-yl 5-((2-(1H-indol-3-yl)ethyl)amino)-3-methyl-5- oxopentanoate (11e).}

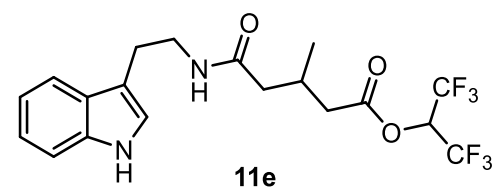

11e was prepared according to General procedure A, starting from tryptamine ( $2.00 \mathrm{~g}$, $12.5 \mathrm{mmol}, 1.0$ equiv.) and 3-methylglutaric anhydride (1.60 g, $12.5 \mathrm{mmol}, 1.0$ equiv.) in DMF $(50 \mathrm{~mL})$. The reaction mixture was subsequently treated with EDCI (4.78 g, $25.0 \mathrm{mmol}, 2.0$ equiv.), DMAP (305 mg, $2.50 \mathrm{mmol}, 0.2$ equiv.) and HFIP (6.60 mL, 62.5 mmol, 5.0 equiv.). Purification by silica gel column chromatography (pentane/EtOAc $=3: 2)$ afforded $11 \mathrm{e}(3.21 \mathrm{~g}, 7.33 \mathrm{mmol}, 58 \%)$ as a white solid.

${ }^{1} \mathbf{H}$ NMR $\left(500 \mathrm{MHz}, \mathrm{CD}_{2} \mathrm{Cl}_{2}\right) \delta[\mathrm{ppm}]=8.33(\mathrm{~s}, 1 \mathrm{H}), 7.61-7.59(\mathrm{~m}, 1 \mathrm{H}), 7.38(\mathrm{dt}, J=$ 
8.2, $1.0 \mathrm{~Hz}, 1 \mathrm{H}), 7.18$ (ddd, $J=8.2,7.0,1.2 \mathrm{~Hz}, 1 \mathrm{H}), 7.10$ (ddd, $J=7.9,7.0,1.0 \mathrm{~Hz}$, 1H), 7.06-7.02 (m, 1H), 5.87-5.82 (m, 1H), $5.64(\mathrm{~s}, 1 \mathrm{H}), 3.57(\mathrm{td}, J=6.8,5.7 \mathrm{~Hz}, 2 \mathrm{H})$, $2.96(\mathrm{td}, J=6.9,0.9 \mathrm{~Hz}, 2 \mathrm{H}), 2.64(\mathrm{dd}, J=15.3,5.3 \mathrm{~Hz}, 1 \mathrm{H}), 2.51-2.43(\mathrm{~m}, 1 \mathrm{H}), 2.38$ $(\mathrm{dd}, J=15.4,8.0 \mathrm{~Hz}, 1 \mathrm{H}), 2.13(\mathrm{dd}, J=14.4,6.8 \mathrm{~Hz}, 1 \mathrm{H}), 2.04(\mathrm{dd}, J=14.4,7.1 \mathrm{~Hz}$, $1 \mathrm{H}), 0.99(\mathrm{~d}, J=6.7 \mathrm{~Hz}, 3 \mathrm{H})$.

${ }^{13} \mathbf{C ~ N M R}\left(126 \mathrm{MHz}, \mathrm{CD}_{2} \mathrm{Cl}_{2}\right) \delta[\mathrm{ppm}]=171.1,169.8,136.9,127.8,122.6,122.4,120.5$ $(\mathrm{q}, J=282.1 \mathrm{~Hz}), 119.7,119.0,113.3,111.6,67.2-66.1(\mathrm{~m}), 43.0,40.1,39.9,28.2,25.8$, 19.5 .

${ }^{19}$ F NMR $\left(471 \mathrm{MHz}, \mathrm{CD}_{2} \mathrm{Cl}_{2}\right) \delta[\mathrm{ppm}]=-73.59(\mathrm{~d}, J=12.9 \mathrm{~Hz})$.

HRMS-ESI: calcd. for $\mathrm{C}_{19} \mathrm{H}_{20} \mathrm{~F}_{6} \mathrm{~N}_{2} \mathrm{O}_{3} \mathrm{Na}[\mathrm{M}+\mathrm{Na}]^{+}$: 461.1270 ; found: 461.1269 .

FT-IR: $v\left[\mathrm{~cm}^{-1}\right]=3328,1780,1649,1288,1244,1198,1110,933,745$.

\section{1,1,1,3,3,3-Hexafluoropropan-2-yl 5-((2-(1H-indol-3-yl)ethyl)amino)-2-methyl-5-} oxopentanoate (11f) and 1,1,1,3,3,3-hexafluoropropan-2-yl 5-((2-(1H-indol-3yl)ethyl)amino)-4-methyl-5-oxopentanoate (11g).

11f and 11g were prepared according to General procedure A, starting from tryptamine (500 mg, $3.12 \mathrm{mmol}, 1.0$ equiv.) and 2-methylglutaric anhydride (400 mg, $3.12 \mathrm{mmol}$, 1.0 equiv.) in DMF $(10.0 \mathrm{~mL})$. The reaction mixture was subsequently treated with EDCI (1.19 g, 6.25 mmol, 2.0 equiv.), DMAP (76.4 mg, $0.625 \mathrm{mmol}, 0.2$ equiv.) and HFIP (1.65 mL, $15.6 \mathrm{mmol}, 5.0$ equiv.). Purification by silica gel column chromatography (pentane/EtOAc $=3: 2)$ afforded $11 \mathrm{f}(190 \mathrm{mg}, 0.434 \mathrm{mmol}, 14 \%)$ as a light brown solid and $\mathbf{1 1 g}$ (135 $\mathrm{mg}, 0.308 \mathrm{mmol}, 10 \%)$ as a light brown solid.

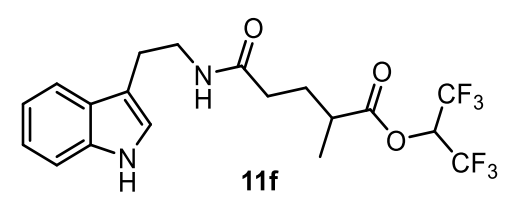

${ }^{1} \mathbf{H}$ NMR $\left(500 \mathrm{MHz}, \mathrm{CD}_{2} \mathrm{Cl}_{2}\right) \delta[\mathrm{ppm}]=8.23(\mathrm{~s}, 1 \mathrm{H}), 7.62-7.57(\mathrm{~m}, 1 \mathrm{H}), 7.38(\mathrm{dd}, J=$ 8.1, $1.0 \mathrm{~Hz}, 1 \mathrm{H}), 7.18(\mathrm{ddd}, J=8.1,7.0,1.2 \mathrm{~Hz}, 1 \mathrm{H}), 7.10$ (ddd, $J=8.0,7.0,1.0 \mathrm{~Hz}$, $1 \mathrm{H}), 7.06(\mathrm{~d}, J=2.4 \mathrm{~Hz}, 1 \mathrm{H}), 5.85-5.80(\mathrm{~m}, 1 \mathrm{H}), 5.55(\mathrm{~s}, 1 \mathrm{H}), 3.55(\mathrm{td}, J=6.8,5.7 \mathrm{~Hz}$, 2H), 2.99-2.93 (m, 2H), 2.75-2.65 (m, 1H), 2.19-2.06 (m, 2H), 2.00-1.93 (m, 1H), $1.88-1.80(\mathrm{~m}, 1 \mathrm{H}), 1.22(\mathrm{~d}, J=7.0 \mathrm{~Hz}, 3 \mathrm{H})$. 
${ }^{13} \mathbf{C ~ N M R}\left(126 \mathrm{MHz}, \mathrm{CD}_{2} \mathrm{Cl}_{2}\right) \delta[\mathrm{ppm}]=173.3,171.6,136.9,127.8,122.5,122.4,121.0$ (d, $J=279.2 \mathrm{~Hz}), 119.7,119.0,113.4,111.6,67.2-66.1$ (m), 40.1, 38.8, 33.8, 29.1, 25.7, 16.9 .

${ }^{19}$ F NMR $\left(471 \mathrm{MHz}, \mathrm{CD}_{2} \mathrm{Cl}_{2}\right) \delta[\mathrm{ppm}]=-73.64(\mathrm{~d}, J=28.6 \mathrm{~Hz})$.

HRMS-ESI: calcd. for $\mathrm{C}_{19} \mathrm{H}_{20} \mathrm{~F}_{6} \mathrm{~N}_{2} \mathrm{O}_{3} \mathrm{Na}[\mathrm{M}+\mathrm{Na}]^{+}$: 461.1270; found: 461.1270.

FT-IR: $v\left[\mathrm{~cm}^{-1}\right]=3328,1781,1635,1457,1388,1288,1241,1200,1109,932,741$.

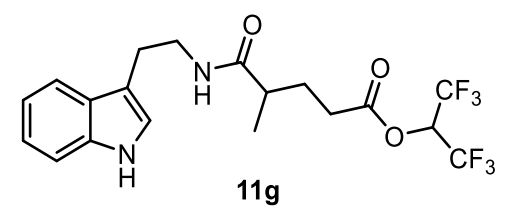

${ }^{1} \mathbf{H}$ NMR $\left(600 \mathrm{MHz}, \mathrm{CDCl}_{3}\right) \delta[\mathrm{ppm}]=8.44(\mathrm{~s}, 1 \mathrm{H}), 7.61(\mathrm{dt}, J=7.9,0.9 \mathrm{~Hz}, 1 \mathrm{H}), 7.36$ (dt, $J=8.2,0.9 \mathrm{~Hz}, 1 \mathrm{H}), 7.21(\mathrm{ddd}, J=8.2,7.0,1.2 \mathrm{~Hz}, 1 \mathrm{H}), 7.13$ (ddd, $J=8.0,7.0$, $1.0 \mathrm{~Hz}, 1 \mathrm{H}), 6.99(\mathrm{~d}, J=2.2 \mathrm{~Hz}, 1 \mathrm{H}), 5.79-5.68(\mathrm{~m}, 1 \mathrm{H}), 5.69(\mathrm{t}, J=5.9 \mathrm{~Hz}, 1 \mathrm{H}), 3.61$ (td, $J=6.8,5.8 \mathrm{~Hz}, 2 \mathrm{H}), 2.99-2.96(\mathrm{~m}, 2 \mathrm{H}), 2.50$ (ddd, $J=16.6,8.7,6.0 \mathrm{~Hz}, 1 \mathrm{H}), 2.41$ (ddd, $J=16.6,8.5,7.0 \mathrm{~Hz}, 1 \mathrm{H}), 2.15-2.09(\mathrm{~m}, 1 \mathrm{H}), 2.01-1.95(\mathrm{~m}, 1 \mathrm{H}), 1.76-1.70(\mathrm{~m}$, 1H), $1.12(\mathrm{~d}, J=6.9 \mathrm{~Hz}, 3 \mathrm{H})$.

${ }^{13} \mathbf{C}$ NMR $\left(151 \mathrm{MHz}, \mathrm{CDCl}_{3}\right) \delta[\mathrm{ppm}]=175.2,170.2,136.6,127.4,122.3,122.2,120.5$ (d, $J=274.1 \mathrm{~Hz}), 119.5,118.7,112.7,111.5,66.9-66.0(\mathrm{~m}), 40.2,39.8,31.0,28.6,25.4$, 17.9 .

${ }^{19}$ F NMR $\left(565 \mathrm{MHz}, \mathrm{CDCl}_{3}\right) \delta[\mathrm{ppm}]=-73.18(\mathrm{~d}, J=6.5 \mathrm{~Hz})$.

HRMS-ESI: calcd. for $\mathrm{C}_{19} \mathrm{H}_{20} \mathrm{~F}_{6} \mathrm{~N}_{2} \mathrm{O}_{3} \mathrm{Na}[\mathrm{M}+\mathrm{Na}]^{+}$: 461.1270; found: 461.1271 .

FT-IR: $v\left[\mathrm{~cm}^{-1}\right]=3275,2925,2844,1638,1398,1328,1303,1213,1180,742$.

\section{1,1,1,3,3,3-Hexafluoropropan-2-yl 5-((2-(1H-indol-3-yl)ethyl)amino)-3,3-dimeth-} yl-5-oxopentanoate (11h).

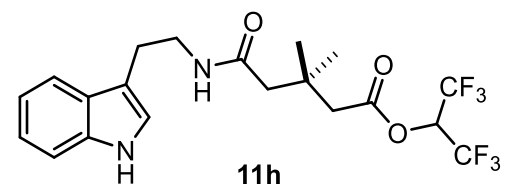

11h was prepared according to General procedure A, starting from tryptamine (400 mg, $2.50 \mathrm{mmol}, 1.0$ equiv.) and 3,3-dimethylglutaric anhydride (355 mg, 2.50 
mmol, 1.0 equiv.) in DMF $(10.0 \mathrm{~mL})$. The reaction mixture was subsequently treated with EDCI (955 mg, $5.00 \mathrm{mmol}, 2.0$ equiv.), DMAP (61.1 mg, $500 \mu \mathrm{mol}, 0.2$ equiv.) and HFIP (1.32 mL, $12.5 \mathrm{mmol}, 5.0$ equiv.). Purification by silica gel column chromatography (pentane/EtOAc $=3: 2)$ afforded $\mathbf{1 1 h}(697 \mathrm{mg}, 1.54 \mathrm{mmol}, 62 \%)$ as a white solid.

${ }^{1} \mathbf{H}$ NMR $\left(500 \mathrm{MHz}, \mathrm{CDCl}_{3}\right) \delta[\mathrm{ppm}]=8.21(\mathrm{~s}, 1 \mathrm{H}), 7.60(\mathrm{dt}, J=7.9,0.9 \mathrm{~Hz}, 1 \mathrm{H}), 7.37$ (dt, $J=8.1,0.9 \mathrm{~Hz}, 1 \mathrm{H}), 7.21$ (ddd, $J=8.2,7.0,1.2 \mathrm{~Hz}, 1 \mathrm{H}), 7.12$ (ddd, $J=8.0,7.0$, $1.0 \mathrm{~Hz}, 1 \mathrm{H}), 7.01(\mathrm{~d}, J=2.4 \mathrm{~Hz}, 1 \mathrm{H}), 5.81(\mathrm{~s}, 1 \mathrm{H}), 5.80-5.72(\mathrm{~m}, 1 \mathrm{H}), 3.61(\mathrm{td}, J=6.8$, $5.7 \mathrm{~Hz}, 2 \mathrm{H}), 2.97(\mathrm{td}, J=6.9,0.9 \mathrm{~Hz}, 2 \mathrm{H}), 2.56(\mathrm{~s}, 2 \mathrm{H}), 2.16(\mathrm{~s}, 2 \mathrm{H}), 1.08(\mathrm{~s}, 6 \mathrm{H})$.

${ }^{13} \mathbf{C ~ N M R}\left(126 \mathrm{MHz}, \mathrm{CDCl}_{3}\right) \delta[\mathrm{ppm}]=170.7,169.3,136.6,127.4,122.3,122.1,120.6$ (q, $J=283.3 \mathrm{~Hz}), 119.6,118.8,113.0,111.4,66.8-65.7$ (m) , 47.3, 43.8, 39.7, 33.5, 28.0, 25.4 .

${ }^{19}$ F NMR $\left(565 \mathrm{MHz}, \mathrm{CDCl}_{3}\right) \delta[\mathrm{ppm}]=-72.98(\mathrm{~d}, J=6.4 \mathrm{~Hz})$.

HRMS-ESI: calcd. for $\mathrm{C}_{20} \mathrm{H}_{23} \mathrm{~F}_{6} \mathrm{~N}_{2} \mathrm{O}_{3}[\mathrm{M}+\mathrm{H}]^{+}$: 453.1608; found: 453.1611 .

FT-IR: $v\left[\mathrm{~cm}^{-1}\right]=3238,2963,1779,1647,1522,1388,1289,1236,1198,1109,746$.

\section{(S)-1,1,1,3,3,3-Hexafluoropropan-2-yl-5-((3-(1H-indol-3-yl)-1-methoxy-1-oxopro-} pan-2-yl)amino)-5-oxopentanoate (11i).

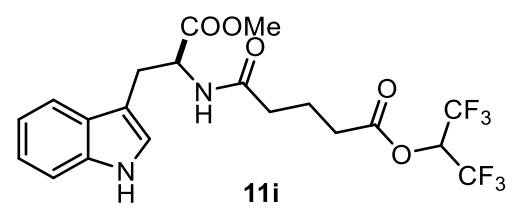

11i was prepared according to General procedure A, starting from (S)-tryptophan methyl ester (300 mg, $1.38 \mathrm{mmol}, 1.0$ equiv.) and glutaric anhydride (157 mg, $1.38 \mathrm{mmol}, 1.0$ equiv.) in DMF $(10.0 \mathrm{~mL})$. The reaction mixture was subsequently treated with EDCI (526 mg, $2.75 \mathrm{mmol}, 2.0$ equiv.), DMAP (33.6 mg, $0.275 \mathrm{mmol}$, 0.2 equiv.) and HFIP (727 $\mu \mathrm{L}, 6.88 \mathrm{mmol}, 5.0$ equiv.). Purification by silica gel column chromatography (pentane/EtOAc $=3: 2)$ afforded $11 i(150 \mathrm{mg}, 0.311 \mathrm{mmol}, 23 \%)$ as a white solid.

${ }^{1} \mathbf{H}$ NMR $\left(500 \mathrm{MHz}, \mathrm{CDCl}_{3}\right) \delta[\mathrm{ppm}]=8.32(\mathrm{~s}, 1 \mathrm{H}), 7.53-7.50(\mathrm{~m}, 1 \mathrm{H}), 7.34(\mathrm{dt}, J=$ 8.1, $0.9 \mathrm{~Hz}, 1 \mathrm{H}), 7.19$ (ddd, $J=8.2,7.0,1.2 \mathrm{~Hz}, 1 \mathrm{H}), 7.11(\mathrm{ddd}, J=8.0,7.0,1.0 \mathrm{~Hz}$, 
1H), $6.95(\mathrm{~d}, J=2.4 \mathrm{~Hz}, 1 \mathrm{H}), 6.05(\mathrm{~d}, J=8.0 \mathrm{~Hz}, 1 \mathrm{H}), 5.78-5.73(\mathrm{~m}, 1 \mathrm{H}), 4.95(\mathrm{dt}, J$ $=7.9,5.5 \mathrm{~Hz}, 1 \mathrm{H}), 3.70(\mathrm{~s}, 3 \mathrm{H}), 3.36-3.26(\mathrm{~m}, 2 \mathrm{H}), 2.49(\mathrm{t}, J=7.4 \mathrm{~Hz}, 2 \mathrm{H}), 2.18(\mathrm{t}, J$ $=7.4 \mathrm{~Hz}, 2 \mathrm{H}), 1.98-1.92(\mathrm{~m}, 2 \mathrm{H})$.

${ }^{13} \mathbf{C ~ N M R}\left(126 \mathrm{MHz}, \mathrm{CDCl}_{3}\right) \delta[\mathrm{ppm}]=172.5,171.4,170.0,136.3,127.8,122.8,122.4$, $120.5(\mathrm{q}, J=278.7 \mathrm{~Hz}), 119.8,118.5,111.5,110.0,67.0-65.9$ (m), 53.1, 52.5, 34.6, $32.2,27.7,20.1$.

${ }^{19}$ F NMR $\left(471 \mathrm{MHz}, \mathrm{CDCl}_{3}\right) \delta[\mathrm{ppm}]=-73.19(\mathrm{~d}, J=6.4 \mathrm{~Hz})$.

HRMS-ESI: calcd. for $\mathrm{C}_{20} \mathrm{H}_{20} \mathrm{~F}_{6} \mathrm{~N}_{2} \mathrm{O}_{5} \mathrm{Na}[\mathrm{M}+\mathrm{Na}]^{+}$: 505.1168; found: 505.1191 .

FT-IR: $v\left[\mathrm{~cm}^{-1}\right]$ 3624, 3376, 2953, 1779, 1736, 1661, 1518, 1440, 1289, 1228, 1200 , $1110,781,753$.

$[\boldsymbol{\alpha}]_{\mathbf{D}}^{\mathbf{2 6}}=+9.2\left(c=0.7, \mathrm{CHCl}_{3}\right)$.

\section{1,1,1,3,3,3-Hexafluoropropan-2-yl 2-(2-((2-(1H-indol-3-yl)ethyl)amino)-2-oxoeth-} yl)benzoate (11j).

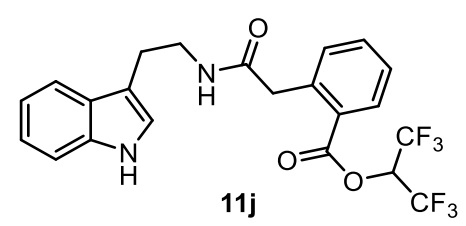

$\mathbf{1 1} \mathbf{j}$ was prepared according to General procedure $\mathbf{A}$, starting from tryptamine (150 mg, $0.938 \mathrm{mmol}, 1.0$ equiv.) and homophthalic anhydride (152 mg, $0.938 \mathrm{mmol}, 1.0$ equiv.) in DMF (5.0 mL). The reaction mixture was subsequently treated with EDCI (197 mg, $1.03 \mathrm{mmol}, 2.0$ equiv.), DMAP (22.9 mg, $0.188 \mathrm{mmol}, 0.2$ equiv.) and HFIP (495 $\mu \mathrm{L}$, 4.69 mmol, 5.0 equiv.). Purification by silica gel column chromatography (pentane/EtOAc $=5: 2)$ afforded $\mathbf{1 1} \mathbf{j}(32.0 \mathrm{mg}, 678 \mu \mathrm{mol}, 7 \%)$ as a white solid.

${ }^{1} \mathbf{H}$ NMR $\left(500 \mathrm{MHz}, \mathrm{CDCl}_{3}\right) \delta[\mathrm{ppm}]=8.06(\mathrm{~s}, 1 \mathrm{H}), 8.02(\mathrm{dd}, J=7.9,1.5 \mathrm{~Hz}, 1 \mathrm{H})$, 7.60-7.54 (m, 2H), $7.43(\mathrm{dd}, J=7.8,1.3 \mathrm{~Hz}, 1 \mathrm{H}), 7.40(\mathrm{td}, J=7.7,1.3 \mathrm{~Hz}, 1 \mathrm{H}), 7.34$ (dd, $J=8.2,1.0 \mathrm{~Hz}, 1 \mathrm{H}), 7.19(\mathrm{ddd}, J=8.1,7.0,1.2 \mathrm{~Hz}, 1 \mathrm{H}), 7.10$ (ddd, $J=8.1,7.0$, $1.0 \mathrm{~Hz}, 1 \mathrm{H}), 6.90(\mathrm{~d}, J=2.3 \mathrm{~Hz}, 1 \mathrm{H}), 5.96(\mathrm{~s}, 1 \mathrm{H}), 5.90-5.68(\mathrm{~m}, 1 \mathrm{H}), 3.83(\mathrm{~s}, 2 \mathrm{H})$, $3.56(\mathrm{td}, J=6.9,5.7 \mathrm{~Hz}, 2 \mathrm{H}), 2.98-2.86(\mathrm{~m}, 2 \mathrm{H})$.

${ }^{13} \mathbf{C ~ N M R}\left(126 \mathrm{MHz}, \mathrm{CDCl}_{3}\right) \delta[\mathrm{ppm}]=170.0,163.8,138.8,136.5,134.6,132.9,131.6$, 
$127.8,127.5,125.9,122.3,122.1,120.6$ (q, $J=282.9 \mathrm{~Hz}), 119.6,118.8,113.1,111.3$, 67.5-66.4 (m), 42.2, 40.1, 25.2.

${ }^{19}$ F NMR $\left(471 \mathrm{MHz}, \mathrm{CDCl}_{3}\right) \delta[\mathrm{ppm}]=-72.97$. (It should be a doublet peak, but due to the resolution, only singlet peak was observed.)

HRMS-ESI: calcd. for $\mathrm{C}_{22} \mathrm{H}_{18} \mathrm{~F}_{6} \mathrm{~N}_{2} \mathrm{O}_{3} \mathrm{Na}[\mathrm{M}+\mathrm{Na}]^{+}$: 495.1114; found: 495.1118 .

FT-IR: $v\left[\mathrm{~cm}^{-1}\right]=3306,1755,1651,1551,1360,1242,1193,1104,1019,915,715$.

\section{1,1,1,3,3,3-Hexafluoropropan-2-yl 6-((2-(1H-indol-3-yl)ethyl)amino)-6-oxohexan- oate (11k).}

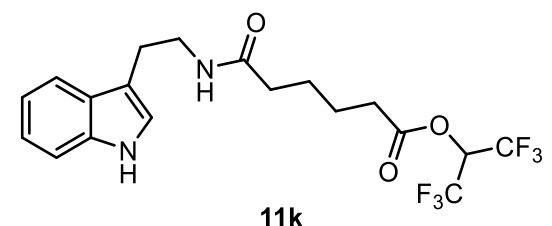

11k was prepared according to General procedure A, starting from tryptamine (400 mg, $2.50 \mathrm{mmol}, 1.0$ equiv.) and oxepane-2,7-dione (320 mg, $2.50 \mathrm{mmol}, 1.0$ equiv.) in DMF $(10.0 \mathrm{~mL})$. The reaction mixture was subsequently treated with EDCI (955 $\mathrm{mg}$, $5.00 \mathrm{mmol}, 2.0$ equiv.), DMAP (61.1 mg, $0.500 \mathrm{mmol}, 0.2$ equiv.) and HFIP (1.32 mL, 12.5 mmol, 5.0 equiv.). Purification by silica gel column chromatography (pentane/EtOAc $=3: 2)$ afforded 11k (289 $\mathrm{mg}, 0.660 \mathrm{mmol}, 26 \%)$ as a white solid.

${ }^{1} \mathbf{H}$ NMR $\left(500 \mathrm{MHz}, \mathrm{CDCl}_{3}\right) \delta[\mathrm{ppm}]=8.73(\mathrm{~s}, 1 \mathrm{H}), 7.59(\mathrm{~d}, J=7.9 \mathrm{~Hz}, 1 \mathrm{H}), 7.35(\mathrm{~d}$, $J=8.1 \mathrm{~Hz}, 1 \mathrm{H}), 7.21(\mathrm{ddd}, J=8.1,7.0,1.2 \mathrm{~Hz}, 1 \mathrm{H}), 7.15-7.08(\mathrm{~m}, 1 \mathrm{H}), 6.96(\mathrm{~d}, J=$ $2.3 \mathrm{~Hz}, 1 \mathrm{H}), 5.87-5.75(\mathrm{~m}, 2 \mathrm{H}), 3.61-3.57(\mathrm{~m}, 2 \mathrm{H}), 2.96(\mathrm{t}, J=6.8 \mathrm{~Hz}, 2 \mathrm{H}), 2.46(\mathrm{t}, J$ $=6.7 \mathrm{~Hz}, 2 \mathrm{H}), 2.15-2.01(\mathrm{~m}, 2 \mathrm{H}), 1.67-1.62(\mathrm{~m}, 4 \mathrm{H})$.

${ }^{13} \mathbf{C ~ N M R}\left(126 \mathrm{MHz}, \mathrm{CDCl}_{3}\right) \delta[\mathrm{ppm}]=172.6,170.3,136.6,127.5,123.9,122.2,120.6$ (q, $J=284.4 \mathrm{~Hz}), 119.4,118.7,112.7,111.5,67.0-65.9(\mathrm{~m}), 40.0,36.0,33.0,25.4,24.8$, 24.1.

${ }^{19}$ F NMR $\left(565 \mathrm{MHz}, \mathrm{CDCl}_{3}\right) \delta[\mathrm{ppm}]=-73.24(\mathrm{~d}, J=6.3 \mathrm{~Hz})$.

HRMS-ESI: calcd. for $\mathrm{C}_{19} \mathrm{H}_{20} \mathrm{~F}_{6} \mathrm{~N}_{2} \mathrm{O}_{3} \mathrm{Na}[\mathrm{M}+\mathrm{Na}]^{+}$: 461.1270 ; found: 461.1285 .

FT-IR: $v\left[\mathrm{~cm}^{-1}\right]=2928,2855,1713,1659,1626,1442,1329,1230,745$. 
$N$-(2-(1H-indol-3-yl)ethyl)-8-((1,1,1,3,3,3-hexafluoropropan-2-yl)oxy)-7-oxooctanamide (11).

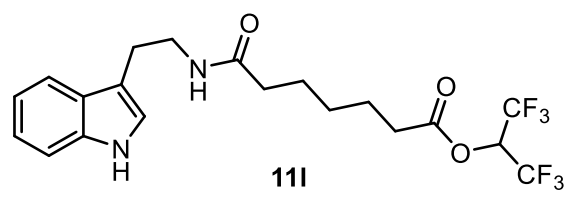

11 was prepared according to General procedure A, starting from tryptamine (400 mg, $2.50 \mathrm{mmol}, 1.0$ equiv.) and oxocane-2,8-dione (355 mg, $2.50 \mathrm{mmol}, 1.0$ equiv.) in DMF $(10.0 \mathrm{~mL})$. The reaction mixture was subsequently treated with EDCI (955 mg, $5.00 \mathrm{mmol}, 2.0$ equiv.), DMAP (61.1 mg, $0.500 \mathrm{mmol}, 0.2$ equiv.) and HFIP (1.32 mL, 12.5 mmol, 5.0 equiv.). Purification by silica gel column chromatography (pentane/EtOAc = 3:2) afforded 111 (300 $\mathrm{mg}, 0.644 \mathrm{mmol}, 26 \%)$ as a white solid.

${ }^{1} \mathbf{H}$ NMR $\left(500 \mathrm{MHz}, \mathrm{CDCl}_{3}\right) \delta[\mathrm{ppm}]=8.62(\mathrm{~s}, 1 \mathrm{H}), 7.60-7.58(\mathrm{~m}, 1 \mathrm{H}), 7.36(\mathrm{dt}, J=$ 8.1, $0.9 \mathrm{~Hz}, 1 \mathrm{H}), 7.20$ (ddd, $J=8.2,7.0,1.2 \mathrm{~Hz}, 1 \mathrm{H}), 7.11(\mathrm{ddd}, J=8.0,7.0,1.1 \mathrm{~Hz}$, $1 \mathrm{H}), 6.98(\mathrm{~d}, J=2.3 \mathrm{~Hz}, 1 \mathrm{H}), 5.80-5.75(\mathrm{~m}, 1 \mathrm{H}), 5.70(\mathrm{t}, J=5.8 \mathrm{~Hz}, 1 \mathrm{H}), 3.59(\mathrm{td}, J=$ 6.8, $5.7 \mathrm{~Hz}, 2 \mathrm{H}), 2.96(\mathrm{td}, J=6.8,0.9 \mathrm{~Hz}, 2 \mathrm{H}), 2.44(\mathrm{t}, J=7.5 \mathrm{~Hz}, 2 \mathrm{H}), 2.08(\mathrm{t}, J=7.5$ $\mathrm{Hz}, 2 \mathrm{H}), 1.71-1.52(\mathrm{~m}, 4 \mathrm{H}), 1.34-1.20$ (m, 2H).

${ }^{13} \mathbf{C ~ N M R}\left(126 \mathrm{MHz}, \mathrm{CDCl}_{3}\right) \delta[\mathrm{ppm}]=172.9,170.4,136.6,127.5,122.3,122.2,120.6$ (q, $J=281.4 \mathrm{~Hz}), 119.5,118.7,112.8,111.5,67.0-65.9(\mathrm{~m}), 39.9,36.4,33.1,28.4,25.4$, $25.2,24.3$

${ }^{19}$ F NMR $\left(565 \mathrm{MHz}, \mathrm{CDCl}_{3}\right) \delta[\mathrm{ppm}]=-73.23(\mathrm{~d}, J=6.4 \mathrm{~Hz})$.

HRMS-ESI: calcd. for $\mathrm{C}_{20} \mathrm{H}_{22} \mathrm{~F}_{6} \mathrm{~N}_{2} \mathrm{O}_{3} \mathrm{Na}[\mathrm{M}+\mathrm{Na}]^{+}$: 475.1427; found: 475.1442 .

FT-IR: $v\left[\mathrm{~cm}^{-1}\right]=3286,2934,2870,1778,1648,1532,1387,1288,1236,1199,1110$, 745.

1,1,1,3,3,3-Hexafluoropropan-2-yl 5-((3-(1 H-indol-3-yl)propyl)amino)-5-oxopentanoate $(11 \mathrm{~m})$

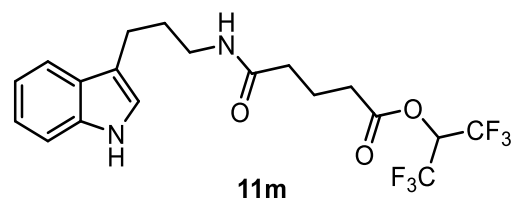


11m was prepared according to General procedure A, starting from $1 H$-indole-3propanamine ( $85.0 \mathrm{mg}, 0.489 \mathrm{mmol}, 1.0$ equiv.) and glutaric anhydride (55.7 mg, 0.489 mmol, 1.0 equiv.) in DMF $(2.0 \mathrm{~mL})$. The reaction mixture was subsequently treated with EDCI (187 mg, 0.977 mmol, 2.0 equiv.), DMAP (11.9 mg, $98.0 \mu \mathrm{mol}, 0.2$ equiv.) and HFIP (258 $\mu \mathrm{L}, 2.44 \mathrm{mmol}, 5.0$ equiv.). Purification by silica gel column chromatography (pentane/EtOAc $=3: 2)$ afforded $11 \mathrm{~m}(120 \mathrm{mg}, 0.274 \mathrm{mmol}, 56 \%)$ as a white solid.

${ }^{1} \mathbf{H}$ NMR $\left(500 \mathrm{MHz}, \mathrm{CDCl}_{3}\right) \delta[\mathrm{ppm}]=8.11(\mathrm{~s}, 1 \mathrm{H}), 7.59-7.57(\mathrm{~m}, 1 \mathrm{H}), 7.36-7.34(\mathrm{~m}$, 1H), 7.19 (ddd, $J=8.2,7.0,1.2 \mathrm{~Hz}, 1 \mathrm{H}), 7.14-7.10(\mathrm{~m}, 1 \mathrm{H}), 6.98(\mathrm{dd}, J=2.3,1.2 \mathrm{~Hz}$, $1 \mathrm{H}), 5.80-5.75(\mathrm{~m}, 1 \mathrm{H}), 5.46(\mathrm{~s}, 1 \mathrm{H}), 3.32(\mathrm{td}, J=7.1,5.9 \mathrm{~Hz}, 2 \mathrm{H}), 2.80(\mathrm{td}, J=7.3$, $0.9 \mathrm{~Hz}, 2 \mathrm{H}), 2.55$ (t, $J=7.2 \mathrm{~Hz}, 2 \mathrm{H}), 2.12$ (t, $J=7.3 \mathrm{~Hz}, 2 \mathrm{H}), 2.00-1.89$ (m, 4H).

${ }^{13} \mathbf{C ~ N M R}\left(126 \mathrm{MHz}, \mathrm{CDCl}_{3}\right) \delta[\mathrm{ppm}]=171.5,170.1,136.6,127.4,122.2,121.6,119.4$, $118.8,115.5,111.3,66.8-66.3(\mathrm{~m}), 39.6,34.9,32.4,29.8,22.8,20.4$. (Due to the resolution or/and overlapping, the resonance of $\mathrm{CF}_{3}$ was not observed.)

${ }^{19}$ F NMR $\left(471 \mathrm{MHz}, \mathrm{CDCl}_{3}\right) \delta[\mathrm{ppm}]=-73.18(\mathrm{~d}, J=6.4 \mathrm{~Hz})$.

HRMS-ESI: calcd. for $\mathrm{C}_{19} \mathrm{H}_{20} \mathrm{~F}_{6} \mathrm{~N}_{2} \mathrm{O}_{3} \mathrm{Na}[\mathrm{M}+\mathrm{Na}]^{+}: 461.1270$; found: 461.1287 .

FT-IR: $v\left[\mathrm{~cm}^{-1}\right]=3404,3295,2925,1780,1649,1385,1356,1288,1228,1200,1110$, 744.

1,1,1,3,3,3-Hexafluoropropan-2-yl 5-((4-(1H-indol-3-yl)butyl)amino)-5-oxopentanoate (11n).

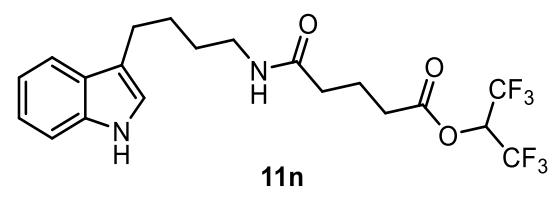

11n was prepared according to General procedure A, starting from $1 H$-indole-3butanamine (120 mg, $0.638 \mathrm{mmol}, 1.0$ equiv.) and glutaric anhydride (72.8 $\mathrm{mg}$, $0.638 \mathrm{mmol}, 1.0$ equiv.) in DMF $(2.0 \mathrm{~mL})$. The reaction mixture was subsequently treated with EDCI (244 mg, 1.28 mmol, 2.0 equiv.), DMAP (15.6 mg, 0.128 mmol, 0.2 equiv.) and HFIP (337 $\mu \mathrm{L}, 3.19$ mmol, 5.0 equiv.). Purification by silica gel column chromatography (pentane/EtOAc $=3: 2)$ afforded $11 \mathrm{n}(140 \mathrm{mg}, 0.310 \mathrm{mmol}, 48 \%)$ as a 
white solid.

${ }^{1} \mathbf{H}$ NMR $\left(500 \mathrm{MHz}, \mathrm{CDCl}_{3}\right) \delta[\mathrm{ppm}]=8.06(\mathrm{~s}, 1 \mathrm{H}), 7.62-7.56(\mathrm{~m}, 1 \mathrm{H}), 7.35(\mathrm{dt}, J=$ 8.2, $0.9 \mathrm{~Hz}, 1 \mathrm{H}$ ), 7.19 (ddd, $J=8.1,7.0,1.2 \mathrm{~Hz}, 1 \mathrm{H}$ ), 7.11 (ddd, $J=8.0,7.0,1.0 \mathrm{~Hz}$, $1 \mathrm{H}), 6.96(\mathrm{dd}, J=2.3,1.0 \mathrm{~Hz}, 1 \mathrm{H}), 5.80-5.75(\mathrm{~m}, 1 \mathrm{H}), 5.43(\mathrm{~s}, 1 \mathrm{H}), 3.26(\mathrm{td}, J=7.2$, $5.7 \mathrm{~Hz}, 2 \mathrm{H}), 2.78(\mathrm{td}, J=7.4,0.9 \mathrm{~Hz}, 2 \mathrm{H}), 2.57(\mathrm{t}, J=7.2 \mathrm{~Hz}, 2 \mathrm{H}), 2.16(\mathrm{t}, J=7.3 \mathrm{~Hz}$, 2H), 2.01 (q, $J=7.3 \mathrm{~Hz}, 2 \mathrm{H}), 1.80-1.69(\mathrm{~m}, 2 \mathrm{H}), 1.60-1.54(\mathrm{~m}, 2 \mathrm{H})$.

${ }^{13} \mathrm{C} \mathrm{NMR}\left(126 \mathrm{MHz}, \mathrm{CDCl}_{3}\right) \delta[\mathrm{ppm}]=171.5,170.1,136.5,127.5,122.0,121.5,120.5$ (d, $J=282.6 \mathrm{~Hz}), 119.2,119.0,116.3,111.3,66.7-66.2$ (m), 39.6, 34.9, 32.4, 29.4, 27.4, $24.81,20.4$.

${ }^{19}$ F NMR $\left(471 \mathrm{MHz}, \mathrm{CDCl}_{3}\right) \delta[\mathrm{ppm}]=-73.17(\mathrm{~d}, J=6.3 \mathrm{~Hz})$.

HRMS-ESI: calcd. for $\mathrm{C}_{20} \mathrm{H}_{22} \mathrm{~F}_{6} \mathrm{~N}_{2} \mathrm{O}_{3} \mathrm{Na}[\mathrm{M}+\mathrm{Na}]^{+}$: 475.1427 ; found: 475.1436 .

FT-IR: $v\left[\mathrm{~cm}^{-1}\right]=3401,2924,2854,1781,1651,1288,1228,1200,1110,927,906$, 744.

1,1,1,3,3,3-Hexafluoropropan-2-yl 3,3-dimethyl-5-oxo-5-(phenethylamino)pentanoate (110).<smiles>CC(CC(=O)NCCc1ccccc1)CC(=O)OC(C(F)(F)F)C(F)(F)F</smiles>

110 was prepared according to General procedure A, starting from phenethylamine (300 mg, $2.48 \mathrm{mmol}, 1.0$ equiv.) and 3,3-dimethylglutaric anhydride (352 mg, $2.48 \mathrm{mmol}, 1.0$ equiv.) in DMF $(10.0 \mathrm{~mL})$. The reaction mixture was subsequently treated with EDCI (947 mg, 4.96 mmol, 2.0 equiv.), DMAP (60.6 mg, 0.496 mmol, 0.2 equiv.) and HFIP (1.31 mL, $12.4 \mathrm{mmol}, 5.0$ equiv.). Purification by silica gel column chromatography (pentane/EtOAc $=5: 2)$ afforded $110(452 \mathrm{mg}, 1.09 \mathrm{mmol}, 44 \%)$ as a white solid.

${ }^{1} \mathbf{H}$ NMR $\left(500 \mathrm{MHz}, \mathrm{CDCl}_{3}\right) \delta[\mathrm{ppm}]=7.31-7.27$ (m, 2H), 7.24-7.17 (m, 3H), 5.80$5.71(\mathrm{~m}, 2 \mathrm{H}), 3.54(\mathrm{td}, J=7.0,5.8 \mathrm{~Hz}, 2 \mathrm{H}), 2.82(\mathrm{t}, J=6.9 \mathrm{~Hz}, 2 \mathrm{H}), 2.50(\mathrm{~s}, 2 \mathrm{H}), 2.17$ (s, 2H), $1.08(\mathrm{~s}, 6 \mathrm{H})$.

${ }^{13} \mathbf{C ~ N M R}\left(126 \mathrm{MHz}, \mathrm{CDCl}_{3}\right) \delta[\mathrm{ppm}]=170.6,169.4,138.9,128.8,128.8,126.7,120.5$ (q, $J=279.6 \mathrm{~Hz}), 66.8-65.7(\mathrm{~m}), 47.2,43.7,40.4,35.7,33.5,28.0$. 
${ }^{19}$ F NMR $\left(471 \mathrm{MHz}, \mathrm{CDCl}_{3}\right) \delta[\mathrm{ppm}]=-73.02(\mathrm{~d}, J=6.3 \mathrm{~Hz})$.

HRMS-ESI: calcd. for $\mathrm{C}_{18} \mathrm{H}_{21} \mathrm{~F}_{6} \mathrm{NO}_{3} \mathrm{Na}[\mathrm{M}+\mathrm{Na}]^{+}$: 436.1318; found: 436.1335 .

FT-IR: $v\left[\mathrm{~cm}^{-1}\right]=3309,1967,1645,1543,1282,1268,1200,1107$.

\section{1,1,1,3,3,3-Hexafluoropropan-2-yl 5-((4-methoxyphenethyl)amino)-3,3-dimethyl- 5-oxopentanoate (11p).}<smiles>COc1ccc(CCNC(=O)CC(C)(C)CC(=O)OC(C(F)(F)F)C(F)(F)F)cc1</smiles>

$11 p$ was prepared according to General procedure A, starting from 4methoxyphenethylamine (300 mg, $1.99 \mathrm{mmol}, 1.0$ equiv.) and 3,3-dimethylglutaric anhydride (282 mg, $1.99 \mathrm{mmol}, 1.0$ equiv.) in DMF (10.0 mL). The reaction mixture was subsequently treated with EDCI (759 mg, 3.97 mmol, 2.0 equiv.), DMAP (48.5 mg, $0.397 \mathrm{mmol}, 0.2$ equiv.) and HFIP (1.05 mL, $9.93 \mathrm{mmol}, 5.0$ equiv.). Purification by silica gel column chromatography (pentane/EtOAc = 5:2) afforded 11p (523 mg, 1.18 mmol, $59 \%$ ) as a white solid.

${ }^{1} \mathbf{H}$ NMR $\left(500 \mathrm{MHz}^{\mathrm{CDCl}} 3\right) \delta[\mathrm{ppm}]=7.13-7.06(\mathrm{~m}, 2 \mathrm{H}), 6.85-6.79(\mathrm{~m}, 2 \mathrm{H}), 5.72-$ $5.70(\mathrm{~m}, 2 \mathrm{H}), 3.78(\mathrm{~s}, 3 \mathrm{H}), 3.49(\mathrm{td}, J=7.0,5.8 \mathrm{~Hz}, 2 \mathrm{H}), 2.75(\mathrm{t}, J=7.0 \mathrm{~Hz}, 2 \mathrm{H}), 2.53$ (s, 2H), 2.17 (s, 2H), 1.08 (s, 6H).

${ }^{13} \mathbf{C ~ N M R}\left(126 \mathrm{MHz}, \mathrm{CDCl}_{3}\right) \delta[\mathrm{ppm}]=170.6,169.4,158.5,130.8,129.8,120.6(\mathrm{q}, J$ $=291.0 \mathrm{~Hz}), 114.2,66.6-66.0(\mathrm{~m}), 55.3,47.2$, 43.7, 40.6, 34.8, 33.5, 28.0.

${ }^{19}$ F NMR $\left(471 \mathrm{MHz}, \mathrm{CDCl}_{3}\right) \delta[\mathrm{ppm}]=-73.03(\mathrm{~d}, J=6.3 \mathrm{~Hz})$.

HRMS-ESI: calcd. for $\mathrm{C}_{19} \mathrm{H}_{23} \mathrm{~F}_{6} \mathrm{NO}_{4} \mathrm{Na}[\mathrm{M}+\mathrm{Na}]^{+}$: 466.1423; found: 466.1442 .

FT-IR: $v\left[\mathrm{~cm}^{-1}\right]=2970,1738,1515,1440,1366,1217,1105$.

1,1,1,3,3,3-Hexafluoropropan-2-yl 5-((3,4-dimethoxyphenethyl)amino)-3,3-dimethyl-5-oxopentanoate (11q).<smiles>COc1ccc(CCNC(=O)CC(C)(C)CC(=O)OC(C(F)(F)F)C(F)(F)F)cc1OC</smiles> 
$11 q$ was prepared according to General procedure A, starting from 3,4dimethoxyphenethylamine (500 mg, $2.76 \mathrm{mmol}, 1.0$ equiv.) and 3,3-dimethylglutaric anhydride (392 mg, $2.76 \mathrm{mmol}, 1.0$ equiv.) in DMF (15.0 mL). The reaction mixture was subsequently treated with EDCI (1.05 g, $5.52 \mathrm{mmol}, 2.0$ equiv.), DMAP (67.5 mg, $0.552 \mathrm{mmol}, 0.2$ equiv.) and HFIP (1.46 mL, $13.8 \mathrm{mmol}, 5.0$ equiv.). Purification by silica gel column chromatography (pentane/EtOAc $=5: 2)$ afforded 11q $(670 \mathrm{mg}$, $1.42 \mathrm{mmol}, 51 \%$ ) as a white solid.

${ }^{1} \mathbf{H}$ NMR $\left(500 \mathrm{MHz}, \mathrm{CDCl}_{3}\right) \delta[\mathrm{ppm}]=6.80-6.77(\mathrm{~m}, 1 \mathrm{H}), 6.71(\mathrm{~d}, J=7.6 \mathrm{~Hz}, 2 \mathrm{H})$, 5.80-5.70 (m, 2H), $3.86(\mathrm{~s}, 3 \mathrm{H}), 3.85(\mathrm{~s}, 3 \mathrm{H}), 3.51(\mathrm{td}, J=7.0,5.8 \mathrm{~Hz}, 2 \mathrm{H}), 2.75(\mathrm{t}, J$ $=7.0 \mathrm{~Hz}, 2 \mathrm{H}), 2.54(\mathrm{~s}, 2 \mathrm{H}), 2.18(\mathrm{~s}, 2 \mathrm{H}), 1.08(\mathrm{~s}, 6 \mathrm{H})$.

${ }^{13} \mathbf{C}$ NMR $\left(126 \mathrm{MHz}, \mathrm{CDCl}_{3}\right) \delta[\mathrm{ppm}]=170.6,169.4,149.2,147.8,131.3,120.7,120.5$ (q, $J=280.8 \mathrm{~Hz}), 111.8,111.4,66.5-65.7$ (m), 56.0, 55.9, 47.2, 43.7, 40.5, 35.3, 33.5, 28.1 .

${ }^{19}$ F NMR $\left(471 \mathrm{MHz}, \mathrm{CDCl}_{3}\right) \delta[\mathrm{ppm}]=-73.01(\mathrm{~d}, J=6.5 \mathrm{~Hz})$.

HRMS-ESI: calcd. for $\mathrm{C}_{20} \mathrm{H}_{25} \mathrm{~F}_{6} \mathrm{NO}_{5} \mathrm{Na}[\mathrm{M}+\mathrm{Na}]^{+}$: 496.1529; found: 496.1518 .

FT-IR: $v\left[\mathrm{~cm}^{-1}\right]=2371,2354,1649,1518,1288,1243,1107$.

\section{1,1,1,3,3,3-Hexafluoropropan-2-yl 5-((3,4-dimethoxyphenethyl)amino)-5-oxopen- tanoate (11r).}<smiles>COc1ccc(CCNC(=O)CCCC(=O)OC(C(F)(F)F)C(F)(F)F)cc1OC</smiles>

11r was prepared according to General procedure A, starting from 3,4dimethoxyphenethylamine (500 mg, $2.76 \mathrm{mmol}, 1.0$ equiv.) and glutaric anhydride (315 mg, $2.76 \mathrm{mmol}, 1.0$ equiv.) in DMF $(15.0 \mathrm{~mL})$. The reaction mixture was subsequently treated with EDCI (1.05 g, $5.52 \mathrm{mmol}, 2.0$ equiv.), DMAP (67.5 mg, $0.552 \mathrm{mmol}, 0.2$ equiv.) and HFIP (1.46 mL, $13.8 \mathrm{mmol}, 5.0$ equiv.). Purification by silica gel column chromatography (pentane/EtOAc $=5: 2)$ afforded 11r $(670 \mathrm{mg}$, $1.42 \mathrm{mmol}, 55 \%)$ as a white solid.

${ }^{1} \mathbf{H}$ NMR $\left(500 \mathrm{MHz}, \mathrm{CDCl}_{3}\right) \delta[\mathrm{ppm}]=6.79(\mathrm{~d}, J=8.7 \mathrm{~Hz}, 1 \mathrm{H}), 6.72-6.67(\mathrm{~m}, 2 \mathrm{H})$, 
5.77-5.72 (m, 1H), $5.54(\mathrm{~s}, 1 \mathrm{H}), 3.85(\mathrm{~d}, J=3.5 \mathrm{~Hz}, 6 \mathrm{H}), 3.49(\mathrm{td}, J=7.0,5.9 \mathrm{~Hz}, 2 \mathrm{H})$, $2.74(\mathrm{t}, J=7.0 \mathrm{~Hz}, 2 \mathrm{H}), 2.57(\mathrm{t}, J=7.2 \mathrm{~Hz}, 2 \mathrm{H}), 2.18(\mathrm{t}, J=7.2 \mathrm{~Hz}, 2 \mathrm{H}), 2.04-1.94(\mathrm{~m}$, $2 \mathrm{H})$.

${ }^{13} \mathbf{C ~ N M R}\left(126 \mathrm{MHz}, \mathrm{CDCl}_{3}\right) \delta[\mathrm{ppm}]=171.5,170.0,149.2,147.9,131.3,120.7,120.5$ (q, $J=279.6 \mathrm{~Hz}), 111.9,111.5,67.0-65.9$ (m), 56.0, 55.9, 40.8, 35.3, 34.8, 32.4, 20.4. ${ }^{19}$ F NMR $\left(565 \mathrm{MHz}, \mathrm{CDCl}_{3}\right) \delta[\mathrm{ppm}]=-73.22(\mathrm{~d}, J=6.4 \mathrm{~Hz})$.

HRMS-ESI: calcd. for $\mathrm{C}_{18} \mathrm{H}_{21} \mathrm{~F}_{6} \mathrm{NO}_{5} \mathrm{Na}[\mathrm{M}+\mathrm{Na}]^{+}$: 468.1216 ; found: 468.1217 . FT-IR: $v\left[\mathrm{~cm}^{-1}\right]=2942,1682,1602,1513,1377,1341,1272,1162,752$.

\section{1,1,1,3,3,3-Hexafluoropropan-2-yl 2-(2-((3,4-dimethoxyphenethyl)amino)-2-oxo- ethyl)benzoate (11s).}<smiles>COc1ccc(CCNC(=O)Cc2ccccc2C(=O)OC(C(F)(F)F)C(F)(F)F)cc1OC</smiles>

$11 \mathrm{~s}$ was prepared according to General procedure A, starting from 3,4dimethoxyphenethylamine (300 mg, $1.66 \mathrm{mmol}, 1.0$ equiv.) and homophthalic anhydride (268 mg, $1.66 \mathrm{mmol}, 1.0$ equiv.) in DMF (10.0 mL). The reaction mixture was subsequently treated with EDCI (633 mg, $3.31 \mathrm{mmol}, 2.0$ equiv.), DMAP (40.5 mg, $0.331 \mathrm{mmol}, 0.2$ equiv.) and HFIP (1.39 mL, $8.29 \mathrm{mmol}, 5.0$ equiv.). Purification by silica gel column chromatography (pentane/EtOAc $=5: 2)$ afforded 11s $(189 \mathrm{mg}$, $0.383 \mathrm{mmol}, 23 \%$ ) as a white solid.

${ }^{1} \mathbf{H}$ NMR $\left(600 \mathrm{MHz}, \mathrm{CDCl}_{3}\right) \delta[\mathrm{ppm}]=8.00(\mathrm{dd}, J=7.9,1.4 \mathrm{~Hz}, 1 \mathrm{H}), 7.55(\mathrm{td}, J=7.6$, $1.4 \mathrm{~Hz}, 1 \mathrm{H}), 7.40(\mathrm{dd}, J=7.8,1.3 \mathrm{~Hz}, 1 \mathrm{H}), 7.37(\mathrm{td}, J=7.7,1.3 \mathrm{~Hz}, 1 \mathrm{H}), 6.69(\mathrm{~d}, J=$ $8.1 \mathrm{~Hz}, 1 \mathrm{H}), 6.63(\mathrm{~d}, J=2.0 \mathrm{~Hz}, 1 \mathrm{H}), 6.58(\mathrm{dd}, J=8.1,2.0 \mathrm{~Hz}, 1 \mathrm{H}), 6.07$ (t, $J=5.8 \mathrm{~Hz}$, 1H), 5.96-5.90 (m, 1H), $3.81(\mathrm{~s}, 2 \mathrm{H}), 3.80(\mathrm{~s}, 3 \mathrm{H}), 3.79(\mathrm{~s}, 3 \mathrm{H}), 3.42(\mathrm{td}, J=7.1,5.7$ $\mathrm{Hz}, 2 \mathrm{H}), 2.67$ (t, $J=7.1 \mathrm{~Hz}, 2 \mathrm{H})$.

${ }^{13} \mathbf{C}$ NMR $\left(151 \mathrm{MHz}, \mathrm{CDCl}_{3}\right) \delta[\mathrm{ppm}]=169.8,163.7,148.9,147.6,138.7,134.4,132.7$, $131.4,131.3,127.6,125.7,120.6,120.5$ (q, $J=286.3 \mathrm{~Hz}), 111.9,111.2,67.3-66.4(\mathrm{~m})$, 55.8, 55.7, 41.9, 40.8, 35.0.

${ }^{19}$ F NMR $\left(565 \mathrm{MHz}, \mathrm{CDCl}_{3}\right) \delta[\mathrm{ppm}]=-72.99(\mathrm{~d}, J=6.9 \mathrm{~Hz})$. 
HRMS-ESI: calcd. for $\mathrm{C}_{22} \mathrm{H}_{21} \mathrm{~F}_{6} \mathrm{NO}_{5} \mathrm{Na}[\mathrm{M}+\mathrm{Na}]^{+}$: 516.1216; found: 516.1201.

FT-IR: $v\left[\mathrm{~cm}^{-1}\right]=2937,1755,1652,1515,1237,1194,1109,1096,1025,913,715$.

\section{1,1,1,3,3,3-Hexafluoropropan-2-yl 2-(2-oxo-2-(phenethylamino)ethyl)benzoate} (11t).<smiles>O=C(Cc1ccccc1C(=O)OC(F)(F)F)NCCc1ccccc1</smiles>

11t was prepared according to General procedure A, starting from phenethylamine (250 mg, $2.07 \mathrm{mmol}, 1.0$ equiv.) and homophthalic anhydride (335 mg, $2.07 \mathrm{mmol}, 1.0$ equiv.) in DMF (10.0 mL). The reaction mixture was subsequently treated with EDCI (789 mg, 4.13 mmol, 2.0 equiv.), DMAP (50.5 mg, 0.413 mmol, 0.2 equiv.) and HFIP (1.09 mL, $10.3 \mathrm{mmol}, 5.0$ equiv.). Purification by silica gel column chromatography (pentane/EtOAc $=5: 2)$ afforded 11t (189 $\mathrm{mg}, 0.436 \mathrm{mmol}, 21 \%)$ as a white solid.

${ }^{1} \mathbf{H}$ NMR $\left(700 \mathrm{MHz}, \mathrm{CDCl}_{3}\right) \delta[\mathrm{ppm}]=8.05(\mathrm{dd}, J=8.0,1.5 \mathrm{~Hz}, 1 \mathrm{H}), 7.59(\mathrm{td}, J=7.6$, $1.5 \mathrm{~Hz}, 1 \mathrm{H}), 7.45$ (dd, $J=7.8,1.3 \mathrm{~Hz}, 1 \mathrm{H}), 7.41$ (td, $J=7.7,1.3 \mathrm{~Hz}, 1 \mathrm{H}), 7.25-7.20$ (m, 2H), 7.20-7.16 (m, 1H), 7.09-7.03 (m, 2H), $6.11(\mathrm{t}, J=5.9 \mathrm{~Hz}, 1 \mathrm{H}), 5.96-5.92(\mathrm{~m}$, 1H), $3.84(\mathrm{~s}, 2 \mathrm{H}), 3.47(\mathrm{td}, J=7.0,5.8 \mathrm{~Hz}, 2 \mathrm{H}), 2.75(\mathrm{t}, J=7.0 \mathrm{~Hz}, 2 \mathrm{H})$.

${ }^{13} \mathbf{C}$ NMR $\left(176 \mathrm{MHz}, \mathrm{CDCl}_{3}\right) \delta[\mathrm{ppm}]=169.9,163.8,138.85,138.78,134.5,132.8$, $131.5,128.7,128.5,127.7,126.4,125.8,120.6$ (q, $J=282.8 \mathrm{~Hz}), 67.5-66.3(\mathrm{~m}), 42.0$, $40.8,35.5$.

${ }^{19}$ F NMR $\left(376 \mathrm{MHz}, \mathrm{CDCl}_{3}\right) \delta[\mathrm{ppm}]=-72.94(\mathrm{~d}, J=12.5 \mathrm{~Hz})$.

HRMS-ESI: calcd. for $\mathrm{C}_{20} \mathrm{H}_{17} \mathrm{~F}_{6} \mathrm{NO}_{3} \mathrm{Na}[\mathrm{M}+\mathrm{Na}]^{+}: 456.1005$; found: 456.1012 .

FT-IR: $v\left[\mathrm{~cm}^{-1}\right]=3305,1754,1651,1549,1359,1239,1190,1099,1018,914,899$, 714.

1,1,1,3,3,3-Hexafluoropropan-2-yl 5-((2-(furan-3-yl)ethyl)amino)-5-oxopentanoate (11u).

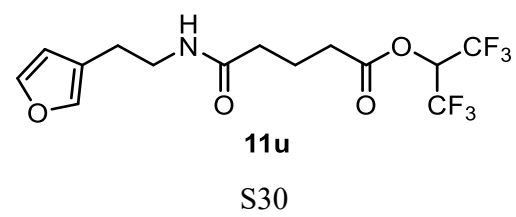


11u was prepared according to General procedure A, starting from 2-(furan-3yl)ethan-1-amine (180 mg, $1.62 \mathrm{mmol}, 1.0$ equiv.) and glutaric anhydride (185 mg, $1.62 \mathrm{mmol}, 1.0$ equiv.) in DMF $(5.0 \mathrm{~mL})$. The reaction mixture was subsequently treated with EDCI (620 mg, 3.24 mmol, 2.0 equiv.), DMAP (39.6 mg, 0.324 mmol, 0.2 equiv.) and HFIP ( $857 \mu \mathrm{L}, 8.11 \mathrm{mmol}, 5.0$ equiv.). Purification by silica gel column chromatography (pentane/EtOAc $=5: 2)$ afforded $11 \mathbf{u}(200 \mathrm{mg}, 0.533 \mathrm{mmol}, 33 \%)$ as a light yellow solid.

${ }^{1} \mathbf{H}$ NMR $\left(700 \mathrm{MHz}, \mathrm{CDCl}_{3}\right) \delta[\mathrm{ppm}]=7.36(\mathrm{t}, J=1.7 \mathrm{~Hz}, 1 \mathrm{H}), 7.25-7.24(\mathrm{~m}, 1 \mathrm{H})$, $6.27(\mathrm{dd}, J=1.9,0.9 \mathrm{~Hz}, 1 \mathrm{H}), 5.77-5.73(\mathrm{~m}, 1 \mathrm{H}), 5.63(\mathrm{~s}, 1 \mathrm{H}), 3.44(\mathrm{td}, J=6.9,5.8$ $\mathrm{Hz}, 2 \mathrm{H}), 2.62(\mathrm{td}, J=6.9,1.0 \mathrm{~Hz}, 2 \mathrm{H}), 2.56(\mathrm{t}, J=7.3 \mathrm{~Hz}, 2 \mathrm{H}), 2.20(\mathrm{t}, J=7.3 \mathrm{~Hz}, 2 \mathrm{H})$, $2.01-1.97(\mathrm{~m}, 2 \mathrm{H})$.

${ }^{13} \mathbf{C ~ N M R}\left(176 \mathrm{MHz}, \mathrm{CDCl}_{3}\right) \delta[\mathrm{ppm}]=171.5,170.0,143.4,139.7,121.8,120.5(\mathrm{q}, J$ $=282.0 \mathrm{~Hz}), 110.8,66.9-66.1(\mathrm{~m}), 39.5,34.8,32.4,25.0,20.4$.

${ }^{19}$ F NMR $\left(565 \mathrm{MHz}, \mathrm{CDCl}_{3}\right) \delta[\mathrm{ppm}]=-73.29(\mathrm{~d}, J=6.5 \mathrm{~Hz})$.

HRMS-ESI: calcd. for $\mathrm{C}_{14} \mathrm{H}_{15} \mathrm{~F}_{6} \mathrm{NO}_{4} \mathrm{Na}[\mathrm{M}+\mathrm{Na}]^{+}$: 398.0797; found: 398.0811 .

FT-IR: $v\left[\mathrm{~cm}^{-1}\right]=2920,2850,1780,1659,1447,1386,1288,1227,1198,1110,924$, 789.

\section{1,1,1,3,3,3-Hexafluoropropan-2-yl 5-((2-(furan-2-yl)ethyl)amino)-5-oxopentano- ate (11v).}

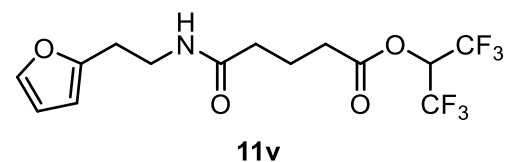

11v was prepared according to General procedure A, starting from 2-(furan-2yl)ethan-1-amine (180 mg, $1.62 \mathrm{mmol}, 1.0$ equiv.) and glutaric anhydride (185 mg, $1.62 \mathrm{mmol}, 1.0$ equiv.) in DMF $(5.0 \mathrm{~mL})$. The reaction mixture was subsequently treated with EDCI (620 mg, 3.24 mmol, 2.0 equiv.), DMAP (39.6 mg, 0.324 mmol, 0.2 equiv.) and HFIP ( $857 \mu \mathrm{L}, 8.11 \mathrm{mmol}, 5.0$ equiv.). Purification by silica gel column chromatography (pentane/EtOAc $=3: 2)$ afforded $11 \mathbf{v}(250 \mathrm{mg}, 0.667 \mathrm{mmol}, 41 \%)$ as a light yellow solid. 
${ }^{1} \mathbf{H}$ NMR $\left(700 \mathrm{MHz}, \mathrm{CDCl}_{3}\right) \delta[\mathrm{ppm}]=7.33(\mathrm{dd}, J=1.9,0.8 \mathrm{~Hz}, 1 \mathrm{H}), 6.32-6.28(\mathrm{~m}$, 1H), 6.07-6.06 (m, 1H), 5.78-5.74 (m, 1H), $5.58(\mathrm{~s}, 1 \mathrm{H}), 3.59-3.49(\mathrm{~m}, 2 \mathrm{H}), 2.85(\mathrm{td}$, $J=6.5,0.8 \mathrm{~Hz}, 2 \mathrm{H}), 2.58(\mathrm{t}, J=7.3 \mathrm{~Hz}, 2 \mathrm{H}), 2.22$ (t, $J=7.2 \mathrm{~Hz}, 2 \mathrm{H}), 2.06-1.97$ (m, $2 \mathrm{H})$.

${ }^{13} \mathbf{C}$ NMR $\left(176 \mathrm{MHz}, \mathrm{CDCl}_{3}\right) \delta[\mathrm{ppm}]=171.5,170.1,153.1,141.8,120.6(\mathrm{~d}, J=281.9$ $\mathrm{Hz}), 110.5,106.6,66.7-66.3$ (m), 38.3, 34.9, 32.4, 28.2, 20.4.

${ }^{19}$ F NMR $\left(376 \mathrm{MHz}, \mathrm{CDCl}_{3}\right) \delta[\mathrm{ppm}]=-73.19(\mathrm{~d}, J=6.0 \mathrm{~Hz})$.

HRMS-ESI: calcd. for $\mathrm{C}_{14} \mathrm{H}_{15} \mathrm{~F}_{6} \mathrm{NO}_{4} \mathrm{Na}[\mathrm{M}+\mathrm{Na}]^{+}$: 398.0797; found: 398.0786.

FT-IR: $v\left[\mathrm{~cm}^{-1}\right]=3314,2930,1781,1647,1551,1386,1288,1232,1200,1111,907$, 780.

\section{1,1,1,3,3,3-Hexafluoropropan-2-yl 5-oxo-5-((2-(thiophen-3-yl)ethyl)amino)penta- noate (11w).}<smiles>O=C(CCCC(=O)OC(C(F)(F)F)C(F)(F)F)NCCc1ccsc1</smiles>

11w was prepared according to General procedure A, starting from 2-(thiophen-3yl)ethan-1-amine (150 mg, $1.18 \mathrm{mmol}, 1.0$ equiv.) and glutaric anhydride (135 mg, $1.18 \mathrm{mmol}, 1.0$ equiv.) in DMF $(5.0 \mathrm{~mL})$. The reaction mixture was subsequently treated with EDCI (451 mg, $2.36 \mathrm{mmol}, 2.0$ equiv.), DMAP (28.9 mg, $0.236 \mathrm{mmol}$, 0.2 equiv.) and HFIP (624 $\mu \mathrm{L}, 5.91 \mathrm{mmol}, 5.0$ equiv.). Purification by silica gel column chromatography (pentane/EtOAc = 3:2) afforded $\mathbf{1 1} \mathbf{w}(200 \mathrm{mg}, 0.512 \mathrm{mmol}, 43 \%)$ as a white solid.

${ }^{1} \mathbf{H}$ NMR $\left(500 \mathrm{MHz}, \mathrm{CDCl}_{3}\right) \delta[\mathrm{ppm}]=7.29-7.24(\mathrm{~m}, 1 \mathrm{H}), 6.97-6.98(\mathrm{~m}, 1 \mathrm{H}), 6.93$ $(\mathrm{dd}, J=4.9,1.3 \mathrm{~Hz}, 1 \mathrm{H}), 5.77-5.71(\mathrm{~m}, 1 \mathrm{H}), 5.63(\mathrm{~s}, 1 \mathrm{H}), 3.53-3.48(\mathrm{~m}, 2 \mathrm{H}), 2.83(\mathrm{t}$, $J=6.9 \mathrm{~Hz}, 2 \mathrm{H}), 2.54(\mathrm{td}, J=7.2,0.8 \mathrm{~Hz}, 2 \mathrm{H}), 2.18(\mathrm{t}, J=7.2 \mathrm{~Hz}, 2 \mathrm{H}), 2.05-1.93(\mathrm{~m}$, $2 \mathrm{H})$.

${ }^{13}$ C NMR $\left(126 \mathrm{MHz}, \mathrm{CDCl}_{3}\right) \delta[\mathrm{ppm}]=171.5,170.0,139.1,128.1,126.2,121.5,120.5$ $(\mathrm{q}, J=282.0 \mathrm{~Hz}), 67.0-65.9(\mathrm{~m}), 39.9,34.8,32.3,30.3,20.4$.

${ }^{19}$ F NMR $\left(471 \mathrm{MHz}, \mathrm{CDCl}_{3}\right) \delta[\mathrm{ppm}]=-73.24(\mathrm{~d}, J=6.4 \mathrm{~Hz})$. 
HRMS-ESI: calcd. for $\mathrm{C}_{14} \mathrm{H}_{15} \mathrm{~F}_{6} \mathrm{NO}_{3} \mathrm{SK}[\mathrm{M}+\mathrm{K}]^{+}$: 430.0309; found: 430.0328 .

FT-IR: $v\left[\mathrm{~cm}^{-1}\right]=3319,1770,1638,1534,1385,1281,1201,1106,720$.

\section{1,1,1,3,3,3-Hexafluoropropan-2-yl 5-((2-(benzo[b]thiophen-3-yl)ethyl)amino)-5- oxopentanoate (11x).}

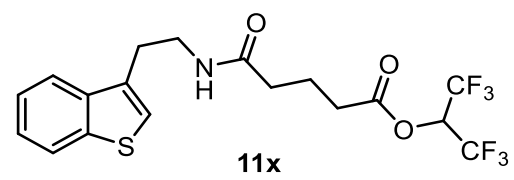

11x was prepared according to General procedure A, starting from 2(benzo[b]thiophen-3-yl)ethan-1-amine (140 mg, $0.791 \mathrm{mmol}, 1.0$ equiv.) and glutaric anhydride (90.2 mg, $0.791 \mathrm{mmol}, 1.0$ equiv.) in DMF (3.0 mL). The reaction mixture was subsequently treated with EDCI (302 mg, $1.58 \mathrm{mmol}, 2.0$ equiv.), DMAP (19.3 mg, $0.158 \mathrm{mmol}, 0.2$ equiv.) and HFIP (418 $\mu \mathrm{L}, 3.95 \mathrm{mmol}, 5.0$ equiv.). Purification by silica gel column chromatography (pentane/EtOAc $=2: 1)$ afforded 11x $(183 \mathrm{mg}$, $0.415 \mathrm{mmol}, 52 \%$ ) as a white solid.

${ }^{1} \mathbf{H}$ NMR $\left(700 \mathrm{MHz}, \mathrm{CDCl}_{3}\right) \delta[\mathrm{ppm}]=7.86(\mathrm{dt}, J=7.8,1.0 \mathrm{~Hz}, 1 \mathrm{H}), 7.77(\mathrm{dt}, J=8.0$, $1.0 \mathrm{~Hz}, 1 \mathrm{H}), 7.39$ (ddd, $J=8.0,7.0,1.2 \mathrm{~Hz}, 1 \mathrm{H}), 7.35$ (ddd, $J=8.2,7.0,1.3 \mathrm{~Hz}, 1 \mathrm{H})$, $7.14(\mathrm{~s}, 1 \mathrm{H}), 5.80-5.73(\mathrm{~m}, 2 \mathrm{H}), 3.60(\mathrm{td}, J=7.0,5.9 \mathrm{~Hz}, 2 \mathrm{H}), 3.05(\mathrm{td}, J=6.9,1.0 \mathrm{~Hz}$, 2H), $2.55(\mathrm{t}, J=7.3 \mathrm{~Hz}, 2 \mathrm{H}), 2.17(\mathrm{t}, J=7.3 \mathrm{~Hz}, 2 \mathrm{H}), 2.01-1.97(\mathrm{~m}, 2 \mathrm{H})$.

${ }^{13} \mathbf{C ~ N M R}\left(176 \mathrm{MHz}, \mathrm{CDCl}_{3}\right) \delta[\mathrm{ppm}]=171.6,170.0,140.6,138.8,133.4,124.5,124.2$, 123.0, 122.5, 121.6, 120.5 (q, $J=282.7 \mathrm{~Hz}), 66.9-66.1$ (m), 39.1, 34.8, 32.4, 28.7, 20.3. ${ }^{19}$ F NMR $\left(376 \mathrm{MHz}, \mathrm{CDCl}_{3}\right) \delta[\mathrm{ppm}]=-73.20$. (It should be a doublet peak, but due to the resolution, only singlet peak was observed.)

HRMS-ESI: calcd. for $\mathrm{C}_{18} \mathrm{H}_{17} \mathrm{~F}_{6} \mathrm{NO}_{3} \mathrm{SNa}[\mathrm{M}+\mathrm{Na}]^{+}$: 464.0725; found: 464.0745.

FT-IR: $v\left[\mathrm{~cm}^{-1}\right]=2928,2850,1736,1698,1215,747$.

\section{1,1,1,3,3,3-Hexafluoropropan-2-yl 5-((2-(6-methoxy-1H-indol-3-yl)ethyl)amino)- 3-methyl-5-oxopentanoate (11y).}




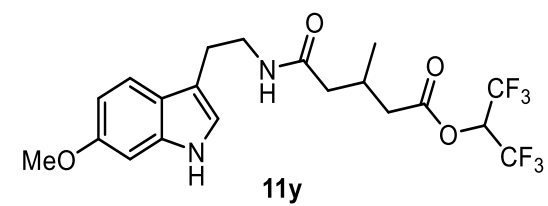

$11 y$ was prepared according to General procedure A, starting from 6methoxytrytamine (100 mg, $0.526 \mathrm{mmol}, 1.0$ equiv.) and glutaric anhydride (67.4 mg, $0.526 \mathrm{mmol}, 1.0$ equiv.) in DMF (3.0 mL). The reaction mixture was subsequently treated with EDCI (201 mg, $1.05 \mathrm{mmol}, 2.0$ equiv.), DMAP (12.9 mg, $0.105 \mathrm{mmol}$, 0.2 equiv.) and HFIP (278 $\mu \mathrm{L}, 2.63 \mathrm{mmol}, 5.0$ equiv.). Purification by silica gel column chromatography (pentane/EtOAc $=3: 2)$ afforded $11 \mathbf{y}(150 \mathrm{mg}, 0.320 \mathrm{mmol}, 61 \%)$ as a white solid.

${ }^{1} \mathbf{H}$ NMR $\left(700 \mathrm{MHz}, \mathrm{CDCl}_{3}\right) \delta[\mathrm{ppm}]=8.19(\mathrm{~s}, 1 \mathrm{H}), 7.44(\mathrm{~d}, J=8.6 \mathrm{~Hz}, 1 \mathrm{H}), 6.89$ $6.87(\mathrm{~m}, 1 \mathrm{H}), 6.84(\mathrm{~d}, J=2.3 \mathrm{~Hz}, 1 \mathrm{H}), 6.79(\mathrm{dd}, J=8.6,2.3 \mathrm{~Hz}, 1 \mathrm{H}), 5.79-5.75(\mathrm{~m}$, $1 \mathrm{H}), 5.66(\mathrm{~s}, 1 \mathrm{H}), 3.83(\mathrm{~d}, J=0.7 \mathrm{~Hz}, 3 \mathrm{H}), 3.58(\mathrm{dt}, J=8.5,6.8 \mathrm{~Hz}, 2 \mathrm{H}), 2.95-2.89(\mathrm{~m}$, 2H), 2.65-2.59 (m, 1H), 2.52-2.48 (m, 1H), 2.48-2.34 (m, 1H), $2.13(\mathrm{dd}, J=14.3,6.9$ Hz, 1H), 2.03 (ddd, $J=14.5,7.4,1.7 \mathrm{~Hz}, 1 \mathrm{H}), 0.99$ (d, $J=6.8 \mathrm{~Hz}, 3 \mathrm{H})$.

${ }^{13} \mathbf{C ~ N M R}\left(176 \mathrm{MHz}, \mathrm{CDCl}_{3}\right) \delta[\mathrm{ppm}]=171.1,169.4,156.8,137.3,121.9,120.9,120.5$ (q, $J=282.4 \mathrm{~Hz}), 119.3,112.9,109.6,94.9,66.8-66.0(\mathrm{~m}), 55.8,42.8,39.8,39.5,27.9$, $25.5,19.4$.

${ }^{19}$ F NMR $\left(565 \mathrm{MHz}, \mathrm{CDCl}_{3}\right) \delta[\mathrm{ppm}]=-73.11(\mathrm{~d}, J=7.7 \mathrm{~Hz})$.

HRMS-ESI: calcd. for $\mathrm{C}_{20} \mathrm{H}_{22} \mathrm{~F}_{6} \mathrm{~N}_{2} \mathrm{O}_{4} \mathrm{Na}[\mathrm{M}+\mathrm{Na}]^{+}$: 491.1374; found: 491.1376 .

FT-IR: $v\left[\mathrm{~cm}^{-1}\right]$ 2958, 1776, 1648, 1386, 1288, 1200, 1110, 937, 812.

\subsubsection{Additional substrates}

\section{1,1,1,3,3,3-Hexafluoropropan-2-yl 4-((2-(1H-indol-3-yl)ethyl)amino)-4-oxobuta- noate (S1).}

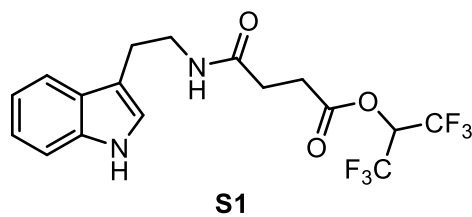

S1 was prepared according to General procedure A, starting from tryptamine ( $250 \mathrm{mg}$, $1.56 \mathrm{mmol}, 1.0$ equiv.) and succinic anhydride (156 mg, $1.56 \mathrm{mmol}, 1.0$ equiv.) in DMF 
$(5.0 \mathrm{~mL})$. The reaction mixture was subsequently treated with EDCI $(328 \mathrm{mg}$, $1.72 \mathrm{mmol}, 2.0$ equiv.), DMAP (38.2 mg, $0.313 \mathrm{mmol}, 0.2$ equiv.) and HFIP (825 $\mu \mathrm{L}$, 7.81 mmol, 5.0 equiv.). Purification by silica gel column chromatography (pentane/EtOAc $=3: 2)$ afforded $\mathbf{S 1}(286 \mathrm{mg}, 0.698 \mathrm{mmol}, 45 \%)$ as a white solid.

${ }^{1} \mathbf{H}$ NMR $\left(600 \mathrm{MHz}, \mathrm{CDCl}_{3}\right) \delta[\mathrm{ppm}]=8.22(\mathrm{~s}, 1 \mathrm{H}), 7.59(\mathrm{dd}, J=7.9,1.1 \mathrm{~Hz}, 1 \mathrm{H})$, $7.37(\mathrm{dt}, J=8.1,0.9 \mathrm{~Hz}, 1 \mathrm{H}), 7.22(\mathrm{ddd}, J=8.2,7.0,1.2 \mathrm{~Hz}, 1 \mathrm{H}), 7.13$ (ddd, $J=8.0$, 7.0, $1.0 \mathrm{~Hz}, 1 \mathrm{H}), 7.01(\mathrm{~d}, J=2.3 \mathrm{~Hz}, 1 \mathrm{H}), 5.79-5.75(\mathrm{~m}, 1 \mathrm{H}), 5.63(\mathrm{~d}, J=5.6 \mathrm{~Hz}, 1 \mathrm{H})$, $3.60(\mathrm{q}, J=6.5 \mathrm{~Hz}, 2 \mathrm{H}), 2.96(\mathrm{td}, J=6.8,0.9 \mathrm{~Hz}, 2 \mathrm{H}), 2.84(\mathrm{t}, J=6.9 \mathrm{~Hz}, 2 \mathrm{H}), 2.44(\mathrm{t}$, $J=6.9 \mathrm{~Hz}, 2 \mathrm{H})$.

${ }^{13} \mathbf{C ~ N M R}\left(151 \mathrm{MHz}, \mathrm{CDCl}_{3}\right) \delta[\mathrm{ppm}]=170.2,170.0,136.6,127.4,122.4,122.2,120.5$ $(\mathrm{d}, J=283.0 \mathrm{~Hz}), 119.6,118.8,112.9,111.4,67.2-66.3$ (m), 40.1, 30.4, 28.7, 25.3.

${ }^{19}$ F NMR $\left(565 \mathrm{MHz}, \mathrm{CDCl}_{3}\right) \delta[\mathrm{ppm}]=-73.14(\mathrm{~d}, J=6.6 \mathrm{~Hz})$.

HRMS-ESI: calcd. for $\mathrm{C}_{17} \mathrm{H}_{16} \mathrm{~F}_{6} \mathrm{~N}_{2} \mathrm{O}_{3} \mathrm{Na}[\mathrm{M}+\mathrm{Na}]^{+}$: 433.0957 ; found: 433.0974 .

FT-IR: $v\left[\mathrm{~cm}^{-1}\right]=3422,3328,1754,1633,1384,1286,1201,1108,905,738$.

\section{1,1,1,3,3,3-Hexafluoropropan-2-yl $\quad 5$-(((1H-indol-3-yl)methyl)amino)-5-oxopent- anoate (S2).}

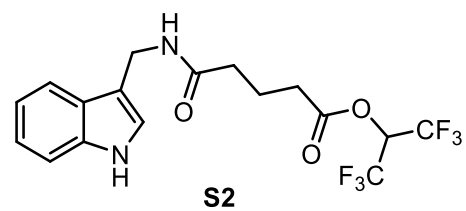

S2 was prepared according to General procedure A, starting from $1 H$-indole-3methanamine (310 mg, $2.12 \mathrm{mmol}, 1.0$ equiv.) and glutaric anhydride (242 mg, $2.12 \mathrm{mmol}, 1.0$ equiv.) in DMF $(10.0 \mathrm{~mL})$. The reaction mixture was subsequently treated with EDCI (811 mg, $4.25 \mathrm{mmol}, 2.0$ equiv.), DMAP (51.9 mg, $0.425 \mathrm{mmol}$, 0.2 equiv.) and HFIP (1.12 mL, $10.6 \mathrm{mmol}, 5.0$ equiv.). Purification by silica gel column chromatography (pentane/EtOAc $=3: 2)$ afforded $\mathbf{S 2}(300 \mathrm{mg}, 0.732 \mathrm{mmol}, 34 \%)$ as a white solid.

${ }^{1} \mathbf{H}$ NMR $\left(700 \mathrm{MHz} \mathrm{CDCl}_{3}\right) \delta[\mathrm{ppm}]=8.28(\mathrm{~s}, 1 \mathrm{H}), 7.62-7.60(\mathrm{~m}, 1 \mathrm{H}), 7.39(\mathrm{dt}, J=$ 8.1, 0.9 Hz, 1H), $7.23(\mathrm{ddd}, J=8.1,7.0,1.2 \mathrm{~Hz}, 1 \mathrm{H}), 7.18-7.12(\mathrm{~m}, 2 \mathrm{H}), 5.79-5.72(\mathrm{~m}$, 1H), $5.70(\mathrm{~s}, 1 \mathrm{H}), 4.62(\mathrm{dd}, J=5.2,0.8 \mathrm{~Hz}, 2 \mathrm{H}), 2.60(\mathrm{t}, J=7.3 \mathrm{~Hz}, 2 \mathrm{H}), 2.23(\mathrm{t}, J=$ 
$7.2 \mathrm{~Hz}, 2 \mathrm{H}), 2.07-2.03(\mathrm{~m}, 2 \mathrm{H})$.

${ }^{13} \mathbf{C ~ N M R}\left(176 \mathrm{MHz}, \mathrm{CDCl}_{3}\right) \delta[\mathrm{ppm}]=171.3,170.1,136.5,126.6,123.4,122.7,120.5$ $(\mathrm{d}, J=286.2 \mathrm{~Hz}), 120.1,118.8,112.7,111.5,66.9-66.1$ (m), 35.3, 34.9, 32.5, 20.5.

${ }^{19}$ F NMR $\left(565 \mathrm{MHz}, \mathrm{CDCl}_{3}\right) \delta[\mathrm{ppm}]=-73.20(\mathrm{~d}, J=6.3 \mathrm{~Hz})$.

HRMS-ESI: calcd. for $\mathrm{C}_{17} \mathrm{H}_{16} \mathrm{~F}_{6} \mathrm{~N}_{2} \mathrm{O}_{3} \mathrm{Na}[\mathrm{M}+\mathrm{Na}]^{+}$: 433.0957; found: 433.0972 .

FT-IR: $v\left[\mathrm{~cm}^{-1}\right]=3259,1738,1660,1364,1204,1108,744$.

\section{1,1,1,3,3,3-Hexafluoropropan-2-yl 10-((2-(1H-indol-3-yl)ethyl)amino)-10-oxodec- anoate (S3).}

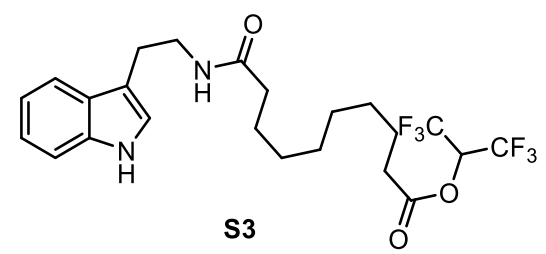

S3 was prepared according to General procedure A, starting from tryptamine (200 $\mathrm{mg}$, $1.25 \mathrm{mmol}, 1.0$ equiv.) and oxacycloundecane-2,11-dione (230 mg, $1.25 \mathrm{mmol}$, 1.0 equiv. $)$ in DMF $(5.0 \mathrm{~mL})$. The reaction mixture was subsequently treated with EDCI (477 mg, 2.50 mmol, 2.0 equiv.), DMAP (30.5 mg, $0.250 \mathrm{mmol}, 0.2$ equiv.) and HFIP (660 $\mu \mathrm{L}, 6.25 \mathrm{mmol}, 5.0$ equiv.). Purification by silica gel column chromatography (pentane/EtOAc $=3: 2)$ afforded $\mathbf{S 3}(150 \mathrm{mg}, 0.304 \mathrm{mmol}, 24 \%)$ as a white solid.

${ }^{1} \mathbf{H}$ NMR $\left(500 \mathrm{MHz}, \mathrm{CDCl}_{3}\right) \delta[\mathrm{ppm}]=8.72(\mathrm{~s}, 1 \mathrm{H}), 7.60(\mathrm{dd}, J=8.0,1.1 \mathrm{~Hz}, 1 \mathrm{H})$, 7.39-7.34 (m, 1H), 7.20 (ddd, $J=8.1,7.0,1.2 \mathrm{~Hz}, 1 \mathrm{H}), 7.11$ (ddd, $J=8.0,7.0,1.0 \mathrm{~Hz}$, $1 \mathrm{H}), 6.98(\mathrm{~d}, J=2.0 \mathrm{~Hz}, 1 \mathrm{H}), 5.85-5.67(\mathrm{~m}, 2 \mathrm{H}), 3.62-3.58(\mathrm{~m}, 2 \mathrm{H}), 2.97(\mathrm{t}, J=6.8$ $\mathrm{Hz}, 2 \mathrm{H}), 2.49(\mathrm{t}, J=7.4 \mathrm{~Hz}, 2 \mathrm{H}), 2.10(\mathrm{t}, J=7.6 \mathrm{~Hz}, 2 \mathrm{H}), 1.69-1.65(\mathrm{~m}, 2 \mathrm{H}), 1.62-$ $1.56(\mathrm{~m}, 2 \mathrm{H}), 1.32-1.25(\mathrm{~m}, 8 \mathrm{H})$.

${ }^{13} \mathbf{C ~ N M R}\left(126 \mathrm{MHz}, \mathrm{CDCl}_{3}\right) \delta[\mathrm{ppm}]=173.4,170.5,136.6,127.5,122.3,122.1,120.5$ (q, $J=282.5 \mathrm{~Hz}), 119.4,118.7,112.8,111.5,66.9-65.8(\mathrm{~m}), 39.9,36.8,33.3,29.2,29.1$, $28.9,28.7,25.7,25.4,24.5$.

${ }^{19}$ F NMR $\left(471 \mathrm{MHz}, \mathrm{CDCl}_{3}\right) \delta[\mathrm{ppm}]=-73.26(\mathrm{~d}, J=5.7 \mathrm{~Hz})$.

HRMS-ESI: calcd. for $\mathrm{C}_{23} \mathrm{H}_{28} \mathrm{~F}_{6} \mathrm{~N}_{2} \mathrm{O}_{3} \mathrm{Na}[\mathrm{M}+\mathrm{Na}]^{+}:$: 17.1896 ; found: 517.1921 .

FT-IR: $v\left[\mathrm{~cm}^{-1}\right]=2939,2921,2146,2042,1779,1649,1289,1228,1202,1111,760$. 


\section{1,1,1,3,3,3-Hexafluoropropan-2-yl (S)-5-((3-(4-hydroxyphenyl)-1-methoxy-1-oxo-}

propan-2-yl)amino)-5-oxopentanoate (S4).<smiles>O=C(CCCC(=O)OC(C(F)(F)F)C(F)(F)F)N[C@@H](Cc1ccc(O)cc1)C(=O)O</smiles>

S4 was prepared according to General procedure A, starting from $(S)$-tyrosine methyl ester (300 mg, $1.54 \mathrm{mmol}, 1.0$ equiv.) and glutaric anhydride (175 mg, $1.54 \mathrm{mmol}$, 1.0 equiv.) in DMF $(10.0 \mathrm{~mL})$. The reaction mixture was subsequently treated with EDCI (588 mg, $3.01 \mathrm{mmol}, 2.0$ equiv.), DMAP (37.6 mg, $0.308 \mathrm{mmol}, 0.2$ equiv.) and HFIP (813 $\mu \mathrm{L}, 7.69$ mmol, 5.0 equiv.). Purification by silica gel column chromatography (pentane/EtOAc $=3: 2)$ afforded $\mathbf{S 4}(170 \mathrm{mg}, 0.370 \mathrm{mmol}, 24 \%)$ as a white solid.

${ }^{1} \mathbf{H}$ NMR $\left(500 \mathrm{MHz}, \mathrm{CDCl}_{3}\right) \delta[\mathrm{ppm}]=7.04(\mathrm{~s}, 1 \mathrm{H}), 6.95-6.89(\mathrm{~m}, 2 \mathrm{H}), 6.75-6.69(\mathrm{~m}$, 2H), $6.15(\mathrm{~d}, J=8.1 \mathrm{~Hz}, 1 \mathrm{H}), 5.79-5.74(\mathrm{~m}, 1 \mathrm{H}), 4.85(\mathrm{ddd}, J=8.1,6.4,5.6 \mathrm{~Hz}, 1 \mathrm{H})$, $3.73(\mathrm{~s}, 3 \mathrm{H}), 3.07(\mathrm{dd}, J=14.1,5.5 \mathrm{~Hz}, 1 \mathrm{H}), 2.95(\mathrm{dd}, J=14.1,6.5 \mathrm{~Hz}, 1 \mathrm{H}), 2.51(\mathrm{td}$, $J=7.3,1.3 \mathrm{~Hz}, 2 \mathrm{H}), 2.25(\mathrm{t}, J=7.5 \mathrm{~Hz}, 2 \mathrm{H}), 1.99-1.93(\mathrm{~m}, 2 \mathrm{H})$.

${ }^{13} \mathbf{C ~ N M R}\left(126 \mathrm{MHz}, \mathrm{CDCl}_{3}\right) \delta[\mathrm{ppm}]=172.5,172.0,170.0,155.7,130.3,127.0,120.5$ $(\mathrm{q}, J=284.3 \mathrm{~Hz}), 115.7,67.1-66.0(\mathrm{~m}), 53.4,52.6,37.2,34.7,32.2,20.2$.

${ }^{19}$ F NMR $\left(471 \mathrm{MHz}, \mathrm{CDCl}_{3}\right) \delta[\mathrm{ppm}]=-73.24(\mathrm{~d}, J=6.7 \mathrm{~Hz})$.

HRMS-ESI: calcd. for $\mathrm{C}_{18} \mathrm{H}_{19} \mathrm{~F}_{6} \mathrm{NO}_{6} \mathrm{~K}[\mathrm{M}+\mathrm{K}]^{+}$: 498.0749 ; found: 498.0774 .

FT-IR: $v\left[\mathrm{~cm}^{-1}\right]=2960,1780,1740,1651,1516,1286,1223,1198,1108,905,733$. $[\boldsymbol{\alpha}]_{\mathbf{D}}^{\mathbf{2 6}}=+19.0\left(c=0.4, \mathrm{CHCl}_{3}\right)$.

\section{1,1,1,3,3,3-Hexafluoropropan-2-yl 5-oxo-5-(phenethylamino)pentanoate (S5).}<smiles>O=C(CCCC(=O)OC(C(F)(F)F)C(F)(F)F)NCCc1ccccc1</smiles>

S5 was prepared according to General procedure A, starting from phenethylamine S37 
(200 mg, $1.65 \mathrm{mmol}, 1.0$ equiv.) and glutaric anhydride (188 mg, $1.65 \mathrm{mmol}, 1.0$ equiv.) in DMF $(5.0 \mathrm{~mL})$. The reaction mixture was subsequently treated with EDCI (631 mg, $3.31 \mathrm{mmol}, 2.0$ equiv.), DMAP (40.4 mg, $0.331 \mathrm{mmol}, 0.2$ equiv.) and HFIP (873 $\mu \mathrm{L}$, 8.26 mmol, 5.0 equiv.). Purification by silica gel column chromatography (pentane/EtOAc $=5: 2)$ afforded S5 (200 $\mathrm{mg}, 0.519 \mathrm{mmol}, 31 \%)$ as a white solid.

${ }^{1} \mathbf{H}$ NMR $\left(500 \mathrm{MHz}, \mathrm{CDCl}_{3}\right) \delta[\mathrm{ppm}]=7.33-7.28(\mathrm{~m}, 2 \mathrm{H}), 7.26-7.21(\mathrm{~m}, 1 \mathrm{H}), 7.18$ (dt, $J=7.9,1.7 \mathrm{~Hz}, 2 \mathrm{H}), 7.79-7.73(\mathrm{~m}, 1 \mathrm{H}), 5.54(\mathrm{~s}, 1 \mathrm{H}), 3.58-3.48(\mathrm{~m}, 2 \mathrm{H}), 2.81(\mathrm{td}$, $J=7.0,1.7 \mathrm{~Hz}, 2 \mathrm{H}), 2.54(\mathrm{td}, J=7.3,1.8 \mathrm{~Hz}, 2 \mathrm{H}), 2.18(\mathrm{td}, J=7.2,1.8 \mathrm{~Hz}, 2 \mathrm{H}), 2.02$ $1.97(\mathrm{~m}, 2 \mathrm{H})$.

${ }^{13} \mathbf{C ~ N M R}\left(126 \mathrm{MHz}, \mathrm{CDCl}_{3}\right) \delta[\mathrm{ppm}]=171.5,170.1,138.8,128.84,128.78,126.7$, $120.5(\mathrm{~d}, J=282.0 \mathrm{~Hz}), 67.0-65.9(\mathrm{~m}), 40.6,35.7,34.8,32.4,20.4$.

${ }^{19}$ F NMR $\left(471 \mathrm{MHz}, \mathrm{CDCl}_{3}\right) \delta[\mathrm{ppm}]=-73.22(\mathrm{~d}, J=6.3 \mathrm{~Hz})$.

HRMS-ESI: calcd. for $\mathrm{C}_{16} \mathrm{H}_{17} \mathrm{~F}_{6} \mathrm{NO}_{3} \mathrm{Na}[\mathrm{M}+\mathrm{Na}]^{+}$: 408.1005 ; found: 408.1007 .

FT-IR: $v\left[\mathrm{~cm}^{-1}\right]=3309,1967,1645,1543,1282,1268,1107$.

\section{1,1,1,3,3,3-Hexafluoropropan-2-yl 5-((4-methoxyphenethyl)amino)-5-oxopenta- noate (S6).}<smiles>COc1ccc(CCNC(=O)CCCC(=O)OC(C(F)(F)F)C(F)(F)F)cc1</smiles>

S6 was prepared according to General procedure A, starting from 4methoxyphenethylamine (200 mg, $1.32 \mathrm{mmol}, 1.0$ equiv.) and glutaric anhydride (151 mg, $1.32 \mathrm{mmol}, 1.0$ equiv.) in DMF $(5.0 \mathrm{~mL})$. The reaction mixture was subsequently treated with EDCI (506 mg, $2.65 \mathrm{mmol}, 2.0$ equiv.), DMAP (32.4 mg, 0.365 mmol, 0.2 equiv.) and HFIP (699 $\mu \mathrm{L}, 6.62 \mathrm{mmol}, 5.0$ equiv.). Purification by silica gel column chromatography (pentane/EtOAc $=5: 2)$ afforded S6 $(225 \mathrm{mg}$, $0.542 \mathrm{mmol}, 41 \%$ ) as a white solid.

${ }^{1} \mathbf{H}$ NMR $\left(500 \mathrm{MHz}, \mathrm{CDCl}_{3}\right) \delta[\mathrm{ppm}]=7.08(\mathrm{~d}, J=8.6 \mathrm{~Hz}, 2 \mathrm{H}), 6.83(\mathrm{~d}, J=8.6 \mathrm{~Hz}$, 2H), 5.78-5.73 (m, 1H), $5.63(\mathrm{~s}, 1 \mathrm{H}), 3.77(\mathrm{~s}, 3 \mathrm{H}), 3.46(\mathrm{td}, J=7.0,5.8 \mathrm{~Hz}, 2 \mathrm{H}), 2.74$ (t, $J=7.0 \mathrm{~Hz}, 2 \mathrm{H}), 2.55(\mathrm{t}, J=7.3 \mathrm{~Hz}, 2 \mathrm{H}), 2.18(\mathrm{t}, J=7.2 \mathrm{~Hz}, 2 \mathrm{H}), 2.01-1.97(\mathrm{~m}$, 
$2 \mathrm{H})$.

${ }^{13} \mathbf{C ~ N M R}\left(126 \mathrm{MHz}, \mathrm{CDCl}_{3}\right) \delta[\mathrm{ppm}]=171.5,170.0,158.4,130.8,129.8,120.5(\mathrm{q}, J$ $=279.4 \mathrm{~Hz}), 114.1,67.0-65.9(\mathrm{~m}), 55.30,55.28,40.8,34.8,32.4,20.4$.

${ }^{19}$ F NMR $\left(471 \mathrm{MHz}, \mathrm{CDCl}_{3}\right) \delta[\mathrm{ppm}]=-73.25(\mathrm{~d}, J=8.6 \mathrm{~Hz})$.

HRMS-ESI: calcd. for $\mathrm{C}_{17} \mathrm{H}_{19} \mathrm{~F}_{6} \mathrm{NO}_{4} \mathrm{Na}[\mathrm{M}+\mathrm{Na}]^{+}$: 438.1110 ; found: 438.1109 .

FT-IR: $v\left[\mathrm{~cm}^{-1}\right]$ 3299, 1767, 1636, 1542, 1282, 1257, 1202, 1107, 1038.

\section{1,1,1,3,3,3-Hexafluoropropan-2-yl 5-0xo-5-((2-(pyridin-2-yl)ethyl)amino)penta- noate (S7).}

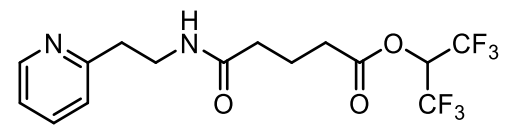

S7

S7 was prepared according to General procedure A, starting from 2-(pyridin-2yl)ethan-1-amine (300 mg, $2.46 \mathrm{mmol}, 1.0$ equiv.) and glutaric anhydride (280 mg, $2.46 \mathrm{mmol}, 1.0$ equiv.) in DMF $(10.0 \mathrm{~mL})$. The reaction mixture was subsequently treated with EDCI (939 mg, 4.92 mmol, 2.0 equiv.), DMAP (60.1 mg, 0.492 mmol, 0.2 equiv.) and HFIP (1.30 mL, $12.3 \mathrm{mmol}, 5.0$ equiv.). Purification by silica gel column chromatography (pentane/EtOAc $=3: 2)$ afforded $\mathbf{S} 7(670 \mathrm{mg}, 1.51 \mathrm{mmol}, 61 \%)$ as a colorless oil.

${ }^{1} \mathbf{H}$ NMR $\left(500 \mathrm{MHz}, \mathrm{CDCl}_{3}\right) \delta[\mathrm{ppm}]=8.44(\mathrm{ddd}, J=4.9,1.9,0.9 \mathrm{~Hz}, 1 \mathrm{H}), 7.56(\mathrm{td}, J$ $=7.7,1.9 \mathrm{~Hz}, 1 \mathrm{H}), 7.14-7.06(\mathrm{~m}, 2 \mathrm{H}), 6.75(\mathrm{~s}, 1 \mathrm{H}), 5.74-5.69(\mathrm{~m}, 1 \mathrm{H}), 3.60(\mathrm{dt}, J=$ 6.6, $5.7 \mathrm{~Hz}, 2 \mathrm{H}), 2.98-2.89(\mathrm{~m}, 2 \mathrm{H}), 2.50(\mathrm{t}, J=7.4 \mathrm{~Hz}, 2 \mathrm{H}), 2.17(\mathrm{t}, J=7.2 \mathrm{~Hz}, 2 \mathrm{H})$, $2.02-1.88(\mathrm{~m}, 2 \mathrm{H})$.

${ }^{13} \mathbf{C ~ N M R}\left(126 \mathrm{MHz}, \mathrm{CDCl}_{3}\right) \delta[\mathrm{ppm}]=171.5,170.0,159.6,149.1,136.7,123.5,121.6$, $120.4(\mathrm{q}, J=279.4 \mathrm{~Hz}), 66.9-65.8(\mathrm{~m}), 38.7,36.9,34.9,32.4,20.4$.

${ }^{19}$ F NMR $\left(471 \mathrm{MHz}, \mathrm{CDCl}_{3}\right) \delta[\mathrm{ppm}]=-73.19(\mathrm{~d}, J=6.5 \mathrm{~Hz})$.

HRMS-ESI: calcd. for $\mathrm{C}_{15} \mathrm{H}_{16} \mathrm{~F}_{6} \mathrm{~N}_{2} \mathrm{O}_{3} \mathrm{Na}[\mathrm{M}+\mathrm{Na}]^{+}$: 409.0957; found: 409.0972.

FT-IR: $v\left[\mathrm{~cm}^{-1}\right]=2950,1781,1652,1288,1230,1199,1110,747$. 


\subsubsection{Reaction optimization}

\begin{tabular}{ccc} 
then base or acid \\
entry \\
\hline $\mathbf{8 a :}: \mathrm{R}=$ methoxy
\end{tabular}

\subsubsection{Substrate scope}

\section{General procedure $C$}

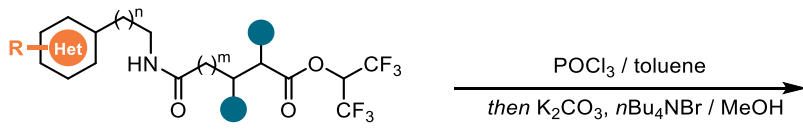

$8,11 a-x$

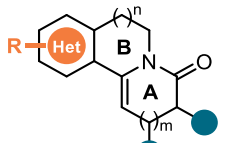

$10,12 a-x$

To a solution of the substrate $\mathbf{8}$ or $\mathbf{1 1 a}-\mathbf{x}$ (1.0 equiv.) in toluene was added $\mathrm{POCl}_{3}$ (1.0 equiv.) dropwise. Then the mixture was heated to $115^{\circ} \mathrm{C}$ (oil bath temperature) until the starting material was fully consumed. After allowing the mixture to cool down to $80{ }^{\circ} \mathrm{C}$ (oil bath temperature), the same volume of $\mathrm{MeOH}, \mathrm{K}_{2} \mathrm{CO}_{3}$ (10.0 equiv.) and $n \mathrm{Bu} 4 \mathrm{NBr}(0.1$ equiv.) were added subsequently. Afterwards the resulting mixture was stirred overnight at $80{ }^{\circ} \mathrm{C}$, cooled to room temperature, filtered through Celite ${ }^{\circledR}$, concentrated under reduced pressure, and purified by silica gel or neutral aluminium 
oxide column chromatography.

(Note: It is not necessary to perform the reaction under argon.)

Methyl 4-(4,9-dihydro-3H-pyrido[3,4-b]indol-1-yl)butanoate (9).

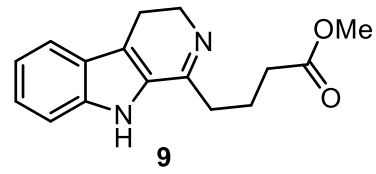

To a solution of the substrate $\mathbf{8 a}(100 \mathrm{mg}, 0.347 \mathrm{mmol}, 1.0$ equiv.) in toluene (3.0 mL), was added $\mathrm{POCl}_{3}\left(31.7 \mu \mathrm{L}, 0.347 \mathrm{mmol}, 1.0\right.$ equiv.). The mixture was heated to $115^{\circ} \mathrm{C}$ (oil bath temperature) until the starting material was fully consumed. Then the mixture was concentrated under reduced pressure and purified by silica gel flash column chromatography (DCM/MeOH=10:1). $9(79.0 \mathrm{mg}, 0.293 \mathrm{mmol}, 84 \%)$ was afforded as a dark yellow solid.

${ }^{1} \mathbf{H}$ NMR $\left(500 \mathrm{MHz}, \mathrm{CDCl}_{3}\right) \delta[\mathrm{ppm}]=11.39(\mathrm{~s}, 1 \mathrm{H}), 7.63(\mathrm{~d}, J=8.2 \mathrm{~Hz}, 1 \mathrm{H}), 7.59$ (dd, $J=8.5,0.9 \mathrm{~Hz}, 1 \mathrm{H}), 7.46(\mathrm{ddd}, J=8.4,6.9,1.2 \mathrm{~Hz}, 1 \mathrm{H}), 7.22$ (ddd, $J=8.1,6.9$, $1.4 \mathrm{~Hz}, 1 \mathrm{H}), 4.02(\mathrm{t}, J=8.8 \mathrm{~Hz}, 2 \mathrm{H}), 3.76(\mathrm{~s}, 3 \mathrm{H}), 3.36-3.28(\mathrm{~m}, 2 \mathrm{H}), 3.26-3.19(\mathrm{~m}$, 2H), 2.66-2.57 (m, 2H), 2.24-2.10 (m, 2H).

${ }^{13} \mathbf{C ~ N M R}\left(126 \mathrm{MHz}, \mathrm{CDCl}_{3}\right) \delta[\mathrm{ppm}]=173.9,169.3,141.7,129.1,125.7,124.3,124.0$, $122.0,121.4,114.1,52.0,42.4,32.8,31.7,23.4,19.5$.

HRMS-ESI: calcd. for $\mathrm{C}_{16} \mathrm{H}_{19} \mathrm{~N}_{2} \mathrm{O}_{2}[\mathrm{M}+\mathrm{H}]^{+}: 271.1441$; found: 271.1455 .

FT-IR: $v\left[\mathrm{~cm}^{-1}\right]=2925,1733,1630,1554,1434,1336,1229,747$.

\section{2,6,7,12-Tetrahydroindolo[2,3-a]quinolizin-4(3H)-one (10).}

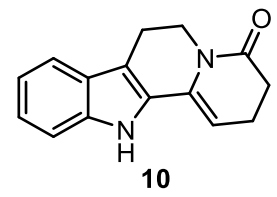

10 was prepared according to General procedure C, starting from 8d (100 mg, 0.236 mmol, 1.0 equiv.) and $\mathrm{POCl}_{3}(21.5 \mu \mathrm{L}, 0.236 \mathrm{mmol}, 1.0$ equiv.) in toluene $(3.0 \mathrm{~mL})$. The reaction mixture was subsequently treated with $\mathrm{MeOH}(3.0 \mathrm{~mL}), \mathrm{K}_{2} \mathrm{CO}_{3}$ (326 mg, $2.36 \mathrm{mmol}, 10$ equiv.) and $n \mathrm{Bu} 4 \mathrm{NBr}$ (7.6 mg, $24.0 \mu \mathrm{mol}, 0.1$ equiv.). 
Purification by silica gel column chromatography (pentane/EtOAc $=2: 1$ ) afforded 10 (51.0 mg, $0.214 \mathrm{mmol}, 90 \%)$ as a yellow solid.

Scale-up reaction starting from $8 \mathbf{d}\left(2.00 \mathrm{~g}, 4.72 \mathrm{mmol}, 1.0\right.$ equiv.) and $\mathrm{POCl}_{3}(430 \mu \mathrm{L}$, $4.72 \mathrm{mmol}, 1.0$ equiv.) in toluene $(80 \mathrm{~mL})$. The reaction mixture was subsequently treated with $\mathrm{MeOH}(80 \mathrm{~mL}), \mathrm{K}_{2} \mathrm{CO}_{3}(6.52 \mathrm{~g}, 47.2 \mathrm{mmol}, 10$ equiv.) and $n \mathrm{Bu} 4 \mathrm{NBr}$ (152 mg, 0.472 mmol, 0.1 equiv.). Purification by silica gel column chromatography (pentane/EtOAc $=2: 1)$ afforded $\mathbf{1 0}(910 \mathrm{mg}, 3.82 \mathrm{mmol}, 81 \%)$ as a yellow solid.

${ }^{1} \mathbf{H}$ NMR $\left(500 \mathrm{MHz}, \mathrm{CDCl}_{3}\right) \delta[\mathrm{ppm}]=7.97(\mathrm{~s}, 1 \mathrm{H}), 7.53-7.51(\mathrm{~m}, 1 \mathrm{H}), 7.34(\mathrm{dt}, J=$ 8.2, $0.9 \mathrm{~Hz}, 1 \mathrm{H}), 7.23(\mathrm{ddd}, J=8.2,7.0,1.1 \mathrm{~Hz}, 1 \mathrm{H}), 7.12(\mathrm{ddd}, J=8.0,7.1,1.0 \mathrm{~Hz}$, $1 \mathrm{H}), 5.49(\mathrm{t}, J=5.0 \mathrm{~Hz}, 1 \mathrm{H}), 4.11(\mathrm{t}, J=6.0 \mathrm{~Hz}, 2 \mathrm{H}), 2.91(\mathrm{t}, J=6.0 \mathrm{~Hz}, 2 \mathrm{H}), 2.70$ $2.59(\mathrm{~m}, 2 \mathrm{H}), 2.53-2.42(\mathrm{~m}, 2 \mathrm{H})$.

${ }^{13} \mathbf{C ~ N M R}\left(126 \mathrm{MHz}, \mathrm{CDCl}_{3}\right) \delta[\mathrm{ppm}]=169.8,137.3,131.4,128.1,126.9,123.6,120.2$, $119.1,112.0,111.1,99.4,39.3,31.9,20.8,19.6$.

HRMS-ESI: calcd. for $\mathrm{C}_{15} \mathrm{H}_{15} \mathrm{~N}_{2} \mathrm{O}[\mathrm{M}+\mathrm{H}]^{+}$: 239.1179; found: 239.1179.

FT-IR: $v\left[\mathrm{~cm}^{-1}\right]=3287,2926,1636,1436,1400,1232,1214,742$.

\section{9-Methoxy-2,6,7,12-tetrahydroindolo[2,3-a]quinolizin-4(3H)-one (12a).}

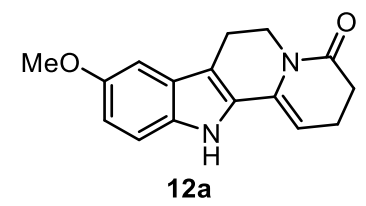

12a was prepared according to General procedure C, starting from 11a (100 mg, 0.220 mmol, 1.0 equiv.) and $\mathrm{POCl}_{3}(20.1 \mu \mathrm{L}, 0.220 \mathrm{mmol}, 1.0$ equiv.) in toluene $(3.0 \mathrm{~mL})$. The reaction mixture was subsequently treated with $\mathrm{MeOH}(3.0 \mathrm{~mL}), \mathrm{K}_{2} \mathrm{CO}_{3}$ (304 mg, $2.20 \mathrm{mmol}, 10$ equiv.) and $n \mathrm{Bu} 4 \mathrm{NBr}$ (7.1 mg, $22.0 \mu \mathrm{mol}, 0.1$ equiv.). Purification by silica gel column chromatography (pentane/EtOAc $=2: 1)$ afforded 12a (45.0 mg, $0.168 \mathrm{mmol}, 76 \%)$ as a yellow solid.

${ }^{1} \mathbf{H}$ NMR $\left(500 \mathrm{MHz} \mathrm{CDCl}_{3}\right) \delta[\mathrm{ppm}]=8.15(\mathrm{~s}, 1 \mathrm{H}), 7.22(\mathrm{dd}, J=8.8,0.6 \mathrm{~Hz}, 1 \mathrm{H})$, $6.94(\mathrm{~d}, J=2.5 \mathrm{~Hz}, 1 \mathrm{H}), 6.87(\mathrm{dd}, J=8.8,2.5 \mathrm{~Hz}, 1 \mathrm{H}), 5.51$ (t, $J=4.9 \mathrm{~Hz}, 1 \mathrm{H}), 4.11$ $(\mathrm{t}, J=6.0 \mathrm{~Hz}, 2 \mathrm{H}), 3.86(\mathrm{~s}, 3 \mathrm{H}), 2.88(\mathrm{t}, J=6.0 \mathrm{~Hz}, 2 \mathrm{H}), 2.63(\mathrm{t}, J=7.8 \mathrm{~Hz}, 2 \mathrm{H}), 2.45$ $(\mathrm{td}, J=7.7,4.9 \mathrm{~Hz}, 2 \mathrm{H})$. 
${ }^{13} \mathbf{C}$ NMR $\left(126 \mathrm{MHz}, \mathrm{CDCl}_{3}\right) \delta[\mathrm{ppm}]=169.9,154.5,132.4,131.4,128.9,127.2,113.8$, $111.9,111.8,100.8,99.2,56.0,39.3,31.9,20.8,19.6$.

HRMS-ESI: calcd. for $\mathrm{C}_{16} \mathrm{H}_{16} \mathrm{~N}_{2} \mathrm{O}_{2} \mathrm{Na}[\mathrm{M}+\mathrm{Na}]^{+}$: 291.1104; found: 291.1105 .

FT-IR: $v\left[\mathrm{~cm}^{-1}\right]=3308,2927,2834,1665,1639,1487,1396,1214,1170,1025,801$, 753.

10-methoxy-2,6,7,12-tetrahydroindolo[2,3-a]quinolizin-4(3H)-one (12b).

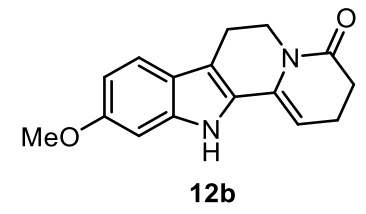

12b was prepared according to General procedure C, starting from $\mathbf{1 1 b}(80.0 \mathrm{mg}$, $0.176 \mathrm{mmol}, 1.0$ equiv.) and $\mathrm{POCl}_{3}(16.1 \mu \mathrm{L}, 0.176 \mathrm{mmol}, 1.0$ equiv.) in toluene $(3.0 \mathrm{~mL})$. The reaction mixture was subsequently treated with $\mathrm{MeOH}(3.0 \mathrm{~mL}), \mathrm{K}_{2} \mathrm{CO}_{3}$ (243 mg, $1.76 \mathrm{mmol}, 10$ equiv.) and $n \mathrm{Bu} 4 \mathrm{NBr}(5.7 \mathrm{mg}, 18.0 \mu \mathrm{mol}, 0.1$ equiv.). Purification by silica gel column chromatography (pentane/EtOAc $=2: 1$ ) afforded 12b (40.0 mg, $0.149 \mathrm{mmol}, 85 \%)$ as a yellow solid.

${ }^{1} \mathbf{H}$ NMR $\left(500 \mathrm{MHz}, \mathrm{CD}_{2} \mathrm{Cl}_{2}\right) \delta[\mathrm{ppm}]=8.12(\mathrm{~s}, 1 \mathrm{H}), 7.37(\mathrm{~d}, J=8.6 \mathrm{~Hz}, 1 \mathrm{H}), 6.85(\mathrm{~d}$, $J=2.3 \mathrm{~Hz}, 1 \mathrm{H}), 6.75(\mathrm{dd}, J=8.6,2.3 \mathrm{~Hz}, 1 \mathrm{H}), 5.45$ (t, $J=4.9 \mathrm{~Hz}, 1 \mathrm{H}), 4.05$ (t, $J=6.0$ $\mathrm{Hz}, 2 \mathrm{H}), 3.83(\mathrm{~s}, 3 \mathrm{H}), 2.85(\mathrm{t}, J=6.0 \mathrm{~Hz}, 2 \mathrm{H}), 2.57(\mathrm{t}, J=7.7 \mathrm{~Hz}, 2 \mathrm{H}), 2.44(\mathrm{td}, J=$ 7.7, $4.9 \mathrm{~Hz}, 2 \mathrm{H})$.

${ }^{13} \mathbf{C}$ NMR $\left(126 \mathrm{MHz}, \mathrm{CD}_{2} \mathrm{Cl}_{2}\right) \delta[\mathrm{ppm}]=169.8,158.0,138.7,131.7,127.6,121.6,119.9$, 112.2, 110.1, 98.5, 95.0, 55.9, 39.4, 32.3, 21.1, 19.9.

HRMS-ESI: calcd. for $\mathrm{C}_{16} \mathrm{H}_{17} \mathrm{~N}_{2} \mathrm{O}_{2}[\mathrm{M}+\mathrm{H}]^{+}$: 269.1284; found: 269.1284 .

FT-IR: $v\left[\mathrm{~cm}^{-1}\right]=3309,2930,2835,1665,1639,1487,1397,1214,1170,1043,1025$, 754.

10-Bromo-2,6,7,12-tetrahydroindolo[2,3-a]quinolizin-4(3H)-one (12c). 


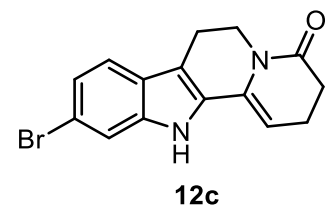

12c was prepared according to General procedure C, starting from 11c (37.0 mg, $74.0 \mu \mathrm{mol}, 1.0$ equiv. $)$ and $\mathrm{POCl}_{3}(6.7 \mu \mathrm{L}, 74.0 \mu \mathrm{mol}, 1.0$ equiv. $)$ in toluene $(2.0 \mathrm{~mL})$. The reaction mixture was subsequently treated with $\mathrm{MeOH}(2.0 \mathrm{~mL}), \mathrm{K}_{2} \mathrm{CO}_{3}(99.0 \mathrm{mg}$, $740 \mu \mathrm{mol}, 10$ equiv.) and $n \mathrm{Bu} 4 \mathrm{NBr}(2.4 \mathrm{mg}, 7.0 \mu \mathrm{mol}, 0.1$ equiv.). Purification by silica gel column chromatography (pentane/EtOAc $=2: 1)$ afforded 12c $(20.0 \mathrm{mg}, 63.0 \mu \mathrm{mol}$, $86 \%$ ) as a yellow solid.

${ }^{1}$ H NMR (700 MHz, $\left.d 6-\mathrm{DMSO}\right) \delta[\mathrm{ppm}]=11.42(\mathrm{~s}, 1 \mathrm{H}), 7.48(\mathrm{dd}, J=1.8,0.6 \mathrm{~Hz}, 1 \mathrm{H})$, $7.44(\mathrm{~d}, J=8.4 \mathrm{~Hz}, 1 \mathrm{H}), 7.13(\mathrm{dd}, J=8.4,1.8 \mathrm{~Hz}, 1 \mathrm{H}), 5.87$ (t, $J=4.9 \mathrm{~Hz}, 1 \mathrm{H}), 3.93$ (t, $J=5.9 \mathrm{~Hz}, 2 \mathrm{H}), 2.81(\mathrm{t}, J=6.0 \mathrm{~Hz}, 2 \mathrm{H}), 2.48(\mathrm{~d}, J=7.7 \mathrm{~Hz}, 2 \mathrm{H}), 2.42-2.36(\mathrm{~m}$, $2 \mathrm{H})$.

${ }^{13}$ C NMR (176 MHz, $d_{6}$-DMSO) $\delta[\mathrm{ppm}]=168.4,138.0,130.5,129.2,125.1,121.9$, $120.3,115.1,113.6,110.0,100.9,38.4,31.1,20.1,18.9$.

HRMS-ESI: calcd. for $\mathrm{C}_{15} \mathrm{H}_{12} \mathrm{BrN}_{2} \mathrm{O}$ [M - H]- 315.0138 ; found: 315.0127 .

FT-IR: $v\left[\mathrm{~cm}^{-1}\right]=2961,2926,1667,1652,1458,1390,1213,1180,1045,1180,1045$, $798,749$.

\section{0-Fluoro-2,6,7,12-tetrahydroindolo[2,3-a]quinolizin-4(3H)-one (12d).}

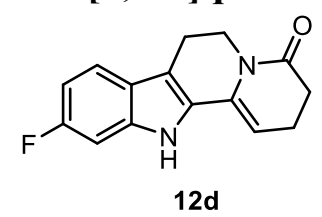

12d was prepared according to General procedure C, starting from 11d (100 mg, 0.226 mmol, 1.0 equiv.) and $\mathrm{POCl}_{3}(20.6 \mu \mathrm{L}, 0.226 \mathrm{mmol}, 1.0$ equiv.) in toluene $(3.0 \mathrm{~mL})$. The reaction mixture was subsequently treated with $\mathrm{MeOH}(3.0 \mathrm{~mL}), \mathrm{K}_{2} \mathrm{CO}_{3}$ (313 mg, $2.26 \mathrm{mmol}, 10$ equiv.) and $n \mathrm{Bu} 4 \mathrm{NBr}$ (7.3 mg, $23.0 \mu \mathrm{mol}, 0.1$ equiv.). Purification by silica gel column chromatography (pentane/EtOAc $=2: 1)$ afforded 12d (49.0 mg, $0.184 \mathrm{mmol}, 81 \%$ ) as a yellow solid.

${ }^{1}$ H NMR $\left(700 \mathrm{MHz}, d_{6-\mathrm{DMSO}}\right) \delta[\mathrm{ppm}]=11.36(\mathrm{~s}, 1 \mathrm{H}), 7.46(\mathrm{dd}, J=8.6,5.5 \mathrm{~Hz}, 1 \mathrm{H})$, S44 
$7.09(\mathrm{dd}, J=10.0,2.3 \mathrm{~Hz}, 1 \mathrm{H}), 6.86(\mathrm{ddd}, J=9.8,8.6,2.4 \mathrm{~Hz}, 1 \mathrm{H}), 5.83(\mathrm{t}, J=4.9 \mathrm{~Hz}$, 1H), $3.93(\mathrm{t}, J=5.9 \mathrm{~Hz}, 2 \mathrm{H}), 2.80(\mathrm{t}, J=6.0 \mathrm{~Hz}, 2 \mathrm{H}), 2.48(\mathrm{t}, J=8.0 \mathrm{~Hz}, 2 \mathrm{H}), 2.38$ (ddd, $J=8.0,6.6,3.2 \mathrm{~Hz}, 2 \mathrm{H}$ ).

${ }^{13}$ C NMR (176 MHz, $d_{6}$-DMSO) $\delta[\mathrm{ppm}]=168.5,159.6(\mathrm{~d}, J=235.6 \mathrm{~Hz}), 137.2(\mathrm{~d}, J$ $=12.7 \mathrm{~Hz}), 130.7,129.0,122.9,119.7(\mathrm{~d}, J=10.7 \mathrm{~Hz}), 109.9,107.4(\mathrm{~d}, J=24.3 \mathrm{~Hz})$, 100.1, 97.2 (d, $J=25.8 \mathrm{~Hz}), 38.4,31.2,20.1,18.9$.

${ }^{19}$ F NMR $\left(471 \mathrm{MHz}, d_{6-\mathrm{DMSO}}\right) \delta[\mathrm{ppm}]=-119.64(\mathrm{~d}, J=11.5 \mathrm{~Hz})$.

HRMS-ESI: calcd. for $\mathrm{C}_{15} \mathrm{H}_{13} \mathrm{FN}_{2} \mathrm{ONa}[\mathrm{M}+\mathrm{Na}]^{+}$: 279.0904; found: 279.0904 .

FT-IR: $v\left[\mathrm{~cm}^{-1}\right]=2954,2920,1681,1457,1329,1205,1174,1043,745$.

2-Methyl-2,6,7,12-tetrahydroindolo[2,3-a]quinolizin-4(3H)-one (12e).

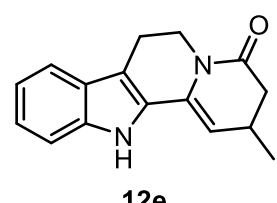

$12 \mathrm{e}$

12e was prepared according to General procedure $\mathbf{C}$, starting from 11e (150 mg, $0.342 \mathrm{mmol}, 1.0$ equiv.) and $\mathrm{POCl}_{3}(31.3 \mu \mathrm{L}, 0.342 \mathrm{mmol}, 1.0$ equiv.) in toluene $(4.0 \mathrm{~mL})$. The reaction mixture was subsequently treated with $\mathrm{MeOH}(4.0 \mathrm{~mL}), \mathrm{K}_{2} \mathrm{CO}_{3}$ (473 mg, $3.42 \mathrm{mmol}, 10$ equiv.) and $n \mathrm{Bu} 4 \mathrm{NBr}(11.0 \mathrm{mg}, 34.0 \mu \mathrm{mol}, 0.1$ equiv.). Purification by silica gel column chromatography (pentane/EtOAc $=2: 1$ ) afforded 12e (70.0 mg, $0.258 \mathrm{mmol}, 75 \%)$ as a yellow solid.

Scale-up reaction starting from $11 \mathrm{e}\left(1.00 \mathrm{~g}, 2.28 \mathrm{mmol}, 1.0\right.$ equiv.) and $\mathrm{POCl}_{3}(208 \mu \mathrm{L}$, $2.28 \mathrm{mmol}, 1.0$ equiv.) in toluene $(30 \mathrm{~mL})$. The reaction mixture was subsequently treated with $\mathrm{MeOH}(30 \mathrm{~mL}), \mathrm{K}_{2} \mathrm{CO}_{3}(3.15 \mathrm{~g}, 22.8 \mathrm{mmol}, 10$ equiv.) and $n \mathrm{Bu} 4 \mathrm{NBr}$ (73.6 mg, $0.228 \mathrm{mmol}, 0.1$ equiv.). Purification by silica gel column chromatography (pentane/EtOAc $=2: 1)$ afforded $12 \mathrm{e}(405 \mathrm{mg}, 1.61 \mathrm{mmol}, 70 \%)$ as a yellow solid.

${ }^{1} \mathbf{H}$ NMR $\left(500 \mathrm{MHz}, \mathrm{CDCl}_{3}\right) \delta[\mathrm{ppm}]=8.43(\mathrm{~s}, 1 \mathrm{H}), 7.51(\mathrm{~d}, J=7.9 \mathrm{~Hz}, 1 \mathrm{H}), 7.34(\mathrm{dd}$, $J=8.2,1.1 \mathrm{~Hz}, 1 \mathrm{H}), 7.24-7.19(\mathrm{~m}, 1 \mathrm{H}), 7.15-7.09(\mathrm{~m}, 1 \mathrm{H}), 5.45(\mathrm{t}, J=4.0 \mathrm{~Hz}, 1 \mathrm{H})$, 4.44-4.38 (m, 1H), 3.84-3.78 (m, 1H), 2.98-2.84 (m, 2H), 2.81-2.65 (m, 2H), 2.39$2.33(\mathrm{~m}, 1 \mathrm{H}), 1.17(\mathrm{~d}, J=6.9 \mathrm{~Hz}, 3 \mathrm{H})$.

${ }^{13} \mathbf{C ~ N M R}\left(126 \mathrm{MHz}, \mathrm{CDCl}_{3}\right) \delta[\mathrm{ppm}]=169.8,137.4,130.1,128.0,126.8,123.6,120.1$, 
$119.1,112.1,111.1,106.0,40.0,39.3,26.2,20.8,20.4$.

HRMS-ESI: calcd. for $\mathrm{C}_{16} \mathrm{H}_{17} \mathrm{~N}_{2} \mathrm{O}[\mathrm{M}+\mathrm{H}]^{+}$: 253.1335; found: 253.1335.

FT-IR: $v\left[\mathrm{~cm}^{-1}\right]=3286,1638,1399,1328,1303,1213,1180,742$.

3-Methyl-2,6,7,12-tetrahydroindolo[2,3-a]quinolizin-4(3H)-one (12f).

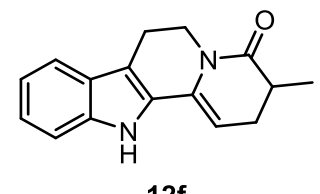

$12 f$

12f was prepared according to General procedure C, starting from 11 (132 mg, $0.301 \mathrm{mmol}, 1.0$ equiv.) and $\mathrm{POCl}_{3}(27.5 \mu \mathrm{L}, 0.301 \mathrm{mmol}, 1.0$ equiv.) in toluene (3.5 mL). The reaction mixture was subsequently treated with $\mathrm{MeOH}(3.5 \mathrm{~mL}), \mathrm{K}_{2} \mathrm{CO}_{3}$ (416 mg, $3.01 \mathrm{mmol}, 10$ equiv.) and $n \mathrm{Bu} 4 \mathrm{NBr}(9.7 \mathrm{mg}, 30.0 \mu \mathrm{mol}, 0.1$ equiv.). Purification by silica gel column chromatography (pentane/EtOAc $=2: 1$ ) afforded $\mathbf{1 2 f}$ (55.0 $\mathrm{mg}, 0.218 \mathrm{mmol}, 72 \%)$ as a yellow solid.

${ }^{1} \mathbf{H}$ NMR $\left(700 \mathrm{MHz}, \mathrm{CD}_{2} \mathrm{Cl}_{2}\right) \delta[\mathrm{ppm}]=8.68(\mathrm{~s}, 1 \mathrm{H}), 7.52-7.51(\mathrm{~m}, 1 \mathrm{H}), 7.36(\mathrm{dt}, J=$ 8.1, $0.9 \mathrm{~Hz}, 1 \mathrm{H}), 7.21(\mathrm{ddd}, J=8.2,7.0,1.2 \mathrm{~Hz}, 1 \mathrm{H}), 7.10(\mathrm{ddd}, J=8.0,7.0,1.0 \mathrm{~Hz}$, 1H), $5.61(\mathrm{dd}, J=5.9,4.0 \mathrm{~Hz}, 1 \mathrm{H}), 4.50(\mathrm{dt}, J=12.7,5.1 \mathrm{~Hz}, 1 \mathrm{H}), 3.72-3.68(\mathrm{~m}, 1 \mathrm{H})$, 2.95-2.85 (m, 2H), 2.67-2.60 (m, 1H), $2.51(\mathrm{ddd}, J=16.9,6.6,5.9 \mathrm{~Hz}, 1 \mathrm{H}), 2.24$ (ddd, $J=16.9,11.4,4.1 \mathrm{~Hz}, 1 \mathrm{H}), 1.27(\mathrm{~d}, J=6.9 \mathrm{~Hz}, 3 \mathrm{H})$.

${ }^{13} \mathbf{C ~ N M R}\left(176 \mathrm{MHz}, \mathrm{CD}_{2} \mathrm{Cl}_{2}\right) \delta[\mathrm{ppm}]=173.1,137.7,131.3,128.7,127.1,123.6,120.2$, $119.2,111.8,111.4,99.2,39.9,36.1,28.0,21.1,16.1$.

HRMS-ESI: calcd. for $\mathrm{C}_{16} \mathrm{H}_{16} \mathrm{~N}_{2} \mathrm{ONa}[\mathrm{M}+\mathrm{Na}]^{+}: 275.1155$; found: 275.1161 .

FT-IR: $v\left[\mathrm{~cm}^{-1}\right]=3275,1638,1452,1398,1328,1303,1213,1180,742$.

1-Methyl-2,6,7,12-tetrahydroindolo[2,3-a]quinolizin-4(3H)-one (12g).

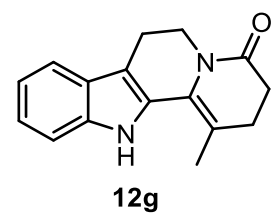

$\mathbf{1 2 g}$ was prepared according to General procedure C, starting from $\mathbf{1 1 g}$ (108 $\mathrm{mg}$, 0.247 mmol, 1.0 equiv.) and $\mathrm{POCl}_{3}(22.5 \mu \mathrm{L}, 0.247 \mathrm{mmol}, 1.0$ equiv.) in toluene $(3.0 \mathrm{~mL})$. The reaction mixture was subsequently treated with $\mathrm{MeOH}(3.0 \mathrm{~mL}), \mathrm{K}_{2} \mathrm{CO}_{3}$ 
(341 mg, $2.47 \mathrm{mmol}, 10$ equiv.) and $n \mathrm{Bu} 4 \mathrm{NBr}$ (7.9 mg, $25.0 \mu \mathrm{mol}, 0.1$ equiv.). Purification by silica gel column chromatography (pentane/EtOAc $=2: 1$ ) afforded $\mathbf{1 2 g}$ (40.0 mg, $0.159 \mathrm{mmol}, 64 \%$ ) as a yellow solid.

${ }^{1} \mathbf{H}$ NMR $\left(700 \mathrm{MHz}, \mathrm{CD}_{2} \mathrm{Cl}_{2}\right) \delta[\mathrm{ppm}]=8.42(\mathrm{~s}, 1 \mathrm{H}), 7.54-7.52(\mathrm{~m}, 1 \mathrm{H}), 7.41(\mathrm{dt}, J=$ 8.1, $0.9 \mathrm{~Hz}, 1 \mathrm{H}), 7.20(\mathrm{ddd}, J=8.2,7.0,1.2 \mathrm{~Hz}, 1 \mathrm{H}), 7.12(\mathrm{ddd}, J=8.0,7.0,1.0 \mathrm{~Hz}$, $1 \mathrm{H}), 4.05(\mathrm{t}, J=5.9 \mathrm{~Hz}, 2 \mathrm{H}), 2.88(\mathrm{t}, J=5.9 \mathrm{~Hz}, 2 \mathrm{H}), 2.57-2.48(\mathrm{~m}, 2 \mathrm{H}), 2.47-2.41$ (m, 2H), $2.29(\mathrm{~d}, J=1.3 \mathrm{~Hz}, 3 \mathrm{H})$.

${ }^{13}$ C NMR $\left(176 \mathrm{MHz}, \mathrm{CD}_{2} \mathrm{Cl}_{2}\right) \delta[\mathrm{ppm}]=169.6,137.4,129.4,126.5,126.3,123.3,120.4$, 119.0, 113.1, 112.8, 111.4, 39.7, 32.0, 29.4, 21.4, 19.8 .

HRMS-ESI: calcd. for $\mathrm{C}_{16} \mathrm{H}_{16} \mathrm{~N}_{2} \mathrm{ONa}[\mathrm{M}+\mathrm{Na}]^{+}: 275.1155$; found: 275.1169 .

FT-IR: $v\left[\mathrm{~cm}^{-1}\right]=3356,1638,1405,1356,1210,1040,744$.

\section{2,2-Dimethyl-2,6,7,12-tetrahydroindolo[2,3-a]quinolizin-4(3H)-one (12h).}

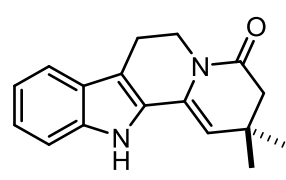

$12 \mathrm{~h}$

12h was prepared according to General procedure C, starting from $11 \mathrm{~h}$ (100 $\mathrm{mg}$, $0.221 \mathrm{mmol}, 1.0$ equiv.) and $\mathrm{POCl}_{3}(20.2 \mu \mathrm{L}, 0.221 \mathrm{mmol}, 1.0$ equiv.) in toluene $(3.0 \mathrm{~mL})$. The reaction mixture was subsequently treated with $\mathrm{MeOH}(3.0 \mathrm{~mL}), \mathrm{K}_{2} \mathrm{CO}_{3}$ (306 mg, $2.21 \mathrm{mmol}, 10$ equiv.) and $n \mathrm{Bu} 4 \mathrm{NBr}$ (7.1 mg, $22.0 \mu \mathrm{mol}, 0.1$ equiv.). Purification by silica gel column chromatography (pentane/EtOAc $=2: 1$ ) afforded $\mathbf{1 2 h}$ (49.0 mg, $0.184 \mathrm{mmol}, 83 \%$ ) as a yellow solid.

${ }^{1} \mathbf{H}$ NMR $\left(500 \mathrm{MHz}, \mathrm{CDCl}_{3}\right) \delta[\mathrm{ppm}]=8.63(\mathrm{~s}, 1 \mathrm{H}), 7.53-7.51(\mathrm{~m}, 1 \mathrm{H}), 7.34(\mathrm{dt}, J=$ 8.2, $0.9 \mathrm{~Hz}, 1 \mathrm{H}), 7.23(\mathrm{ddd}, J=8.2,7.1,1.2 \mathrm{~Hz}, 1 \mathrm{H}), 7.12(\mathrm{ddd}, J=8.0,7.1,1.0 \mathrm{~Hz}$, 1H), $5.44(\mathrm{~s}, 1 \mathrm{H}), 4.14(\mathrm{t}, J=6.0 \mathrm{~Hz}, 2 \mathrm{H}), 2.93(\mathrm{t}, J=6.0 \mathrm{~Hz}, 2 \mathrm{H}), 2.52(\mathrm{~s}, 2 \mathrm{H}), 1.18$ $(\mathrm{s}, 6 \mathrm{H})$.

${ }^{13} \mathbf{C ~ N M R}\left(126 \mathrm{MHz}, \mathrm{CDCl}_{3}\right) \delta[\mathrm{ppm}]=169.7,137.4,128.9,128.0,126.8,123.5,120.0$, 119.0, 112.1, 111.1, 110.7, 46.5, 39.4, 30.8, 28.2, 20.7.

HRMS-ESI: calcd. for $\mathrm{C}_{17} \mathrm{H}_{18} \mathrm{~N}_{2} \mathrm{ONa}[\mathrm{M}+\mathrm{Na}]^{+}$: 289.1311 ; found: 289.1311 . 
FT-IR: $v\left[\mathrm{~cm}^{-1}\right]=2930,1666,1646,1395,1215,752,740$.

(S)-Methyl-4-oxo-2,3,4,6,7,12-hexahydroindolo[2,3-a]quinolizine-6-carboxylate (12i).

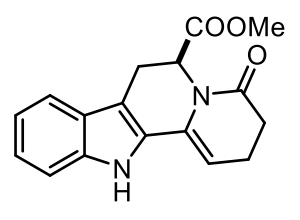

$12 \mathbf{i}$

12i was prepared according to General procedure C, starting from 11i (69 mg, 0.143 mmol, 1.0 equiv.) and $\mathrm{POCl}_{3}(13.1 \mu \mathrm{L}, 0.134 \mathrm{mmol}, 1.0$ equiv.) in toluene $(2.0 \mathrm{~mL})$. The reaction mixture was subsequently treated with $\mathrm{MeOH}(2.0 \mathrm{~mL}), \mathrm{K}_{2} \mathrm{CO}_{3}$ (198 mg, $1.43 \mathrm{mmol}, 10$ equiv.) and $n \mathrm{Bu} 4 \mathrm{NBr}$ (4.6 mg, $14.0 \mu \mathrm{mol}, 0.1$ equiv.). Purification by silica gel column chromatography (pentane/EtOAc $=1: 1)$ afforded $\mathbf{1 2 i}$ (30.0 mg, $0.101 \mathrm{mmol}, 71 \%$ ) as a yellow solid.

${ }^{1} \mathbf{H}$ NMR $\left(700 \mathrm{MHz}, \mathrm{CDCl}_{3}\right) \delta[\mathrm{ppm}]=8.02(\mathrm{~s}, 1 \mathrm{H}), 7.53-7.51(\mathrm{~m}, 1 \mathrm{H}), 7.33-7.29(\mathrm{~m}$, 1H), 7.23-7.20 (m, 1H), $7.12(\mathrm{ddd}, J=8.0,7.1,1.0 \mathrm{~Hz}, 1 \mathrm{H}), 5.90(\mathrm{dd}, J=6.5,1.5 \mathrm{~Hz}$, 1H), 5.55 (dt, $J=8.0,3.8 \mathrm{~Hz}, 1 \mathrm{H}), 3.60-3.58(\mathrm{~m}, 4 \mathrm{H}), 3.14$ (dd, $J=16.1,6.5 \mathrm{~Hz}, 1 \mathrm{H})$, 2.76-2.71 (m, 1H), 2.67-2.61 (m, 2H), 2.50-2.40 (m, 1H).

${ }^{13} \mathbf{C ~ N M R}\left(176 \mathrm{MHz}, \mathrm{CDCl}_{3}\right) \delta[\mathrm{ppm}]=171.3,170.1,137.4,130.2,127.8,126.8,123.8$, 120.3, 119.2, 111.1, 109.0, 100.0, 52.8, 50.9, 31.6, 23.1, 19.6.

HRMS-ESI: calcd. for $\mathrm{C}_{17} \mathrm{H}_{16} \mathrm{~N}_{2} \mathrm{O}_{3} \mathrm{Na}[\mathrm{M}+\mathrm{Na}]^{+}: 319.1053$; found: 319.1044 .

FT-IR: $v\left[\mathrm{~cm}^{-1}\right]=2928,2850,1736,1698,1366,1331,1215,747$.

$[\alpha]_{\mathrm{D}}^{26}=+4.6\left(c=0.2, \mathrm{CHCl}_{3}\right)$.

8,13-Dihydroindolo[2',3':3,4]pyrido[1,2-b]isoquinolin-5(7H)-one (12j).

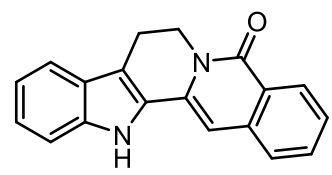

12j nortetoyobyrine

$\mathbf{1 2} \mathbf{j}$ was prepared according to General procedure $\mathbf{C}$, starting from $\mathbf{1 1} \mathbf{j}$ (50.0 mg, S48 
0.106 mmol, 1.0 equiv.) and $\mathrm{POCl}_{3}(9.7 \mu \mathrm{L}, 0.106 \mathrm{mmol}, 1.0$ equiv.) in toluene $(1.5 \mathrm{~mL})$. The reaction mixture was subsequently treated with $\mathrm{MeOH}(1.5 \mathrm{~mL}), \mathrm{K}_{2} \mathrm{CO}_{3}$

(146 mg, $1.06 \mathrm{mmol}, 10$ equiv.) and $n \mathrm{Bu} 4 \mathrm{NBr}(3.4 \mathrm{mg}, 11.0 \mu \mathrm{mol}, 0.1$ equiv.). Purification by silica gel column chromatography (pentane/EtOAc $=3: 1$ ) afforded $\mathbf{1 2} \mathbf{j}$ (25.0 mg, $87.4 \mu \mathrm{mol}, 82 \%$ ) as a light yellow solid.

${ }^{1} \mathbf{H}$ NMR $\left(500 \mathrm{MHz}, d_{6}\right.$-DMSO) $\delta[\mathrm{ppm}]=11.69(\mathrm{~s}, 1 \mathrm{H}), 8.24(\mathrm{dd}, J=8.0,1.3 \mathrm{~Hz}, 1 \mathrm{H})$, $7.71(\mathrm{td}, J=7.5,1.3 \mathrm{~Hz}, 1 \mathrm{H}), 7.61(\mathrm{dd}, J=16.4,7.8 \mathrm{~Hz}, 2 \mathrm{H}), 7.45(\mathrm{dd}, J=18.8,7.9$ $\mathrm{Hz}, 2 \mathrm{H}), 7.21(\mathrm{ddd}, J=8.2,6.9,1.2 \mathrm{~Hz}, 1 \mathrm{H}), 7.10-7.04(\mathrm{~m}, 2 \mathrm{H}), 4.40(\mathrm{t}, J=6.5 \mathrm{~Hz}$, 2H), 3.09 (t, $J=6.6 \mathrm{~Hz}, 2 \mathrm{H})$.

${ }^{13}$ C NMR (126 MHz, $d_{6}$-DMSO) $\delta[\mathrm{ppm}]=161.2,138.0,136.2,132.6,132.4,128.2$, $127.5,126.1,126.0,125.5,124.5,123.5,119.5,119.1,112.5,111.6,99.0,40.4,19.3$

HRMS-ESI: calcd. for $\mathrm{C}_{19} \mathrm{H}_{13} \mathrm{~N}_{2} \mathrm{O}[\mathrm{M}-\mathrm{H}]^{-}: 285.1033$; found: 285.1038 .

FT-IR: $v\left[\mathrm{~cm}^{-1}\right]=2923,2853,1738,1650,1614,1595,1365,1217,742$.

\section{2,3,4,7,8,13-Hexahydro-5H-azepino[1',2':1,2]pyrido[3,4-b]indol-5-one (12k).}

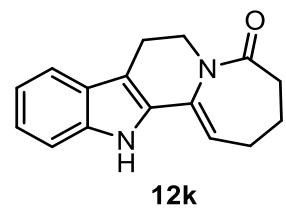

12k was prepared according to General procedure C, starting from 11k (200 mg, 0.457 mmol, 1.0 equiv.) and $\mathrm{POCl}_{3}(41.7 \mu \mathrm{L}, 0.457 \mathrm{mmol}, 1.0$ equiv.) in toluene $(5.0 \mathrm{~mL})$. The reaction mixture was subsequently treated with $\mathrm{MeOH}(5.0 \mathrm{~mL}), \mathrm{K}_{2} \mathrm{CO}_{3}$ (631 mg, $4.57 \mathrm{mmol}, 10$ equiv.) and $n \mathrm{Bu} 4 \mathrm{NBr}$ (14.7 mg, $46.0 \mu \mathrm{mol}, 0.1$ equiv.). Purification by silica gel column chromatography (pentane/EtOAc $=2: 1$ ) afforded $\mathbf{1 2 k}$ (30.0 $\mathrm{mg}, 0.119 \mathrm{mmol}, 26 \%$ ) as a yellow solid.

${ }^{1} \mathbf{H}$ NMR $\left(700 \mathrm{MHz}, \mathrm{CD}_{2} \mathrm{Cl}_{2}\right) \delta[\mathrm{ppm}]=9.10(\mathrm{~s}, 1 \mathrm{H}), 7.48-7.46(\mathrm{~m}, 1 \mathrm{H}), 7.35(\mathrm{dt}, J=$ 8.1, $0.9 \mathrm{~Hz}, 1 \mathrm{H}), 7.18$ (ddd, $J=8.2,7.0,1.2 \mathrm{~Hz}, 1 \mathrm{H}), 7.07$ (ddd, $J=8.0,7.0,1.0 \mathrm{~Hz}$, $1 \mathrm{H}), 6.08(\mathrm{t}, J=7.3 \mathrm{~Hz}, 1 \mathrm{H}), 4.07(\mathrm{t}, J=5.9 \mathrm{~Hz}, 2 \mathrm{H}), 2.84(\mathrm{t}, J=5.9 \mathrm{~Hz}, 2 \mathrm{H}), 2.55(\mathrm{t}$, $J=7.1 \mathrm{~Hz}, 2 \mathrm{H}), 2.37$ (q, $J=7.5 \mathrm{~Hz}, 2 \mathrm{H}), 2.20-2.16(\mathrm{~m}, 2 \mathrm{H})$.

${ }^{13} \mathbf{C}$ NMR $\left(176 \mathrm{MHz}, \mathrm{CD}_{2} \mathrm{Cl}_{2}\right) \delta[\mathrm{ppm}]=175.8,139.5,136.6,131.7,129.4,125.3,122.0$, $121.1,114.1,114.0,113.4,43.4,37.6,32.1,26.1,23.9$. 
HRMS-ESI: calcd. for $\mathrm{C}_{16} \mathrm{H}_{16} \mathrm{~N}_{2} \mathrm{ONa}[\mathrm{M}+\mathrm{Na}]^{+}$: 275.1155; found: 275.1152.

FT-IR: $v\left[\mathrm{~cm}^{-1}\right]$ 2928, 2855, 1713, 1659, 1625, 1442, 1329, 1230, 1170, 745.

\section{2,4,5,8,9,14-Hexahydroazocino[1',2':1,2]pyrido[3,4-b]indol-6(3H)-one (12l).}

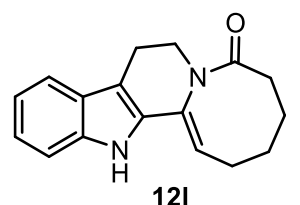

12l was prepared according to General procedure C, starting from 111 (200 mg, 0.429 mmol, 1.0 equiv.) and $\mathrm{POCl}_{3}(39.2 \mu \mathrm{L}, 0.429 \mathrm{mmol}, 1.0$ equiv.) in toluene $(5.0 \mathrm{~mL})$. The reaction mixture was subsequently treated with $\mathrm{MeOH}(5.0 \mathrm{~mL}), \mathrm{K}_{2} \mathrm{CO}_{3}$ (593 mg, $4.29 \mathrm{mmol}, 10$ equiv.) and $n \mathrm{Bu} 4 \mathrm{NBr}$ (13.8 mg, $43.0 \mu \mathrm{mol}, 0.1$ equiv.). Purification by silica gel column chromatography (pentane/EtOAc $=2: 1)$ afforded 12 I (50.0 mg, $0.168 \mathrm{mmol}, 39 \%$ ) as a yellow solid.

${ }^{1} \mathbf{H}$ NMR $\left(700 \mathrm{MHz}, \mathrm{CD}_{2} \mathrm{Cl}_{2}\right) \delta[\mathrm{ppm}]=8.34(\mathrm{~s}, 1 \mathrm{H}), 7.47-7.46(\mathrm{~m}, 1 \mathrm{H}), 7.34(\mathrm{dt}, J=$ 8.1, $0.9 \mathrm{~Hz}, 1 \mathrm{H}), 7.18(\mathrm{ddd}, J=8.2,7.1,1.2 \mathrm{~Hz}, 1 \mathrm{H}), 7.08$ (ddd, $J=8.0,7.0,1.0 \mathrm{~Hz}$, $1 \mathrm{H}), 5.77$ (t, $J=8.2 \mathrm{~Hz}, 1 \mathrm{H}), 5.07$ (ddd, $J=12.5,5.4,1.2 \mathrm{~Hz}, 1 \mathrm{H}), 3.18-3.14(\mathrm{~m}, 1 \mathrm{H})$, $2.97(\mathrm{ddd}, J=15.7,12.4,5.5 \mathrm{~Hz}, 1 \mathrm{H}), 2.76(\mathrm{ddd}, J=15.9,4.3,1.2 \mathrm{~Hz}, 1 \mathrm{H}), 2.55$ (td, $J$ $=12.2,1.6 \mathrm{~Hz}, 1 \mathrm{H}), 2.49-2.39(\mathrm{~m}, 2 \mathrm{H}), 2.10(\mathrm{dddd}, J=14.2,11.9,8.9,1.3 \mathrm{~Hz}, 1 \mathrm{H})$, 1.99-1.90 (m, 1H), 1.69-1.55 (m, 2H), 1.39-1.28 (m, 1H).

${ }^{13} \mathbf{C ~ N M R}\left(176 \mathrm{MHz}, \mathrm{CD}_{2} \mathrm{Cl}_{2}\right) \delta[\mathrm{ppm}]=174.0,137.3,133.1,130.4,127.6,123.4,120.2$, 119.1, 116.0, 112.3, 111.3, 42.8, 35.0, 26.6, 25.5, 25.1, 22.1 .

HRMS-ESI: calcd. for $\mathrm{C}_{17} \mathrm{H}_{18} \mathrm{~N}_{2} \mathrm{ONa}[\mathrm{M}+\mathrm{Na}]^{+}$: 289.1311 ; found: 289.1315 .

FT-IR: $v\left[\mathrm{~cm}^{-1}\right]=3260,2928,2854,1620,1450,1406,1230,1170,7432$.

\section{2,3,6,7,8,13-Hexahydro-4H-pyrido $\left[1^{\prime}, 2^{\prime}: 1,2\right]$ azepino $[3,4-b]$ indol-4-one (12m).}

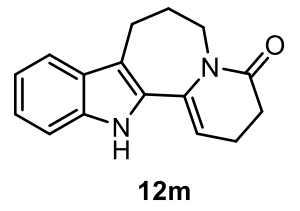

$\mathbf{1 2} \mathbf{m}$ was prepared according to General procedure C, starting from $\mathbf{1 1} \mathbf{m}(50.0 \mathrm{mg}$, S50 
0.114 mmol, 1.0 equiv.) and $\mathrm{POCl}_{3}(10.4 \mu \mathrm{L}, 0.114 \mathrm{mmol}, 1.0$ equiv.) in toluene $(1.5 \mathrm{~mL})$. The reaction mixture was subsequently treated with $\mathrm{MeOH}(1.5 \mathrm{~mL}), \mathrm{K}_{2} \mathrm{CO}_{3}$ (158 mg, $1.14 \mathrm{mmol}, 10$ equiv.) and $n \mathrm{Bu} 4 \mathrm{NBr}(3.7 \mathrm{mg}, 11.0 \mu \mathrm{mol}, 0.1$ equiv.). Purification by silica gel column chromatography (pentane/EtOAc $=2: 1$ ) afforded $\mathbf{1 2} \mathbf{m}$ (15.0 $\mathrm{mg}, 59.5 \mu \mathrm{mol}, 52 \%)$ as a yellow solid.

${ }^{1} \mathbf{H}$ NMR $\left(700 \mathrm{MHz}, \mathrm{CDCl}_{3}\right) \delta[\mathrm{ppm}]=8.00(\mathrm{~s}, 1 \mathrm{H}), 7.55-7.54(\mathrm{~m}, 1 \mathrm{H}), 7.33(\mathrm{dt}, J=$ 8.1, $0.9 \mathrm{~Hz}, 1 \mathrm{H}), 7.21(\mathrm{ddd}, J=8.2,7.0,1.2 \mathrm{~Hz}, 1 \mathrm{H}), 7.13(\mathrm{ddd}, J=8.0,7.0,1.0 \mathrm{~Hz}$, $1 \mathrm{H}), 5.54(\mathrm{t}, J=5.1 \mathrm{~Hz}, 1 \mathrm{H}), 3.91-3.83(\mathrm{~m}, 2 \mathrm{H}), 2.93(\mathrm{t}, J=7.3 \mathrm{~Hz}, 2 \mathrm{H}), 2.60(\mathrm{dd}, J=$ 8.6, 6.7 Hz, 2H), 2.48-2.39 (m, 2H), 2.10-2.06 (m, 2H).

${ }^{13} \mathbf{C ~ N M R}\left(176 \mathrm{MHz}, \mathrm{CDCl}_{3}\right) \delta[\mathrm{ppm}]=170.6,137.2,136.0,129.2,128.5,123.2,120.1$, $118.9,113.3,110.9,104.4,42.2,31.9,26.2,20.1,19.8$.

HRMS-ESI: calcd. for $\mathrm{C}_{16} \mathrm{H}_{16} \mathrm{~N}_{2} \mathrm{ONa}[\mathrm{M}+\mathrm{Na}]^{+}:$275.1155; found: 275.1156.

FT-IR: $v\left[\mathrm{~cm}^{-1}\right]$ 2925, 2858, 1653, 1398, 1328, 1260, 1239, 1171, 745.

\section{2,6,7,8,9,14-Hexahydropyrido[1',2':1,2] azocino[3,4-b]indol-4(3H)-one (12n).}

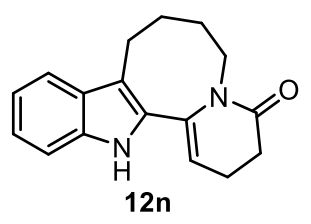

12n was prepared according to General procedure $\mathbf{C}$, starting from 11n (39.0 mg, $86.0 \mu \mathrm{mol}, 1.0$ equiv.) and $\mathrm{POCl}_{3}(7.9 \mu \mathrm{L}, 86.0 \mu \mathrm{mol}, 1.0$ equiv. $)$ in toluene $(1.5 \mathrm{~mL})$. The reaction mixture was subsequently treated with $\mathrm{MeOH}(1.5 \mathrm{~mL}), \mathrm{K}_{2} \mathrm{CO}_{3}(119 \mathrm{mg}$, $863 \mu \mathrm{mol}, 10$ equiv. $)$ and $n \mathrm{Bu} 4 \mathrm{NBr}(2.8 \mathrm{mg}, 9.0 \mu \mathrm{mol}, 0.1$ equiv.). Purification by silica gel column chromatography (pentane/EtOAc $=2: 1)$ afforded $12 \mathbf{n}(10.0 \mathrm{mg}, 37.6 \mu \mathrm{mol}$, $43 \%)$ as a yellow solid.

${ }^{1} \mathbf{H}$ NMR $\left(700 \mathrm{MHz}, \mathrm{CDCl}_{3}\right) \delta[\mathrm{ppm}]=7.83(\mathrm{~s}, 1 \mathrm{H}), 7.55(\mathrm{dt}, J=7.9,0.9 \mathrm{~Hz}, 1 \mathrm{H}), 7.33$ (dt, $J=8.1,0.9 \mathrm{~Hz}, 1 \mathrm{H}), 7.22$ (ddd, $J=8.1,7.0,1.1 \mathrm{~Hz}, 1 \mathrm{H}), 7.14$ (ddd, $J=8.0,7.0$, $1.0 \mathrm{~Hz}, 1 \mathrm{H}), 5.44(\mathrm{t}, J=4.9 \mathrm{~Hz}, 1 \mathrm{H}), 2.92-2.88(\mathrm{~m}, 2 \mathrm{H}), 2.63(\mathrm{dd}, J=8.4,7.3 \mathrm{~Hz}, 2 \mathrm{H})$, 2.46-2.42 (m, 2H), 1.93-1.80 (m, 6H).

${ }^{13} \mathbf{C ~ N M R}\left(176 \mathrm{MHz}, \mathrm{CDCl}_{3}\right) \delta[\mathrm{ppm}]=170.2,136.9,135.2,128.6,128.0,122.9,119.8$, 
$118.8,114.9,110.7,106.4,43.3,31.9,28.9,24.1,23.6,20.0$.

HRMS-ESI: calcd. for $\mathrm{C}_{17} \mathrm{H}_{18} \mathrm{~N}_{2} \mathrm{ONa}[\mathrm{M}+\mathrm{Na}]^{+}$: 289.1311 ; found: 289.1388 .

FT-IR: $v\left[\mathrm{~cm}^{-1}\right]=2954,2922,2853,1737,1661,1458,1378,1217,1033,743$.

\section{2,2-Dimethyl-2,3,6,7-tetrahydro-4H-pyrido[2,1-a]isoquinolin-4-one (12o).}<smiles>CC1(C)C=C2c3ccccc3CCN2C(=O)C1</smiles>

120 was prepared according to General procedure C, starting from 110 (138 mg, 0.334 mmol, 1.0 equiv.) and $\mathrm{POCl}_{3}(76.2 \mu \mathrm{L}, 0.835 \mathrm{mmol}, 2.5$ equiv.) in toluene $(4.0 \mathrm{~mL})$. The reaction mixture was subsequently treated with $\mathrm{MeOH}(4.0 \mathrm{~mL}), \mathrm{K}_{2} \mathrm{CO}_{3}$ (462 mg, $3.34 \mathrm{mmol}, 10$ equiv.) and $n \mathrm{Bu} 4 \mathrm{NBr}$ (10.8 mg, $33.0 \mu \mathrm{mol}, 0.1$ equiv.). Purification by neutral aluminium oxide column chromatography (pentane/DCM = 1:1) afforded 120 (25.0 $\mathrm{mg}, 0.110 \mathrm{mmol}, 33 \%)$ as a white solid.

${ }^{1} \mathbf{H}$ NMR $\left(700 \mathrm{MHz}, \mathrm{C}_{6} \mathrm{D}_{6}\right) \delta[\mathrm{ppm}]=7.36-7.33(\mathrm{~m}, 1 \mathrm{H}), 7.01-6.95(\mathrm{~m}, 2 \mathrm{H}), 6.76-$ $6.72(\mathrm{~m}, 1 \mathrm{H}), 5.34(\mathrm{~s}, 1 \mathrm{H}), 3.79(\mathrm{dd}, J=6.4,5.4 \mathrm{~Hz}, 2 \mathrm{H}), 2.32(\mathrm{~d}, J=0.8 \mathrm{~Hz}, 2 \mathrm{H}), 2.31$ $(\mathrm{td}, J=5.9,0.9 \mathrm{~Hz}, 2 \mathrm{H}), 0.92(\mathrm{~s}, 6 \mathrm{H})$.

${ }^{13} \mathrm{C}$ NMR $\left(176 \mathrm{MHz}, \mathrm{C}_{6} \mathrm{D}_{6}\right) \delta[\mathrm{ppm}]=168.3,135.1,134.1,130.9,128.4,128.4,126.8$, $124.3,112.7,46.0,38.3,30.5,29.4,27.9$.

HRMS-ESI: calcd. for $\mathrm{C}_{15} \mathrm{H}_{17} \mathrm{NONa}[\mathrm{M}+\mathrm{Na}]^{+}: 250.1202$; found: 250.1208 .

FT-IR: $v\left[\mathrm{~cm}^{-1}\right]=2948,2925,2858,1974,1733,1626,1492,1465,1429,1320,1208$, 1126,743 .

\section{0-Methoxy-2,2-dimethyl-2,3,6,7-tetrahydro-4H-pyrido[2,1-a]isoquinolin-4-one} (12p).

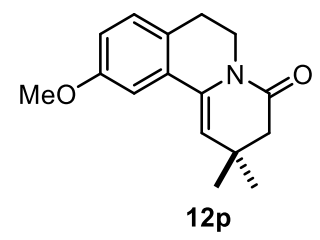


12p was prepared according to General procedure C, starting from 11p $(80.0 \mathrm{mg}$, 0.181 mmol, 1.0 equiv.) and $\mathrm{POCl}_{3}(16.5 \mu \mathrm{L}, 0.181 \mathrm{mmol}, 1.0$ equiv.) in toluene $(3.0 \mathrm{~mL})$. The reaction mixture was subsequently treated with $\mathrm{MeOH}(3.0 \mathrm{~mL}), \mathrm{K}_{2} \mathrm{CO}_{3}$ (250 mg, $1.81 \mathrm{mmol}, 10$ equiv.) and $n \mathrm{Bu}_{4} \mathrm{NBr}$ (5.8 mg, $18 \mu \mathrm{mol}, 0.1$ equiv.). Purification by neutral aluminium oxide column chromatography (pentane/DCM = 1:1) afforded 12p (20.0 mg, $77.8 \mu \mathrm{mol}, 43 \%)$ as a white solid.

${ }^{1}$ H NMR $\left(700 \mathrm{MHz}, \mathrm{C}_{6} \mathrm{D}_{6}\right) \delta[\mathrm{ppm}]=6.70(\mathrm{dd}, J=8.3,0.42 \mathrm{~Hz}, 1 \mathrm{H}), 6.66(\mathrm{dd}, J=8.3$, $2.5 \mathrm{~Hz}, 1 \mathrm{H}), 5.40$ (d, $J=0.9 \mathrm{~Hz}, 1 \mathrm{H}), 3.88-3.82(\mathrm{~m}, 2 \mathrm{H}), 3.32(\mathrm{~s}, 3 \mathrm{H}), 2.35-2.29(\mathrm{~m}$, $4 \mathrm{H}), 0.91(\mathrm{~s}, 6 \mathrm{H})$. (Due to the solvent peak overlapping, one resonance is missing. But the ABX system could be proved by the coupling constant of the rest two Hs.)

${ }^{13}$ C NMR (176 MHz, C6 $\left.\mathrm{D}_{6}\right) \delta[\mathrm{ppm}]=168.4,159.1,134.3,131.9,129.4,127.6,114.2$, $112.9,109.6,54.9,46.0,38.6,30.5,28.7,27.9$.

HRMS-ESI: calcd. for $\mathrm{C}_{16} \mathrm{H}_{19} \mathrm{NO}_{2} \mathrm{Na}[\mathrm{M}+\mathrm{Na}]^{+}$: 280.1308 ; found: 280.1301 .

FT-IR: $v\left[\mathrm{~cm}^{-1}\right]=2926,2854,1746,1655,1605,1518,1466,1360,1271,1232,1193$, $1140,1109,1029,910$.

\section{9,10-Dimethoxy-2,2-dimethyl-2,3,6,7-tetrahydro-4H-pyrido[2,1-a]isoquinolin-4- one (12q).}<smiles>COc1cc2c(cc1OC)C1=CC(C)(C)CC(=O)N1CC2</smiles>

12q was prepared according to General procedure C, starting from 11q (150 mg, 0.317 mmol, 1.0 equiv.) and $\mathrm{POCl}_{3}(28.9 \mu \mathrm{L}, 0.317 \mathrm{mmol}, 1.0$ equiv.) in toluene $(5.0 \mathrm{~mL})$. The reaction mixture was subsequently treated with $\mathrm{MeOH}(5.0 \mathrm{~mL}), \mathrm{K}_{2} \mathrm{CO}_{3}$ (438 mg, $3.17 \mathrm{mmol}, 10$ equiv.) and $n \mathrm{Bu} 4 \mathrm{NBr}$ (10.2 mg, $32.0 \mu \mathrm{mol}, 0.1$ equiv.). Purification by silica gel column chromatography (pentane/EtOAc $=3: 1$ ) afforded 12q (80.5 $\mathrm{mg}, 0.280 \mathrm{mmol}, 88 \%)$ as a yellow solid.

${ }^{1} \mathbf{H}$ NMR $\left(500 \mathrm{MHz} \mathrm{CDCl}_{3}\right) \delta[\mathrm{ppm}]=7.02(\mathrm{~s}, 1 \mathrm{H}), 6.61(\mathrm{~s}, 1 \mathrm{H}), 5.47(\mathrm{~s}, 1 \mathrm{H}), 3.91-$ $3.89(\mathrm{~m}, 5 \mathrm{H}), 3.88(\mathrm{~s}, 3 \mathrm{H}), 2.77(\mathrm{t}, J=5.9 \mathrm{~Hz}, 2 \mathrm{H}), 2.42(\mathrm{~s}, 2 \mathrm{H}), 1.14(\mathrm{~s}, 6 \mathrm{H})$. 
${ }^{13} \mathbf{C}$ NMR $\left(126 \mathrm{MHz}, \mathrm{CDCl}_{3}\right) \delta[\mathrm{ppm}]=169.7,149.3,148.2,133.2,127.6,122.3,112.0$, $110.8,106.8,56.2,56.1,46.0,38.5,30.5,28.9,28.2$.

HRMS-ESI: calcd. for $\mathrm{C}_{17} \mathrm{H}_{21} \mathrm{NO}_{3} \mathrm{Na}[\mathrm{M}+\mathrm{Na}]^{+}: 310.1413$; found: 310.1428 .

FT-IR: $v\left[\mathrm{~cm}^{-1}\right]=2964,2934,1775,1645,1516,1388,1358,1287,1263,1234,1198$, $1109,937$.

9,10-Dimethoxy-2,3,6,7-tetrahydro-4H-pyrido[2,1-a]isoquinolin-4-one (12r).<smiles>COc1cc2c(cc1OC)C1=CCCC(=O)N1CC2</smiles>

$12 r$

12r was prepared according to General procedure C, starting from $11 \mathbf{r}$ (100 mg, $0.225 \mathrm{mmol}, 1.0$ equiv.) and $\mathrm{POCl}_{3}(20.5 \mu \mathrm{L}, 0.225 \mathrm{mmol}, 1.0$ equiv.) in toluene $(3.0 \mathrm{~mL})$. The reaction mixture was subsequently treated with $\mathrm{MeOH}(3.0 \mathrm{~mL}), \mathrm{K}_{2} \mathrm{CO}_{3}$ (311 mg, $2.25 \mathrm{mmol}, 10$ equiv.) and $n \mathrm{Bu} 4 \mathrm{NBr}$ (7.2 mg, $22 \mu \mathrm{mol}, 0.1$ equiv.). Purification by neutral aluminium oxide column chromatography (pentane/DCM =1:1) afforded 12r (44.0 mg, $0.170 \mathrm{mmol}, 76 \%)$ as a yellow solid.

${ }^{1} \mathbf{H}$ NMR $\left(500 \mathrm{MHz}, \mathrm{C}_{6} \mathrm{D}_{6}\right) \delta[\mathrm{ppm}]=6.92(\mathrm{~s}, 1 \mathrm{H}), 6.20(\mathrm{~s}, 1 \mathrm{H}), 5.31(\mathrm{td}, J=5.0,1.9$ $\mathrm{Hz}, 1 \mathrm{H}), 3.93(\mathrm{td}, J=5.9,1.8 \mathrm{~Hz}, 2 \mathrm{H}), 3.46$ (s, 3H), 3.36 (s, 3H), 2.41 (td, $J=7.9,1.8$ $\mathrm{Hz}, 2 \mathrm{H}), 2.38-2.31(\mathrm{~m}, 2 \mathrm{H}), 2.00-1.95(\mathrm{~m}, 2 \mathrm{H})$.

${ }^{13}$ C NMR (126 MHz, C6 6 6) $\delta[\mathrm{ppm}]=169.0,150.4,149.2,136.4,127.8,123.0,111.6$, $108.4,99.7,55.9,55.5,38.6,31.6,29.0,19.9$.

HRMS-ESI: calcd. for $\mathrm{C}_{15} \mathrm{H}_{17} \mathrm{NO}_{3} \mathrm{Na}[\mathrm{M}+\mathrm{Na}]^{+}: 282.1100$; found: 282.1106.

FT-IR: $v\left[\mathrm{~cm}^{-1}\right]=2942,1682,1602,1513,1465,1377,1341,1272,1202,1179,1162$, 752.

2,3-Dimethoxy-5,6-dihydro-8H-isoquinolino[3,2-a]isoquinolin-8-one (12s).<smiles>COc1cc2c(cc1OC)-c1cc3ccccc3c(=O)n1CC2</smiles>

12s was prepared according to General procedure C, starting from 11s (193 mg, $0.391 \mathrm{mmol}, 1.0$ equiv.) and $\mathrm{POCl}_{3}(35.7 \mu \mathrm{L}, 0.391 \mathrm{mmol}, 1.0$ equiv.) in toluene S54 
$(5.0 \mathrm{~mL})$. The reaction mixture was subsequently treated with $\mathrm{MeOH}(5.0 \mathrm{~mL}), \mathrm{K}_{2} \mathrm{CO}_{3}$ (541 mg, 3.92 mmol, 10 equiv.) and $n \mathrm{Bu} 4 \mathrm{NBr}$ (12.6 mg, $39.0 \mu \mathrm{mol}, 0.1$ equiv.). Purification by silica gel column chromatography (pentane/EtOAc $=3: 1)$ afforded $12 \mathrm{~s}$ (100 mg, $0.326 \mathrm{mmol}, 83 \%)$ as a yellow solid.

${ }^{1} \mathbf{H}$ NMR $\left(600 \mathrm{MHz}, \mathrm{CDCl}_{3}\right) \delta[\mathrm{ppm}]=8.41(\mathrm{dt}, J=8.1,1.7 \mathrm{~Hz}, 1 \mathrm{H}), 7.62-7.59(\mathrm{~m}$, 1H), 7.58-7.53 (m, 1H), 7.44-7.40 (m, 1H), $7.26(\mathrm{~d}, J=2.1 \mathrm{~Hz}, 1 \mathrm{H}), 6.86(\mathrm{t}, J=2.1$ Hz, 1H), 6.73 (d, $J=2.4 \mathrm{~Hz}, 1 \mathrm{H}), 4.39-4.32$ (m, 2H), 3.98 (s, 3H), 3.93 (s, 3H), 2.93 $(\mathrm{m}, 2 \mathrm{H})$.

${ }^{13} \mathbf{C ~ N M R}\left(151 \mathrm{MHz}, \mathrm{CDCl}_{3}\right) \delta[\mathrm{ppm}]=162.3,150.5,148.6,137.5,136.8,132.4,128.8$, $128.1,126.3,126.0,124.7,122.4,110.6,108.0,101.5,56.4,56.1,39.8,28.2$.

HRMS-ESI: calcd. for $\mathrm{C}_{19} \mathrm{H}_{17} \mathrm{NO}_{3} \mathrm{Na}[\mathrm{M}+\mathrm{Na}]^{+}: 330.1100$ found: 330.1115 .

FT-IR: $v\left[\mathrm{~cm}^{-1}\right]=3001,2937,2839,1644,1606,1512,1466,1362,1269,1234,1104$, $1018,754$.

\section{5,6-Dihydro-8H-isoquinolino[3,2-a]isoquinolin-8-one (12t).}

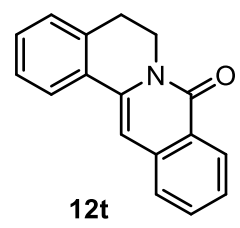

12t was prepared according to General procedure C, starting from 11t (217 mg, $0.501 \mathrm{mmol}, 1.0$ equiv.) and $\mathrm{POCl}_{3}(45.7 \mu \mathrm{L}, 0.501 \mathrm{mmol}, 1.0$ equiv.) in toluene $(6.0 \mathrm{~mL})$. The reaction mixture was subsequently treated with $\mathrm{MeOH}(6.0 \mathrm{~mL}), \mathrm{K}_{2} \mathrm{CO}_{3}$ (693 mg, $5.01 \mathrm{mmol}, 10$ equiv.) and $n \mathrm{Bu} 4 \mathrm{NBr}(16.2 \mathrm{mg}, 50.0 \mu \mathrm{mol}, 0.1$ equiv.). Purification by silica gel column chromatography (pentane/EtOAc $=5: 1)$ afforded 12t (60 mg, $0.243 \mathrm{mmol}, 48 \%$ ) as a yellow solid.

${ }^{1} \mathbf{H}$ NMR $\left(600 \mathrm{MHz}, \mathrm{CD}_{2} \mathrm{Cl}_{2}\right) \delta[\mathrm{ppm}]=8.36(\mathrm{dd}, J=8.1,1.3 \mathrm{~Hz}, 1 \mathrm{H}), 7.90-7.84(\mathrm{~m}$, 1H), 7.65 (ddd, $J=8.2,6.9,1.4 \mathrm{~Hz}, 1 \mathrm{H}), 7.63-7.60(\mathrm{~m}, 1 \mathrm{H}), 7.46$ (ddd, $J=8.1,6.9,1.4$ Hz, 1H), 7.41-7.34 (m, 2H), 7.33-7.27 (m, 1H), 7.05 (s, 1H), 4.37-4.30 (m, 2H), 3.06$2.98(\mathrm{~m}, 2 \mathrm{H})$. 
${ }^{13} \mathrm{C} \mathrm{NMR}\left(151 \mathrm{MHz}, \mathrm{CD}_{2} \mathrm{Cl}_{2}\right) \delta[\mathrm{ppm}]=162.2,138.0,137.0,136.1,132.6,130.7,129.7$, $128.4,128.1,127.8,126.8,126.7,125.4,125.3,102.8,40.0,28.9$.

HRMS-ESI: calcd. for $\mathrm{C}_{17} \mathrm{H}_{13} \mathrm{NONa}[\mathrm{M}+\mathrm{Na}]^{+}: 270.0889$; found: 270.0903 .

FT-IR: $v\left[\mathrm{~cm}^{-1}\right]=2945,2896,1647,1619,1596,1491,1341,1315,1166,766$.

\section{4,5,8,9-Tetrahydro-7H-furo[2,3-a]quinolizin-7-one (12u).}

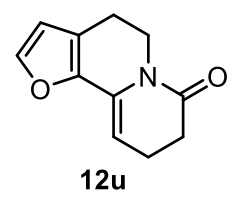

12u was prepared according to General procedure C, starting from $11 \mathbf{u}(43.0 \mathrm{mg}$, $0.115 \mathrm{mmol}, 1.0$ equiv.) and $\mathrm{POCl}_{3}(10.5 \mu \mathrm{L}, 0.115 \mathrm{mmol}, 1.0$ equiv.) in toluene $(1.5 \mathrm{~mL})$. The reaction mixture was subsequently treated with $\mathrm{MeOH}(1.5 \mathrm{~mL}), \mathrm{K}_{2} \mathrm{CO}_{3}$ (158 mg, $1.15 \mathrm{mmol}, 10$ equiv.) and $n \mathrm{Bu}_{4} \mathrm{NBr}$ (3.7 $\mathrm{mg}, 11 \mu \mathrm{mol}, 0.1$ equiv.). Purification by neutral aluminium oxide column chromatography (pentane/DCM =1:1) afforded 12u (18.0 mg, $95.2 \mu \mathrm{mol}, 83 \%)$ as a yellow solid.

${ }^{1}$ H NMR $\left(700 \mathrm{MHz}, \mathrm{C}_{6} \mathrm{D}_{6}\right) \delta[\mathrm{ppm}]=6.90(\mathrm{t}, J=1.7 \mathrm{~Hz}, 1 \mathrm{H}), 5.80(\mathrm{t}, J=1.7 \mathrm{~Hz}, 1 \mathrm{H})$, $5.45(\mathrm{t}, J=5.0 \mathrm{~Hz}, 1 \mathrm{H}), 3.82(\mathrm{t}, J=6.0 \mathrm{~Hz}, 2 \mathrm{H}), 2.30(\mathrm{t}, J=7.7 \mathrm{~Hz}, 2 \mathrm{H}), 2.06(\mathrm{t}, J=$ $6.0 \mathrm{~Hz}, 2 \mathrm{H}), 1.85(\mathrm{td}, J=7.7,5.0 \mathrm{~Hz}, 2 \mathrm{H})$.

${ }^{13}$ C NMR (176 MHz, $\left.\mathrm{C}_{6} \mathrm{D}_{6}\right) \delta[\mathrm{ppm}]=168.4,145.3,142.6,130.4,118.2,110.8,98.1$, $38.7,32.0,21.6,19.5$.

HRMS-ESI: calcd. for $\mathrm{C}_{11} \mathrm{H}_{11} \mathrm{NO}_{2} \mathrm{Na}[\mathrm{M}+\mathrm{Na}]^{+}:$212.0682; found: 212.0675 .

FT-IR: $v\left[\mathrm{~cm}^{-1}\right]=2929,2855,1725,1553,1457,1380,1335,1181,1019,742$.

\section{4,5,8,9-Tetrahydro-7H-furo[3,2-a]quinolizin-7-one (12v).}

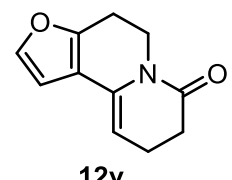

12v was prepared according to General procedure $\mathbf{C}$, starting from $11 \mathbf{v}$ (40.0 $\mathrm{mg}$, 0.107 mmol, 1.0 equiv.) and $\mathrm{POCl}_{3}(9.7 \mu \mathrm{L}, 0.107 \mathrm{mmol}, 1.0$ equiv.) in toluene $(1.5 \mathrm{~mL})$. The reaction mixture was subsequently treated with $\mathrm{MeOH}(1.5 \mathrm{~mL}), \mathrm{K}_{2} \mathrm{CO}_{3}$ 
(147 mg, $1.07 \mathrm{mmol}, 10$ equiv.) and $n \mathrm{Bu} 4 \mathrm{NBr}(3.4 \mathrm{mg}, 11 \mu \mathrm{mol}, 0.1$ equiv.). Purification by neutral aluminium oxide column chromatography (pentane/DCM =1:1) afforded $12 \mathrm{v}(10.0 \mathrm{mg}, 54.0 \mu \mathrm{mol}, 51 \%)$ as a yellow solid.

${ }^{1} \mathbf{H}$ NMR $\left(700 \mathrm{MHz}, \mathrm{C}_{6} \mathrm{D}_{6}\right) \delta[\mathrm{ppm}]=6.90(\mathrm{dd}, J=2.0,0.7 \mathrm{~Hz}, 1 \mathrm{H}), 6.11(\mathrm{~d}, J=2.0$ $\mathrm{Hz}, 1 \mathrm{H}), 4.93(\mathrm{t}, J=4.9 \mathrm{~Hz}, 1 \mathrm{H}), 3.81(\mathrm{t}, J=6.1 \mathrm{~Hz}, 2 \mathrm{H}), 2.33(\mathrm{t}, J=7.7 \mathrm{~Hz}, 2 \mathrm{H})$, $2.22-2.18(\mathrm{~m}, 2 \mathrm{H}), 1.87-1.83(\mathrm{~m}, 2 \mathrm{H})$.

${ }^{13}$ C NMR (176 MHz, $\left.\mathrm{C}_{6} \mathrm{D}_{6}\right) \delta[\mathrm{ppm}]=168.7,150.0,142.3,132.5,115.2,106.3,100.0$, $38.3,32.2,23.0,19.8$.

(Note: as the product owns a vinylogous fragment, which is highly reactive, the NMR shows some impurity, thought we used quite mild isolation method.)

HRMS-ESI: calcd. for $\mathrm{C}_{11} \mathrm{H}_{11} \mathrm{NO}_{2} \mathrm{Na}[\mathrm{M}+\mathrm{Na}]^{+}:$212.0682; found: 212.0688 .

FT-IR: $v\left[\mathrm{~cm}^{-1}\right]=2925,1697,1368,1301,1221,1168,1122,1079,1041,746$.

\section{4,5,8,9-Tetrahydro-7H-thieno[2,3-a]quinolizin-7-one (12w).}

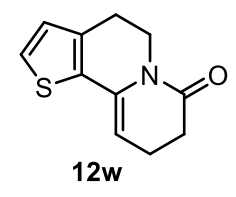

12w was prepared according to General procedure $\mathbf{C}$, starting from $\mathbf{1 1 w}(48.0 \mathrm{mg}$, 0.123 mmol, 1.0 equiv.) and $\mathrm{POCl}_{3}(11.2 \mu \mathrm{L}, 0.123 \mathrm{mmol}, 1.0$ equiv.) in toluene $(1.5 \mathrm{~mL})$. The reaction mixture was subsequently treated with $\mathrm{MeOH}(1.5 \mathrm{~mL}), \mathrm{K}_{2} \mathrm{CO}_{3}$ (170 mg, $1.23 \mathrm{mmol}, 10$ equiv.) and $n \mathrm{Bu} 4 \mathrm{NBr}$ (4.0 mg, $12 \mu \mathrm{mol}, 0.1$ equiv.). Purification by neutral aluminium oxide column chromatography (pentane/DCM = 1:1) afforded 12w (23.0 mg, $0.112 \mathrm{mmol}, 91 \%)$ as a yellow solid.

${ }^{1}$ H NMR $\left(500 \mathrm{MHz}, \mathrm{C}_{6} \mathrm{D}_{6}\right) \delta[\mathrm{ppm}]=6.61(\mathrm{dd}, J=5.1,2.0 \mathrm{~Hz}, 1 \mathrm{H}), 6.31(\mathrm{dd}, J=5.1$, $2.0 \mathrm{~Hz}, 1 \mathrm{H}), 5.20(\mathrm{td}, J=5.0,2.0 \mathrm{~Hz}, 1 \mathrm{H}), 3.83(\mathrm{td}, J=6.0,2.0 \mathrm{~Hz}, 2 \mathrm{H}), 2.29(\mathrm{td}, J=$ 7.8, 1.9 Hz, 2H), 2.17 (td, $J=6.0,2.0 \mathrm{~Hz}, 2 \mathrm{H}), 1.79$ (tdd, $J=7.5,4.9,1.9 \mathrm{~Hz}, 2 \mathrm{H})$.

${ }^{13}$ C NMR $\left(126 \mathrm{MHz}, \mathrm{C}_{6} \mathrm{D}_{6}\right) \delta[\mathrm{ppm}]=168.9,136.6,133.9,132.4,128.0,124.5,101.1$, 39.0, 32.3, 25.6, 20.2.

HRMS-ESI: calcd. for $\mathrm{C}_{11} \mathrm{H}_{11} \mathrm{NOSNa}[\mathrm{M}+\mathrm{Na}]^{+}$: 228.0453; found: 228.0453 .

FT-IR: $v\left[\mathrm{~cm}^{-1}\right]=2930,1681,1433,1367,1331,1304,1225,1171,1136,1035,875$, 
764.

2,3,6,7-tetrahydro-4H-benzo[4,5] thieno[2,3-a]quinolizin-4-one (12x).

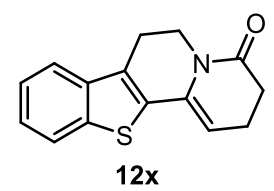

12x was prepared according to General procedure C, starting from 11x (30.0 mg, $68.0 \mu \mathrm{mol}, 1.0$ equiv. $)$ and $\mathrm{POCl}_{3}(6.2 \mu \mathrm{L}, 68 \mu \mathrm{mol}, 1.0$ equiv.) in toluene (1.5 mL). The reaction mixture was subsequently treated with $\mathrm{MeOH}(1.5 \mathrm{~mL}), \mathrm{K}_{2} \mathrm{CO}_{3}$ (94.0 mg, 0.680 mmol, 10 equiv.) and $n \mathrm{Bu} 4 \mathrm{NBr}(2.2 \mathrm{mg}, 7.0 \mu \mathrm{mol}, 0.1$ equiv.). Purification by neutral aluminium oxide column chromatography (pentane/DCM $=1: 1$ ) afforded $\mathbf{1 2} \mathbf{w}$ (15.0 mg, $58.8 \mu \mathrm{mol}, 86 \%$ ) as a yellow solid.

${ }^{1} \mathbf{H}$ NMR $\left(700 \mathrm{MHz}, \mathrm{CD}_{2} \mathrm{Cl}_{2}\right) \delta[\mathrm{ppm}]=7.79(\mathrm{ddd}, J=7.8,1.3,0.7 \mathrm{~Hz}, 1 \mathrm{H}), 7.63(\mathrm{ddd}$, $J=7.8,1.4,0.7 \mathrm{~Hz}, 1 \mathrm{H}), 7.40-7.36(\mathrm{~m}, 1 \mathrm{H}), 7.36-7.33(\mathrm{~m}, 1 \mathrm{H}), 5.66(\mathrm{t}, J=5.0 \mathrm{~Hz}$, $1 \mathrm{H}), 4.08(\mathrm{t}, J=6.0 \mathrm{~Hz}, 2 \mathrm{H}), 2.99-2.90(\mathrm{~m}, 2 \mathrm{H}), 2.58(\mathrm{t}, J=7.8 \mathrm{~Hz}, 2 \mathrm{H}), 2.51-2.40$ $(\mathrm{m}, 2 \mathrm{H})$.

${ }^{13} \mathbf{C}$ NMR $\left(176 \mathrm{MHz}, \mathrm{CD}_{2} \mathrm{Cl}_{2}\right) \delta[\mathrm{ppm}]=169.3,139.1,133.5,131.7,130.8,125.8,125.0$, $122.8,121.9,104.6,38.4,31.9,23.6,20.2$. (One $\mathrm{sp}^{2}$ carbon is missing, probably due to the resonance overlap.)

HRMS-ESI: calcd. for $\mathrm{C}_{15} \mathrm{H}_{13} \mathrm{NOSNa}[\mathrm{M}+\mathrm{Na}]^{+}: 278.0610$; found: 278.0624 .

FT-IR: $v[\mathrm{~cm}-1]=2929,2876,1652,1458,1398,1382,1329,1215,1045,745$.

10-Methoxy-2-methyl-2,6,7,12-tetrahydroindolo[2,3-a]quinolizin-4(3H)-one (13).

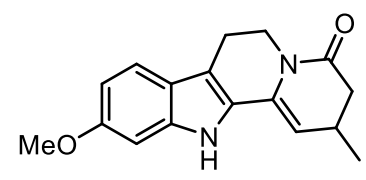

13

13 was prepared according to General procedure C, starting from 11y (100 mg, 0.214 mmol, 1.0 equiv.) and $\mathrm{POCl}_{3}(19.5 \mu \mathrm{L}, 0.214 \mathrm{mmol}, 1.0$ equiv.) in toluene $(3.0 \mathrm{~mL})$. The reaction mixture was subsequently treated with $\mathrm{MeOH}(3.0 \mathrm{~mL}), \mathrm{K}_{2} \mathrm{CO}_{3}$ (295 mg, 2.14 mmol, 10 equiv.) and $n \mathrm{Bu}_{4} \mathrm{NBr}$ (6.9 mg, $21 \mu \mathrm{mol}, 0.1$ equiv.). 
Purification by silica gel column chromatography (pentane/EtOAc $=2: 1$ ) afforded 13 (47.0 mg, $0.167 \mathrm{mmol}, 78 \%)$ as a yellow solid.

${ }^{1} \mathbf{H}$ NMR $\left(700 \mathrm{MHz}, \mathrm{CD}_{2} \mathrm{Cl}_{2}\right) \delta[\mathrm{ppm}]=8.37(\mathrm{~s}, 1 \mathrm{H}), 7.37(\mathrm{dt}, J=8.6,0.6 \mathrm{~Hz}, 1 \mathrm{H})$, $6.85(\mathrm{dd}, J=2.3,0.5 \mathrm{~Hz}, 1 \mathrm{H}), 6.75(\mathrm{dd}, J=8.6,2.3 \mathrm{~Hz}, 1 \mathrm{H}), 5.37(\mathrm{~d}, J=4.0 \mathrm{~Hz}, 1 \mathrm{H})$, $4.34(\mathrm{dt}, J=12.7,5.3 \mathrm{~Hz}, 1 \mathrm{H}), 3.83(\mathrm{~s}, 3 \mathrm{H}), 3.79-3.75(\mathrm{~m}, 1 \mathrm{H}), 2.88-2.83(\mathrm{~m}, 2 \mathrm{H})$, 2.79-2.71 (m, 1H), $2.65(\mathrm{ddd}, J=15.4,5.9,1.0 \mathrm{~Hz}, 1 \mathrm{H}), 2.32(\mathrm{ddd}, J=15.4,10.3,0.7$ $\mathrm{Hz}, 1 \mathrm{H}), 1.15(\mathrm{~d}, J=7.0 \mathrm{~Hz}, 3 \mathrm{H})$.

${ }^{13} \mathrm{C} \mathrm{NMR}\left(176 \mathrm{MHz}, \mathrm{CD}_{2} \mathrm{Cl}_{2}\right) \delta[\mathrm{ppm}]=169.7,158.0,138.7,130.4,127.4,121.5,119.9$, $112.4,110.0,105.2,95.0,55.9,40.4,39.4,26.5,21.0,20.5$.

HRMS-EI: calcd. for $\mathrm{C}_{17} \mathrm{H}_{18} \mathrm{~N}_{2} \mathrm{O}_{2}[\mathrm{M}]^{+}:$282.1362; found: 282.1363 .

FT-IR: $v\left[\mathrm{~cm}^{-1}\right]$ 2952, 2931, 1641, 1625, 1454, 1398, 1262, 1159, 1038, 770.

\subsubsection{Detail and consideration of the annulation of the additional substrates}

The annulation of the additional substrates can be divided into two parts. One is no observation of product by the NMR (group I), the other is NMR showed the product, but liable to decompose (group II). 


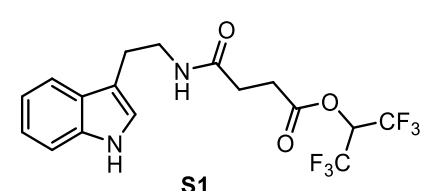

s1

annulation

$\checkmark$

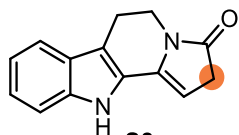

S8<smiles>O=C(CCCC(=O)OC(F)(F)C(F)(F)F)N[C@@H](Cc1ccc(O)cc1)C(=O)O</smiles>

$\stackrel{54}{\text { annulation }}$

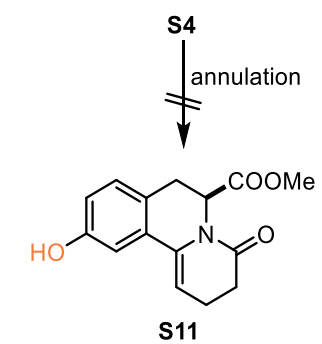

s11<smiles>O=C(CCCC(=O)OC(F)(F)F)NCc1c[nH]c2ccccc12</smiles>

S2

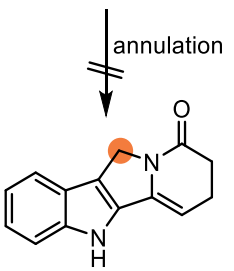

s9

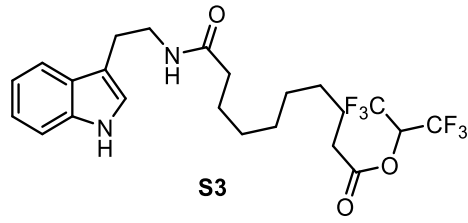

annulation

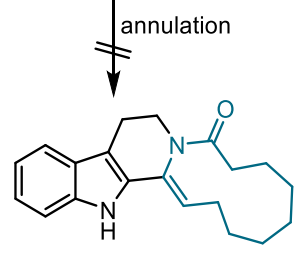

S10<smiles>O=C(CCCC(=O)OC(C(F)(F)F)C(F)(F)F)NCCc1ccccn1</smiles>

s7

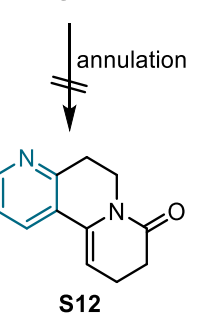

Scheme S-1: Group I examples

There was no proof that the annulation products of S1-3 were formed. We proposed the S8 and S9 have a reactive methylene group, which might cause $\mathbf{S 8}$ and $\mathbf{S 9}$ unstable under the reaction condition. S12 contains an 11-membered ring with a $\mathrm{C}-\mathrm{C}$ double bond. The unsuccessful result of $\mathbf{S 3}$ could be attributed to the difficulty of the macrolactamization or the geometry of the $\mathrm{C}-\mathrm{C}$ double bond. Failure of the annulation of S4 could be caused by the reaction between the free hydroxyl group and the phosphorus (V) reagent. Unsuccessful annulation of $\mathbf{S 7}$ indicates that the reaction is relied on electrophilicity of the heterocycles. (Scheme S-1) 
<smiles>O=C(CCCC(=O)OC(C(F)(F)F)C(F)(F)F)NCCc1ccccc1</smiles>

S5 annulation $\vdots$<smiles>O=C1CCC=C2c3ccccc3CCN12</smiles>

S13<smiles>COc1ccc(CCNC(=O)CCCC(=O)OC(C(F)(F)F)C(F)(F)F)cc1</smiles>

s6

annulation

i<smiles>COc1ccc2c(c1)C1=CCCC(=O)N1CC2</smiles>

S14<smiles>CCCCCCCc1ccccc1CCNC(=O)CCCC(=O)OC(C(F)(F)F)C(F)(F)F</smiles>

endo-dig cycliziation
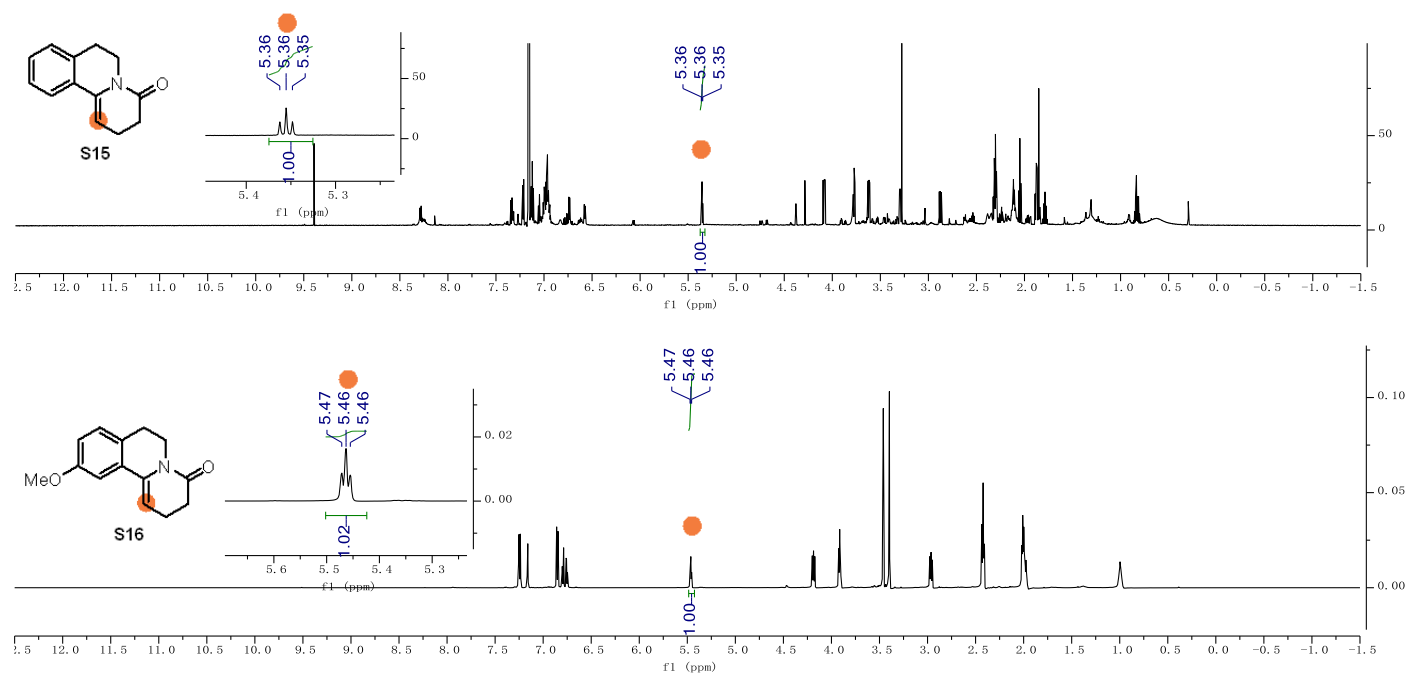

Scheme S-2: Group II examples

The reaction of $\mathbf{S 5}$ and $\mathbf{S 6}$ showed the identical peak of this type of compound on the ${ }^{1} \mathrm{H}$ NMR, but there are some impurities which might be the side products formed during the reaction period or in the isolation. We hypothesized that the electrophilicity of the benzene ring influenced the reaction rate of the endo-dig cyclization. If the rate of the endo-dig cyclization is slow, some competition reactions could happen, leading to side products. In the exo-trig cyclization stage, the Thorpe-Ingold effect could increase the rate and drive the whole reaction equilibrium to form the desired product. (Scheme S2)

In general, these compounds containing the $[2,3-a]$ quinolizinone motif are sensitive to acid, in some case even the $\mathrm{CDCl}_{3}$ can lead to partly decomposition. The stability is dependent on the fused ring system. According to our experience, the indole fused compounds are stable. But the larger ring system is relatively unstable. The other heterocycles mentioned above, without another aromatic ring like 12s and 12t, are 
relatively unstable. 


\subsubsection{Natural products and analogues synthesis}

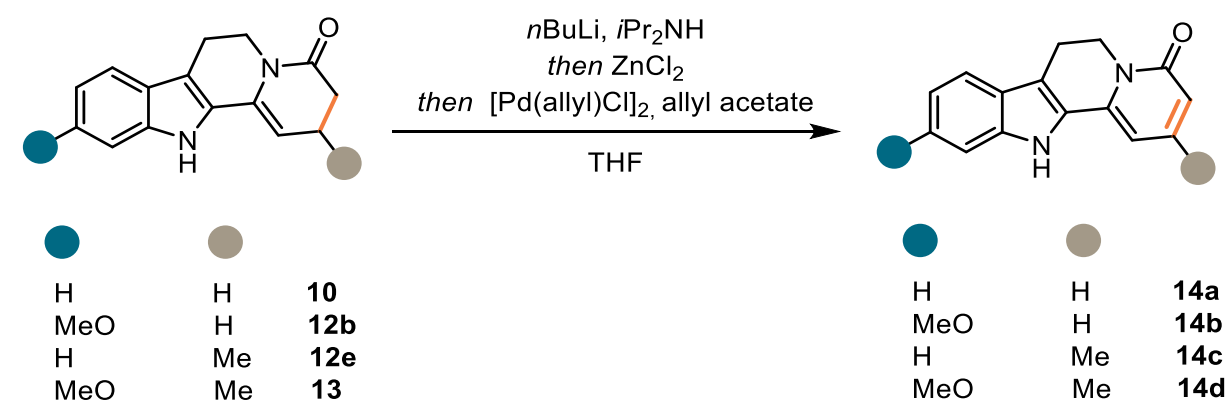

\section{General procedure $D^{1}$}

To a $0{ }^{\circ} \mathrm{C}$ solution of diisopropylamine (10.0 equiv.) in THF was added $n \mathrm{BuLi}(2.5 \mathrm{M}$ in hexane, 10.0 equiv.). The reaction mixture was stirred for $1 \mathrm{~h}$. A solution of enamide (1.0 equiv.) in THF was added dropwise into the resulting mixture. The reaction mixture was stirred for $1 \mathrm{~h}$. $\mathrm{ZnCl}_{2}$ (1.9 $\mathrm{M}$ in 2-Me-THF, 3.5 equiv.) was added and stirred for $1 \mathrm{~h}$. A stock solution of $[\mathrm{Pd}(\text { allyl }) \mathrm{Cl}]_{2}(2.5 \mathrm{~mol} \%)$ and allyl acetate (1.2 equiv. $)$ in THF was next added at the same temperature. The reaction mixture was moved into a preheated $60{ }^{\circ} \mathrm{C}$ oil bath and stirred for $3 \mathrm{~d}$. The resulting mixture was cooled to room temperature and quenched by $\mathrm{NH}_{4} \mathrm{Cl}$ (sat. aq.), diluted with $\mathrm{DCM}$, and the organic phase was separated. The aqueous phase was extracted with DCM and the combined organic layers were washed with brine, dried over $\mathrm{MgSO}_{4}$, filtered, and concentrated under reduced pressure by rotary evaporation and purified by silica gel column chromatography.

(Note: Preparation of $[\mathrm{Pd}($ allyl $) \mathrm{Cl}] 2$, allyl acetate stock solution: [Pd(allyl)Cl]2 (27 mg, $0.075 \mathrm{mmol}$ ) was weighed into a flame dried vial, the vial was evacuated and backfilled with argon (this process was repeated 3 times). Allyl acetate $(0.39 \mathrm{~mL}, 0.36 \mathrm{~g}, 3.6 \mathrm{mmol})$ and THF (2.6 mL) were added sequentially and the solution was stirred for $0.5 \mathrm{~h}$.) 


\section{7,12-Dihydroindolo[2,3-a]quinolizin-4(6H)-one (14a).}

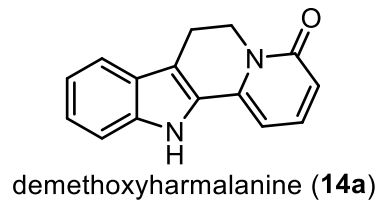

14a was prepared according to General procedure D, starting from diisopropylamine (0.29 mL, $2.1 \mathrm{mmol}, 10.0$ equiv.) in THF $(5.0 \mathrm{~mL})$ was added $n \operatorname{BuLi}(2.5 \mathrm{M}$ in hexane, $0.84 \mathrm{~mL}, 2.1 \mathrm{mmol}, 10.0$ equiv.). A solution of 10 (50 mg, $0.21 \mathrm{mmol}, 1.0$ equiv.) in THF (5.0 mL) was added dropwise into the resulting mixture. Then the reaction mixture was treated with $\mathrm{ZnCl}_{2}$ (1.9 $\mathrm{M}$ in 2-Me-THF, $0.39 \mathrm{~mL}, 0.73 \mathrm{mmol}, 3.5$ equiv.). Afterwards a stock solution of $[\mathrm{Pd}(\text { allyl }) \mathrm{Cl}]_{2}(1.9 \mathrm{mg}, 5.1 \mu \mathrm{mol}, 2.5 \mathrm{~mol} \%)$ and allyl acetate $(27 \mu \mathrm{L}, 25 \mu \mathrm{mol}, 1.2$ equiv.) in THF (1.0 mL) was next added. Purification by silica gel column chromatography (pentane/EtOAc $=1: 2)$ afforded 14a $(38 \mathrm{mg}$, $0.16 \mathrm{mmol}, 76 \%$ ) as a yellow solid.

${ }^{1} \mathbf{H}$ NMR $\left(700 \mathrm{MHz}, \mathrm{CD}_{3} \mathrm{OD}\right) \delta[\mathrm{ppm}]=8.10(\mathrm{dt}, J=8.0,1.0 \mathrm{~Hz}, 1 \mathrm{H}), 8.06(\mathrm{dd}, J=$ 9.0, 7.1 Hz, 1H), $7.93(\mathrm{dt}, J=8.3,0.9 \mathrm{~Hz}, 1 \mathrm{H}), 7.76$ (ddd, $J=8.2,7.0,1.1 \mathrm{~Hz}, 1 \mathrm{H})$, $7.61(\mathrm{ddd}, J=8.0,7.0,0.9 \mathrm{~Hz}, 1 \mathrm{H}), 7.23$ (dd, $J=7.1,1.2 \mathrm{~Hz}, 1 \mathrm{H}), 6.99$ (dd, $J=8.9$, $1.2 \mathrm{~Hz}, 1 \mathrm{H}), 4.95(\mathrm{t}, J=7.0 \mathrm{~Hz}, 2 \mathrm{H}), 3.64(\mathrm{dd}, J=7.5,6.5 \mathrm{~Hz}, 2 \mathrm{H})$.

${ }^{13} \mathrm{C}$ NMR $\left(176 \mathrm{MHz}, \mathrm{CD}_{3} \mathrm{OD}\right) \delta[\mathrm{ppm}]=165.0,141.2,140.3,140.0,128.6,126.9$, $125.5,121.1,120.4,117.8,115.3,112.8,102.4,42.1,20.3$

HRMS-ESI: calcd. for $\mathrm{C}_{15} \mathrm{H}_{13} \mathrm{~N}_{2} \mathrm{O}[\mathrm{M}+\mathrm{H}]^{+}$: 237.1023; found: 237.1021 .

FT-IR: $v\left[\mathrm{~cm}^{-1}\right]=2927,2185,1652,1568,1539,1500,1452,1324,1143,777,740$.

10-Methoxy-7,12-dihydroindolo[2,3-a]quinolizin-4(6H)-one (14b).

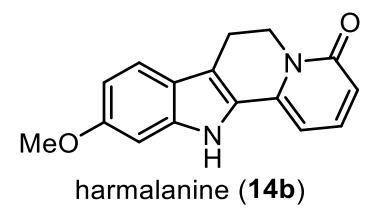

14b was prepared according to General procedure D, starting from diisopropylamine (0.13 mL, $0.93 \mathrm{mmol}, 10.0$ equiv.) in THF (2.5 mL) was added $n \mathrm{BuLi}(2.5 \mathrm{M}$ in hexane, $0.37 \mathrm{~mL}, 0.93 \mathrm{mmol}, 10.0$ equiv.). A solution of $\mathbf{1 2 b}(25 \mathrm{mg}, 93 \mu \mathrm{mol}, 1.0$ equiv.) in THF (2.5 mL) was added dropwise into the resulting mixture. Then the reaction mixture 
was treated with $\mathrm{ZnCl}_{2}$ (1.9 $\mathrm{M}$ in 2-Me-THF, $0.17 \mathrm{~mL}, 0.33 \mathrm{mmol}, 3.5$ equiv.). After that, the stock solution of $[\mathrm{Pd}(\text { allyl }) \mathrm{Cl}]_{2}(0.85 \mathrm{mg}, 2.3 \mu \mathrm{mol}, 2.5 \mathrm{~mol} \%)$ and allyl acetate (11.2 $\mu \mathrm{L}, 0.11 \mathrm{mmol}, 1.2$ equiv.) in THF $(0.5 \mathrm{~mL})$ was next added. Purification by silica gel column chromatography (pentane/EtOAc $=1: 2)$ afforded 14a $(15 \mathrm{mg}, 56 \mu \mathrm{mol}$, $60 \%$ ) as a yellow solid.

${ }^{1} \mathbf{H}$ NMR $\left(500 \mathrm{MHz}, \mathrm{CDCl}_{3}\right) \delta[\mathrm{ppm}]=8.27(\mathrm{~s}, 1 \mathrm{H}), 7.46(\mathrm{~d}, J=8.7 \mathrm{~Hz}, 1 \mathrm{H}), 7.33(\mathrm{dd}$, $J=9.1,7.0 \mathrm{~Hz}, 1 \mathrm{H}), 6.87(\mathrm{~d}, J=2.2 \mathrm{~Hz}, 1 \mathrm{H}), 6.83(\mathrm{dd}, J=8.7,2.3 \mathrm{~Hz}, 1 \mathrm{H}), 6.48(\mathrm{dd}$, $J=9.1,1.2 \mathrm{~Hz}, 1 \mathrm{H}), 6.25(\mathrm{dd}, J=7.0,1.2 \mathrm{~Hz}, 1 \mathrm{H}), 4.43(\mathrm{t}, J=6.9 \mathrm{~Hz}, 2 \mathrm{H}), 3.87(\mathrm{~s}$, $3 \mathrm{H}), 3.06(\mathrm{t}, J=7.0 \mathrm{~Hz}, 2 \mathrm{H})$.

${ }^{13} \mathrm{C}$ NMR $\left(126 \mathrm{MHz}, \mathrm{CD}_{3} \mathrm{OD}\right) \delta[\mathrm{ppm}]=165.0,159.9,141.4,141.2,140.2,127.5$, $121.3,121.2,116.9,115.8,111.9,101.7,95.3,55.9,42.0,20.4$.

HRMS-ESI: calcd. for $\mathrm{C}_{16} \mathrm{H}_{15} \mathrm{~N}_{2} \mathrm{O}_{2}[\mathrm{M}+\mathrm{H}]^{+}:$267.1128; found: 267.1127.

FT-IR: $v\left[\mathrm{~cm}^{-1}\right]=2922,2853,1651,1567,1540,1457,1375,1271,1201,1161,1143$, 798.

\section{2-Methyl-7,12-dihydroindolo[2,3-a]quinolizin-4(6H)-one (14c).}

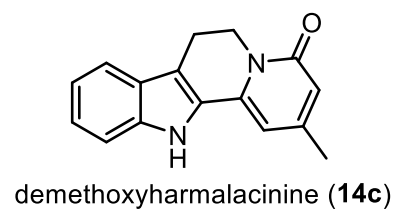

14c was prepared according to General procedure $\mathbf{D}$, starting from diisopropylamine (0.17 mL, $1.2 \mathrm{mmol}, 10.0$ equiv.) in THF (3.0 mL) was added $n \operatorname{BuLi}(2.5 \mathrm{M}$ in hexane, $0.48 \mathrm{~mL}, 1.2 \mathrm{mmol}, 10.0$ equiv.). A solution of $12 \mathrm{e}$ (30 mg, $0.12 \mathrm{mmol}, 1.0$ equiv.) in THF $(3.0 \mathrm{~mL})$ was added dropwise into the resulting mixture. Then the reaction mixture was treated with $\mathrm{ZnCl}_{2}$ (1.9 M in 2-Me-THF, $0.22 \mathrm{~mL}, 0.42 \mathrm{mmol}, 3.5$ equiv.). After that, the stock solution of $[\mathrm{Pd}(\text { allyl }) \mathrm{Cl}]_{2}(1.1 \mathrm{mg}, 3.0 \mu \mathrm{mol}, 2.5 \mathrm{~mol} \%)$ and allyl acetate ( $15 \mu \mathrm{L}, 0.14 \mathrm{mmol}, 1.2$ equiv.) in THF $(0.5 \mathrm{~mL})$ was next added. Purification by silica gel column chromatography (pentane/EtOAc $=1: 2)$ afforded 14c $(21 \mathrm{mg}, 84 \mu \mathrm{mol}$, $71 \%$ ) as a yellow solid.

${ }^{1} \mathbf{H}$ NMR $\left(500 \mathrm{MHz}, \mathrm{CDCl}_{3}+\mathrm{CD}_{3} \mathrm{OD}(\mathrm{v} / \mathrm{v}=1: 1)\right) \delta[\mathrm{ppm}]=7.82-7.76(\mathrm{~m}, 1 \mathrm{H}), 7.65$ $(\mathrm{dd}, J=8.3,1.1 \mathrm{~Hz}, 1 \mathrm{H}), 7.48(\mathrm{ddd}, J=8.2,7.1,1.3 \mathrm{~Hz}, 1 \mathrm{H}), 7.34(\mathrm{ddd}, J=8.0,7.0$, 
$1.1 \mathrm{~Hz}, 1 \mathrm{H}), 6.83(\mathrm{~d}, J=1.8 \mathrm{~Hz}, 1 \mathrm{H}), 6.59-6.52(\mathrm{~m}, 1 \mathrm{H}), 4.62(\mathrm{t}, J=7.0 \mathrm{~Hz}, 2 \mathrm{H}), 3.34$ $(\mathrm{t}, J=6.9 \mathrm{~Hz}, 2 \mathrm{H}), 2.51(\mathrm{~s}, 3 \mathrm{H})$.

${ }^{13} \mathbf{C ~ N M R}\left(126 \mathrm{MHz}, \mathrm{CDCl}_{3}+\mathrm{CD}_{3} \mathrm{OD}(\mathrm{v} / \mathrm{v}=1: 1)\right) \delta[\mathrm{ppm}]=163.2,151.5,138.4,137.3$, 127.0, 125.2, 123.9, 119.6, 118.9, 115.2, 113.6, 111.4, 103.7, 40.4, 20.5, 19.1 .

HRMS-ESI: calcd. for $\mathrm{C}_{16} \mathrm{H}_{13} \mathrm{~N}_{2} \mathrm{O}$ [M - H] $]^{-}$: 249.1033; found: 249.1035.

FT-IR: $v\left[\mathrm{~cm}^{-1}\right]=2924,1649,1583,1568,1543,1499,1364,1148,743$.

10-Methoxy-2-methyl-7,12-dihydroindolo[2,3-a]quinolizin-4(6H)-one (14d).

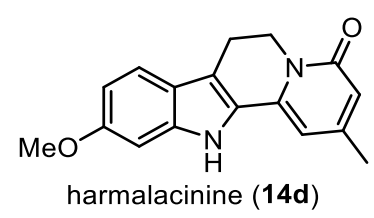

14d was prepared according to General procedure $\mathbf{D}$, starting from diisopropylamine (0.35 mL, $2.5 \mathrm{mmol}, 15.0$ equiv.) in THF $(3.0 \mathrm{~mL})$ was added $n \mathrm{BuLi}(2.5 \mathrm{M}$ in hexanes, $1.0 \mathrm{~mL}, 2.5 \mathrm{mmol}, 15.0$ equiv.). A solution of $\mathbf{1 3}$ (47 mg, $0.17 \mathrm{mmol}, 1.0$ equiv.) in THF $(3.0 \mathrm{~mL})$ was added dropwise into the resulting mixture. Then the reaction mixture was treated with $\mathrm{ZnCl}_{2}(1.9 \mathrm{M}$ in 2-Me-THF, $0.44 \mathrm{~mL}, 0.83 \mathrm{mmol}, 3.5$ equiv.). After that, the stock solution of $[\mathrm{Pd}(\text { allyl }) \mathrm{Cl}]_{2}(0.15 \mathrm{mg}, 4.2 \mu \mathrm{mol}, 2.5 \mathrm{~mol} \%)$ and allyl acetate (22 $\mu \mathrm{L}, 0.20 \mathrm{mmol}, 1.2$ equiv.) in THF $(0.5 \mathrm{~mL})$ was next added. Purification by silica gel column chromatography (pentane/EtOAc = 1:2) afforded 14d $(36 \mathrm{mg}, 0.129 \mathrm{mmol}$, $77 \%$ ) as a yellow solid.

${ }^{1} \mathbf{H}$ NMR $\left(500 \mathrm{MHz}, \mathrm{CDCl}_{3}\right) \delta[\mathrm{ppm}]=9.11(\mathrm{~s}, 1 \mathrm{H}), 7.44(\mathrm{~d}, J=8.7 \mathrm{~Hz}, 1 \mathrm{H}), 6.87(\mathrm{~d}$, $J=2.2 \mathrm{~Hz}, 1 \mathrm{H}), 6.82(\mathrm{dd}, J=8.7,2.3 \mathrm{~Hz}, 1 \mathrm{H}), 6.31(\mathrm{t}, J=1.4 \mathrm{~Hz}, 1 \mathrm{H}), 6.26(\mathrm{~d}, J=1.7$ $\mathrm{Hz}, 1 \mathrm{H}), 4.41(\mathrm{t}, J=6.9 \mathrm{~Hz}, 2 \mathrm{H}), 3.85$ (s, 3H), $3.03(\mathrm{t}, J=6.9 \mathrm{~Hz}, 2 \mathrm{H}), 2.17$ (s, 3H).

${ }^{13} \mathbf{C ~ N M R}\left(126 \mathrm{MHz}, \mathrm{CDCl}_{3}\right) \delta[\mathrm{ppm}]=163.2,158.5,150.2,139.6,137.2,126.7120 .5$, 120.4, 116.4, $115.2111 .0,102.0,94.9,55.8,40.4,21.5,19.9$.

HRMS-ESI: calcd. for $\mathrm{C}_{17} \mathrm{H}_{17} \mathrm{~N}_{2} \mathrm{O}_{2}[\mathrm{M}+\mathrm{H}]^{+}$: 281.1284; found: 281.1284.

FT-IR: $v\left[\mathrm{~cm}^{-1}\right]$ 3184, 2922, 1651, 1626, 1567, 1460, 1374, 1270, 1201, 1139, 1031, $814,753$. 


\subsubsection{Optimization of regioselective dehydrogenation}

With the classic dehydrogenation methods, the reactions turned out to be unsatisfying.

The best result was obtained with LDA, sulfinimidoyl chloride system. We consiedered that the enolation was a reasonable strategy to afford the regioselectivity.

Table S-1: Dehydrogenation by classic conditions

\begin{tabular}{|c|c|c|c|}
\hline entry & conditions & solvent & yield \\
\hline 1 & $\mathrm{MnO}_{2}$ & DCM & trace \\
\hline 2 & $\gamma-\mathrm{MnO}_{2}$ & DCM & trace \\
\hline 3 & NBS, BPO & $\mathrm{CDCl}_{3}$ & no conversion \\
\hline 4 & $\mathrm{~S}$, Dowtherm ${ }^{\circledR} \mathrm{A}$ & -- & complex mixture \\
\hline 5 & DDQ & Dioxane & complex mixture \\
\hline 6 & $\mathrm{Cu}(\mathrm{OAc})_{2}, \mathrm{Na}_{2} \mathrm{CO}_{3}, \mathrm{py}, \mathrm{O}_{2}$ & toluene & no conversion \\
\hline 7 & $\mathrm{CuBr}_{2}$ & $\mathrm{MeCN}$ & no conversion \\
\hline 8 & $\mathrm{Pd}(\mathrm{OAc})_{2}, \mathrm{O}_{2}$ & DMSO & trace \\
\hline 9 & $\mathrm{~V}(\mathrm{acac})_{2}, \mathrm{TFA}, \mathrm{Bu}_{4} \mathrm{NBr}, \mathrm{O}_{2}$ & Dioxane & complex mixture \\
\hline 10 & LiTMP, sulfinimidoyl chloride & THF & complex mixture \\
\hline 11 & NaHMDS, sulfinimidoyl chloride & THF & trace \\
\hline 12 & MeLi, sulfinimidoyl chloride & THF & $14 \%$ \\
\hline 13 & LDA, PhSeCl & THF & trace \\
\hline 14 & LDA, PhsooMe & THF & complex mixture \\
\hline 15 & LDA, sulfinimidoyl chloride & THF & $33 \%$ \\
\hline
\end{tabular}

With the protocol reported by Newhouse et al., initially the resulted in conversion. Finally, LDA was the best base for the reaction affording $76 \%$ yield.

Table S-2: Optimization of Newhouse's procedure

\begin{tabular}{|c|c|c|c|c|}
\hline entry & Step 1 & Step 2 & Step 3 & yield $^{[\mathrm{a}]}$ \\
\hline 1 & LiCyan (2.5 eq.), $-40{ }^{\circ} \mathrm{C}$ & $\mathrm{ZnCl}_{2}$ (3.5 eq.) & {$[\mathrm{Pd}](2.5 \mathrm{~mol} \%), 14 \mathrm{~h}$} & $S M: P=9.8: 1$ \\
\hline 2 & LiCyan (2.5 eq.),$-40^{\circ} \mathrm{C}$ & $\mathrm{ZnCl}_{2}$ (3.5 eq.) & {$[\mathrm{Pd}](2.5 \mathrm{~mol} \%), 38 \mathrm{~h}$} & $S M: P=5.1: 1$ \\
\hline 3 & LiCyan (2.5 eq.),$-40{ }^{\circ} \mathrm{C}$ & $\mathrm{ZnCl}_{2}$ (3.5 eq.) & [Pd] $(2.5 \mathrm{~mol} \%), 62 \mathrm{~h}$ & $S M: P=6.5: 1$ \\
\hline 4 & LiCyan (2.5 eq.),$-40^{\circ} \mathrm{C}$ & $\mathrm{ZnCl}_{2}$ (3.5 eq.) & [Pd] $(2.5 \mathrm{~mol} \%), 86 \mathrm{~h}$ & $S M: P=2.1: 1$ \\
\hline 5 & LiCyan (5.0 eq.), $-40^{\circ} \mathrm{C}$ & $\mathrm{ZnCl}_{2}$ (7.0 eq.) & [Pd] $(5.0 \mathrm{~mol} \%), 14 \mathrm{~h}$ & $S M: P=3.9: 1$ \\
\hline 6 & LiCyan (5.0 eq.), $-40^{\circ} \mathrm{C}$ & $\mathrm{ZnCl}_{2}$ (7.0 eq.) & [Pd] $(5.0 \mathrm{~mol} \%), 38 \mathrm{~h}$ & $S M: P=3.8: 1$ \\
\hline 7 & LiCyan (5.0 eq.), $-40^{\circ} \mathrm{C}$ & $\mathrm{ZnCl}_{2}$ (7.0 eq.) & [Pd] $(5.0 \mathrm{~mol} \%), 62 \mathrm{~h}$ & $S M: P=3.4: 1$ \\
\hline 8 & LiCyan (5.0 eq.),$-40^{\circ} \mathrm{C}$ & $\mathrm{ZnCl}_{2}$ (7.0 eq.) & [Pd] $(5.0 \mathrm{~mol} \%), 86 \mathrm{~h}$ & $S M: P=2.3: 1$ \\
\hline 9 & LiCyan (2.5 eq.), $0^{\circ} \mathrm{C}$ & $\mathrm{ZnCl}_{2}$ (3.5 eq.) & [Pd] $(2.5 \mathrm{~mol} \%), 14 \mathrm{~h}$ & $S M: P=36: 1$ \\
\hline 10 & LiCyan (2.5 eq.), $0{ }^{\circ} \mathrm{C}$ & $\mathrm{ZnCl}_{2}$ (3.5 eq.) & {$[\mathrm{Pd}](2.5 \mathrm{~mol} \%), 75 \mathrm{~h}$} & $S M: P=9.4: 1$ \\
\hline 11 & LiCyan (2.5 eq.), $0{ }^{\circ} \mathrm{C}$ & $\mathrm{ZnCl}_{2}$ (3.5 eq.) & [Pd] $(2.5 \mathrm{~mol} \%), 96 \mathrm{~h}$ & $S M: P=9.5: 1$ \\
\hline 12 & LiTMP (2.5 eq.), $0^{\circ} \mathrm{C}$ & $\mathrm{ZnCl}_{2}$ (3.5 eq.) & [Pd] $(2.5 \mathrm{~mol} \%), 14 \mathrm{~h}$ & $S M: P=4.0: 1$ \\
\hline 13 & LiTMP (2.5 eq.), $0^{\circ} \mathrm{C}$ & $\mathrm{ZnCl}_{2}$ (3.5 eq.) & [Pd] $(2.5 \mathrm{~mol} \%), 75 \mathrm{~h}$ & $S M: P=2.3: 1$ \\
\hline 14 & LiTMP (2.5 eq.), $0^{\circ} \mathrm{C}$ & $\mathrm{ZnCl}_{2}$ (3.5 eq.) & [Pd] $(2.5 \mathrm{~mol} \%), 96 \mathrm{~h}$ & $S M: P=2.4: 1$ \\
\hline 15 & LiCyan ( 10 eq.), $-40^{\circ} \mathrm{C}$ & $\mathrm{ZnCl}_{2}$ (3.5 eq.) & [Pd] $(2.5 \mathrm{~mol} \%), 21 \mathrm{~h}$ & $S M: P=3: 1$ \\
\hline 16 & LiCyan (10 eq.), $-40^{\circ} \mathrm{C}$ & $\mathrm{ZnCl}_{2}$ (3.5 eq.) & {$[\mathrm{Pd}](2.5 \mathrm{~mol} \%), 51 \mathrm{~h}$} & $S M: P=2.9: 1$ \\
\hline 17 & LiCyan (10 eq.), $-40^{\circ} \mathrm{C}$ & $\mathrm{ZnCl}_{2}$ (3.5 eq.) & [Pd] $(2.5 \mathrm{~mol} \%), 88 \mathrm{~h}$ & $S M: P=1.5: 1$ \\
\hline 18 & LiTMP (10 eq.), $0{ }^{\circ} \mathrm{C}$ & $\mathrm{ZnCl}_{2}$ (3.5 eq.) & [Pd] $(2.5 \mathrm{~mol} \%), 21 \mathrm{~h}$ & $S M: P=1.7: 1$ \\
\hline 19 & LiTMP (10 eq.), $0^{\circ} \mathrm{C}$ & $\mathrm{ZnCl}_{2}$ (3.5 eq.) & [Pd] $(2.5 \mathrm{~mol} \%), 51 \mathrm{~h}$ & $S M: P=2.0: 1$ \\
\hline 20 & LiTMP (10 eq.), $0{ }^{\circ} \mathrm{C}$ & $\mathrm{ZnCl}_{2}$ (3.5 eq.) & [Pd] $(2.5 \mathrm{~mol} \%), 88 \mathrm{~h}$ & $S M: P=0.5: 1$ \\
\hline 21 & $\operatorname{LDA}\left(10\right.$ eq.), $0^{\circ} \mathrm{C}$ & $\mathrm{ZnCl}_{2}$ (3.5 eq.) & [Pd] $(2.5 \mathrm{~mol} \%), 88 \mathrm{~h}$ & $76 \%{ }^{[\mathrm{b}]}$ \\
\hline
\end{tabular}

[a] ratio was determined by NMR; [b] isolated yield 


\subsection{Reductive pathway}

\section{2,3,6,7,12,12b-Hexahydroindolo[2,3-a]quinolizin-4(1H)-one (rac-15).}

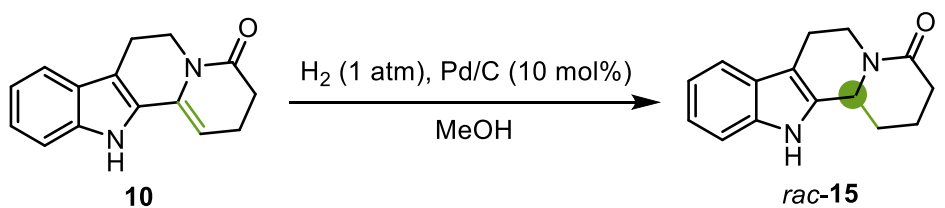

To a solution of enamide 10 (0.10 g, $0.42 \mathrm{mmol}, 1.0$ equiv.) in $\mathrm{MeOH}(10 \mathrm{~mL})$, was added $\mathrm{Pd} / \mathrm{C}$ (10\% Pd on charcoal, $45 \mathrm{mg}, 40 \mu \mathrm{mol}, 0.1$ equiv.). Then hydrogen was charged to the reaction mixture with a balloon. After the mixture was stirred overnight, it was filtered through Celite ${ }^{\circledR}$, and concentrated under reduced pressure. Purification by silica gel column chromatography (pentane/EtOAc $=1: 1)$ afforded rac-15 $(76 \mathrm{mg}$, $0.32 \mathrm{mmol}, 75 \%$ ) as a light yellow powder.

\section{2,3,6,7,12,12b-hexahydroindolo[2,3-a]quinolizin-4(1H)-one $((S)-15)$.}

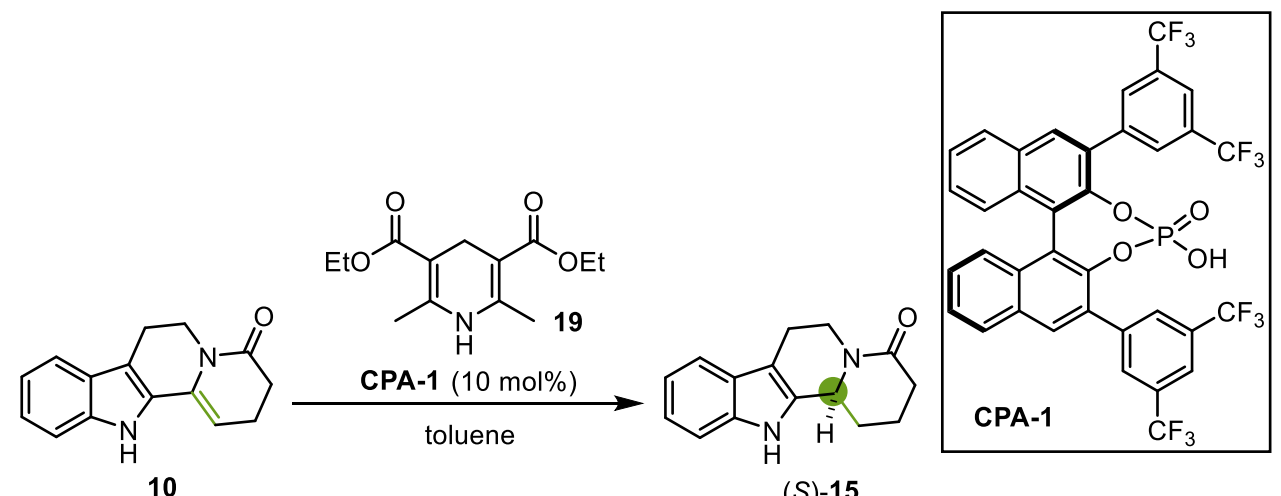

To a mixture of enamide 10 (26 mg, $0.11 \mathrm{mmol}, 1.0$ equiv.) and Hantzsch ester 19 (42 mg, $0.16 \mathrm{mmol}, 1.5$ equiv.) in toluene $(2.5 \mathrm{~mL})$, was added a solution of CPA-1 (8.4 mg, $10 \mu \mathrm{mol}, 0.1$ equiv.) in toluene $(1.0 \mathrm{~mL})$. Then the reaction mixture was immediately moved to a pre-heated $60^{\circ} \mathrm{C}$ oil bath. After stirring for $24 \mathrm{~h}$, the resulting mixture was quenched by $\mathrm{NaHCO}_{3}$ (sat. aq.), diluted with DCM. The organic phase was separated. The aqueous phase was extracted with DCM and the combined organic layers were washed with brine, dried over $\mathrm{MgSO}_{4}$, filtered, and concentrated under reduced pressure. Purification by silica gel column chromatography (pentane/EtOAc $=$ 1:1) afforded $(\boldsymbol{S})-15$ (16 mg, $70 \mu \mathrm{mol}, 61 \%, 99 \%$ (b.r.s.m.), 80\% ee) as a light yellow powder. 
${ }^{1} \mathbf{H}$ NMR $\left(500 \mathrm{MHz}, \mathrm{CDCl}_{3}\right) \delta[\mathrm{ppm}]=8.09(\mathrm{~s}, 1 \mathrm{H}), 7.51(\mathrm{dd}, J=7.8,1.1 \mathrm{~Hz}, 1 \mathrm{H})$, 7.34 (dt, $J=8.0,0.9 \mathrm{~Hz}, 1 \mathrm{H}), 7.18$ (ddd, $J=8.2,7.0,1.2 \mathrm{~Hz}, 1 \mathrm{H}), 7.13$ (ddd, $J=8.0$, 7.0, $1.0 \mathrm{~Hz}, 1 \mathrm{H}), 5.23-5.14(\mathrm{~m}, 1 \mathrm{H}), 4.82-4.75(\mathrm{~m}, 1 \mathrm{H}), 2.87(\mathrm{~m}, 2 \mathrm{H}), 2.81-2.73(\mathrm{~m}$, 1H), 2.62-2.57 (m, 1H), 2.50-2.43 (m, 1H), 2.40 (td, $J=11.6,5.8 \mathrm{~Hz}, 1 \mathrm{H}), 2.02-1.93$ (m, 1H), 1.92-1.83 (m, 1H), 1.83-1.73 (m, 1H).

${ }^{13} \mathbf{C ~ N M R}\left(126 \mathrm{MHz}, \mathrm{CDCl}_{3}\right) \delta[\mathrm{ppm}]=169.5,136.3,133.4,127.0,122.3,120.0,118.6$, $111.1,109.8,54.6,40.4,32.5,29.2,21.1,19.5$.

HRMS-EI: calcd. for $\mathrm{C}_{15} \mathrm{H}_{16} \mathrm{~N}_{2} \mathrm{O}$ [M] $]^{+}$: 240.1257; found: 240.1259 .

FT-IR: $v\left[\mathrm{~cm}^{-1}\right]=3223,2923,2853,1615,1470,1445,1415,1266,1235,741$.

$[\boldsymbol{\alpha}]_{\mathbf{D}}^{26}=-191.2\left(c=0.8, \mathrm{CHCl}_{3}\right) ;$ Lit. $^{2}:[\boldsymbol{\alpha}]_{\mathbf{D}}^{25}=-220.2\left(c=0.10 \mathrm{CHCl}_{3}\right)(94 \% e e)$

HPLC: $10 \%$ EtOH/Hexane, Chiralpak IA, $1.0 \mathrm{~mL} / \mathrm{min}$

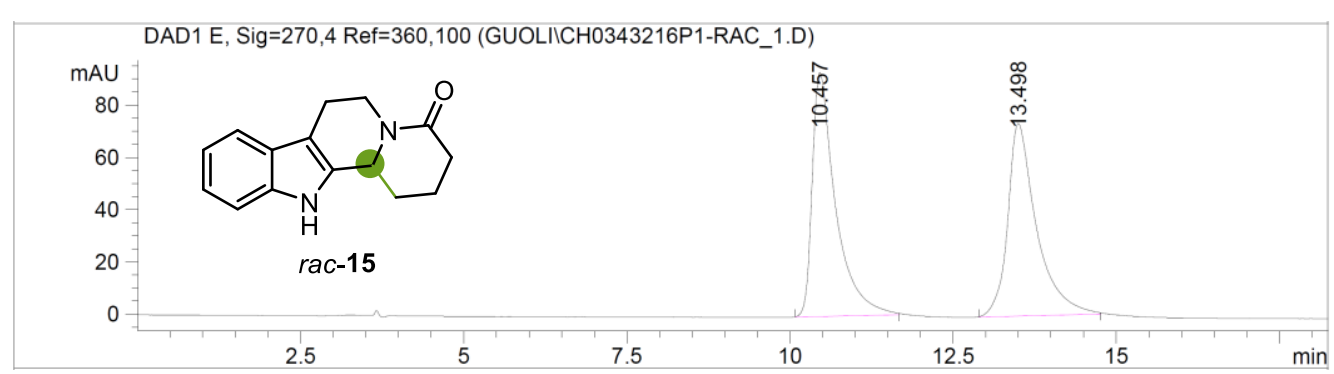

Signal 3: DAD1 E, Sig=270,4 Ref=360,100

\begin{tabular}{|c|c|c|c|c|c|c|}
\hline $\begin{array}{c}\text { Peak } \\
\#\end{array}$ & $\begin{array}{c}\text { RetTime } \\
\text { [min] }\end{array}$ & Type & $\begin{array}{l}\text { Width } \\
\text { [min] }\end{array}$ & $\begin{array}{c}\text { Area } \\
{\left[\mathrm{mAU}^{*} \mathrm{~s}\right]}\end{array}$ & $\begin{array}{l}\text { Height } \\
\text { [mAU] }\end{array}$ & $\begin{array}{c}\text { Area } \\
\frac{\circ}{0}\end{array}$ \\
\hline \multicolumn{7}{|c|}{$----|-------|$} \\
\hline 1 & 10.457 & BB & 0.3627 & 2324.59595 & 93.30048 & 50.6335 \\
\hline 2 & 13.498 & $\mathrm{BB}$ & 0.4409 & 2266.42432 & 73.67532 & 49.3665 \\
\hline Tota & $S:$ & & & 4591.02026 & 166.97581 & \\
\hline
\end{tabular}

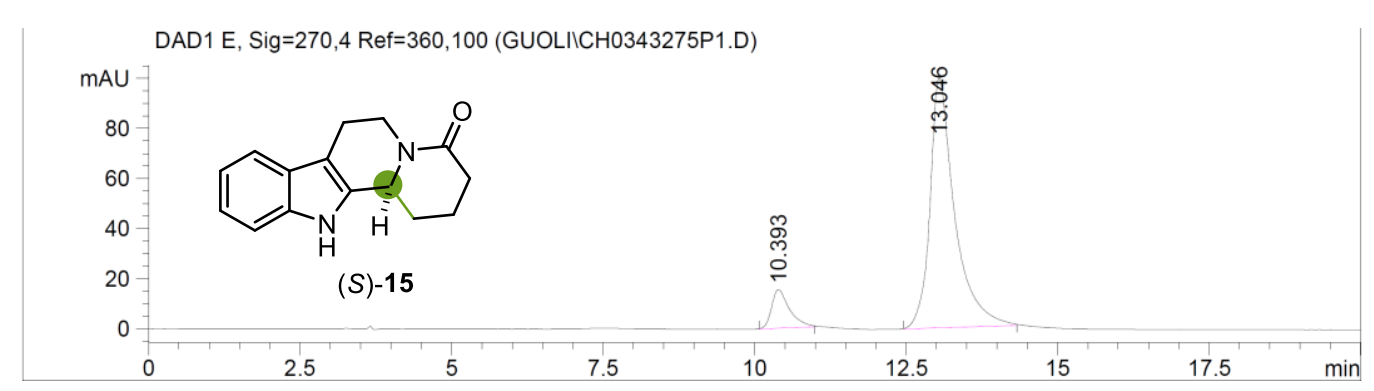

[2] P. Mondal, N. P. Argade J. Org. Chem. 2013, 78, 6802-6808 


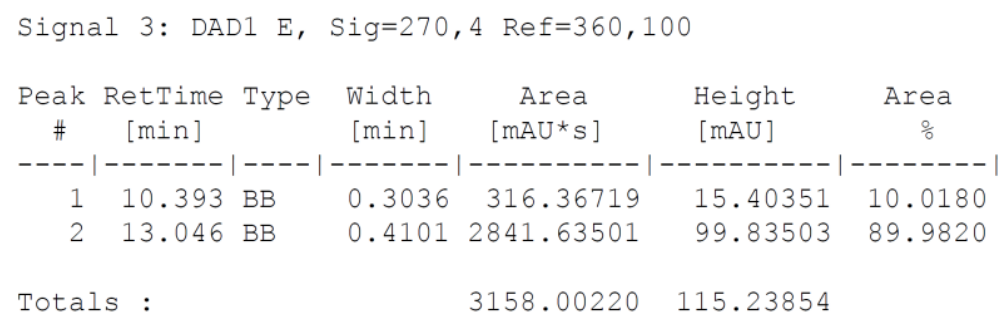

6,7,12,12b-Tetrahydroindolo[2,3-a]quinolizin-4(1H)-one (16). ${ }^{[3]}$

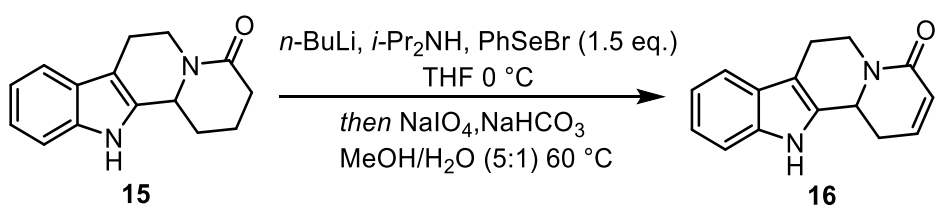

Diisopropylamine (234 $\mu \mathrm{L}, 1.67 \mathrm{mmol}, 10.0$ equiv.) was added to dry THF (5.0 mL) at $0{ }^{\circ} \mathrm{C} . n \mathrm{BuLi}(2.5 \mathrm{M}$ in hexane, $667 \mu \mathrm{L}, 1.67 \mathrm{mmol}, 10.0$ equiv.) was added and the mixture was stirred for $30 \mathrm{~min}$ before cooling to $-78^{\circ} \mathrm{C}$. A solution of $\mathrm{rac}-\mathbf{1 5}(40.0 \mathrm{mg}$, $0.167 \mathrm{mmol}, 1.0$ equiv. $)$ in THF $(2.0 \mathrm{~mL})$ was added dropwise. The reaction was stirred at $-78{ }^{\circ} \mathrm{C}$ for $1 \mathrm{~h}$. A solution of $\mathrm{PhSeBr}(59.0 \mathrm{mg}, 250 \mu \mathrm{mol}, 1.5$ equiv. $)$ in THF (2.0 mL) was the added dropwise and the reaction mixture was stirred for $24 \mathrm{~h}$. The reaction was quenched by $\mathrm{NH}_{4} \mathrm{Cl}$ (sat. aq.) and extracted with DCM. The combined organic phases were washed with brine, dried over $\mathrm{MgSO}_{4}$, filtered and concentrated under reduced pressure. The crude selenide was dissolved in methanol $(5.0 \mathrm{~mL})$ and water $(1.0 \mathrm{~mL})$. $\mathrm{NaIO}_{4}\left(71.0 \mathrm{mg}, 333 \mu \mathrm{mol}, 2.0\right.$ equiv.) and $\mathrm{NaHCO}_{3}(16.8 \mathrm{mg}, 200 \mu \mathrm{mol}, 1.2$ equiv.) were added and the reaction was heated at $60{ }^{\circ} \mathrm{C}$ for $24 \mathrm{~h}$. The reaction was quenched by $\mathrm{NaHCO}_{3}$ (sat. aq.) and extracted with DCM. The combined organic phases were washed with brine, dried over $\mathrm{MgSO}_{4}$ and concentrated under reduced pressure. The crude product was purified by silica gel column chromatography $($ pentane/EtOAc $=1: 1)$ to give 16 (16.0 mg, $67.2 \mu \mathrm{mol}, 40 \%)$ as light yellow powder.

${ }^{1}$ H NMR $\left(500 \mathrm{MHz}, d_{6}\right.$-DMSO) $\delta[\mathrm{ppm}]=10.96(\mathrm{~s}, 1 \mathrm{H}), 7.44(\mathrm{dd}, J=7.9,1.1 \mathrm{~Hz}, 1 \mathrm{H})$, $7.33(\mathrm{dt}, J=8.0,0.9 \mathrm{~Hz}, 1 \mathrm{H}), 7.08$ (ddd, $J=8.2,7.1,1.2 \mathrm{~Hz}, 1 \mathrm{H}), 6.99$ (ddd, $J=8.0$, 7.1, $1.0 \mathrm{~Hz}, 1 \mathrm{H}), 6.81(\mathrm{ddd}, J=9.7,6.2,2.1 \mathrm{~Hz}, 1 \mathrm{H}), 5.92(\mathrm{dd}, J=9.7,2.8 \mathrm{~Hz}, 1 \mathrm{H})$, $4.91-4.82(\mathrm{~m}, 1 \mathrm{H}), 4.78(\mathrm{ddd}, J=13.6,5.2,1.9 \mathrm{~Hz}, 1 \mathrm{H}), 3.02(\mathrm{ddd}, J=17.7,6.3,4.9$ 
$\mathrm{Hz}, 1 \mathrm{H}), 2.88-2.75(\mathrm{~m}, 2 \mathrm{H}), 2.67(\mathrm{~m}, 1 \mathrm{H}), 2.32-2.19(\mathrm{~m}, 1 \mathrm{H})$.

${ }^{13}$ C NMR (126 MHz, $d_{6}$-DMSO) $\delta[\mathrm{ppm}]=164.1,139.4,136.3,133.6,126.1,124.7$, $121.1,118.6,117.9,111.1,107.0,51.2,38.2,30.3,20.5$.

HRMS-EI: calcd. for $\mathrm{C}_{15} \mathrm{H}_{14} \mathrm{~N}_{2} \mathrm{O}$ [M] $]^{+}$: 238.1100; found: 238.1099 .

FT-IR: $v\left[\mathrm{~cm}^{-1}\right]=3780,2918,2849,1737,1655,1601,1436,1324,1303,817,740$. 
2.5. Total synthesis of pegamunine $\mathrm{A}$

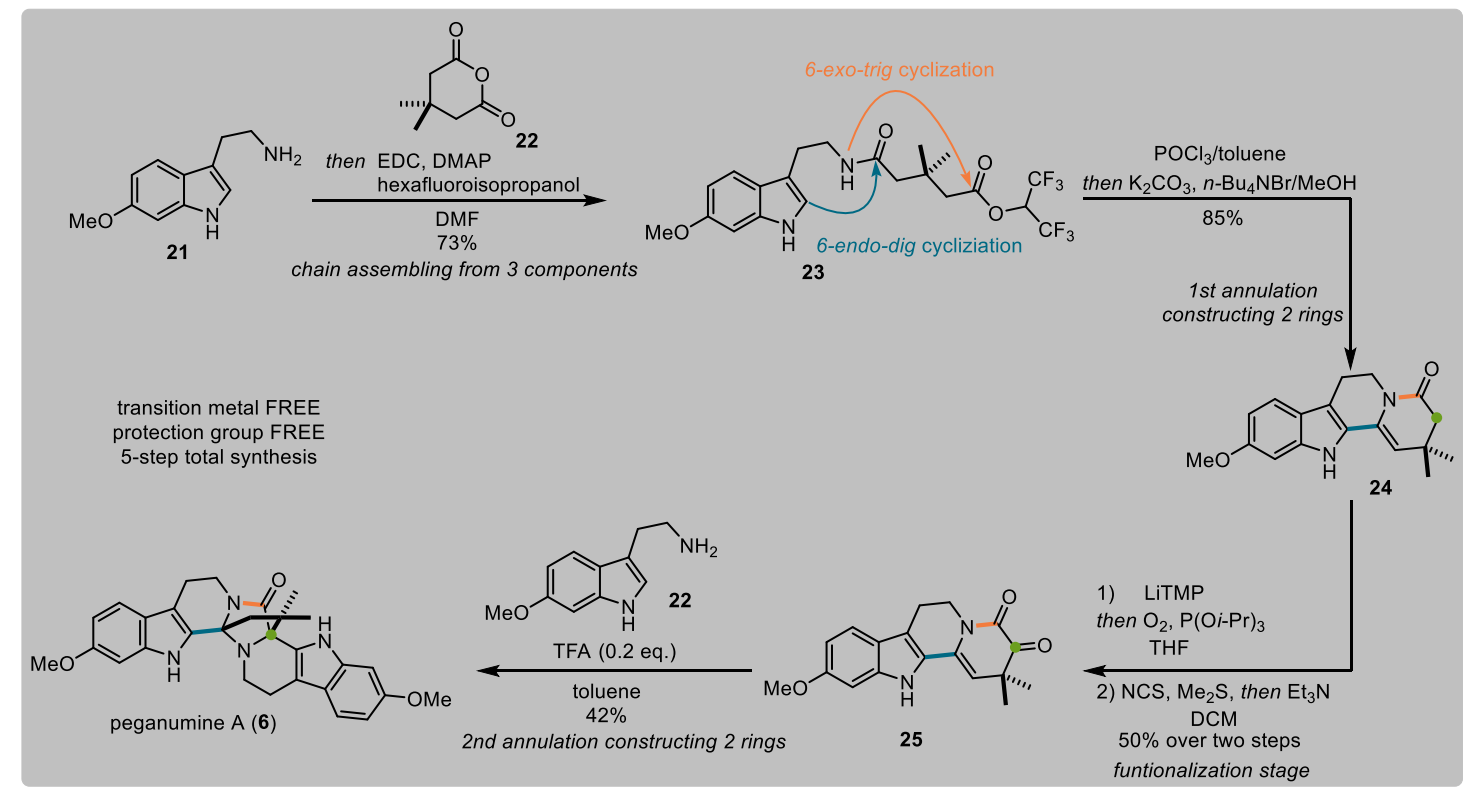

\subsubsection{Preparation of ketoamide}

\section{1,1,1,3,3,3-Hexafluoropropan-2-yl 5-((2-(6-methoxy-1H-indol-3-yl)ethyl)amino)-}

\section{3,3-dimethyl-5-oxopentanoate (24).}
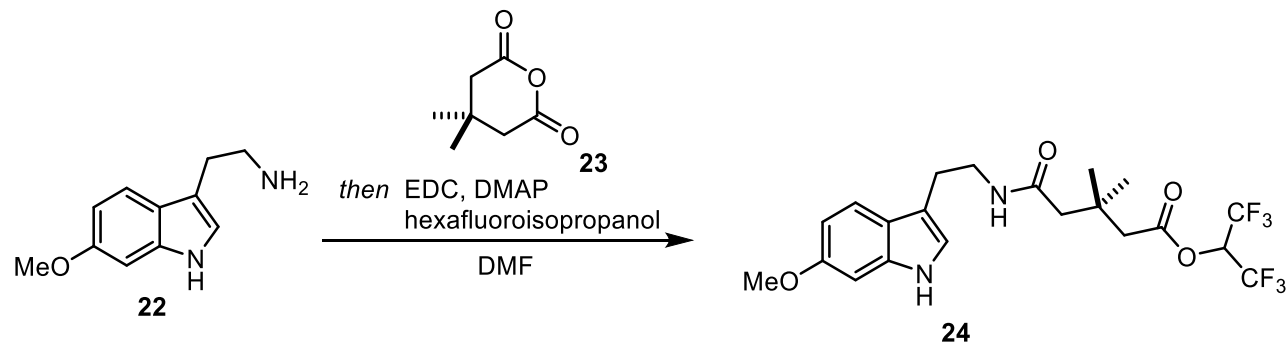

24 was prepared according to General procedure A, starting from 6methoxytryptamine (200 mg, $1.05 \mathrm{mmol}, 1.0$ equiv.) and 3,3-dimethylglutaric anhydride (150 mg, $1.05 \mathrm{mmol}, 1.0$ equiv.) in DMF (5.0 mL). The reaction mixture was subsequently treated with EDCI (402 mg, $2.11 \mathrm{mmol}, 2.0$ equiv.), DMAP (25.7 mg, $0.211 \mathrm{mmol}, 0.2$ equiv.) and $\operatorname{HFIP}(556 \mu \mathrm{L}, 5.26 \mathrm{mmol}, 5.0$ equiv.). Purification by silica gel column chromatography (pentane/EtOAc = 3:2) afforded 24 (370 mg, $0.768 \mathrm{mmol}, 73 \%$ ) as a yellow solid.

${ }^{1} \mathrm{H}$ NMR $\left(500 \mathrm{MHz}, \mathrm{CD}_{2} \mathrm{Cl}_{2}\right) \delta[\mathrm{ppm}]=8.13(\mathrm{~s}, 1 \mathrm{H}), 7.45(\mathrm{~d}, J=8.6 \mathrm{~Hz}, 1 \mathrm{H}), 6.92(\mathrm{dt}$, $J=2.1,1.0 \mathrm{~Hz}, 1 \mathrm{H}), 6.86(\mathrm{~d}, J=2.2 \mathrm{~Hz}, 1 \mathrm{H}), 6.75(\mathrm{dd}, J=8.6,2.3 \mathrm{~Hz}, 1 \mathrm{H}), 5.86-5.78$ 
$(\mathrm{m}, 1 \mathrm{H}), 5.75(\mathrm{~s}, 1 \mathrm{H}), 3.82(\mathrm{~s}, 3 \mathrm{H}), 3.54(\mathrm{td}, J=6.9,5.7 \mathrm{~Hz}, 2 \mathrm{H}), 2.94-2.87(\mathrm{~m}, 2 \mathrm{H})$, $2.62(\mathrm{~s}, 2 \mathrm{H}), 2.15(\mathrm{~s}, 2 \mathrm{H}), 1.08(\mathrm{~s}, 6 \mathrm{H})$.

${ }^{13} \mathbf{C ~ N M R}\left(126 \mathrm{MHz}, \mathrm{CD}_{2} \mathrm{Cl}_{2}\right) \delta[\mathrm{ppm}]=170.7,169.5,157.1,137.7,122.2,121.2,119.6$, $113.3,109.8,94.9,66.7-66.2(\mathrm{~m}), 55.9,47.5,44.0,39.9,33.5,27.9,25.8$. (Due to the resolution or/and overlapping, the resonance of $\mathrm{CF}_{3}$ is missing.)

${ }^{19} \mathbf{F}$ NMR $\left(471 \mathrm{MHz}, \mathrm{CD}_{2} \mathrm{Cl}_{2}\right) \delta[\mathrm{ppm}]=-73.47$.

HRMS-EI: calcd. for $\mathrm{C}_{21} \mathrm{H}_{24} \mathrm{~F}_{6} \mathrm{~N}_{2} \mathrm{O}_{4} \mathrm{Na}[\mathrm{M}+\mathrm{Na}]^{+}$: 505.1532; found: 505.1533 .

FT-IR: $v\left[\mathrm{~cm}^{-1}\right]=2969,2941,1766,1737,1649,1456,1360,1289,1200,1109,771$.

\section{0-Methoxy-2,2-dimethyl-2,6,7,12-tetrahydroindolo[2,3-a]quinolizin-4(3H)-one} (25)

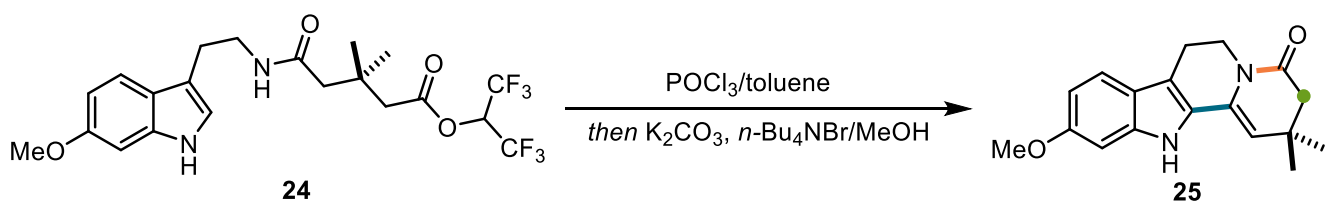

25 was prepared according to General procedure C, starting from 24 (100 mg, 0.207 mmol, 1.0 equiv.) and $\mathrm{POCl}_{3}(18.9 \mu \mathrm{L}, 0.207 \mathrm{mmol}, 1.0$ equiv.) in toluene $(3.0 \mathrm{~mL})$. The reaction mixture was subsequently treated with $\mathrm{MeOH}(3.0 \mathrm{~mL}), \mathrm{K}_{2} \mathrm{CO}_{3}$ (287 mg, $2.08 \mathrm{mmol}, 10$ equiv.) and $n \mathrm{Bu} 4 \mathrm{NBr}(6.7 \mathrm{mg}, 0.021 \mathrm{mmol}, 0.1$ equiv.). Purification by silica gel column chromatography (pentane/EtOAc $=2: 1$ ) afforded 25 (49.0 mg, $0.165 \mathrm{mmol}, 85 \%$ ) as a yellow solid.

${ }^{1} \mathbf{H}$ NMR $\left(700 \mathrm{MHz}, \mathrm{CDCl}_{3}\right) \delta[\mathrm{ppm}]=8.09(\mathrm{~s}, 1 \mathrm{H}), 7.37(\mathrm{~d}, J=8.5 \mathrm{~Hz}, 1 \mathrm{H}), 6.83(\mathrm{~s}$, $1 \mathrm{H}), 6.78(\mathrm{~d}, J=8.5 \mathrm{~Hz}, 1 \mathrm{H}), 5.27(\mathrm{~s}, 1 \mathrm{H}), 4.09(\mathrm{t}, J=5.9 \mathrm{~Hz}, 2 \mathrm{H}), 3.85(\mathrm{~s}, 3 \mathrm{H}), 2.88$ $(\mathrm{t}, J=5.9 \mathrm{~Hz}, 2 \mathrm{H}), 2.48(\mathrm{~s}, 2 \mathrm{H}), 1.16(\mathrm{~s}, 6 \mathrm{H})$.

${ }^{13} \mathbf{C ~ N M R}\left(176 \mathrm{MHz}, \mathrm{CDCl}_{3}\right) \delta[\mathrm{ppm}]=169.6,157.6,138.3,128.9,126.9,121.3,119.7$, $112.4,109.7,109.5,95.0,55.8,46.6,39.3,30.8,28.3,20.8$.

HRMS-EI: calcd. for $\mathrm{C}_{18} \mathrm{H}_{20} \mathrm{~N}_{2} \mathrm{O}_{2}[\mathrm{M}]^{+}:$296.1519; found: 296.1520 .

FT-IR: $v\left[\mathrm{~cm}^{-1}\right]=2954,2919,2850,1736,1663,1628,1461,1365,1230,12174,1149$. 


\section{3-Hydroxy-10-methoxy-2,2-dimethyl-2,6,7,12-tetrahydroindolo[2,3-a]quinolizin-}

4(3H)-one (S15).
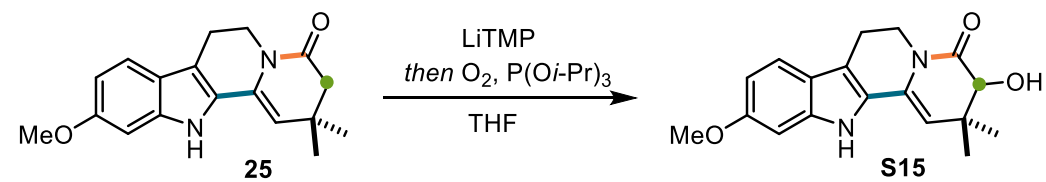

To a solution of tetracyclic lactam 25 (100 mg, 0.34 mmol, 1.0 equiv.) in THF, was added LiTMP (1.0 M solution in THF, $3.40 \mathrm{~mL}, 3.38 \mathrm{mmol}, 10.0$ equiv.) at $-78^{\circ} \mathrm{C}$. The resulting mixture was stirred at that temperature for $1 \mathrm{~h}$ before $\mathrm{P}(\mathrm{OMe})_{3}(250 \mu \mathrm{L}$, $1.01 \mathrm{mmol}, 2.5$ equiv.) was added. $\mathrm{O}_{2}$ was bubbled through the mixture for $20 \mathrm{~min}$, and the mixture was stirred for additional $2 \mathrm{~h}$ under $\mathrm{O}_{2}$ atmosphere. Then the reaction mixture was quenched with saturated $\mathrm{NaHCO}_{3}$ (sat. aq.). The aqueous phase was extracted with DCM. The combined organic phases were washed with brine, dried with $\mathrm{MgSO}_{4}$, filtered and concentrated under reduced pressure. The crude product was purified by silica gel column chromatography (pentane/EtOAc $=3: 2$ ) afforded $\mathbf{S 1 5}$ (75.0 mg, $0.240 \mathrm{mmol}, 71 \%$ ) as yellow solid.

${ }^{1} \mathbf{H}$ NMR $\left(500 \mathrm{MHz}, \mathrm{CDCl}_{3}\right) \delta[\mathrm{ppm}]=8.09(\mathrm{~s}, 1 \mathrm{H}), 7.37(\mathrm{~d}, J=8.6 \mathrm{~Hz}, 1 \mathrm{H}), 6.82(\mathrm{~d}$, $J=2.2 \mathrm{~Hz}, 1 \mathrm{H}), 6.79(\mathrm{dd}, J=8.6,2.2 \mathrm{~Hz}, 1 \mathrm{H}), 5.27(\mathrm{~s}, 1 \mathrm{H}), 4.86(\mathrm{ddd}, J=12.7,5.2$, $2.1 \mathrm{~Hz}, 1 \mathrm{H}), 4.08(\mathrm{~d}, J=2.5 \mathrm{~Hz}, 1 \mathrm{H}), 3.89(\mathrm{~d}, J=2.6 \mathrm{~Hz}, 1 \mathrm{H}), 3.85(\mathrm{~s}, 3 \mathrm{H}), 3.28(\mathrm{td}, J$ $=12.1,5.4 \mathrm{~Hz}, 1 \mathrm{H}), 2.96-2.83(\mathrm{~m}, 2 \mathrm{H}), 1.30(\mathrm{~s}, 3 \mathrm{H}), 1.00(\mathrm{~s}, 3 \mathrm{H})$.

${ }^{13} \mathrm{C} \mathrm{NMR}\left(126 \mathrm{MHz}, \mathrm{CDCl}_{3}\right) \delta[\mathrm{ppm}]=171.7,157.9,138.4,127.8,126.1,121.2,119.8$, $112.4,110.1,109.3,94.9,75.0,55.8,40.7,35.2,26.5,20.6,19.9$.

HRMS-ESI: calcd. for $\mathrm{C}_{18} \mathrm{H}_{20} \mathrm{~N}_{2} \mathrm{O}_{3} \mathrm{Na}[\mathrm{M}+\mathrm{Na}]^{+}: 335.1366$; found: 335.1367 .

FT-IR: $v\left[\mathrm{~cm}^{-1}\right]=3343,2958,2924,1653,1393,1360,1328,1231,1151,1045,1027$, $920,743$.

10-Methoxy-2,2-dimethyl-2,6,7,12-tetrahydroindolo[2,3-a]quinolizine-3,4-dione (26). ${ }^{[4]}$

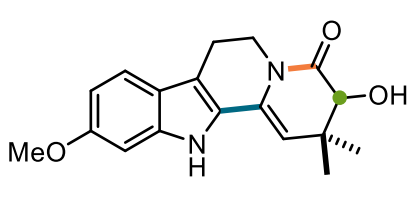

S15

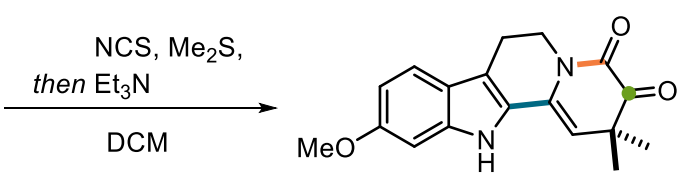

26 
To a solution of NCS (171 mg, $1.28 \mathrm{mmol}, 5.0$ equiv.) in DCM $(5.0 \mathrm{~mL})$ at $0{ }^{\circ} \mathrm{C}$ was added $\mathrm{Me}_{2} \mathrm{~S}$ ( $471 \mu \mathrm{L}, 6.41 \mathrm{mmol}, 25$ equiv.) and the mixture was stirred at $-78^{\circ} \mathrm{C}$ for $1 \mathrm{~h}$. The $\mathbf{S 1 5}$ (80 mg, $0.256 \mathrm{mmol}, 1.0$ equiv.) in DCM (2.0 mL) was added dropwise. The resulting mixture was stirred at $-78^{\circ} \mathrm{C}$ for another two hours before triethylamine (712 $\mu \mathrm{L}, 5.13 \mathrm{mmol}, 20$ equiv.) was added. Then the mixture was stirred at $-78^{\circ} \mathrm{C}$ for 2 h. The mixture was quenched with $\mathrm{NH}_{4} \mathrm{Cl}$ (sat. aq.) and extracted with DCM. The combined organic phases were washed with brine, dried over $\mathrm{MgSO}_{4}$, filtered and concentrated under reduced pressure. The crude product was purified by silica gel column chromatography (pentane/EtOAc $=3: 2)$ afforded $26(57.0 \mathrm{mg}, 0.184 \mathrm{mmol}$, $71 \%)$ as an orange solid.

${ }^{1} \mathbf{H}$ NMR $\left(700 \mathrm{MHz}, \mathrm{CDCl}_{3}\right) \delta[\mathrm{ppm}]=8.08(\mathrm{~s}, 1 \mathrm{H}), 7.42-7.37(\mathrm{~m}, 1 \mathrm{H}), 6.87-6.84(\mathrm{~m}$, $1 \mathrm{H}), 6.80(\mathrm{dd}, J=8.6,2.2 \mathrm{~Hz}, 1 \mathrm{H}), 5.31(\mathrm{~s}, 1 \mathrm{H}), 4.20(\mathrm{t}, J=6.0 \mathrm{~Hz}, 2 \mathrm{H}), 3.86(\mathrm{~s}, 3 \mathrm{H})$, $2.98(\mathrm{t}, J=6.0 \mathrm{~Hz}, 2 \mathrm{H}), 1.39(\mathrm{~s}, 6 \mathrm{H})$.

${ }^{13} \mathbf{C ~ N M R}\left(176 \mathrm{MHz}, \mathrm{CDCl}_{3}\right) \delta[\mathrm{ppm}]=196.1,158.1,157.4,138.7,127.0,125.9,121.0$, $120.0,113.0,110.3,106.0,95.1,55.9,44.9,40.5,25.0,20.4$.

HRMS-EI: calcd. for $\mathrm{C}_{18} \mathrm{H}_{18} \mathrm{~N}_{2} \mathrm{O}_{3}[\mathrm{M}]^{+}: 310.1312$; found: 310.1314 .

FT-IR: $v\left[\mathrm{~cm}^{-1}\right]$ 3284(br), 2956, 2925, 1642, 1392, 1305, 1184, 1143, 1047, 799, 756.

\subsubsection{Racemic protection group free annulation reaction}

\section{Imine pre-formation one-pot procedure. ${ }^{[4]}$}

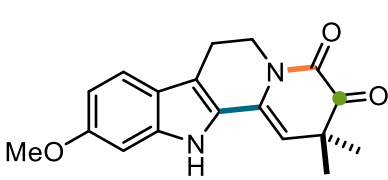

26

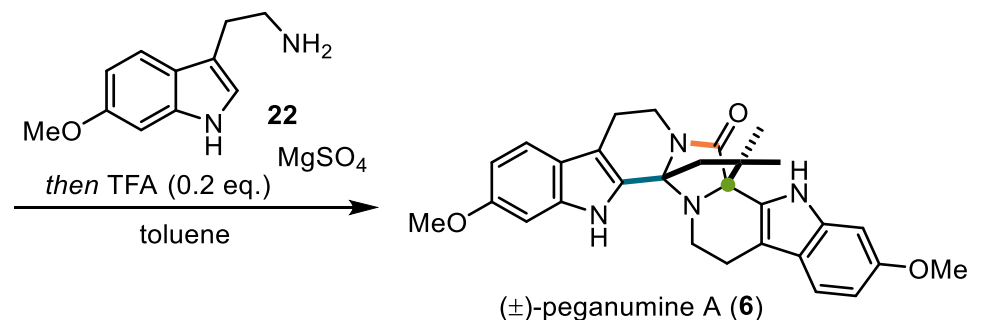

$( \pm)$-peganumine $\mathrm{A}(\mathbf{6})$

A mixture of ketoenamide 25 (56.0 mg, $0.181 \mathrm{mmol}, 1.0$ equiv.), 6-methoxytryptamine (22) (41.2 mg, 0.217 mmol, 1.2 equiv.) and $\mathrm{MgSO}_{4}$ (217 mg, $1.81 \mathrm{mmol}, 10.0$ equiv.) in toluene $(10 \mathrm{~mL})$ was heated to $115^{\circ} \mathrm{C}$ (oil bath temperature) overnight. The reaction 
mixture was cooled to room temperature and TFA $(2.8 \mu \mathrm{L}, 36 \mu \mathrm{mol}, 0.2$ equiv.) were added. The mixture was then heated to reflux for 2 days, before the reaction was quenched with saturated $\mathrm{NaHCO}_{3}$ (sat. aq.). The aqueous phase was extracted with DCM. The combined organic phases were washed with brine, dried with $\mathrm{MgSO}_{4}$, filtered and concentrated under reduced pressure. The crude product was purified by silica gel column chromatography (pentane/EtOAc $=3: 2)$ afforded 6 (34.0 mg, $70.5 \mu \mathrm{mol}, 39 \%$ ) as light yellow solid.

\section{Cascade procedure.}

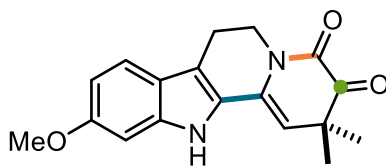

25

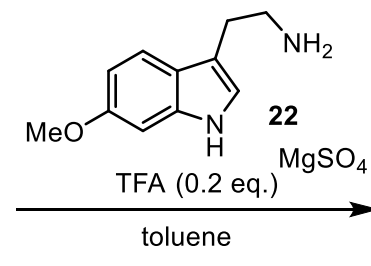

To a mixture of ketoenamide 25 (35.0 mg, 0.113 mmol, 1.0 equiv.), 6methoxytryptamine (22) (25.7 mg, 0.135 mmol, 1.2 equiv.) and $\mathrm{MgSO}_{4}$ (136 mg, 1.13 mmol, 10.0 equiv.) in $10 \mathrm{~mL}$ toluene under argon atmosphere, was added TFA $(1.7 \mu \mathrm{L}$, $23 \mu$ mol, 0.2 equiv.). The resulting reaction mixture was heated to $115^{\circ} \mathrm{C}$ (oil bath temperature) for 2 days. Then the reaction was quenched by $\mathrm{NaHCO}_{3}$ (sat. aq.). The aqueous phase was extracted with DCM. The combined organic phases were washed with brine, dried with $\mathrm{MgSO}_{4}$, filtered and concentrated under reduced pressure. The crude product was purified by silica gel column chromatography (pentane/EtOAc $=3: 2$ ) afforded 6 (23.0 mg, $47.7 \mu \mathrm{mol}, 42 \%)$ as a light yellow solid.

\subsubsection{Enantioselective protection group free annulation reaction}

\subsubsection{Catalyst preparation}

S17 was prepared from S16 by a procedure of Lee et al.. ${ }^{[5]}$ The spectroscopic data are in agreement with literature. $\mathbf{2 8}$ was synthesized from $\mathbf{S 1 7}$ following the procedure of Seidel et al.. ${ }^{[6]}$ The spectroscopic data are in agreement with literature. 
<smiles>NC1CCCCC1N</smiles><smiles>CC(F)(F)c1cc(N=C=S)cc(C(F)(F)F)c1</smiles><smiles>N[C@H]1CCCCC1NC(=S)Nc1cc(C(F)(F)F)cc(C(F)(F)F)c1</smiles><smiles>Cc1c(Br)c(Br)c(Br)c(Br)c1C(=O)N[C@H]1CCCC[C@H]1NC(=S)Nc1cc(C(F)(F)F)cc(C(F)(F)F)c1</smiles>

Scheme S-3: Synthesis of CBSBA.

1,2,3,4,5-pentacarboxycyclopentadiene (PCCP) derived pentamenthyl ester 29 was synthesized from $\mathbf{S 1 8}$ according to a procedure by Lambert et al.. ${ }^{[7]}$ The spectroscopic data are in agreement with literature.

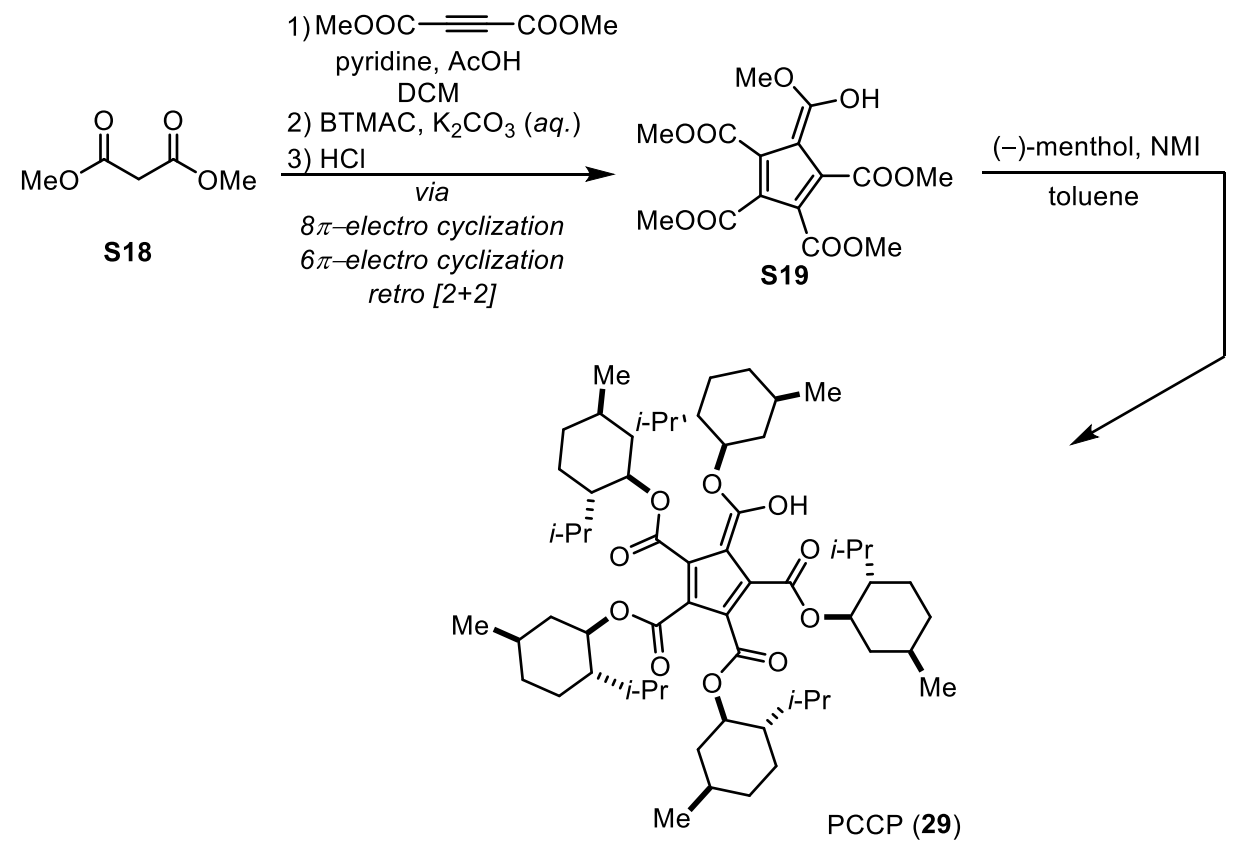

Scheme S-4: Synthesis of PCCP.

\section{Chiral DSIs:}

Generally, the synthesis of S20 needs 7 steps from BINOL. With the S14 in hand, DSIs could be obtained by Suzuki coupling with boronic acid. ${ }^{[8]}$ 


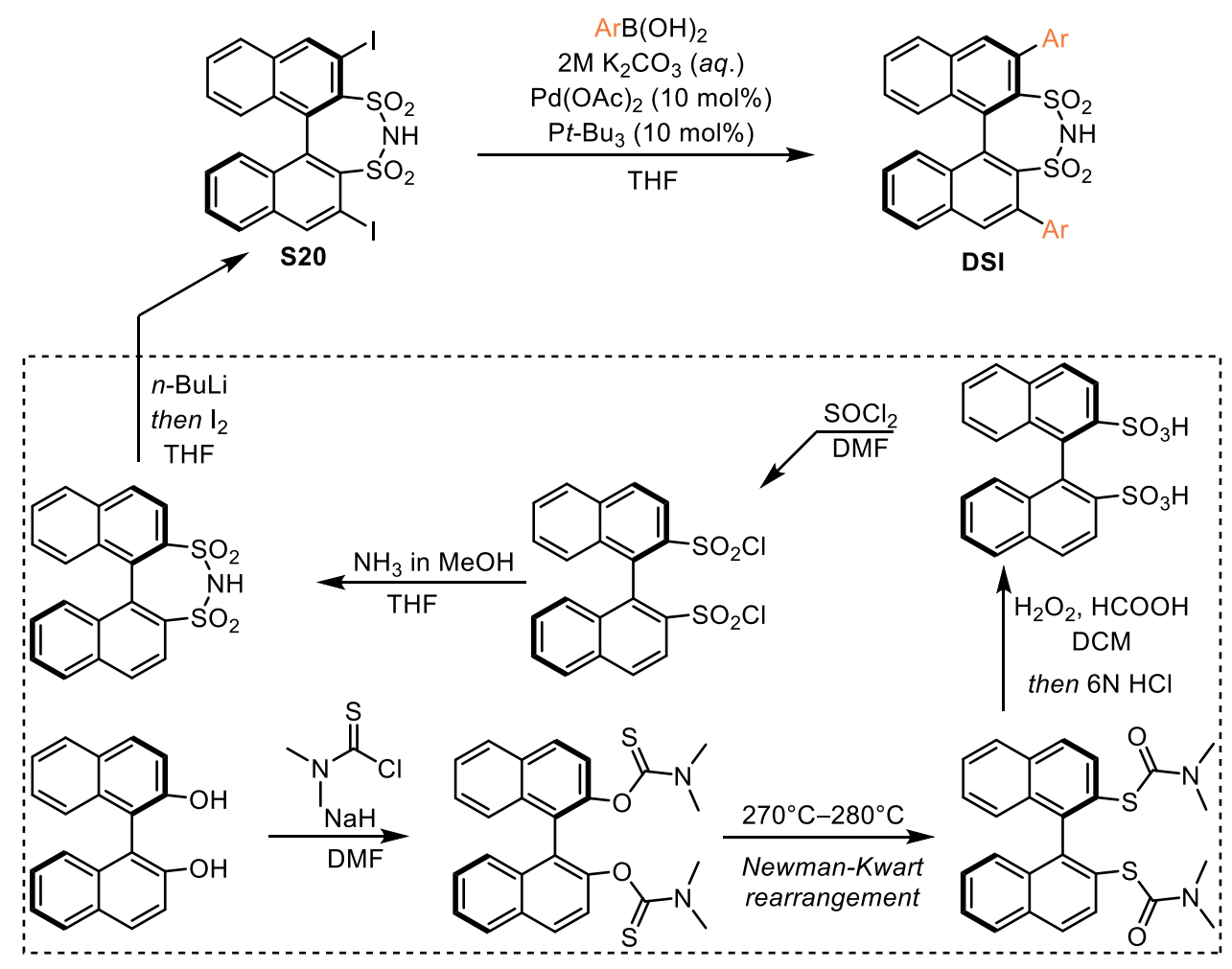

Scheme S-5: Synthesis of DSIs.

The readily available DSIs:

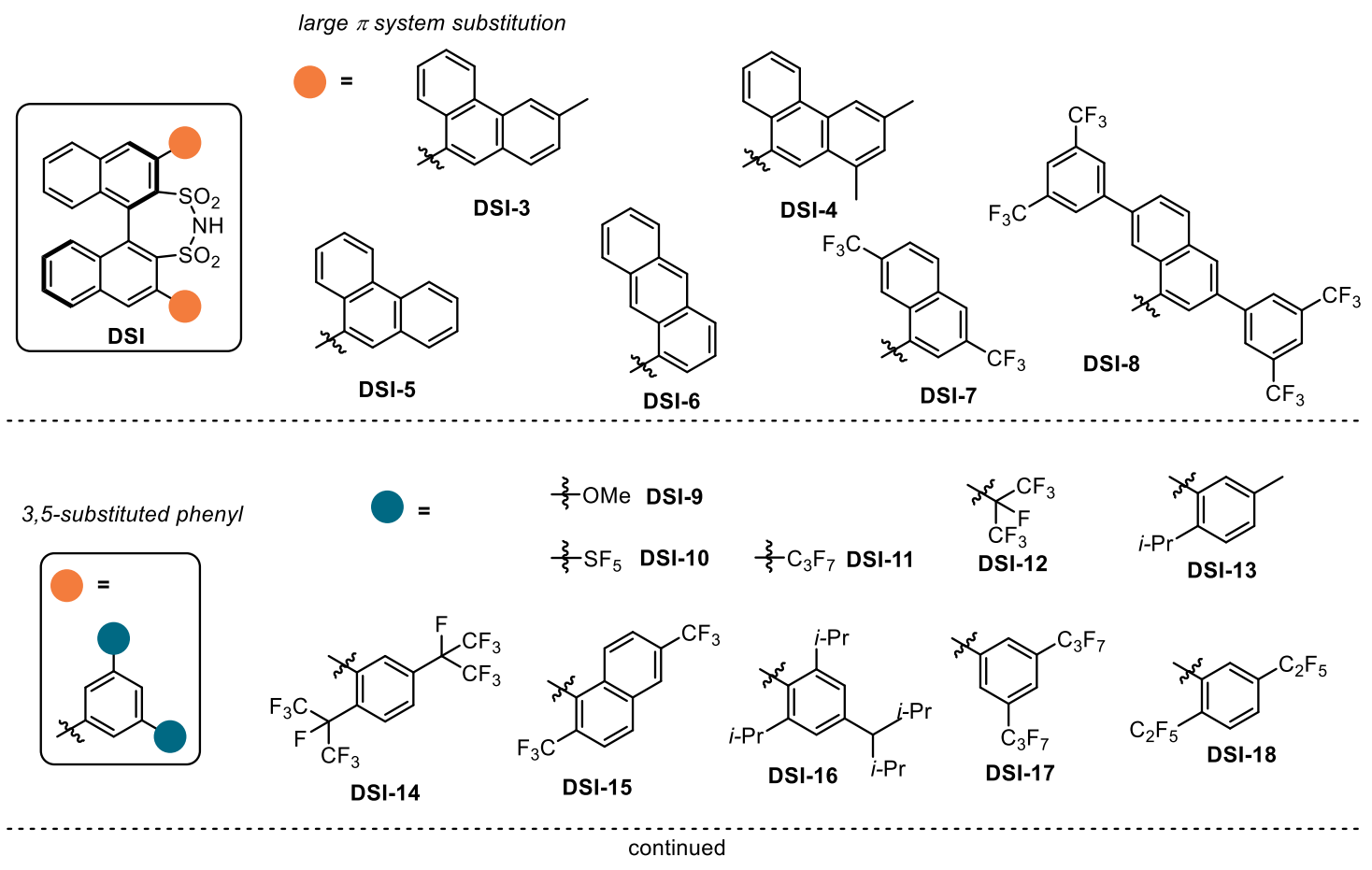



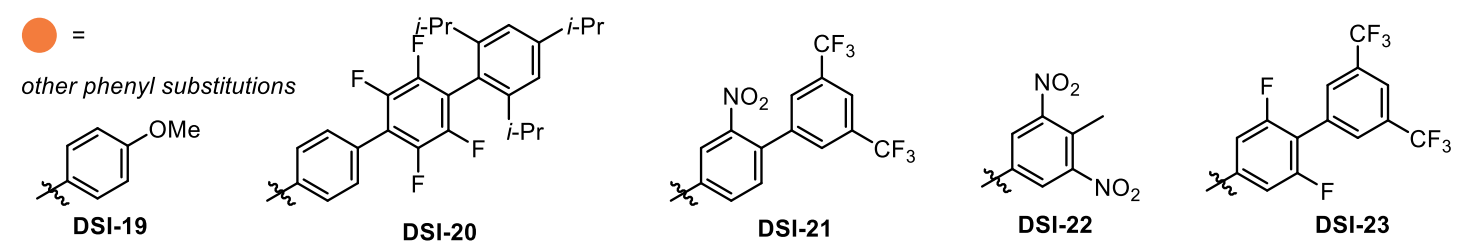

$=\quad$ longer linear substitutions

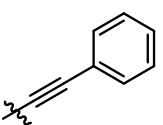

DSI-24

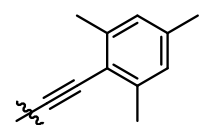

DSI-25

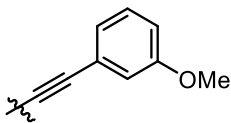

DSI-26

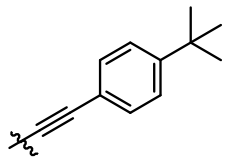

DSI-27

Figure S-1: Screened the readily available DSIs.

\section{General procedure $\mathbf{E}$}

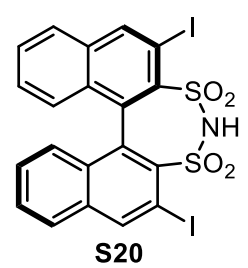

S20

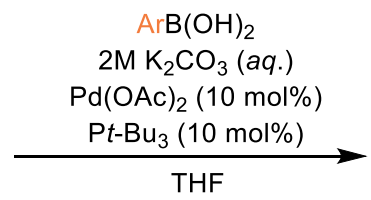

$\mathrm{Pd}(\mathrm{OAc})_{2}(10 \mathrm{~mol} \%)$

$\underset{\mathrm{THF}}{\mathrm{Pt}-\mathrm{Bu}_{3}(10 \mathrm{~mol} \%)}$

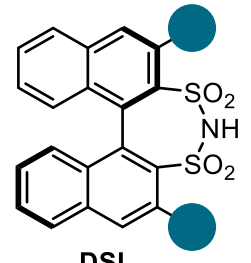

DSI

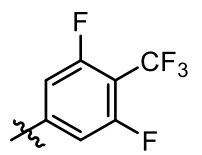

DSI-2 (32)<smiles>Fc1cc(F)cc(I)c1</smiles>

DSI-28 (S21)<smiles>Cc1cc(F)c(F)c(F)c1</smiles>

DSI-29 (S22)<smiles>O=[N+]([O-])c1cc(I)cc([N+](=O)[O-])c1</smiles>

DSI-30 (S23)

$2 \mathrm{M} \mathrm{K}_{2} \mathrm{CO}_{3}$ was added to a solution of $(R)$-DSI iodide (S20) (1.0 equiv.), boronic acid (3.0 equiv.), $\mathrm{Pd}(\mathrm{OAc}) 2$ ( 0.1 equiv.) in THF at room temperature. The reaction flask was degassed and filled with argon three times before $\mathrm{P} t$ - $\mathrm{Bu}_{3}(1.0 \mathrm{M}$ in toluene, 0.1 equiv.) was added. The reaction mixture was then put to a preheated $85^{\circ} \mathrm{C}$ oil bath for $24 \mathrm{~h}$. $10 \%$ aqueous $\mathrm{HCl}$ solution was added and the reaction mixture was extracted three times with DCM. The combined organic layers were dried over $\mathrm{MgSO}_{4}$ and concentrated under reduced pressure. The residue was purified by column chromatography on silica gel (pentane/EtOAc $=3: 2$ ) to give a white solid which was dissolved in DCM and stirred with $4 \mathrm{~N} \mathrm{HCl}$ for $1 \mathrm{~h}$. The organic layer was then separated, dried over $\mathrm{MgSO}_{4}$ and concentrated under reduced pressure to give the desired compound as a white solid. 


\section{3,3,5,5-tetraoxide (S21).}

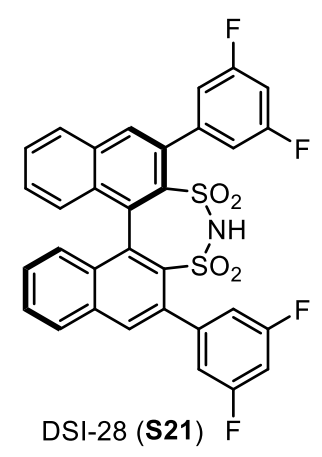

S21 was prepared according to General procedure E, starting from S20 (50 mg, $77 \mu \mathrm{mol}, 1.0$ equiv.), (3,5-difluorophenyl)boronic acid (38 mg, $0.23 \mathrm{mmol}, 3.0$ equiv.) and $\mathrm{Pd}(\mathrm{OAc})_{2}(1.7 \mathrm{mg}, 8.0 \mu \mathrm{mol}, 0.1$ equiv. $)$ in THF $(3.0 \mathrm{~mL})$. After degassing, the mixture was treated with $\mathrm{P}_{-}-\mathrm{Bu}_{3}(1.0 \mathrm{M}$ in toluene, $7.7 \mu \mathrm{L}, 8.0 \mu \mathrm{mol}, 0.1$ equiv.). Purification by silica gel column chromatography (pentane/EtOAc $=2: 1)$ afforded $\mathbf{S 2 1}$ (28 mg, $45 \mu \mathrm{mol}, 58 \%$ ) as a white solid.

${ }^{1} \mathbf{H}$ NMR $\left(500 \mathrm{MHz}, \mathrm{CDCl}_{3}\right) \delta[\mathrm{ppm}]=8.02(\mathrm{~d}, J=9.0 \mathrm{~Hz}, 4 \mathrm{H}), 7.72(\mathrm{ddd}, J=8.2,6.8$, $1.1 \mathrm{~Hz}, 2 \mathrm{H}), 7.45$ (ddd $J=8.4,6.9,1.2 \mathrm{~Hz}, 2 \mathrm{H}), 7.16$ (d, $J=8.6 \mathrm{~Hz}, 2 \mathrm{H}), 7.08$ (d, $J=$ $8.6 \mathrm{~Hz}, 2 \mathrm{H}), 6.99$ (d, $J=8.6 \mathrm{~Hz}, 2 \mathrm{H}), 6.90-6.85(\mathrm{~m}, 2 \mathrm{H}), 5.76(\mathrm{~s}, 1 \mathrm{H})$.

${ }^{13} \mathbf{C}$ NMR $\left(126 \mathrm{MHz}, \mathrm{CDCl}_{3}\right) \delta[\mathrm{ppm}]=162.6,162.5,162.1,162.0,160.6,160.5,160.1$, 160.0, 141.2 141.1, 141.0, 137.5, 133.6, 133.4, 132.6, 131.1, 130.8, 129.4, 128.0, 127.7, $127.2,113.1,112.9,111.3,111.1,102.8,102.6,102.4$. (Due to the complexity of the FC coupling, the coupling constants were not calculated.)

${ }^{19}$ F NMR (471 MHz, $\left.\mathrm{CDCl}_{3}\right) \delta[\mathrm{ppm}]=-109.97,-110.59$.

HRMS-ESI: calcd. for $\mathrm{C}_{32} \mathrm{H}_{16} \mathrm{~F}_{4} \mathrm{NO}_{4} \mathrm{~S}_{2}[\mathrm{M}-\mathrm{H}]^{-}$: 618.0462; found: 618.0472.

FT-IR: $v\left[\mathrm{~cm}^{-1}\right]$ 3158(br), 2927, 1617, 1529, 1439, 1423, 1362, 1340, 1319, 1044, 845, 751. 


\section{3,3,5,5-tetraoxide (S22).}

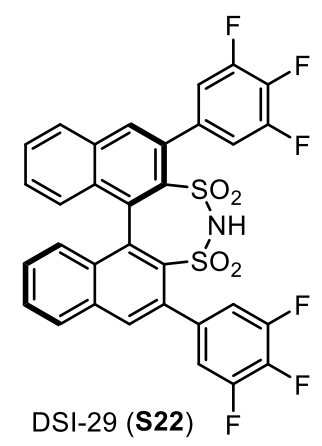

S22 was prepared according to General procedure E, starting from $\mathbf{S 2 0}$ (50 mg, $77 \mu \mathrm{mol}, 1.0$ equiv.), (3,4,5-trifluorophenyl)boronic acid (41 mg, $0.23 \mathrm{mmol}, 3.0$ equiv.) and $\mathrm{Pd}(\mathrm{OAc})_{2}(1.7 \mathrm{mg}, 8.0 \mu \mathrm{mol}, 0.1$ equiv. $)$ in THF $(3.0 \mathrm{~mL})$. After degassing, the mixture was treated with $\mathrm{P} t-\mathrm{Bu}_{3}(1.0 \mathrm{M}$ in toluene, $7.7 \mu \mathrm{L}, 8.0 \mu \mathrm{mol}, 0.1$ equiv.). Purification by silica gel column chromatography (pentane/EtOAc $=2: 1$ ) afforded $\mathbf{S 2 3}$ (36 mg, $54 \mu \mathrm{mol}, 70 \%$ ) as a white solid.

${ }^{1} \mathbf{H}$ NMR $\left(500 \mathrm{MHz}, \mathrm{CDCl}_{3}\right) \delta[\mathrm{ppm}]=8.02(\mathrm{~d}, J=8.7 \mathrm{~Hz}, 4 \mathrm{H}), 7.74(\mathrm{ddd}, J=8.2,6.9$, $1.1 \mathrm{~Hz}, 2 \mathrm{H}), 7.46$ (ddd, $J=8.4,6.9,1.3 \mathrm{~Hz}, 2 \mathrm{H}), 7.20-7.01(\mathrm{~m}, 6 \mathrm{H})$.

${ }^{13} \mathbf{C ~ N M R}\left(126 \mathrm{MHz}, \mathrm{CDCl}_{3}\right) \delta[\mathrm{ppm}]=151.8,151.5,149.9,149.8,149.5,149.5,138.7$, 135.0, 134.9, 134.9, 134.9, 134.8, 134.8, 134.5, 134.0, 133.9, 132.3, 131.9, 130.7, 129.3, $128.8,128.3,115.3,115.1,113.6,113.5$. (Due to the complexity of the F-C coupling, the coupling constants were not calculated.)

${ }^{19}$ F NMR $\left(471 \mathrm{MHz}, \mathrm{CDCl}_{3}\right) \delta[\mathrm{ppm}]=(-138.15)-(-140.64)(\mathrm{m}),-167.04(\mathrm{t}, J=19.9$ $\mathrm{Hz})$.

HRMS-ESI: calcd. for $\mathrm{C}_{32} \mathrm{H}_{14} \mathrm{~F}_{6} \mathrm{NO}_{4} \mathrm{~S}_{2}[\mathrm{M}-\mathrm{H}]^{-}$: 654.0274; found: 654.0282.

FT-IR: $v\left[\mathrm{~cm}^{-1}\right]$ 3161(br), 2924, 1738, 1618, 1529, 1424, 1362, 1217, 1045, 848, 750. 
3,3,5,5-tetraoxide (S23).

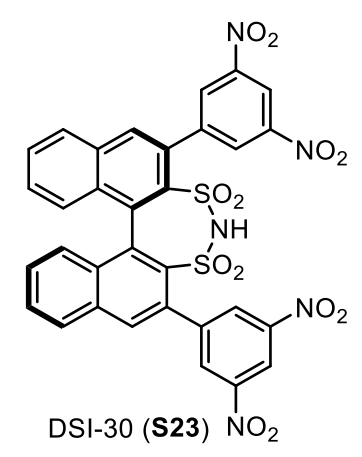

S23 was prepared according to General procedure E, starting from S20 (15 mg, $23 \mu \mathrm{mol}, 1.0$ equiv.), (3,5-dinitrophenyl)boronic acid (15 mg, $0.70 \mathrm{mmol}, 3.0$ equiv.) and $\mathrm{Pd}(\mathrm{OAc})_{2}(0.50 \mathrm{mg}, 2.0 \mu \mathrm{mol}, 0.1$ equiv. $)$ in THF $(1.0 \mathrm{~mL})$. After degassing, the mixture was treated with $\mathrm{P} t-\mathrm{Bu}_{3}(1.0 \mathrm{M}$ in toluene, $2.3 \mu \mathrm{L}, 2.0 \mu \mathrm{mol}, 0.1$ equiv.). Purification by silica gel column chromatography (pentane/EtOAc $=2: 1$ ) afforded $\mathbf{S 2 3}$ (10 mg, $14 \mu \mathrm{mol}, 59 \%$ ) as a white solid.

${ }^{1} \mathbf{H}$ NMR $\left(500 \mathrm{MHz}, \mathrm{CD}_{2} \mathrm{Cl}_{2}\right) \delta[\mathrm{ppm}]=9.05(\mathrm{t}, J=2.1 \mathrm{~Hz}, 2 \mathrm{H}), 8.76-8.61(\mathrm{~m}, 4 \mathrm{H})$, $8.12(\mathrm{~d}, J=6.6 \mathrm{~Hz}, 4 \mathrm{H}), 7.82(\mathrm{t}, J=7.6 \mathrm{~Hz}, 2 \mathrm{H}), 7.55(\mathrm{dd}, J=8.8,6.8 \mathrm{~Hz}, 2 \mathrm{H}), 7.25$ $(\mathrm{d}, J=8.6 \mathrm{~Hz}, 2 \mathrm{H})$.

${ }^{13} \mathbf{C ~ N M R}\left(126 \mathrm{MHz}, \mathrm{CD}_{2} \mathrm{Cl}_{2}\right) \delta[\mathrm{ppm}]=148.2,147.6,142.7,138.9,134.6,134.5,132.5$, $132.1,131.8,131.2,130.9,130.0,129.3,129.2,128.4,118.5$.

HRMS-ESI: calcd. for $\mathrm{C}_{32} \mathrm{H}_{16} \mathrm{~N}_{5} \mathrm{O}_{12} \mathrm{~S}_{2}[\mathrm{M}-\mathrm{H}]^{-}:$726.0256; found: 726.0242 .

FT-IR: $v\left[\mathrm{~cm}^{-1}\right]$ 2924, 1738, 1541, 1343, 1175, 831, 750.

(R)-2,6-Bis(3,5-difluoro-4-(trifluoromethyl)phenyl)-4H-dinaphtho[2,1-d:1',2'$f][1,3,2]$ dithiazepine 3,3,5,5-tetraoxide (32).

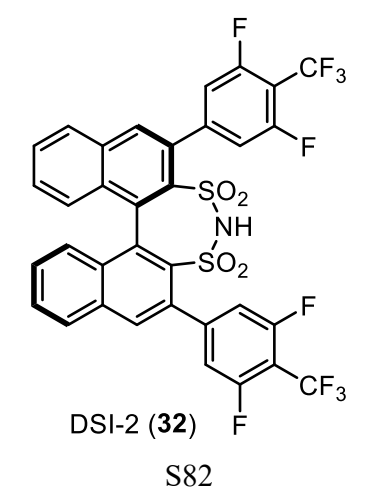


32 was prepared according to General procedure E, starting from S20 (300 mg, 0.464 mmol, 1.0 equiv.), (3,5-difluoro-4-(trifluoromethyl)phenyl)boronic acid (315 mg, $1.39 \mathrm{mmol}, 3.0$ equiv.) and $\mathrm{Pd}(\mathrm{OAc}) 2(10.4 \mathrm{mg}, 46.4 \mu \mathrm{mol}, 0.1$ equiv. $)$ in THF (15 mL). After degassing, the mixture was treated with $\mathrm{P} t$-Bu3 $(1.0 \mathrm{M}$ in toluene, $46.4 \mu \mathrm{L}$, $46.4 \mu \mathrm{mol}, \quad 0.1$ equiv.). Purification by silica gel column chromatography (pentane/EtOAc $=2: 1)$ afforded $32(320 \mathrm{mg}, 0.424 \mathrm{mmol}, 91 \%)$ as a white solid.

${ }^{1} \mathbf{H}$ NMR $\left(500 \mathrm{MHz}, \mathrm{CDCl}_{3}\right) \delta[\mathrm{ppm}]=8.07-7.97(\mathrm{~m}, 4 \mathrm{H}), 7.76(\mathrm{t}, J=7.6 \mathrm{~Hz}, 2 \mathrm{H})$, 7.54-7.46 (m, 2H), 7.24-7.07 (m, 6H).

${ }^{13} \mathbf{C ~ N M R}\left(126 \mathrm{MHz}, \mathrm{CDCl}_{3}\right) \delta[\mathrm{ppm}]=160.4,159.9,158.3,157.8,145.6,138.5,134.2$, $133.5,133.1,132.1,131.4,130.8,129.5,128.9,128.2,122.8,120.7,115.2,115.0,113.6$ 113.5. (Due to the complexity of the $\mathrm{F}-\mathrm{C}$ coupling, the coupling constants were not calculated.)

${ }^{19}$ F NMR $\left(471 \mathrm{MHz}, \mathrm{CDCl}_{3}\right) \delta[\mathrm{ppm}]=-56.16(\mathrm{t}, J=21.7 \mathrm{~Hz}),-111.17(\mathrm{dq}, J=329.9$, $22.4,21.7 \mathrm{~Hz})$.

HRMS-ESI: calcd. for $\mathrm{C}_{34} \mathrm{H}_{14} \mathrm{~F}_{10} \mathrm{NO}_{4} \mathrm{~S}_{2}[\mathrm{M}-\mathrm{H}]^{-}$: 754.0219; found: 754.0210.

FT-IR: $v\left[\mathrm{~cm}^{-1}\right]$ 3075, 1641, 1493, 1429, 1352, 1306, 1137, 1046, 856, 753.

2.5.3.2. Optimization for enantioselective protection group free cascade reaction

General Procedure for the small scale $(0.50 \mathrm{mg}$ or $1.00 \mathrm{mg})$ screening reactions

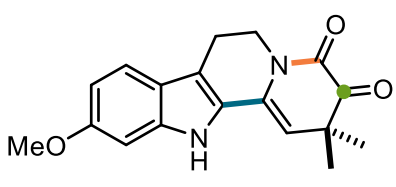

25

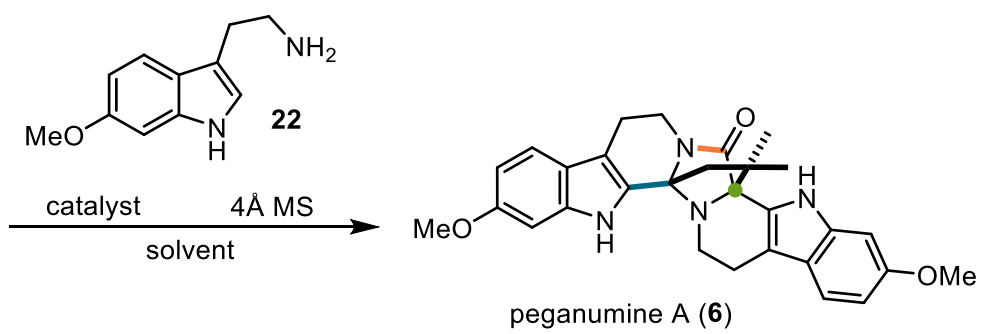

peganumine $A(\mathbf{6})$

A mixture of ketoamide 25 (1.00 mg, $3.00 \mu$ mol, 1.0 equiv.), 6-methoxytryptamine (22) (0.75 mg, $3.60 \mu \mathrm{mol}, 1.2$ equiv.) and $4 \AA \mathrm{MS}$ in corresponding solvent $(0.5 \mathrm{~mL})$ was stirred at room temperature under argon in sealed vial. A solution of corresponding catalyst in the same solvent was added to the reaction mixture and the reaction mixture was heated up to the corresponding temperature for the set duration. The reaction 
mixture was cooled to room temperature and purified by silica gel column chromatography (pentane/EtOAc $=3: 2$ ) to yield the pure product as solid.

(Note: since the ketoamide is not a fine power and cannot dissolve in toluene (the frequently used solvent), the practical way to prepare the $1.00 \mathrm{mg}$ or $0.50 \mathrm{mg}$ ketoamide was that, using fine balance to take $10.00 \mathrm{mg}$ ketoamide which was then dissolved in $5.0 \mathrm{~mL}$ DCM (10.0 $\mathrm{mL}$ for $0.50 \mathrm{mg}$ preparation), then adding $0.5 \mathrm{~mL}$ of such solvent to the $1.5 \mathrm{~mL}$ glass reaction vials, finally removing the DCM by rotary evaporator. And the catalyst was prepared in the corresponding solvent to load into the reaction.)

Table S-3: Screening of DSIs from catalyst pool of AK List

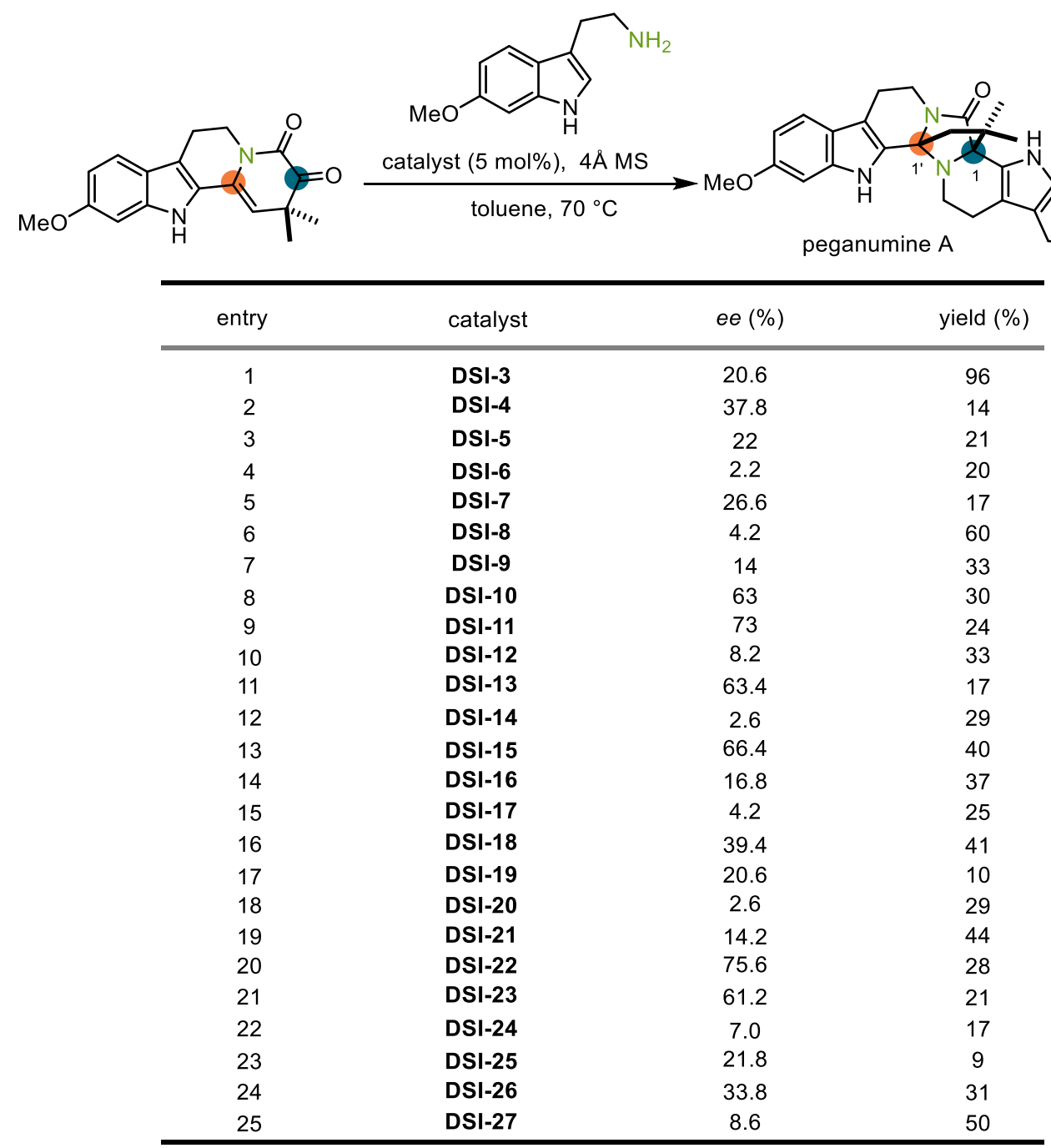




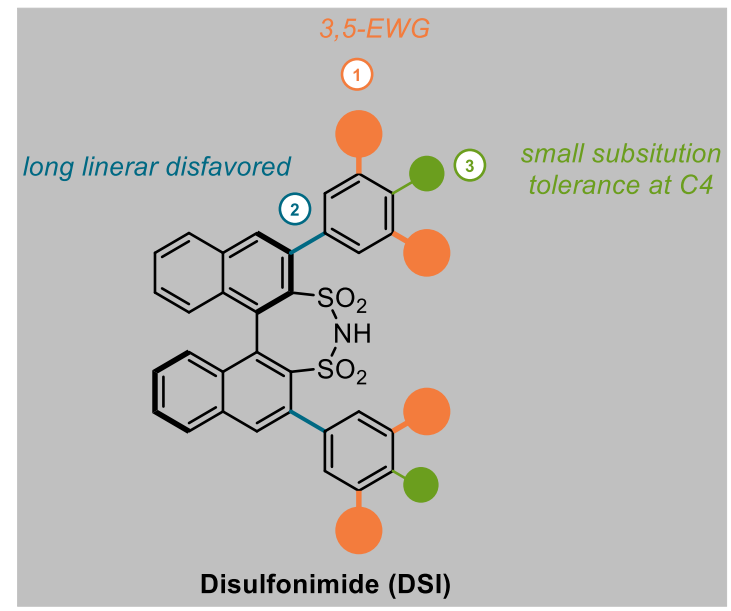

Figure S-2: Proposed SAR of DSIs

Table S-4: Screening for the newly designed DSIs

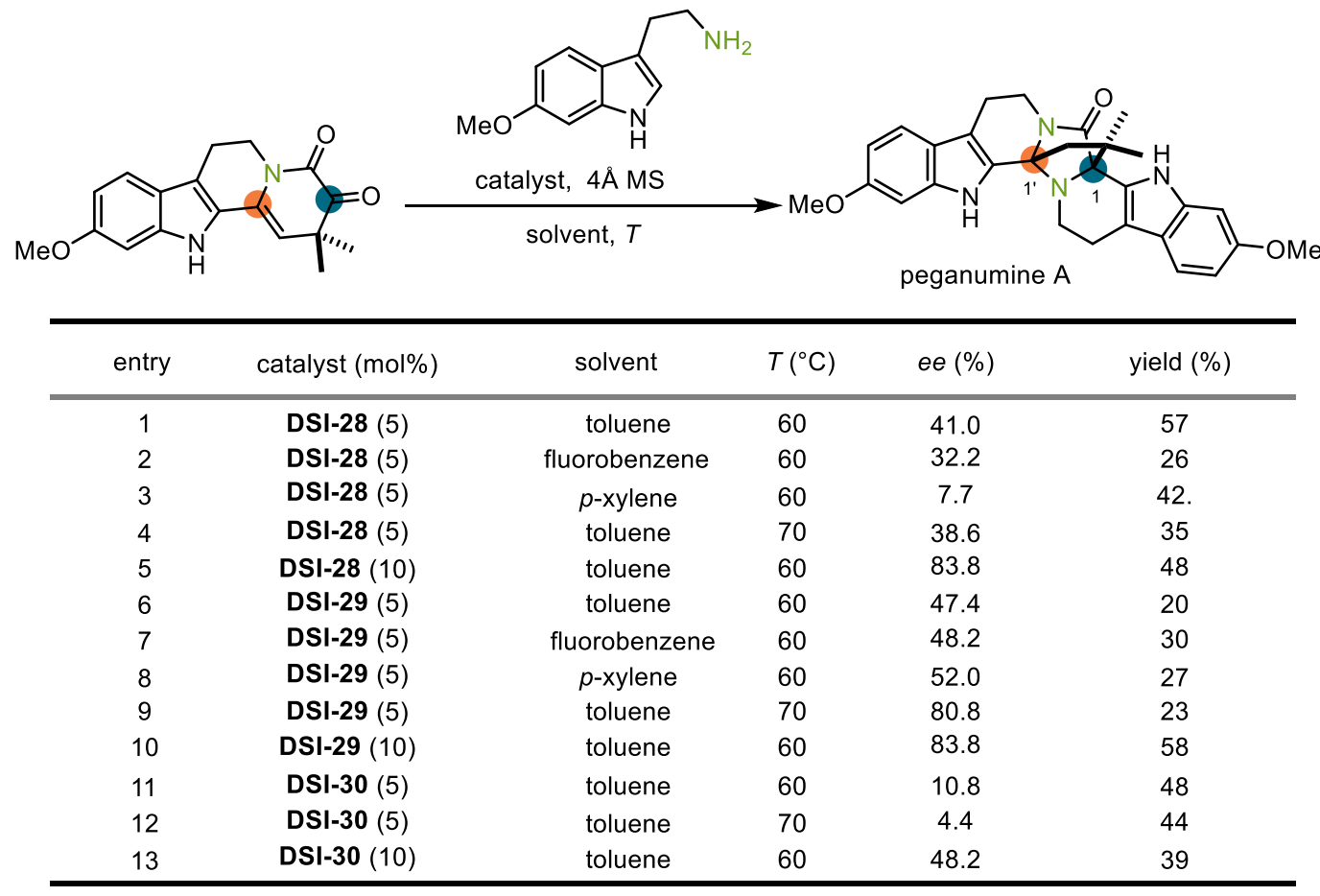


Table S-5: Optimization with DSI-2

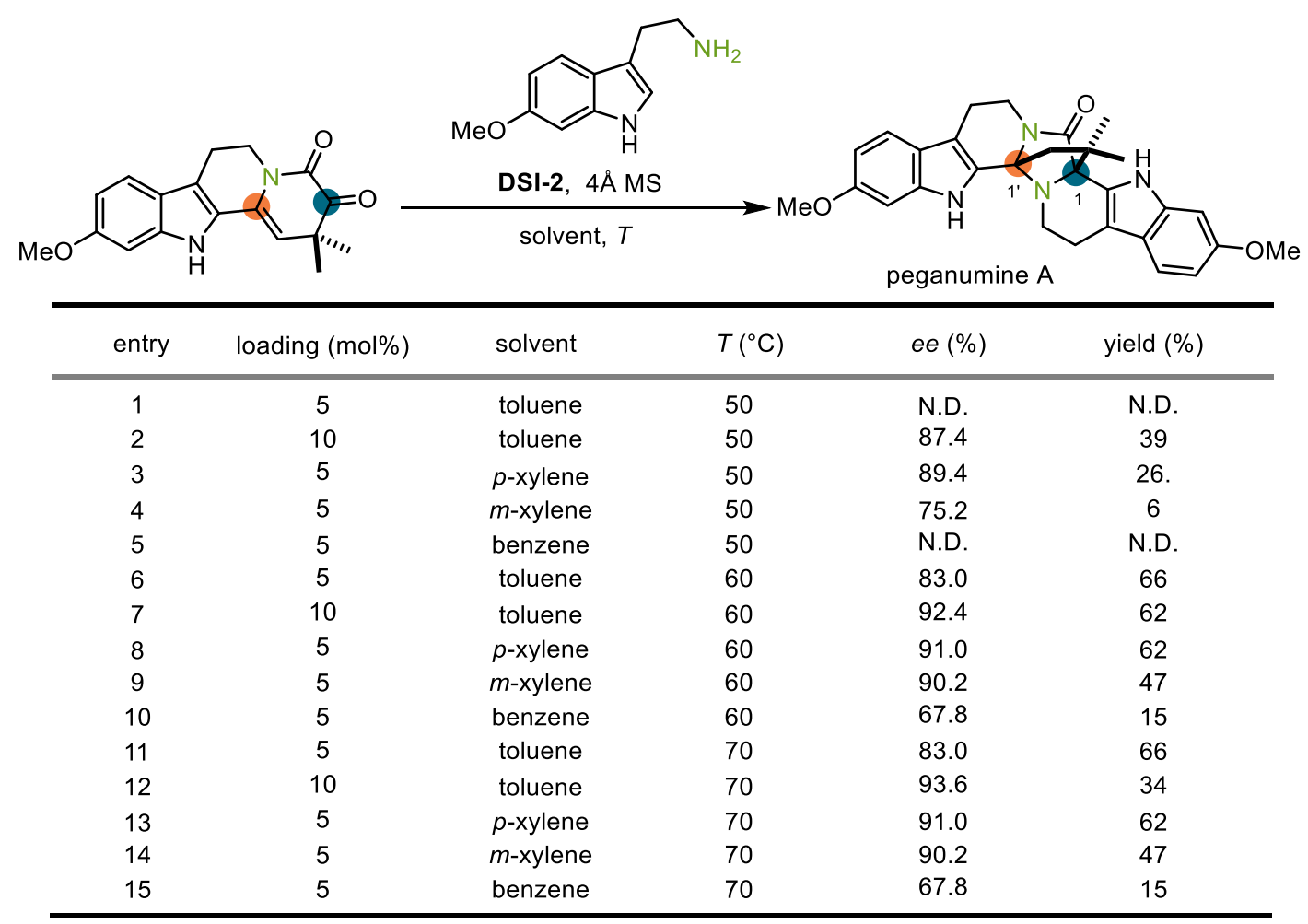

Procedure for the scale-up reaction:

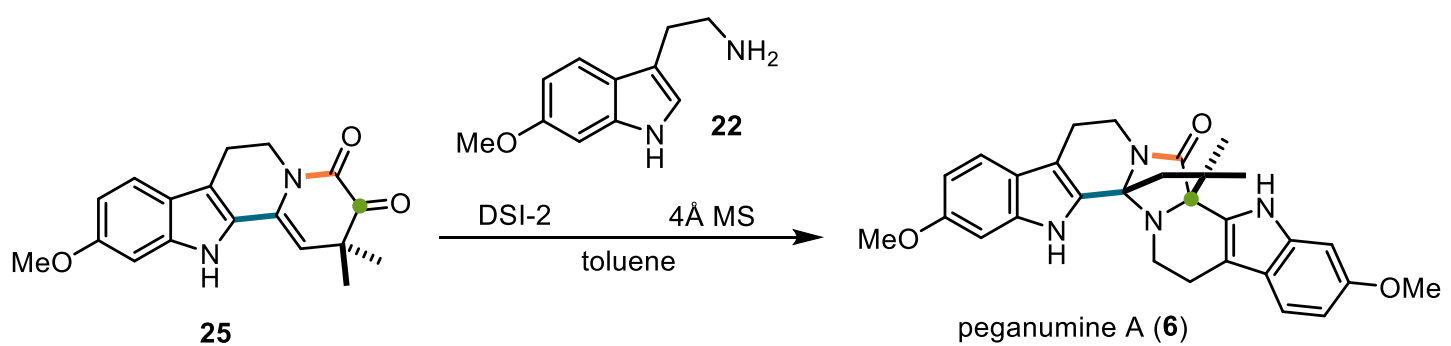

A mixture of ketoamide 25 (66.0 mg, $0.213 \mathrm{mmol}, 1.0$ equiv.), 6-methoxytryptamine (48.5 mg, $0.255 \mathrm{mmol}, 1.2$ equiv.) and $4 \AA$ molecular sieves $(50.0 \mathrm{mg})$ in toluene $(50 \mathrm{~mL})$ was stirred at room temperature. A solution of DSI-2 $(16.1 \mathrm{mg}, 21.3 \mu \mathrm{mol}$, 0.1 equiv.) in toluene $(5.0 \mathrm{~mL})$ was added to the reaction mixture and the reaction mixture was stirred at $60{ }^{\circ} \mathrm{C}$ for $60 \mathrm{~h}$. After the reaction mixture was cooled to room temperature, filtered through Celite ${ }^{\circledR}$, washed by $\mathrm{NaHCO}_{3}$ (sat. aq.), dried over $\mathrm{MgSO}_{4}$ and concentrated under reduced pressure. The crude product was purified by silica gel column chromatography (pentane/EtOAc $=3: 2)$ afforded $\mathbf{6}(83.3 \mathrm{mg}, 0.173 \mathrm{mmol}, 81 \%)$ as a white solid. 


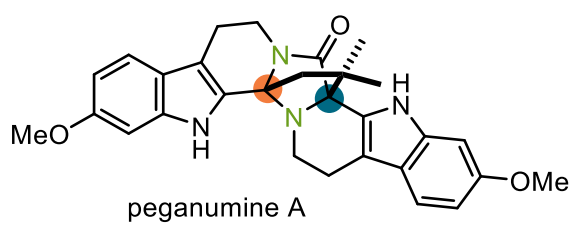

${ }^{1} \mathbf{H}$ NMR $\left(700 \mathrm{MHz}, \mathbf{C D}_{\mathbf{3}}\right.$ OD) $\delta[\mathrm{ppm}]=7.38(\mathrm{~d}, J=8.7 \mathrm{~Hz}, 1 \mathrm{H}), 7.26(\mathrm{~d}, J=8.6 \mathrm{~Hz}$, 1H), $6.99(\mathrm{~d}, J=2.2 \mathrm{~Hz}, 1 \mathrm{H}), 6.93(\mathrm{~d}, J=2.2 \mathrm{~Hz}, 1 \mathrm{H}), 6.73(\mathrm{dd}, J=8.7,2.2 \mathrm{~Hz}, 1 \mathrm{H})$, $6.67(\mathrm{dd}, J=8.6,2.3 \mathrm{~Hz}, 1 \mathrm{H}), 4.14(\mathrm{dd}, J=13.0,5.8 \mathrm{~Hz}, 1 \mathrm{H}), 3.83(\mathrm{~s}, 3 \mathrm{H}), 3.82(\mathrm{~s}$, 3H), 3.21-3.16 (m, 1H), $2.98(\mathrm{dd}, J=15.4,4.4 \mathrm{~Hz}, 1 \mathrm{H}), 2.90-2.76(\mathrm{~m}, 2 \mathrm{H}), 2.69(\mathrm{dd}$, $J=15.5,3.5 \mathrm{~Hz}, 1 \mathrm{H}), 2.59(\mathrm{dd}, J=10.8,5.3 \mathrm{~Hz}, 1 \mathrm{H}), 2.48-2.41(\mathrm{~m}, 1 \mathrm{H}), 2.39(\mathrm{~d}, J=$ $11.5 \mathrm{~Hz}, 1 \mathrm{H}), 1.94$ (d, $J=11.5 \mathrm{~Hz}, 1 \mathrm{H}), 1.44$ (s, 3H), 1.24 (s, 3H).

${ }^{13}$ C NMR $\left(176 \mathrm{MHz}, \mathbf{C D}_{3}\right.$ OD) $\delta[\mathrm{ppm}]=175.2,158.3,157.6,139.5,139.4,127.8$, $126.4,122.3,122.2,119.9,119.2,112.9,112.2,110.5,109.8,96.0,95.7,81.2,79.7$, 56.0, 55.9, 51.9, 41.7, 41.6, 37.3, 27.3, 26.3, 22.5, 22.1.

${ }^{1} \mathbf{H}$ NMR $\left(500 \mathrm{MHz}, \boldsymbol{d}_{6}\right.$-DMSO) $\delta[\mathrm{ppm}]=11.24(\mathrm{~s}, 1 \mathrm{H}), 10.77(\mathrm{~s}, 1 \mathrm{H}), 7.38(\mathrm{~d}, J=$ $8.6 \mathrm{~Hz}, 1 \mathrm{H}), 7.25(\mathrm{~d}, J=8.6 \mathrm{~Hz}, 1 \mathrm{H}), 6.93(\mathrm{~d}, J=2.3 \mathrm{~Hz}, 1 \mathrm{H}), 6.88(\mathrm{~d}, J=2.3 \mathrm{~Hz}, 1 \mathrm{H})$, $6.70(\mathrm{dd}, J=8.6,2.3 \mathrm{~Hz}, 1 \mathrm{H}), 6.63(\mathrm{dd}, J=8.6,2.3 \mathrm{~Hz}, 1 \mathrm{H}), 4.00(\mathrm{dd}, J=12.9,5.8 \mathrm{~Hz}$, $1 \mathrm{H}), 3.78(\mathrm{~s}, 3 \mathrm{H}), 3.77$ (s, 3H), $3.10(\mathrm{td}, J=12.6,4.5 \mathrm{~Hz}, 1 \mathrm{H}), 2.90(\mathrm{dd}, J=15.3,4.2$ Hz, 1H), 2.76-2.69 (m, 1H), 2.69-2.65 (m, 1H), 2.64-2.60 (m, 1H), 2.48-2.43 (m, 1H), $2.34(\mathrm{dd}, J=10.8,4.9 \mathrm{~Hz}, 1 \mathrm{H}), 2.30(\mathrm{~d}, J=11.3 \mathrm{~Hz}, 1 \mathrm{H}), 1.88(\mathrm{~d}, J=11.2 \mathrm{~Hz}, 1 \mathrm{H})$, $1.38(\mathrm{~s}, 3 \mathrm{H}), 1.15(\mathrm{~s}, 3 \mathrm{H})$.

${ }^{13}$ C NMR (126 MHz, $d_{6}$-DMSO) $\delta[\mathrm{ppm}]=171.3,156.1,155.4,137.6,137.5,127.3$, 125.7, 120.5, 120.4, 119.0, 118.2, 111.2, 109.5, 109.0, 108.3, 94.9, 94.7, 78.8, 77.4, $55.23,55.18,50.4,40.1,39.9,35.6,26.8,26.0,21.0,20.9$.

HRMS-ESI: calcd. for $\mathrm{C}_{29} \mathrm{H}_{31} \mathrm{~N}_{4} \mathrm{O}_{3}[\mathrm{M}+\mathrm{H}]^{+}$: 483.2391 ; found: 483.2384 .

FT-IR: $v\left(\mathrm{~cm}^{-1}\right)$ 2954, 2923, 2857, 1736, 1457, 1366, 1217, 820.

HPLC: $40 \%$ PrOH/Heptane, IA, $1.0 \mathrm{~mL} / \mathrm{min}$ 


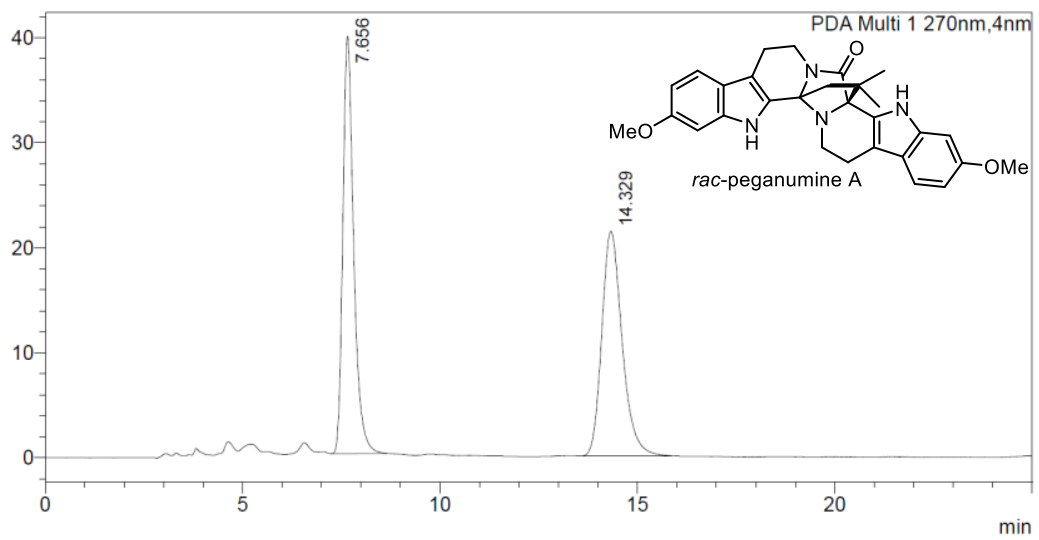

<Peak Table>

PDA Ch1 270nm
\begin{tabular}{|r|r|r|r|r|r|}
\hline Peak\# Ret. Time & \multicolumn{1}{|c|}{ Area } & \multicolumn{1}{|c|}{ Area\% } & Height & Name \\
\hline 1 & 7.656 & 759375 & 49.795 & 39781 & \\
\hline 2 & 14.329 & 765637 & 50.205 & 21402 & \\
\hline Total & & 1525012 & 100.000 & 61184 & \\
\hline
\end{tabular}

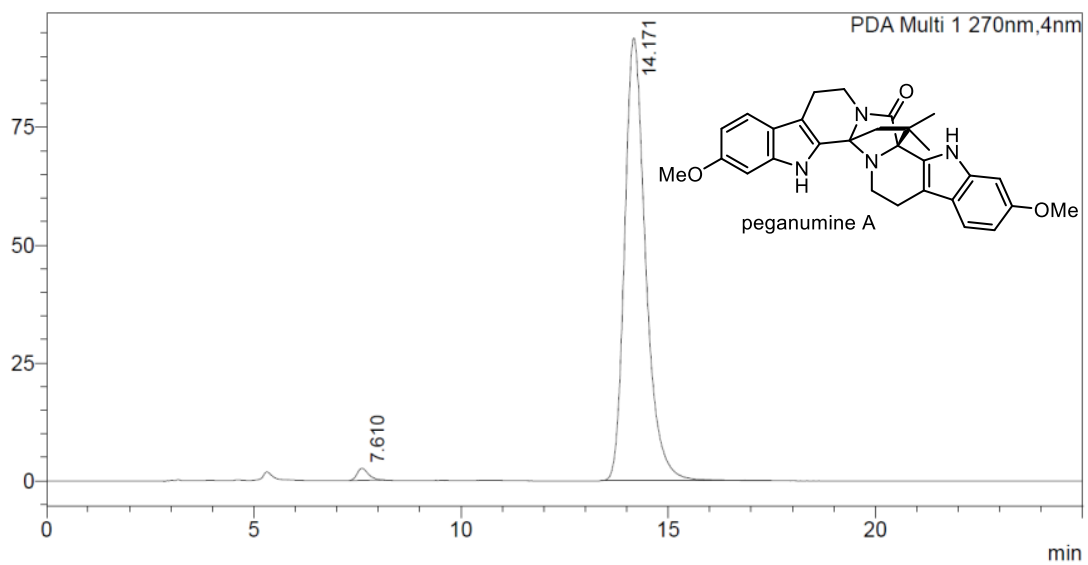

<Peak Table>

PDA Ch1 270nm

PDA Ch1 270nm
\begin{tabular}{|r|r|r|r|r|l|}
\hline Peak\# Ret. Time & Area & Area\% & Height & Name \\
\hline 1 & 7.610 & 48338 & 1.441 & 2562 & \\
\hline 2 & 14.171 & 3305614 & 98.559 & 93896 & \\
\hline Total & & 3353952 & 100.000 & 96458 & \\
\hline
\end{tabular}

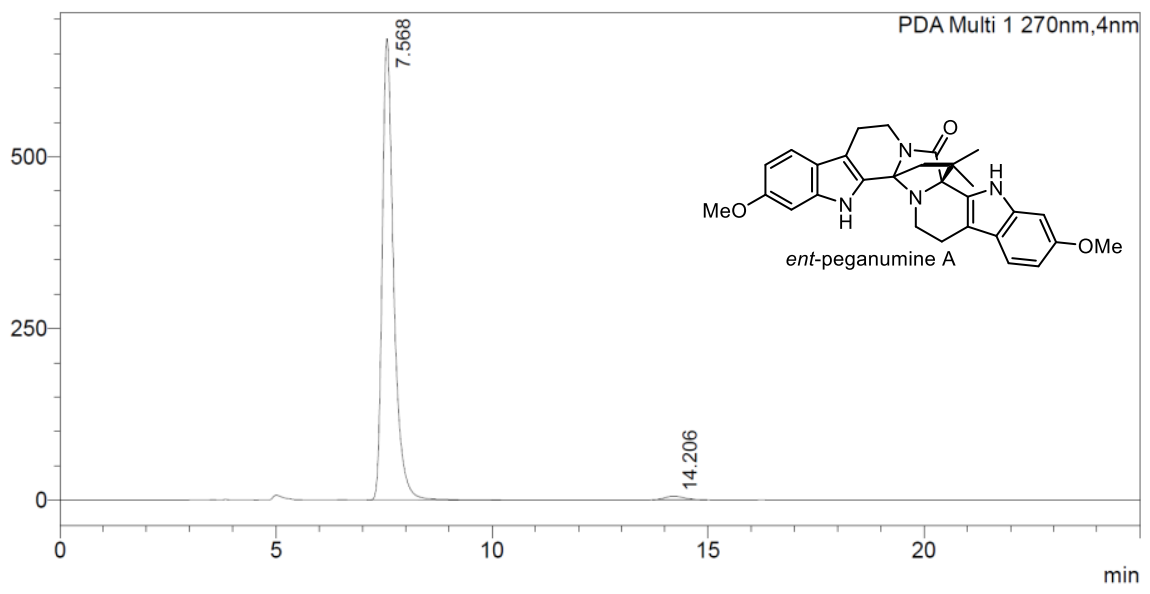

<Peak Table>

PDA Ch1 270nm

\begin{tabular}{|r|r|r|r|r|l|}
\hline Peak\# Ret. Time & \multicolumn{1}{c|}{ Area } & Area\% & Height & Name \\
\hline 1 & 7.568 & 12333154 & 98.548 & 671511 & \\
\hline 2 & 14.206 & 181749 & 1.452 & 5539 & \\
\hline Total & & 12514902 & 100.000 & 677050 & \\
\hline
\end{tabular}


2.5.3.4. NMR comparison between Hua's isolation and our synthesis

\begin{tabular}{|c|c|c|c|c|c|}
\hline \multicolumn{3}{|c|}{${ }^{1} \mathrm{H}-\mathrm{NMR}(\mathrm{ppm})$ in $d_{6}$-DMSO } & \multicolumn{3}{|c|}{${ }^{13} \mathrm{C}-\mathrm{NMR}(\mathrm{ppm})$ in $d_{6}$-DMSO } \\
\hline $\begin{array}{l}\text { Hua's isolation } \\
\qquad(600 \mathrm{MHz})\end{array}$ & $\begin{array}{c}\text { Ours } \\
(500 \mathrm{MHz})\end{array}$ & $\triangle \delta$ & $\begin{array}{l}\text { Hua's isolation } \\
(150 \mathrm{MHz})\end{array}$ & $\begin{array}{c}\text { Ours } \\
(125 \mathrm{MHz})\end{array}$ & $\triangle \delta$ \\
\hline 11.25 (br.s, 1H) & 11.24 (br.s, 1H) & -0.01 & 171.4 & 171.3 & -0.1 \\
\hline 10.77 (br.s, 1H) & 10.77 (br.s, 1H) & 0 & 156.1 & 156.1 & 0 \\
\hline $\begin{array}{c}7.38(\mathrm{~d}, J=8.6 \\
\mathrm{Hz}, 1 \mathrm{H})\end{array}$ & $\begin{array}{c}7.38(\mathrm{~d}, J=8.6 \\
\mathrm{Hz}, 1 \mathrm{H})\end{array}$ & 0 & 155.4 & 155.4 & 0 \\
\hline $\begin{array}{c}7.24(\mathrm{~d}, J=8.6 \\
\mathrm{Hz}, 1 \mathrm{H})\end{array}$ & $\begin{array}{c}7.25(\mathrm{~d}, J=8.6 \\
\mathrm{Hz}, 1 \mathrm{H})\end{array}$ & 0.01 & 137.6 & 137.6 & 0 \\
\hline $\begin{array}{c}6.93(\mathrm{~d}, J=1.6 \\
\mathrm{Hz}, 1 \mathrm{H})\end{array}$ & $\begin{array}{c}6.93(\mathrm{~d}, J=2.3 \\
\mathrm{Hz}, 1 \mathrm{H})\end{array}$ & 0 & 137.5 & 137.5 & 0 \\
\hline $\begin{array}{c}6.87(\mathrm{~d}, J=1.8 \\
\mathrm{Hz}, 1 \mathrm{H})\end{array}$ & $\begin{array}{c}\mathbf{6 . 8 8}(\mathrm{d}, J=2.3 \\
\mathrm{Hz}, 1 \mathrm{H})\end{array}$ & 0.01 & 127.3 & 127.3 & 0 \\
\hline $\begin{array}{c}\mathbf{6 . 7 0}(\mathrm{dd}, J= \\
8.6,1.8 \mathrm{~Hz}, 1 \mathrm{H})\end{array}$ & $\begin{array}{c}\mathbf{6 . 7 0}(\mathrm{dd}, J= \\
8.6,2.3 \mathrm{~Hz}, 1 \mathrm{H})\end{array}$ & 0 & 125.7 & 125.7 & 0 \\
\hline $\begin{array}{c}\mathbf{6 . 6 3}(\mathrm{dd}, J= \\
8.6,1.6 \mathrm{~Hz}, 1 \mathrm{H})\end{array}$ & $\begin{array}{c}\mathbf{6 . 6 3}(\mathrm{dd}, J= \\
8.6,2.3 \mathrm{~Hz}, 1 \mathrm{H})\end{array}$ & 0 & 120.5 & 120.5 & 0 \\
\hline $\begin{array}{c}\mathbf{4 . 0 0}(\mathrm{dd}, J= \\
12.6,5.7 \mathrm{~Hz} \\
1 \mathrm{H})\end{array}$ & $\begin{array}{c}\mathbf{4 . 0 0}(\mathrm{dd}, J= \\
12.9,5.8 \mathrm{~Hz} \\
1 \mathrm{H})\end{array}$ & 0 & 120.4 & 120.4 & 0 \\
\hline $3.78(\mathrm{~s}, 3 \mathrm{H})$ & $3.78(\mathrm{~s}, 3 \mathrm{H})$ & 0 & 119.0 & 119.0 & 0 \\
\hline $3.77(\mathrm{~s}, 3 \mathrm{H})$ & $3.77(\mathrm{~s}, 3 \mathrm{H})$ & 0 & 118.2 & 118.2 & 0 \\
\hline $\begin{array}{c}3.09(\mathrm{td}, J= \\
12.6,4.4 \mathrm{~Hz} \\
1 \mathrm{H})\end{array}$ & $\begin{array}{c}\mathbf{3 . 1 0}(\mathrm{td}, J= \\
12.6,4.5 \mathrm{~Hz} \\
1 \mathrm{H})\end{array}$ & 0.01 & 111.3 & 111.2 & -0.1 \\
\hline $\begin{array}{c}2.90(\mathrm{dd}, J= \\
15.1,4.4 \mathrm{~Hz} \\
1 \mathrm{H})\end{array}$ & $\begin{array}{c}2.90(\mathrm{dd}, J= \\
15.3,4.2 \mathrm{~Hz} \\
1 \mathrm{H})\end{array}$ & 0 & 109.5 & 109.5 & 0 \\
\hline $\begin{array}{c}2.70(\mathrm{ddd}, J= \\
15.1,12.6,5.7 \\
\mathrm{~Hz}, 1 \mathrm{H})\end{array}$ & $\begin{array}{c}\mathbf{2 . 7 6}-\mathbf{2 . 6 9}(\mathrm{m}, \\
1 \mathrm{H})\end{array}$ & & 109.0 & 109.0 & 0 \\
\hline
\end{tabular}




\begin{tabular}{|c|c|c|c|c|c|}
\hline $\begin{array}{c}2.64(\mathrm{dd}, J= \\
11.0,4.9 \mathrm{~Hz}, \\
1 \mathrm{H})\end{array}$ & $\begin{array}{c}\text { 2.69-2.65 (m, } \\
1 \mathrm{H})\end{array}$ & & 108.3 & 108.3 & 0 \\
\hline $\begin{array}{c}\mathbf{2 . 6 3}(\mathrm{dd}, J= \\
11.0,4.9 \mathrm{~Hz}, \\
1 \mathrm{H})\end{array}$ & $\begin{array}{c}\mathbf{2 . 6 4 - 2 . 6 0}(\mathrm{m}, \\
1 \mathrm{H})\end{array}$ & & 94.9 & 94.9 & 0 \\
\hline $\begin{array}{c}2.45(\mathrm{dd}, J= \\
10.9,4.9 \mathrm{~Hz}, \\
1 \mathrm{H})\end{array}$ & $\begin{array}{c}\mathbf{2 . 4 8}-\mathbf{2 . 4 3}(\mathrm{m}, \\
1 \mathrm{H})\end{array}$ & & 94.7 & 94.7 & 0 \\
\hline $\begin{array}{c}2.34(\mathrm{dd}, J= \\
10.9,4.9 \mathrm{~Hz}, \\
1 \mathrm{H})\end{array}$ & $\begin{array}{c}2.34(\mathrm{dd}, J= \\
10.8,4.9 \mathrm{~Hz} \\
1 \mathrm{H})\end{array}$ & 0 & 78.8 & 78.8 & 0 \\
\hline $\begin{array}{c}2.30(\mathrm{~d}, J= \\
10.9 \mathrm{~Hz}, 1 \mathrm{H})\end{array}$ & $\begin{array}{c}2.30(\mathrm{~d}, J= \\
11.3 \mathrm{~Hz}, 1 \mathrm{H})\end{array}$ & 0 & 77.4 & 77.4 & 0 \\
\hline $\begin{array}{c}\mathbf{1 . 8 8}(\mathrm{d}, J= \\
10.9 \mathrm{~Hz}, 1 \mathrm{H})\end{array}$ & $\begin{array}{c}\mathbf{1 . 8 8}(\mathrm{d}, J= \\
11.2 \mathrm{~Hz}, 1 \mathrm{H})\end{array}$ & 0 & $55.2(55.23)$ & $55.2(55.23)$ & 0 \\
\hline $\mathbf{1 . 3 8}(\mathrm{s}, 3 \mathrm{H})$ & $\mathbf{1 . 3 8}(\mathrm{s}, 3 \mathrm{H})$ & 0 & $55.2(55.18)$ & $55.2(55.18)$ & 0 \\
\hline \multirow[t]{8}{*}{$\mathbf{1 . 1 5}(\mathrm{s}, 3 \mathrm{H})$} & $1.15(\mathrm{~s}, 3 \mathrm{H})$ & 0 & 50.4 & 50.4 & 0 \\
\hline & & & 40.0 & 40.1 & 0.1 \\
\hline & & & 40.0 & 39.9 & -0.1 \\
\hline & & & 35.6 & 35.6 & 0 \\
\hline & & & 26.8 & 26.8 & 0 \\
\hline & & & 26.0 & 26.0 & 0 \\
\hline & & & 21.0 & 21.0 & 0 \\
\hline & & & 20.9 & 20.9 & 0 \\
\hline
\end{tabular}


2.5.3.5. NMR comparison between Zhu's synthesis and our synthesis

\begin{tabular}{|c|c|c|c|c|c|}
\hline \multicolumn{3}{|c|}{${ }^{1} \mathrm{H}-\mathrm{NMR}(\mathrm{ppm})$ in $\mathrm{CD}_{3} \mathrm{OD}$} & \multicolumn{3}{|c|}{${ }^{13} \mathrm{C}-\mathrm{NMR}(\mathrm{ppm})$ in $\mathrm{CD}_{3} \mathrm{OD}$} \\
\hline $\begin{array}{c}\text { Zhu's } \\
(400 \mathrm{MHz})\end{array}$ & $\begin{array}{c}\text { Ours } \\
(500 \mathrm{MHz})\end{array}$ & $\triangle \delta$ & $\begin{array}{c}\text { Zhu's } \\
\text { (101 MHz) }\end{array}$ & $\begin{array}{c}\text { Ours } \\
(176 \mathrm{MHz})\end{array}$ & $\triangle \delta$ \\
\hline $\begin{array}{c}7.38(\mathrm{~d}, J=8.6 \\
\mathrm{Hz}, 1 \mathrm{H})\end{array}$ & $\begin{array}{c}7.38(\mathrm{~d}, J=8.6 \\
\mathrm{Hz}, 1 \mathrm{H})\end{array}$ & 0 & 175.2 & 175.2 & 0 \\
\hline $\begin{array}{c}7.26(\mathrm{~d}, J=8.5 \\
\mathrm{Hz}, 1 \mathrm{H})\end{array}$ & $\begin{array}{c}7.26(\mathrm{~d}, J=8.6 \\
\mathrm{Hz}, 1 \mathrm{H})\end{array}$ & 0 & 158.4 & 158.3 & -0.1 \\
\hline $\begin{array}{c}6.99(\mathrm{~d}, J=2.3 \\
\mathrm{Hz}, 1 \mathrm{H})\end{array}$ & $\begin{array}{c}6.99(\mathrm{~d}, J=2.2 \\
\mathrm{Hz}, 1 \mathrm{H})\end{array}$ & 0 & 157.6 & 157.6 & 0 \\
\hline $\begin{array}{c}6.93(\mathrm{~d}, J=2.2 \\
\mathrm{Hz}, 1 \mathrm{H})\end{array}$ & $\begin{array}{c}6.93(\mathrm{~d}, J=2.2 \\
\mathrm{Hz}, 1 \mathrm{H})\end{array}$ & 0 & 139.5 & 139.5 & 0 \\
\hline $\begin{array}{c}\mathbf{6 . 7 3}(\mathrm{dd}, J= \\
8.6,2.3 \mathrm{~Hz}, 1 \mathrm{H})\end{array}$ & $\begin{array}{c}\mathbf{6 . 7 3}(\mathrm{dd}, J= \\
8.6,2.2 \mathrm{~Hz}, 1 \mathrm{H})\end{array}$ & 0 & 139.4 & 139.4 & 0 \\
\hline $\begin{array}{c}\mathbf{6 . 6 7}(\mathrm{dd}, J= \\
8.6,2.3 \mathrm{~Hz}, 1 \mathrm{H})\end{array}$ & $\begin{array}{c}\mathbf{6 . 6 7}(\mathrm{dd}, J= \\
8.5,2.3 \mathrm{~Hz}, 1 \mathrm{H})\end{array}$ & 0 & 127.8 & 127.8 & 0 \\
\hline $\begin{array}{c}\mathbf{4 . 1 4}(\mathrm{dd}, J= \\
13.0,5.8 \mathrm{~Hz} \\
1 \mathrm{H})\end{array}$ & $\begin{array}{c}\mathbf{4 . 1 4}(\mathrm{dd}, J= \\
13.0,5.8 \mathrm{~Hz} \\
1 \mathrm{H})\end{array}$ & 0 & 126.5 & 126.4 & -0.1 \\
\hline $\mathbf{3 . 8 3}(\mathrm{s}, 3 \mathrm{H})$ & $\mathbf{3 . 8 3}(\mathrm{s}, 3 \mathrm{H})$ & 0 & 122.3 & 122.3 & 0 \\
\hline $3.82(\mathrm{~s}, 3 \mathrm{H})$ & $3.82(\mathrm{~s}, 3 \mathrm{H})$ & 0 & 122.2 & 122.2 & 0 \\
\hline $\begin{array}{c}\mathbf{3 . 1 8}(\mathrm{td}, J= \\
12.3,4.7 \mathrm{~Hz} \\
1 \mathrm{H})\end{array}$ & $\begin{array}{c}\text { 3.21-3.16 }(\mathrm{m}, \\
1 \mathrm{H})\end{array}$ & & 120.0 & 119.9 & -0.1 \\
\hline $\begin{array}{c}2.98(\mathrm{dd}, J= \\
15.4,4.5 \mathrm{~Hz} \\
1 \mathrm{H})\end{array}$ & $\begin{array}{c}2.98(\mathrm{dd}, J= \\
15.4,4.3 \mathrm{~Hz} \\
1 \mathrm{H})\end{array}$ & 0 & 119.2 & 119.2 & 0 \\
\hline $\begin{array}{c}2.90-2.74(\mathrm{~m}, \\
2 \mathrm{H})\end{array}$ & $\begin{array}{c}2.90-2.76(\mathrm{~m}, \\
2 \mathrm{H})\end{array}$ & & 112.9 & 112.9 & 0 \\
\hline $\begin{array}{c}2.69(\mathrm{dd}, J= \\
15.2,3.8 \mathrm{~Hz} \\
1 \mathrm{H})\end{array}$ & $\begin{array}{c}2.69(\mathrm{dd}, J= \\
15.5,3.5 \mathrm{~Hz} \\
1 \mathrm{H})\end{array}$ & 0 & 112.2 & 112.2 & 0 \\
\hline $\begin{array}{c}2.59(\mathrm{dd}, J= \\
10.8,5.2 \mathrm{~Hz} \\
1 \mathrm{H})\end{array}$ & $\begin{array}{c}2.59(\mathrm{dd}, J= \\
10.8,5.3 \mathrm{~Hz} \\
1 \mathrm{H})\end{array}$ & 0 & 110.5 & 110.5 & 0 \\
\hline
\end{tabular}




\begin{tabular}{|c|c|c|c|c|c|}
\hline $\begin{array}{c}2.44(\mathrm{dd}, J= \\
11.2,4.1 \mathrm{~Hz}, \\
1 \mathrm{H})\end{array}$ & $\begin{array}{c}2.48-2.41(\mathrm{~m}, \\
1 \mathrm{H})\end{array}$ & & 109.8 & 109.8 & 0 \\
\hline $\begin{array}{c}2.39(\mathrm{~d}, J= \\
11.6 \mathrm{~Hz}, 1 \mathrm{H})\end{array}$ & $\begin{array}{c}2.39(\mathrm{~d}, J= \\
11.5 \mathrm{~Hz}, 1 \mathrm{H})\end{array}$ & ( & 96.0 & 96.0 & 0 \\
\hline $\begin{array}{c}\mathbf{1 . 9 4}(\mathrm{d}, J= \\
11.5 \mathrm{~Hz}, 1 \mathrm{H})\end{array}$ & $\begin{array}{c}\mathbf{1 . 9 4}(\mathrm{d}, J= \\
11.5 \mathrm{~Hz}, 1 \mathrm{H})\end{array}$ & ( & 95.7 & 95.7 & 0 \\
\hline $\mathbf{1 . 4 4}(\mathrm{s}, 3 \mathrm{H})$ & $1.44(\mathrm{~s}, 3 \mathrm{H})$ & ( & 81.3 & 81.2 & -0.1 \\
\hline \multirow[t]{11}{*}{$1.24(\mathrm{~s}, 3 \mathrm{H})$} & $1.24(\mathrm{~s}, 3 \mathrm{H})$ & ( & 79.7 & 79.7 & 0 \\
\hline & & & 56.0 & 56.0 & 0 \\
\hline & & & 56.0 & 55.9 & -0.1 \\
\hline & & & 51.9 & 51.9 & 0 \\
\hline & & & 41.7 & 41.7 & 0 \\
\hline & & & 41.6 & 41.6 & 0 \\
\hline & & & 37.3 & 37.3 & 0 \\
\hline & & & 27.3 & 27.3 & 0 \\
\hline & & & 26.3 & 26.3 & 0 \\
\hline & & & 22.5 & 22.5 & 0 \\
\hline & & & 22.1 & 22.1 & 0 \\
\hline
\end{tabular}


2.6. Total synthesis of ilicifoline B

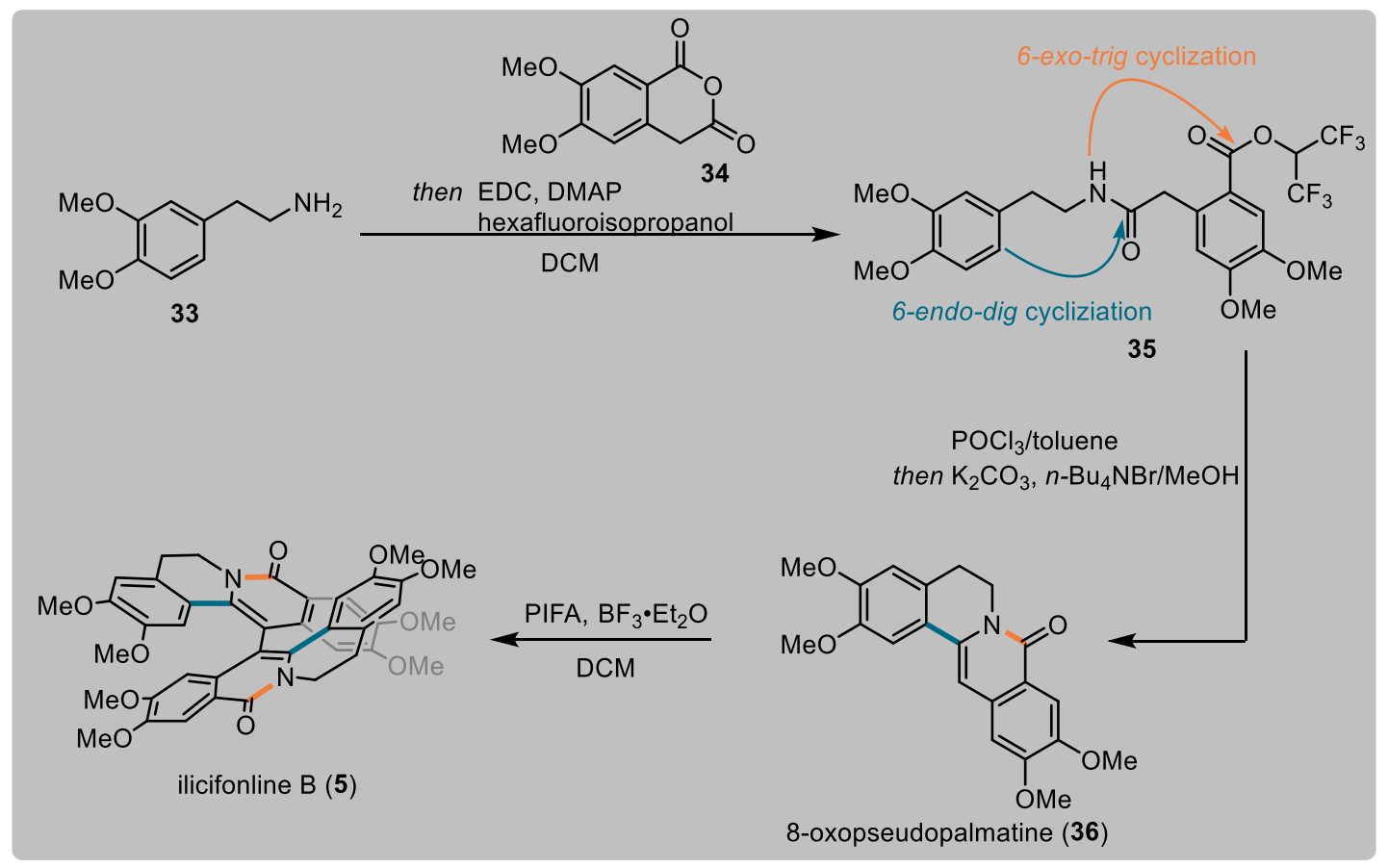

1,1,1,3,3,3-Hexafluoropropan-2-yl 2-(2-((3,4-dimethoxyphenethyl)amino)-2-oxoethyl)-4,5-dimethoxybenzoate (35).

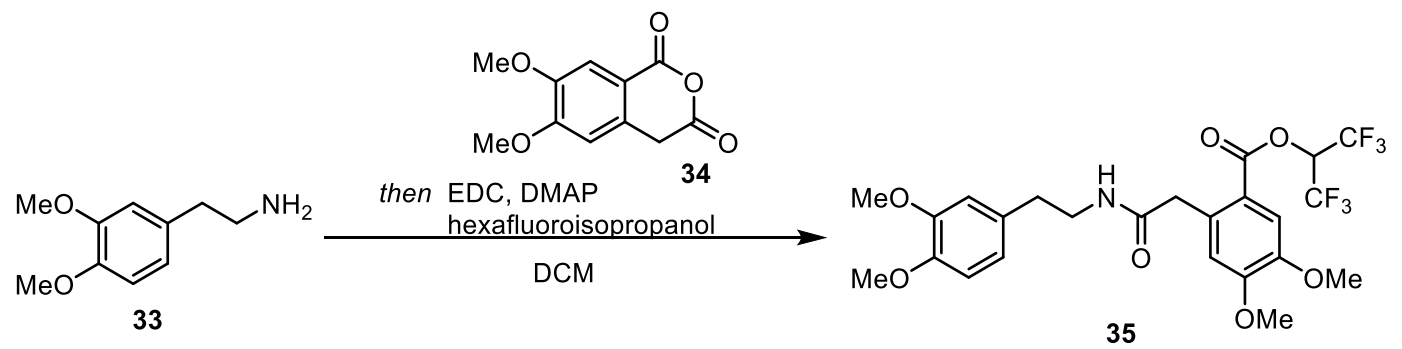

35 was prepared according to General procedure A, starting from 33 (100 mg, $0.552 \mathrm{mmol}, 1.0$ equiv.) and 34 (123 mg, $0.552 \mathrm{mmol}, 1.0$ equiv.) in DCM (5.0 mL). The reaction mixture was subsequently treated with EDCI $(211 \mathrm{mg}, 1.10 \mathrm{mmol}$, 2.0 equiv.), DMAP (13.5 mg, $0.110 \mathrm{mmol}, 0.2$ equiv.) and HFIP (292 $\mu \mathrm{L}, 2.76 \mathrm{mmol}$, 5.0 equiv.). Purification by silica gel column chromatography (pentane/EtOAc $=3: 2$ ) afforded 35 (190 mg, $0.342 \mathrm{mmol}, 62 \%$ ) as a light yellow solid.

${ }^{1} \mathbf{H}$ NMR $\left(700 \mathrm{MHz}, \mathrm{CDCl}_{3}\right) \delta[\mathrm{ppm}]=7.46(\mathrm{~s}, 1 \mathrm{H}), 6.91(\mathrm{~s}, 1 \mathrm{H}), 6.71(\mathrm{~d}, J=8.0 \mathrm{~Hz}$, 1H), $6.65(\mathrm{~d}, J=2.0 \mathrm{~Hz}, 1 \mathrm{H}), 6.59(\mathrm{dd}, J=8.1,2.0 \mathrm{~Hz}, 1 \mathrm{H}), 6.08(\mathrm{~s}, 1 \mathrm{H}), 5.93-5.90$ (m, 1H), $3.95(\mathrm{~s}, 3 \mathrm{H}), 3.91(\mathrm{~s}, 3 \mathrm{H}), 3.83(\mathrm{~s}, 3 \mathrm{H}), 3.82(\mathrm{~s}, 3 \mathrm{H}), 3.79(\mathrm{~s}, 2 \mathrm{H}), 3.43$ (td, $J$ $=7.1,5.7 \mathrm{~Hz}, 2 \mathrm{H}), 2.68(\mathrm{t}, J=7.1 \mathrm{~Hz}, 2 \mathrm{H})$. 
${ }^{13} \mathbf{C}$ NMR $\left(176 \mathrm{MHz}, \mathrm{CDCl}_{3}\right) \delta[\mathrm{ppm}]=170.2,163.4,154.1,149.1,147.9,147.8,134.3$, $131.3,120.69$ (q, $J=283.7 \mathrm{~Hz}), 120.65,116.9,115.0,113.5,112.0,111.2,67.3-$ $66.5(\mathrm{~m}), 56.4,56.1,55.9,42.0,40.9,35.2$. (Based on the intensity of the resonance, the peak at $\delta[\mathrm{ppm}]=55.9$ is considered as two methoxy carbons.)

${ }^{19}$ F NMR $\left(565 \mathrm{MHz}, \mathrm{CDCl}_{3}\right) \delta[\mathrm{ppm}]=-72.98(\mathrm{~d}, J=6.1 \mathrm{~Hz})$.

HRMS-ESI: calcd. for $\mathrm{C}_{24} \mathrm{H}_{25} \mathrm{~F}_{6} \mathrm{NO}_{7} \mathrm{Na}[\mathrm{M}+\mathrm{Na}]^{+}$: 576.1427; found: 576.1401 .

FT-IR: $v\left[\mathrm{~cm}^{-1}\right]$ 2934, 1749, 1674, 1518, 1263, 1234, 1194, 1110, 768.

2,3,10,11-Tetramethoxy-5,6-dihydro-8H-isoquinolino[3,2-a]isoquinolin-8-one (36).<smiles>COc1cc(CC(=O)NCCc2ccc(OC)c(OC)c2)c(C(=O)O[Na])c(C(=O)OC(F)(F)F)c1</smiles><smiles>COc1cc2c(cc1OC)-c1cc3cc(OC)c(OC)cc3c(=O)n1CC2</smiles>

36 was prepared according to General procedure C, starting from 35 (60 mg, $0.11 \mathrm{mmol}, 1.0$ equiv.) and $\mathrm{POCl}_{3}(9.9 \mu \mathrm{L}, 0.11 \mathrm{mmol}, 1.0$ equiv. $)$ in toluene $(3.0 \mathrm{~mL})$. The reaction mixture was subsequently treated with $\mathrm{MeOH}(3.0 \mathrm{~mL}), \mathrm{K}_{2} \mathrm{CO}_{3}(150 \mathrm{mg}$, $1.1 \mathrm{mmol}, 10$ equiv.) and $n \mathrm{Bu} 4 \mathrm{NBr}(3.5 \mathrm{mg}, 0.022 \mathrm{mmol}, 0.1$ equiv.). Purification by silica gel column chromatography (pentane/EtOAc $=1: 1)$ afforded $36(38 \mathrm{mg}$, $0.104 \mathrm{mmol}, 95 \%)$ as a yellow solid.

${ }^{1} \mathbf{H}$ NMR $\left(700 \mathrm{MHz}, \mathrm{CDCl}_{3}\right) \delta[\mathrm{ppm}]=7.81(\mathrm{~s}, 1 \mathrm{H}), 7.25(\mathrm{~s}, 1 \mathrm{H}), 6.94(\mathrm{~s}, 1 \mathrm{H}), 6.83(\mathrm{~s}$, 1H), $6.74(\mathrm{~s}, 1 \mathrm{H}), 4.39-4.34(\mathrm{~m}, 2 \mathrm{H}), 4.02(\mathrm{~s}, 3 \mathrm{H}), 4.01(\mathrm{~s}, 3 \mathrm{H}), 3.98(\mathrm{~s}, 3 \mathrm{H}), 3.94(\mathrm{~s}$, $3 \mathrm{H}), 2.96-2.92(\mathrm{~m}, 2 \mathrm{H})$.

${ }^{13} \mathbf{C ~ N M R}\left(176 \mathrm{MHz}, \mathrm{CDCl}_{3}\right) \delta[\mathrm{ppm}]=161.6,153.7,150.3,149.2,148.6,136.3,132.3$, $128.6,122.7,118.7,110.7,108.0,107.8,106.1,101.3,56.4,56.4,56.2,39.9,28.3$. (Based on the intensity of the resonance, the peak at $\delta[\mathrm{ppm}]=56.2$ is considered as two methoxy carbons.)

HRMS-ESI: calcd. for $\mathrm{C}_{21} \mathrm{H}_{21} \mathrm{NO}_{5} \mathrm{Na}[\mathrm{M}+\mathrm{Na}]^{+}: 390.1312$ found: 390.1323 .

FT-IR: $v\left[\mathrm{~cm}^{-1}\right]$ 2929, 1644, 1594, 1509, 1466, 1426, 1255, 1235, 1097, 1027, 747. 


\section{$2,2^{\prime}, 3,3^{\prime}, 10,10 ', 11,11^{\prime}$-Octamethoxy-5,5',6,6'-tetrahydro-8H,8' $H$-[13,13'-biisoquin-}

olino[3,2-a] isoquinoline]-8,8'-dione (5).

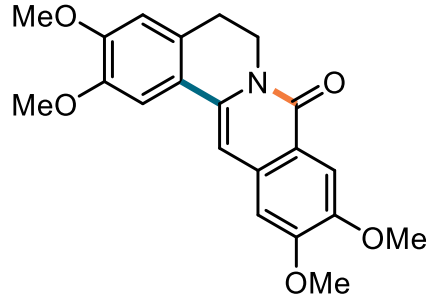

8-oxopseudopalmatine (36)

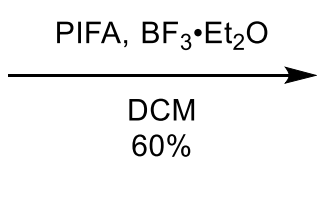

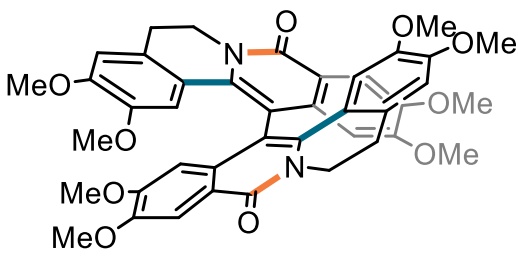

ilicifoline B (5)

A mixture of 8-oxypseudopalmatine (18 mg, $50 \mu \mathrm{mol}, 1.0$ equiv.) and PIFA (16 mg, 0.05 mmol, 1.0 equiv.) in DCM $(2.0 \mathrm{~mL})$, was added $\mathrm{BF}_{3} \bullet \mathrm{Et}_{2} \mathrm{O}(9.7 \mu \mathrm{L}, 0.10 \mathrm{mmol}$, 2.0 equiv.) at $-78^{\circ} \mathrm{C}$. The mixture was stirred for $3 \mathrm{~h}$, then quenched with $\mathrm{NaHCO}_{3}$ (sat. aq.) and diluted with DCM, and the organic phase was separated. The aqueous phase was extracted with DCM and the combined organic layers were washed with brine, dried over $\mathrm{MgSO}_{4}$, filtered, and concentrated under reduced pressure. Purification by silica gel column chromatography (pentane/EtOAc $=1: 1$ to EtOAc) afforded $\mathbf{5}(11 \mathrm{mg}$, $15 \mu \mathrm{mol}, 60 \%$ ) as a yellow solid.

${ }^{1} \mathbf{H}$ NMR $\left(700 \mathrm{MHz}, \mathrm{CDCl}_{3}\right) \delta[\mathrm{ppm}]=7.95(\mathrm{~s}, 2 \mathrm{H}), 6.76(\mathrm{~s}, 2 \mathrm{H}), 6.43(\mathrm{~s}, 2 \mathrm{H}), 6.34(\mathrm{~s}$, 2H), $5.05(\mathrm{dt}, J=13.2,3.8 \mathrm{~Hz}, 2 \mathrm{H}), 4.06(\mathrm{~s}, 6 \mathrm{H}), 3.82(\mathrm{~s}, 6 \mathrm{H}), 3.62(\mathrm{~s}, 6 \mathrm{H}), 3.33(\mathrm{td}, J$ $=13.2,3.0 \mathrm{~Hz}, 2 \mathrm{H}), 3.03(\mathrm{~s}, 6 \mathrm{H}), 2.52(\mathrm{dt}, J=15.1,3.1 \mathrm{~Hz}, 2 \mathrm{H}), 2.13(\mathrm{ddd}, J=14.5$, 12.7, $4.2 \mathrm{~Hz}, 2 \mathrm{H})$.

${ }^{13} \mathbf{C ~ N M R}\left(176 \mathrm{MHz}, \mathrm{CDCl}_{3}\right) \delta[\mathrm{ppm}]=161.0,153.9,149.5,149.2,146.6,137.0,133.6$, 131.3, 122.5, 119.0, 111.6, 110.8, 109.2, 108.1, 105.8, 56.4, 56.3, 56.2, 55.2, 41.4, 29.0. HRMS-EI: calcd. for $\mathrm{C}_{42} \mathrm{H}_{40} \mathrm{~N}_{2} \mathrm{O}_{10} \mathrm{Na}[\mathrm{M}+\mathrm{Na}]^{+}$: 755.2575; found: 755.2576.

FT-IR: $v\left[\mathrm{~cm}^{-1}\right]$ 3004, 2928, 1633, 1607, 1587, 1498, 1464, 1440, 1374, 1271, 1226 , $1133,1096,1008,876,749$. 
NMR comparison between Opatz's synthesis and our synthesis

\begin{tabular}{|c|c|c|c|c|c|}
\hline \multicolumn{3}{|c|}{${ }^{1} \mathrm{H}-\mathrm{NMR}(\mathrm{ppm})$ in $\mathrm{CDCl}_{3}$} & \multicolumn{3}{|c|}{${ }^{13} \mathrm{C}-\mathrm{NMR}$ (ppm) in $\mathrm{CDCl}_{3}$} \\
\hline $\begin{array}{l}\text { Opatz’s } \\
\text { (600 MHz) }\end{array}$ & $\begin{array}{c}\text { Ours } \\
(700 \mathrm{MHz})\end{array}$ & $\triangle \delta$ & $\begin{array}{c}\text { Opatz’s } \\
(150 \mathrm{MHz})\end{array}$ & $\begin{array}{c}\text { Ours } \\
(176 \mathrm{MHz})\end{array}$ & $\triangle \delta$ \\
\hline $7.95(\mathrm{~s}, 2 \mathrm{H})$ & $7.95(\mathrm{~s}, 1 \mathrm{H})$ & 0 & 160.9 & 161.0 & 0.1 \\
\hline $6.75(\mathrm{~s}, 2 \mathrm{H})$ & $6.76(\mathrm{~s}, 2 \mathrm{H})$ & 0.01 & 153.8 & 153.9 & 0.1 \\
\hline $6.42(\mathrm{~s}, 2 \mathrm{H})$ & $6.43(\mathrm{~s}, 2 \mathrm{H})$ & 0.01 & 149.4 & 149.5 & 0.1 \\
\hline $6.33(\mathrm{~s}, 2 \mathrm{H})$ & $\mathbf{6 . 3 4}(\mathrm{s}, 2 \mathrm{H})$ & 0.01 & 149.1 & 149.2 & 0.1 \\
\hline $\begin{array}{c}5.09-4.99(\mathrm{~m}, \\
2 \mathrm{H})\end{array}$ & $\begin{array}{c}\mathbf{5 . 0 5}(\mathrm{dt}, J= \\
13.2,3.8 \mathrm{~Hz} \\
2 \mathrm{H})\end{array}$ & & 137.0 & 137.0 & 0 \\
\hline $4.06(s, 6 H)$ & $4.06(\mathrm{~s}, 6 \mathrm{H})$ & 0 & 133.6 & 133.6 & 0 \\
\hline $3.82(\mathrm{~s}, 6 \mathrm{H})$ & $3.82(\mathrm{~s}, 6 \mathrm{H})$ & 0 & 131.3 & 131.3 & 0 \\
\hline $3.62(\mathrm{~s}, 6 \mathrm{H})$ & $3.62(\mathrm{~s}, 6 \mathrm{H})$ & 0 & 122.5 & 122.5 & 0 \\
\hline $\begin{array}{c}3.40-3.27(\mathrm{~m}, \\
2 \mathrm{H})\end{array}$ & $\begin{array}{c}3.33(\mathrm{td}, J= \\
13.2,3.0 \mathrm{~Hz} \\
2 \mathrm{H})\end{array}$ & & 119.0 & 119.0 & 0 \\
\hline $3.02(\mathrm{~s}, 6 \mathrm{H})$ & $\mathbf{3 . 0 3}(\mathrm{s}, 6 \mathrm{H})$ & 0.01 & 111.5 & 111.6 & 0.1 \\
\hline $\begin{array}{c}2.57-2.47(\mathrm{~m} \\
2 \mathrm{H})\end{array}$ & $\begin{array}{c}2.52(\mathrm{dt}, J= \\
15.1,3.1 \mathrm{~Hz} \\
2 \mathrm{H})\end{array}$ & & 110.8 & 110.8 & 0 \\
\hline $\begin{array}{c}2.19-2.07(m, \\
2 \mathrm{H})\end{array}$ & $\begin{array}{c}\mathbf{2 . 1 3}(\mathrm{ddd}, J= \\
14.5,12.7,4.2 \\
\mathrm{~Hz}, 2 \mathrm{H})\end{array}$ & & 109.1 & 109.2 & 0.1 \\
\hline & & & 108.0 & 108.1 & 0.1 \\
\hline & & & 105.8 & 105.8 & 0 \\
\hline
\end{tabular}




\begin{tabular}{|c|c|c|c|c|c|}
\hline & & & $\mathbf{5 6 . 4}$ & $\mathbf{5 6 . 4}$ & 0 \\
\hline & & & $\mathbf{5 6 . 3}$ & $\mathbf{5 6 . 3}$ & 0 \\
\hline & & & $\mathbf{5 6 . 1}$ & $\mathbf{5 6 . 2}$ & 0.1 \\
\hline & & & $\mathbf{5 5 . 2}$ & $\mathbf{5 5 . 2}$ & 0 \\
\hline & & & $\mathbf{4 1 . 4}$ & $\mathbf{4 1 . 4}$ & 0 \\
\hline
\end{tabular}




\subsubsection{Commercially available amines}<smiles>NCCc1c[nH]c2ccccc12</smiles>

S24<smiles>COc1ccc(CCN)cc1OC</smiles><smiles>COc1ccc2[nH]cc(CCN)c2c1</smiles>

S25<smiles>NCCc1ccccn1</smiles>

S29<smiles>NCCc1ccccc1</smiles>

S26<smiles>COc1ccc(CCN)cc1</smiles>

S27

2.7.2. Noncommercially available amines synthesis by reported methods

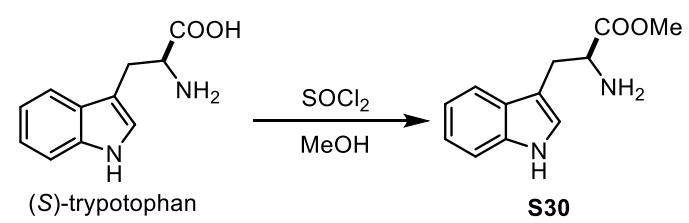

J. Am. Chem. Soc. 2014, 136, 16756

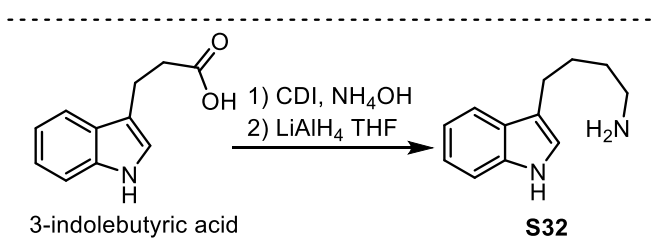

J. Med. Chem. 2004, 47, 3823

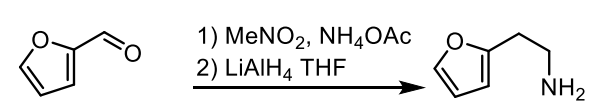

2-furancarboxaldehyde \$34

ACS Catal. 2019, 6, 2610

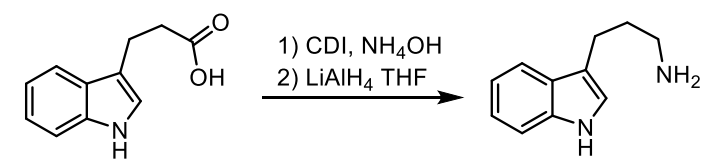

3-indolepropionic acid

S31

J. Med. Chem. 2018, 61, 4004

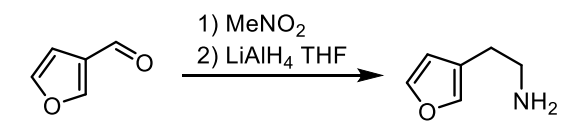

3-furancarboxaldehyde

S33

J. Med. Chem. 2020, 63, 7569

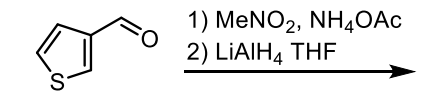

3-thiophencarboxaldehyde

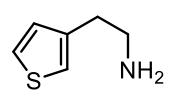

S35

MedChemComm. 2015, 6, 1679

S30 was prepared from $(S)$-trypotophan by the procedure of Carreira et al.. ${ }^{[9]} \mathbf{S 3 1}$ was synthesized from 3-indolepropionic acid according to the procedure of Weinstock et al.. ${ }^{[10]}$ S32 was obtained from 3-indolebutyric acid following the procedure of Mewshaw et al.. ${ }^{[11]} \mathbf{S 3 3}$ was prepared from 3-furancarboxaldehyde via the procedure of Liotta et al.. ${ }^{[12]} \mathbf{S 3 4}$ was prepared from 2-furancarboxaldehyde through the procedure

[9] J.Ruchti, E. M. Carreira J. Am. Chem. Soc. 2014, 136, 16756-16759.

[10] S. Zeeli, T. Weill, E. Finkin-Groner, C. Bejar, M. Melamed, S. Furman, M. Zhenin, A. Nudelman, M. Weinstock J. Med. Chem. 2018, 61, 4004-4019.

[11] R. E. Mewshaw, D. Zhou, P. Zhou, X. Shi, G. Hornby, T. Spangler, R. Scerni, D. Smith, L. E. Schechter, T. H. Andree $J$. Med. Chem. 2004, 47, 3823-3842.

[12] M. P. Epplin, A. Mohan, L. D. Harris, Z. Zhu, K. L. Strong, J. Bacsa, P. Le, D. S. Menaldino, S. F. Traynelis, D. 
of Huang et al.. ${ }^{[13]}$ S35 was prepared from 3-thiophencarboxaldehyde using the procedure of Enzensperger et al.. ${ }^{[14]}$ The spectroscopic data are in agreement with literatures.

\subsubsection{Self-made amines}

The amines (22 and S36-S38) were synthesized from the corresponding heterocycles. The preparation of the aldehydes depended on the starting material supply. For 22, S36 and S37, the aldehydes were synthesized from the corresponding indoles through a Vilsmeier-Haack reaction. For S39, the aldehydes were prepared via bromination and formylation. The Henry reaction with $\mathrm{MeNO}_{2}$ and the reduction by $\mathrm{LiAlH}_{4}$ followed the same procedure.

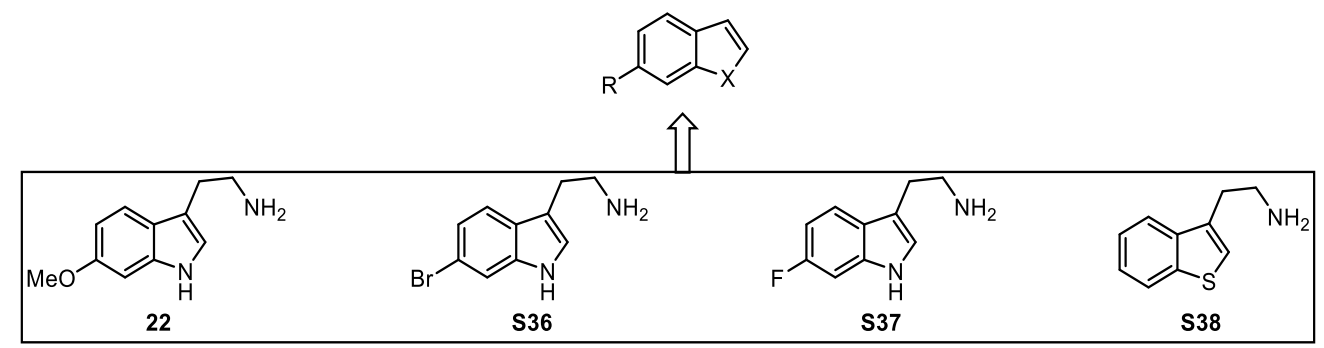

\section{General procedure $\mathbf{F}$}

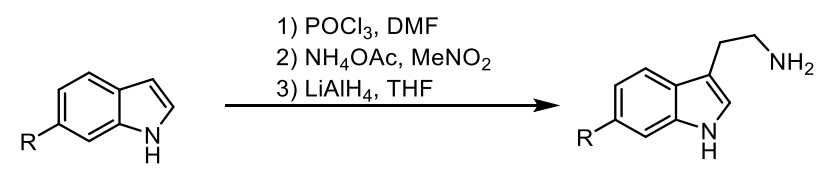

Vilsmeier-Haack reaction: To a dried Schlenk flask with DMF, was added $\mathrm{POCl}_{3}(3.0$ equiv.) dropwise at $0{ }^{\circ} \mathrm{C}$. After the mixture was stirred for $30 \mathrm{~min}$, a solution of corresponding indole (1.0 equiv.) in DMF was added to the reaction mixture dropwise. The resulting mixture was allowed to stir at room temperature for another $2 \mathrm{~h}$. Then the reaction mixture was poured to a flask with ice, and carefully neutralized by $\mathrm{NaHCO}_{3}$ (sat. aq.) until $\mathrm{pH}=7-8$. The suspension was left to rest overnight at room temperature,

C. Liotta J. Med. Chem. 2020, 63, 7569-7600.

[13] J. Liu, P. Chakraborty, H. Zhang, L. Zhong, Z. Wang, X. Huang ACS Catal. 2019, 9, 2610-2617.

[14] M. A. O. Abdel-Fattah, A. H. Abadi, J. Lehmann, P. M. Schweikerta, C. Enzensperger Med. Chem. Commun., 2015, 6, 1679-1686. 
filtered, collected the solid and dried under reduced pressure. This crude product was used without further purification.

Henry reaction: A flask with $\mathrm{NH}_{4} \mathrm{OAc}$ (3.0 equiv.) and corresponding aldehyde (1.0 equiv.) was added $\mathrm{MeNO}_{2}$ (75 equiv.) and the reaction mixture was stirred at $115^{\circ} \mathrm{C}$ for 2h. After allowing the mixture to cool down to room temperature, the reaction mixture was diluted with water and DCM, and the organic phase was separated. The aqueous phase was extracted with DCM. The combined organic layers were washed with brine, dried over $\mathrm{MgSO}_{4}$, filtered, and concentrated under reduced pressure to afford the crude product which was used directly without purification.

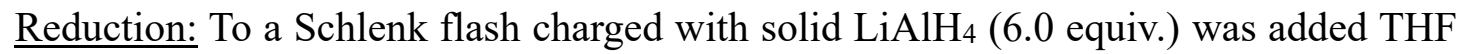
carefully at $0{ }^{\circ} \mathrm{C}$. Then a solution of the crude product of the Henry reaction in THF was added dropwise. The resulting reaction mixture was replaced with an oil bath and heated to $65{ }^{\circ} \mathrm{C}$ for $3 \mathrm{~h}$, then cooled down to $0{ }^{\circ} \mathrm{C}$. Excess $\mathrm{LiAlH}_{4}$ was quenched by the drop-wise addition of $\mathrm{NaOH}$ (aq.) and $\mathrm{H}_{2} \mathrm{O}$. The suspension was allowed to warm to room temperature overnight and then filtered through a plug of Celite ${ }^{\circledR}$. The organic phase was separated. The aqueous phase was extracted with DCM and the combined organic layers were washed with brine, dried over $\mathrm{MgSO}_{4}$, filtered, and concentrated under reduced pressure. The crude material was purified by silica gel column chromatography.

2-(6-Methoxy-1H-indol-3-yl)ethan-1-amine (22).

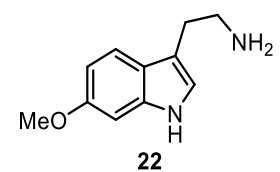

22 was prepared according to General procedure F, starting from 6-methoxyindole (530 mg, 3.60 mmol, 1.0 equiv.) and $\mathrm{POCl}_{3}(1.01 \mathrm{~mL}, 10.8 \mathrm{mmol}, 3.0$ equiv.) in DMF $(3.0 \mathrm{~mL})$. The reaction mixture was subsequently treated with $\mathrm{NH}_{4} \mathrm{OAc}(833 \mathrm{mg}$, 10.8 mmol, 3.0 equiv.), $\mathrm{MeNO}_{2}$ (14.5 mL, 270 mmol, 75 equiv.) and $\mathrm{LiAlH}_{4}(821 \mathrm{mg}$, 10.8 mmol, 6.0 equiv.) in THF $(25 \mathrm{~mL})$. Purification by silica gel column chromatography $(\mathrm{DCM} / \mathrm{MeOH} / \mathrm{TEA}=80: 20: 5)$ afforded $22(270 \mathrm{mg}, 1.42 \mathrm{mmol}, 39 \%)$ 
as a light brown solid.

${ }^{1} \mathbf{H}$ NMR $\left(500 \mathrm{MHz}, \mathrm{CD}_{3} \mathrm{OD}\right) \delta[\mathrm{ppm}]=7.37(\mathrm{~d}, J=8.6 \mathrm{~Hz}, 1 \mathrm{H}), 6.91(\mathrm{~s}, 1 \mathrm{H}), 6.87(\mathrm{~d}$, $J=2.3 \mathrm{~Hz}, 1 \mathrm{H}), 6.67(\mathrm{dd}, J=8.6,2.3 \mathrm{~Hz}, 1 \mathrm{H}), 3.76(\mathrm{~s}, 3 \mathrm{H}), 2.87(\mathrm{t}, J=6.8 \mathrm{~Hz}, 2 \mathrm{H})$, $2.81(\mathrm{t}, J=6.7 \mathrm{~Hz}, 2 \mathrm{H})$.

${ }^{13} \mathrm{C}$ NMR $\left(126 \mathrm{MHz}, \mathrm{CD}_{3} \mathrm{OD}\right) \delta[\mathrm{ppm}]=157.6,138.9,123.2,122.3,119.9,113.3$, $109.9,95.6,56.0,42.9,29.4$.

HRMS-ESI: calcd. for $\mathrm{C}_{11} \mathrm{H}_{15} \mathrm{~N}_{2} \mathrm{O}[\mathrm{M}+\mathrm{H}]^{+}:$191.1179; found: 191.1179.

FT-IR: $v\left[\mathrm{~cm}^{-1}\right]=3401,2924,2834,1627,1456,1305,1262,1200,1160,1026,800$, 753.

2-(6-Fluoro-1H-indol-3-yl)ethan-1-amine (S36).

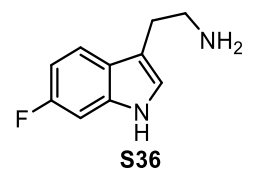

S36 was prepared according to General procedure F, starting from 6-fluoroindole (1.70 g, 12.6 mmol, 1.0 equiv.) and $\mathrm{POCl}_{3}(3.53 \mathrm{~mL}, 37.7 \mathrm{mmol}, 3.0$ equiv.) in DMF $(10.0 \mathrm{~mL})$. The reaction mixture was subsequently treated with $\mathrm{NH}_{4} \mathrm{OAc}(2.91 \mathrm{~g}$, 37.8 mmol, 3.0 equiv.), $\mathrm{MeNO}_{2}\left(50.5 \mathrm{~mL}, 944 \mathrm{mmol}, 75\right.$ equiv.) and $\mathrm{LiAlH}_{4}(2.86 \mathrm{~g}$, 75.5 mmol, 6.0 equiv. $)$ in THF $(80.0 \mathrm{~mL})$. Purification by silica gel column chromatography $(\mathrm{DCM} / \mathrm{MeOH} / \mathrm{TEA}=80: 20: 5)$ afforded S36 (580 mg, $3.26 \mathrm{mmol}$, $26 \%)$ as a brown solid.

${ }^{1} \mathbf{H}$ NMR $\left(500 \mathrm{MHz}, \mathrm{CD}_{3} \mathrm{OD}\right) \delta[\mathrm{ppm}]=7.50(\mathrm{dd}, J=8.7,5.3 \mathrm{~Hz}, 1 \mathrm{H}), 7.13(\mathrm{~s}, 1 \mathrm{H})$, $7.06(\mathrm{dd}, J=9.9,2.3 \mathrm{~Hz}, 1 \mathrm{H}), 6.86-6.77(\mathrm{~m}, 1 \mathrm{H}), 3.11(\mathrm{dd}, J=7.9,6.4 \mathrm{~Hz}, 2 \mathrm{H}), 3.02$ ( $\mathrm{t}, J=7.2 \mathrm{~Hz}, 2 \mathrm{H})$.

${ }^{13} \mathrm{C}$ NMR $\left(126 \mathrm{MHz}, \mathrm{CD}_{3} \mathrm{OD}\right) \delta[\mathrm{ppm}]=161.3(\mathrm{~d}, J=235.3 \mathrm{~Hz}), 138.2(\mathrm{~d}, J=12.4$ $\mathrm{Hz}), 125.1,124.5$ (d, $J=3.3 \mathrm{~Hz}), 119.9$ (d, $J=10.2 \mathrm{~Hz}), 111.7,108.3$ (d, $J=24.9 \mathrm{~Hz})$, $98.3(\mathrm{~d}, J=26.0 \mathrm{~Hz}), 41.8,26.2$.

${ }^{19}$ F NMR $\left(471 \mathrm{MHz}, \mathrm{CD}_{3} \mathrm{OD}\right) \delta[\mathrm{ppm}]=-124.28$.

HRMS-ESI: calcd. for $\mathrm{C}_{10} \mathrm{H}_{12} \mathrm{FN}_{2}[\mathrm{M}+\mathrm{H}]^{+}$: 179.0979; found: 179.0979 .

FT-IR: $v\left[\mathrm{~cm}^{-1}\right]=2918,2840,1626,1456,1345,1225,1144,1099,951,799$. 


\section{2-(6-Bromo-1H-indol-3-yl)ethan-1-amine (S37).}

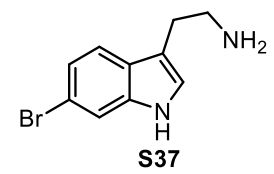

S37 was prepared according to General procedure $\mathbf{F}$, starting from 6-bromoindole (1.0 g, 5.2 mmol, 1.0 equiv.) and $\mathrm{POCl}_{3}(1.4 \mathrm{~mL}, 15 \mathrm{mmol}, 3.0$ equiv.) in DMF (5.0 $\mathrm{mL})$. The reaction mixture was subsequently treated with $\mathrm{NH}_{4} \mathrm{OAc}(1.2 \mathrm{~g}, 15 \mathrm{mmol}$, 3.0 equiv.), $\mathrm{MeNO}_{2}\left(21 \mathrm{~mL}, 386 \mathrm{mmol}, 75\right.$ equiv.) and $\mathrm{LiAlH}_{4}(1.2 \mathrm{~g}, 31 \mathrm{mmol}, 6.0$ equiv.) in THF $(50 \mathrm{~mL})$. Purification by silica gel column chromatography $(\mathrm{DCM} / \mathrm{MeOH} / \mathrm{TEA}=80: 20: 5)$ afforded S37 $(580 \mathrm{mg}, 3.26 \mathrm{mmol}, 63 \%)$ as a light brown solid.

${ }^{1} \mathbf{H}$ NMR $\left(500 \mathrm{MHz}, \mathrm{CD}_{3} \mathrm{OD}\right) \delta[\mathrm{ppm}]=7.50(\mathrm{~d}, J=1.7 \mathrm{~Hz}, 1 \mathrm{H}), 7.45(\mathrm{~d}, J=8.4 \mathrm{~Hz}$, 1H), $7.10(\mathrm{dd}, J=8.4,1.8 \mathrm{~Hz}, 1 \mathrm{H}), 7.08$ (d, $J=0.8 \mathrm{~Hz}, 1 \mathrm{H}), 2.95-2.90(\mathrm{~m}, 2 \mathrm{H}), 2.90$ $-2.85(\mathrm{~m}, 2 \mathrm{H})$.

${ }^{13} \mathrm{C}$ NMR (126 MHz, CD 3 OD) $\delta[\mathrm{ppm}]=139.0,127.7,124.5,122.7,120.7,115.8$, $115.1,113.7,43.0,29.1$

HRMS-ESI: calcd. for $\mathrm{C}_{10} \mathrm{H}_{12} \mathrm{BrN}_{2}[\mathrm{M}+\mathrm{H}]^{+}$: 239.0179; found: 239.0191.

FT-IR: $v\left[\mathrm{~cm}^{-1}\right]=3423,3126,2935,2874,1613,1578,1456,1334,1232,1048,894$, 802,771 .

\section{2-(Benzo[b]thiophen-3-yl)ethan-1-amine (S38).}

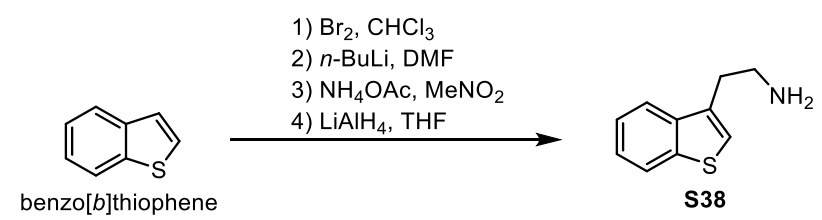

Bromination: ${ }^{[15]}$ Benzo[b]thiophene (2.0 g, $15 \mathrm{mmol}, 1.0$ equiv.) was dissolved in chloroform $(30 \mathrm{~mL})$ and cooled by an ice bath. A solution of bromine $(0.84 \mathrm{~mL}, 16$ mmol, 1.1 equiv. $)$ in chloroform $(10 \mathrm{~mL})$ was slowly added. The reaction mixture was stirred for $1 \mathrm{~h}$ and quenched by $\mathrm{NaOH}\left(1.0 \mathrm{M}\right.$ aq.) and $\mathrm{Na}_{2} \mathrm{~S}_{2} \mathrm{O}_{3}$ (sat. aq.). The resulting mixture was extracted with DCM. After evaporation of the solvent, the intermediate 
was dissolved in ethanol and was added dropwise to a solution of $\mathrm{KOH}$ (sat. EtOH solution, $20 \mathrm{~mL}$ ) was added at ice bath. After refluxing for $2 \mathrm{~h}, \mathrm{H}_{2} \mathrm{O}$ was added and the EtOH was evaporated. The residue was extracted by DCM, washed with brine, dried with $\mathrm{MgSO}_{4}$, filtered and concentrated under reduced pressure to afford crude 3-bromobenzo $[b]$ thiophene as yellow oil. The crude product was used without purification.

Formylation procedure: ${ }^{[16]}$ Crude 3-bromo-benzo[b]thiophene (3.4 g, 16 mmol, 1.0 equiv.) was dissolved in $\mathrm{Et}_{2} \mathrm{O}$ and the solution was cooled to $-78^{\circ} \mathrm{C}$. After the dropwise addition $n \operatorname{BuLi}(2.5 \mathrm{M}$ solution in hexane, $6.4 \mathrm{~mL}, 16 \mathrm{mmol}, 1.0$ equiv.), the mixture was stirred for $30 \mathrm{~min}$. Following the addition of DMF (1.2 mL, $16 \mathrm{mmol}, 1.0$ equiv.) at $-78^{\circ} \mathrm{C}$, the mixture was allowed to warm to room temperature for $1 \mathrm{~h}$. The reaction mixture was quenched by $\mathrm{NH}_{4} \mathrm{Cl}$ (sat. aq.) and extracted with DCM. Combined organic layers were dried over $\mathrm{MgSO}_{4}$, filtered and concentrated under reduced pressure to afford crude 3 - benzo[b]thiophene-3-carbaldehyde as yellow oil..

Henry reaction: A flask with $\mathrm{NH}_{4} \mathrm{OAc}$ (2.3 g, 30 mmol, 3.0 equiv.) and crude 3benzo[b]thiophene-3-carbaldehyde (1.6 g, 9.9 mmol, 1.0 equiv.) was added $\mathrm{MeNO}_{2}$ $(40 \mathrm{~mL})$ and the reaction mixture was stirred at $115^{\circ} \mathrm{C}$ for $2 \mathrm{~h}$. After cooled to room temperature, the reaction mixture was diluted with water and DCM, and the organic phase was separated. The aqueous phase was extracted with DCM and the combined organic layers were washed with brine, dried over $\mathrm{MgSO}_{4}$, filtered and concentrated under reduced pressure to afford the crude 3-(2-nitrovinyl)benzo[b]thiophene which could use directly without purification.

Reduction: To a Schlenk flash charged with solid $\mathrm{LiAlH}_{4}$ (2.2 g, 59 mmol, 6.0 equiv.), was added THF carefully at $0{ }^{\circ} \mathrm{C}$. Then a solution of crude 3-(2nitrovinyl)benzo[b]thiophene in THF $(20 \mathrm{~mL})$ was added dropwise. The resulting reaction mixture was replaced with an oil bath and heated to $65^{\circ} \mathrm{C}$ for $3 \mathrm{~h}$, then cooled first to room temperature and then to $0{ }^{\circ} \mathrm{C}$. Excess $\mathrm{LiAlH}_{4}$ was quenched by the dropwise addition of $\mathrm{NaOH}$ (sat. aq.) and $\mathrm{H}_{2} \mathrm{O}$. The suspension was allowed to warm to room temperature overnight and then filtered through a plug of Celite ${ }^{\circledR}$. The organic 
phase was separated. The aqueous phase was extracted with DCM and the combined organic layers were washed with brine, dried over $\mathrm{MgSO}_{4}$, filtered, and concentrated under reduced pressure. The crude material was purified by silica gel column chromatography (DCM/MeOH/TEA = 80:20:5) afforded S38 (300 mg, 1.69 mmol, 11\% over 4 steps) as a dark brown solid.

${ }^{1} \mathbf{H}$ NMR $\left(500 \mathrm{MHz}, \mathrm{CD}_{3} \mathrm{OD}: \mathrm{CDCl}_{3}(\mathrm{v} / \mathrm{v}=1: 1)\right) \delta[\mathrm{ppm}]=7.54(\mathrm{dt}, J=7.9,1.1 \mathrm{~Hz}$, 1H), 7.46 (dt, $J=8.0,0.9 \mathrm{~Hz}, 1 \mathrm{H}), 7.07$ (ddd, $J=8.0,7.0,1.3 \mathrm{~Hz}, 1 \mathrm{H}), 7.03$ (ddd, $J=$ 8.3, 7.3, 1.3 Hz, 1H), $6.92(\mathrm{~s}, 1 \mathrm{H}), 2.75(\mathrm{~s}, 4 \mathrm{H})$.

${ }^{13} \mathbf{C ~ N M R}\left(126 \mathrm{MHz}, \mathrm{CD}_{3} \mathrm{OD}: \mathrm{CDCl}_{3}(\mathrm{v} / \mathrm{v}=1: 1)\right) \delta[\mathrm{ppm}]=140.2,138.2,132.5,123.9$, $123.6,122.4,122.3,121.0,40.0,30.1$.

HRMS-ESI: calcd. for $\mathrm{C}_{10} \mathrm{H}_{12} \mathrm{NS}[\mathrm{M}+\mathrm{H}]^{+}:$178.0685; found: 178.0693 .

FT-IR: $v\left[\mathrm{~cm}^{-1}\right]=3057,2924,2864,1585,1458,1427,1020,760,732$. 
2.8. Preparation of anhydrides

Commercially available anhydride

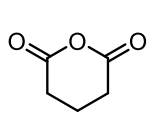

S39<smiles>CC1CC(=O)OC(=O)C1</smiles>

S40<smiles>CC1(C)CC(=O)OC(=O)C1</smiles>

S41<smiles>O=C1Cc2ccccc2C(=O)O1</smiles>

s42

Noncommercially available anhydride<smiles>CC1CCC(=O)OC1=O</smiles>

S43<smiles>O=C1CCCCCC1=O</smiles>

S44<smiles>O=C1CCCCCCC(=O)O1</smiles><smiles>CCCCCCCCC(=O)OC(=O)CCCCCCC</smiles><smiles>COc1cc2c(cc1OC)C(=O)OC(=O)C2</smiles>

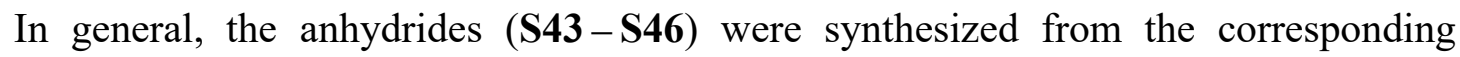
dicarboxylic acids.

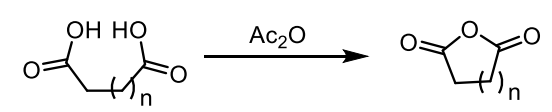

S43 was prepared by the procedure of Wilson et al. ${ }^{[17]}$ S44 was prepared by the procedure of Köper et al. ${ }^{[18]} \mathbf{S 4 5}$ was prepared by the procedure of Sun et al. ${ }^{[19]} \mathbf{S 4 6}$ was prepared by the same procedure as $\mathbf{S 4 5}$.
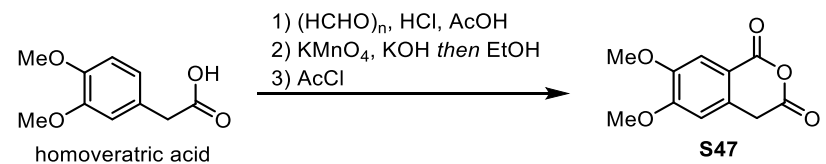

S47 was prepared from homoveratric acid by the procedure of Cushman et al.. ${ }^{[20]}$

[17] D. J. Bennetta, A. J. Blakec, P. A. Cooke, C. R. A. Godfrey, P. L. Pickering, N. S. Simpkins. M. D. Walker, C. Wilson Tetrahedron 2004, 60, 4491-4511.

[18] J. Andersson, J. J. Knobloch, M. V. Perkins, S. A. Holt, I. Köper Langmuir 2017, 33, 4444-4451.

[19] B. Sun, C. Luo, X. Zhang, M. Guo, M. Sun, H. Yu, Q. Chen, W. Yang, M. Wang, S. Zuo, P. Chen, Q. Kan, H. Zhang, Y. Wang, Z. He, J. Sun Nat. Commun. 2019, 10, 3211.

[20] D. E. Beck, K. Agama, C. Marchand, A. Chergui, Y. Pommier, M. Cushman J. Med. Chem. 2014, 57, 14951512 . 


\section{NMR Spectra}

${ }^{1} \mathbf{H}$ NMR $\left(500 \mathrm{MHz}, \mathrm{CDCl}_{3}\right)$ of $\mathbf{8 a}$.

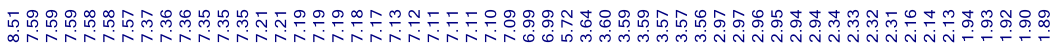

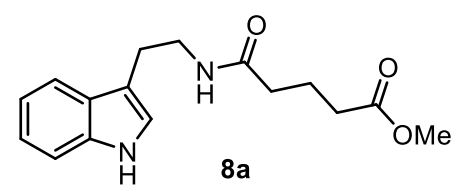

$8 \mathbf{a}$

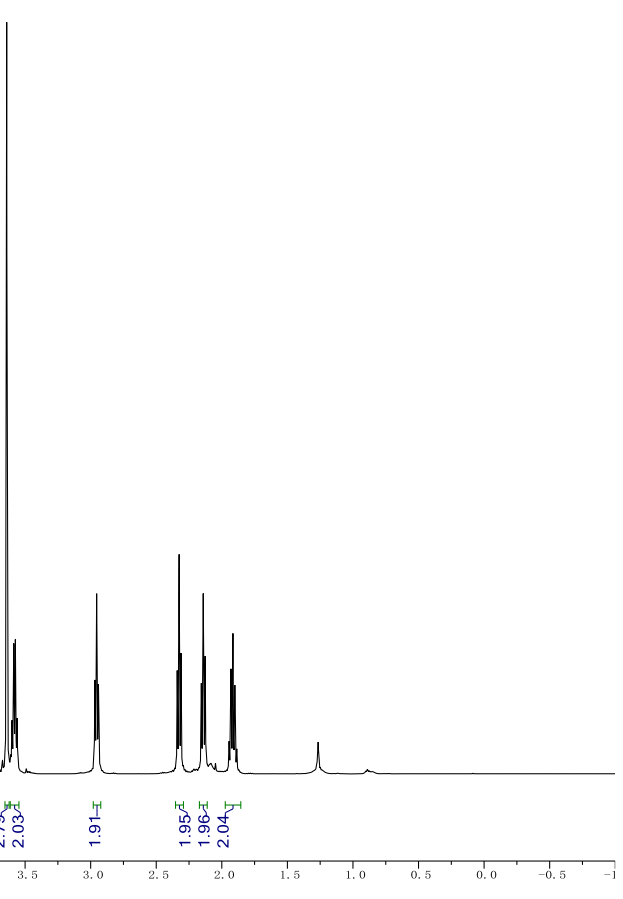

$\left.{ }^{13} \mathbf{C ~ N M R ~ ( 1 2 6 ~ M H z , ~} \mathrm{CDCl}_{3}\right)$ of $\mathbf{8 a}$.

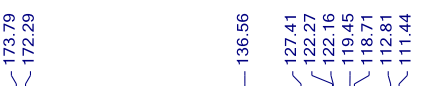

:
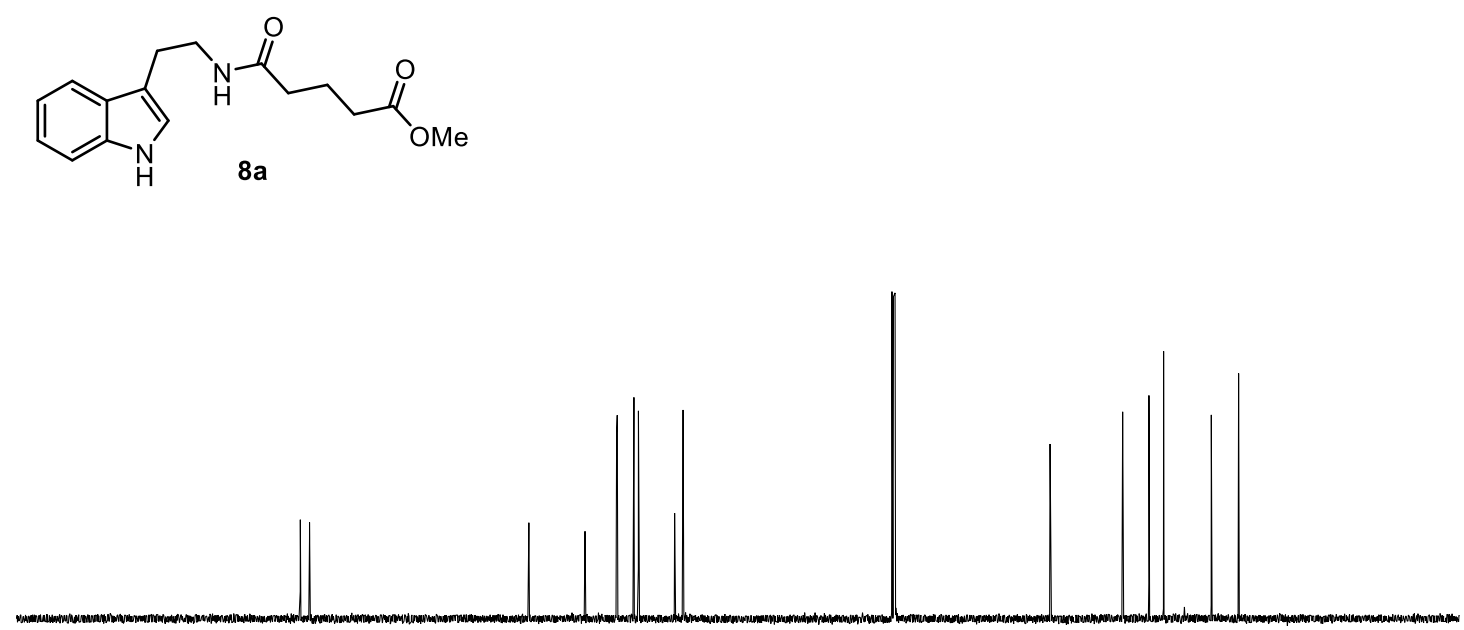

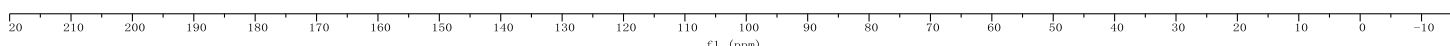


${ }^{1} \mathbf{H}$ NMR (500 MHz, CD 3 OD: $\mathrm{CDCl}_{3}(\mathrm{v} / \mathrm{v}=1: 1)$ ) of $\mathbf{8 b}$.

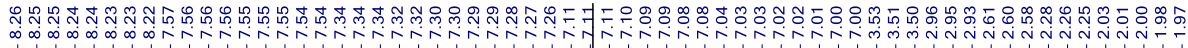<smiles>O=C(CCCC(=O)Oc1ccc([N+](=O)[O-])cc1)NCCc1c[nH]c2ccccc12</smiles>

$8 b$
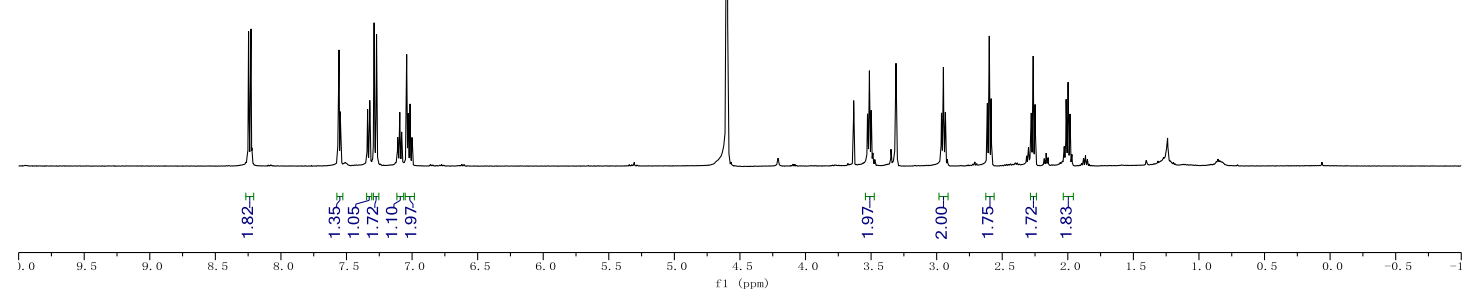

${ }^{13} \mathbf{C}$ NMR (176 MHz, CD $\left.3 \mathrm{OD}: \mathrm{CDCl}_{3}(\mathrm{v} / \mathrm{v}=1: 1)\right) \mathbf{8 b}$.

il<smiles>O=C(CCCC(=O)Oc1ccc([N+](=O)[O-])cc1)NCCc1c[nH]c2ccccc12</smiles>

8b 
${ }^{1} \mathbf{H}$ NMR $\left(500 \mathrm{MHz}, \mathrm{CDCl}_{3}\right)$ of $8 \mathbf{c}$.

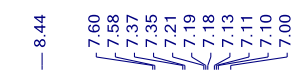

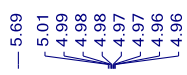
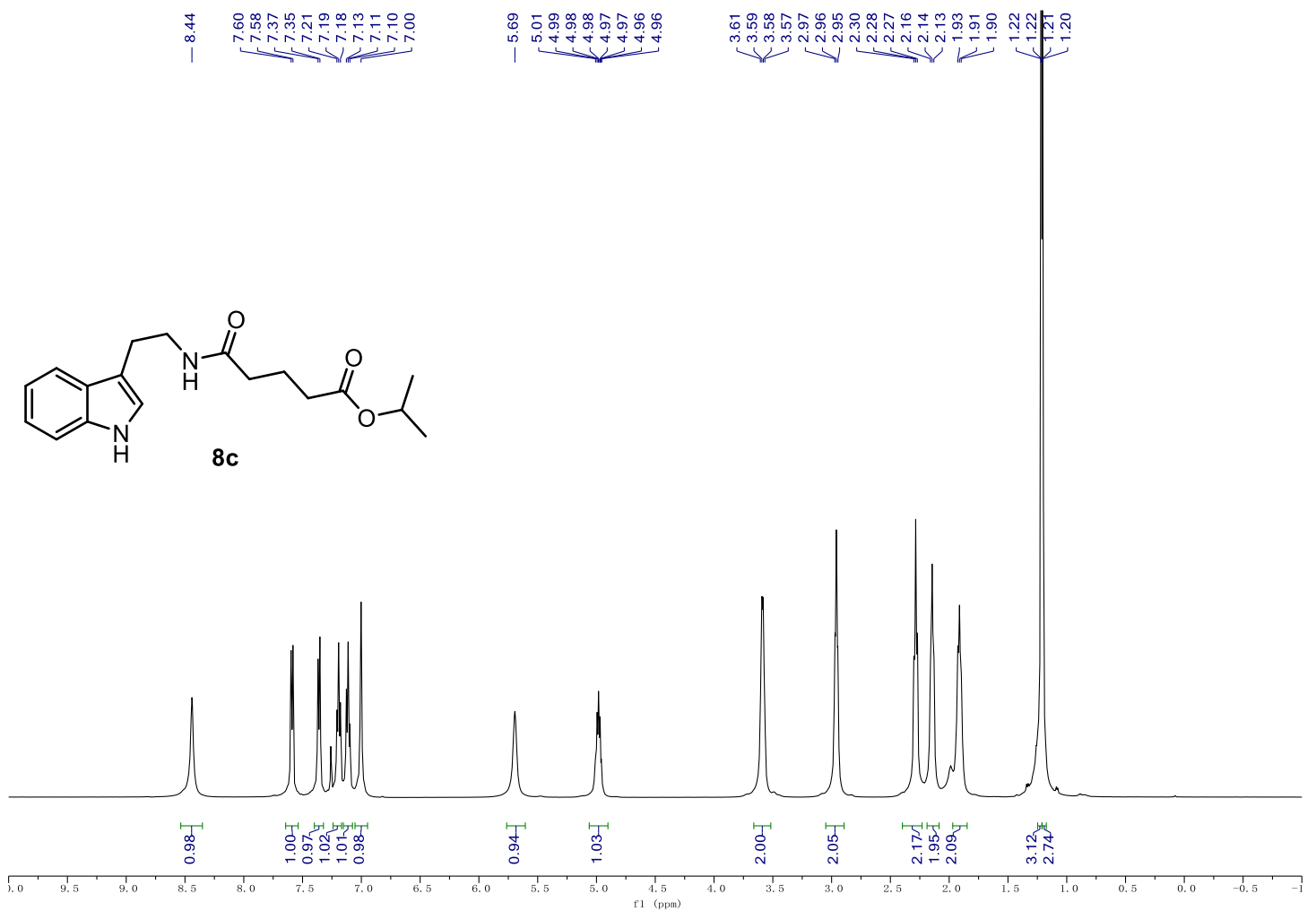

${ }^{13} \mathbf{C ~ N M R}\left(126 \mathrm{MHz}, \mathrm{CDCl}_{3}\right) \mathbf{8 c}$.
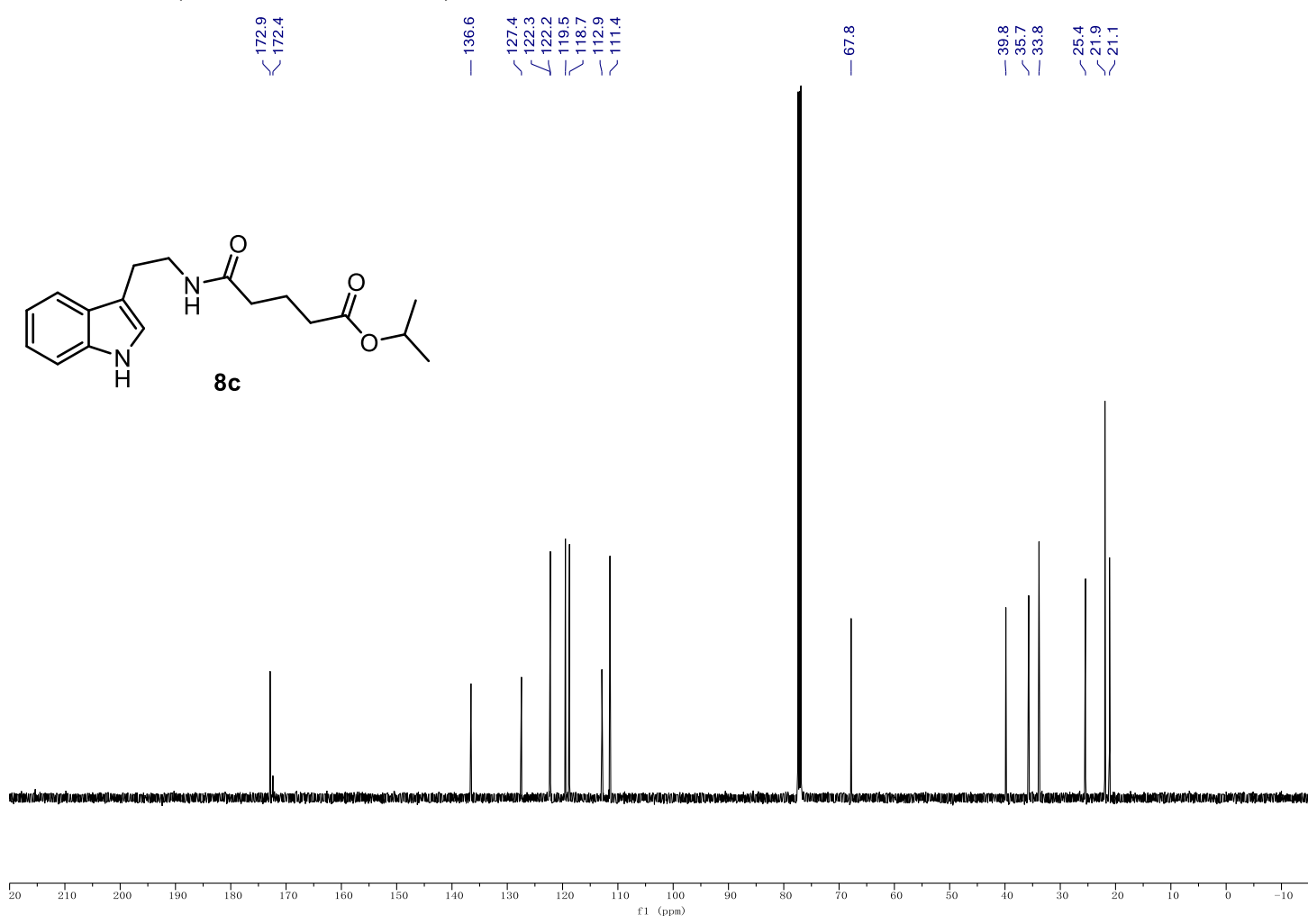

S108 
${ }^{1} \mathbf{H}$ NMR (600 MHz, $\left.\mathrm{CDCl}_{3}\right)$ 8d.

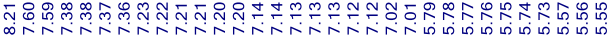

$\underbrace{}_{\mathrm{N}}$

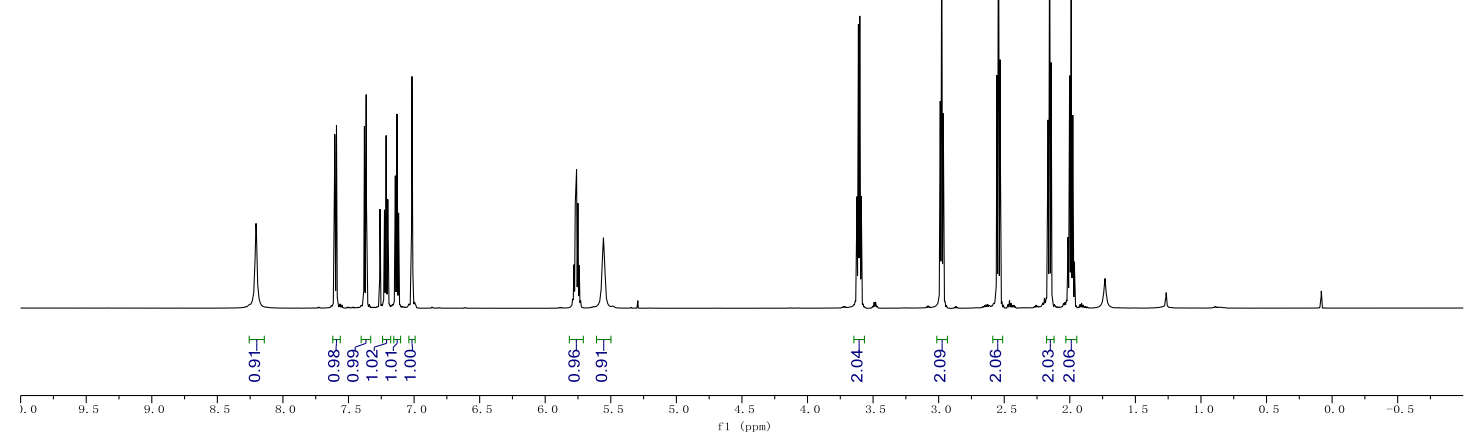

${ }^{13}$ C NMR (151 MHz, $\left.\mathrm{CDCl}_{3}\right)$ 8d.

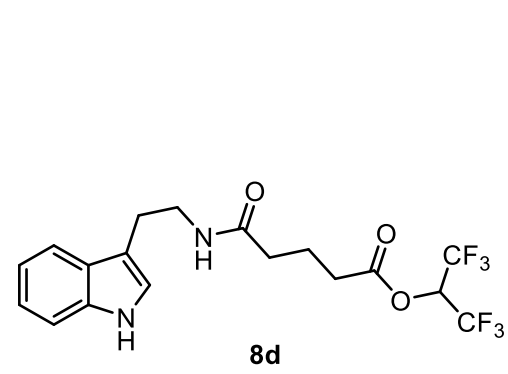

$8 d$
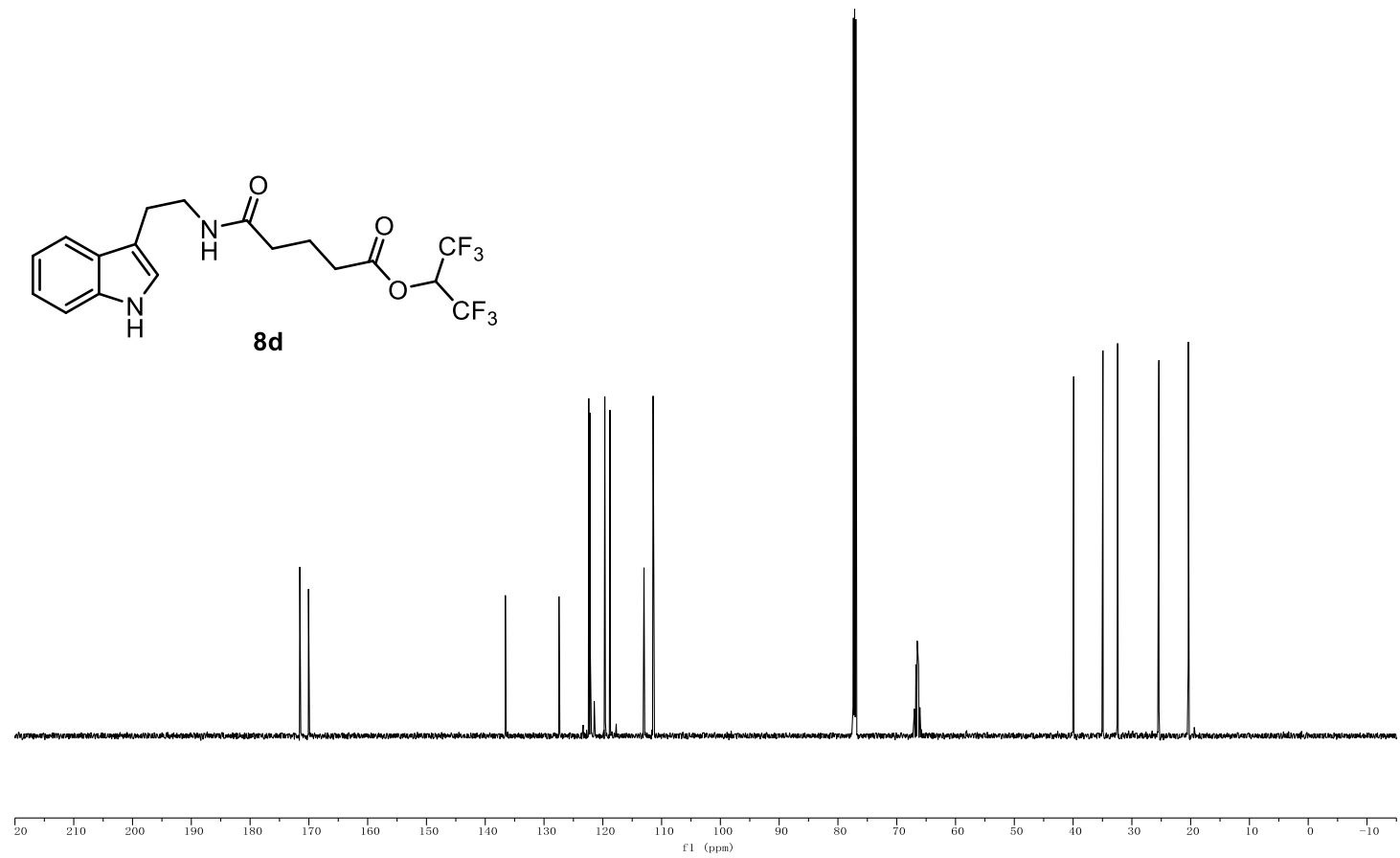
${ }^{19}$ F NMR (565 MHz, $\left.\mathrm{CDCl}_{3}\right)$ 8d.

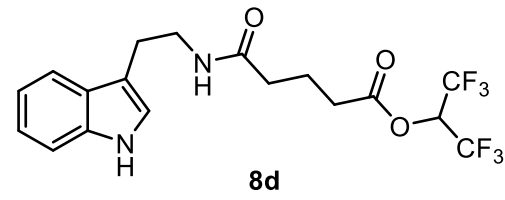

${ }^{1} \mathbf{H}$ NMR (500 MHz, $\left.\mathrm{CDCl}_{3}\right) \mathbf{8 e}$.

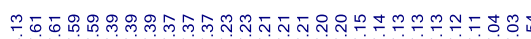
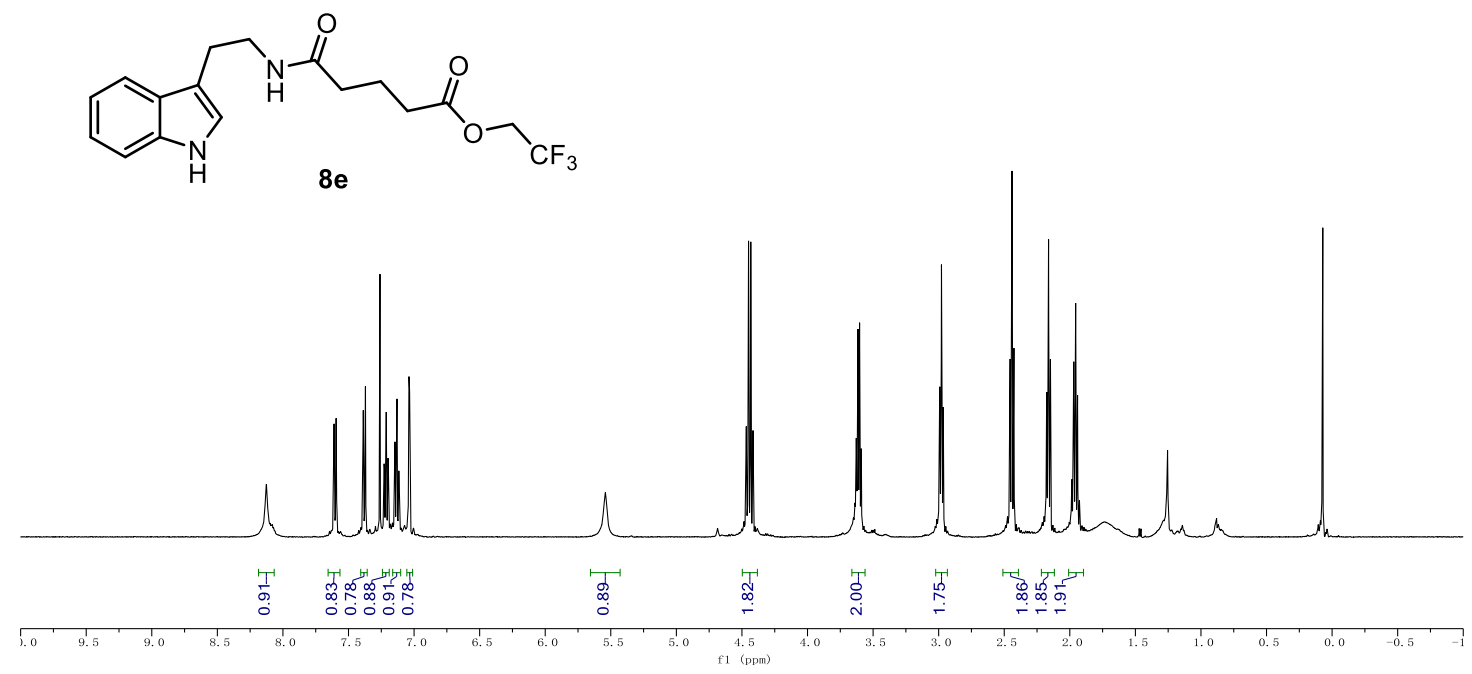
${ }^{13} \mathbf{C ~ N M R}\left(126 \mathrm{MHz}, \mathrm{CDCl}_{3}\right) \mathbf{8 e}$.
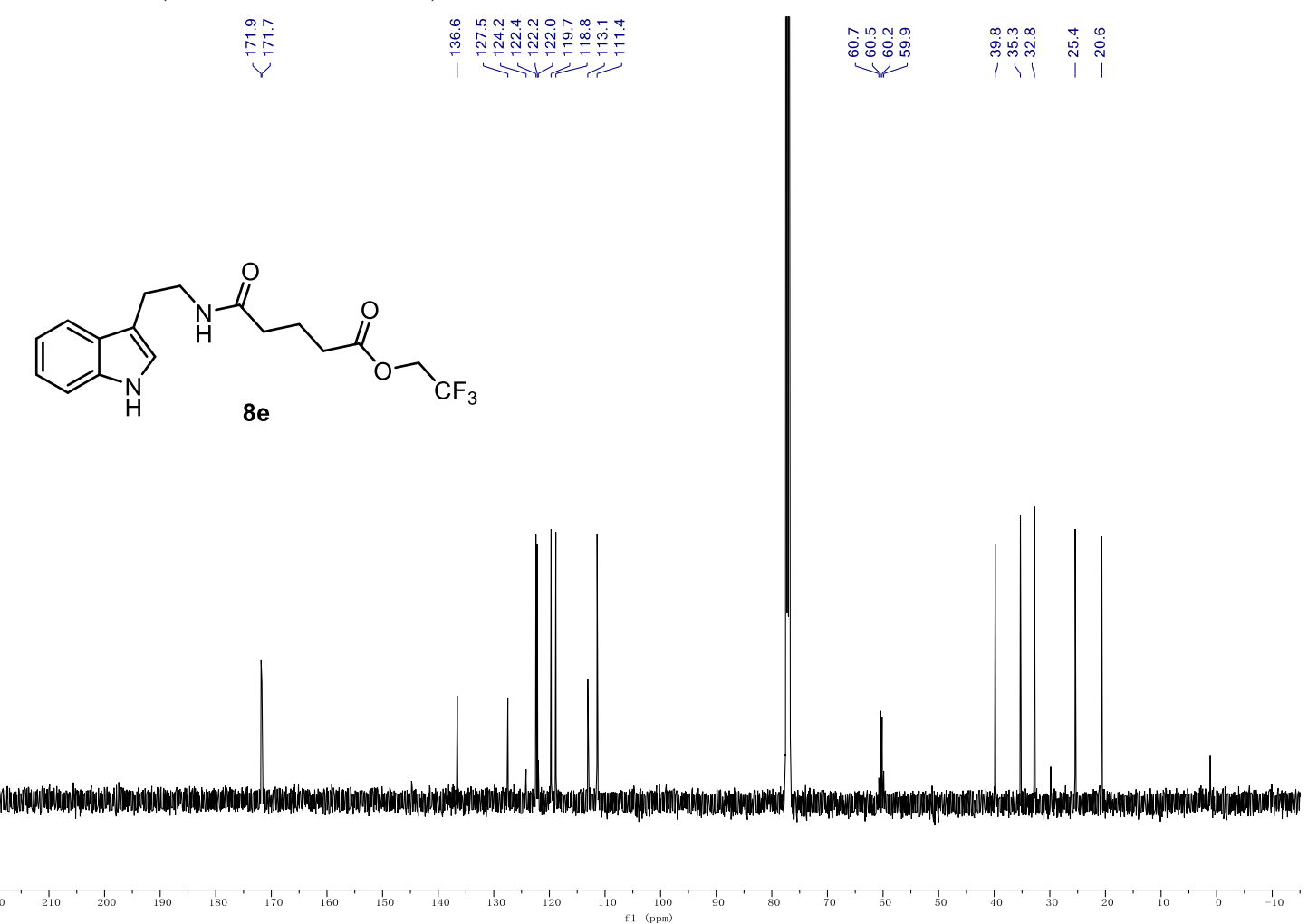

${ }^{19}$ F NMR (471 MHz, $\left.\mathrm{CDCl}_{3}\right) \mathbf{8 e .}$

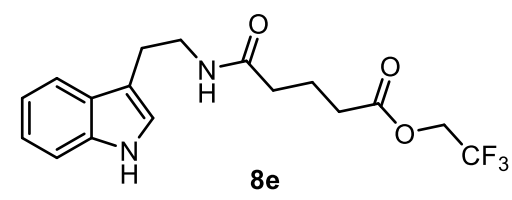


${ }^{1} \mathbf{H}$ NMR (500 MHz, CD 3 OD) 8 f.

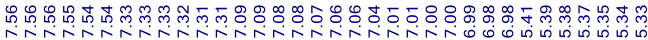

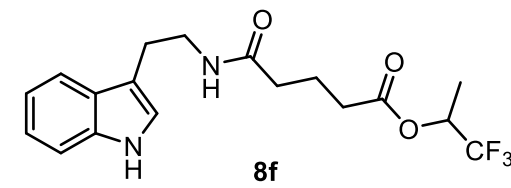

${ }^{13} \mathbf{C}$ NMR (126 MHz, CD $\left.3 \mathrm{OD}\right) \mathbf{8 f}$.
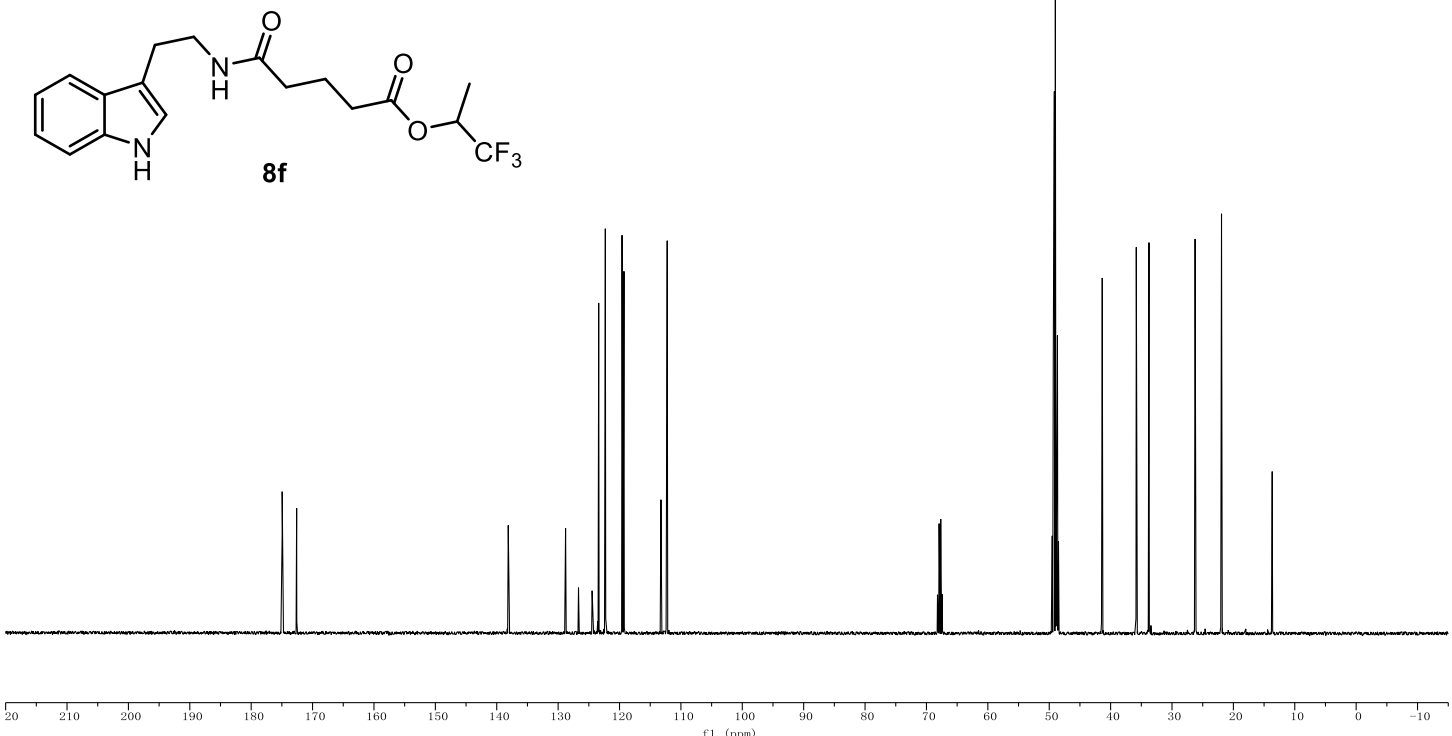
${ }^{19}$ F NMR (471 MHz, CD $\left.3 \mathrm{OD}\right) \mathbf{8 f}$.

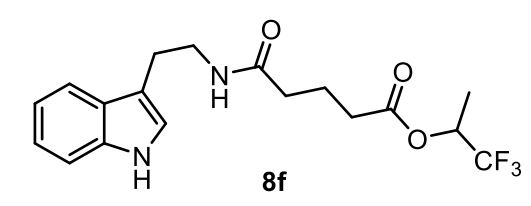

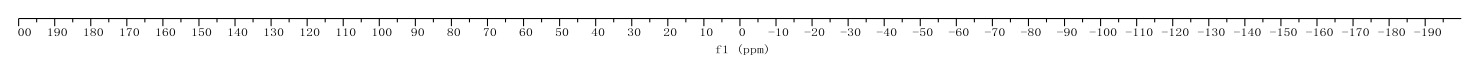

${ }^{1} \mathbf{H}$ NMR (500 MHz, CD3 OD) 8g.

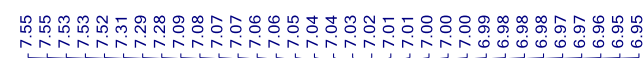

โூ

$\underbrace{m \infty m}$

$\underbrace{C}_{H}$

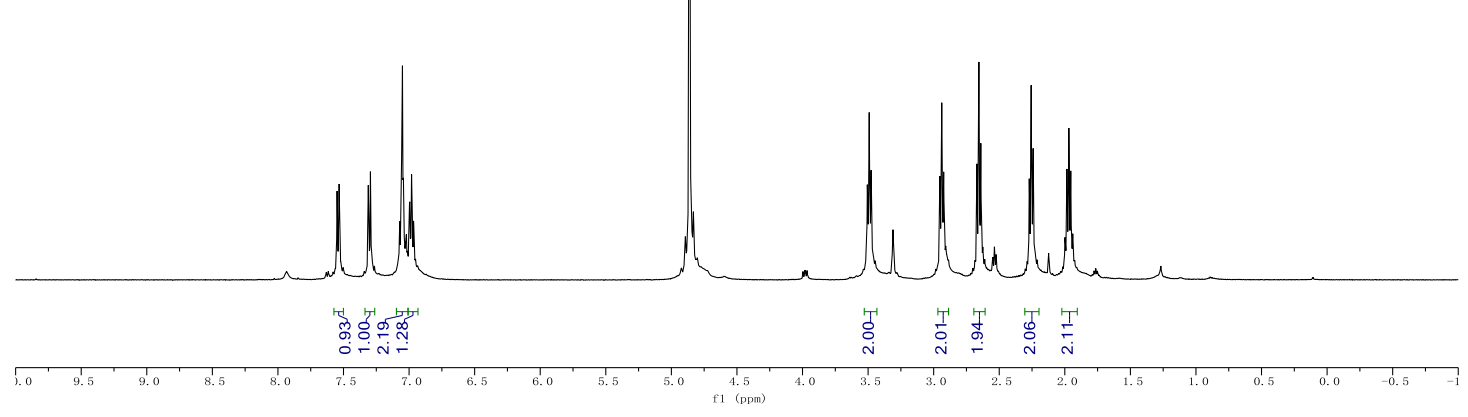


${ }^{\mathbf{1 3}} \mathrm{C}$ NMR (126 MHz, CD $\left.3 \mathrm{OD}\right) \mathbf{8 g}$.

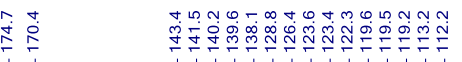

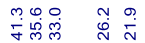

$\underbrace{C}_{H}$
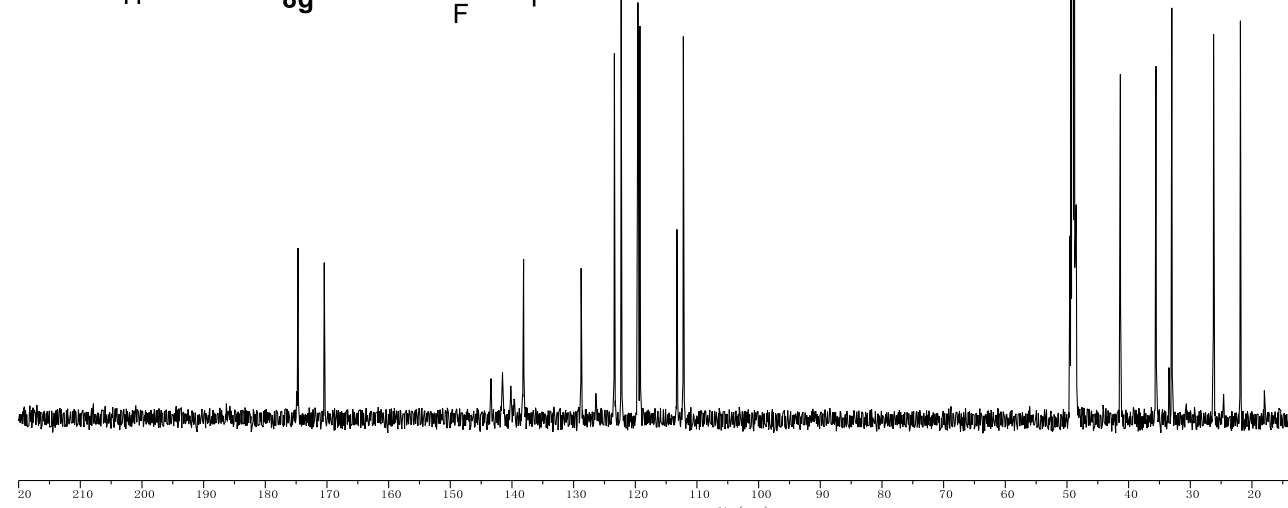

${ }^{19}$ F NMR (471 MHz, CD3OD) 8g.
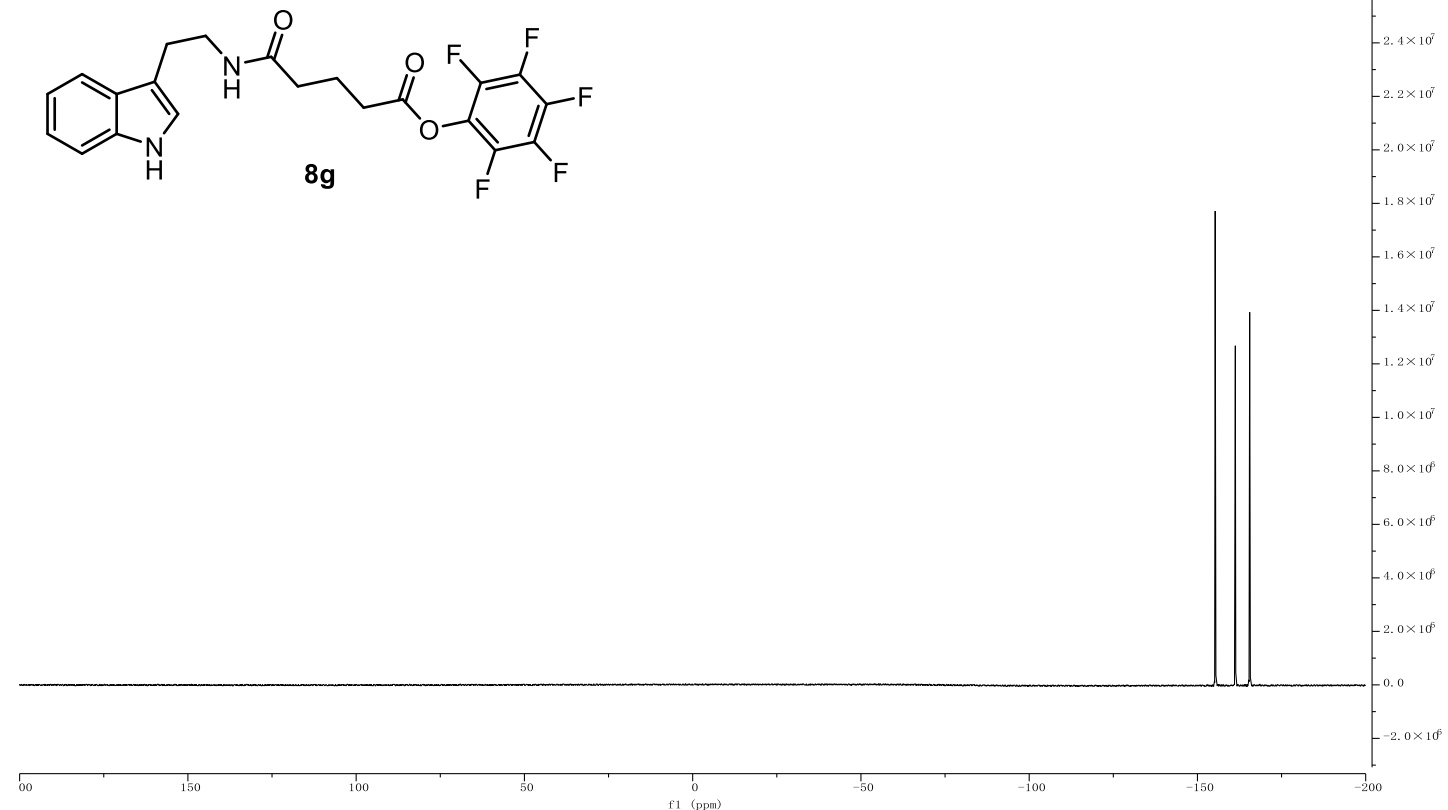
${ }^{\mathbf{1}} \mathbf{H}$ NMR (500 MHz, $\left.\mathrm{CDCl}_{3}\right) \mathbf{8 h}$.

尊

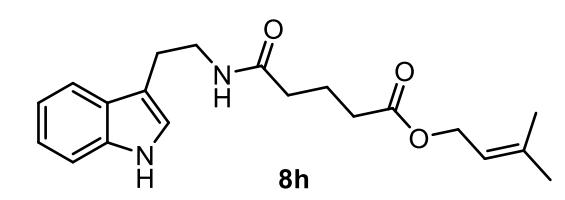

8h

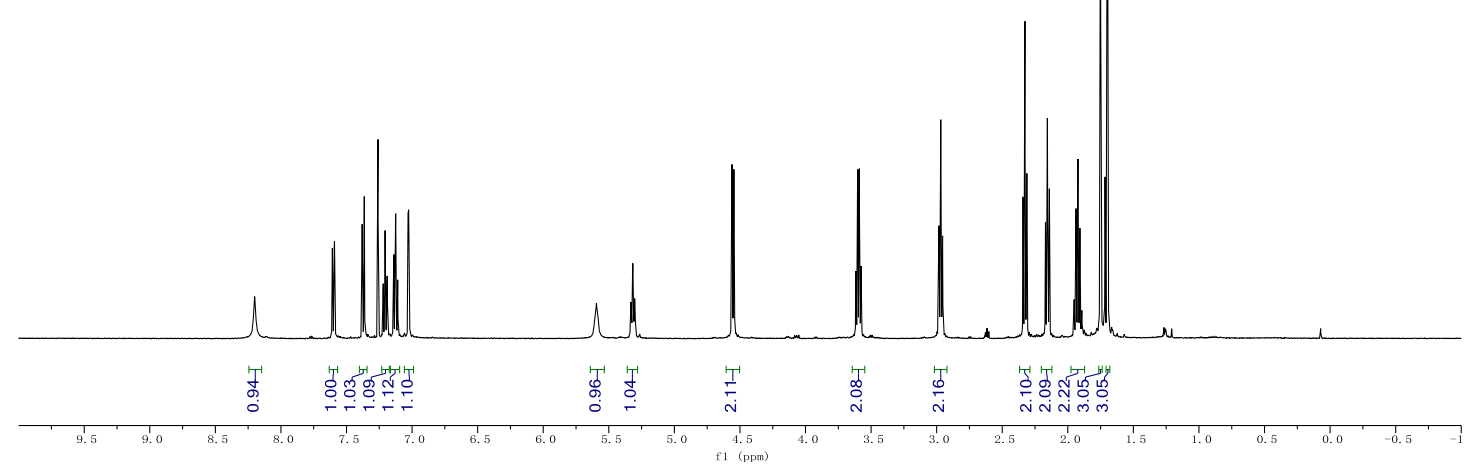

${ }^{13}$ C NMR (126 MHz, $\left.\mathrm{CDCl}_{3}\right) \mathbf{8 h}$.

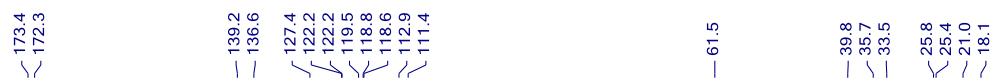<smiles>CC(C)=CCOC(=O)CCCC(=O)NCCc1c[nH]c2ccccc12</smiles>

$8 \mathrm{~h}$ 
${ }^{1} \mathbf{H}$ NMR (500 MHz, $\left.\mathrm{CD}_{2} \mathrm{Cl}_{2}\right) \mathbf{8 i}$.

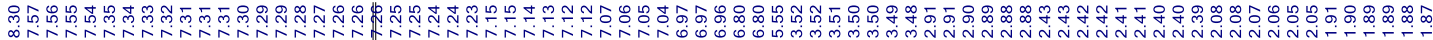

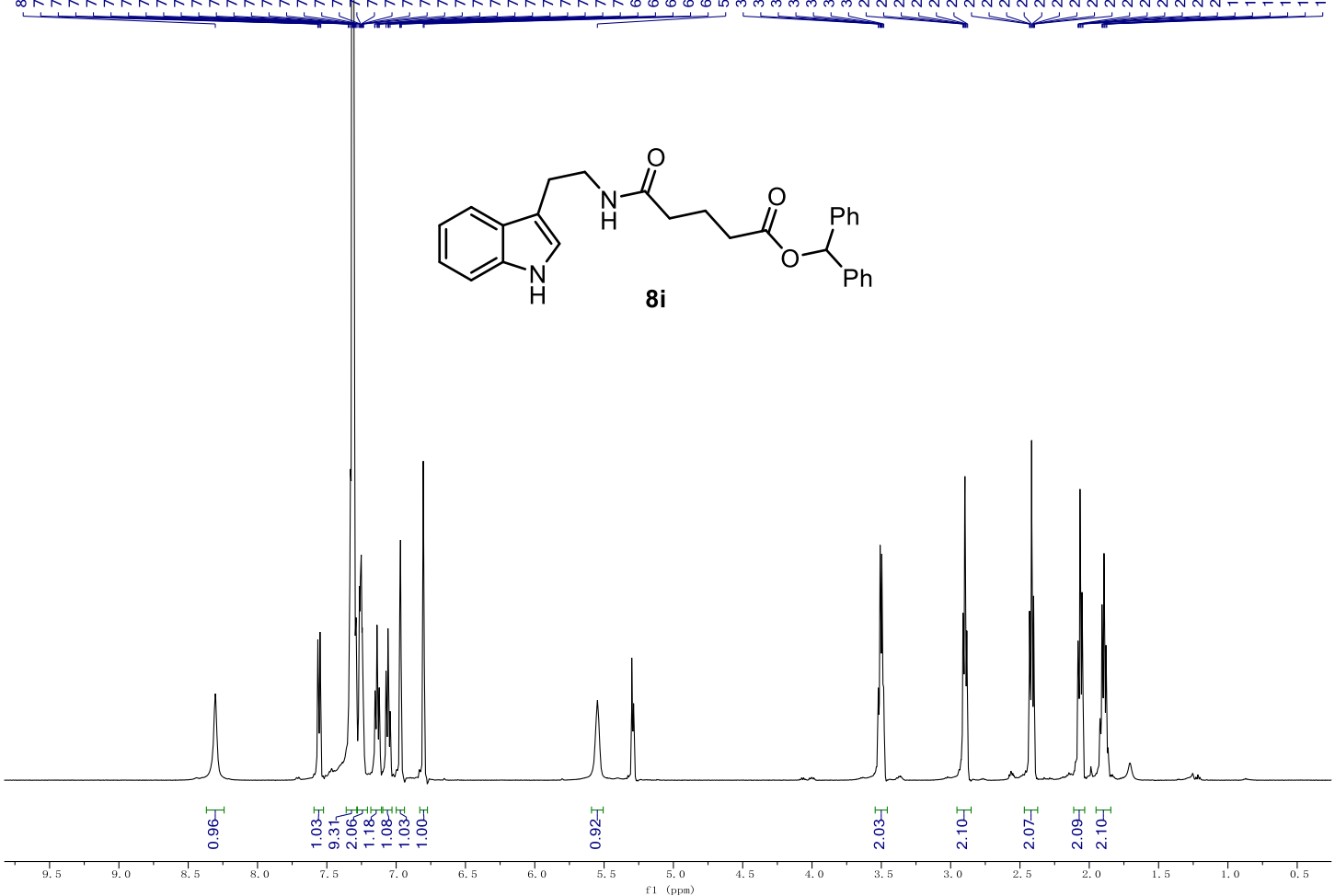

${ }^{13}$ C NMR (126 MHz, $\left.\mathrm{CD}_{2} \mathrm{Cl}_{2}\right) \mathbf{8 i}$.
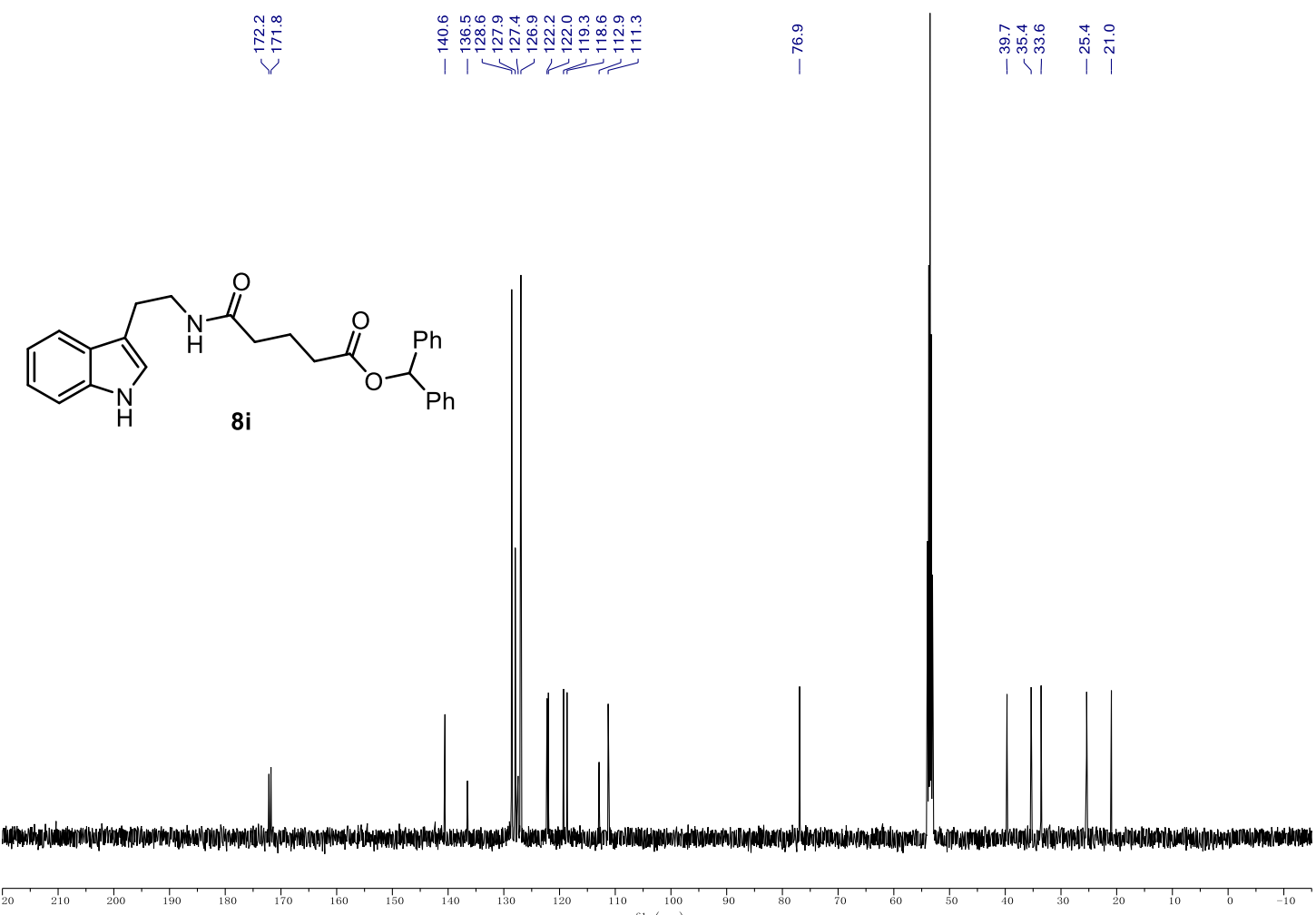
${ }^{1} \mathbf{H}$ NMR $\left(700 \mathrm{MHz}, \mathrm{CDCl}_{3}\right) \mathbf{8 j}$.

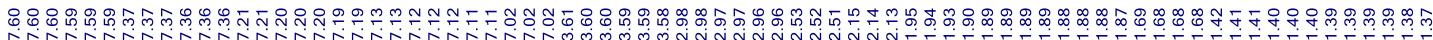

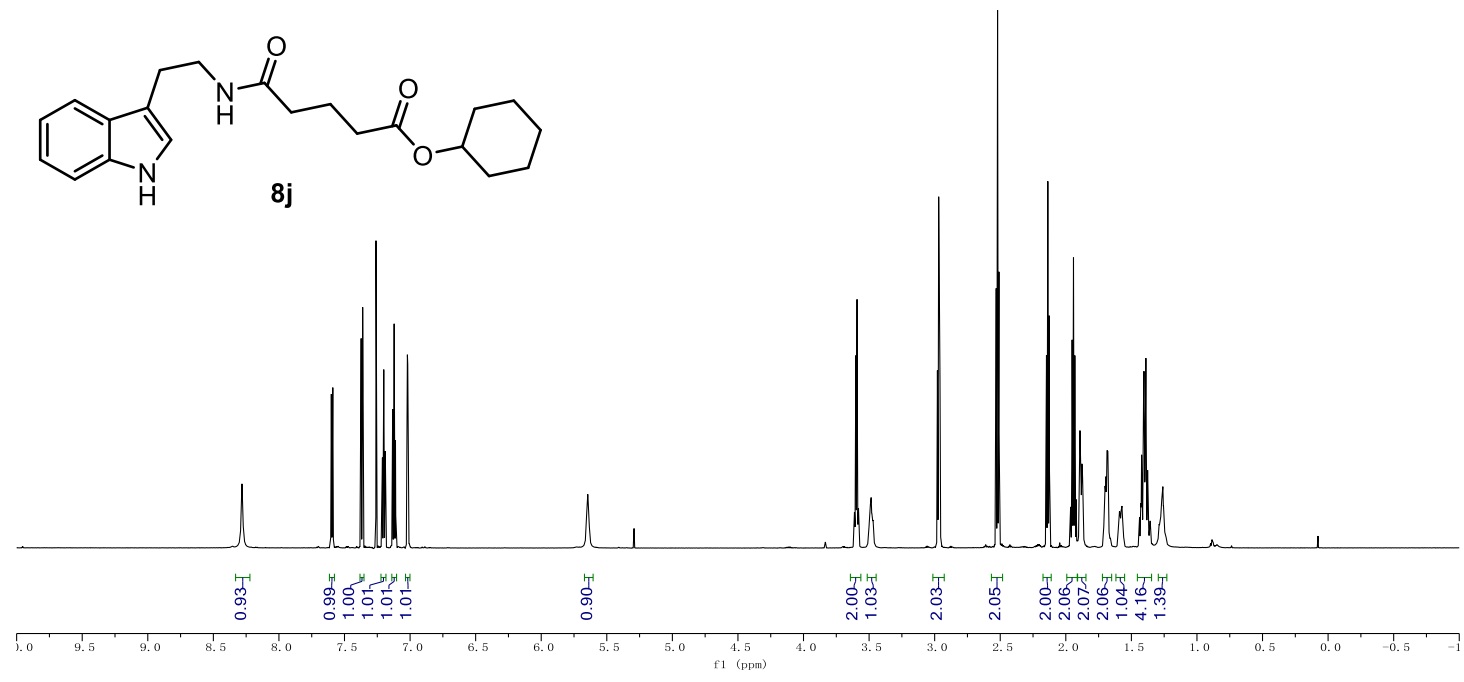

${ }^{13} \mathbf{C ~ N M R}\left(126 \mathrm{MHz}, \mathrm{CDCl}_{3}\right) \mathbf{8 j}$.

商

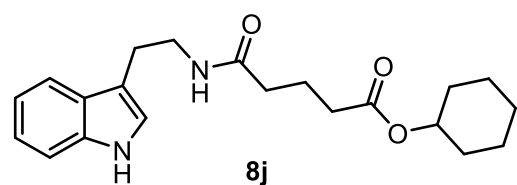

8j
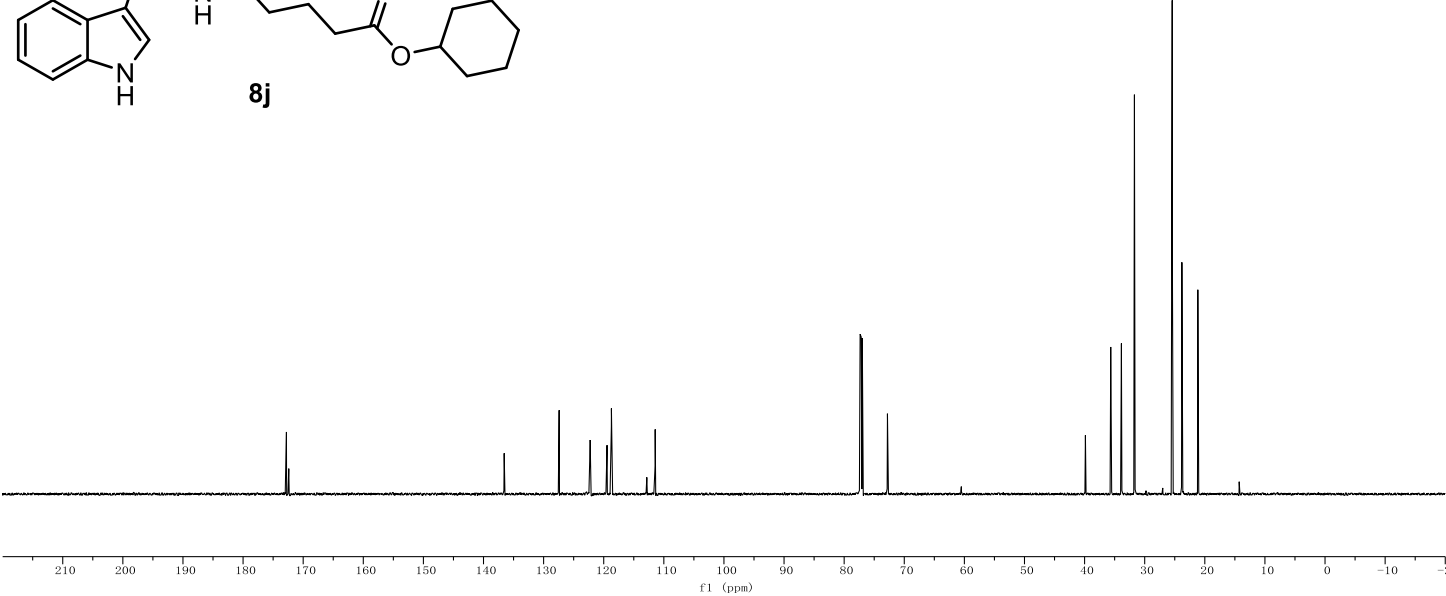
${ }^{1} \mathbf{H}$ NMR (500 MHz, $\left.\mathrm{CDCl}_{3}\right) \mathbf{8 k}$.

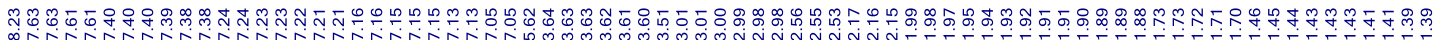
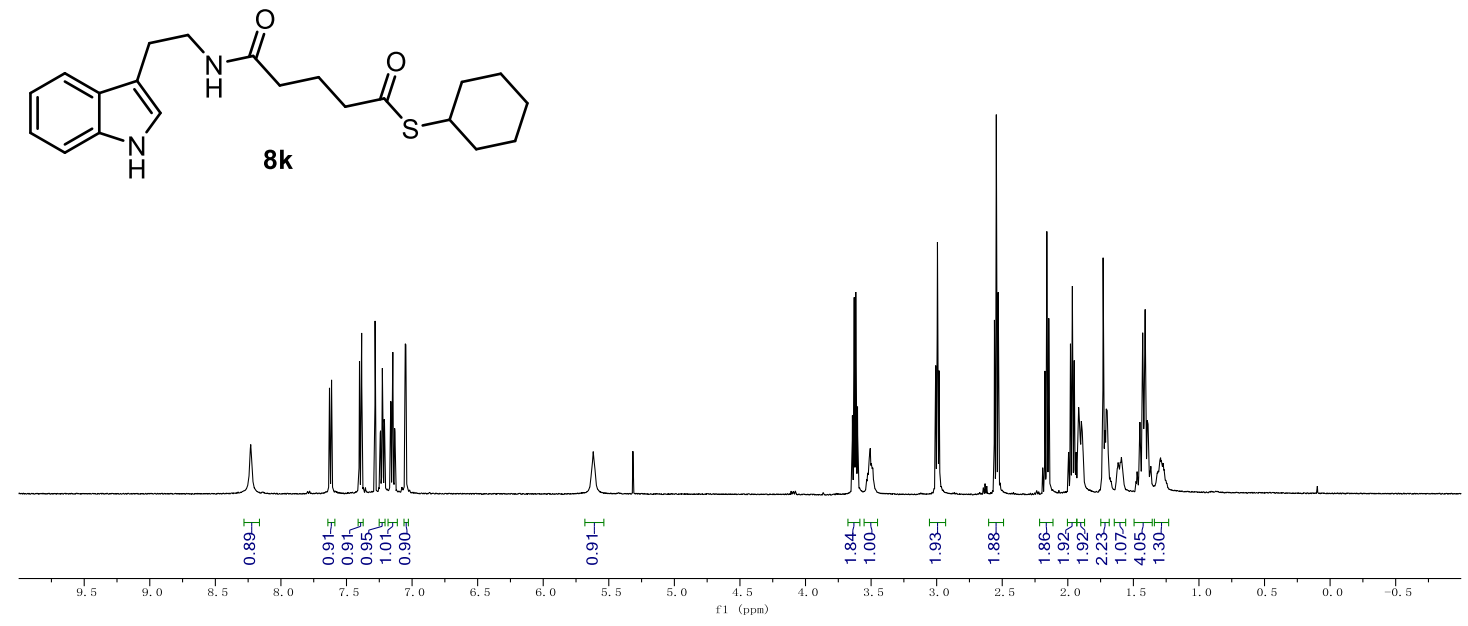

${ }^{13}$ C NMR (176 MHz, $\left.\mathrm{CDCl}_{3}\right)$ 8k.

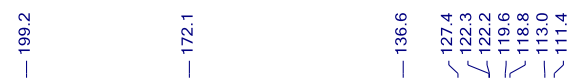

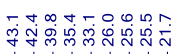

1, 1, 1,

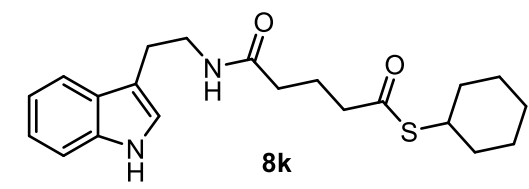

$8 k$ 
${ }^{1}$ H NMR (600 MHz, $\left.\mathrm{CDCl}_{3}\right) \mathbf{8 1}$.

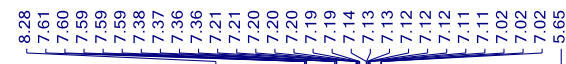

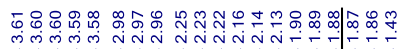

V.

$\overbrace{n}^{n}$

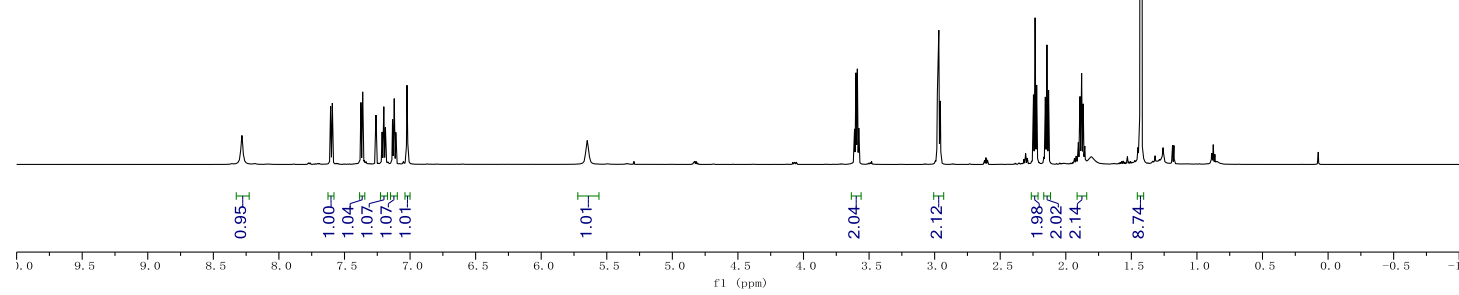

${ }^{13}$ C NMR (151 MHz, $\left.\mathrm{CDCl}_{3}\right) \mathbf{8 1 .}$

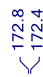

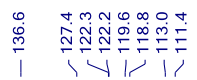

$\stackrel{\substack{\infty \\ \infty}}{1}$

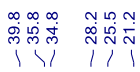

$\overbrace{n}^{n}$

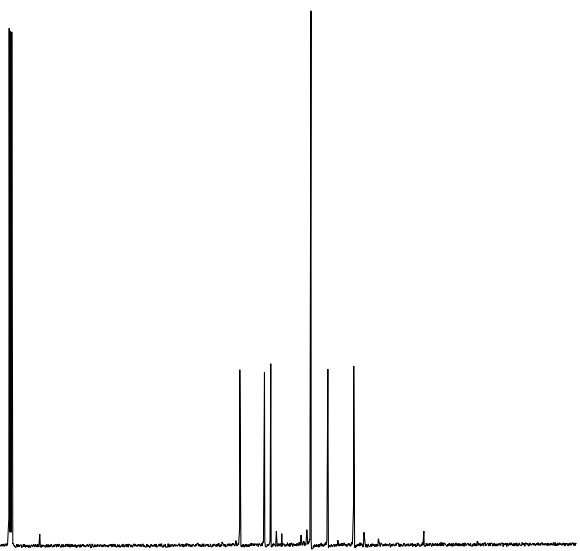

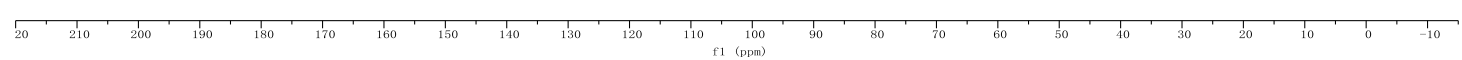


${ }^{1} \mathbf{H}$ NMR (500 MHz, $\left.\mathrm{CDCl}_{3}\right) \mathbf{1 1 a}$.

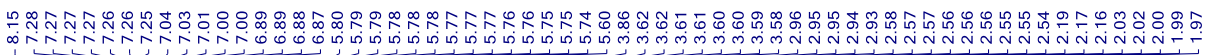

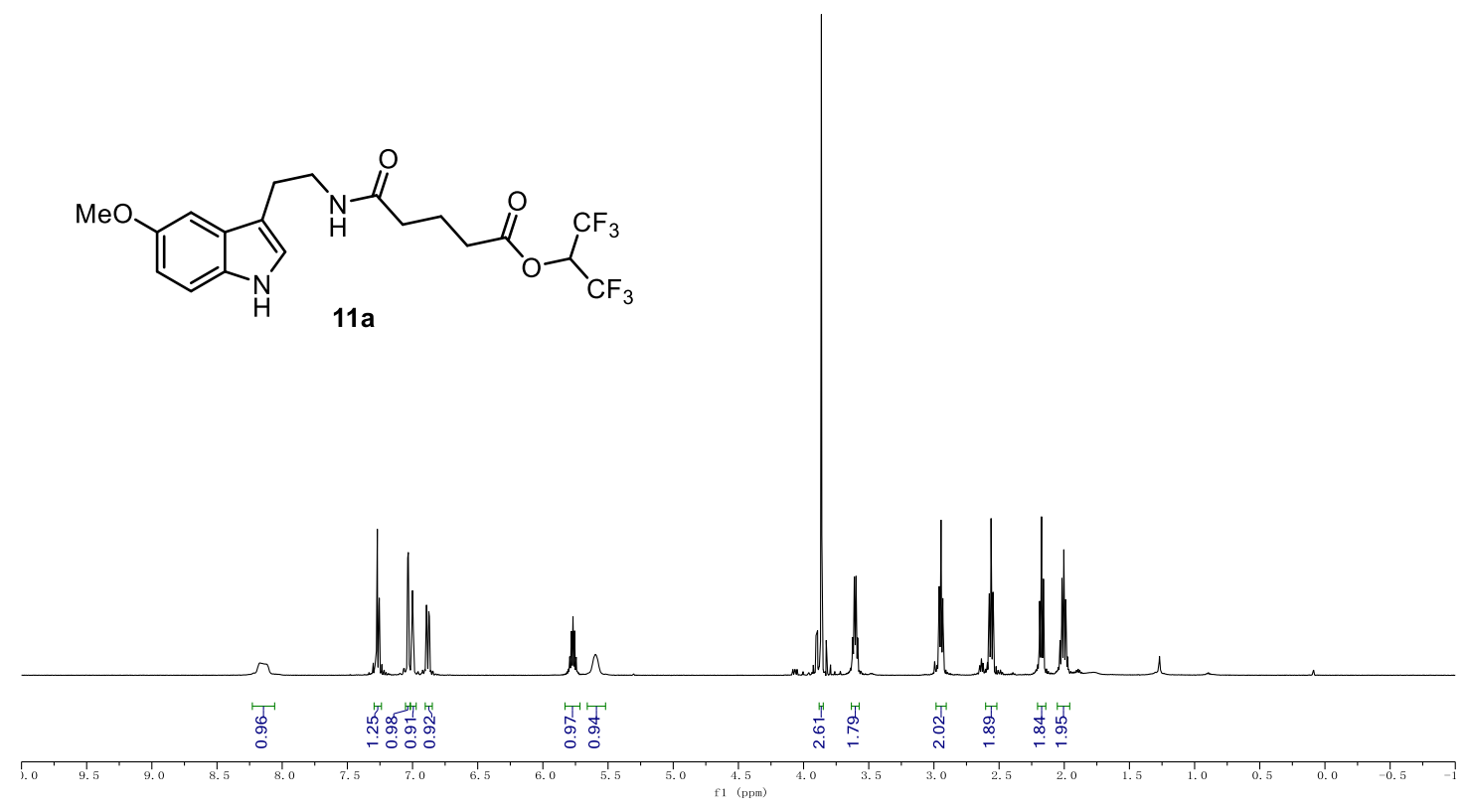

${ }^{13} \mathbf{C}$ NMR (126 MHz, $\left.\mathrm{CDCl}_{3}\right) \mathbf{1 1 a}$.

1)<smiles>COc1ccc2[nH]cc(CCNC(=O)CCCC(=O)OC(C(F)(F)F)C(F)(F)F)c2c1</smiles>

$11 a$ 
${ }^{19}$ F NMR $\left(565 \mathrm{MHz}, \mathrm{CDCl}_{3}\right) \mathbf{1 1 a}$.
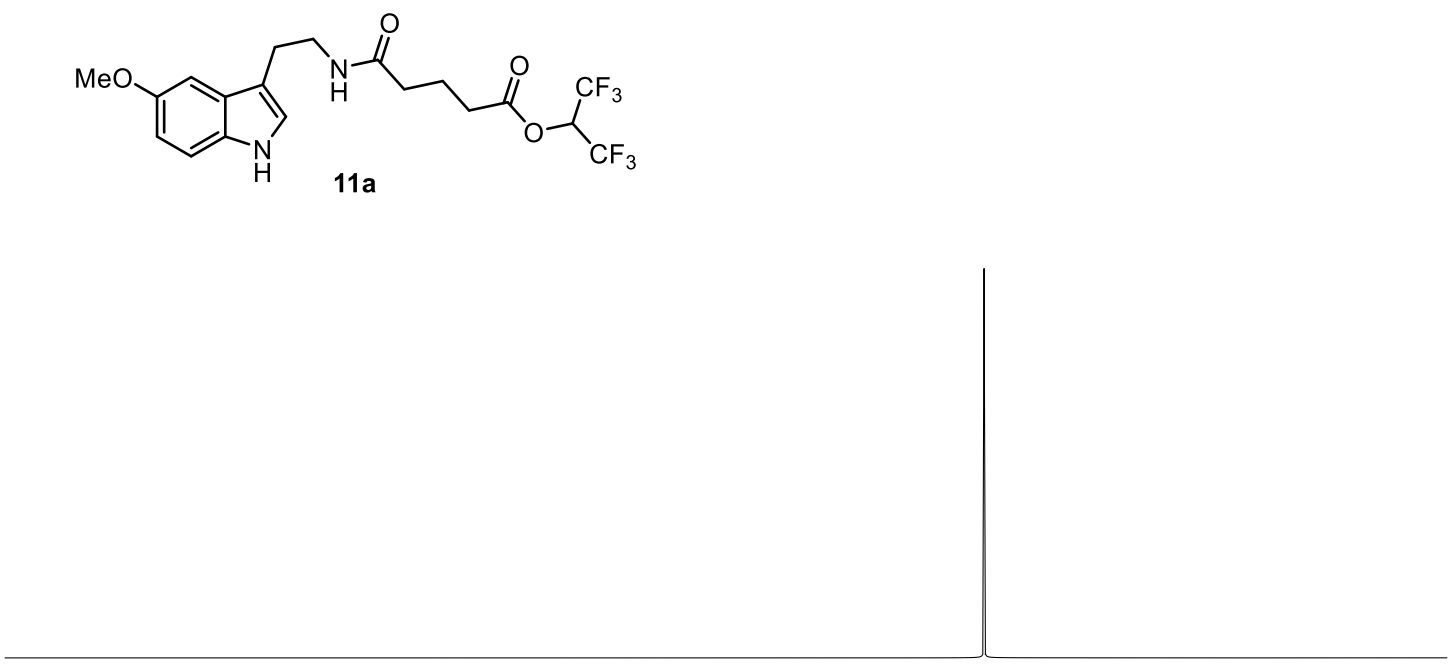

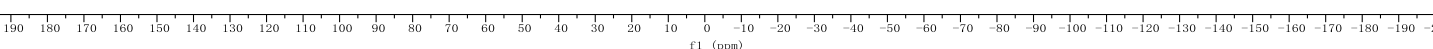

${ }^{1} \mathbf{H}$ NMR (500 MHz, $\left.\mathrm{CD}_{2} \mathrm{Cl}_{2}\right) \mathbf{1 1 b}$.

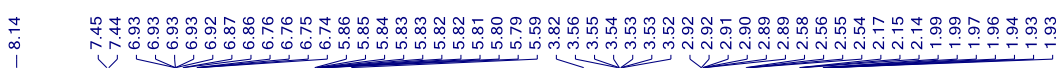<smiles>COc1ccc2c(CCNC(=O)CCCC(=O)OC(C(F)(F)F)C(F)(F)F)c[nH]c2c1</smiles>

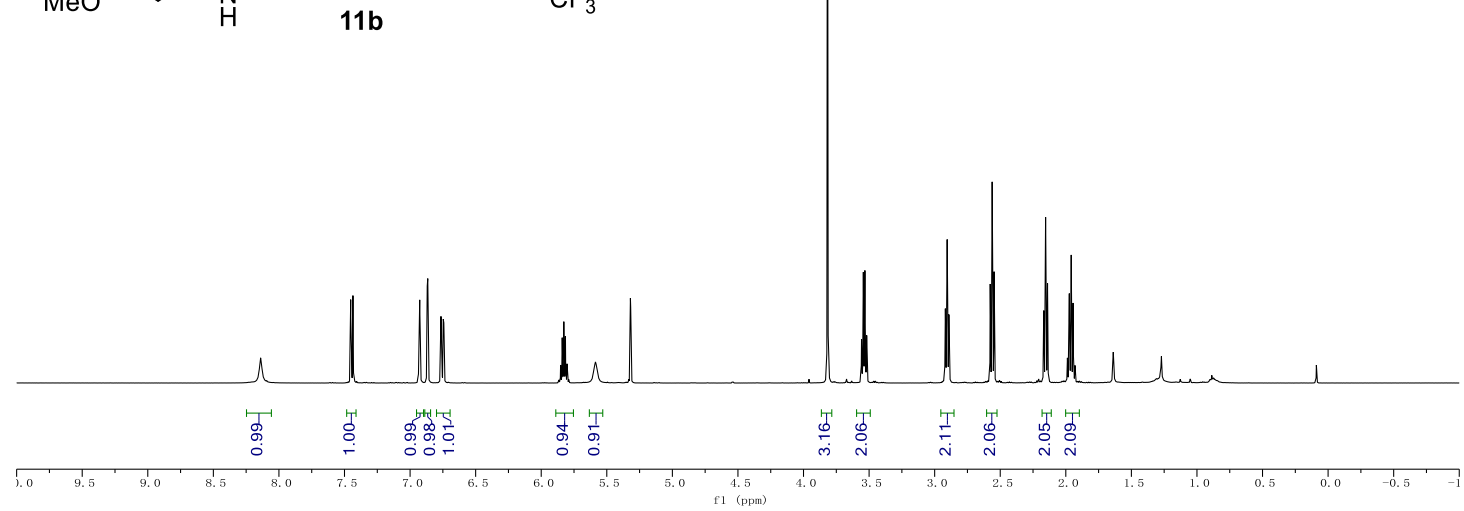


${ }^{13} \mathbf{C}$ NMR (126 MHz, $\left.\mathrm{CD}_{2} \mathrm{Cl}_{2}\right) \mathbf{1 1 b}$.

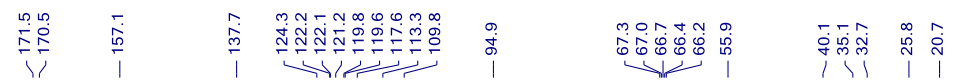

$\overbrace{\mathrm{H}}$
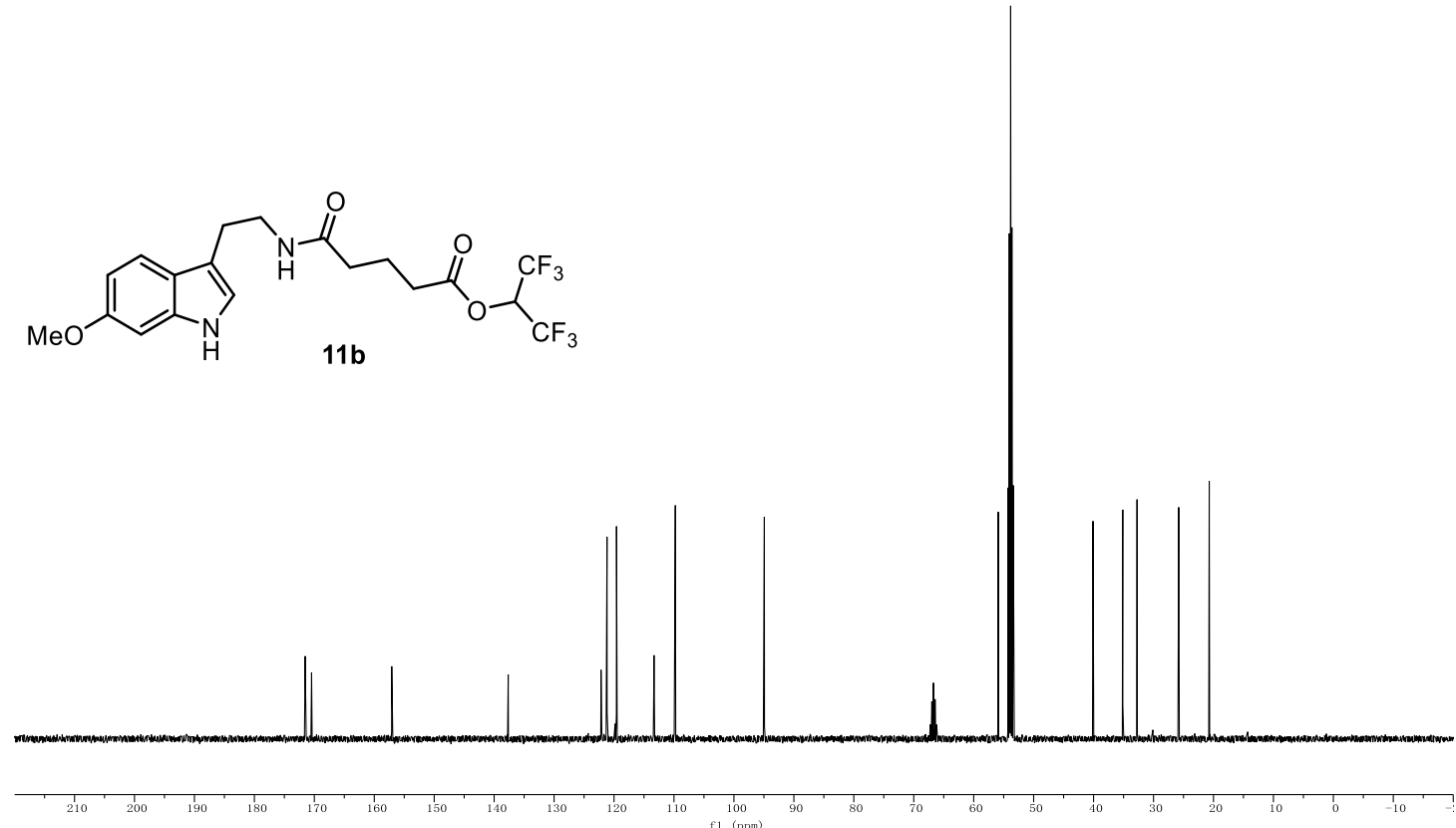

${ }^{19}$ F NMR (471 MHz, $\left.\mathrm{CD}_{2} \mathrm{Cl}_{2}\right) \mathbf{1 1 b}$.

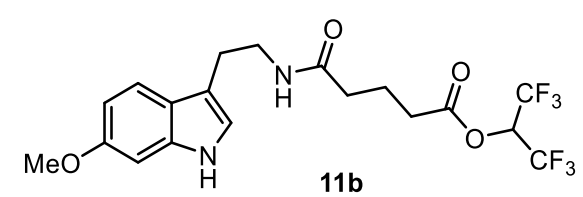


${ }^{1} \mathbf{H}$ NMR (700 MHz, $\left.\mathrm{CDCl}_{3}\right) \mathbf{1 1 c}$.

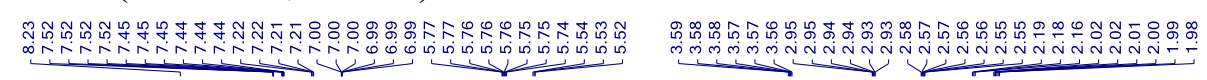
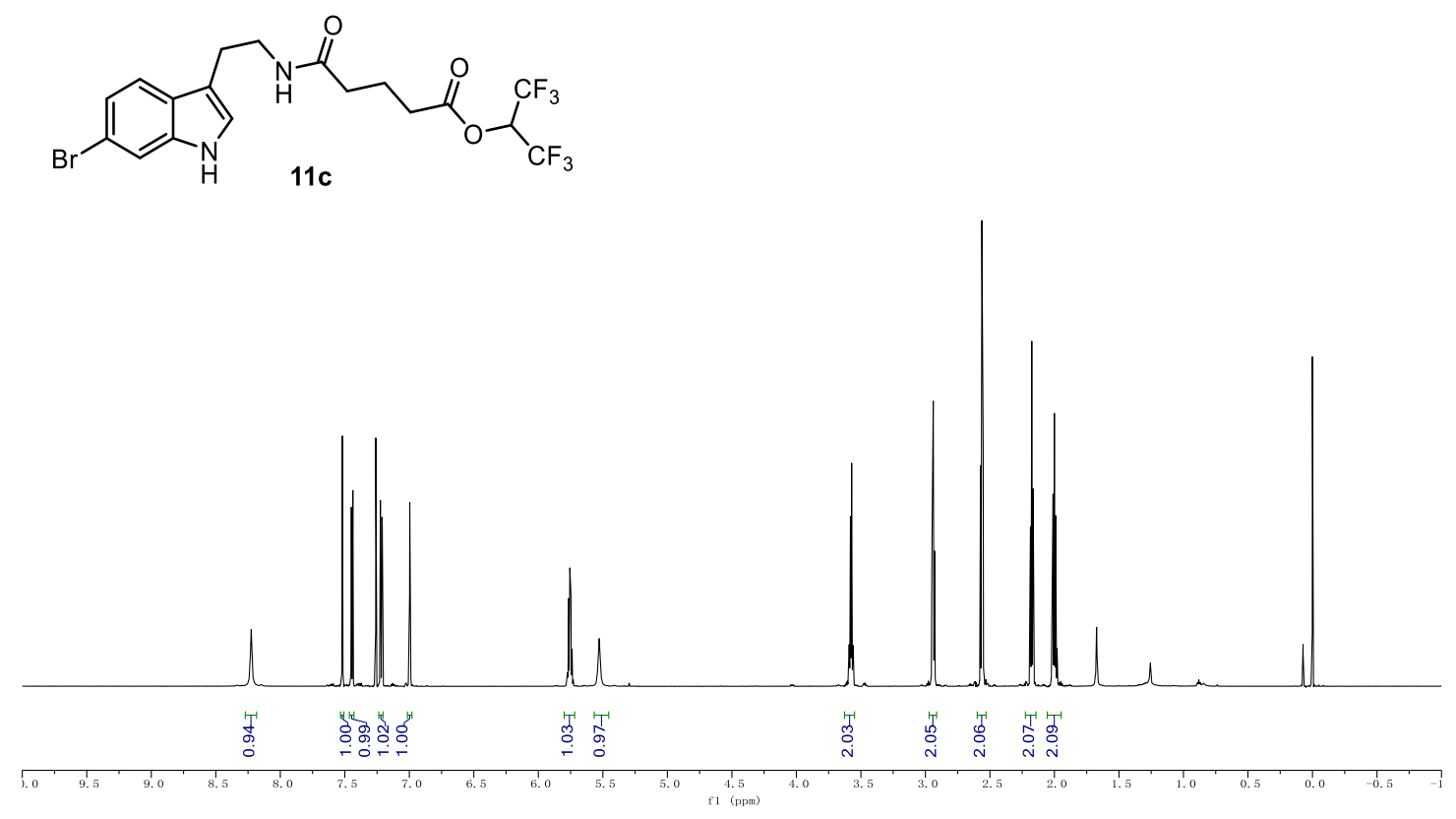

${ }^{13} \mathbf{C ~ N M R}\left(176 \mathrm{MHz}, \mathrm{CDCl}_{3}\right) \mathbf{1 1 c}$.

至

$\sqrt{30.08}$

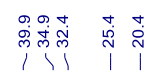
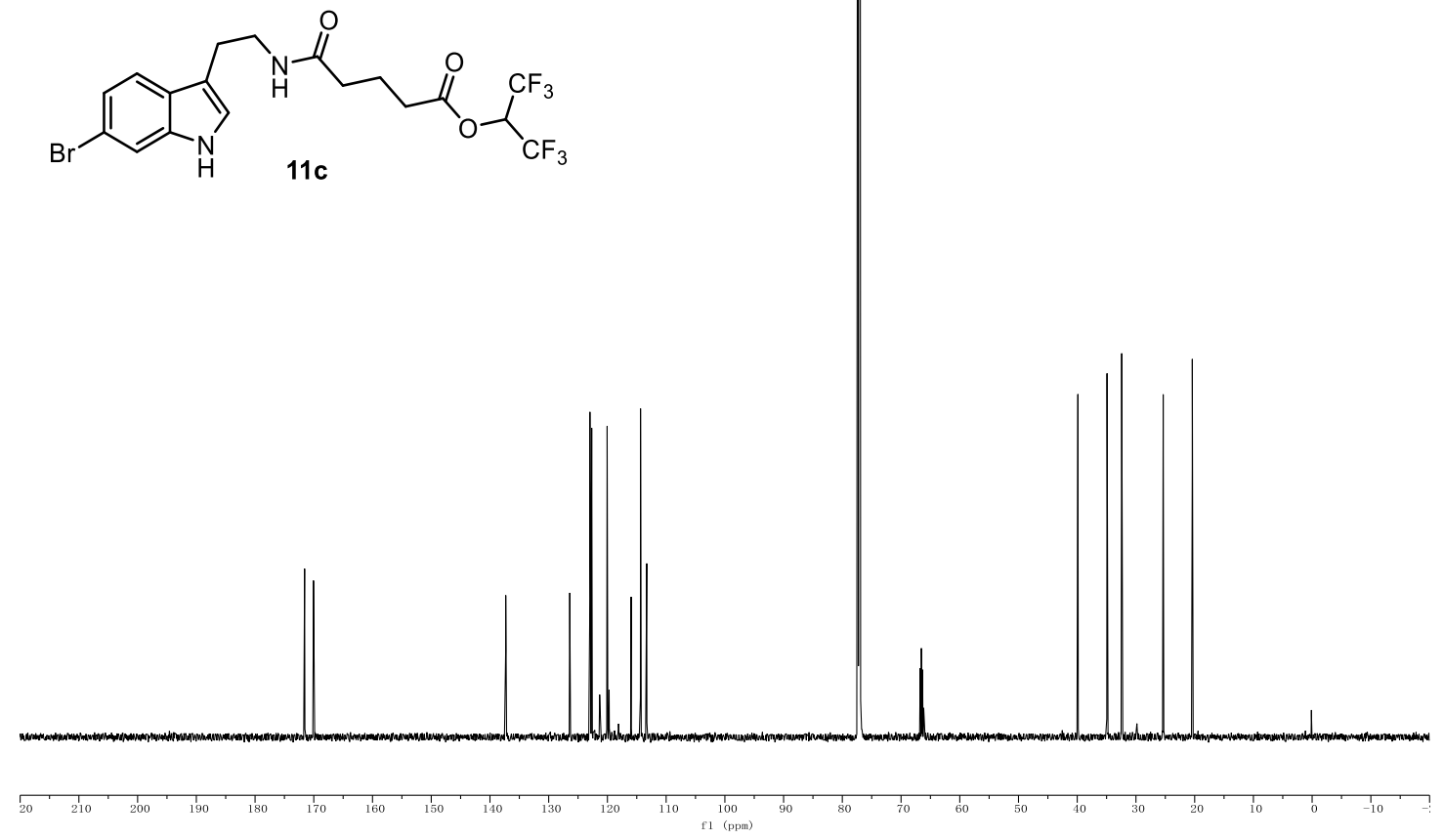

S123 
${ }^{19}$ F NMR (471 MHz, $\left.\mathrm{CDCl}_{3}\right)$ 11c.

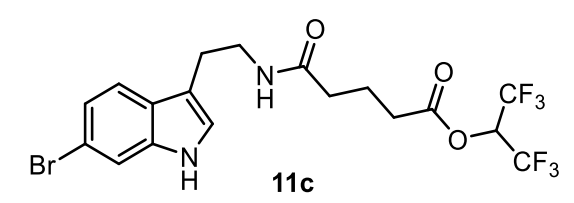

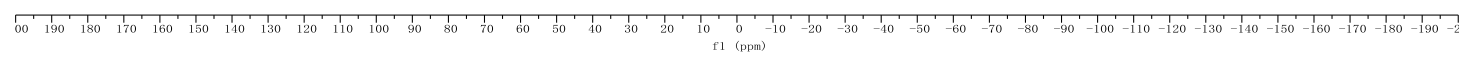

${ }^{1} \mathbf{H}$ NMR $\left(400 \mathrm{MHz}, \mathrm{CDCl}_{3}\right) \mathbf{1 1 d}$.

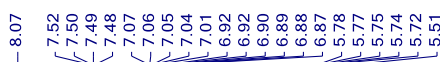

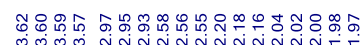

mimm

$\overbrace{\mathrm{H}}^{\mathrm{N}}$

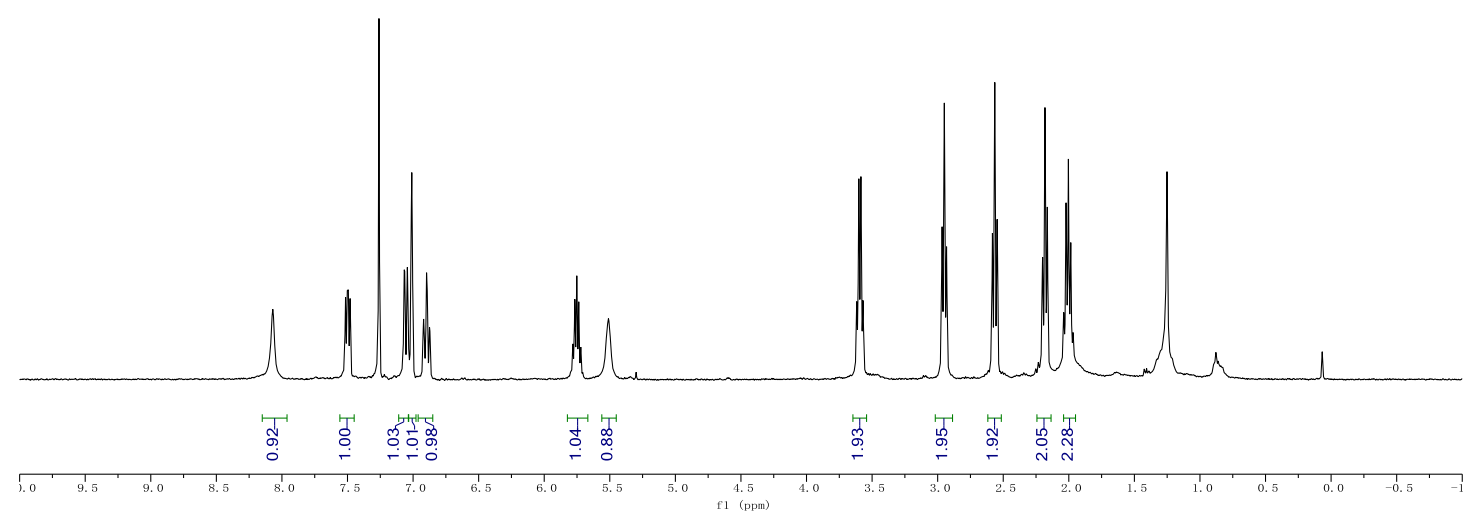


${ }^{13} \mathrm{C}$ NMR (176 MHz, $\left.\mathrm{CDCl}_{3}\right) \mathbf{1 1 d}$.

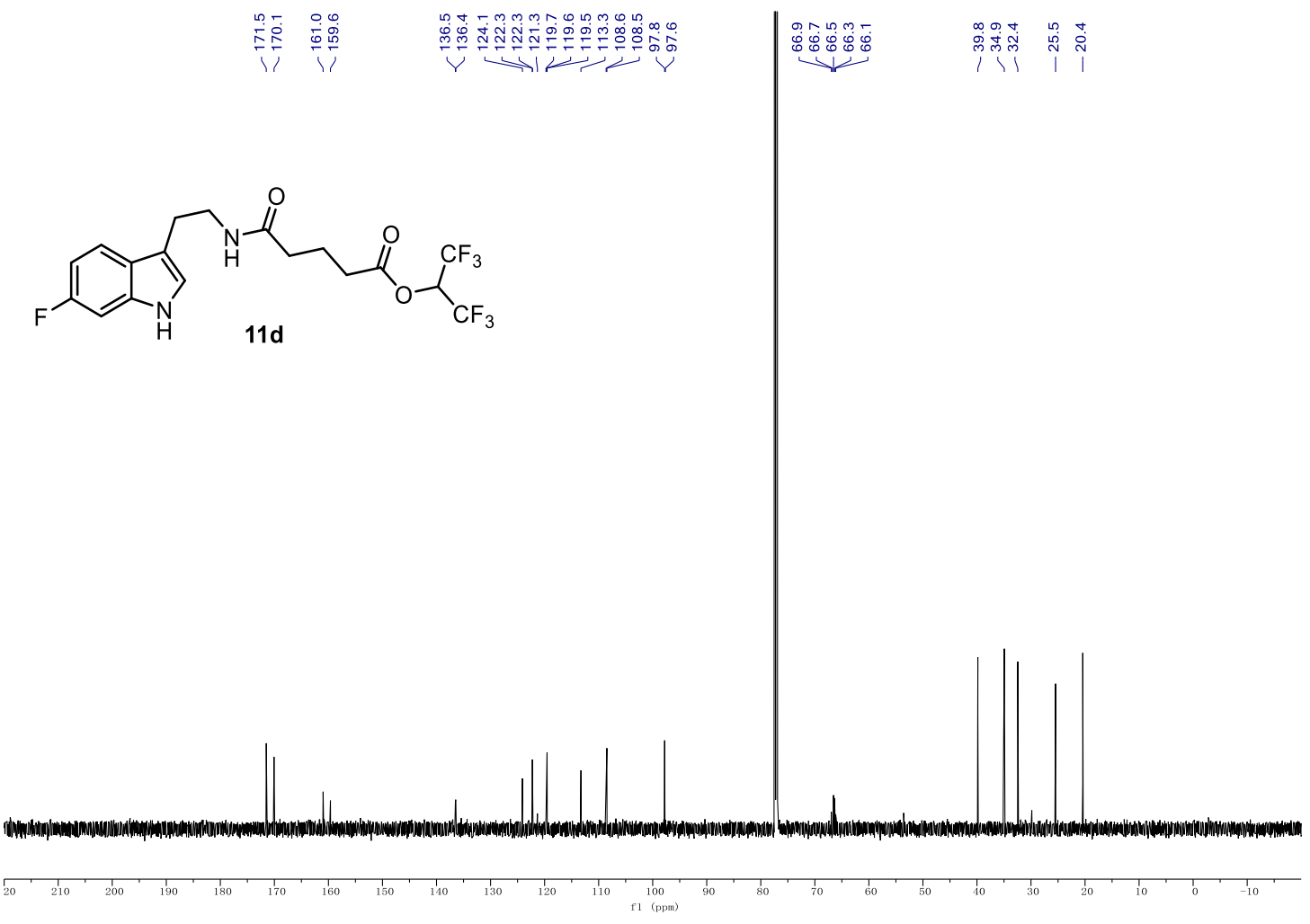

${ }^{19}$ F NMR (471 MHz, $\left.\mathrm{CDCl}_{3}\right) \mathbf{1 1 d}$.
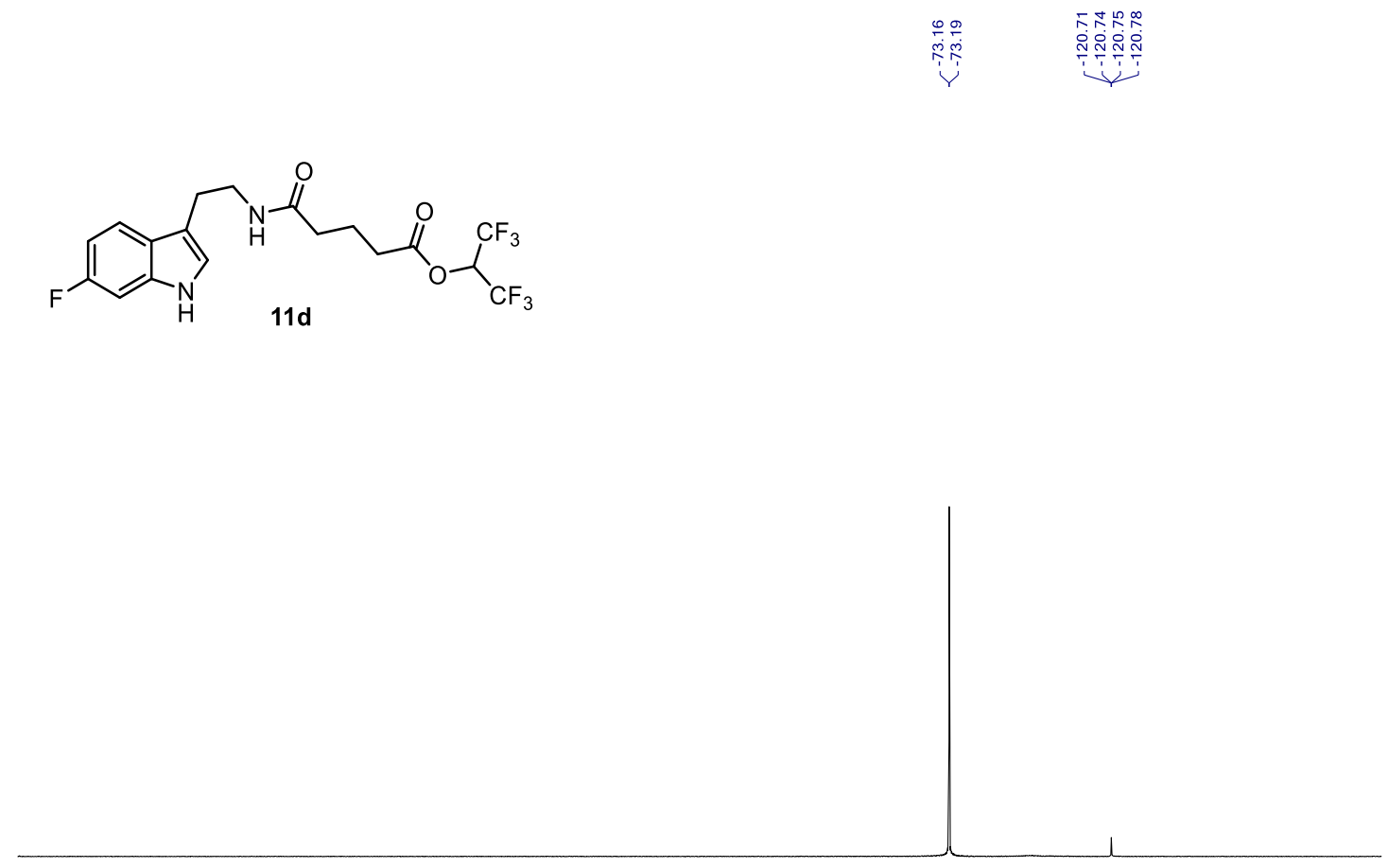

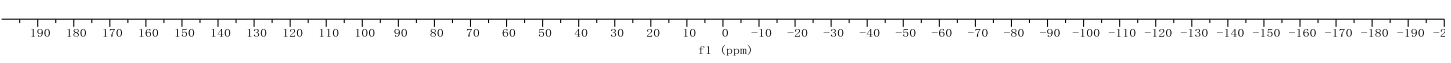


${ }^{1} \mathbf{H}$ NMR (500 MHz, $\left.\mathrm{CD}_{2} \mathrm{Cl}_{2}\right) \mathbf{1 1 e .}$

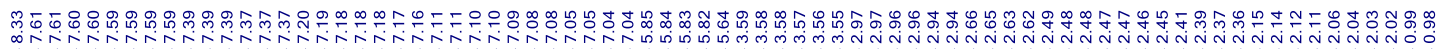

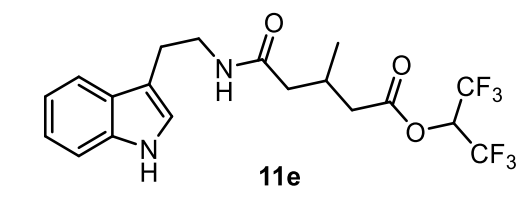

${ }^{13}$ C NMR (126 MHz, $\left.\mathrm{CD}_{2} \mathrm{Cl}_{2}\right) 11 \mathrm{e}$.

登离

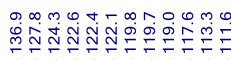

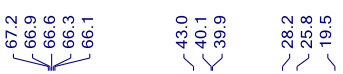

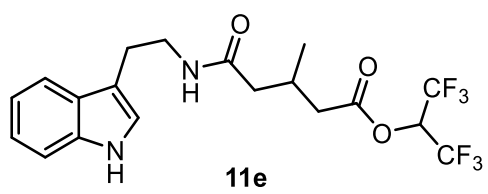

$11 \mathrm{e}$ 
${ }^{19} \mathbf{F}$ NMR $\left(471 \mathrm{MHz}, \mathrm{CD}_{2} \mathrm{Cl}_{2}\right) \mathbf{1 1 e .}$

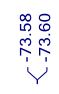

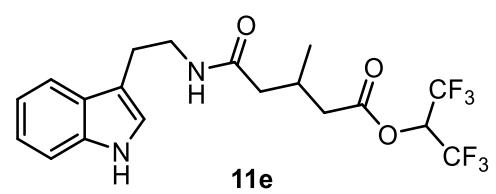

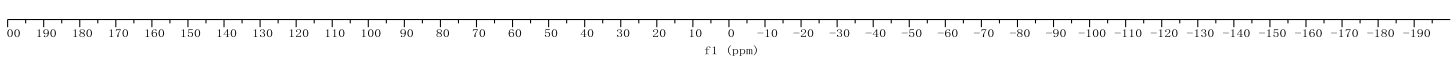

${ }^{1} \mathbf{H}$ NMR (500 MHz, $\left.\mathrm{CD}_{2} \mathrm{Cl}_{2}\right) \mathbf{1 1 f}$.

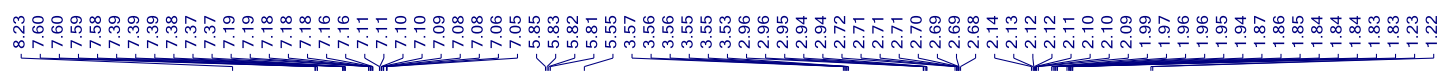
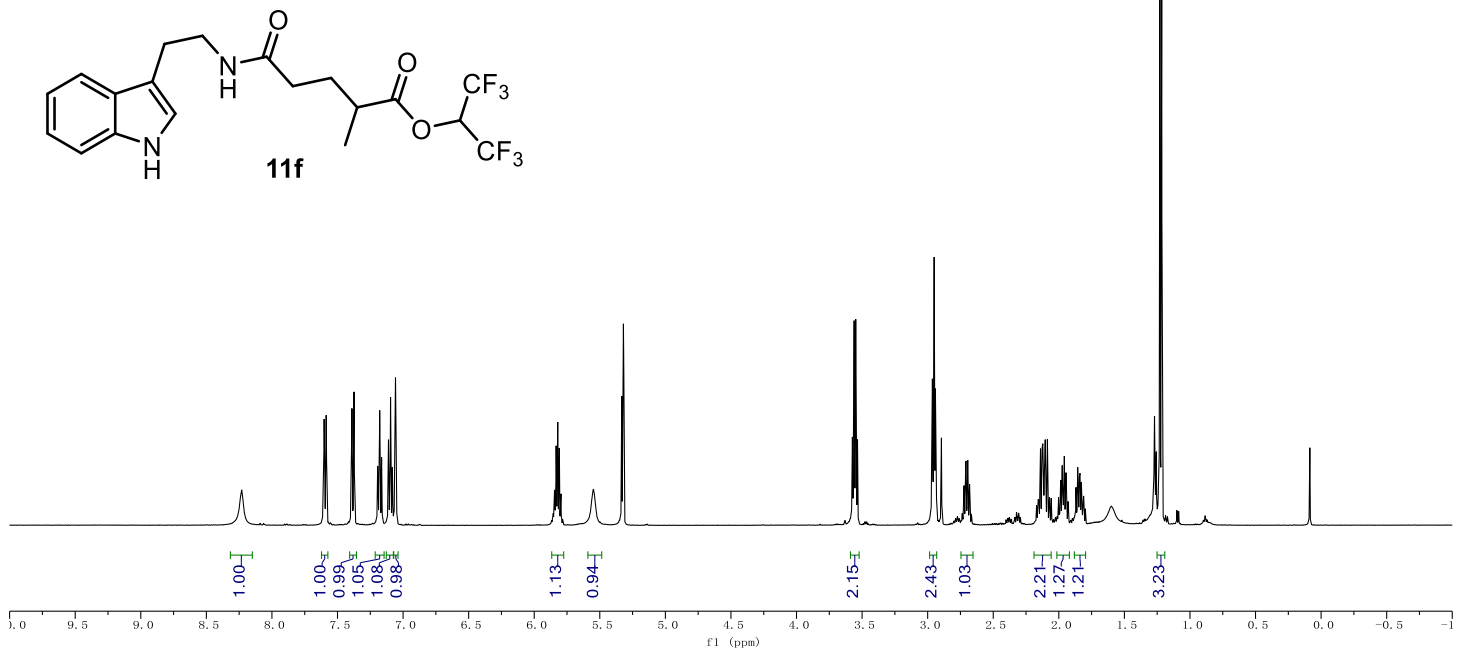

S127 
${ }^{1} \mathbf{H}$ NMR $\left(500 \mathrm{MHz}, \mathrm{CD}_{2} \mathrm{Cl}_{2}\right) \mathbf{1 1 f}$.
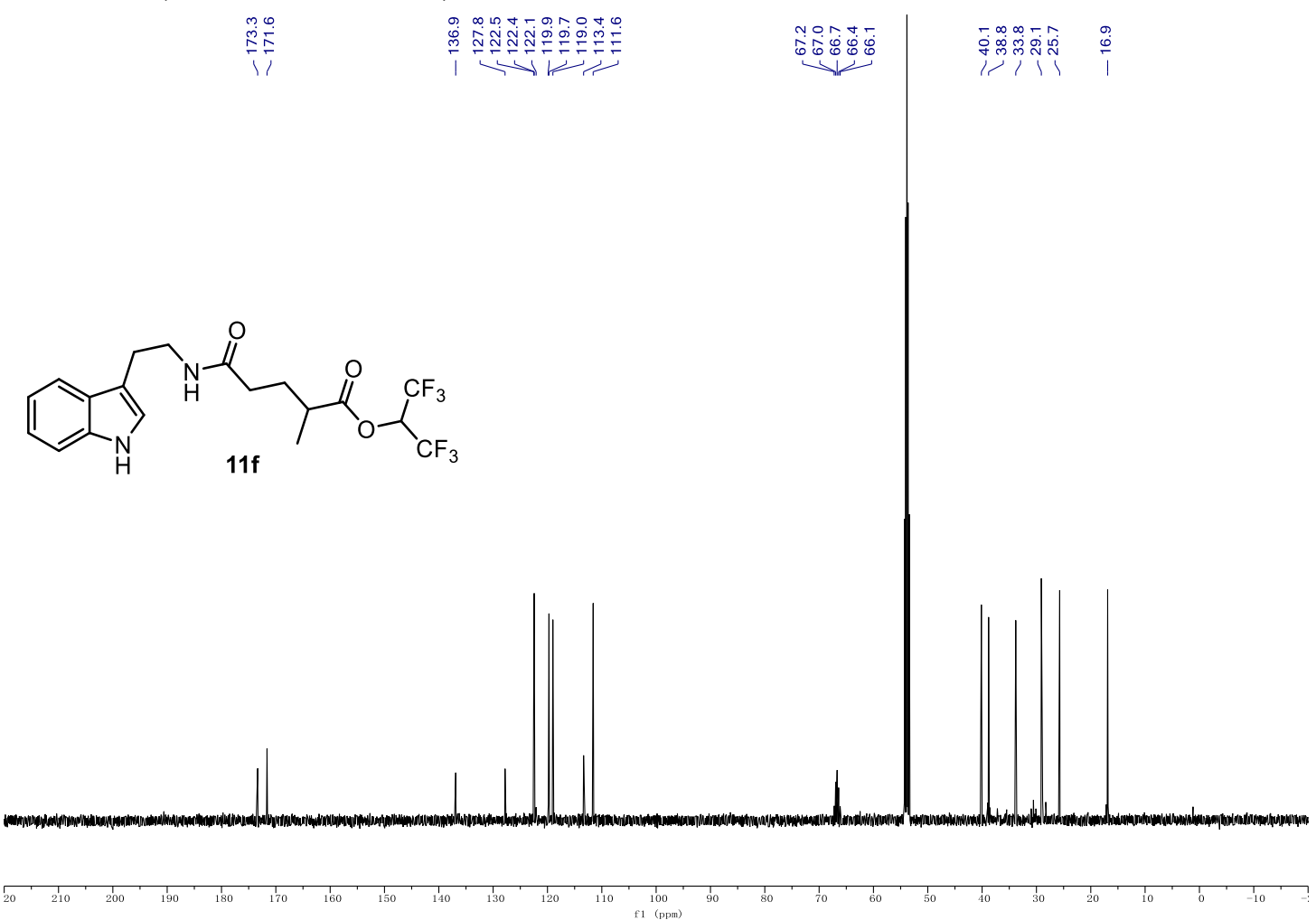

${ }^{1} \mathbf{H}$ NMR $\left(500 \mathrm{MHz}, \mathrm{CD}_{2} \mathrm{Cl}_{2}\right) \mathbf{1 1 f}$.

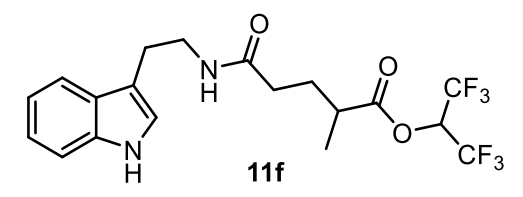


${ }^{1} \mathbf{H}$ NMR (600 MHz, $\left.\mathrm{CDCl}_{3}\right) \mathbf{1 1 g}$.

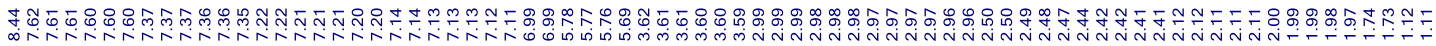<smiles>CC(CCC(=O)OC(C(F)(F)F)C(F)(F)F)C(=O)NCCc1c[nH]c2ccccc12</smiles>

$11 \mathrm{~g}$
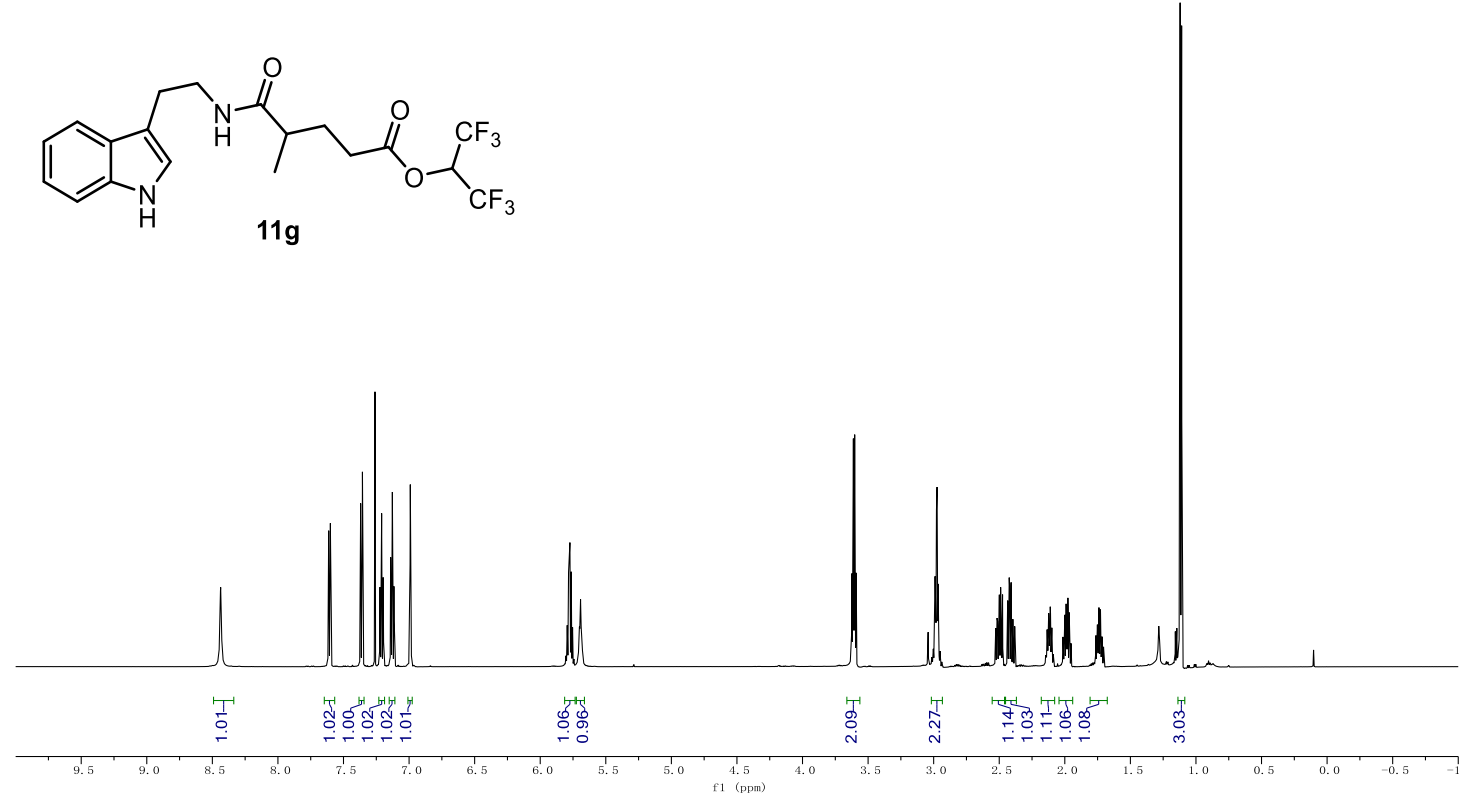

${ }^{13}$ C NMR (151 MHz, $\left.\mathrm{CDCl}_{3}\right) \mathbf{1 1 g}$.

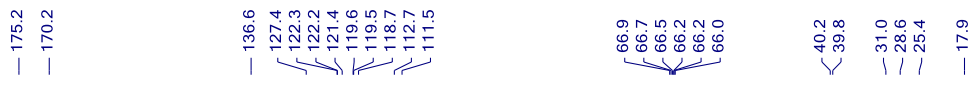

$\overbrace{\mathrm{N}}^{\mathrm{N}}$

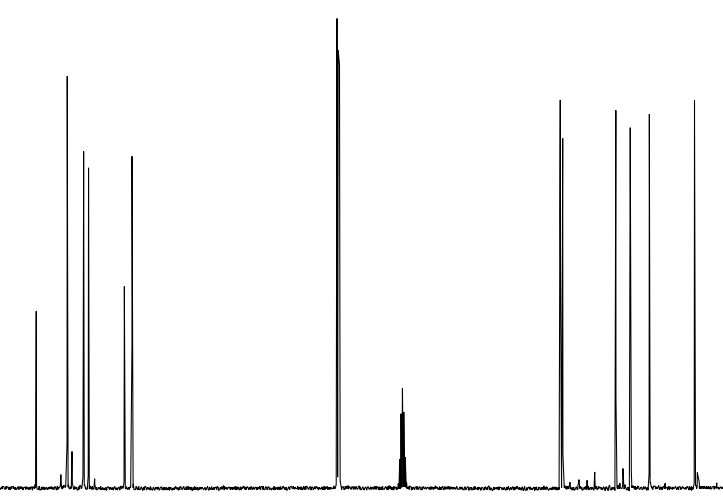

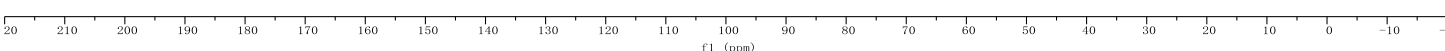


${ }^{19}$ F NMR $\left(565 \mathrm{MHz}, \mathrm{CDCl}_{3}\right)$ 11g.

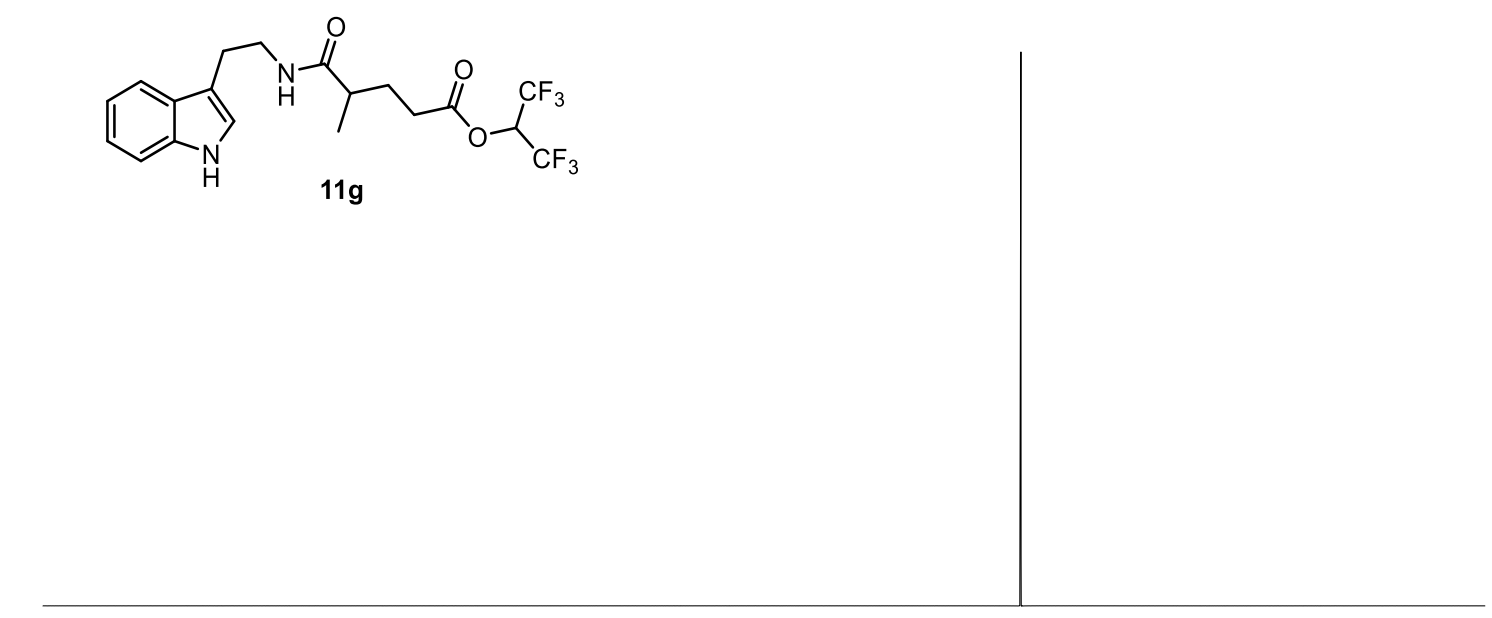

ri

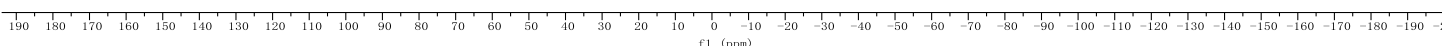

${ }^{1} \mathbf{H}$ NMR (500 MHz, $\left.\mathrm{CDCl}_{3}\right) \mathbf{1 1 h}$.

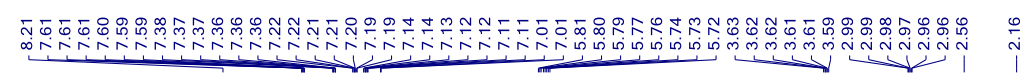
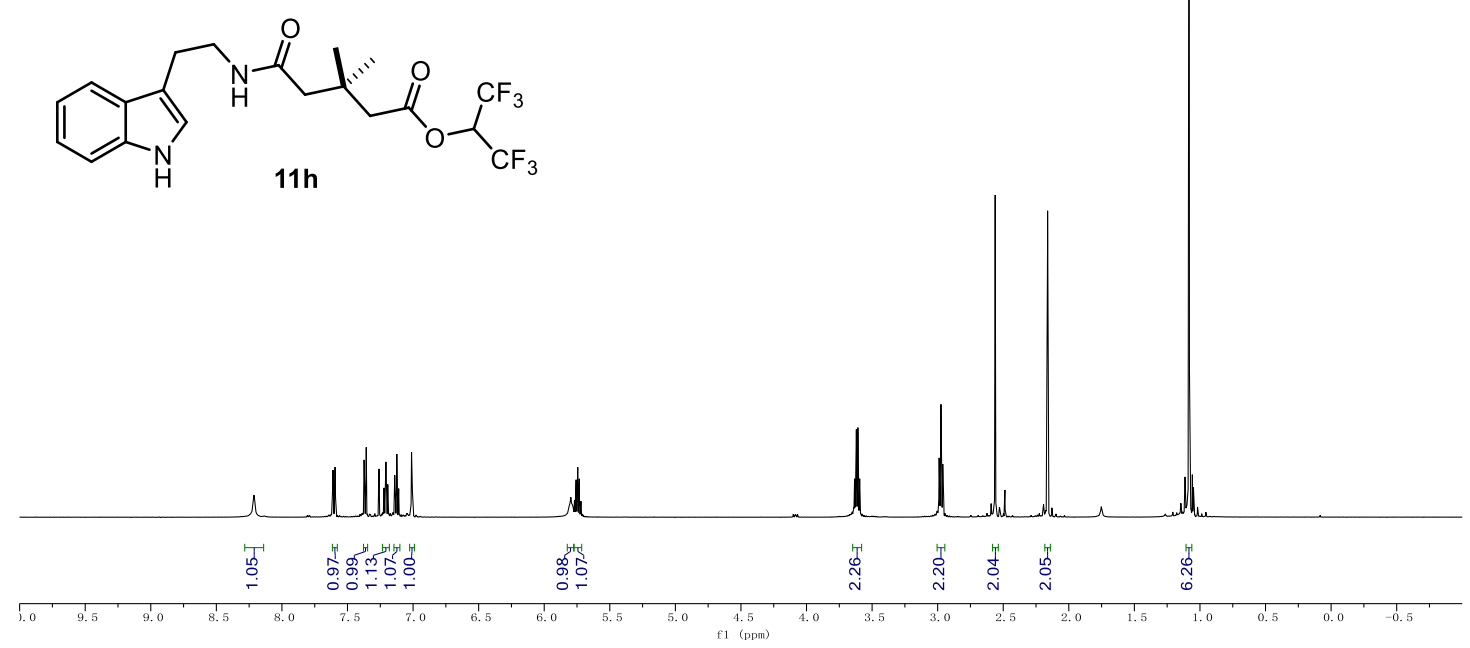
${ }^{13} \mathbf{C ~ N M R}\left(126 \mathrm{MHz}, \mathrm{CDCl}_{3}\right) \mathbf{1 1 h}$.

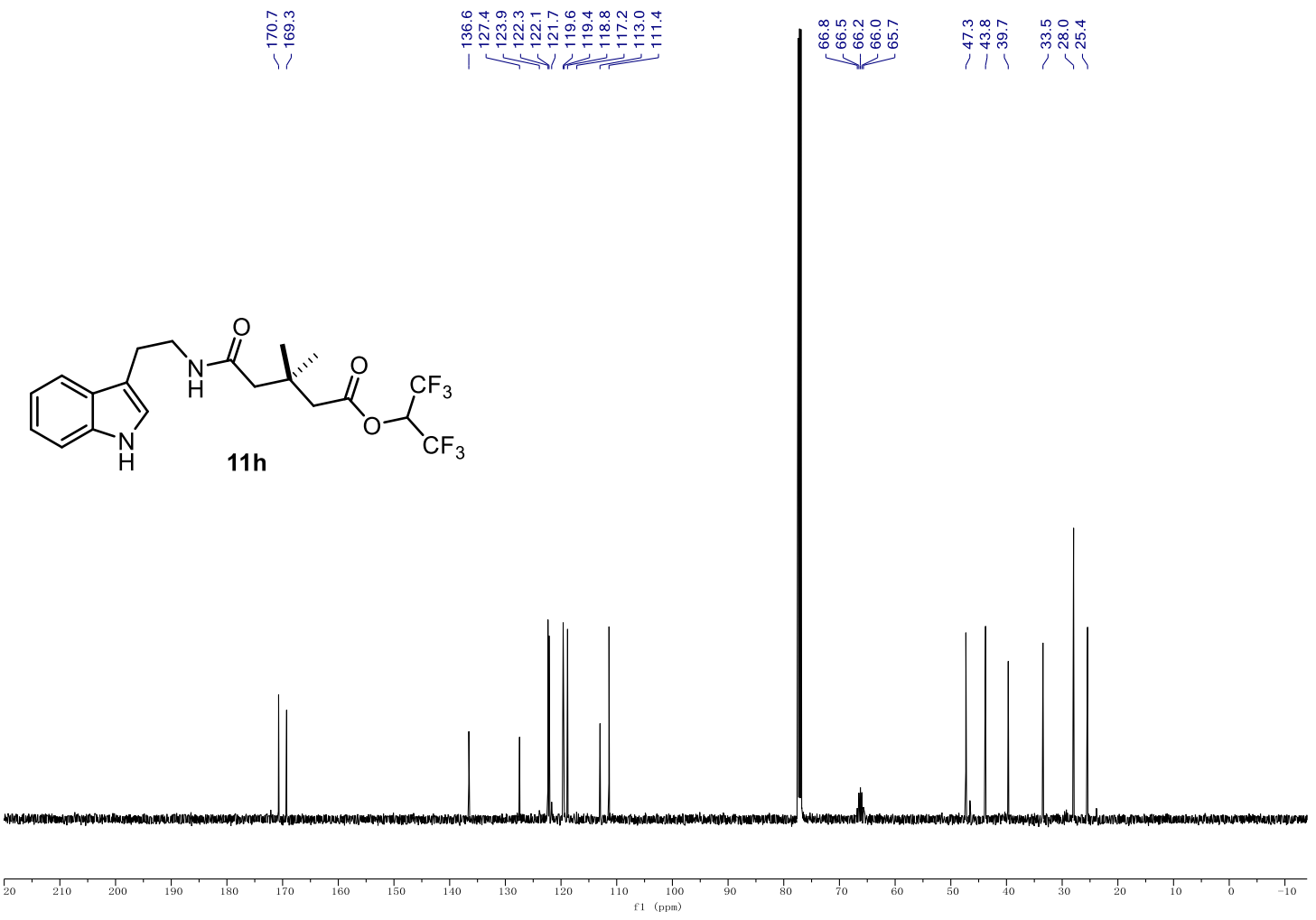

${ }^{19}$ F NMR (565 MHz, $\left.\mathrm{CDCl}_{3}\right) \mathbf{1 1 h}$.

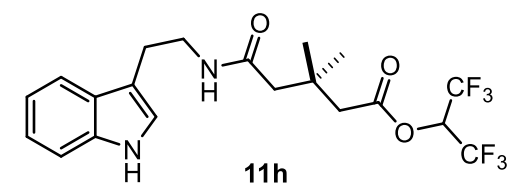


${ }^{1} \mathbf{H}$ NMR (500 MHz, $\left.\mathrm{CDCl}_{3}\right) \mathbf{1 1 i}$.

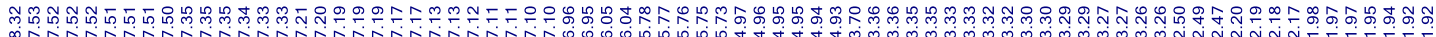

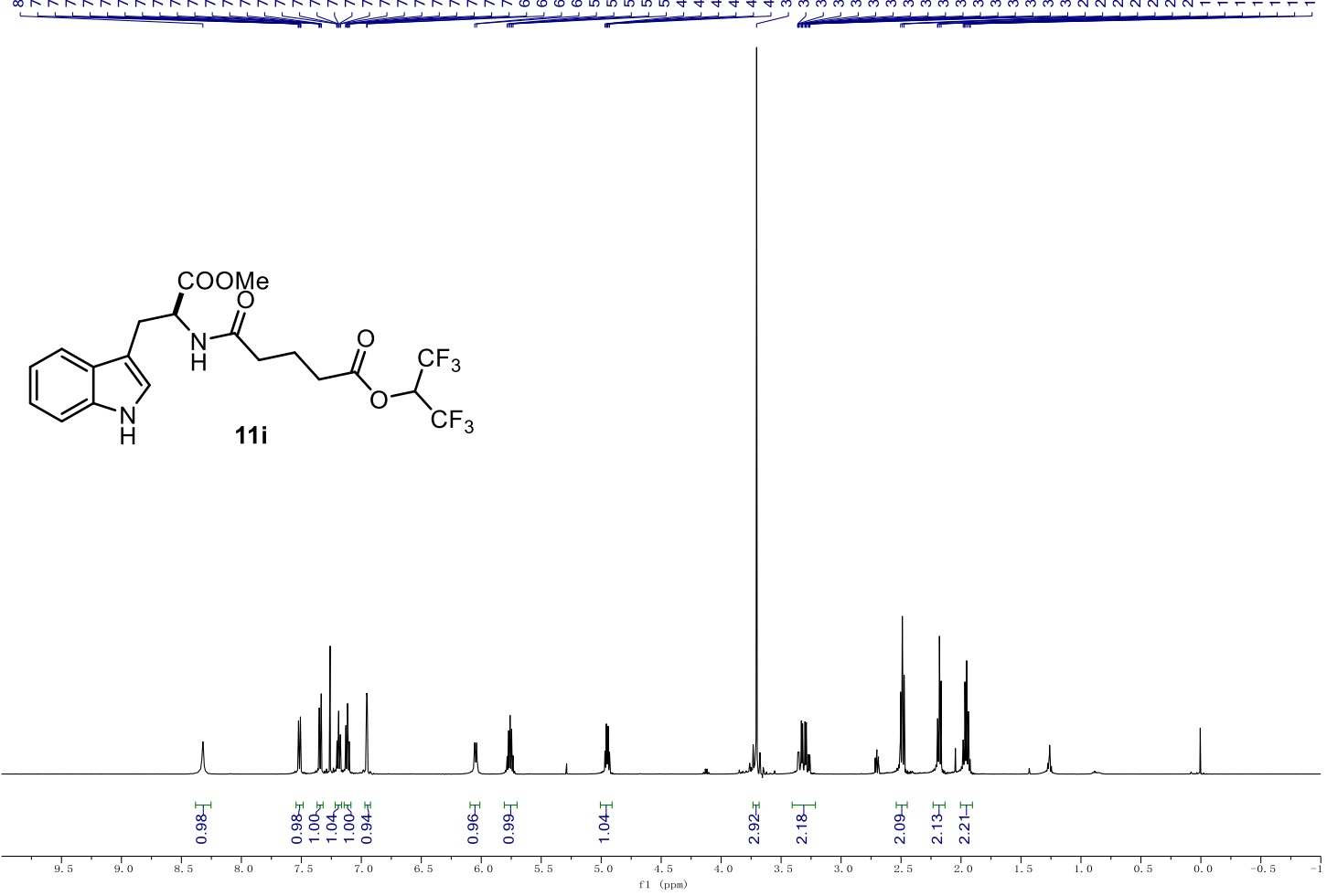

${ }^{13} \mathbf{C}$ NMR (126 MHz, $\left.\mathrm{CDCl}_{3}\right) \mathbf{1 1 i}$.

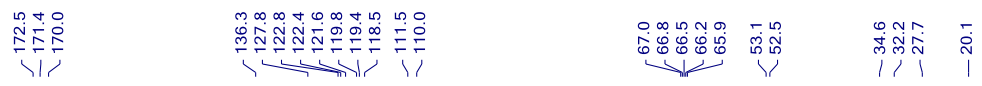<smiles>COC(=O)C(Cc1c[nH]c2ccccc12)NC(=O)CCCC(=O)OC(C(F)(F)F)C(F)(F)F</smiles>

11i

$\mathrm{CF}_{3}$ 
${ }^{19}$ F NMR (471 MHz, $\left.\mathrm{CDCl}_{3}\right) \mathbf{1 1 i}$.

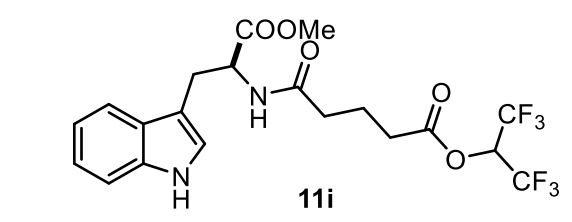

${ }^{\mathbf{1}} \mathbf{H}$ NMR (500 MHz, $\left.\mathrm{CDCl}_{3}\right) \mathbf{1 1 j}$.

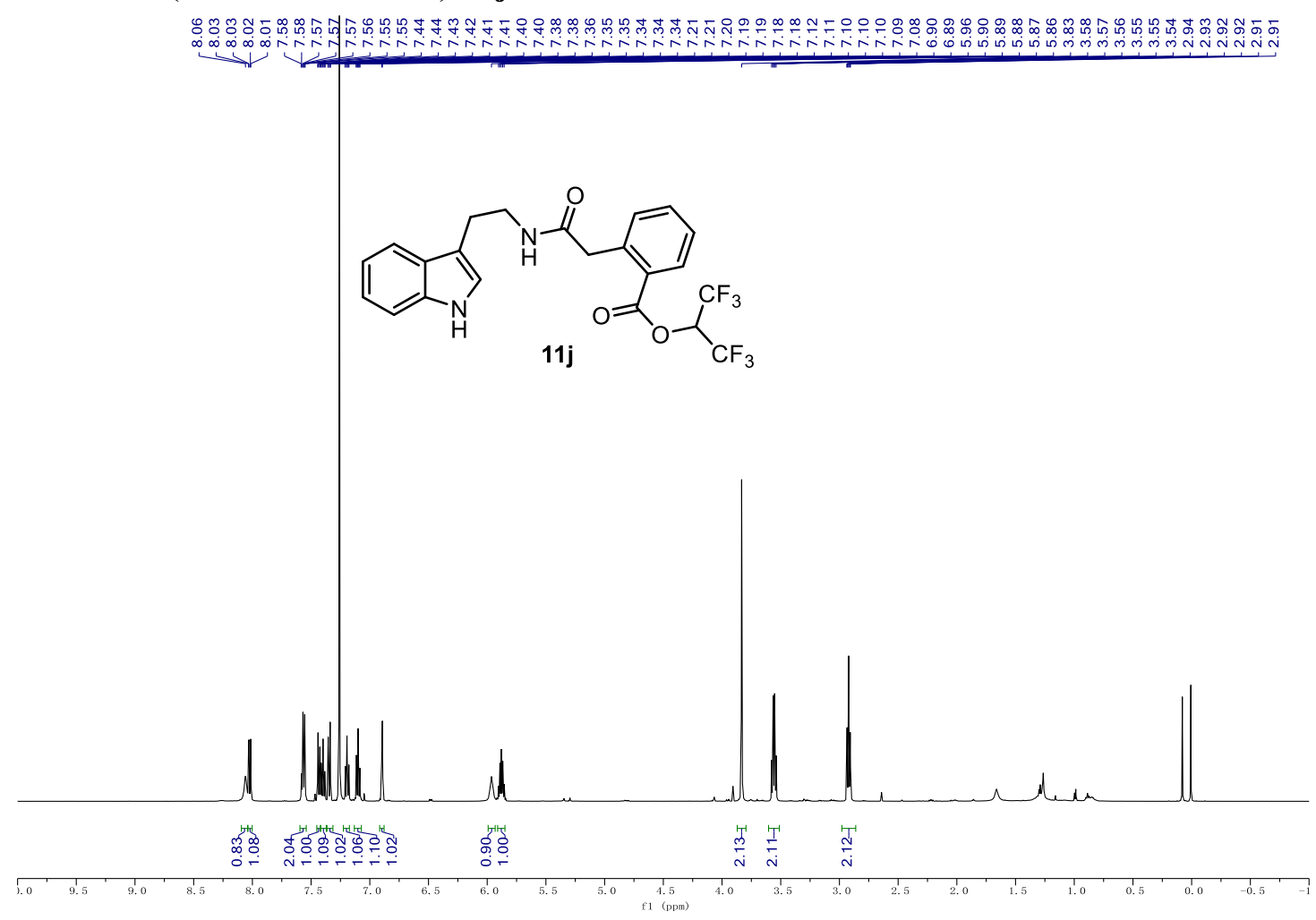


${ }^{13} \mathbf{C}$ NMR (126 MHz, $\left.\mathrm{CDCl}_{3}\right) \mathbf{1 1} \mathbf{j}$.

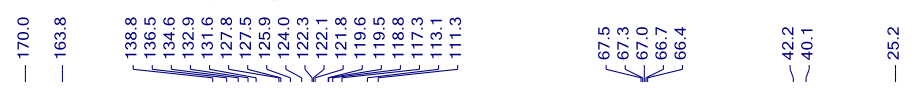
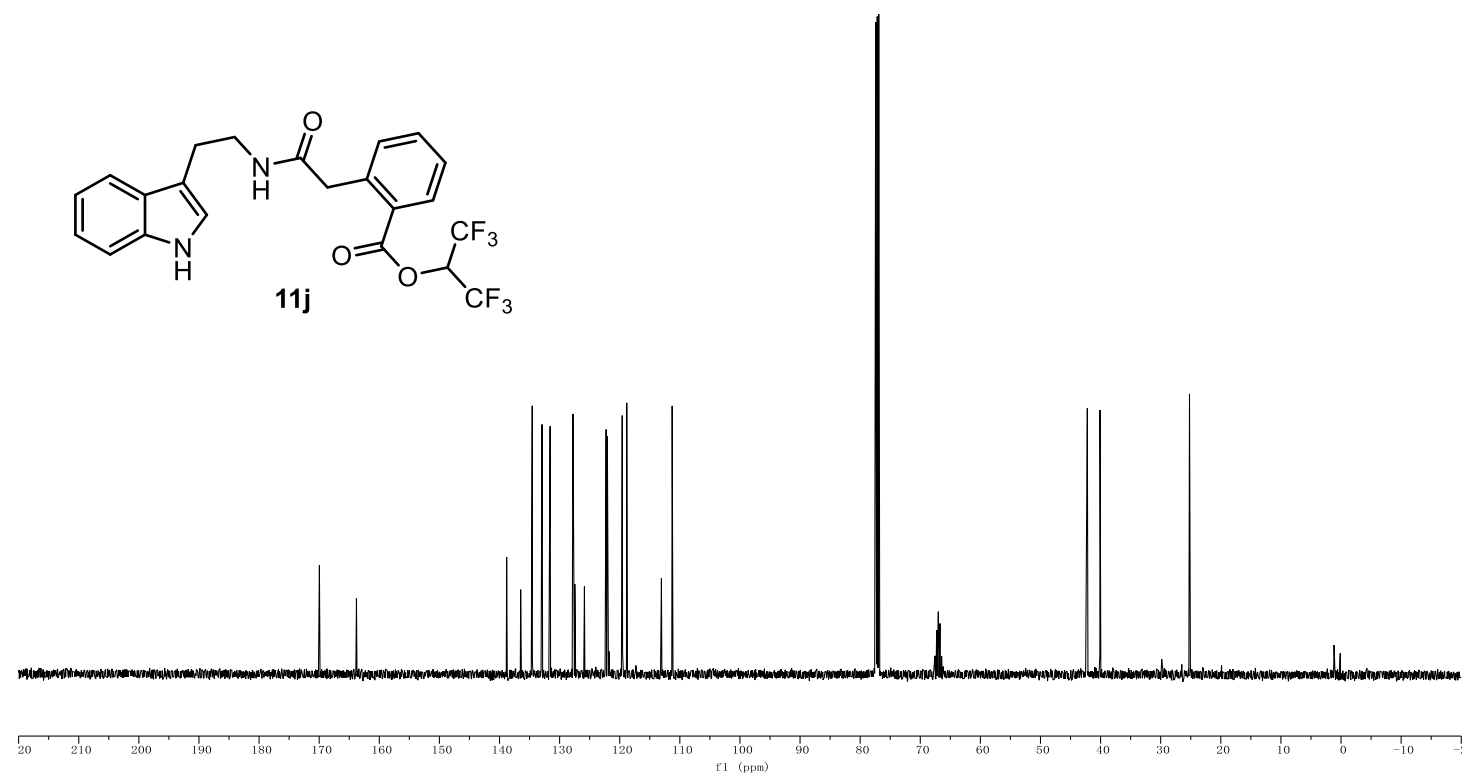

${ }^{19}$ F NMR $\left(471 \mathrm{MHz}, \mathrm{CDCl}_{3}\right) \mathbf{1 1} \mathbf{j}$.

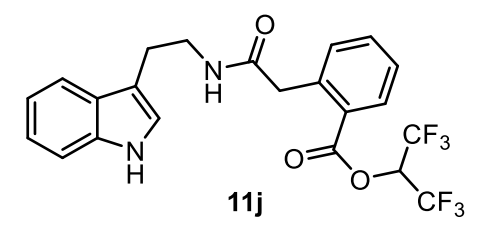


${ }^{1} \mathbf{H}$ NMR $\left(500 \mathrm{MHz}, \mathrm{CDCl}_{3}\right) \mathbf{1 1 k}$.

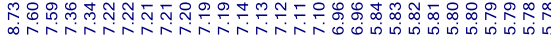

${ }_{11 \mathrm{k}}$
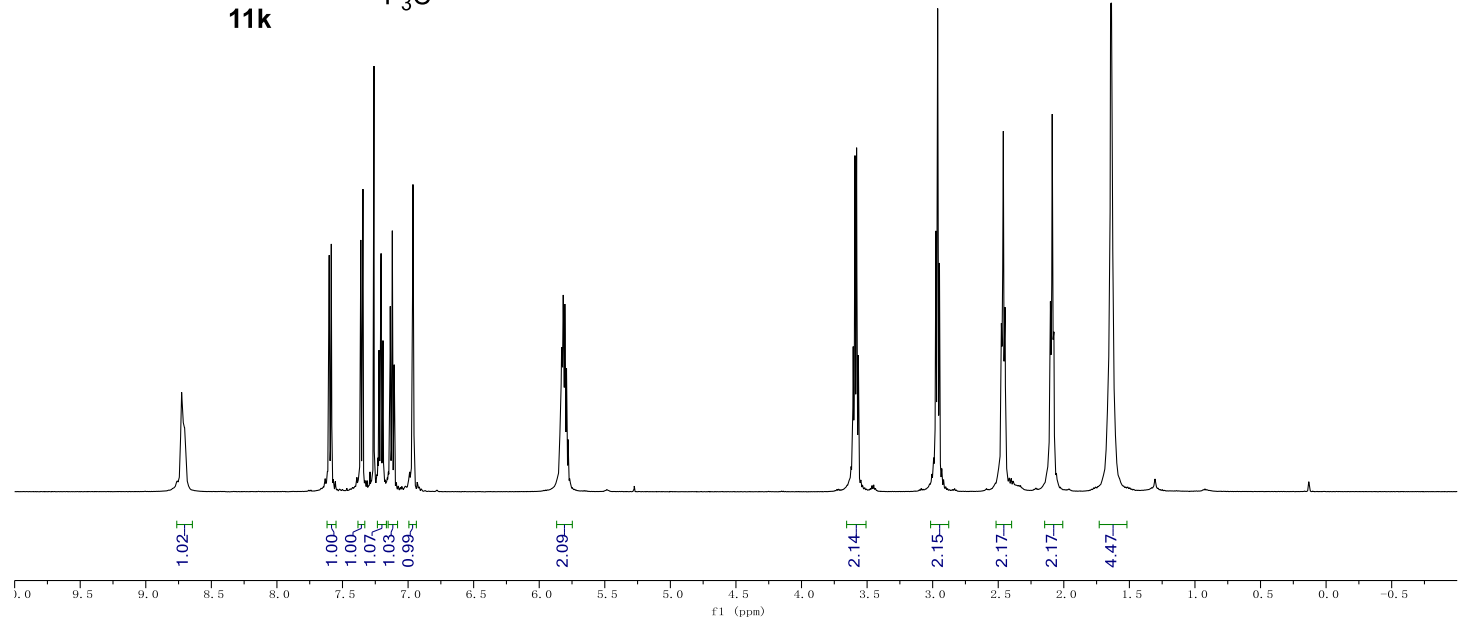

${ }^{13} \mathbf{C ~ N M R}\left(126 \mathrm{MHz}, \mathrm{CDCl}_{3}\right) \mathbf{1 1 k}$.

in

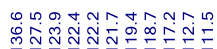

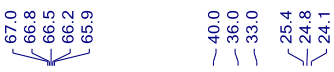

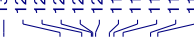

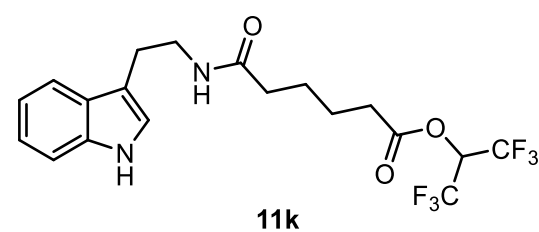
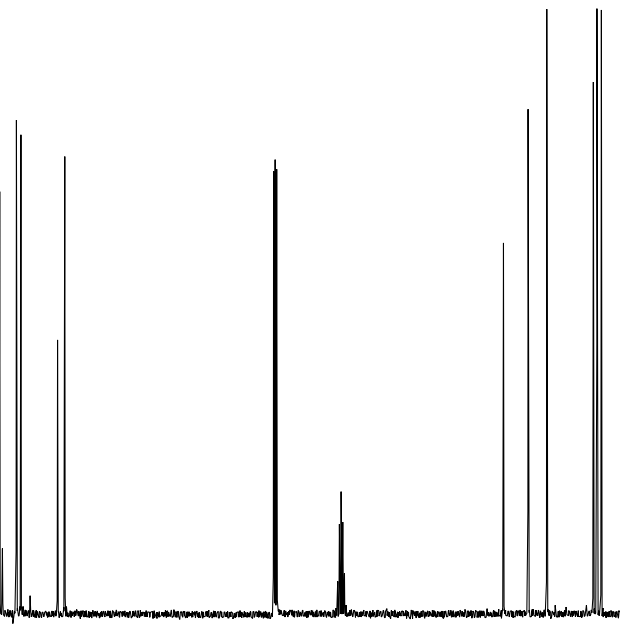

$20 \quad 210$ 
${ }^{19}$ F NMR (565 MHz, $\left.\mathrm{CDCl}_{3}\right)$ 11k.

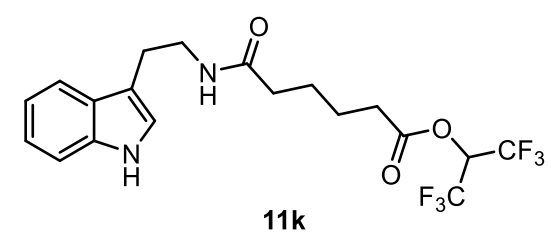

$11 \mathrm{k}$

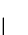

${ }^{1} \mathbf{H}$ NMR $\left(500 \mathrm{MHz}, \mathrm{CDCl}_{3}\right) \mathbf{1 1 1}$.

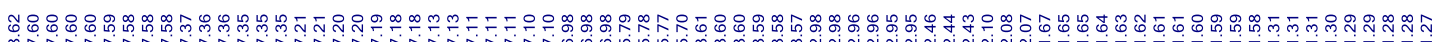<smiles>O=C(CCCCCC(=O)OC(F)(F)F)NCCc1c[nH]c2ccccc12</smiles>

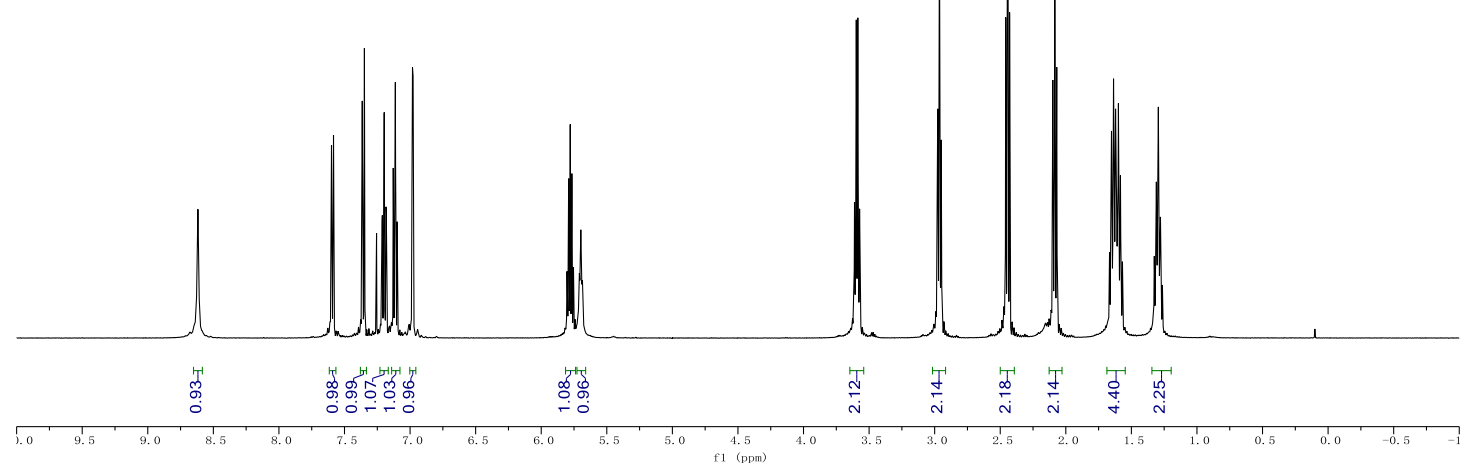


${ }^{13}$ C NMR (126 MHz, $\left.\mathrm{CDCl}_{3}\right) \mathbf{1 1 1}$.

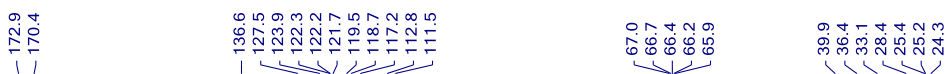

$\overbrace{\mathrm{H}}^{\mathrm{N}} \overbrace{\mathrm{O}}^{\mathrm{N}}$
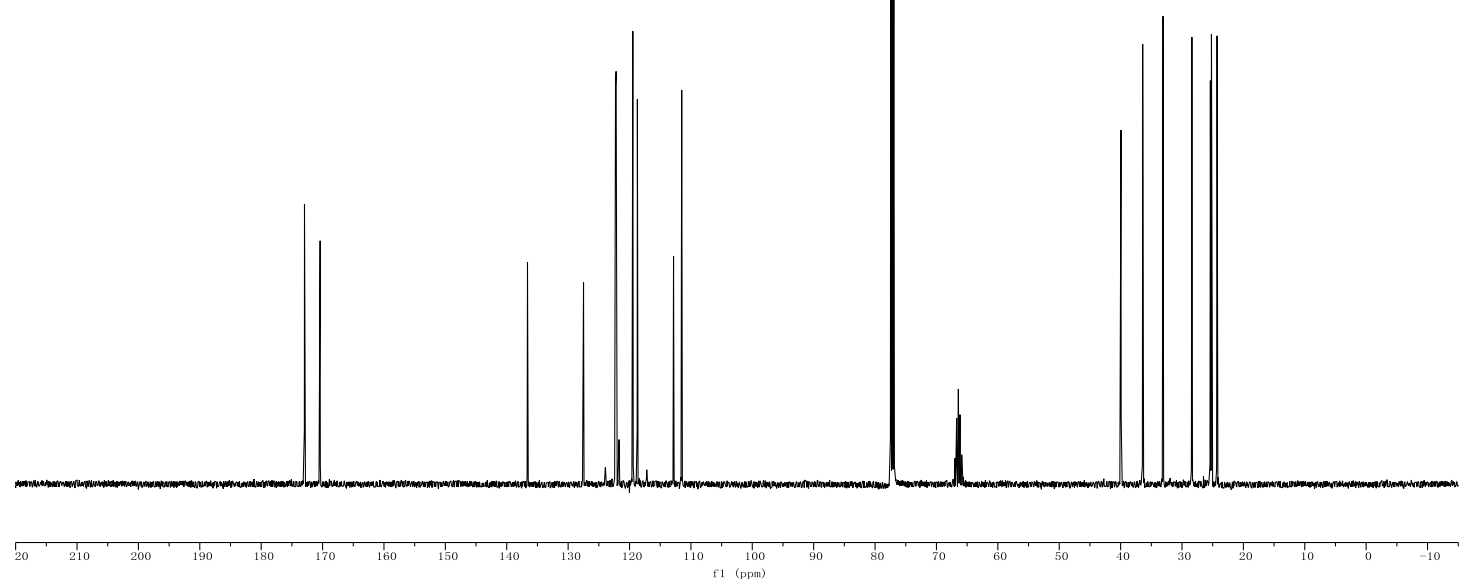

${ }^{19}$ F NMR (565 MHz, $\left.\mathrm{CDCl}_{3}\right) \mathbf{1 1 1}$.

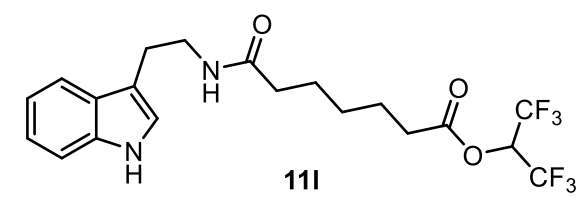

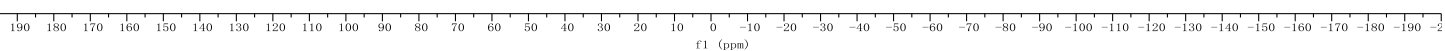


${ }^{1} \mathbf{H}$ NMR $\left(500 \mathrm{MHz}, \mathrm{CDCl}_{3}\right) \mathbf{1 1} \mathbf{m}$.

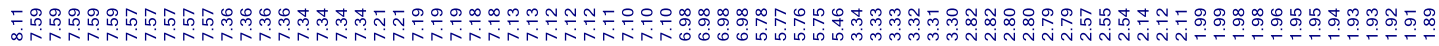<smiles>O=C(CCCC(=O)OC(F)(F)F)NCCCc1c[nH]c2ccccc12</smiles>

$11 \mathrm{~m}$
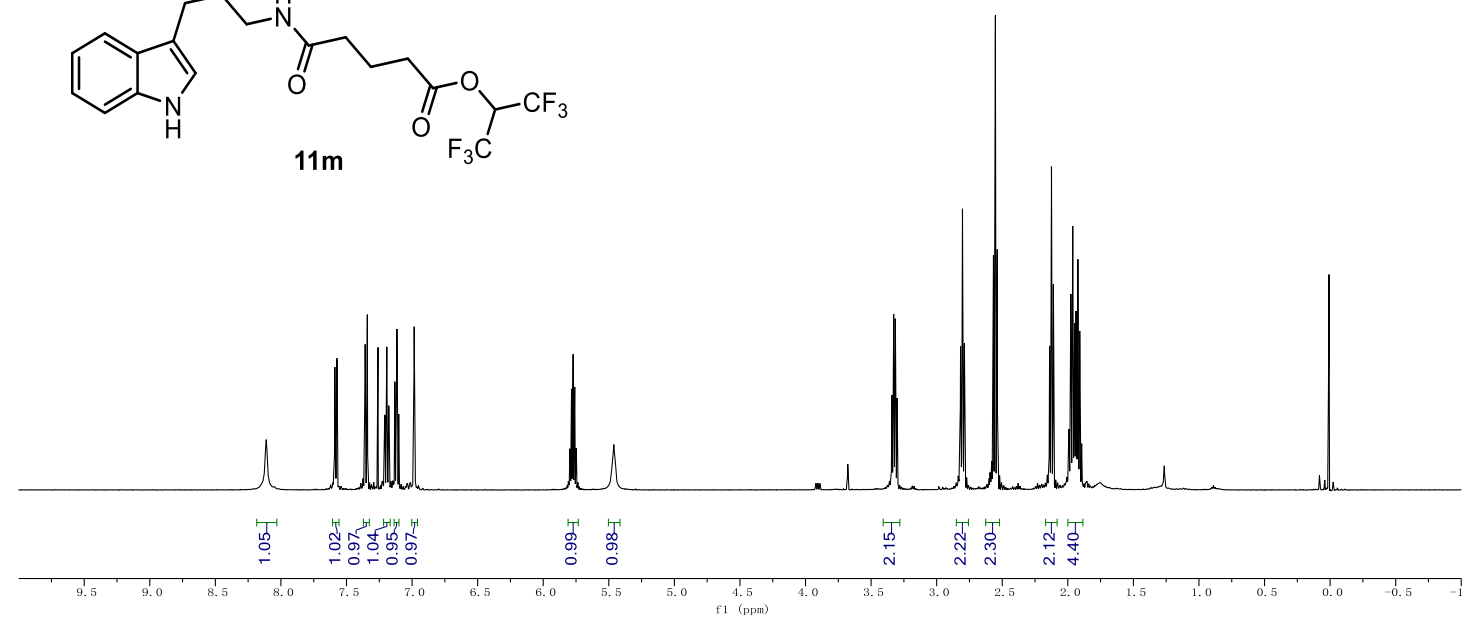

${ }^{13} \mathbf{C ~ N M R}\left(126 \mathrm{MHz}, \mathrm{CDCl}_{3}\right) \mathbf{1 1} \mathbf{m}$.

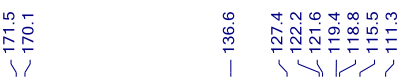

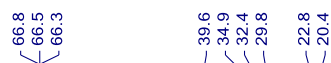

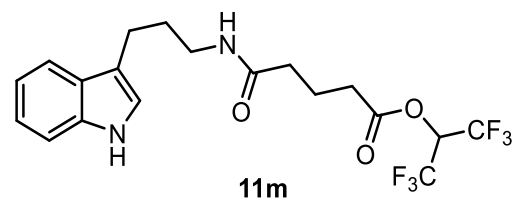

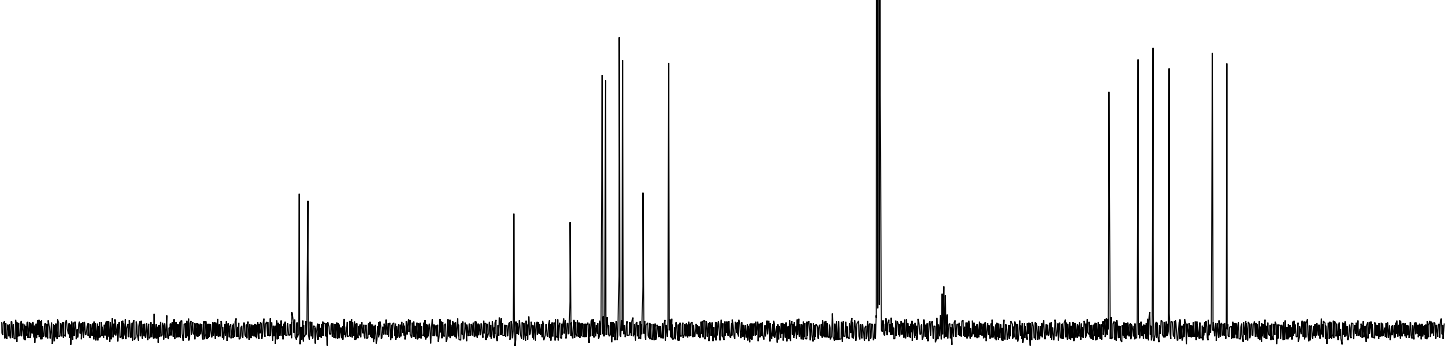

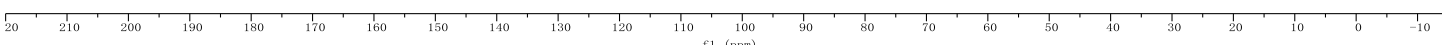


${ }^{19}$ F NMR (471 MHz, $\left.\mathrm{CDCl}_{3}\right) \mathbf{1 1 m}$.

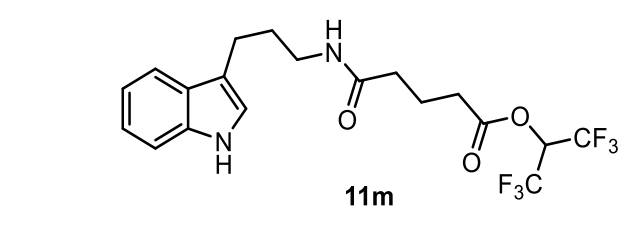

$11 \mathrm{~m}$

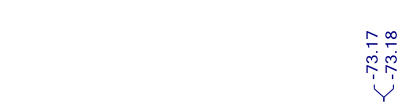

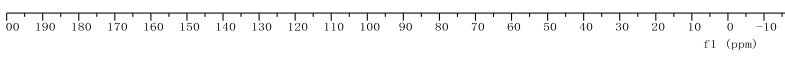

${ }^{1} \mathbf{H}$ NMR (500 MHz, $\left.\mathrm{CDCl}_{3}\right) \mathbf{1 1 n}$.

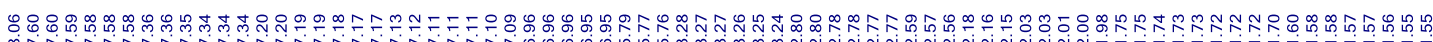

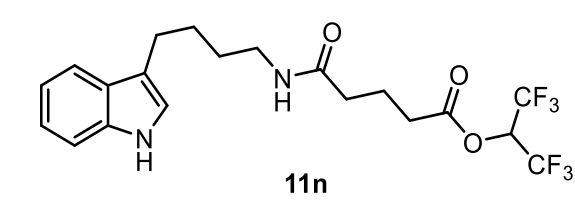


${ }^{13} \mathrm{C}$ NMR (126 MHz, $\left.\mathrm{CDCl}_{3}\right) \mathbf{1 1 n}$.

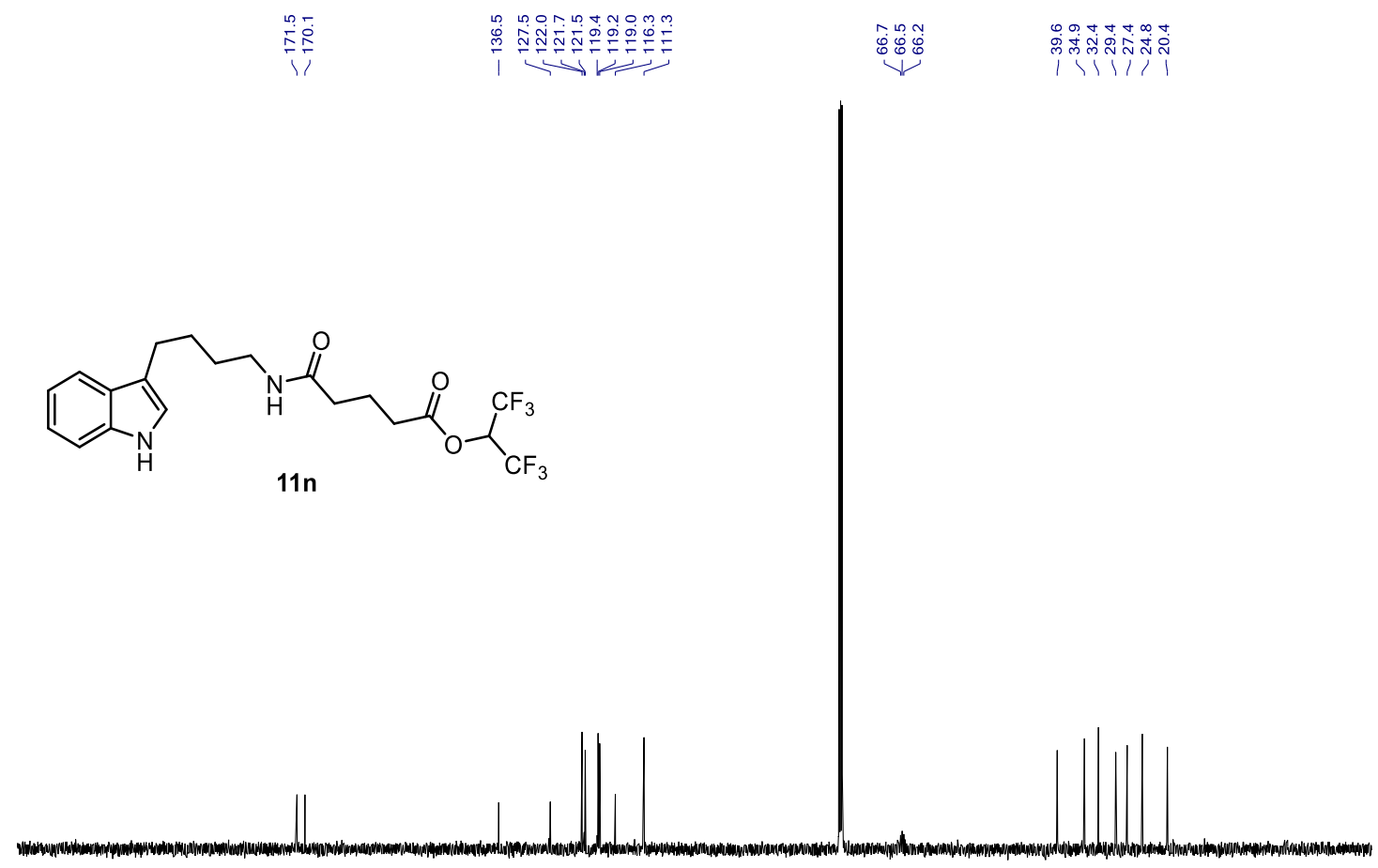

${ }^{19}$ F NMR (471 MHz, $\left.\mathrm{CDCl}_{3}\right) \mathbf{1 1 n}$.

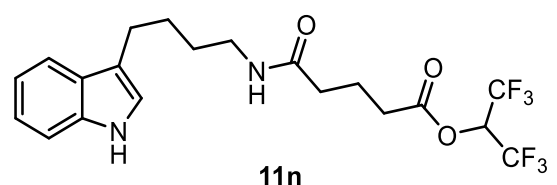


${ }^{1} \mathbf{H}$ NMR (500 MHz, $\left.\mathrm{CDCl}_{3}\right) 110$.

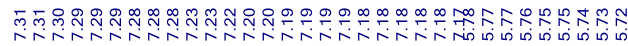<smiles>CC(C)(CC(=O)NCCc1ccccc1)CC(=O)OC(C(F)(F)F)C(F)(F)F</smiles>

${ }^{13}$ C NMR (126 MHz, $\left.\mathrm{CDCl}_{3}\right) \mathbf{1 1 0}$.
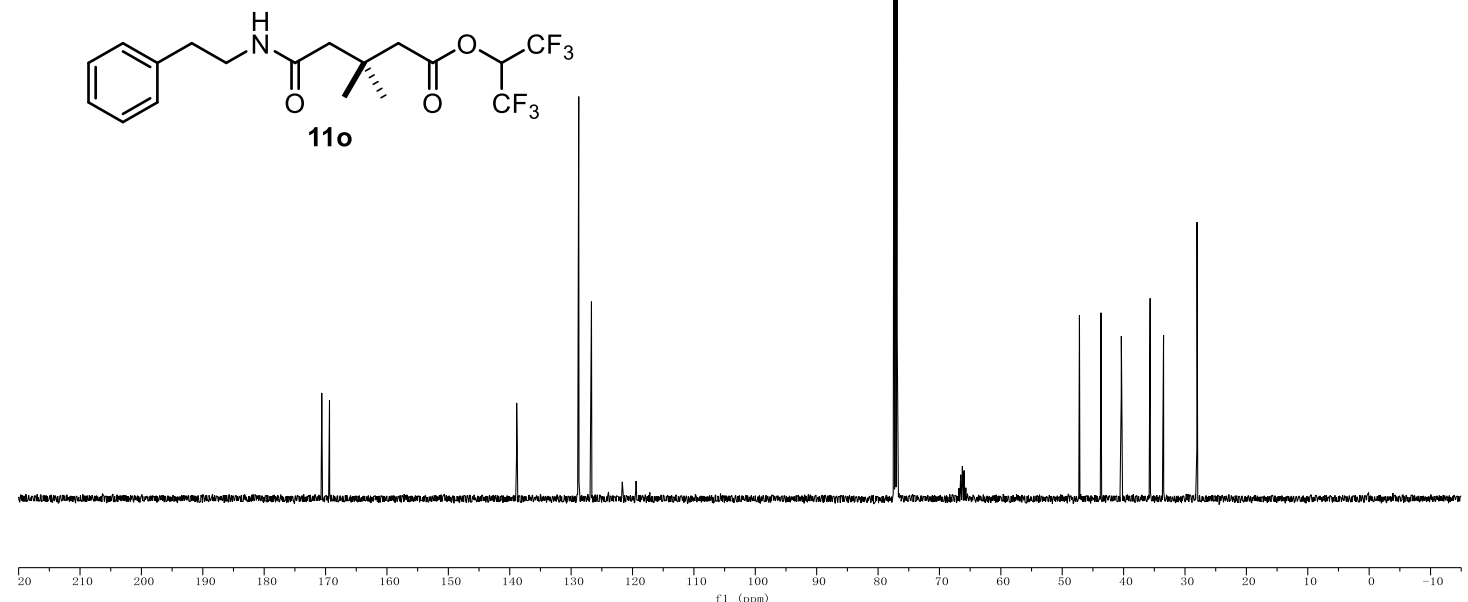
${ }^{19}$ F NMR (471 MHz, $\left.\mathrm{CDCl}_{3}\right) 11 \mathrm{o}$.<smiles>CC(C)(CC(=O)NCCc1ccccc1)CC(=O)OC(C(F)(F)F)C(F)(F)F</smiles>

${ }^{1} \mathbf{H}$ NMR (500 MHz, $\left.\mathrm{CDCl}_{3}\right) \mathbf{1 1 p .}$

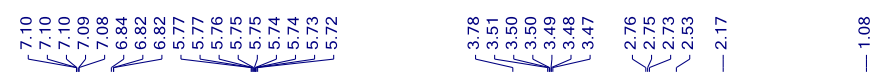

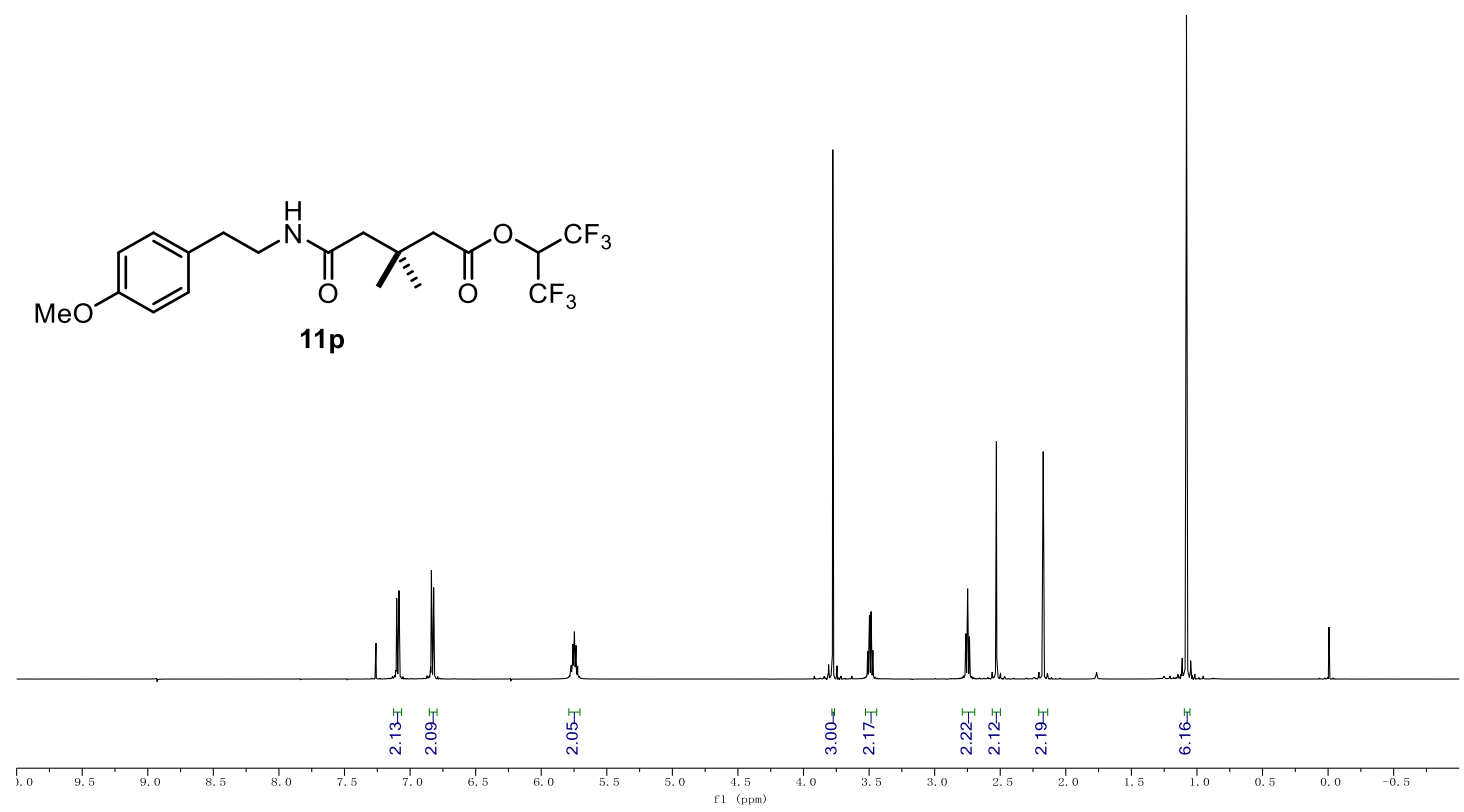


${ }^{13} \mathrm{C}$ NMR (126 MHz, $\left.\mathrm{CDCl}_{3}\right) \mathbf{1 1 p}$.

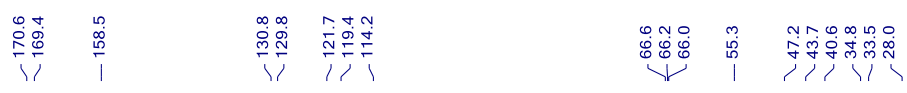

$\overbrace{\mathrm{MeO}}^{\mathrm{H}} \prod_{11 \mathrm{p}}^{\mathrm{O}} \mathrm{O}_{\mathrm{C}}^{\mathrm{CF}} \mathrm{CF}_{3}$

${ }^{19}$ F NMR (471 MHz, $\left.\mathrm{CDCl}_{3}\right) \mathbf{1 1 p .}$

$\overbrace{\mathrm{MeO}}^{\mathrm{N}} \prod_{11 \mathrm{p}}^{\mathrm{O}} \mathrm{Y}_{\mathrm{CF}_{3}}^{\mathrm{CF}_{3}}$ 
${ }^{1} \mathbf{H}$ NMR (500 MHz, $\left.\mathrm{CDCl}_{3}\right)$ 11q.

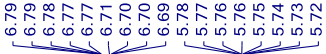

$\overbrace{11 q}^{\mathrm{MeO}}$

${ }^{13} \mathbf{C}$ NMR $\left(126 \mathrm{MHz}, \mathrm{CDCl}_{3}\right) \mathbf{1 1 q}$.

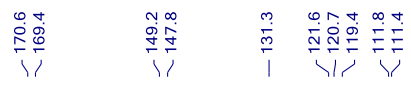

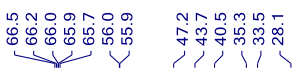

${ }_{\mathrm{MeO}}^{\mathrm{MeO}}$

$11 q$

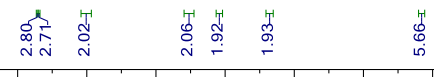

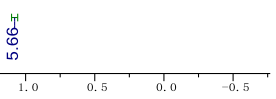

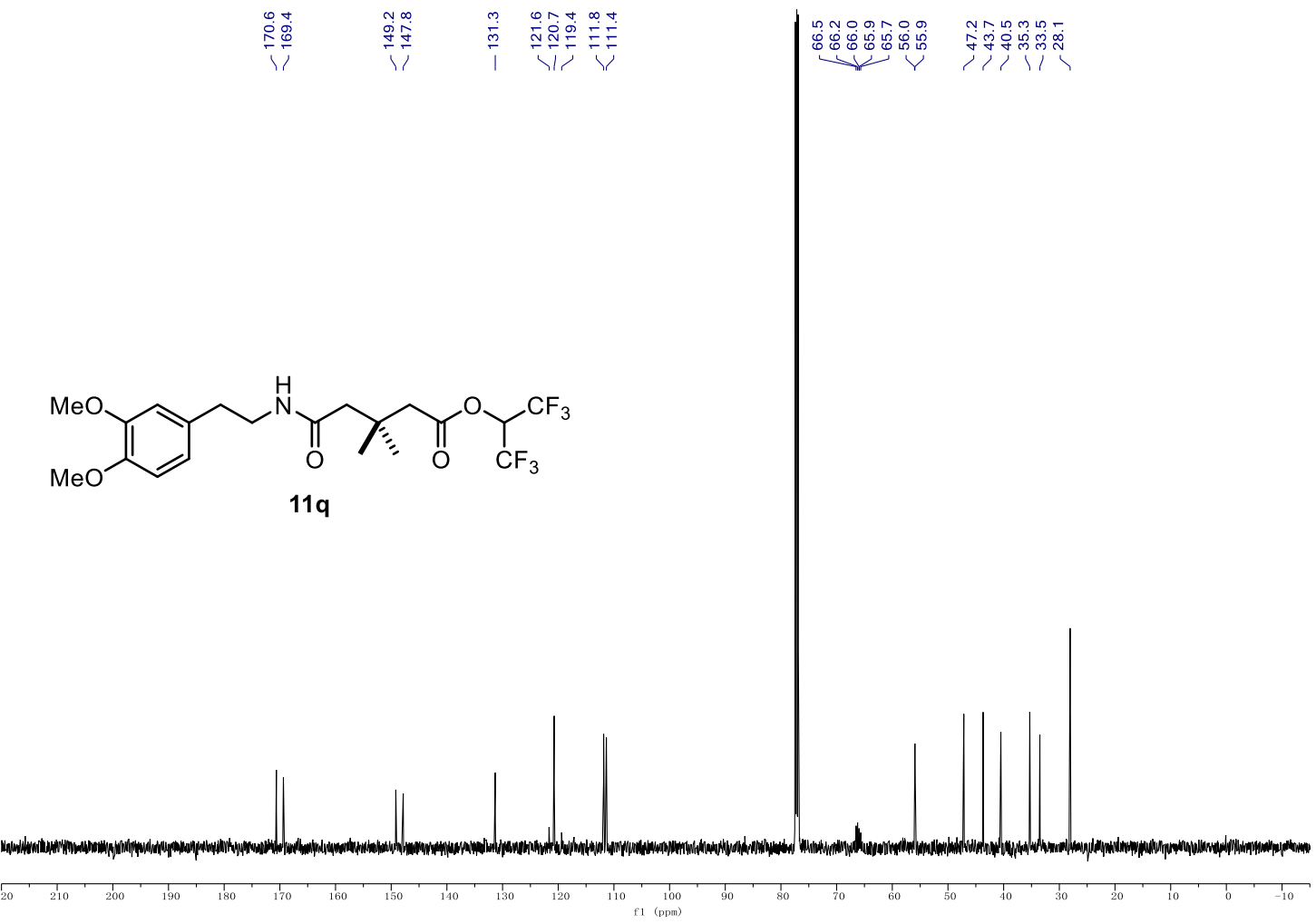


${ }^{19}$ F NMR (471 MHz, $\left.\mathrm{CDCl}_{3}\right)$ 11q.
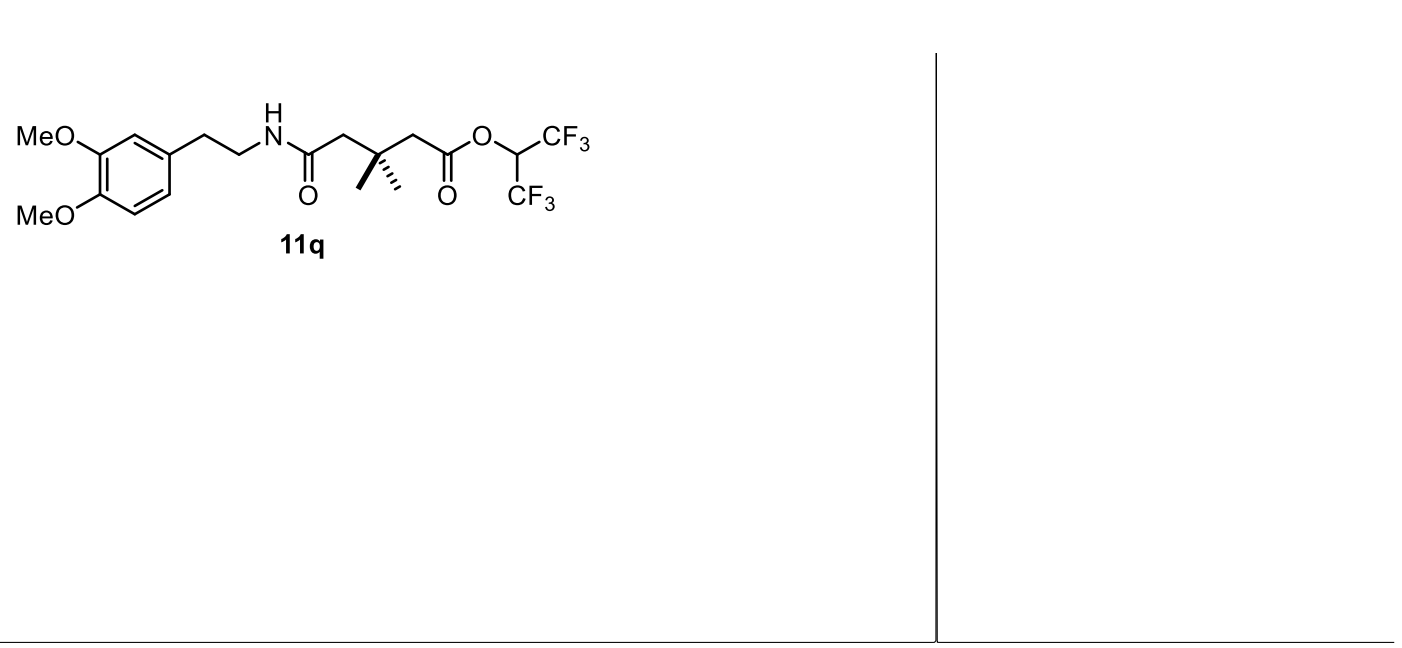

${ }^{1} \mathbf{H}$ NMR $\left(500 \mathrm{MHz}, \mathrm{CDCl}_{3}\right) \mathbf{1 1} \mathbf{r}$.

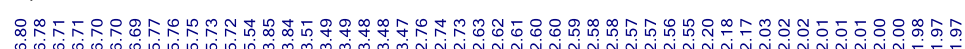

它<smiles>COc1ccc(CCNC(=O)CCCC(=O)OC(C(F)(F)F)C(F)(F)F)cc1OC</smiles>

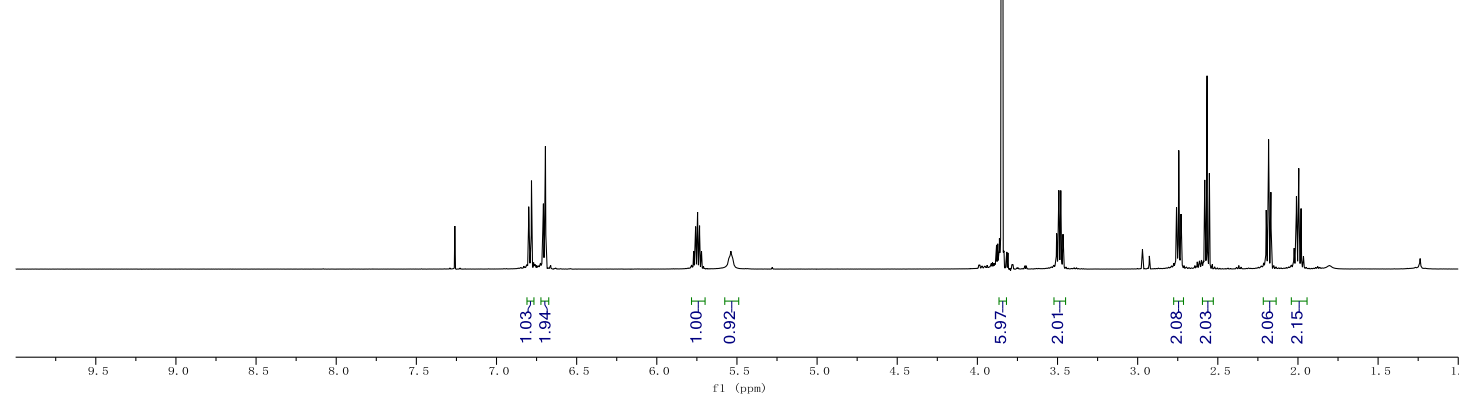


${ }^{13}$ C NMR (126 MHz, $\left.\mathrm{CDCl}_{3}\right) \mathbf{1 1 r}$.

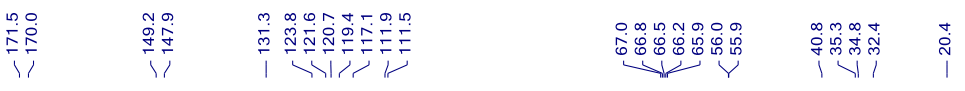<smiles>COc1ccc(CCNC(=O)CCCC(=O)OC(C(F)(F)F)C(F)(F)F)cc1OC</smiles>

$11 \mathrm{r}$

${ }^{19}$ F NMR (565 MHz, $\left.\mathrm{CDCl}_{3}\right)$ 11r.<smiles>COc1ccc(CCNC(=O)CCCC(=O)OC(C(F)(F)F)C(F)(F)F)cc1OC</smiles> 
${ }^{1} \mathbf{H}$ NMR (600 MHz, $\left.\mathrm{CDCl}_{3}\right) \mathbf{1 1 s}$.

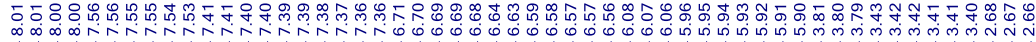

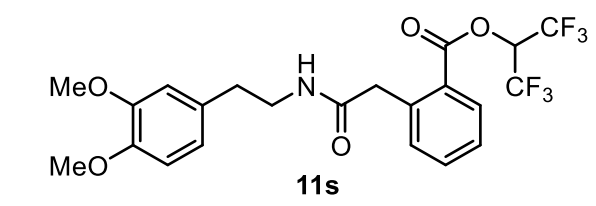

${ }^{13}$ C NMR (151 MHz, $\left.\mathrm{CDCl}_{3}\right) \mathbf{1 1 s}$.

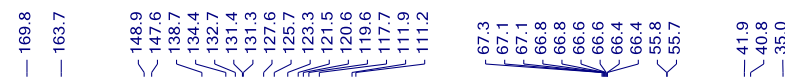

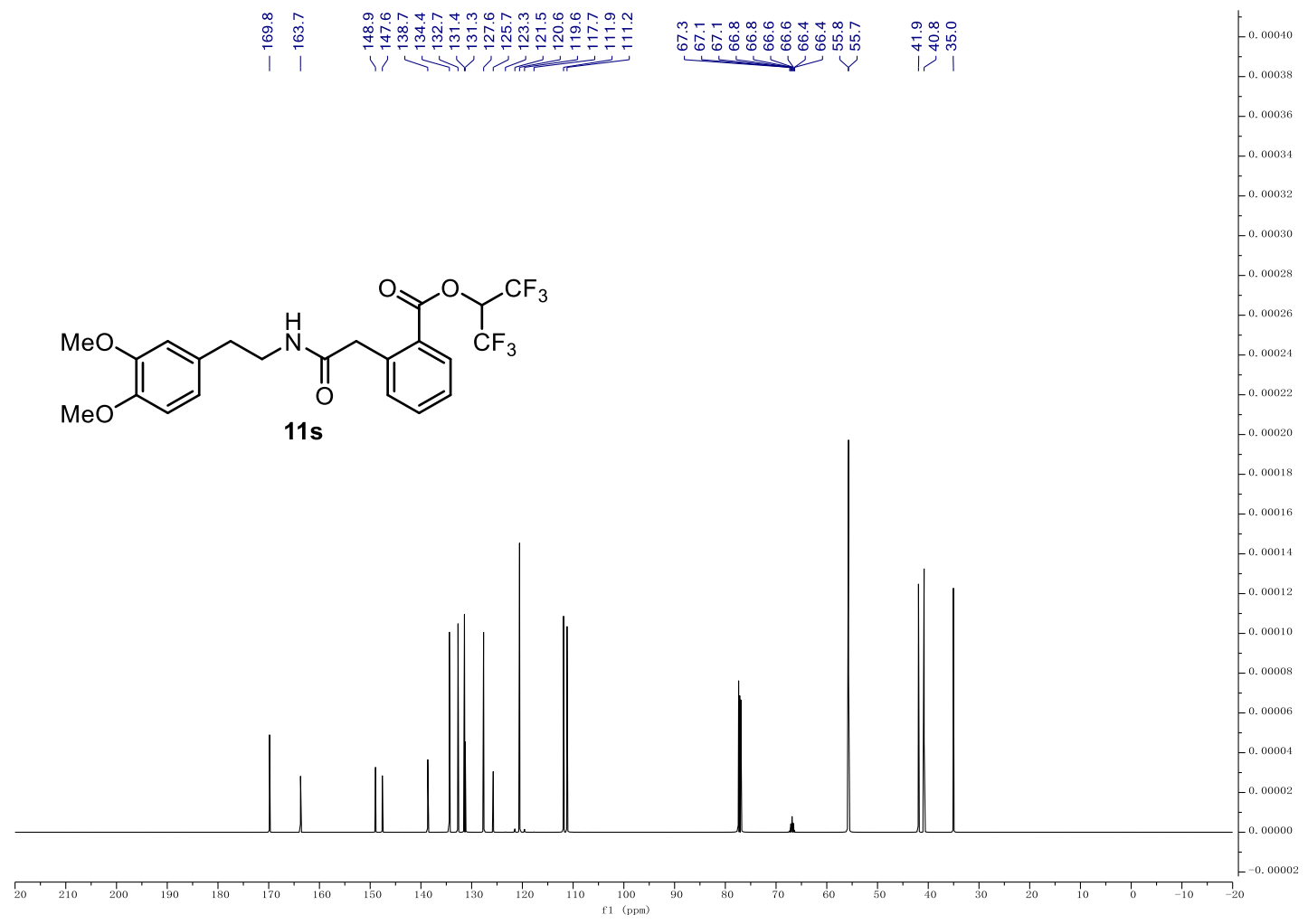

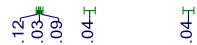

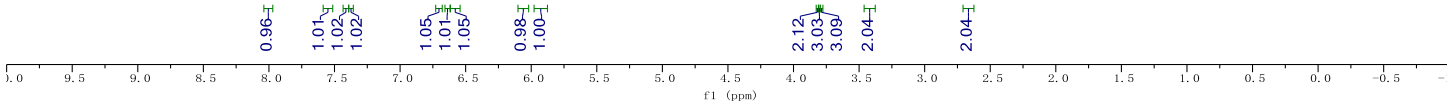


${ }^{19}$ F NMR (565 MHz, $\left.\mathrm{CDCl}_{3}\right)$ 11s.

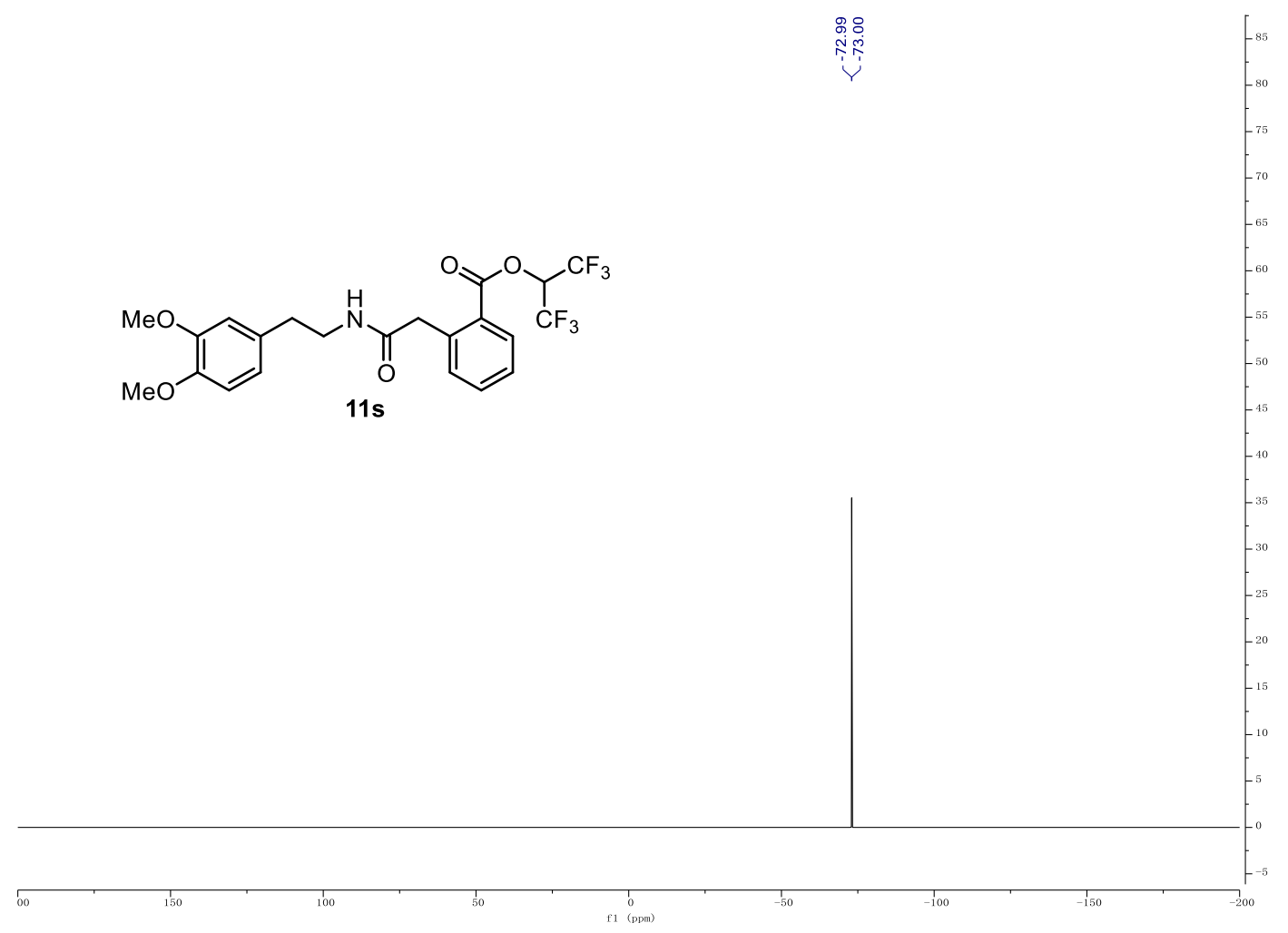

${ }^{1} \mathbf{H}$ NMR $\left(700 \mathrm{MHz}, \mathrm{CDCl}_{3}\right) \mathbf{1 1 t}$.

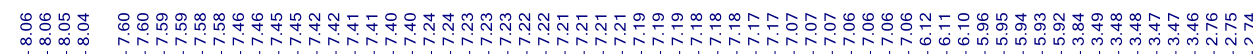<smiles>O=C(Cc1ccccc1C(=O)OC(C(F)(F)F)C(F)(F)F)NCCc1ccccc1</smiles>

$11 t$

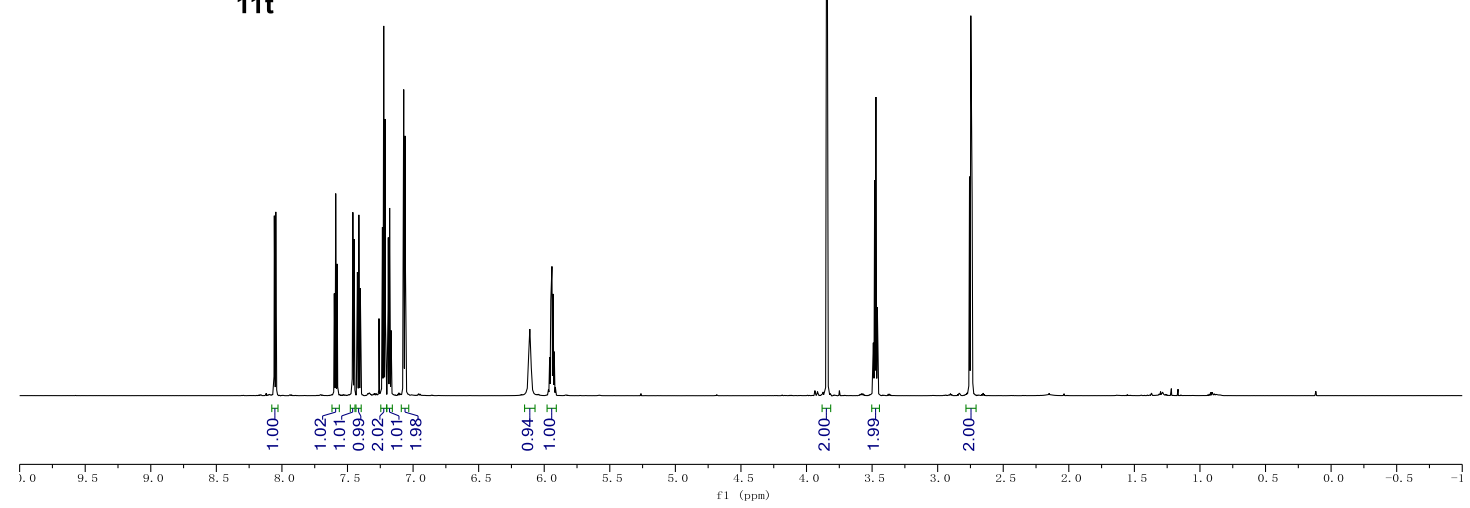


${ }^{13} \mathbf{C}$ NMR (176 MHz, $\left.\mathrm{CDCl}_{3}\right) \mathbf{1 1 t}$.

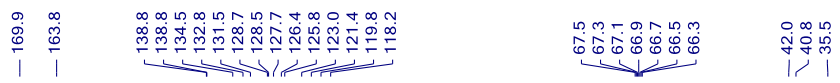

$\left.\underbrace{N}_{11 t}\right|_{1} ^{C}$
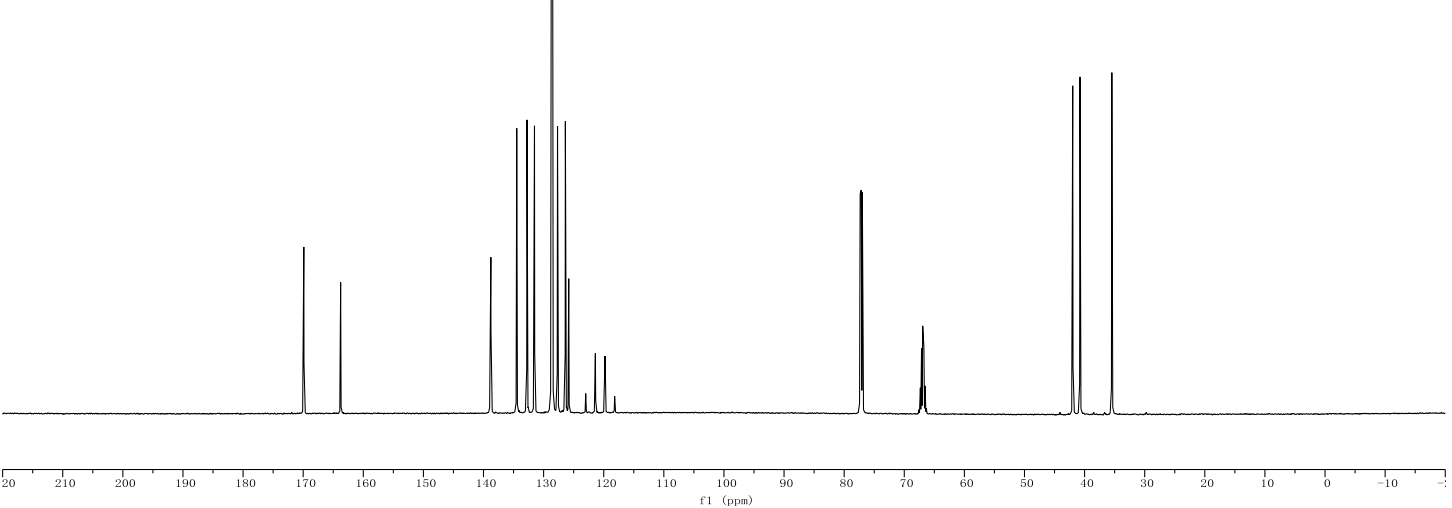

${ }^{19}$ F NMR (376 MHz, $\left.\mathrm{CDCl}_{3}\right) \mathbf{1 1 t}$.<smiles>O=C(Cc1ccccc1C(=O)OC(C(F)(F)F)C(F)(F)F)NCCc1ccccc1</smiles>

$11 \mathrm{t}$ 
${ }^{1} \mathbf{H}$ NMR (700 MHz, $\left.\mathrm{CDCl}_{3}\right) \mathbf{1 1 u}$.

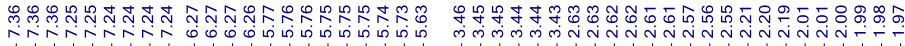<smiles>O=C(CCCC(=O)OC(C(F)(F)F)C(F)(F)F)NCCc1ccoc1</smiles>

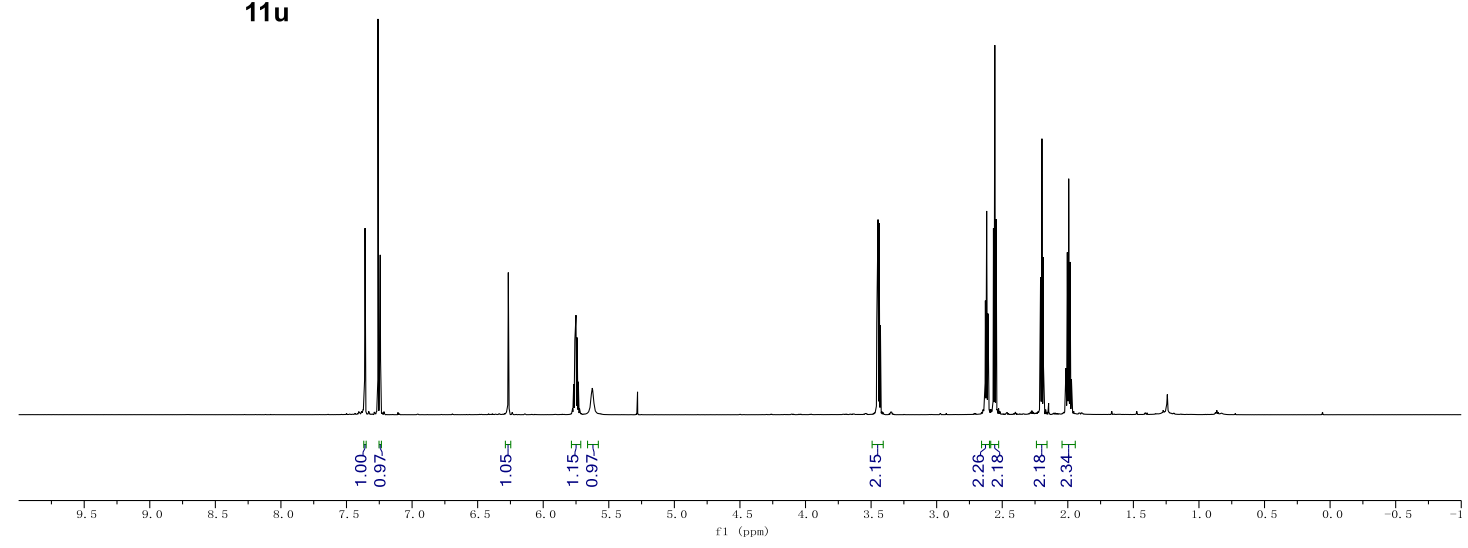

${ }^{13} \mathrm{C}$ NMR (176 MHz, $\left.\mathrm{CDCl}_{3}\right) \mathbf{1 1 u}$.

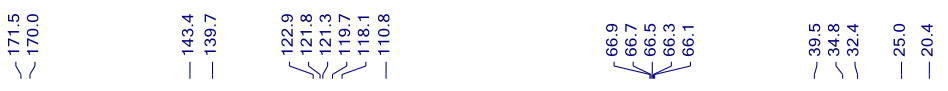<smiles>O=C(CCCC(=O)OC(C(F)(F)F)C(F)(F)F)NCCc1ccoc1</smiles>

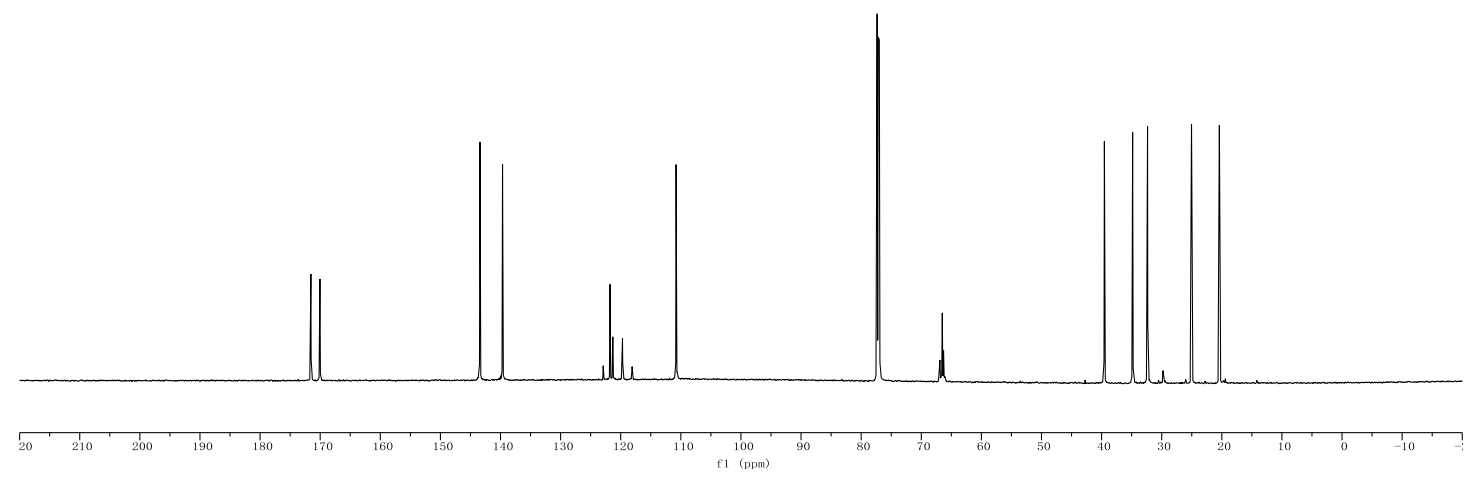


${ }^{19}$ F NMR (565 MHz, $\left.\mathrm{CDCl}_{3}\right) \mathbf{1 1 u}$.<smiles>O=C(CCCC(=O)OC(C(F)(F)F)C(F)(F)F)NCCc1ccoc1</smiles>$$
\text { che }
$$ 
${ }^{13} \mathbf{C}$ NMR (176 MHz, $\left.\mathrm{CDCl}_{3}\right) \mathbf{1 1 v}$.

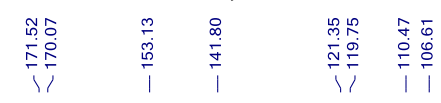

$\underbrace{-}$

ill
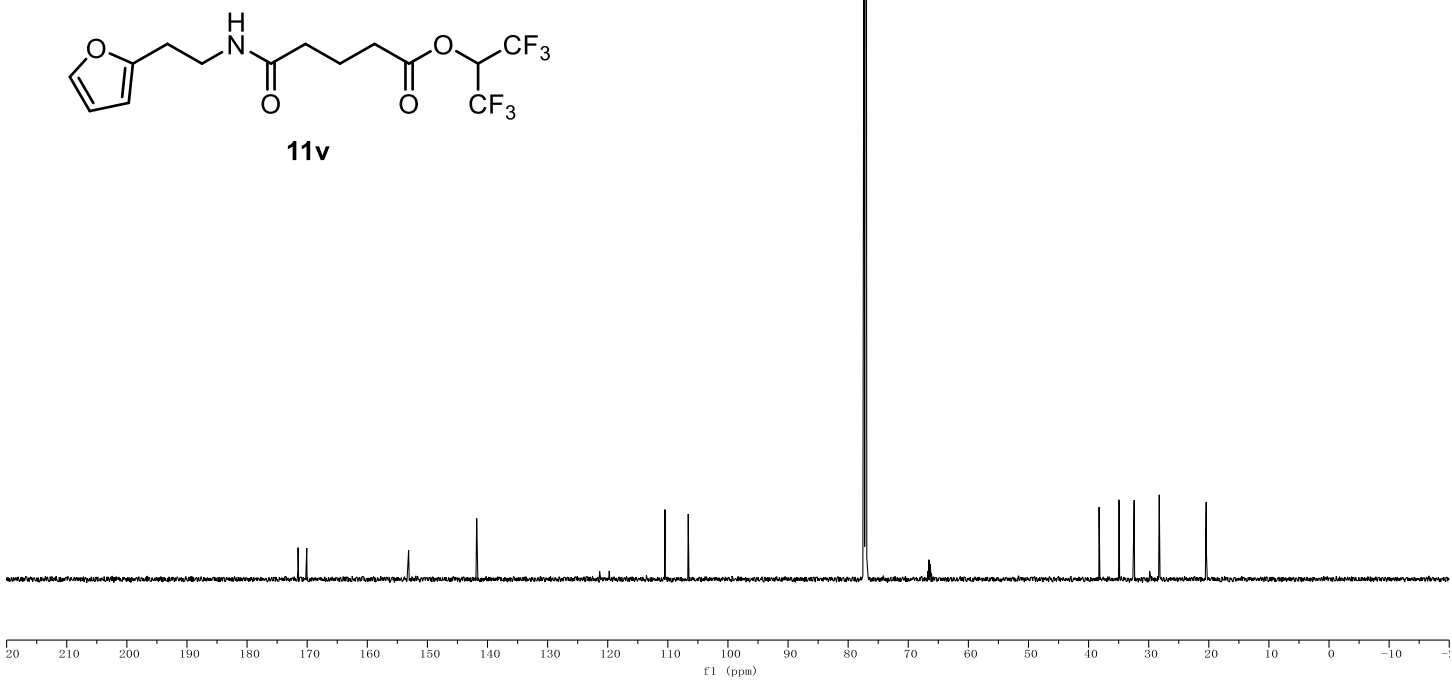

${ }^{19} \mathbf{F}$ NMR $\left(376 \mathrm{MHz}, \mathrm{CDCl}_{3}\right) \mathbf{1 1 v}$.

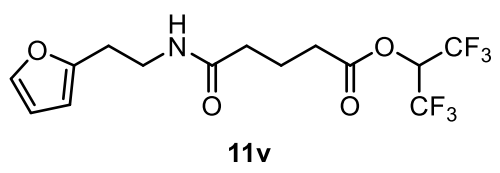

vi

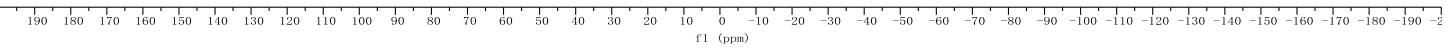

S152 
${ }^{1} \mathbf{H}$ NMR (500 MHz, $\left.\mathrm{CDCl}_{3}\right) \mathbf{1 1 w}$.

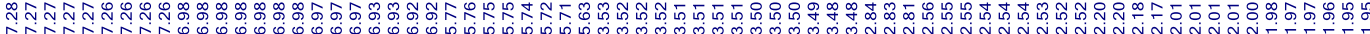<smiles>O=C(CCCC(=O)OC(C(F)(F)F)C(F)(F)F)NCCc1ccsc1</smiles>

${ }^{13} \mathbf{C}$ NMR (126 MHz, $\left.\mathrm{CDCl}_{3}\right) \mathbf{1 1 w}$.

每<smiles>O=C(CCCC(=O)OC(C(F)(F)F)C(F)(F)F)NCCc1ccsc1</smiles>
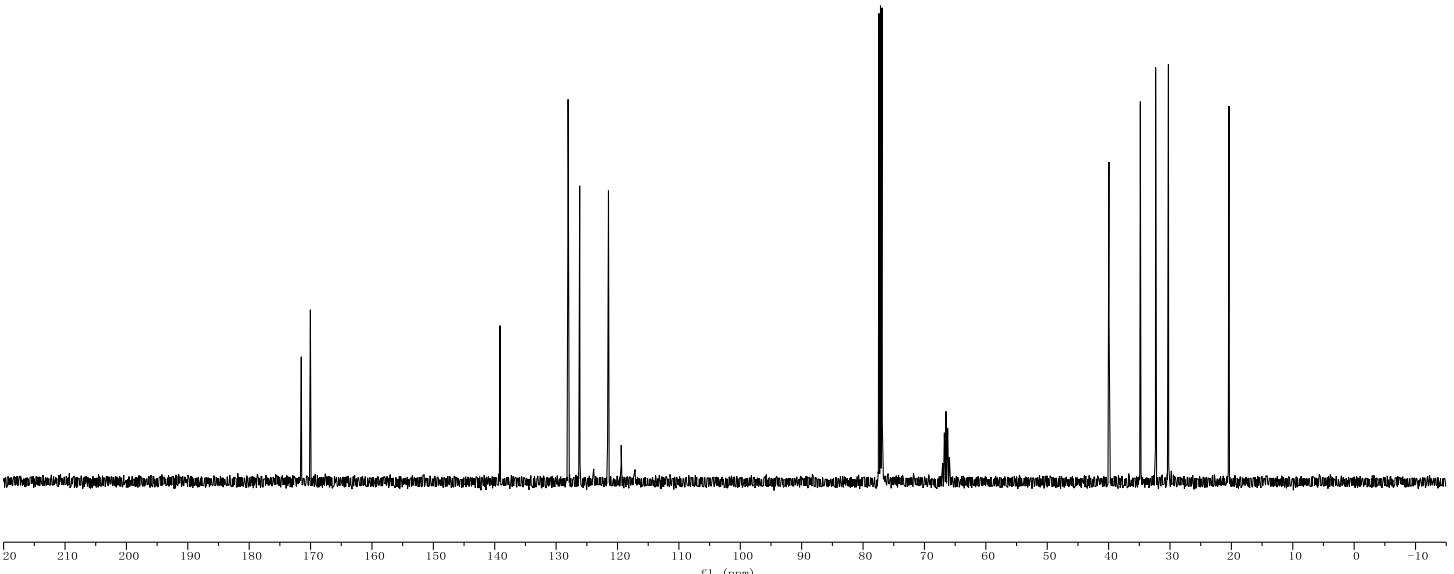
${ }^{19}$ F NMR (471 MHz, $\left.\mathrm{CDCl}_{3}\right) \mathbf{1 1 w}$.

(1)

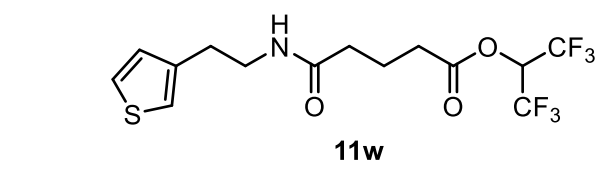

${ }^{1} \mathbf{H}$ NMR $\left(700 \mathrm{MHz}, \mathrm{CDCl}_{3}\right) \mathbf{1 1 x}$.

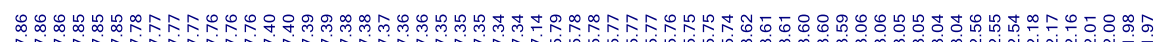

仙
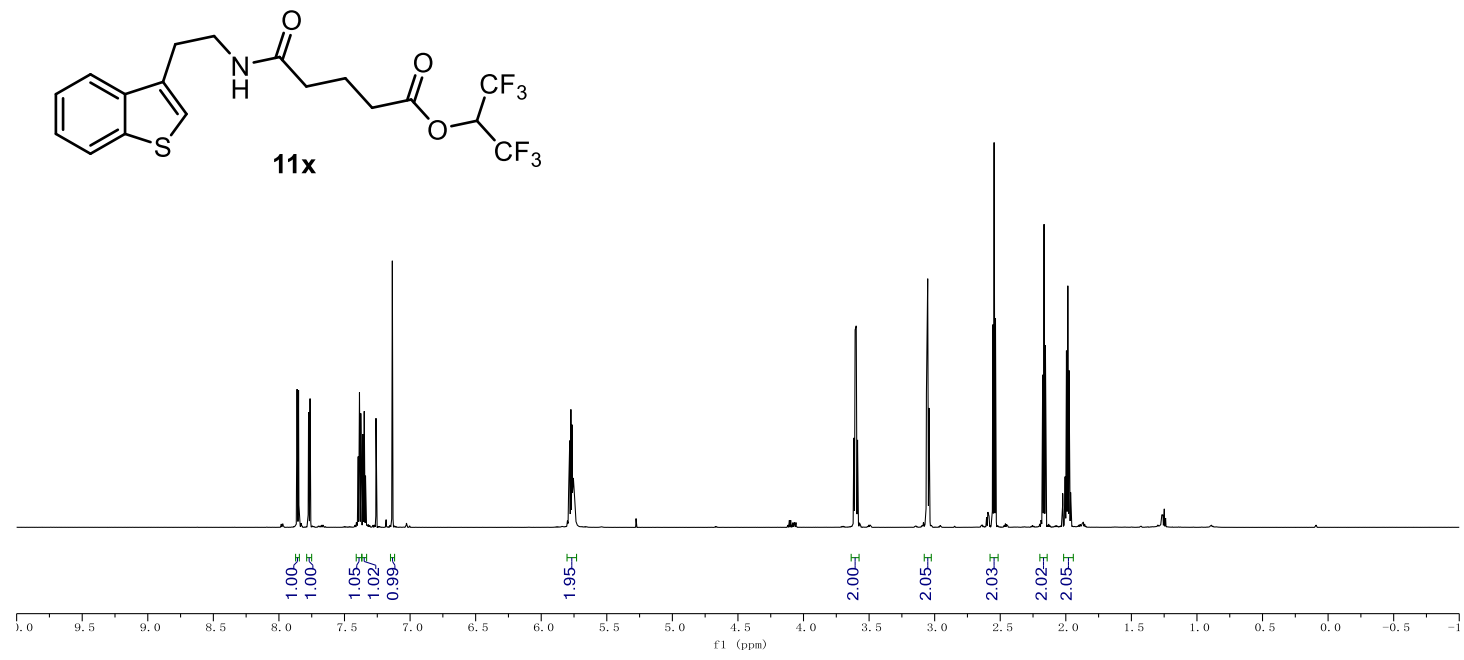
${ }^{13} \mathbf{C}$ NMR $\left(176 \mathrm{MHz}, \mathrm{CDCl}_{3}\right) \mathbf{1 1 x}$.

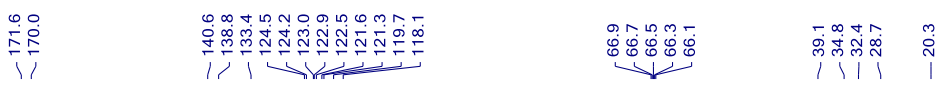<smiles>O=C(CCCC(=O)OC(C(F)(F)F)C(F)(F)F)NCCc1csc2ccccc12</smiles>

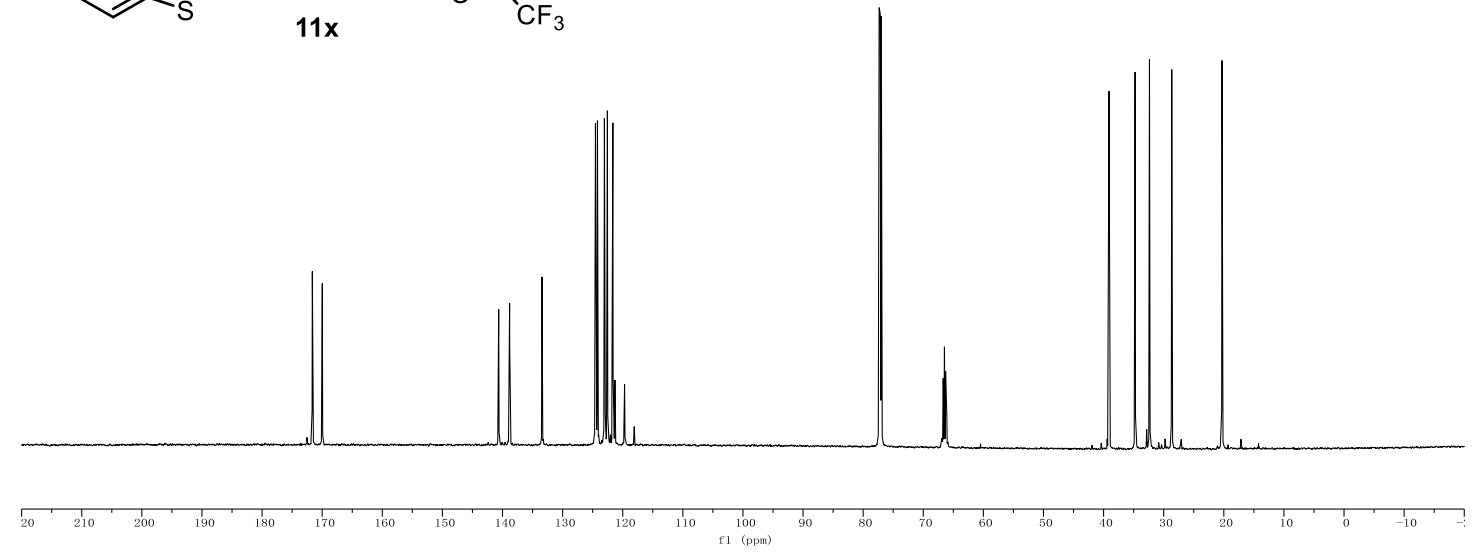

${ }^{19}$ F NMR $\left(376 \mathrm{MHz}, \mathrm{CDCl}_{3}\right) \mathbf{1 1 x}$.

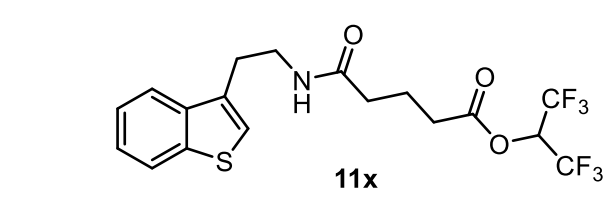

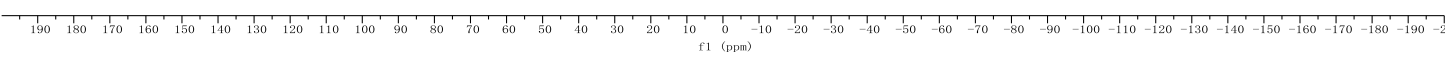


${ }^{1} \mathbf{H}$ NMR $\left(700 \mathrm{MHz}, \mathrm{CDCl}_{3}\right)$ of $\mathbf{1 1 y}$.

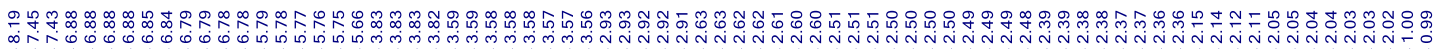

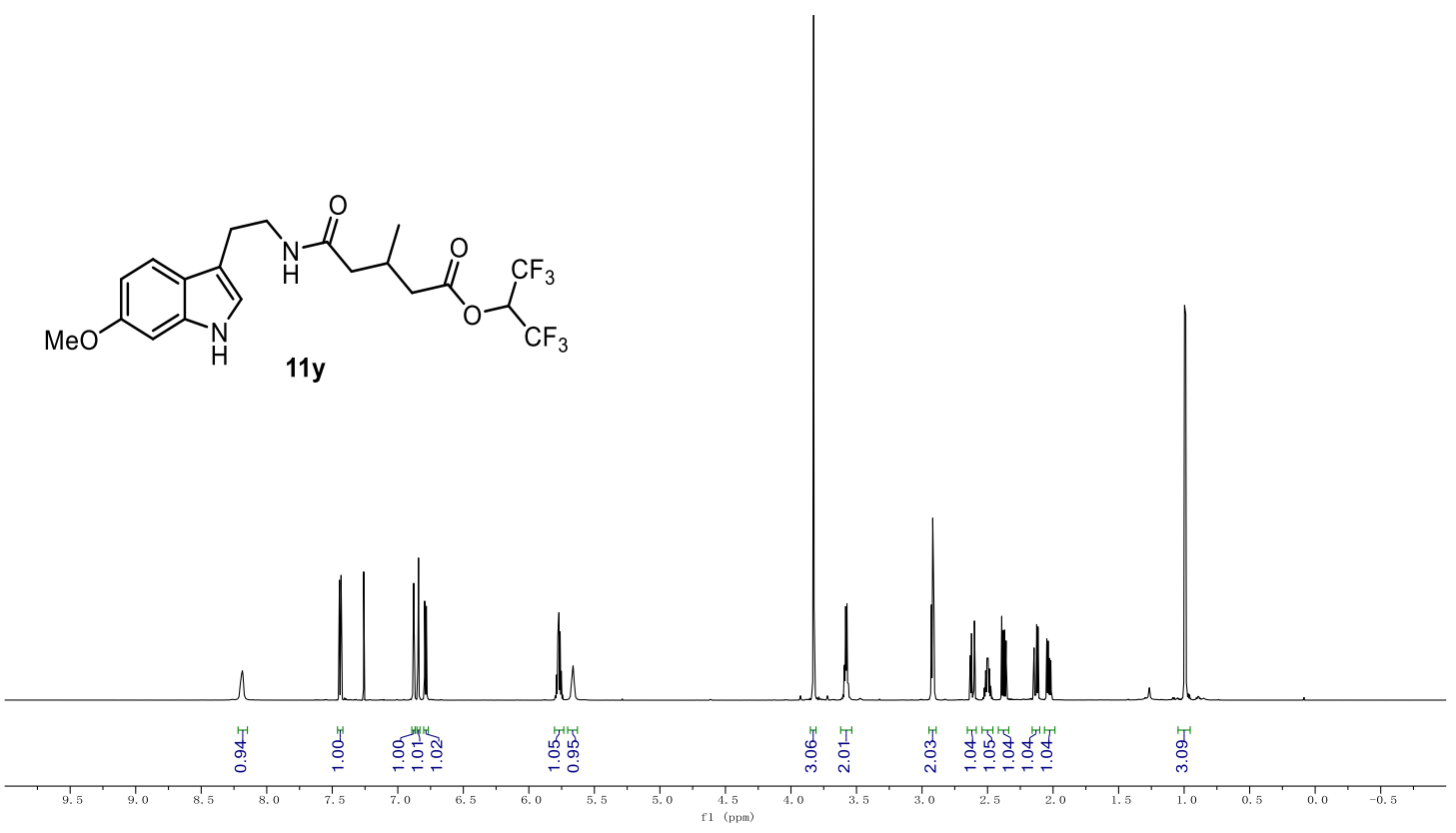

${ }^{13} \mathbf{C}$ NMR $\left(176 \mathrm{MHz}, \mathrm{CDCl}_{3}\right)$ of $\mathbf{1 1 y}$.

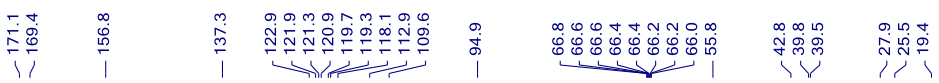
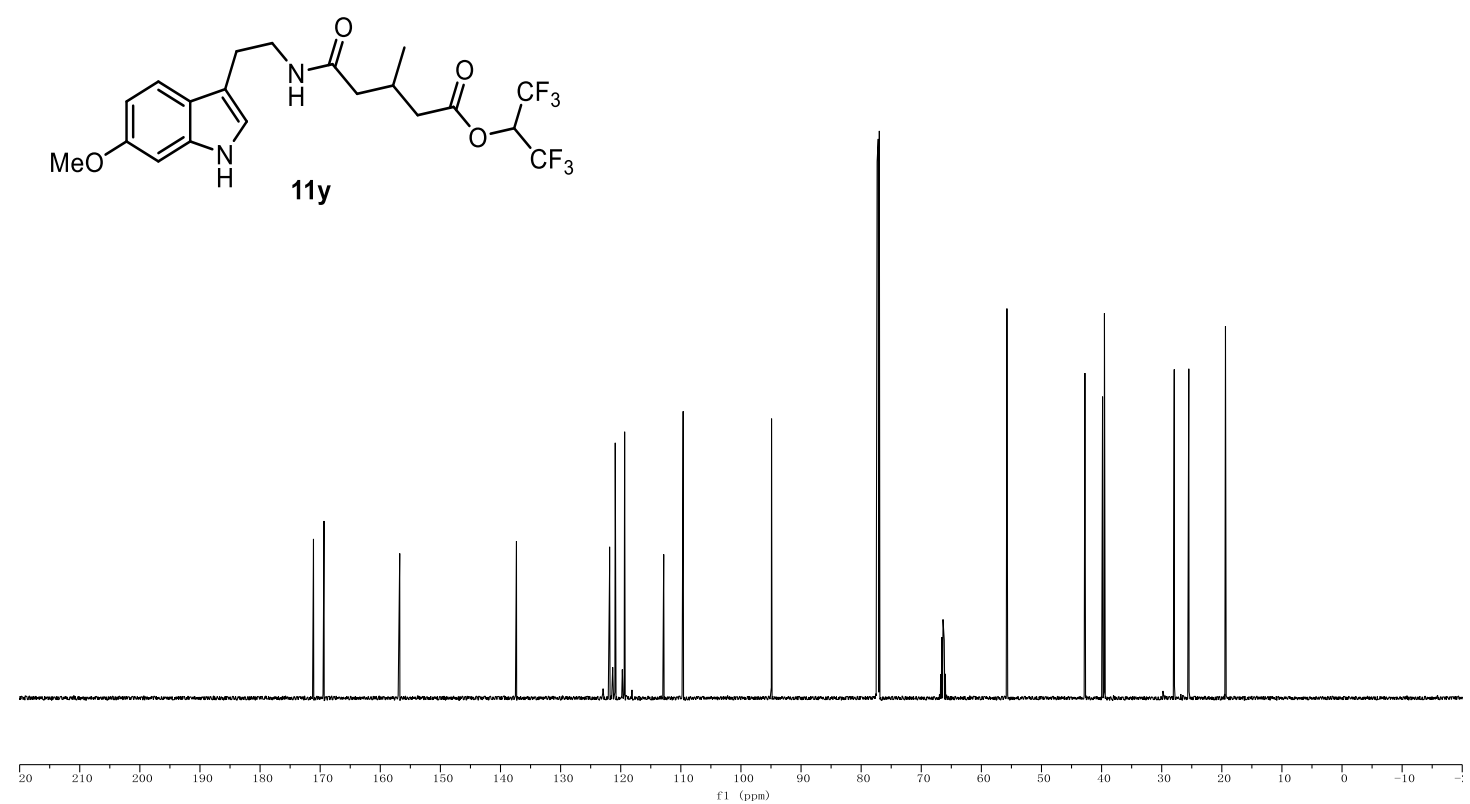
${ }^{19}$ F NMR (376 MHz, $\left.\mathrm{CDCl}_{3}\right)$ of $\mathbf{1 1 y}$.

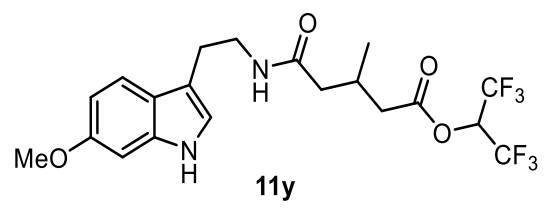

${ }^{1} \mathbf{H}$ NMR (600 MHz, $\left.\mathrm{CDCl}_{3}\right)$ of $\mathbf{S 1}$.

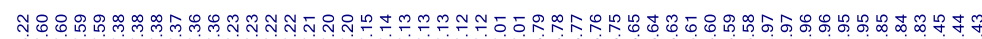

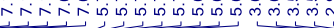
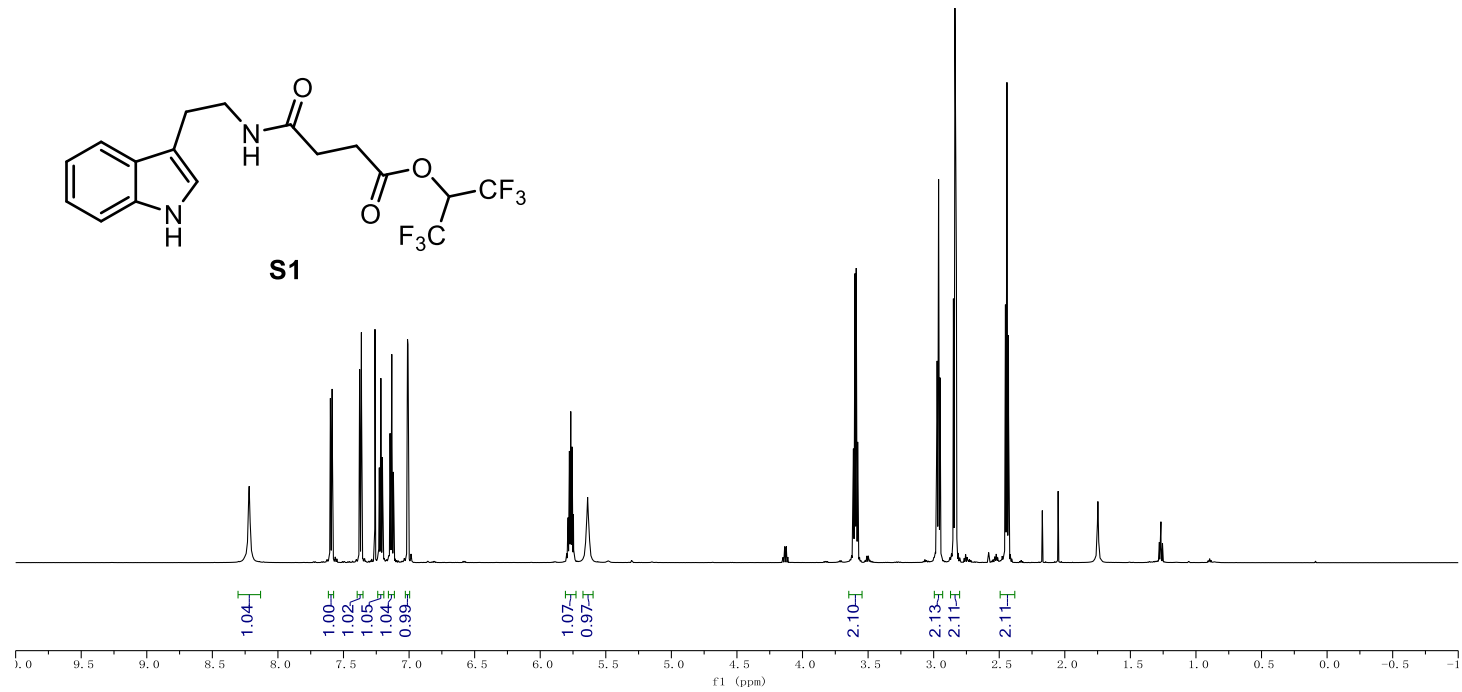
${ }^{13} \mathbf{C}$ NMR (151 MHz, $\left.\mathrm{CDCl}_{3}\right)$ of $\mathbf{S 1}$.
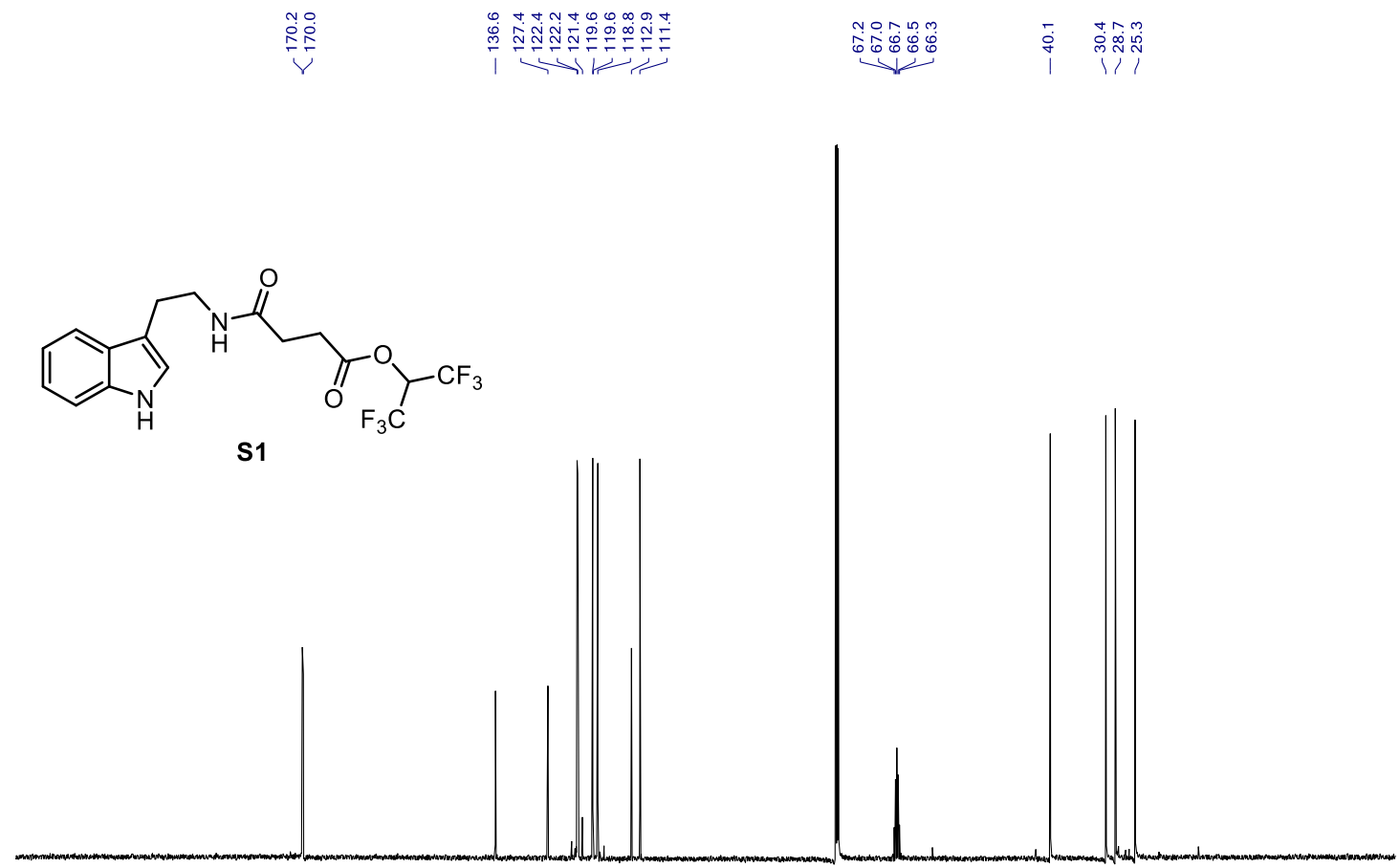

${ }^{19} \mathbf{F}$ NMR $\left(565 \mathrm{MHz}, \mathrm{CDCl}_{3}\right)$ of $\mathbf{S 1}$.

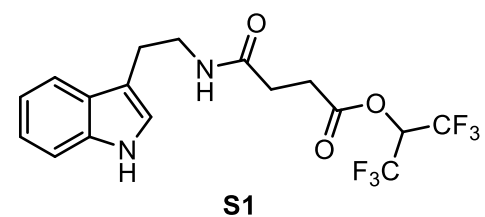

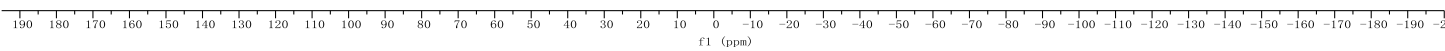


${ }^{1} \mathbf{H}$ NMR $\left(700 \mathrm{MHz}, \mathrm{CDCl}_{3}\right)$ of $\mathbf{S 2}$.

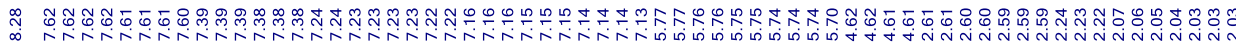<smiles>CC(OC(=O)CCCC(=O)NCc1c[nH]c2ccccc12)C(F)(F)F</smiles>

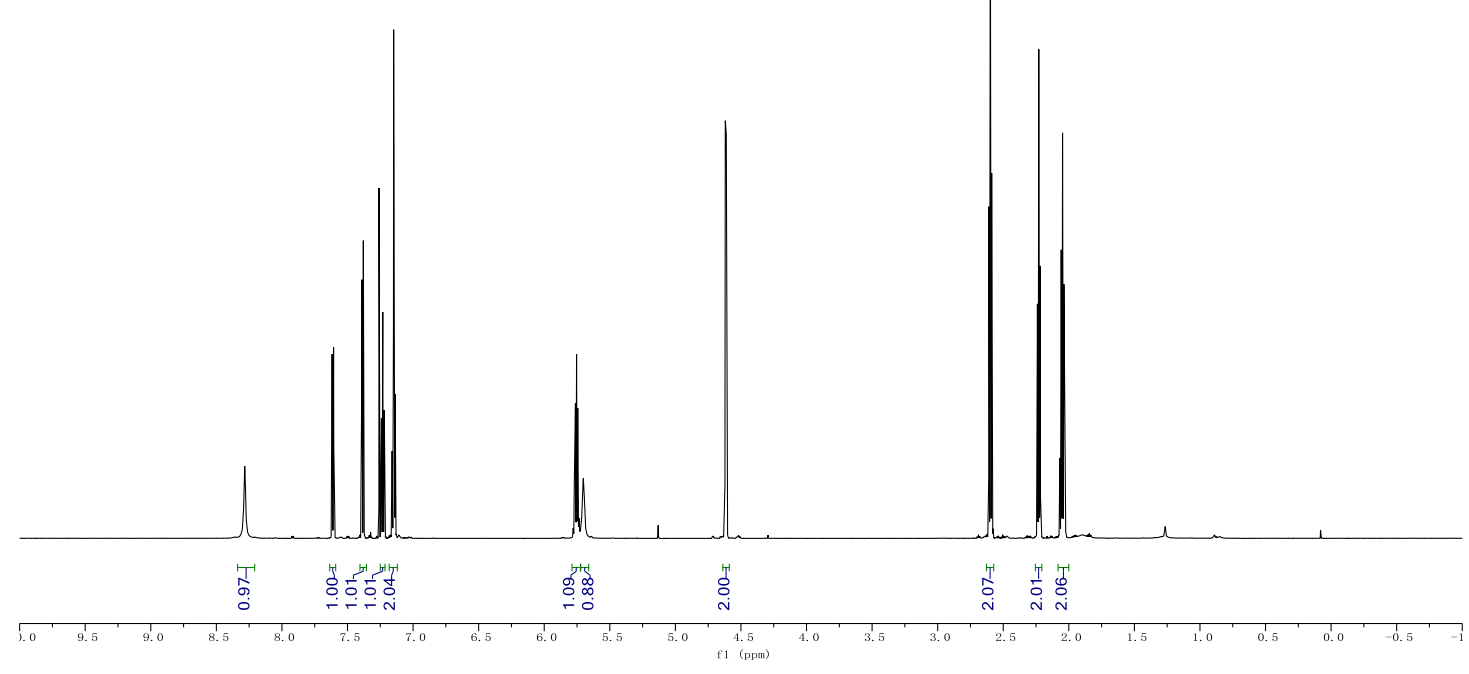

${ }^{13} \mathbf{C}$ NMR (176 MHz, $\left.\mathrm{CDCl}_{3}\right)$ of $\mathbf{S 2}$.

管<smiles>O=C(CCCC(=O)OC(C(F)(F)F)C(F)(F)F)NCc1c[nH]c2ccccc12</smiles>

S2

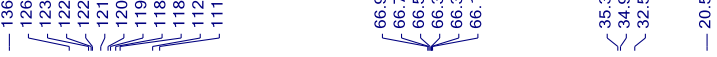
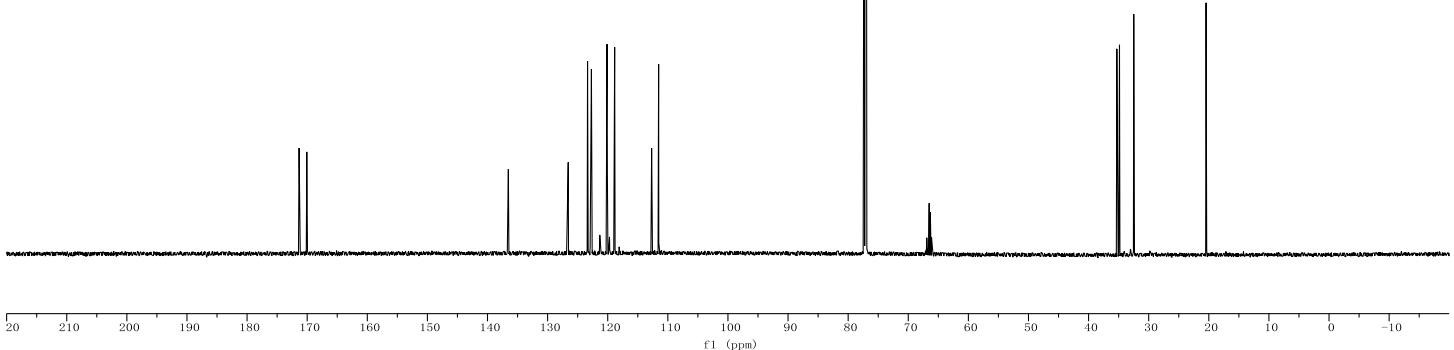
${ }^{19}$ F NMR (565 MHz, $\left.\mathrm{CDCl}_{3}\right)$ of $\mathbf{S 2}$.

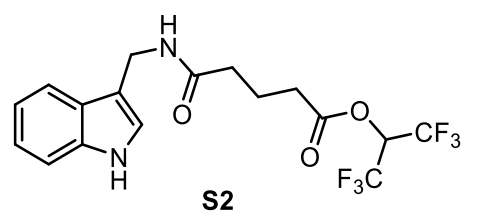

ن 
${ }^{13} \mathbf{C}$ NMR $\left(126 \mathrm{MHz}, \mathrm{CDCl}_{3}\right)$ of $\mathbf{S 3 .}$
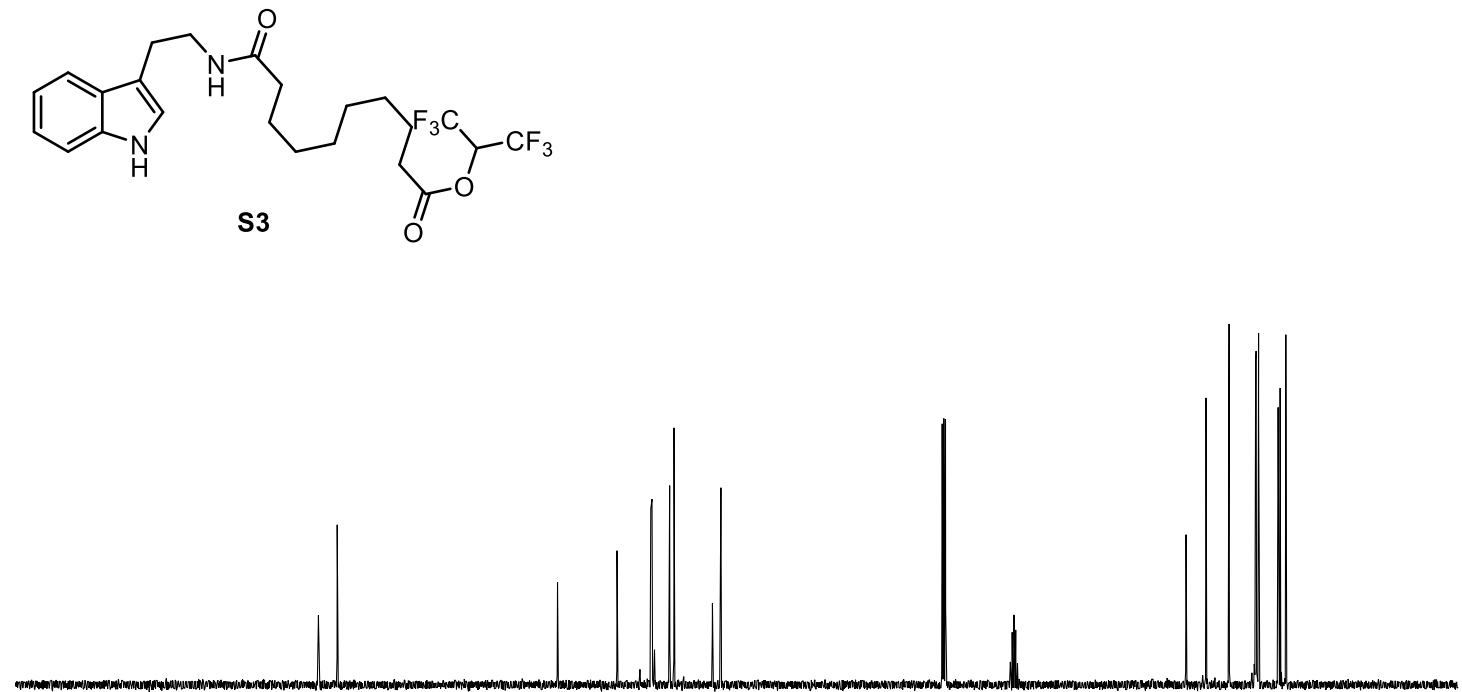

${ }^{19} \mathbf{F}$ NMR $\left(471 \mathrm{MHz}, \mathrm{CDCl}_{3}\right)$ of $\mathbf{S 3}$.<smiles>CC(F)C(F)(F)OC(=O)C[I-]CCCCCCC(=O)NCCc1c[nH]c2ccccc12</smiles> 
${ }^{1} \mathbf{H}$ NMR $\left(500 \mathrm{MHz}, \mathrm{CDCl}_{3}\right)$ of $\mathbf{S 4}$.

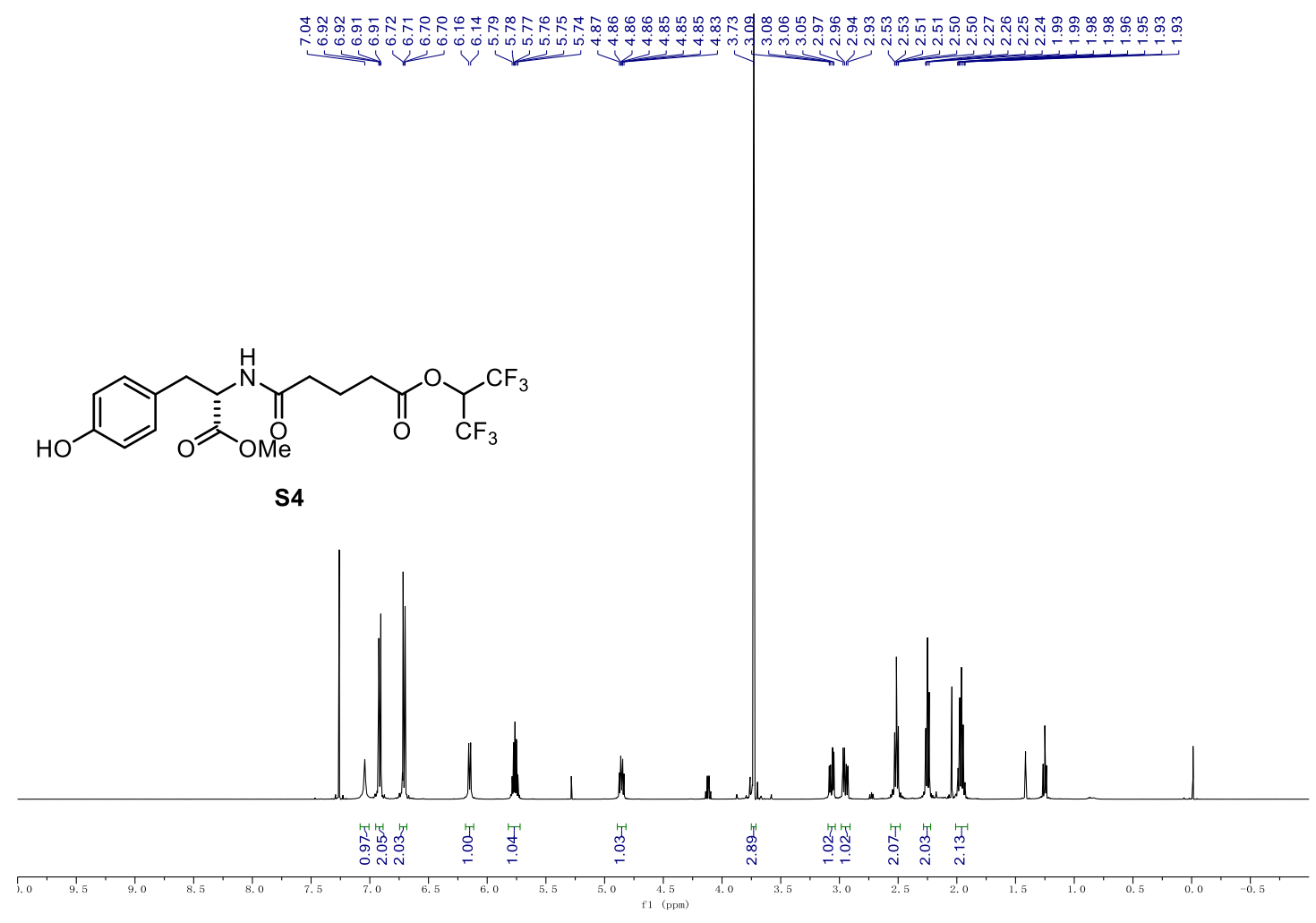

${ }^{13} \mathbf{C}$ NMR (126 MHz, $\left.\mathrm{CDCl}_{3}\right)$ of $\mathbf{S 4}$.

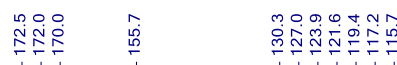

i)

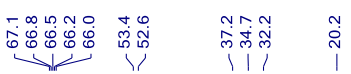

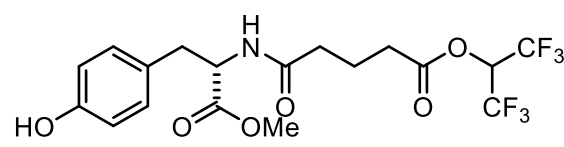

S4

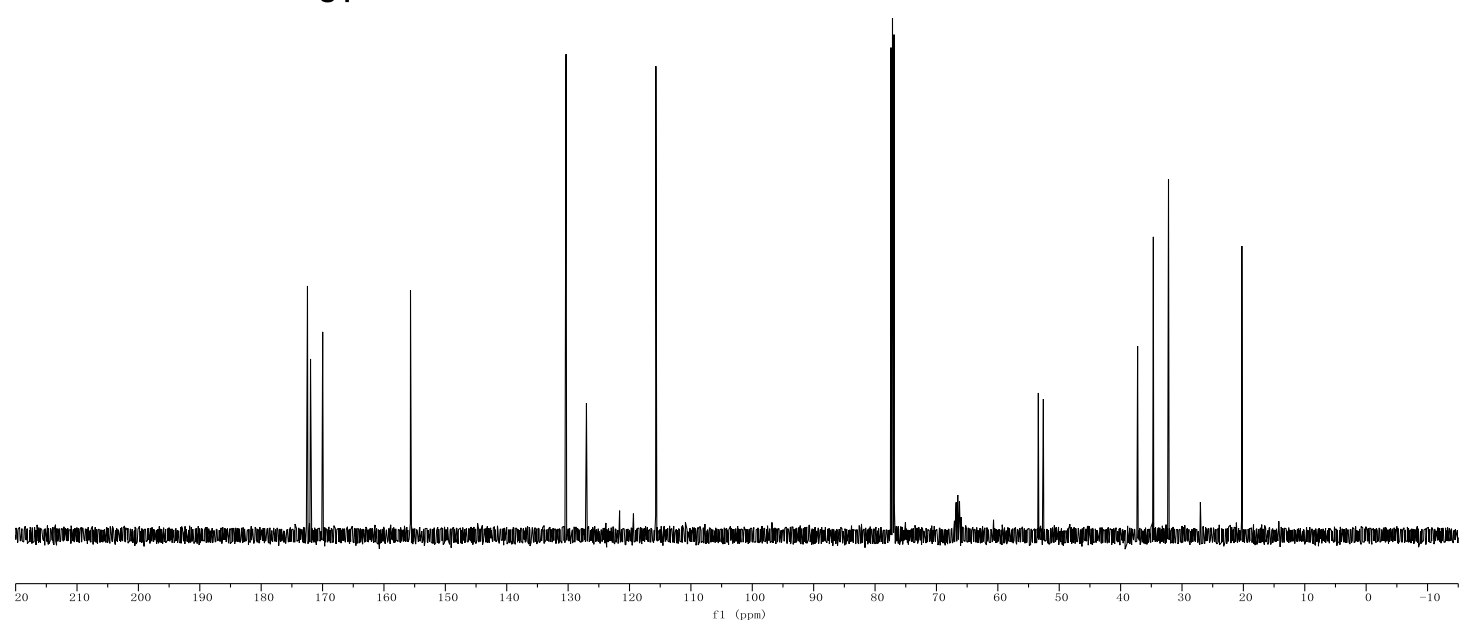


${ }^{19}$ F NMR $\left(471 \mathrm{MHz}, \mathrm{CDCl}_{3}\right)$ of $\mathbf{S 4}$.

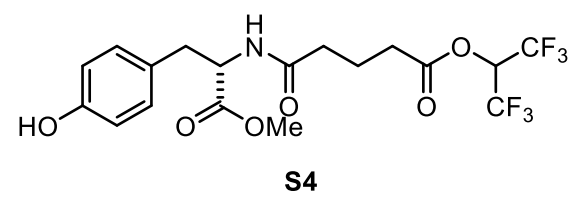

${ }^{1} \mathbf{H}$ NMR $\left(500 \mathrm{MHz}, \mathrm{CDCl}_{3}\right)$ of $\mathbf{S 5}$.

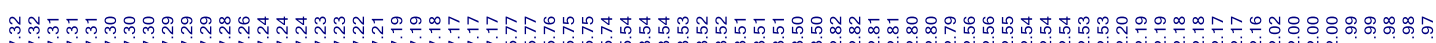<smiles>O=C(CCCC(=O)OC(C(F)(F)F)C(F)(F)F)NCCc1ccccc1</smiles>

S5

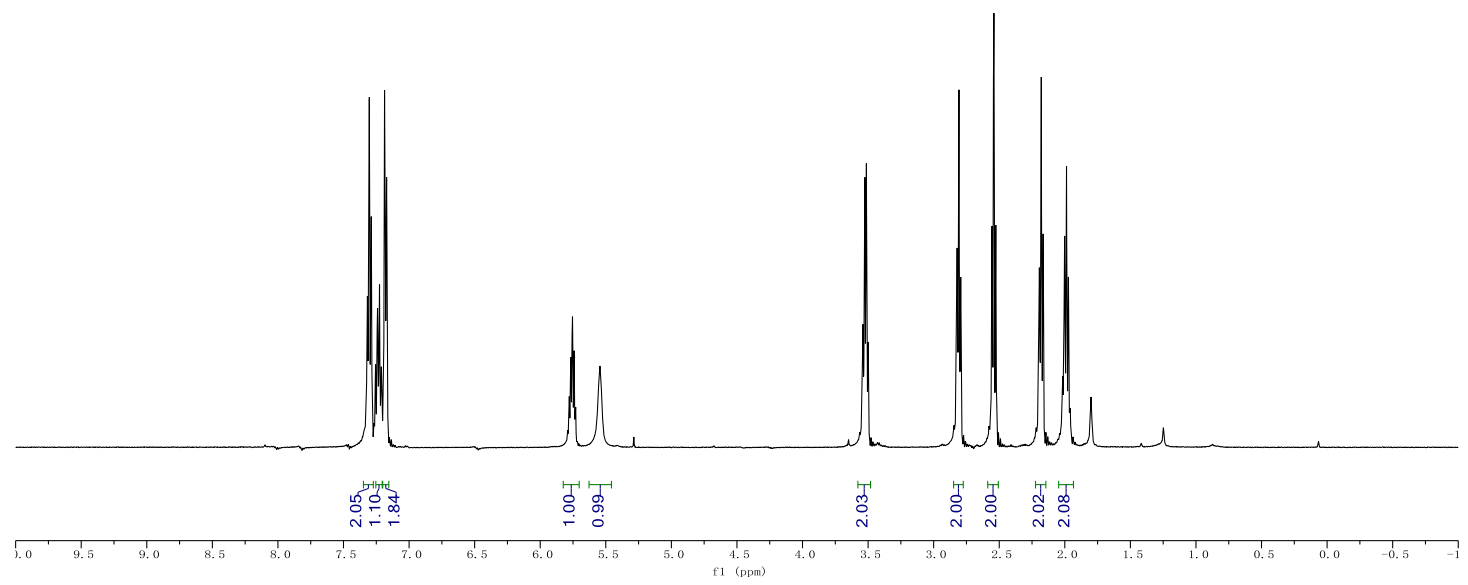


${ }^{13} \mathbf{C}$ NMR (126 MHz, $\left.\mathrm{CDCl}_{3}\right)$ of $\mathbf{S 5}$.

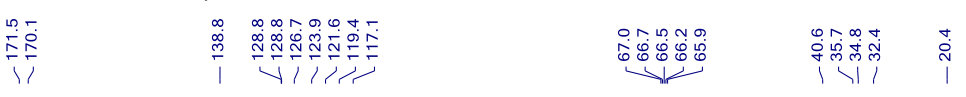

$\overbrace{\mathrm{O}}^{\mathrm{N}} \prod_{\mathrm{CF}_{3}}^{\mathrm{CF}_{3}}$

S5
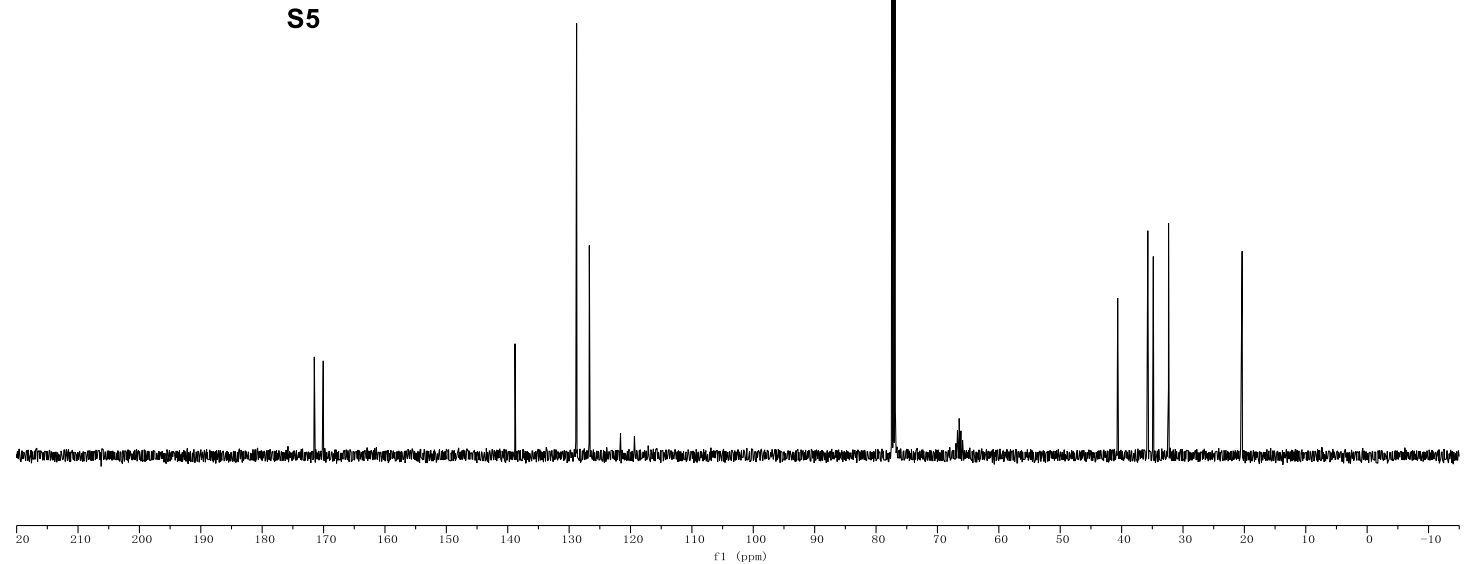

${ }^{19}$ F NMR (471 MHz, $\left.\mathrm{CDCl}_{3}\right)$ of $\mathbf{S 5}$.

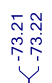<smiles>O=C(CCCC(=O)OC(C(F)(F)F)C(F)(F)F)NCCc1ccccc1</smiles>

300
300 
${ }^{1} \mathbf{H}$ NMR $\left(500 \mathrm{MHz}, \mathrm{CDCl}_{3}\right)$ of $\mathbf{S 6}$.<smiles>[14CH3][14CH3]</smiles>

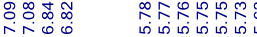

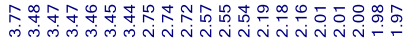

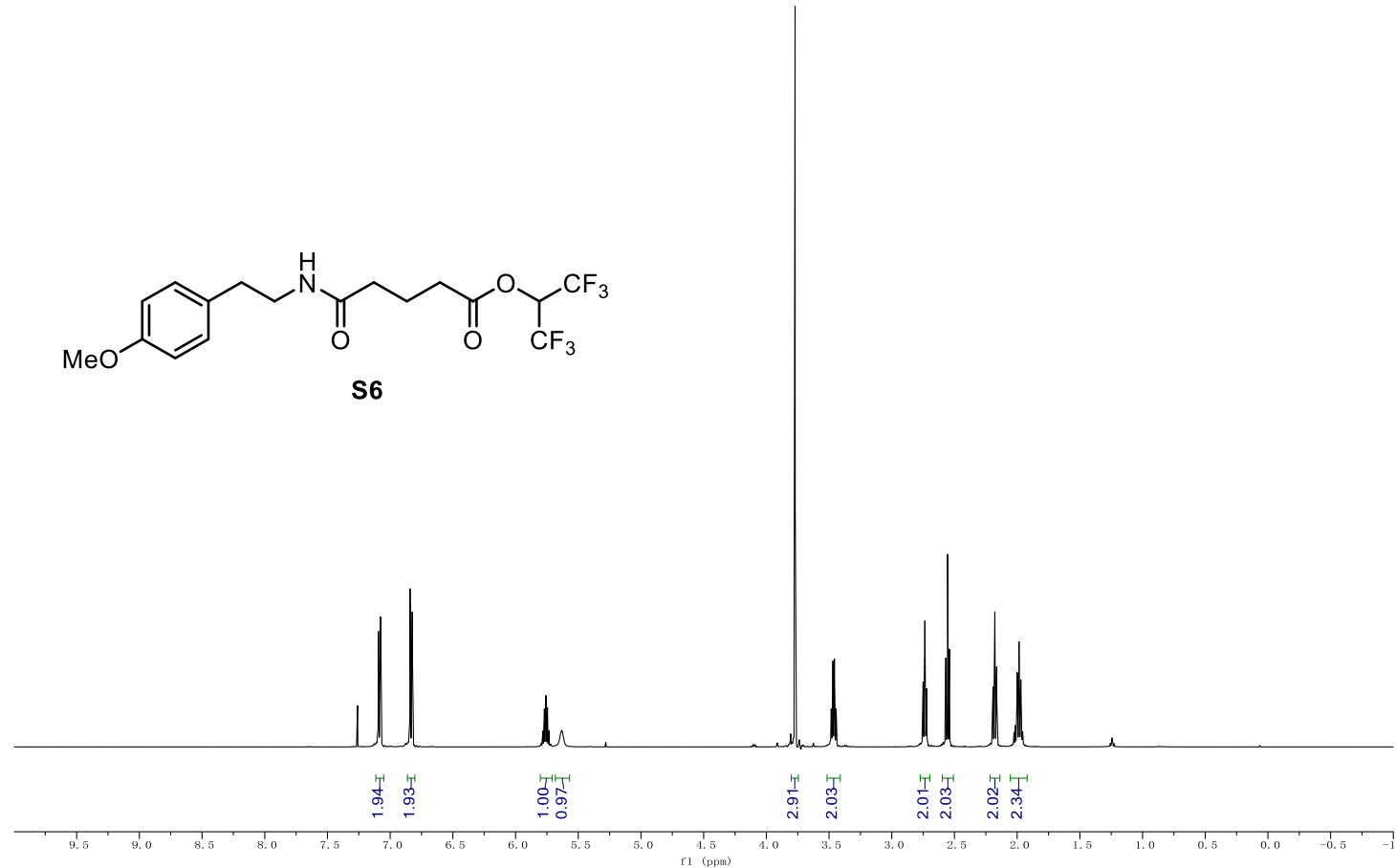

S6

${ }^{13} \mathbf{C ~ N M R}\left(126 \mathrm{MHz}, \mathrm{CDCl}_{3}\right)$ of $\mathbf{S 6}$.

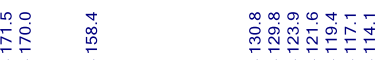

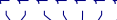

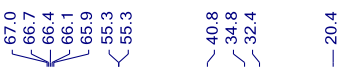<smiles>COc1ccc(CCNC(=O)CCCC(=O)OC(C(F)(F)F)C(F)(F)F)cc1</smiles>

56

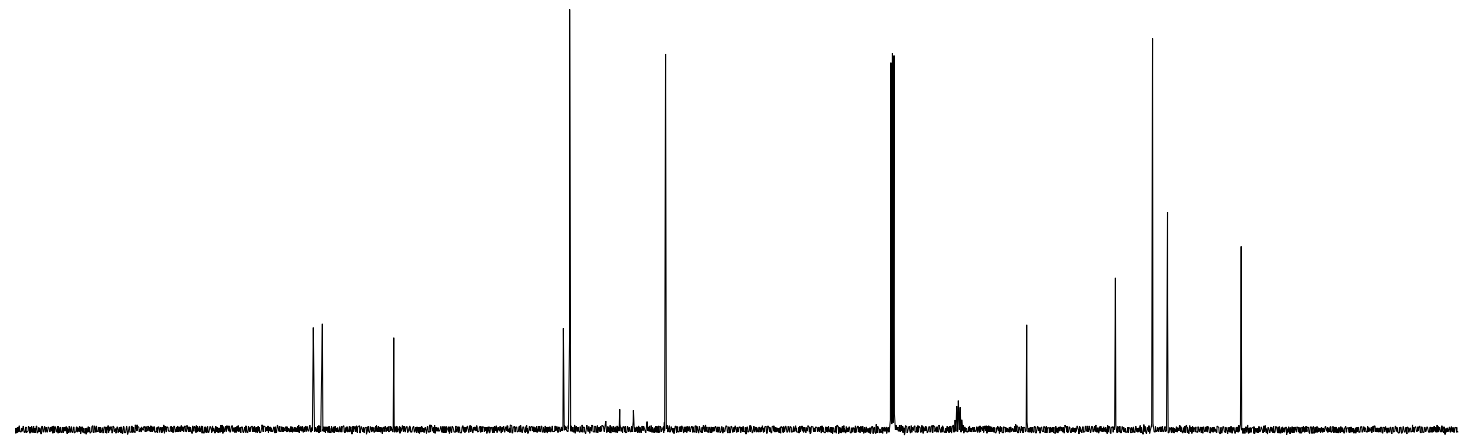

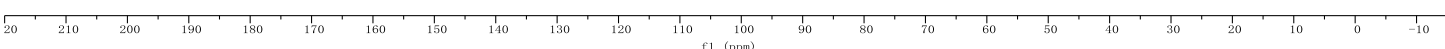


${ }^{19}$ F NMR (471 MHz, $\left.\mathrm{CDCl}_{3}\right)$ of $\mathbf{S 6 .}$

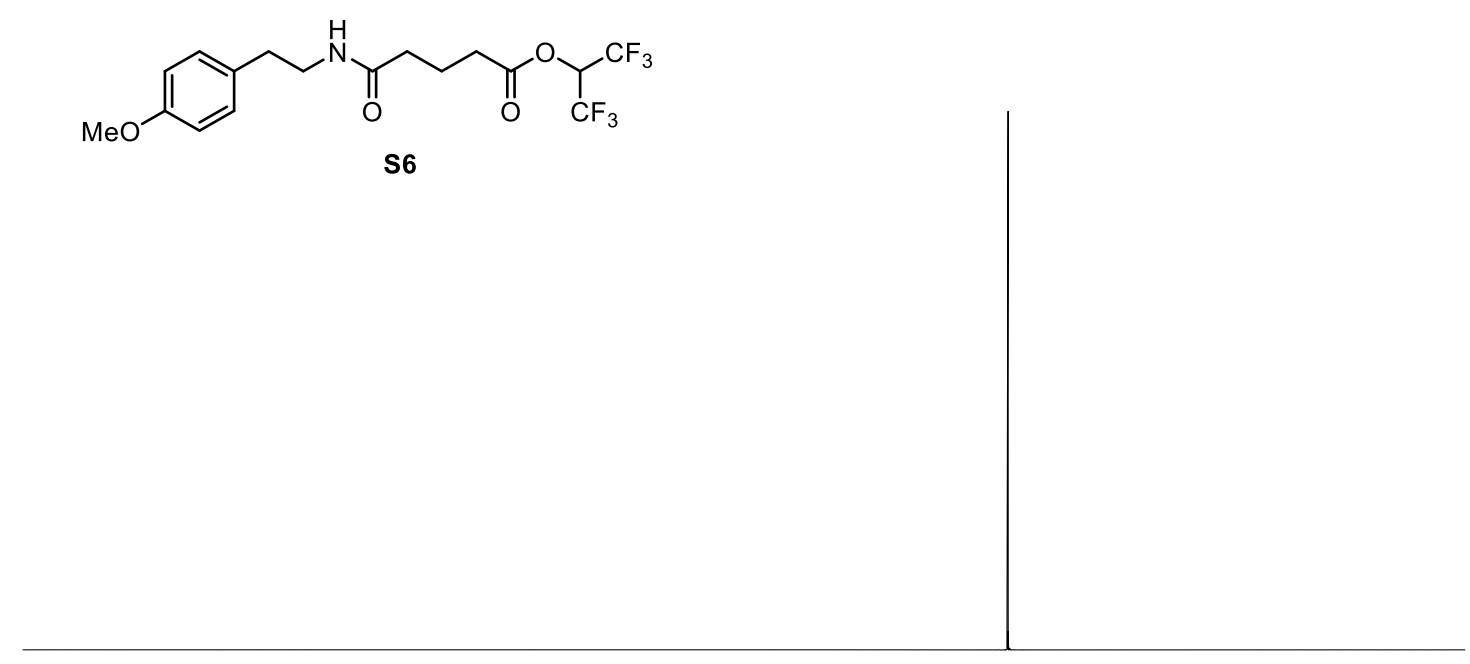

${ }^{1} \mathbf{H}$ NMR (500 MHz, CDCl 3$)$ of $\mathbf{S 7 .}$

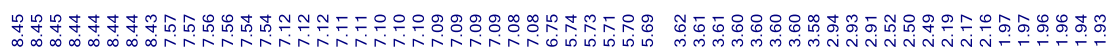
年<smiles>O=C(CCCC(=O)OC(F)(F)F)NCCc1ccccn1</smiles>

S7

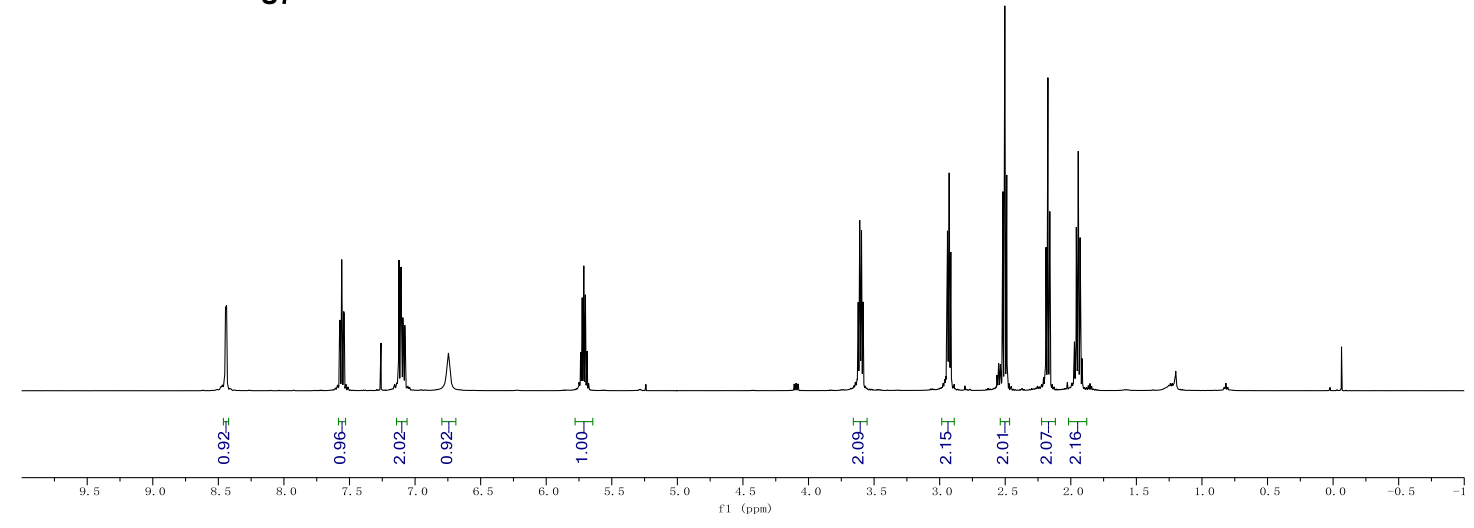


${ }^{13} \mathbf{C}$ NMR $\left(126 \mathrm{MHz}, \mathrm{CDCl}_{3}\right)$ of $\mathbf{S 7}$.
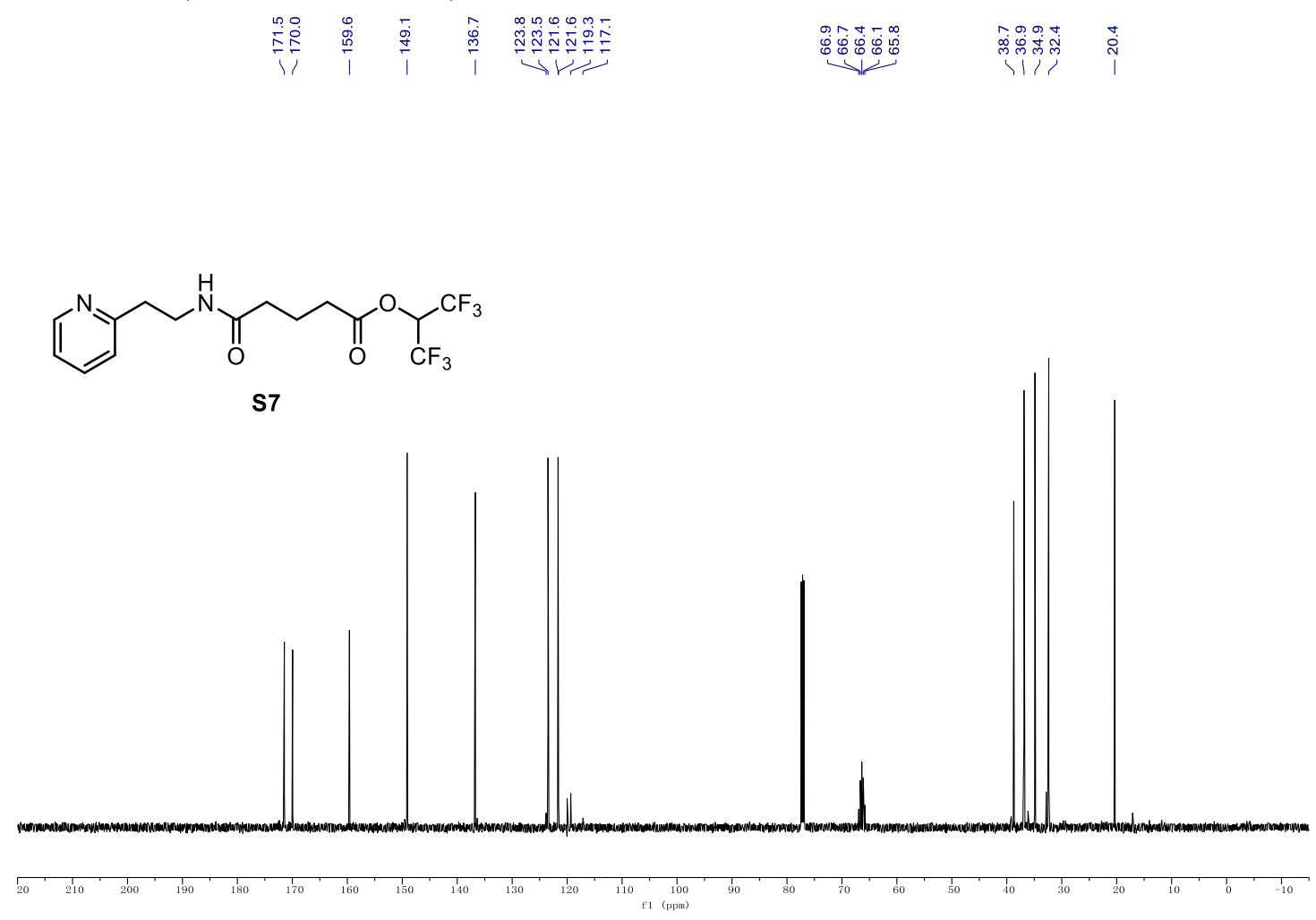

${ }^{19} \mathbf{F}$ NMR $\left(471 \mathrm{MHz}, \mathrm{CDCl}_{3}\right)$ of $\mathbf{S 7}$.<smiles>O=C(CCCC(=O)OC(C(F)(F)F)C(F)(F)F)NCCc1ccccn1</smiles> 
${ }^{1} \mathbf{H}$ NMR (500 MHz, $\left.\mathrm{CDCl}_{3}\right)$ of 9.

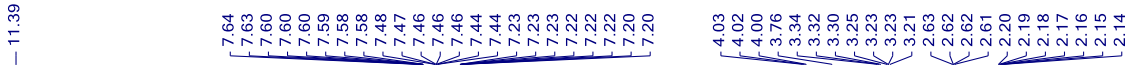
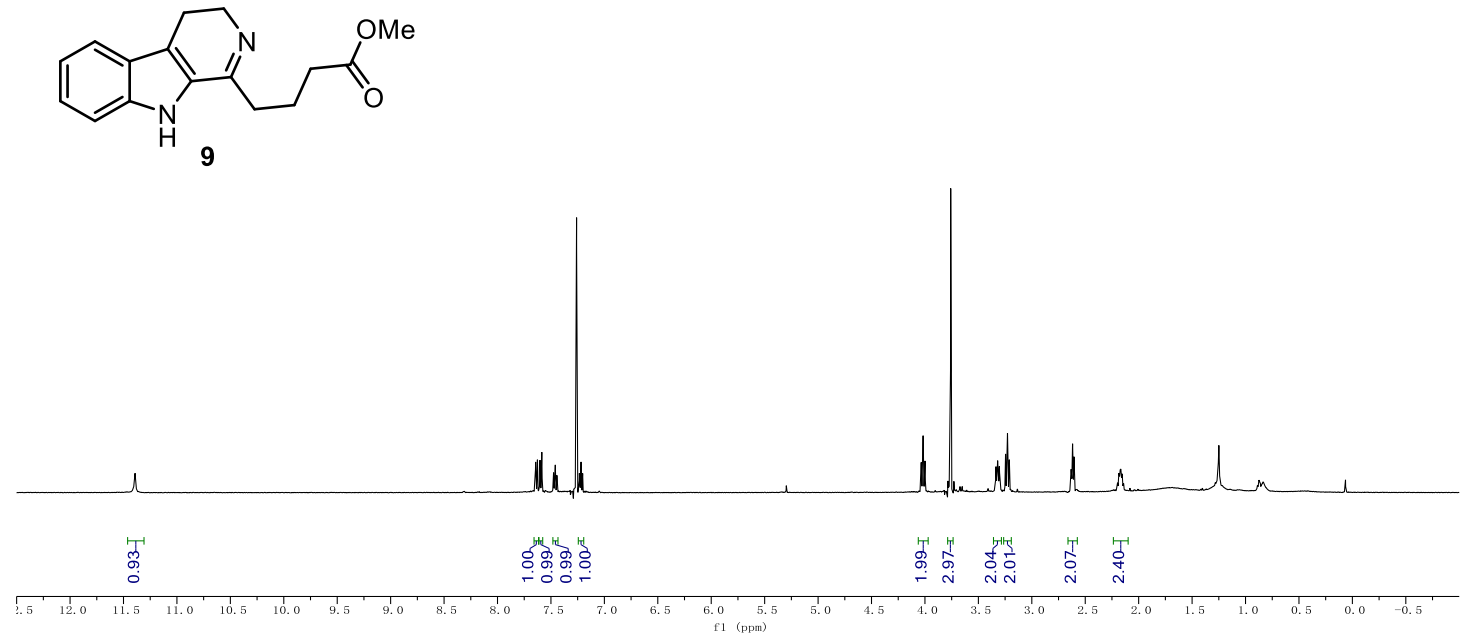

${ }^{13} \mathrm{C}$ NMR (126 MHz, $\left.\mathrm{CDCl}_{3}\right)$ of 9.

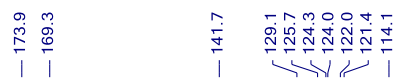

密

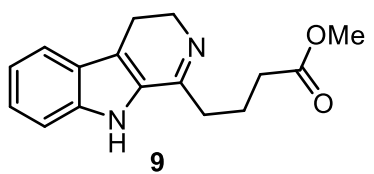


${ }^{1} \mathbf{H}$ NMR $\left(500 \mathrm{MHz}, \mathrm{CDCl}_{3}\right)$ of $\mathbf{1 0}$.

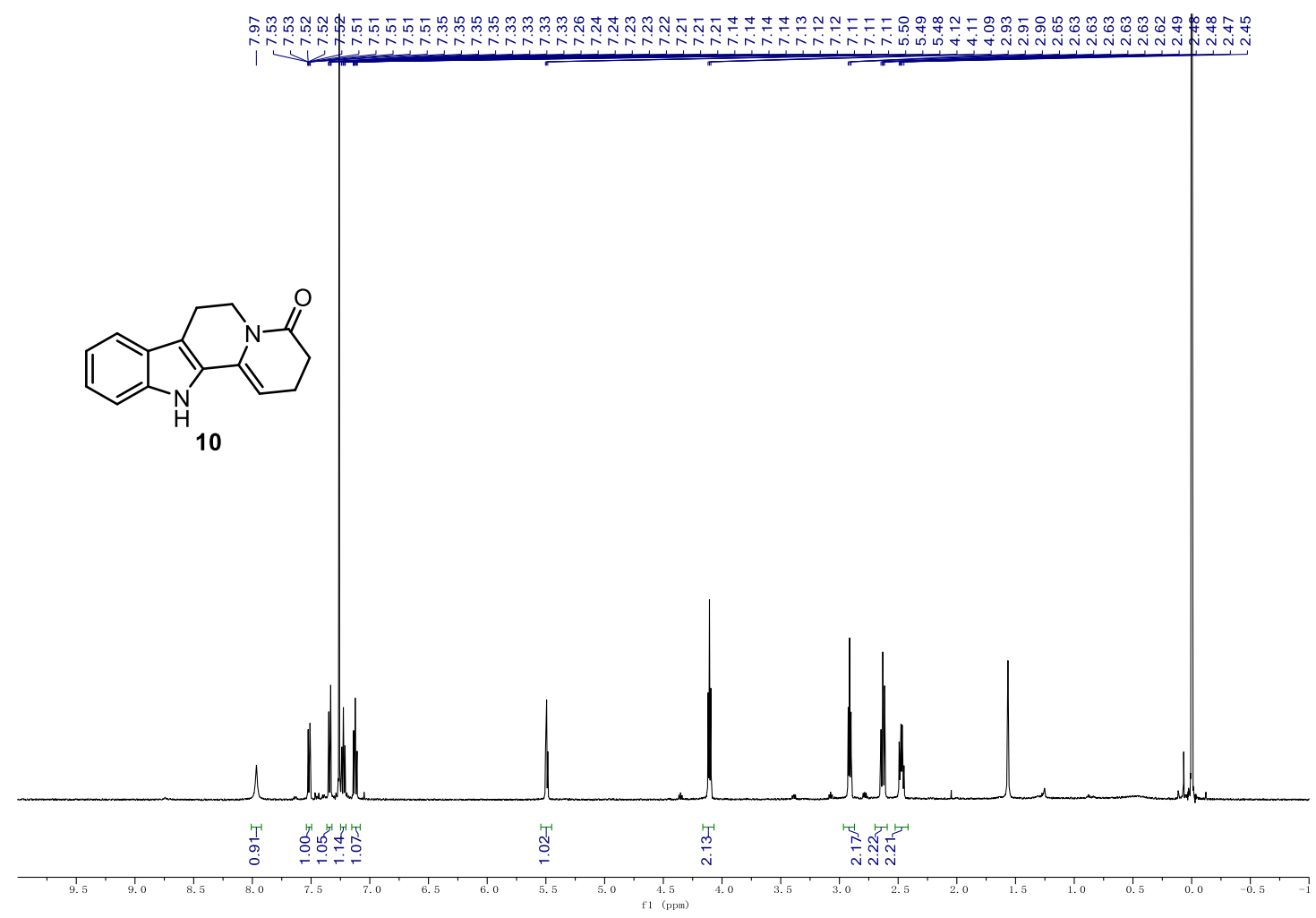

${ }^{13}$ C NMR (126 MHz, $\left.\mathrm{CDCl}_{3}\right)$ of $\mathbf{1 0}$.

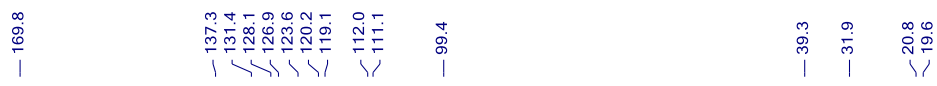<smiles>O=C1CCC=C2c3[nH]c4ccccc4c3CCN12</smiles>

10 
${ }^{\mathbf{1}} \mathbf{H}$ NMR $\left(500 \mathrm{MHz}, \mathrm{CDCl}_{3}\right)$ of 12a.

谓

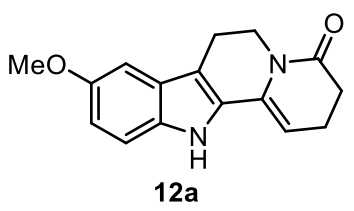

$12 a$

${ }^{13} \mathbf{C}$ NMR $\left(126 \mathrm{MHz}, \mathrm{CDCl}_{3}\right)$ of $\mathbf{1 2 a}$.

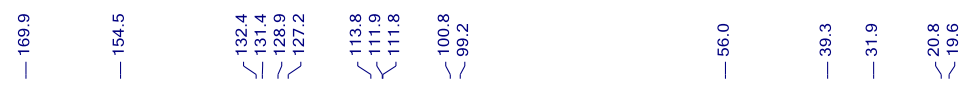<smiles>COc1ccc2[nH]c3c(c2c1)CCN1C(=O)CCC=C31</smiles> 
${ }^{1} \mathbf{H}$ NMR (500 MHz, $\left.\mathrm{CD}_{2} \mathrm{Cl}_{2}\right)$ of $\mathbf{1 2 b}$.

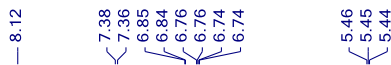

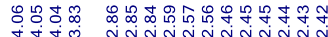

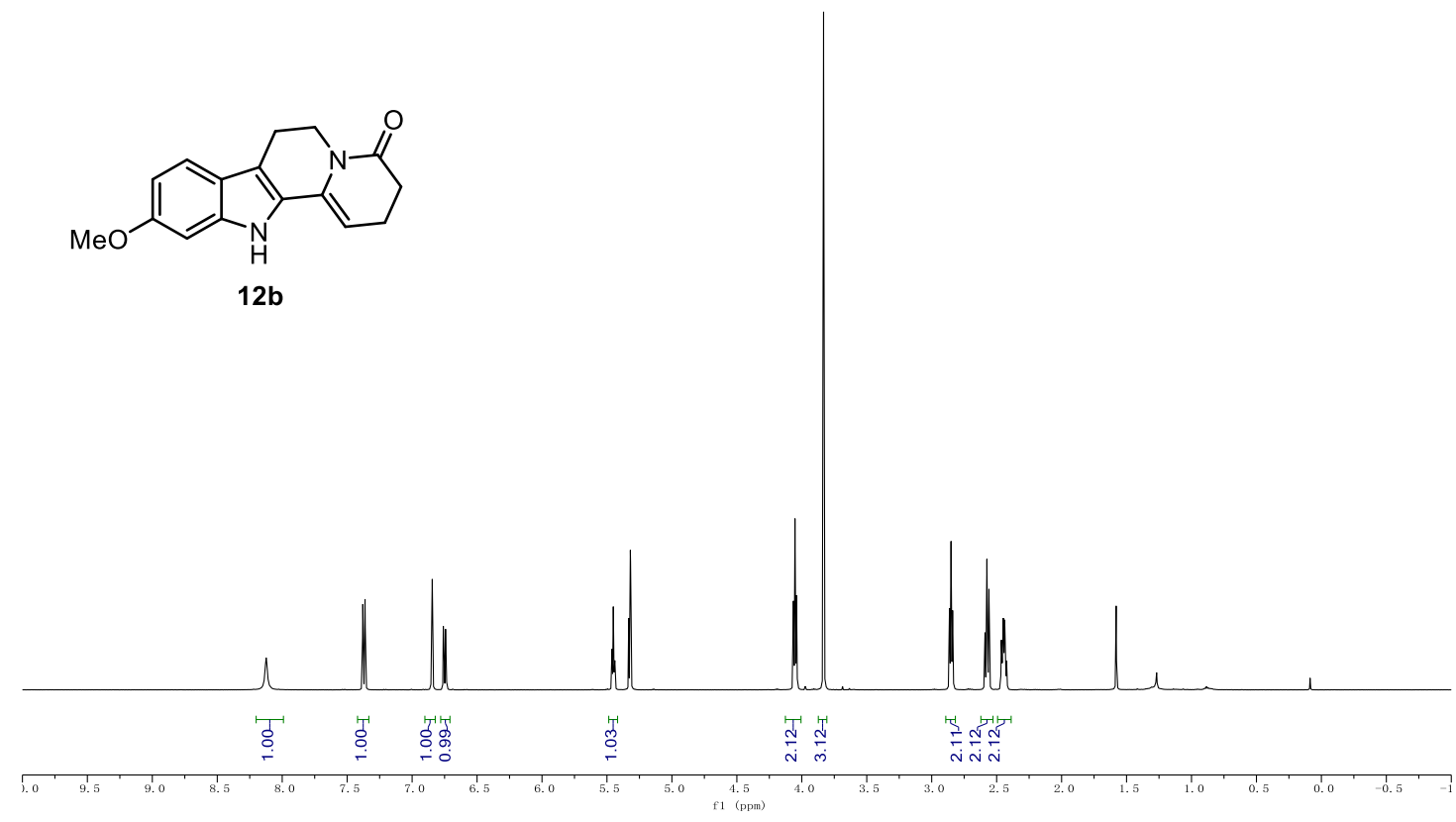

$12 b$

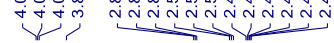

${ }^{13} \mathrm{C} \mathrm{NMR}\left(126 \mathrm{MHz}, \mathrm{CD}_{2} \mathrm{Cl}_{2}\right)$ of $\mathbf{1 2 b}$.

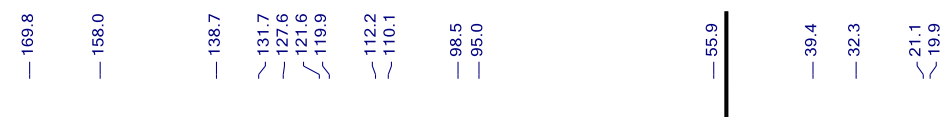

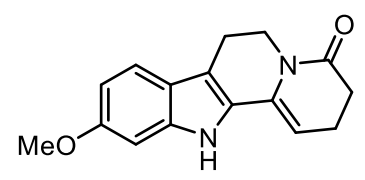

$12 b$

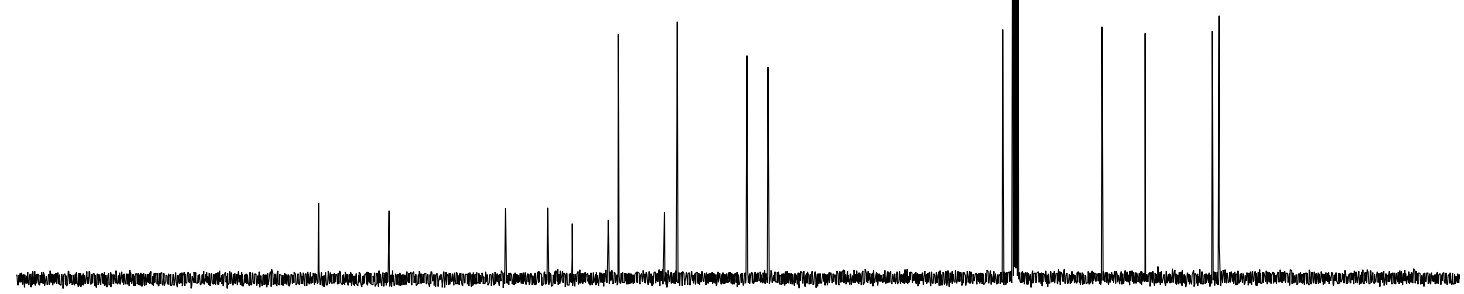


${ }^{1} \mathbf{H}$ NMR (700 MHz, d6-DMSO) of $\mathbf{1 2 c}$

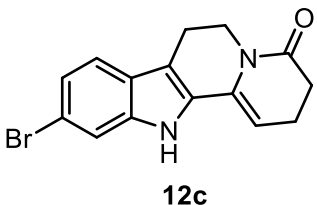

12c

${ }^{13}$ C NMR (176 MHz, $d_{6}$-DMSO) of 12c.

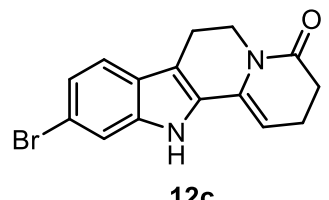

12c

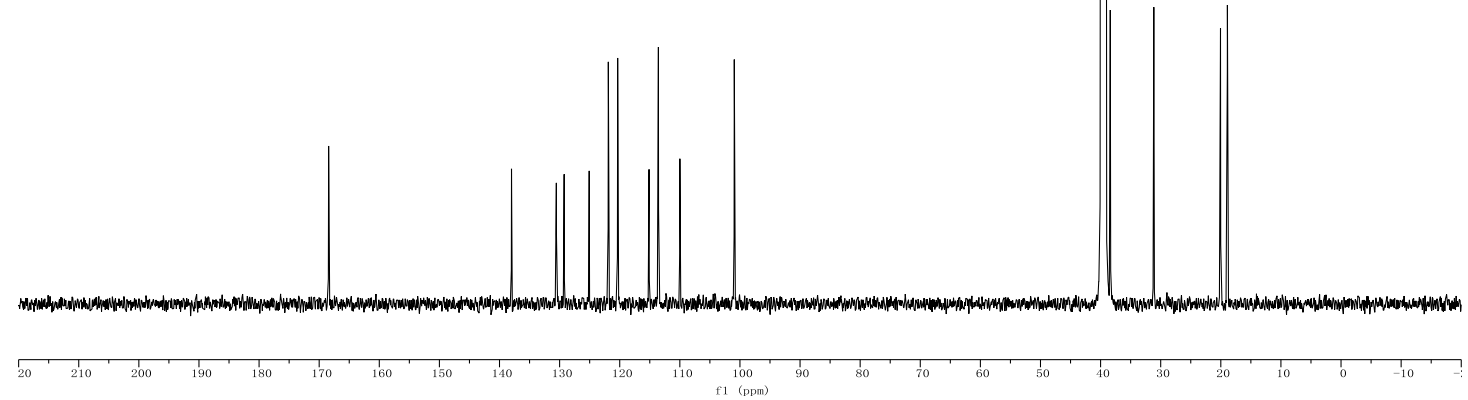


${ }^{1}$ H NMR (700 MHz, d6-DMSO) of 12d.

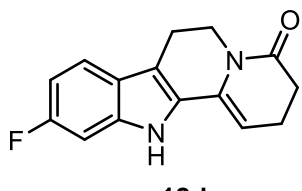

$12 d$

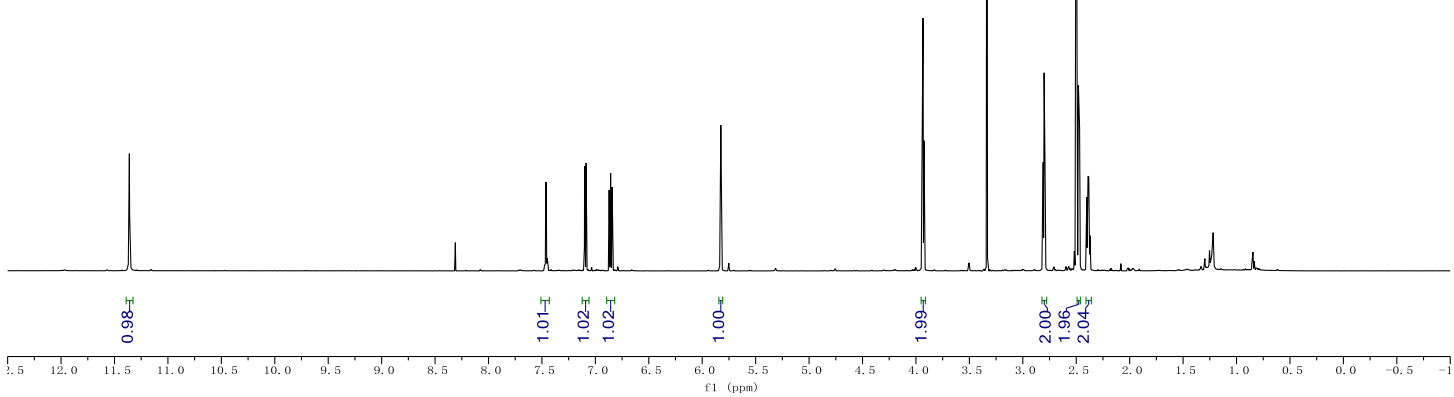

${ }^{13}$ C NMR (176 MHz, d6-DMSO) of 12d.

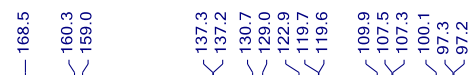

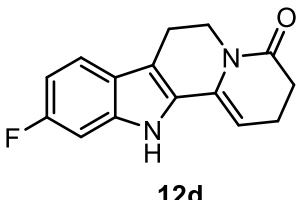

12d

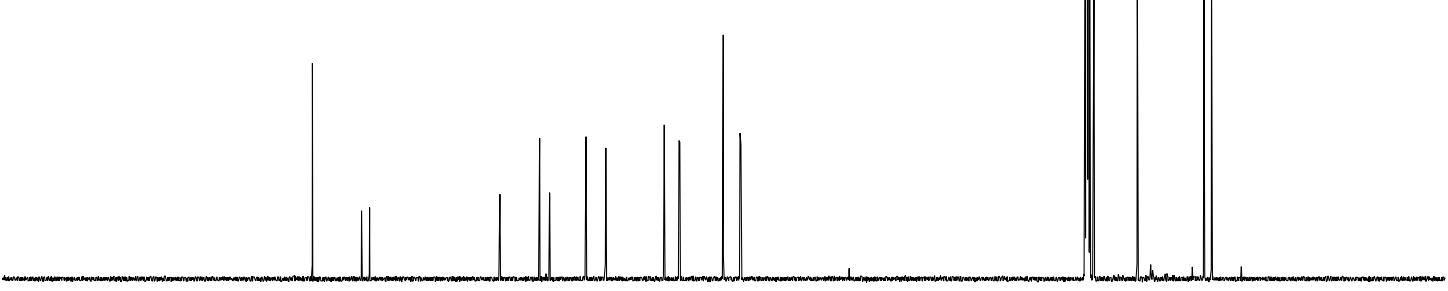


${ }^{19}$ F NMR (471 MHz, $\left.d 6-\mathrm{DMSO}\right)$ of 12d.

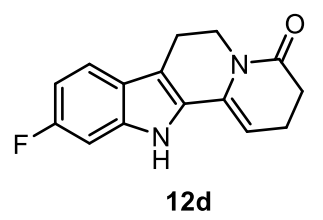

$12 d$ 
${ }^{1} \mathbf{H}$ NMR (500 MHz, $\left.\mathrm{CDCl}_{3}\right)$ of $\mathbf{1 2 e}$.

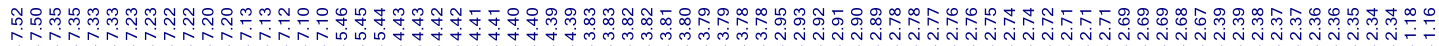<smiles>CC1C=C2c3[nH]c4ccccc4c3CCN2C(=O)C1</smiles>

$12 \mathrm{e}$

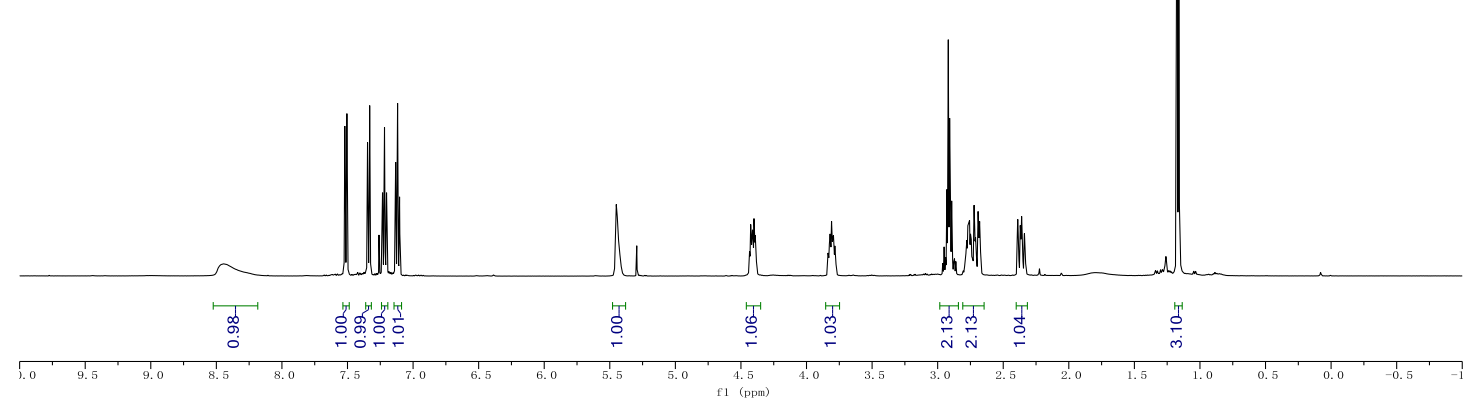

${ }^{13} \mathbf{C ~ N M R}\left(126 \mathrm{MHz}, \mathrm{CDCl}_{3}\right)$ of $\mathbf{1 2 e}$.

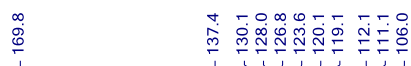

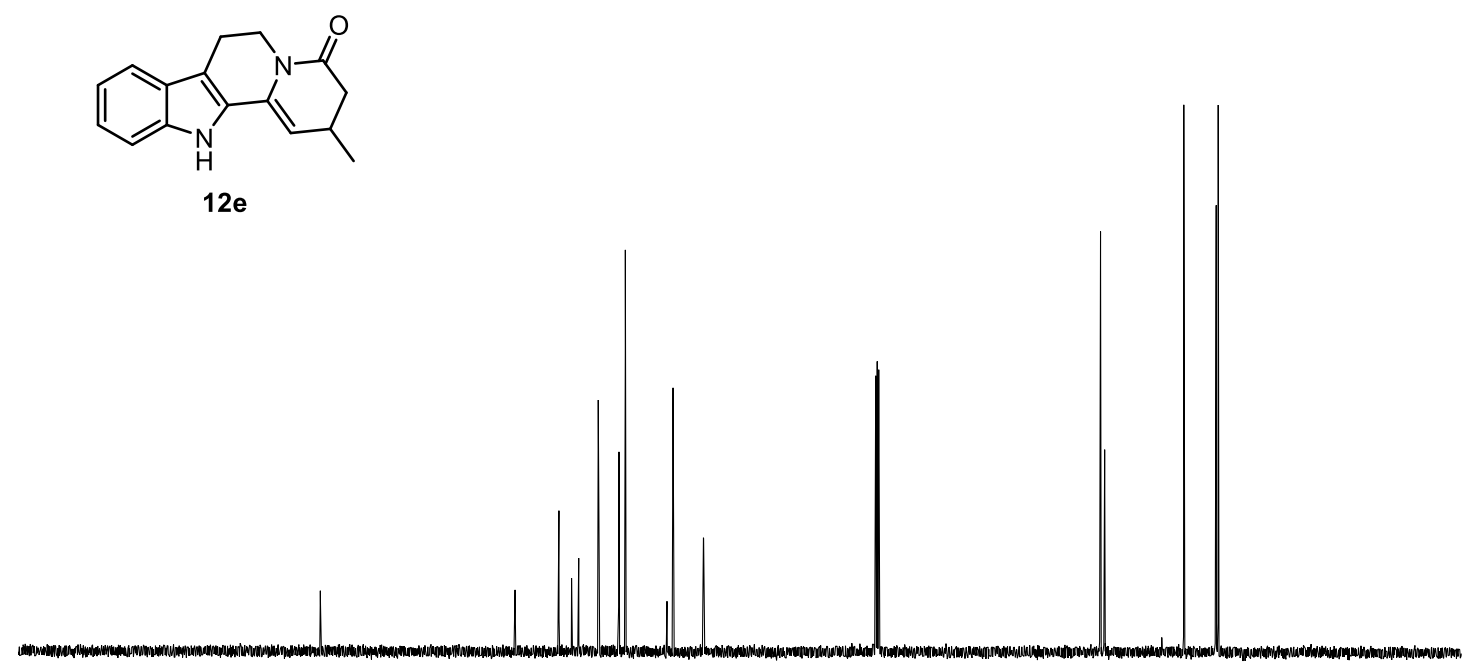

$12 \mathrm{e}$ 
${ }^{1} \mathbf{H}$ NMR (700 MHz, $\left.\mathrm{CD}_{2} \mathrm{Cl}_{2}\right)$ of $\mathbf{1 2 f}$.

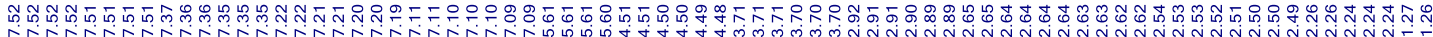
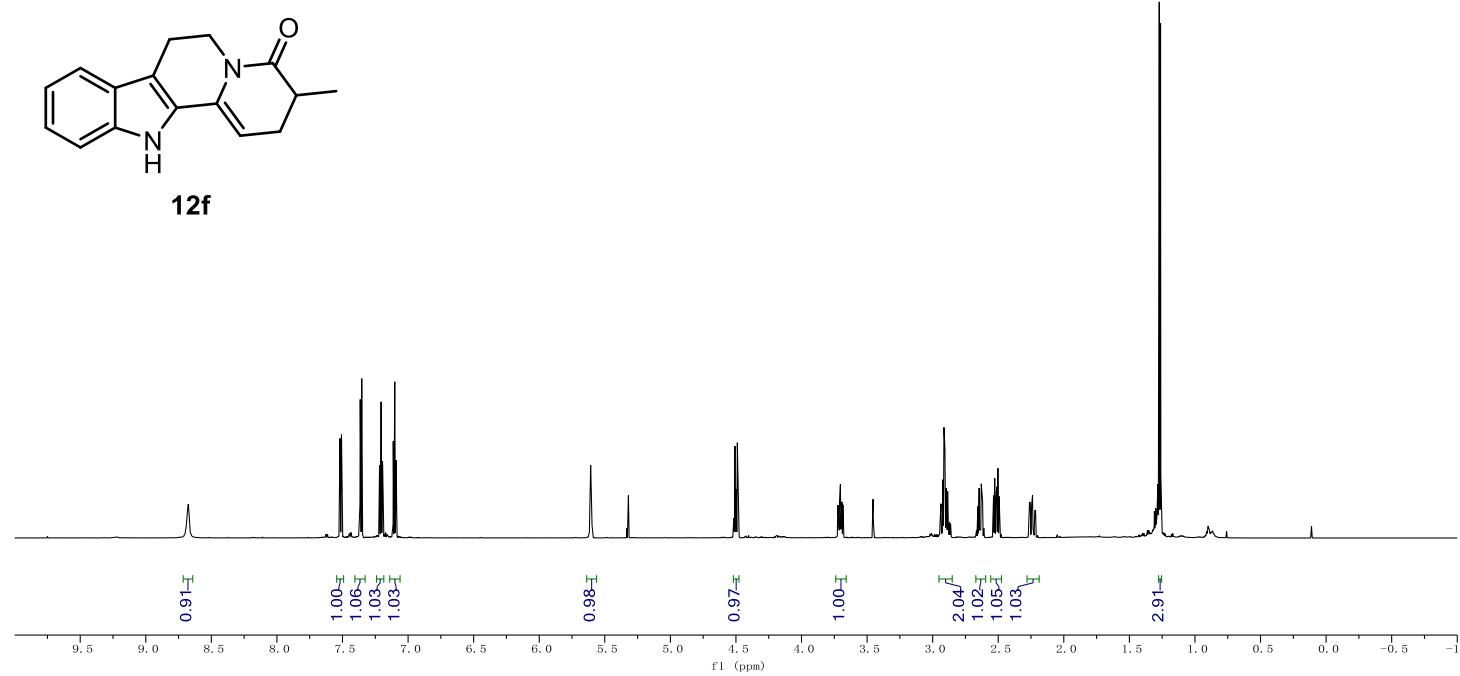

${ }^{13} \mathbf{C}$ NMR (176 MHz, $\left.\mathrm{CD}_{2} \mathrm{Cl}_{2}\right)$ of $12 f$.
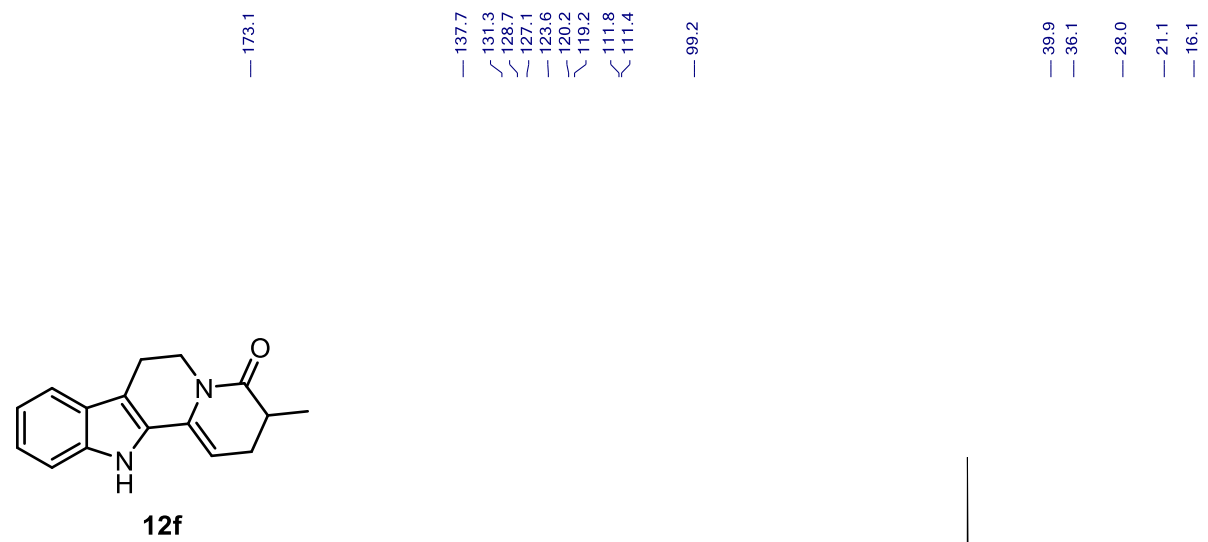

12

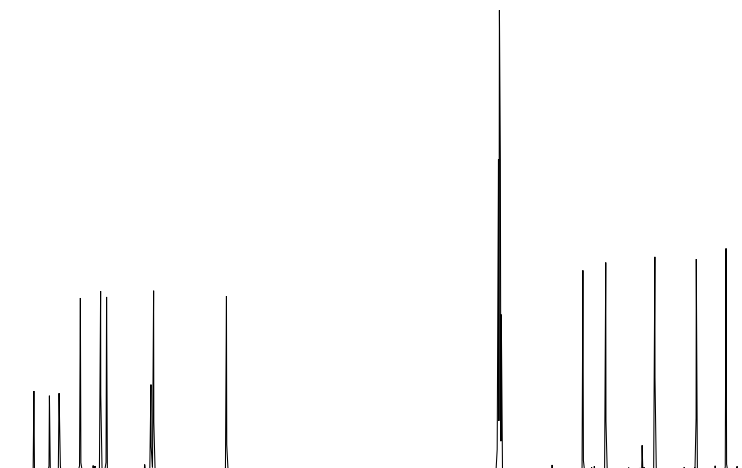


${ }^{1} \mathbf{H}$ NMR (700 MHz, $\left.\mathrm{CD}_{2} \mathrm{Cl}_{2}\right)$ of $\mathbf{1 2 g}$.

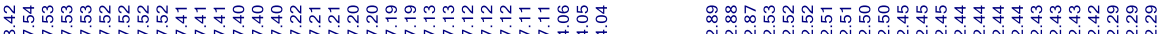

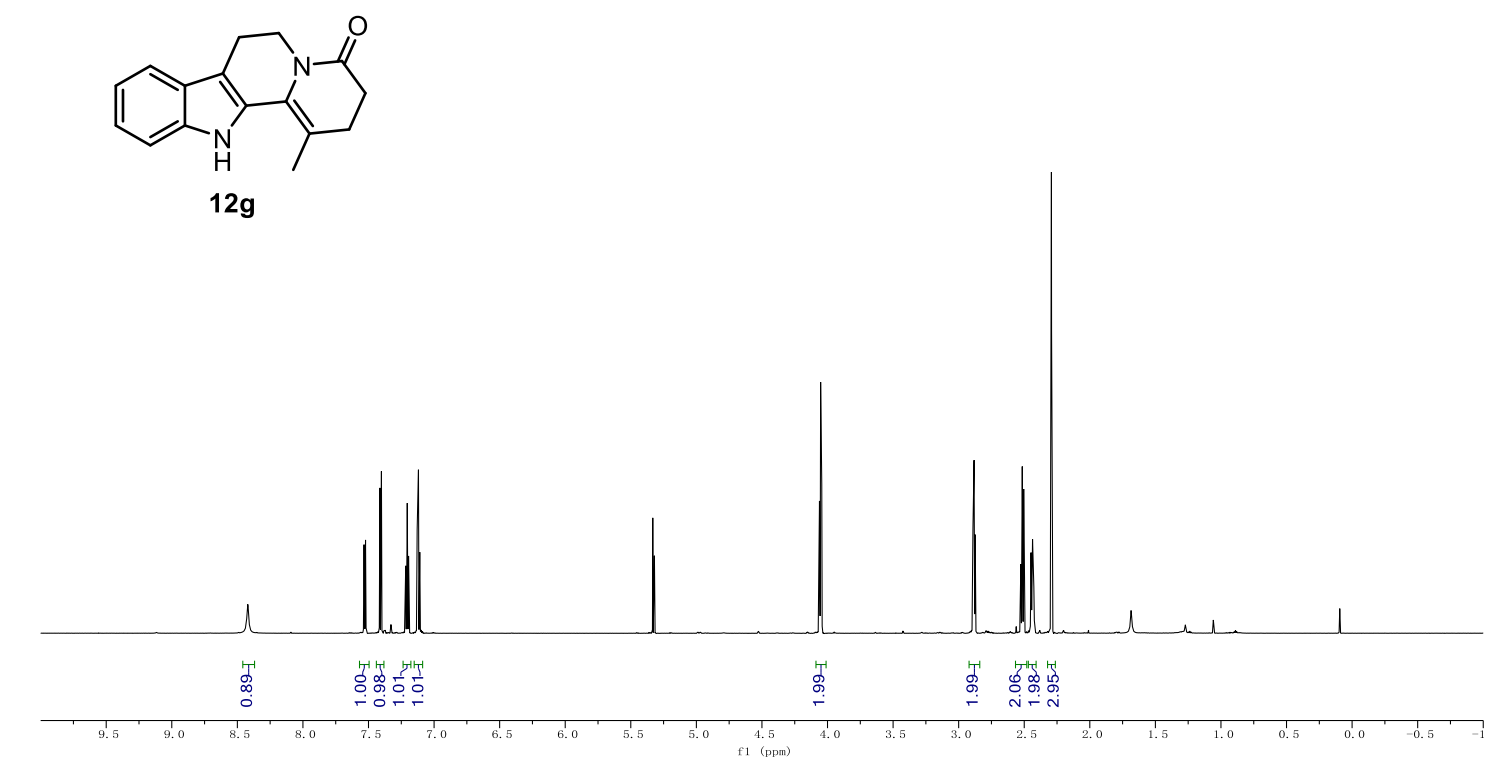

$12 \mathrm{~g}$

${ }^{13} \mathbf{C ~ N M R}\left(176 \mathrm{MHz}, \mathrm{CD}_{2} \mathrm{Cl}_{2}\right)$ of $\mathbf{1 2 g}$.

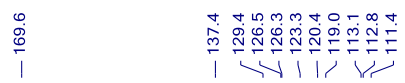

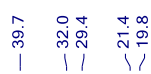

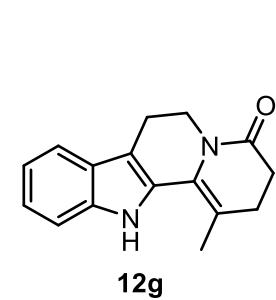

$12 \mathrm{~g}$

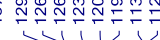
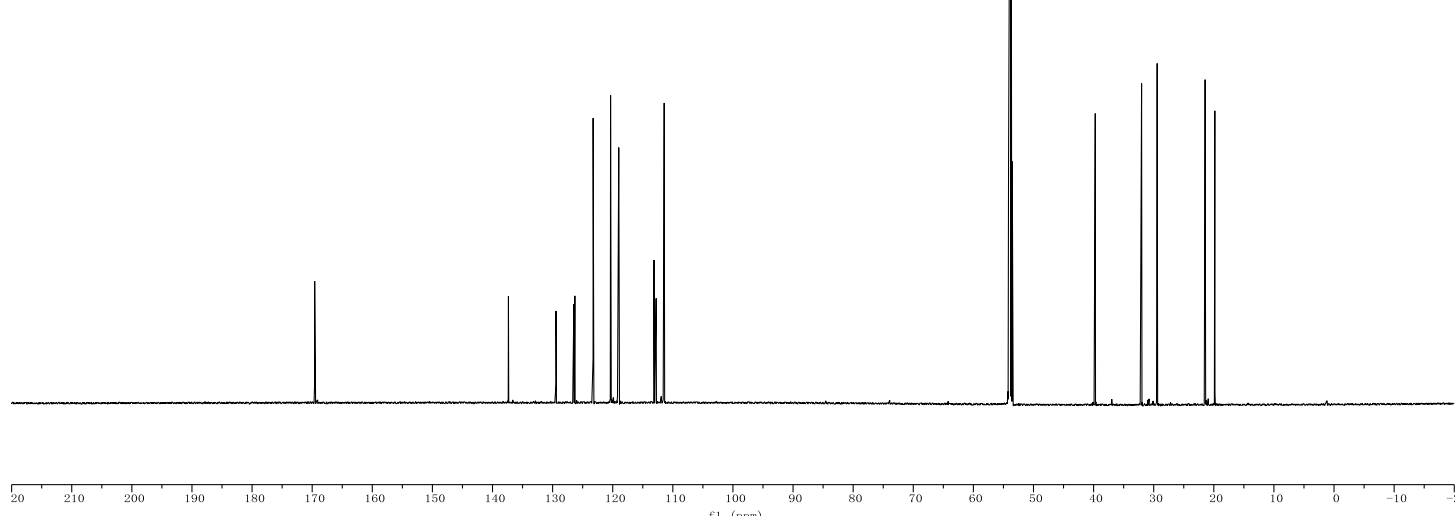
${ }^{1} \mathbf{H} \mathbf{N M R}\left(500 \mathrm{MHz}, \mathrm{CDCl}_{3}\right)$ of $\mathbf{1 2 h}$.

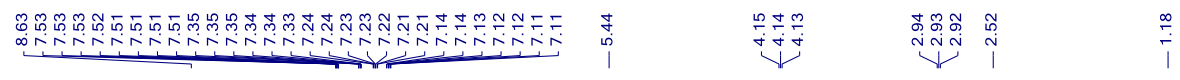<smiles>CC1(C)C=C2c3[nH]c4ccccc4c3CCN2C(=O)C1</smiles>

$12 \mathrm{~h}$

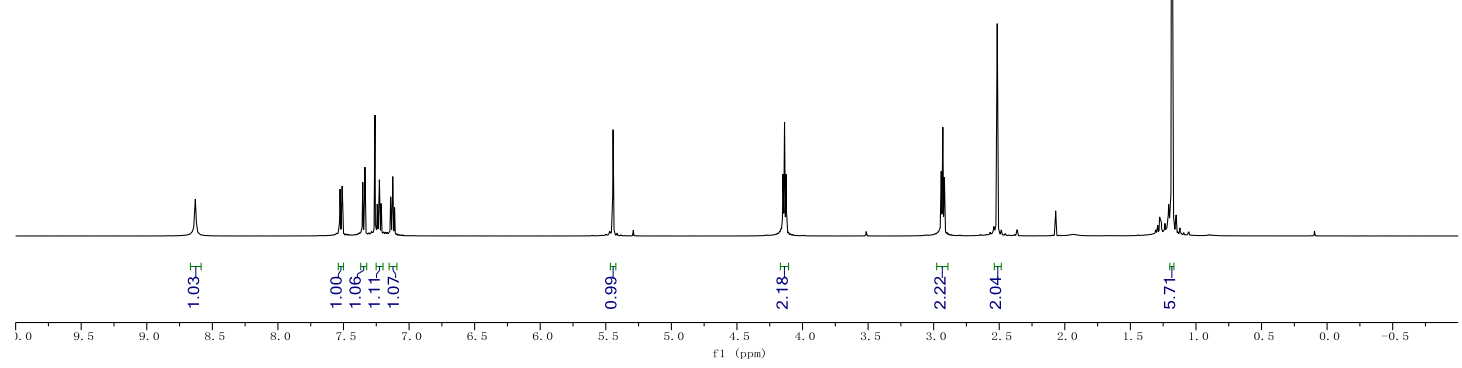

${ }^{13} \mathbf{C ~ N M R}\left(126 \mathrm{MHz}, \mathrm{CDCl}_{3}\right)$ of $\mathbf{1 2 h}$.

然

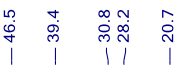

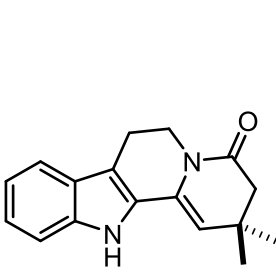

$12 \mathrm{~h}$

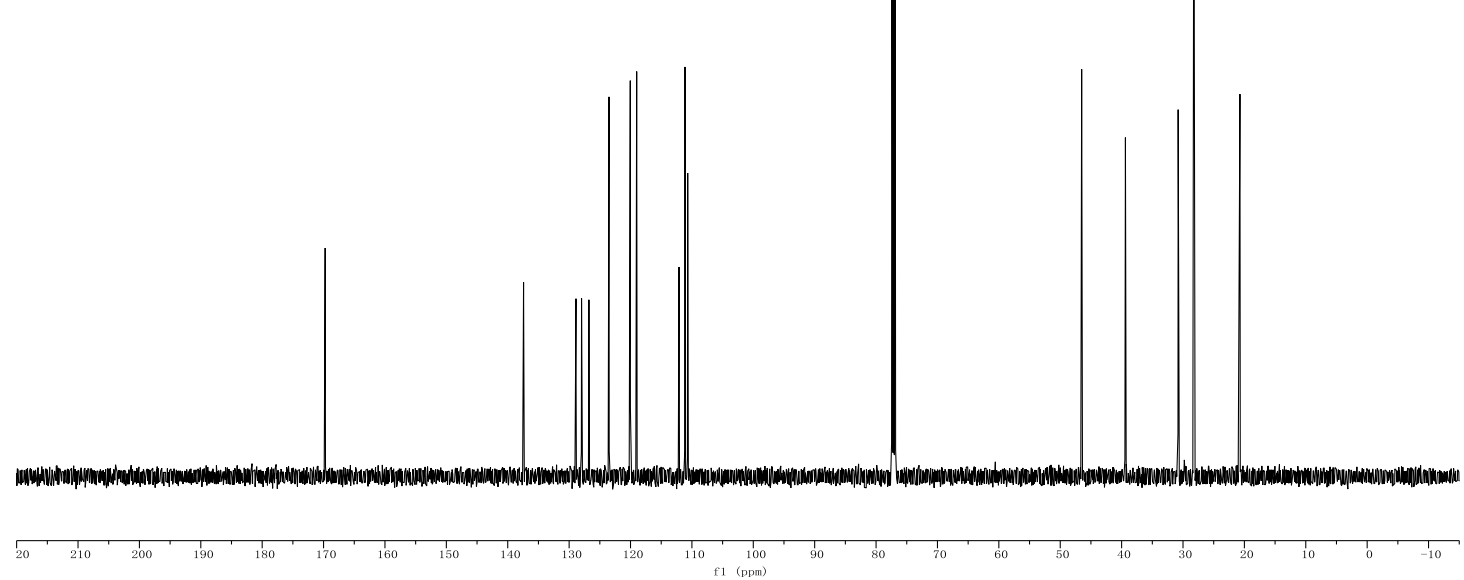


${ }^{1} \mathbf{H}$ NMR $\left(700 \mathrm{MHz}, \mathrm{CDCl}_{3}\right)$ of $\mathbf{1 2 i}$.

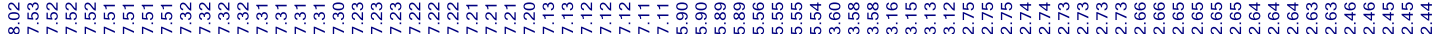

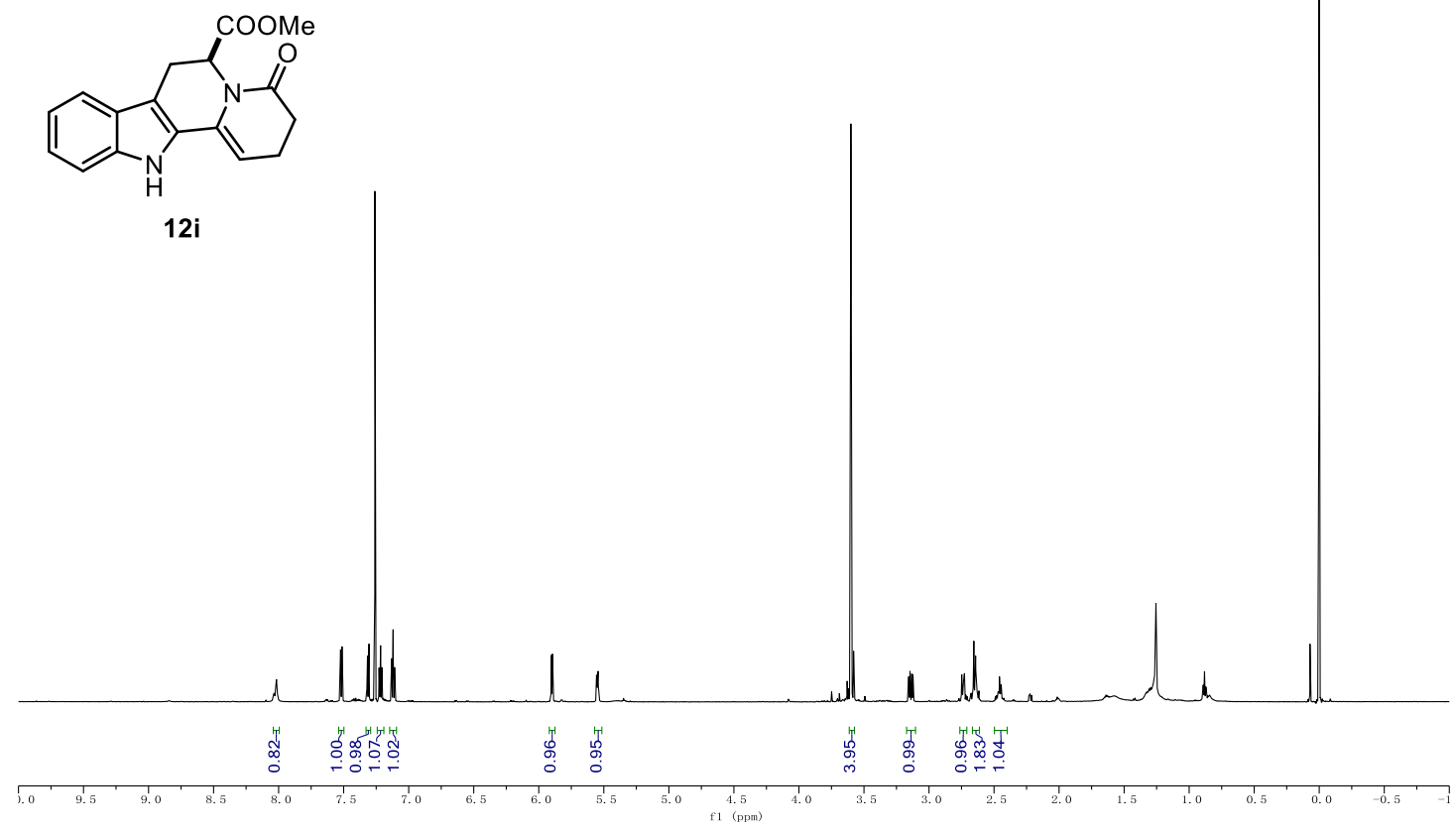

${ }^{13} \mathbf{C ~ N M R}\left(176 \mathrm{MHz}, \mathrm{CDCl}_{3}\right)$ of $\mathbf{1 2 i}$.

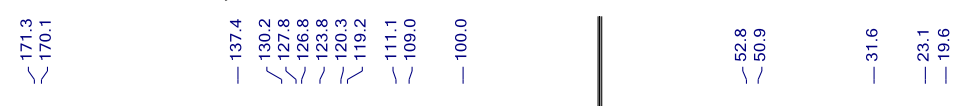<smiles>COC(=O)C1Cc2c([nH]c3ccccc23)C2=CCCC(=O)N21</smiles>

$12 \mathrm{i}$ 
${ }^{1} \mathbf{H}$ NMR (500 MHz, d6-DMSO) of 12j.

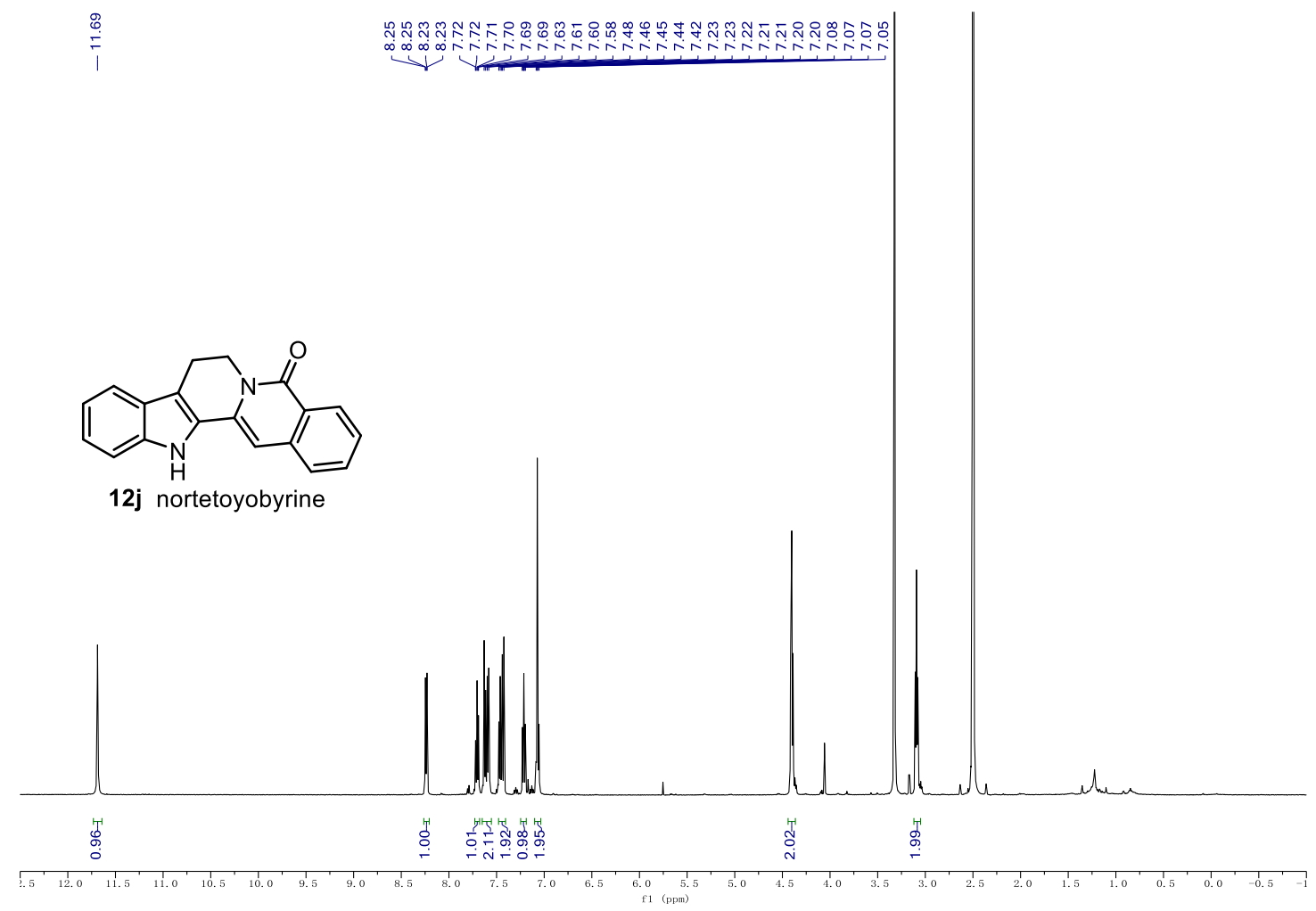

${ }^{13}$ C NMR (126 MHz, $d_{6}$-DMSO) of 12j.

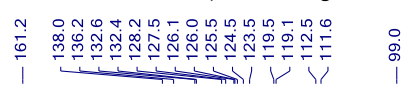

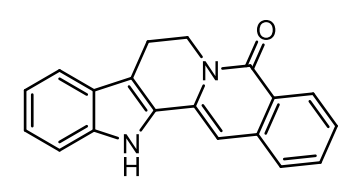

12j nortetoyobyrine

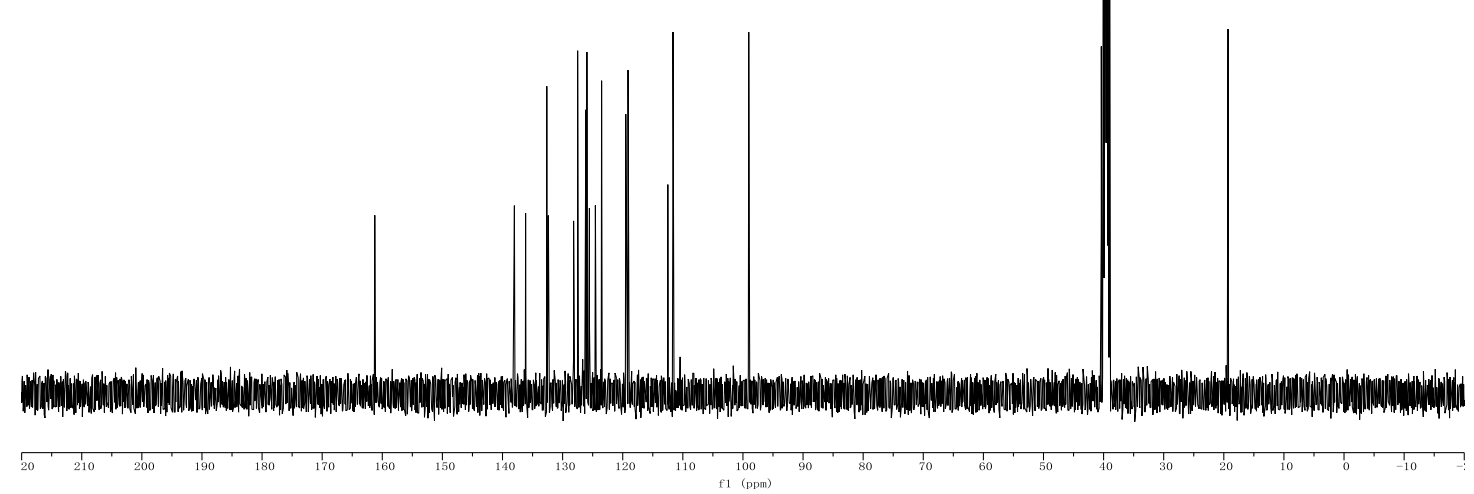


${ }^{1} \mathbf{H}$ NMR $\left(700 \mathrm{MHz}, \mathrm{CD}_{2} \mathrm{Cl}_{2}\right)$ of $\mathbf{1 2 k}$.

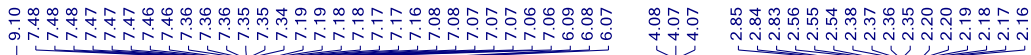

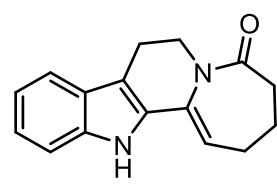

$12 k$

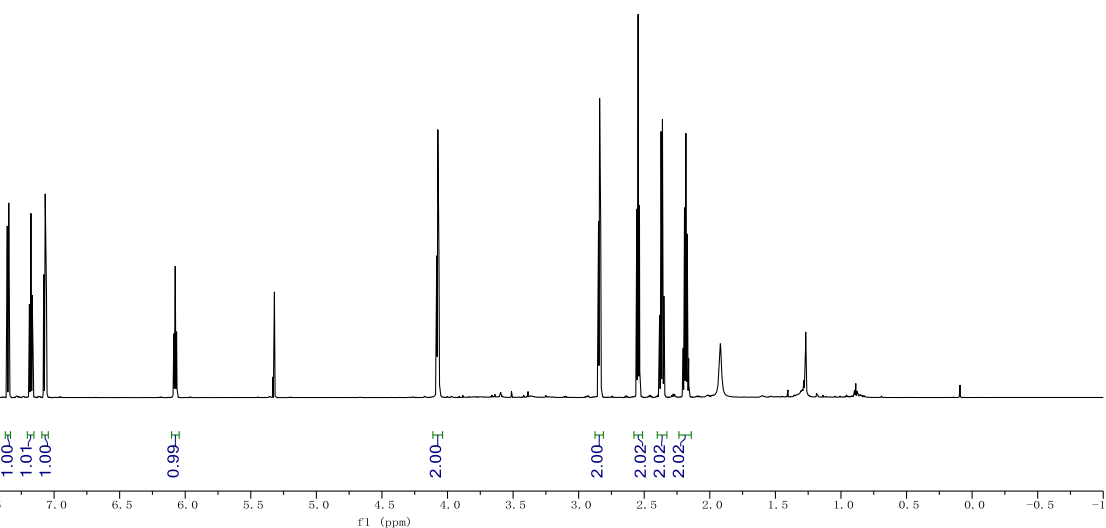

${ }^{13} \mathbf{C ~ N M R}\left(176 \mathrm{MHz}, \mathrm{CD}_{2} \mathrm{Cl}_{2}\right)$ of $\mathbf{1 2 k}$.

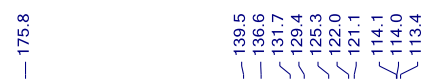

tion

(1)
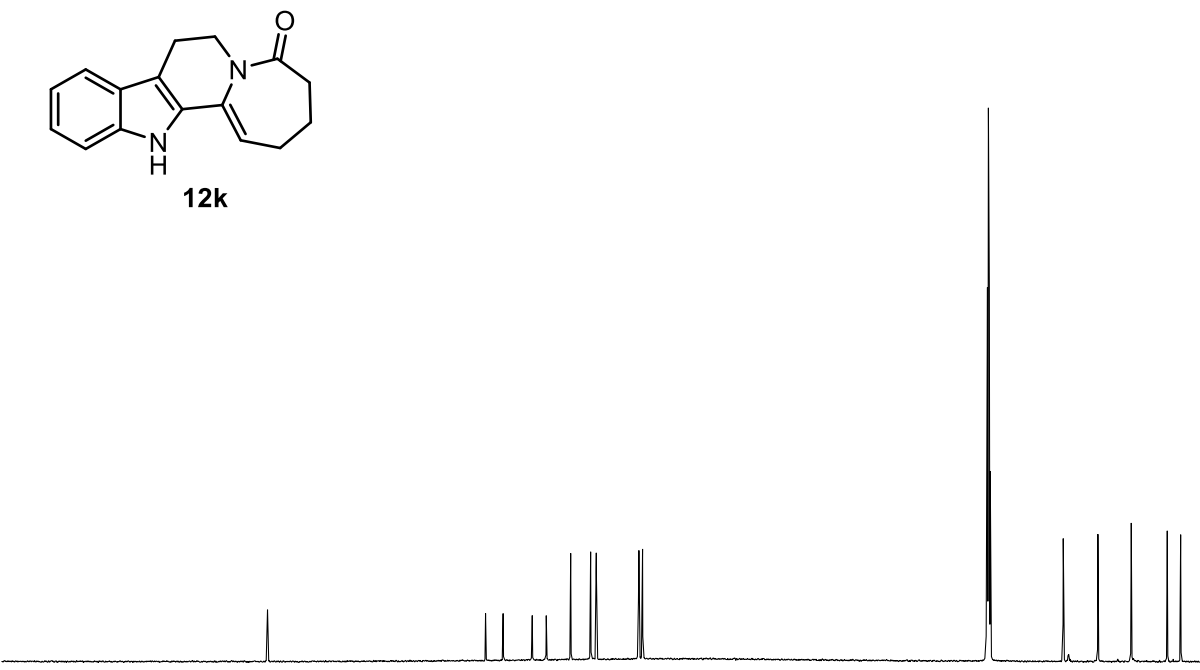
${ }^{1} \mathbf{H}$ NMR (700 MHz, $\mathrm{CD}_{2} \mathrm{Cl}_{2}$ ) of $\mathbf{1 2 l}$.

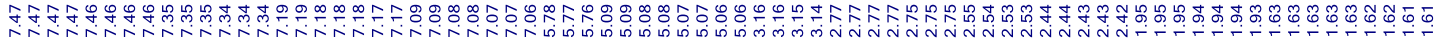<smiles>O=C1CCCCC=C2c3[nH]c4ccccc4c3CCN12</smiles>

121

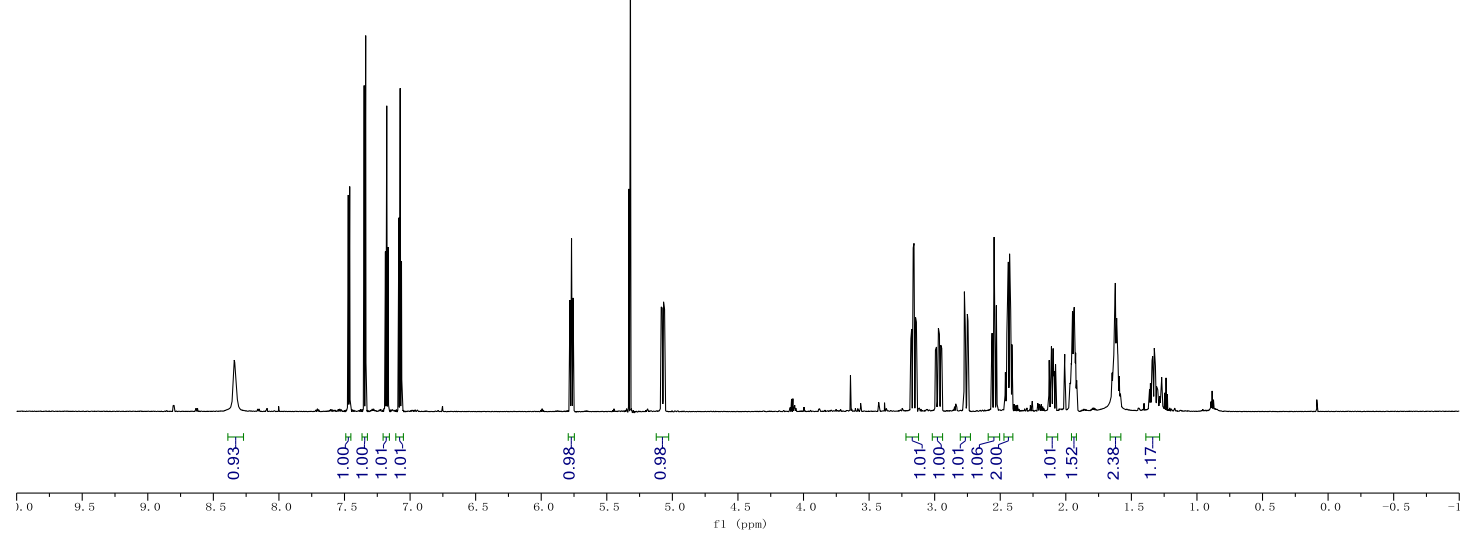

${ }^{13}$ C NMR (176 MHz, $\left.\mathrm{CD}_{2} \mathrm{Cl}_{2}\right)$ of 121.

竞

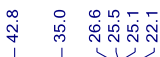<smiles>O=C1CCCCC=C2c3[nH]c4ccccc4c3CCN12</smiles>

12I 
${ }^{1} \mathbf{H}$ NMR (700 MHz, $\left.\mathrm{CDCl}_{3}\right)$ of $\mathbf{1 2 m}$.

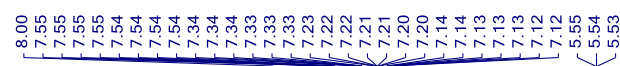

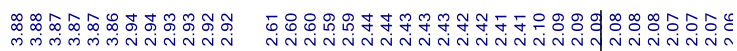

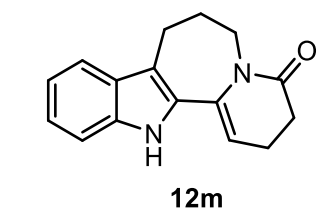

${ }^{13} \mathbf{C}$ NMR $\left(176 \mathrm{MHz}, \mathrm{CD}_{2} \mathrm{Cl}_{2}\right)$ of $\mathbf{1 2 m}$.

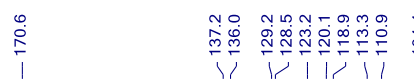

辛

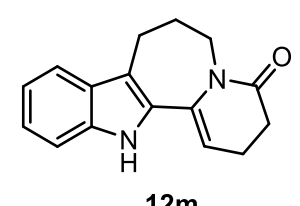

$12 \mathrm{~m}$

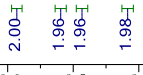


${ }^{1} \mathbf{H}$ NMR (700 MHz, $\left.\mathrm{CDCl}_{3}\right)$ of $\mathbf{1 2 n}$.

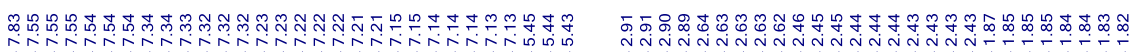

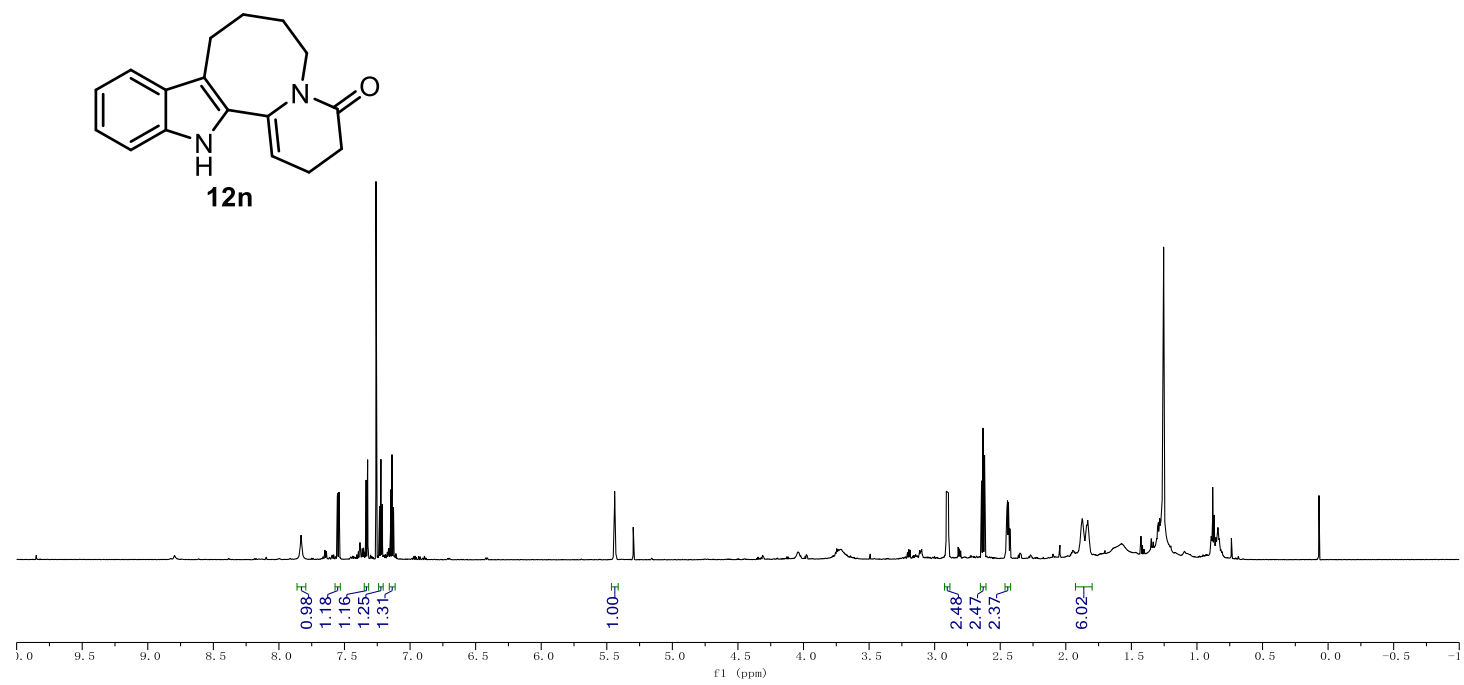

${ }^{13} \mathbf{C ~ N M R}\left(176 \mathrm{MHz}, \mathrm{CDCl}_{3}\right)$ of $\mathbf{1 2 n}$.

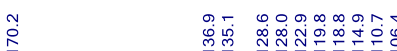

1) 1<smiles>O=C1CCC=C2c3[nH]c4ccccc4c3CCCCN12</smiles> 
${ }^{1}$ H NMR (700 MHz, C6 6 6) of 120.

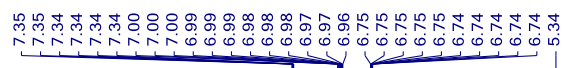

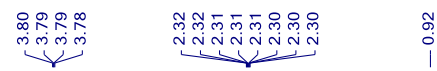

(120

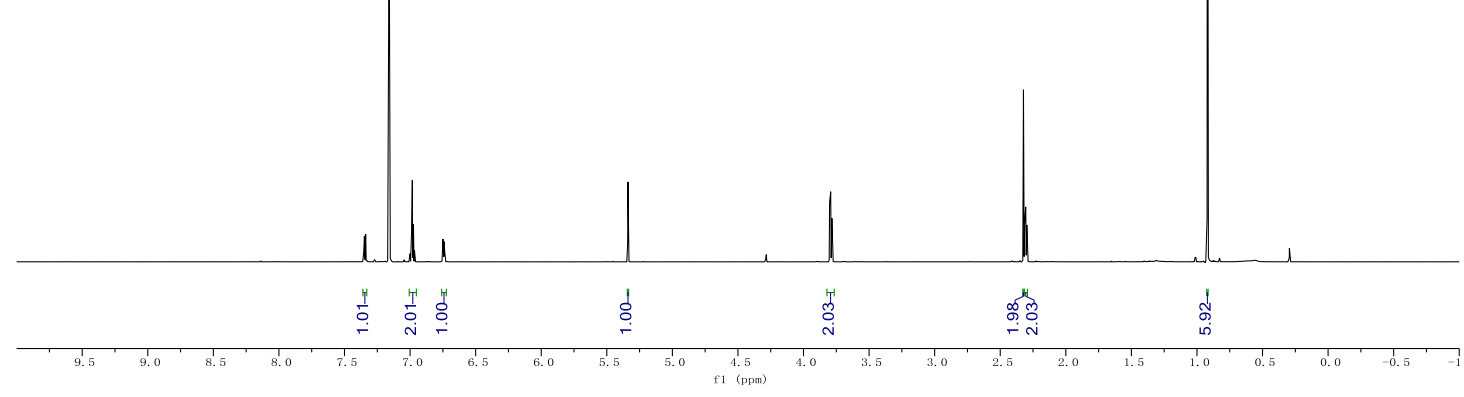

${ }^{13}$ C NMR (176 MHz, C6 6 6) of 120.

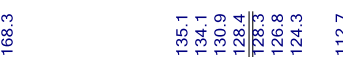

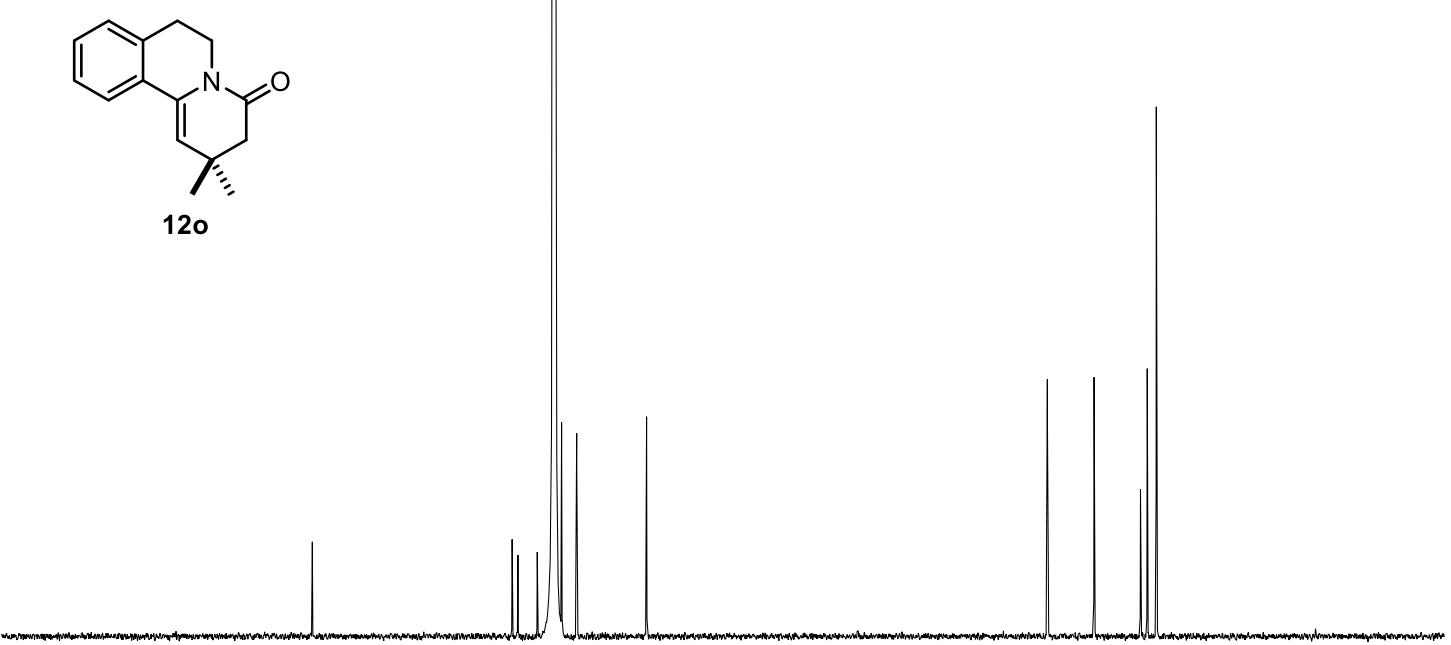

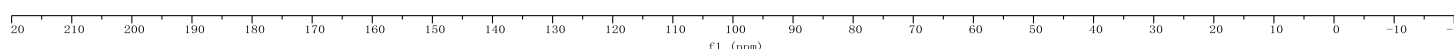


${ }^{1}$ H NMR (700 MHz, C6 6 6) of 12p.

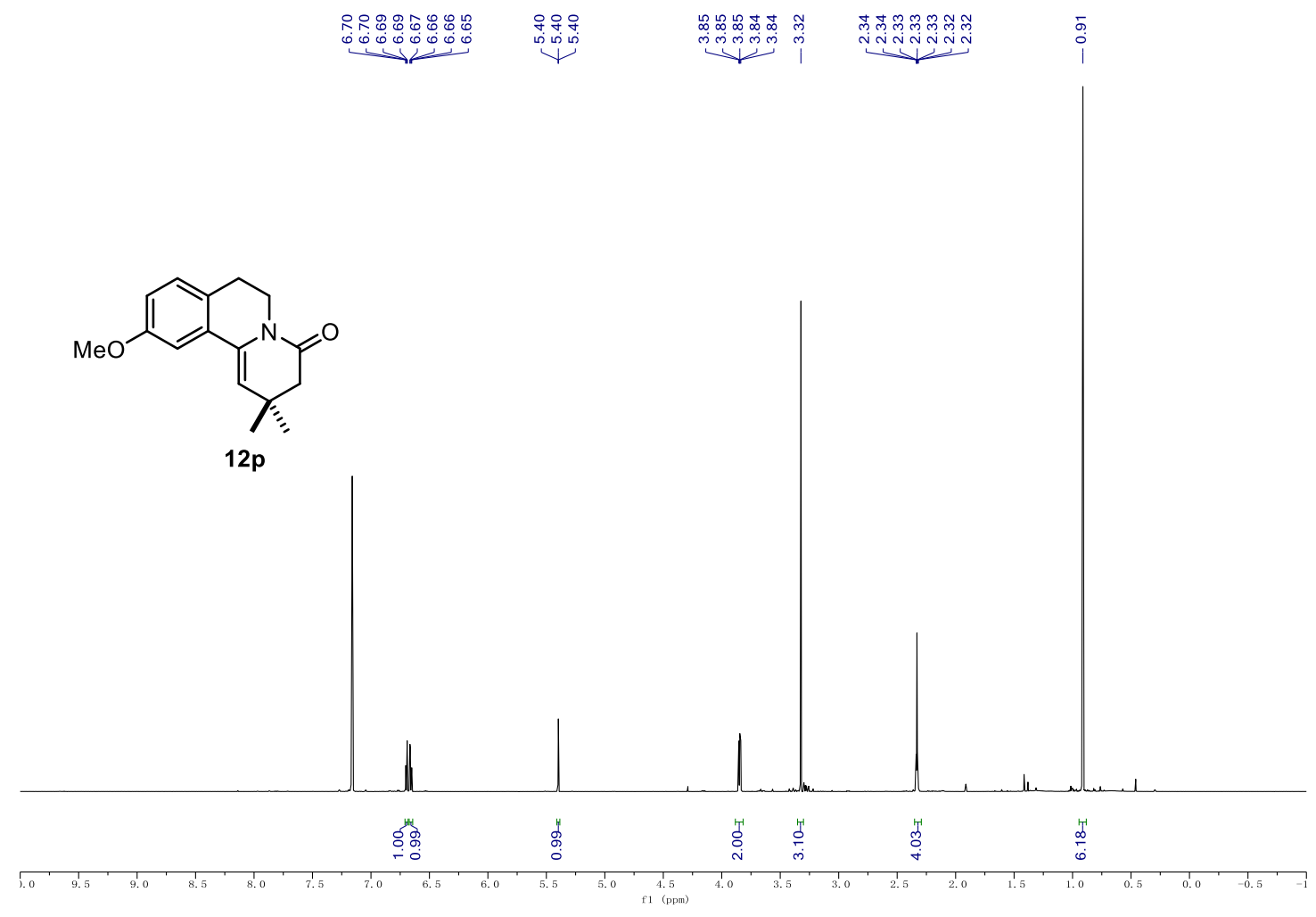

${ }^{13}$ C NMR (176 MHz, C6 6 6) of 12p.
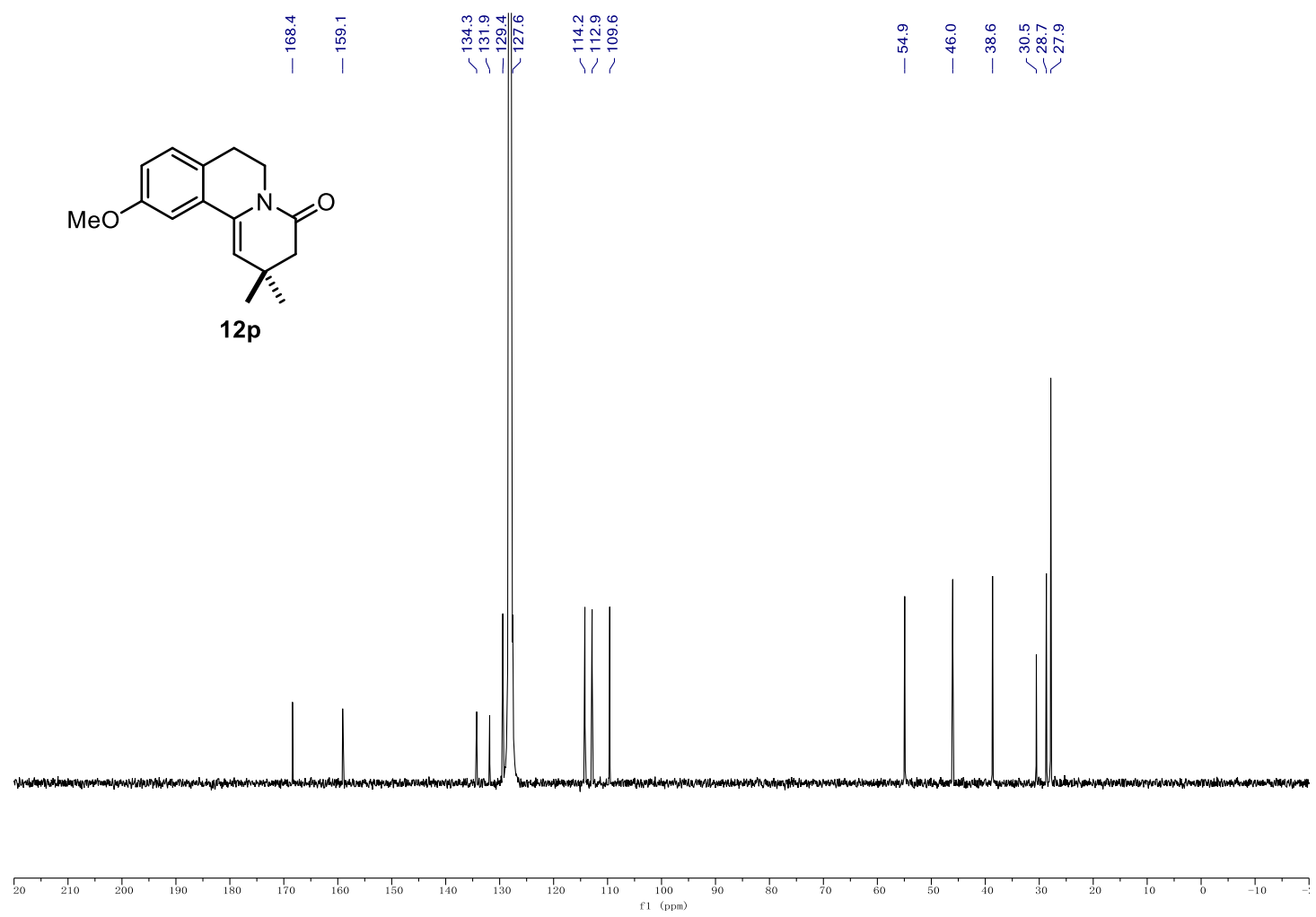
${ }^{1} \mathbf{H}$ NMR (500 MHz, $\left.\mathrm{CDCl}_{3}\right)$ of $\mathbf{1 2 q}$.

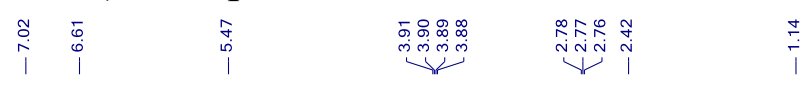<smiles>COc1cc2c(cc1OC)C1=CC(C)(C)CC(=O)N1CC2</smiles>

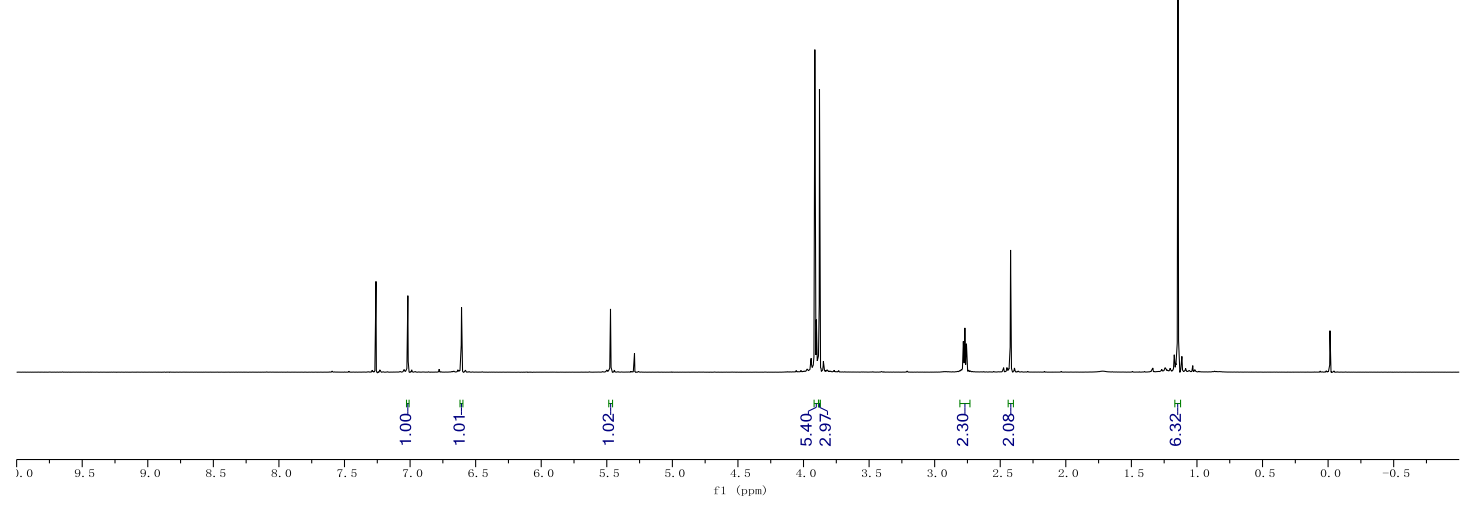

${ }^{13}$ C NMR (126 MHz, $\left.\mathrm{CDCl}_{3}\right)$ of 12q.

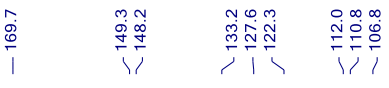<smiles>COc1cc2c(cc1OC)C1=C[C@](C)([14CH3])CC(=O)N1CC2</smiles>

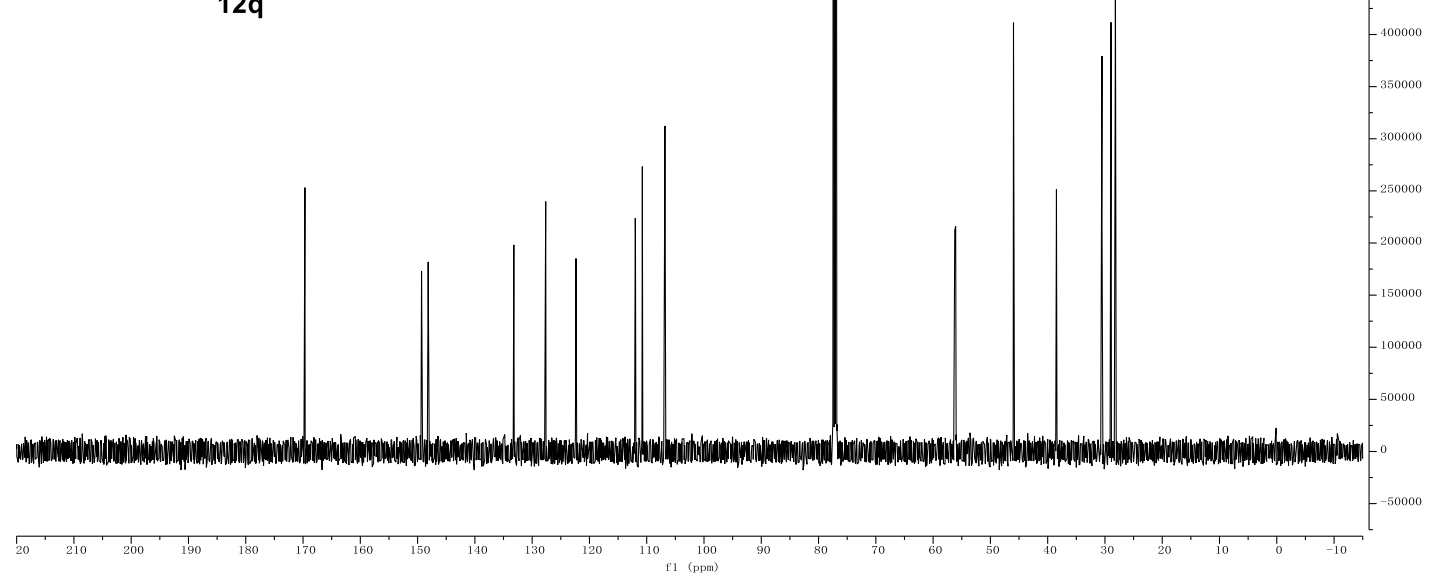


${ }^{1} \mathbf{H}$ NMR (500 MHz, C6 6 6) of 12r.<smiles>COc1cc2c(cc1OC)C1=CCCC(=O)N1CC2</smiles>

$12 r$

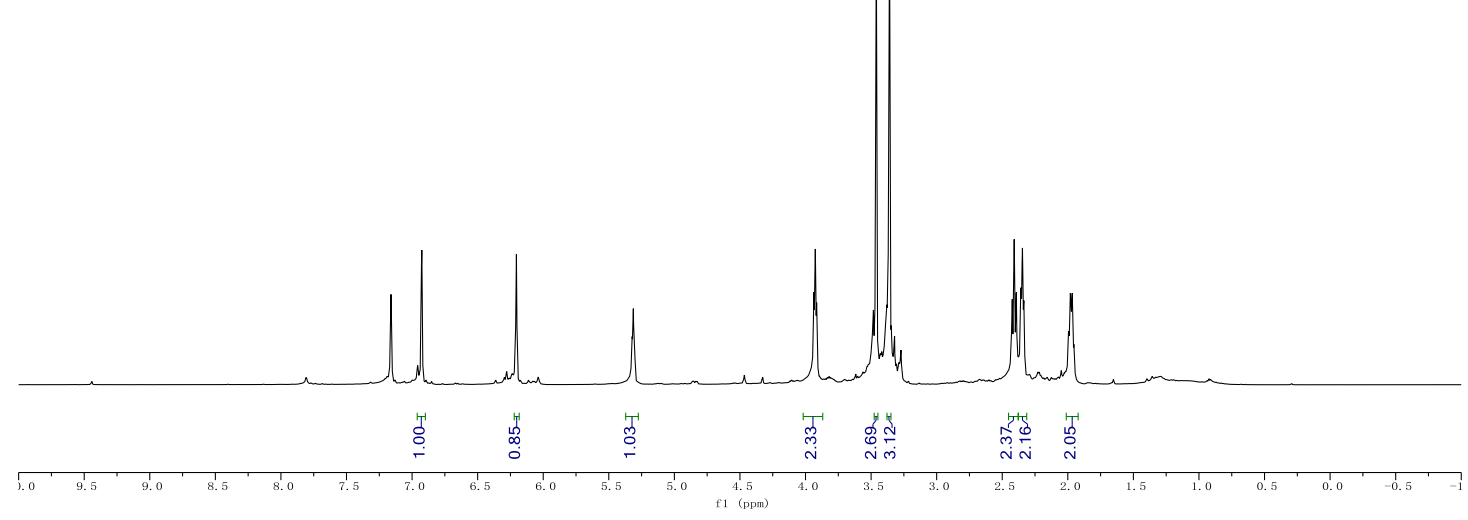

${ }^{13}$ C NMR (126 MHz, $\left.\mathrm{C}_{6} \mathrm{D}_{6}\right)$ of 12r.

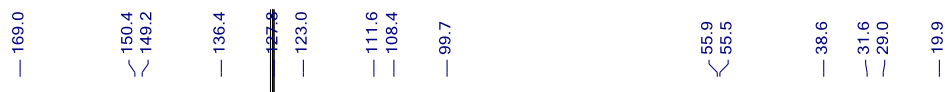<smiles>COc1cc2c(cc1OC)C1=CCCC(=O)N1CC2</smiles>

$12 r$

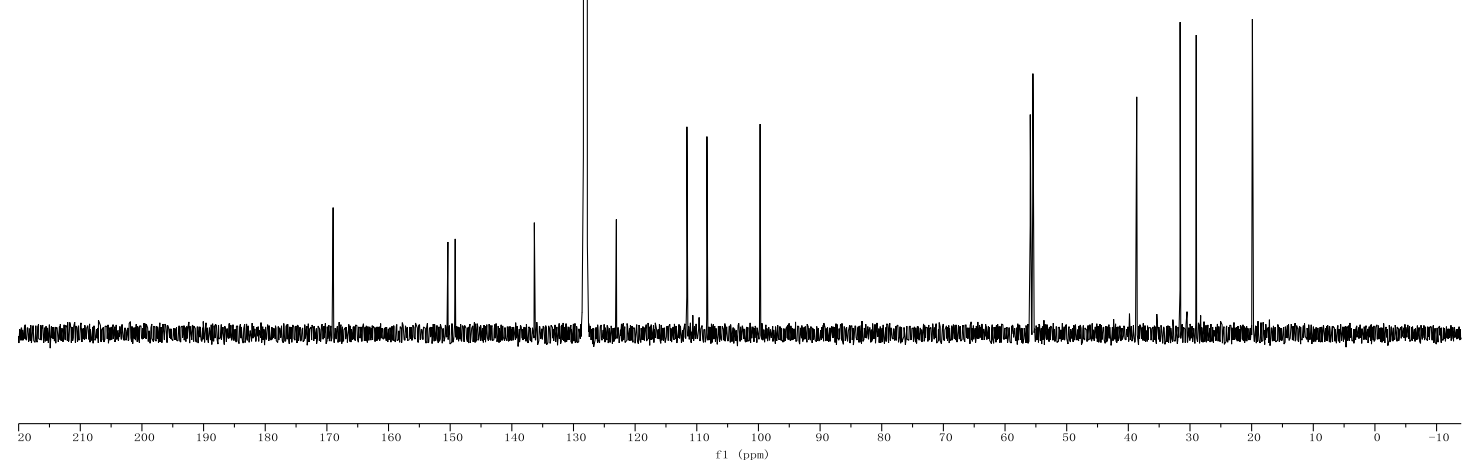


${ }^{1} \mathbf{H}$ NMR $\left(600 \mathrm{MHz}, \mathrm{CDCl}_{3}\right)$ of $\mathbf{1 2 s .}$

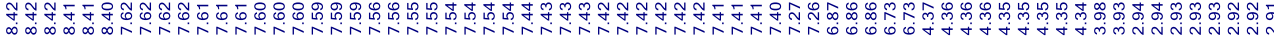

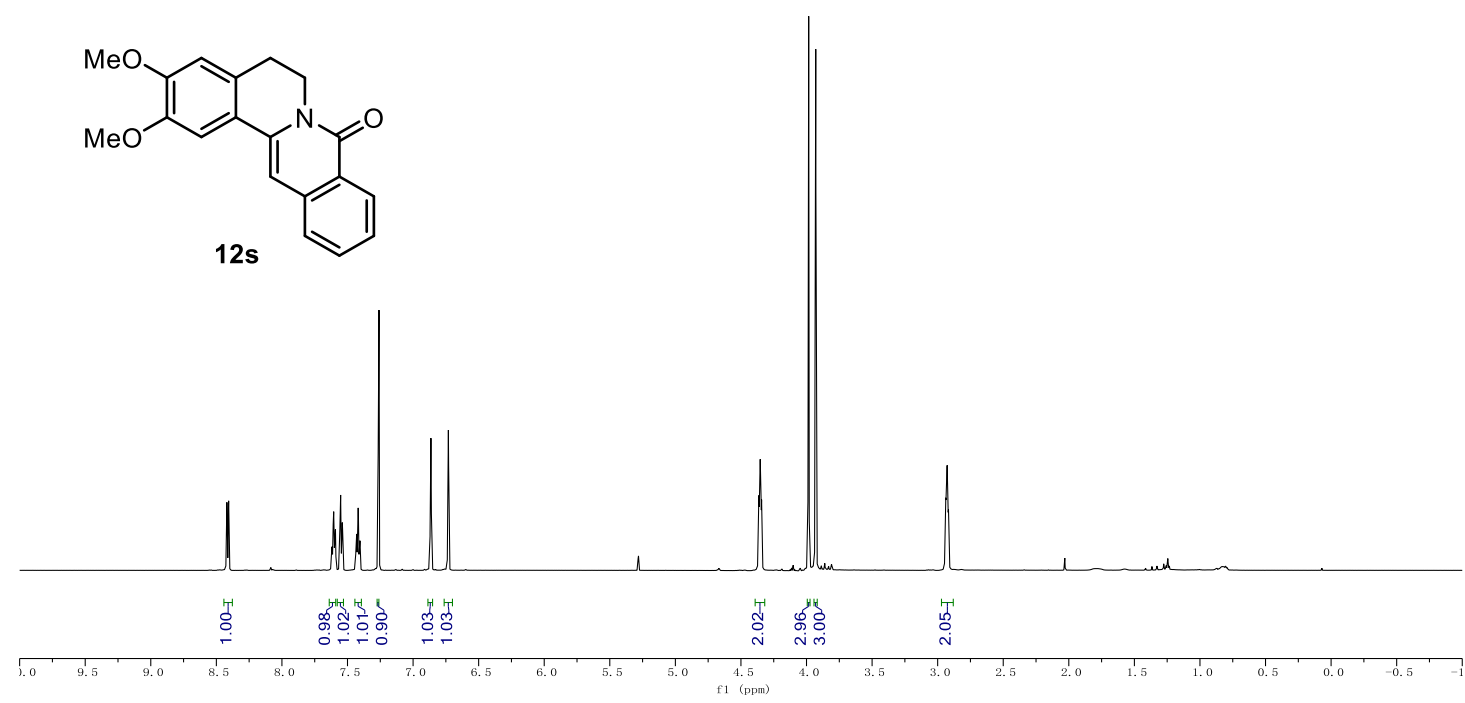

${ }^{13}$ C NMR (151 MHz, $\left.\mathrm{CDCl}_{3}\right)$ of $\mathbf{1 2 s}$.

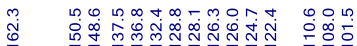

广

$12 \mathrm{~s}$

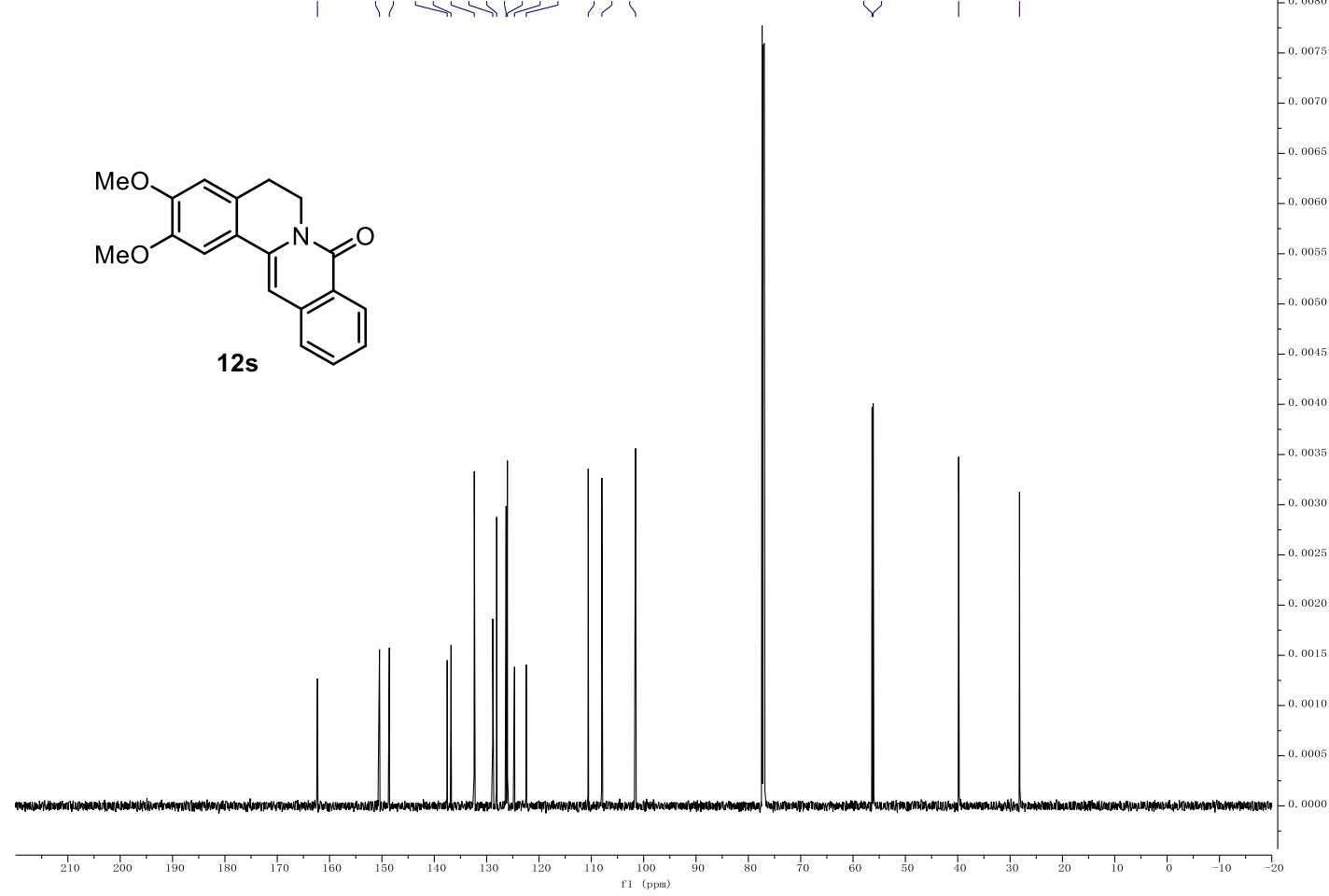

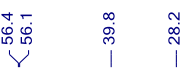


${ }^{1} \mathbf{H}$ NMR $\left(600 \mathrm{MHz}, \mathrm{CD}_{2} \mathrm{Cl}_{2}\right)$ of $\mathbf{1 2 t}$.

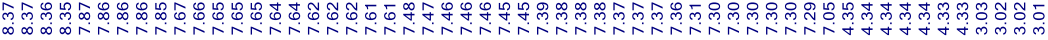<smiles></smiles>

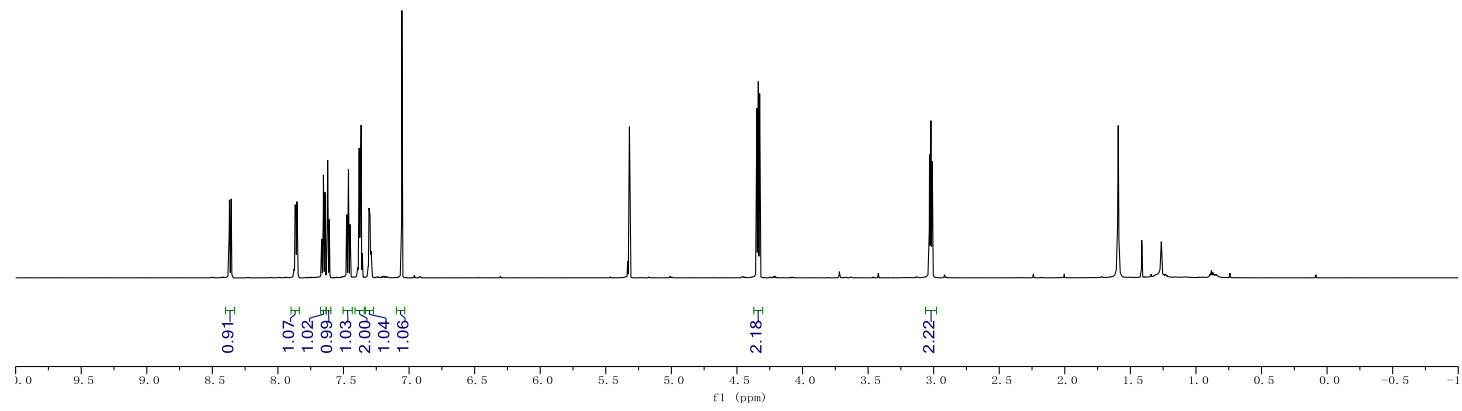

${ }^{13} \mathrm{C}$ NMR (151 MHz, $\left.\mathrm{CD}_{2} \mathrm{Cl}_{2}\right)$ of $\mathbf{1 2 t}$.

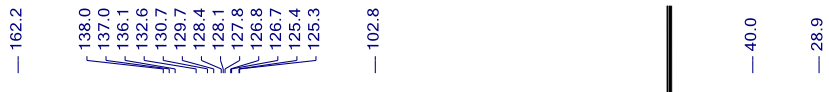<smiles>CCCCCn1c(-c2ccccc2)cc2ccccc2c1=O</smiles> 
${ }^{\mathbf{1}} \mathbf{H}$ NMR (700 MHz, $\left.\mathrm{C}_{6} \mathrm{D}_{6}\right)$ of $\mathbf{1 2 u}$.

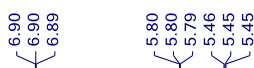

$\underbrace{\mathscr{0} 0}$

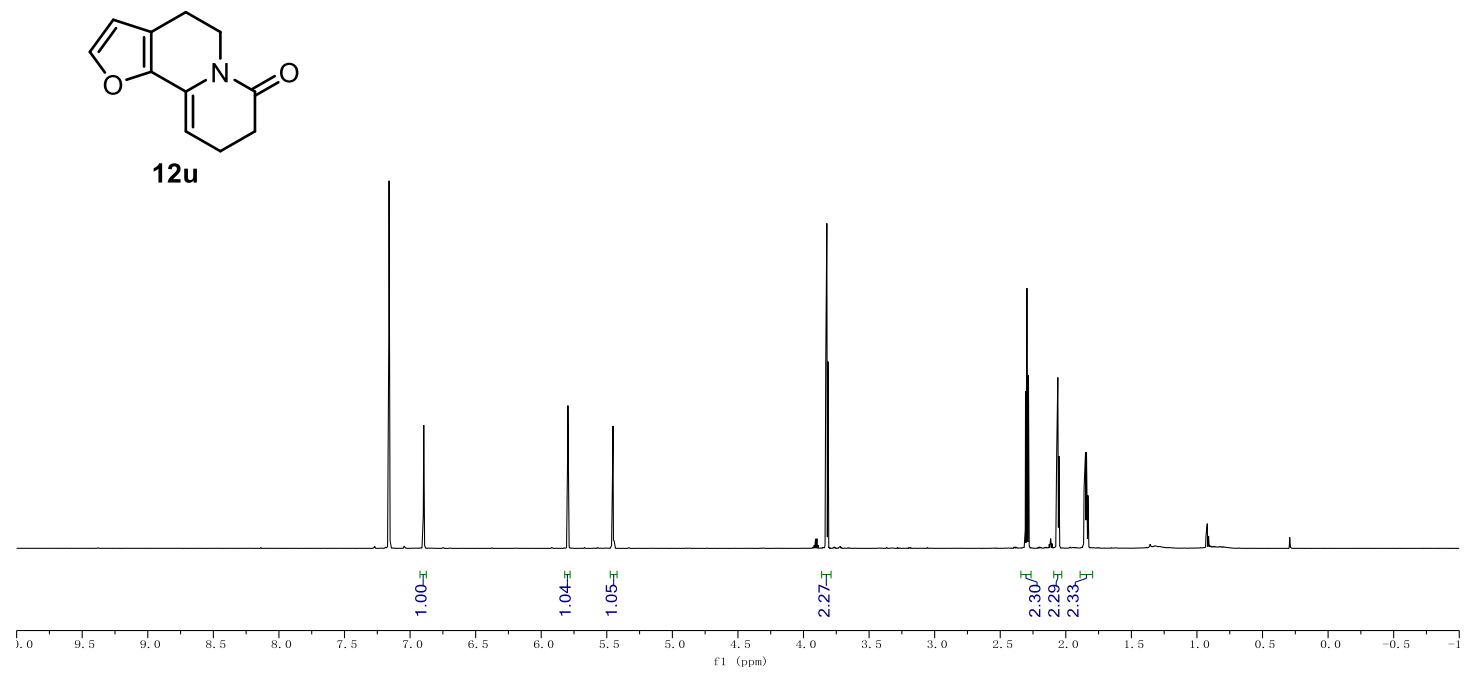

${ }^{13} \mathbf{C}$ NMR (176 MHz, C6 6 6) of $\mathbf{1 2 u}$.
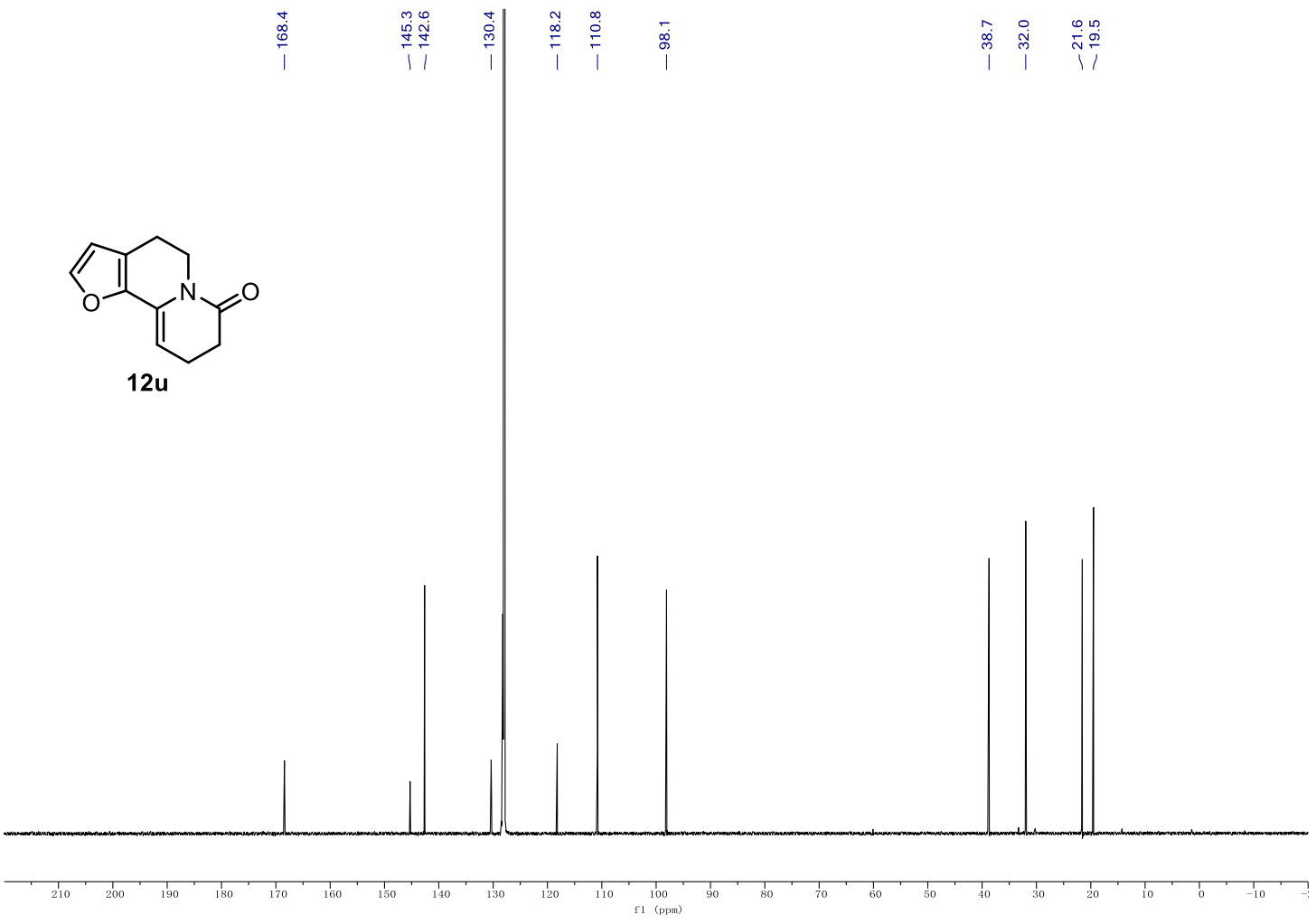
${ }^{\mathbf{1}} \mathbf{H}$ NMR (700 MHz, $\left.\mathrm{C}_{6} \mathrm{D}_{6}\right)$ of $\mathbf{1 2 v}$.

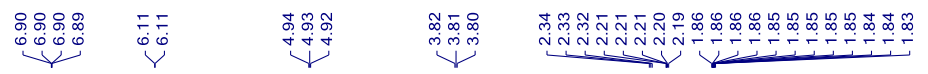

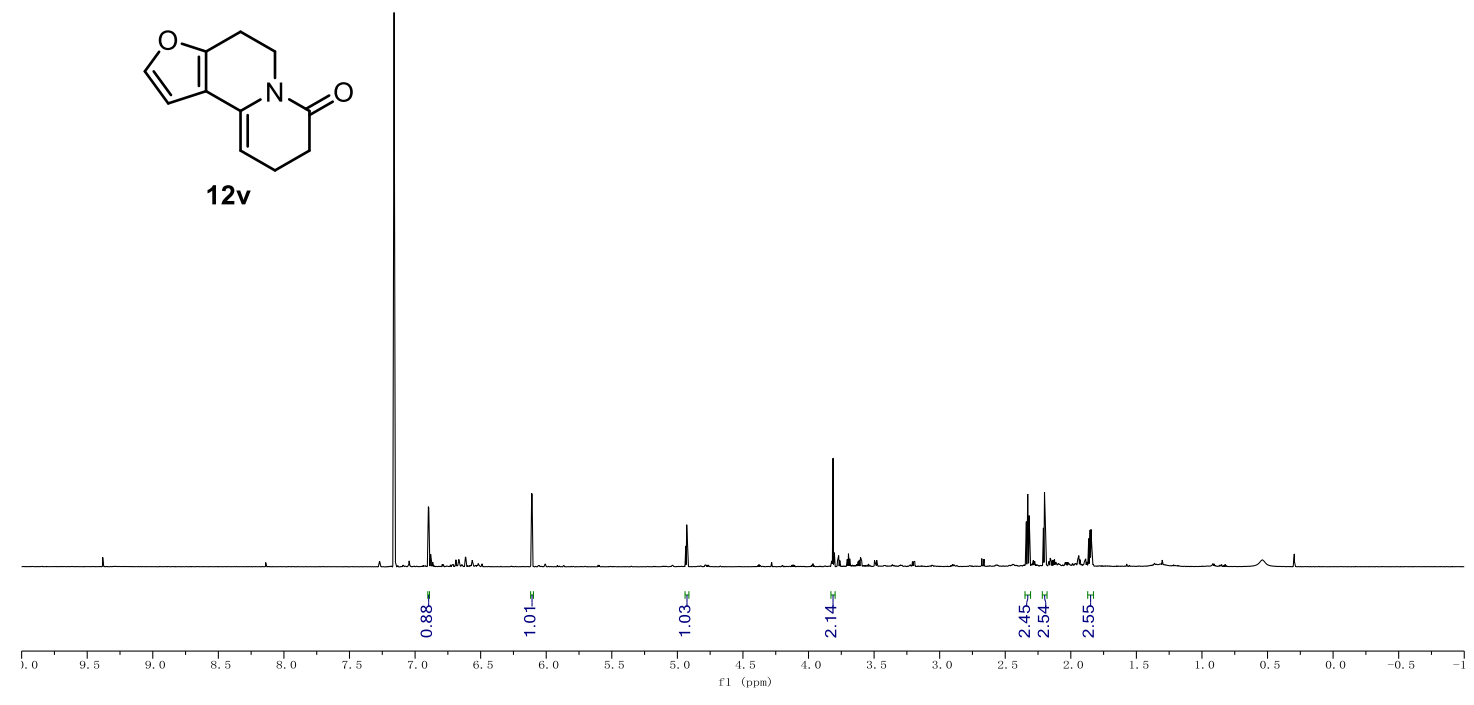

${ }^{13}$ C NMR (176 MHz, C6 6 6) of 12v.
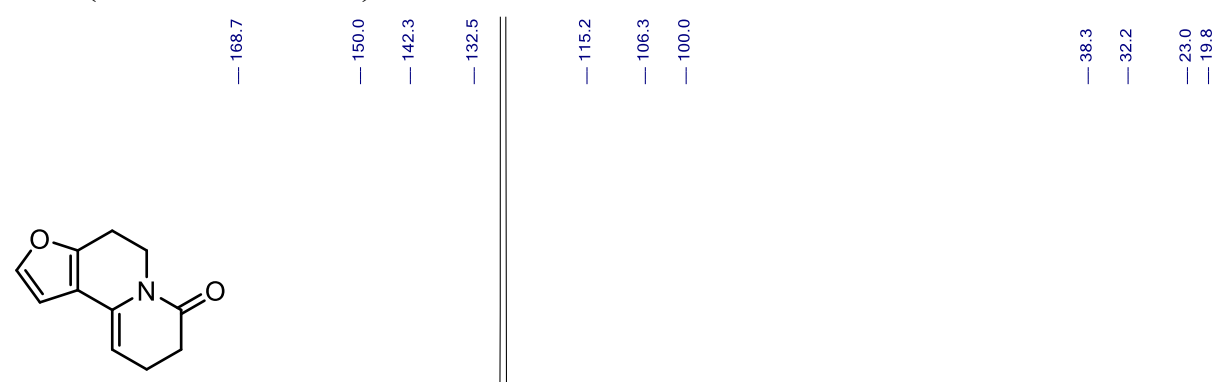

12v
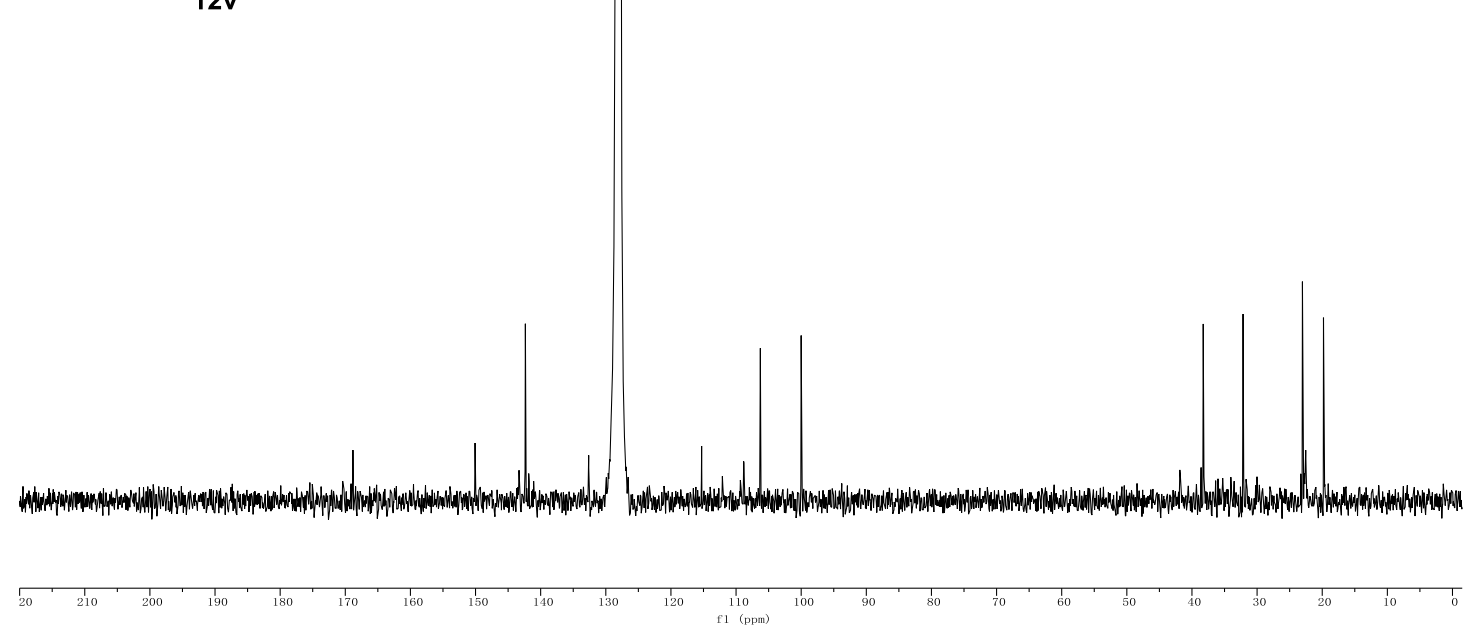
${ }^{\mathbf{1}} \mathbf{H}$ NMR (500 MHz, $\left.\mathrm{C}_{6} \mathrm{D}_{6}\right)$ of $\mathbf{1 2} \mathbf{w}$.

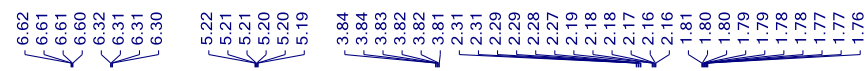<smiles>O=C1CCCCC12CCCCC2</smiles>

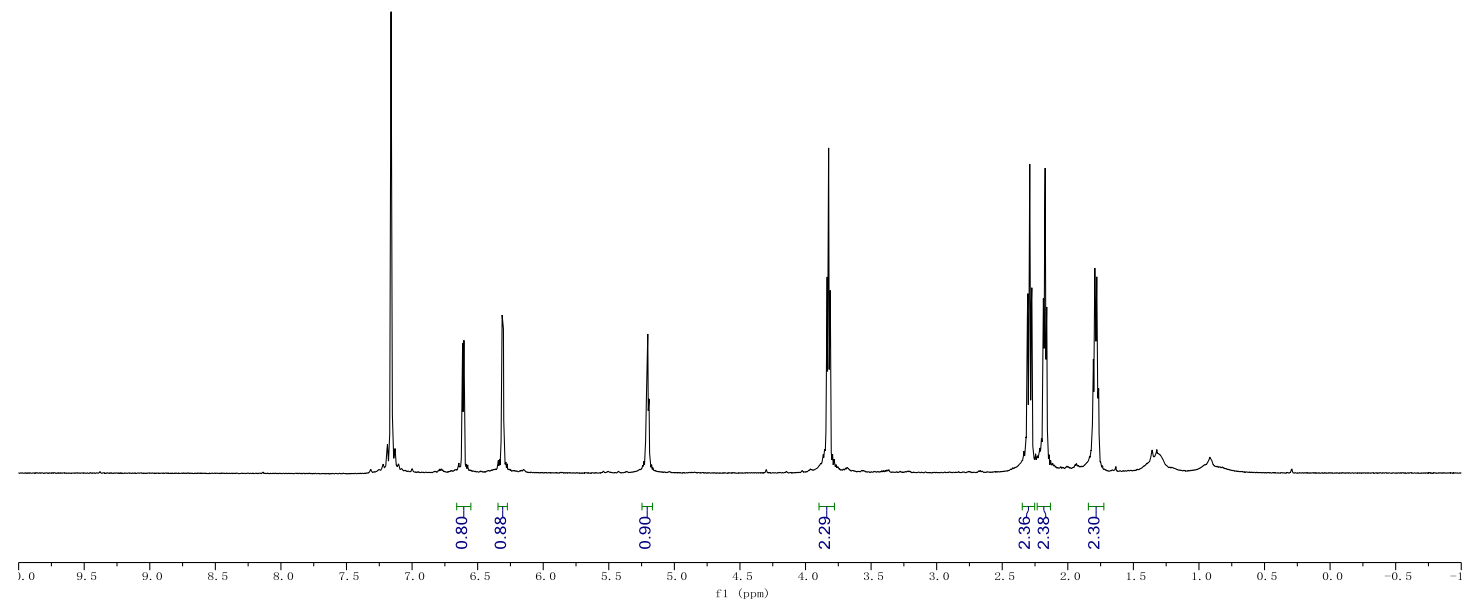

${ }^{13} \mathbf{C}$ NMR (126 MHz, $\left.\mathrm{C}_{6} \mathrm{D}_{6}\right)$ of $\mathbf{1 2 w}$.

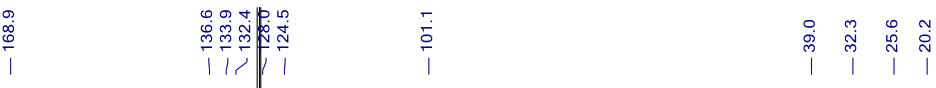<smiles>O=C1CCC=C2c3sccc3CCN12</smiles>

$12 w$

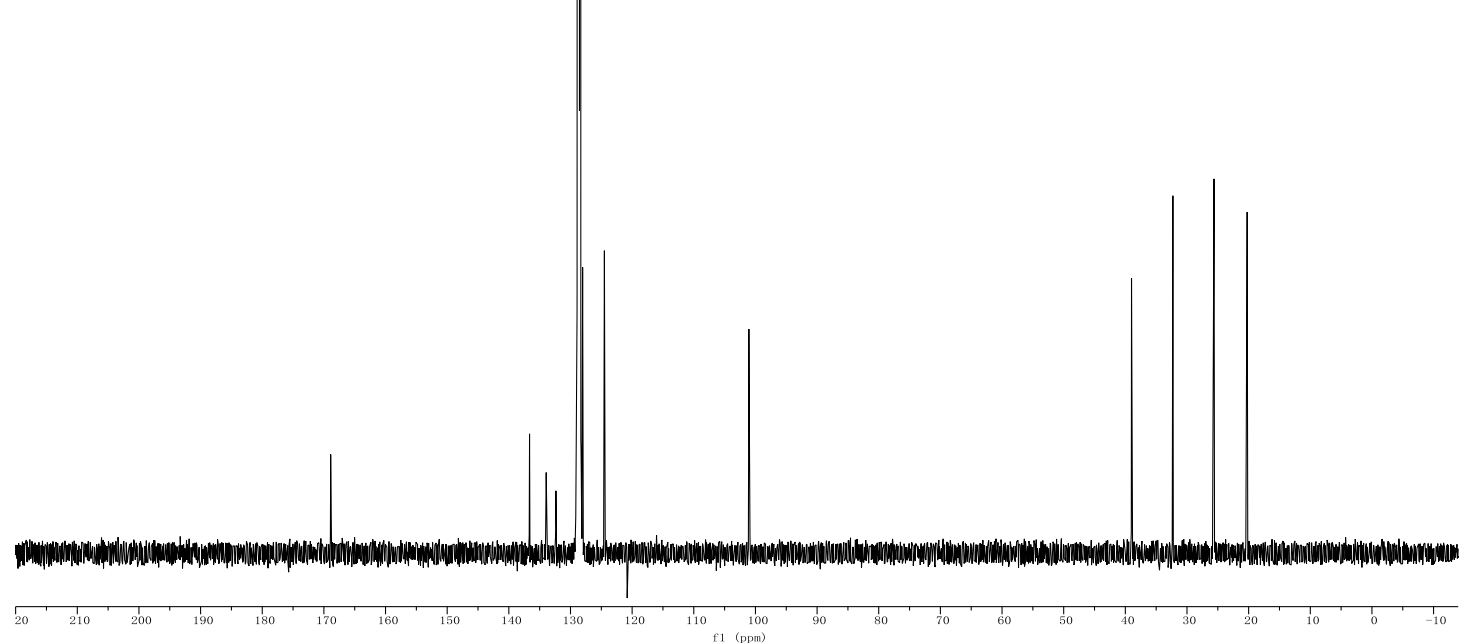


${ }^{1} \mathbf{H}$ NMR $\left(700 \mathrm{MHz}, \mathrm{CD}_{2} \mathrm{Cl}_{2}\right)$ of $\mathbf{1 2 x}$.

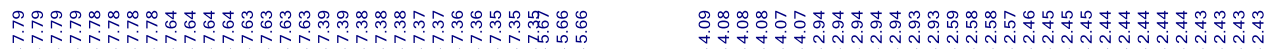
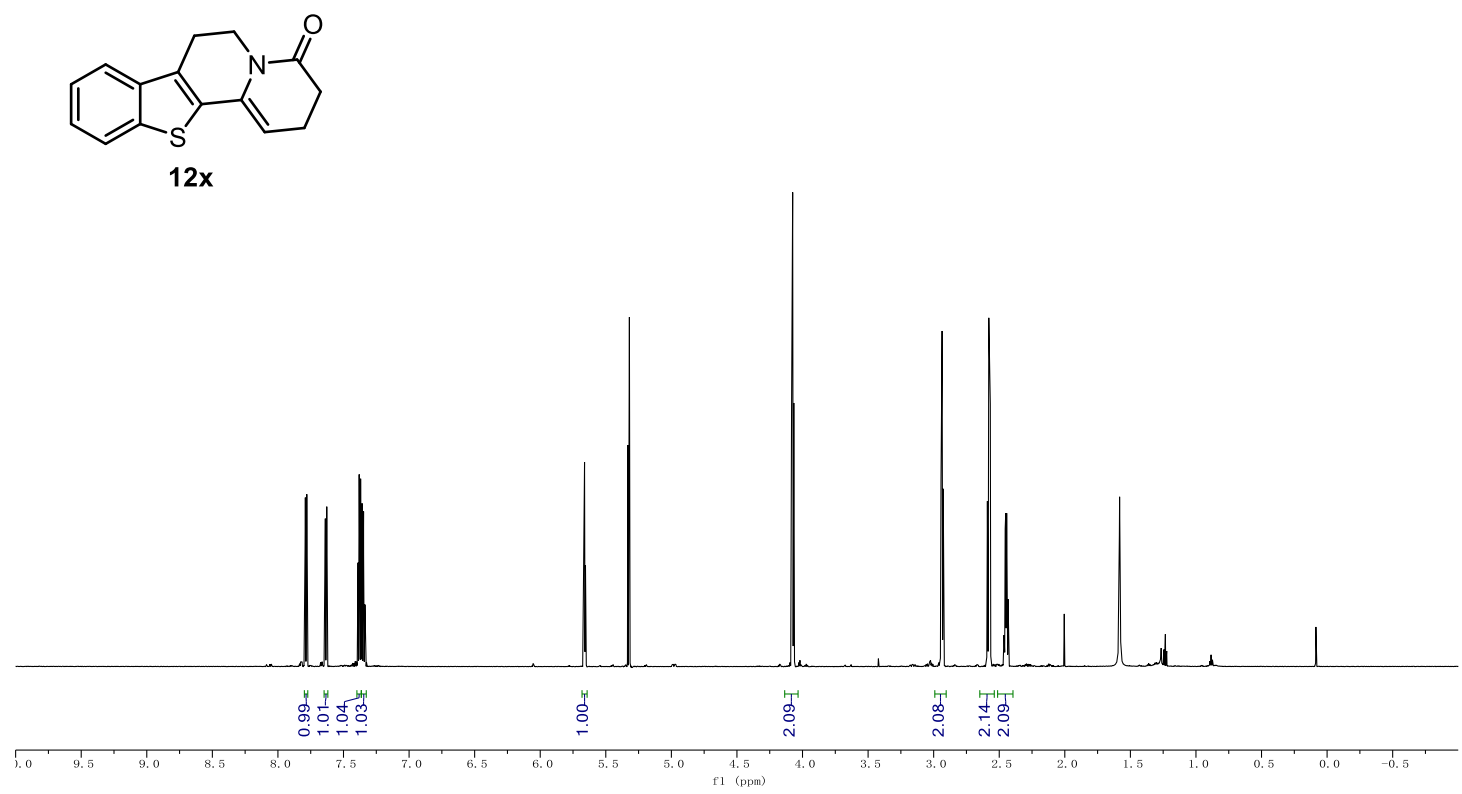

${ }^{13} \mathbf{C}$ NMR (176 MHz, $\left.\mathrm{CD}_{2} \mathrm{Cl}_{2}\right)$ of $\mathbf{1 2 x}$.
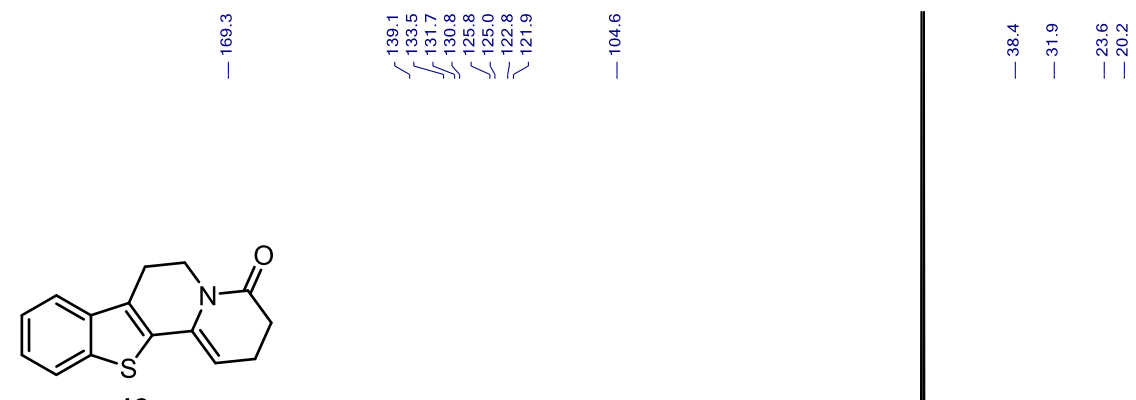

$12 x$ 
${ }^{1} \mathbf{H}$ NMR $\left(700 \mathrm{MHz}, \mathrm{CD}_{2} \mathrm{Cl}_{2}\right)$ of $\mathbf{1 3 .}$

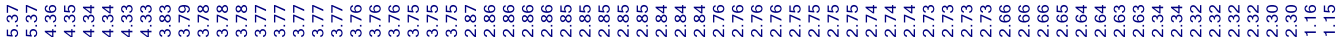

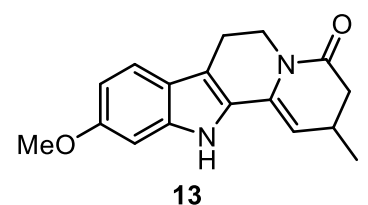

13

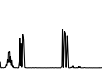

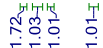

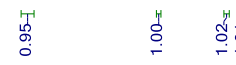

${ }^{13} \mathrm{C}$ NMR (176 MHz, $\left.\mathrm{CD}_{2} \mathrm{Cl}_{2}\right)$ of 13.

它

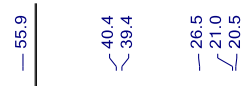

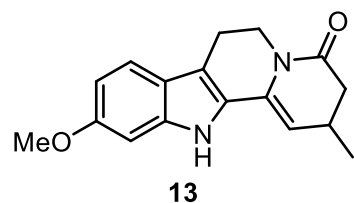

13
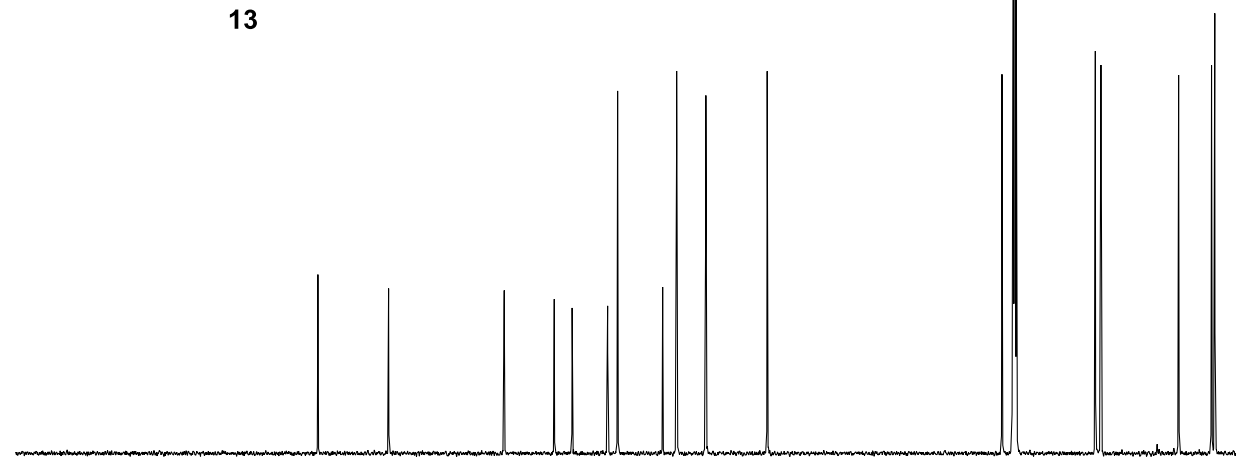

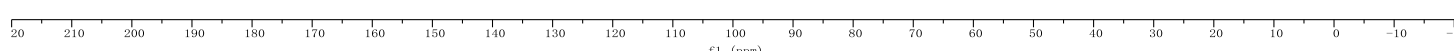


${ }^{1} \mathbf{H}$ NMR (700 MHz, CD3 OD) of 14a.

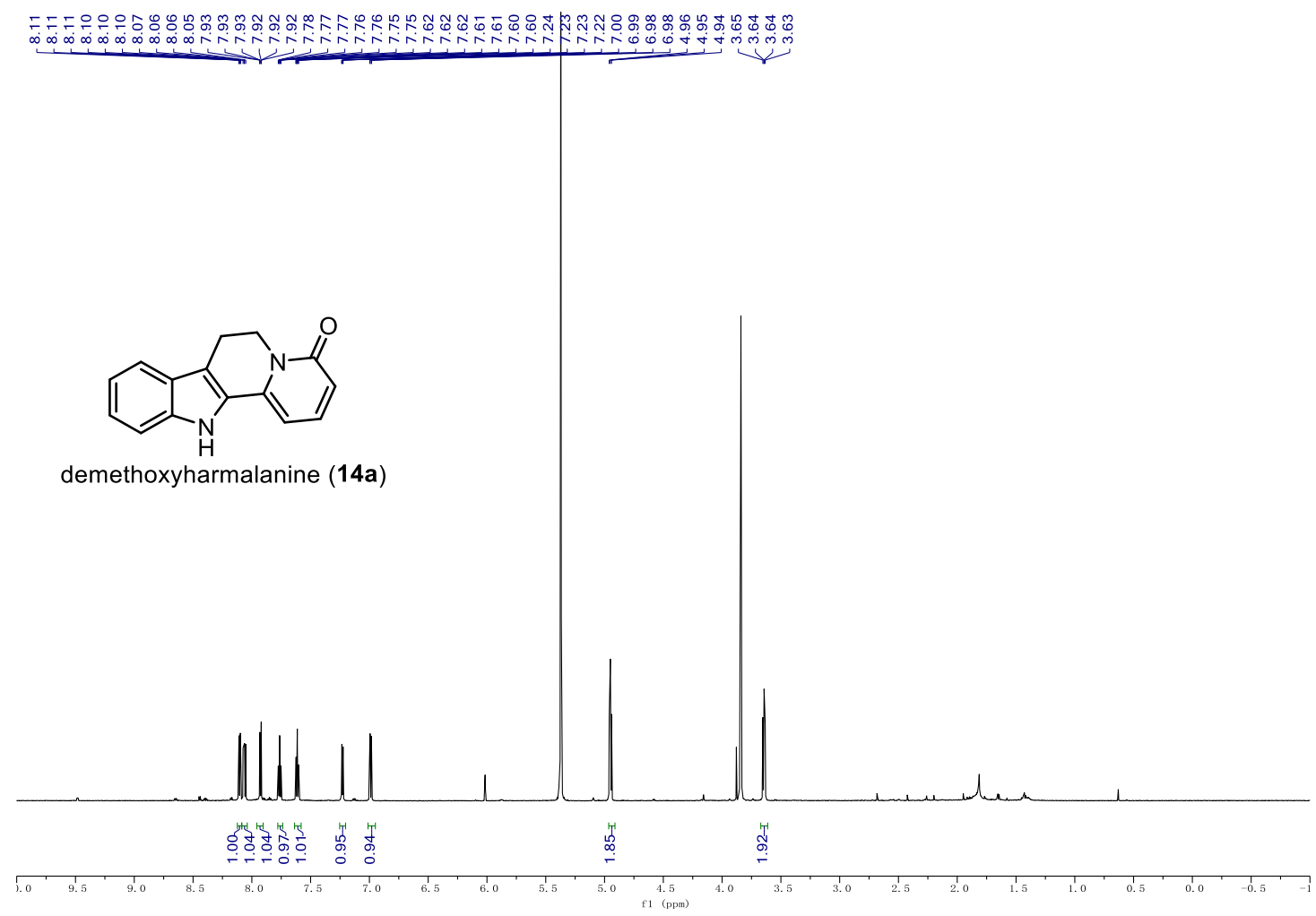

${ }^{13}$ C NMR (176 MHz, $\left.\mathrm{CD}_{3} \mathrm{OD}\right)$ of 14a.

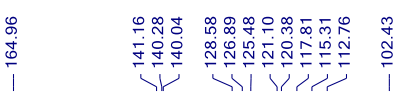

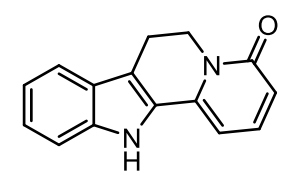

demethoxyharmalanine (14a)

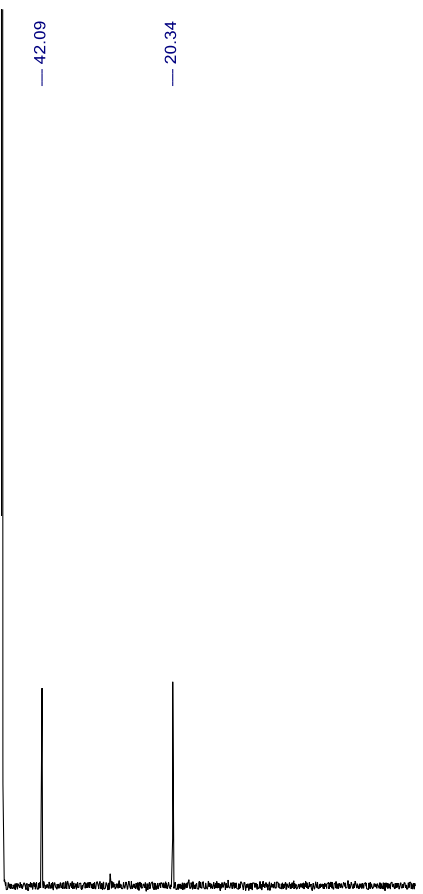

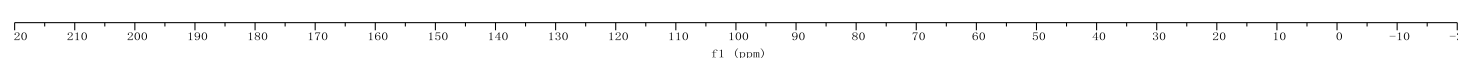


${ }^{1} \mathbf{H}$ NMR (500 MHz, $\left.\mathrm{CDCl}_{3}\right)$ of $\mathbf{1 4 b}$.

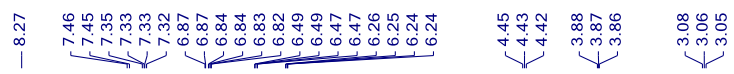

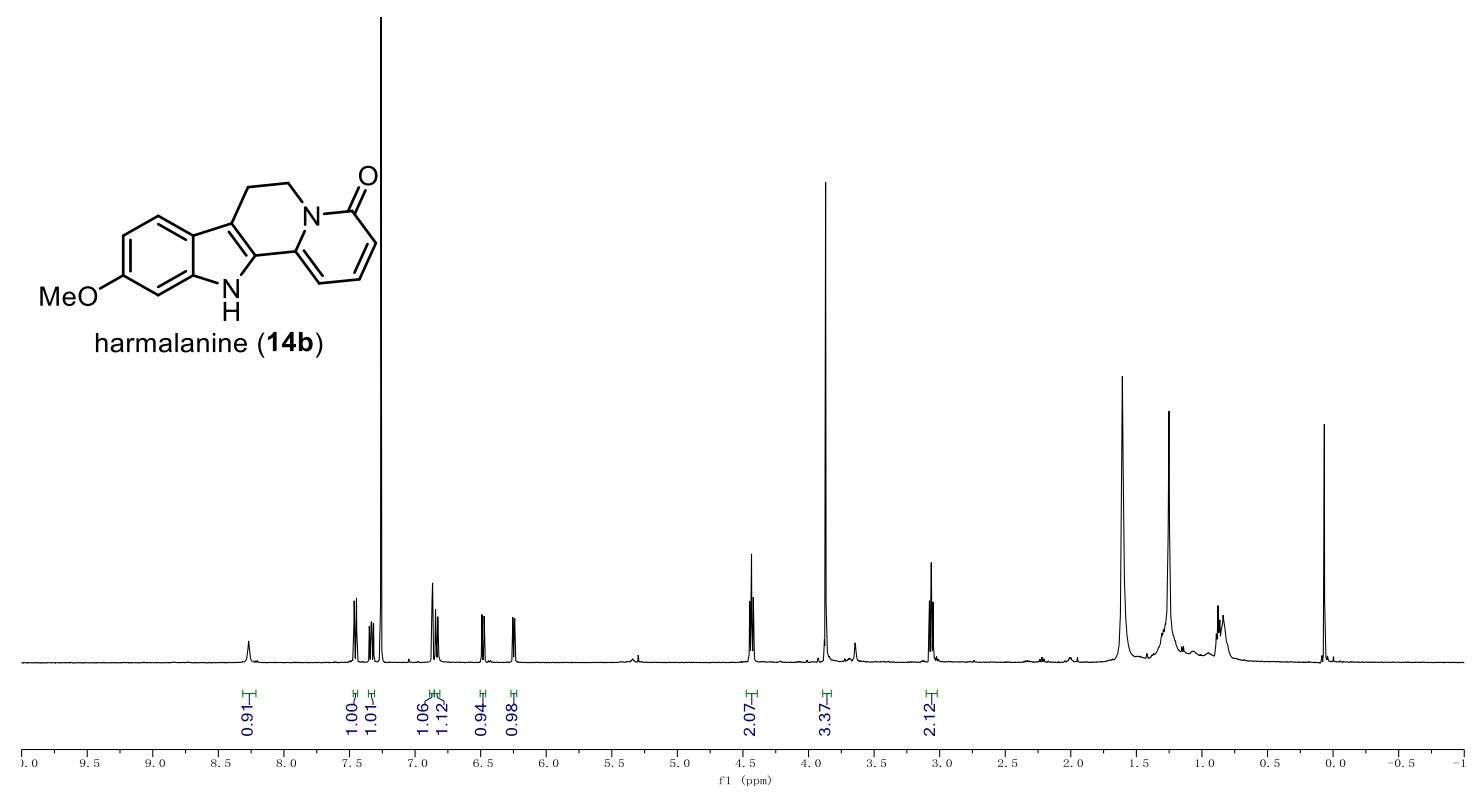

${ }^{13} \mathbf{C}$ NMR (126 MHz, CD $\left.3 \mathrm{OD}\right)$ of $\mathbf{1 4 b .}$

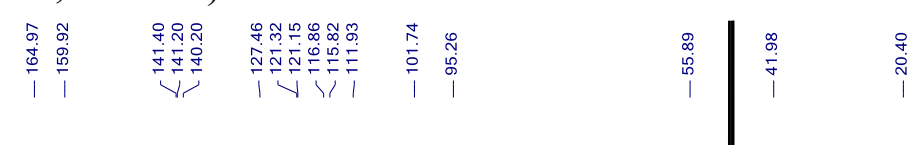<smiles>COc1ccc2c3c([nH]c2c1)-c1cccc(=O)n1CC3</smiles>

harmalanine (14b)
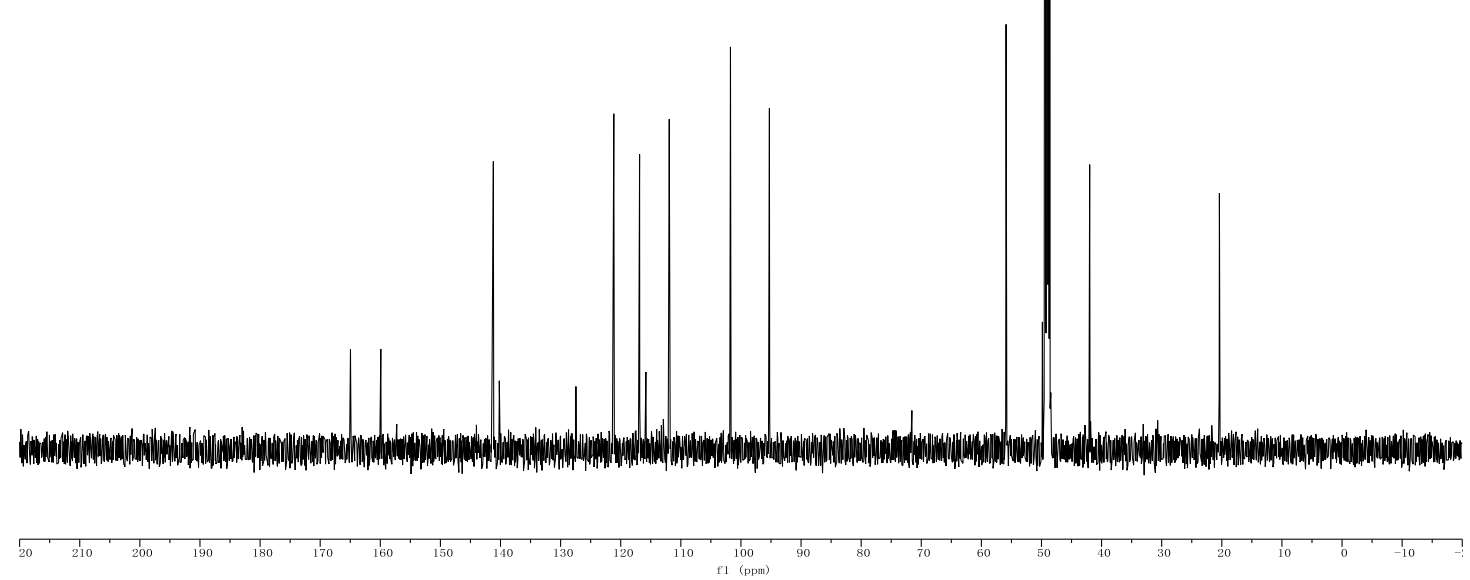
${ }^{1} \mathbf{H}$ NMR (500 MHz, $\mathrm{CDCl}_{3}+\mathrm{CD}_{3} \mathrm{OD}(\mathrm{v} / \mathrm{v}=1: 1)$ ) of $\mathbf{1 4 c}$.

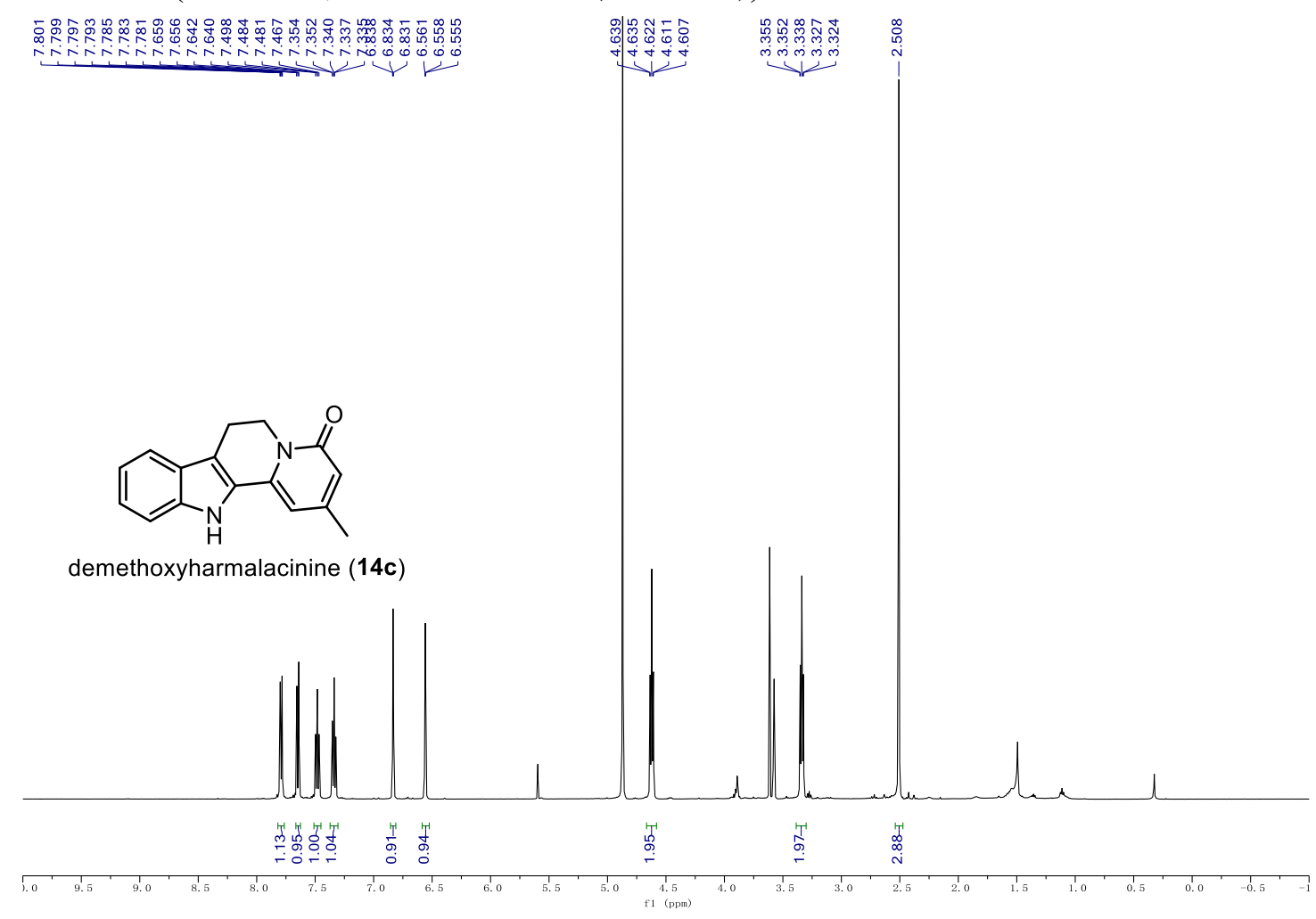

${ }^{13} \mathbf{C ~ N M R}\left(126 \mathrm{MHz}, \mathrm{CDCl}_{3}+\mathrm{CD}_{3} \mathrm{OD}(\mathrm{v} / \mathrm{v}=1: 1)\right)$ of $\mathbf{1 4 c}$.

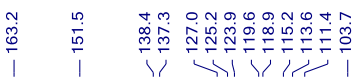

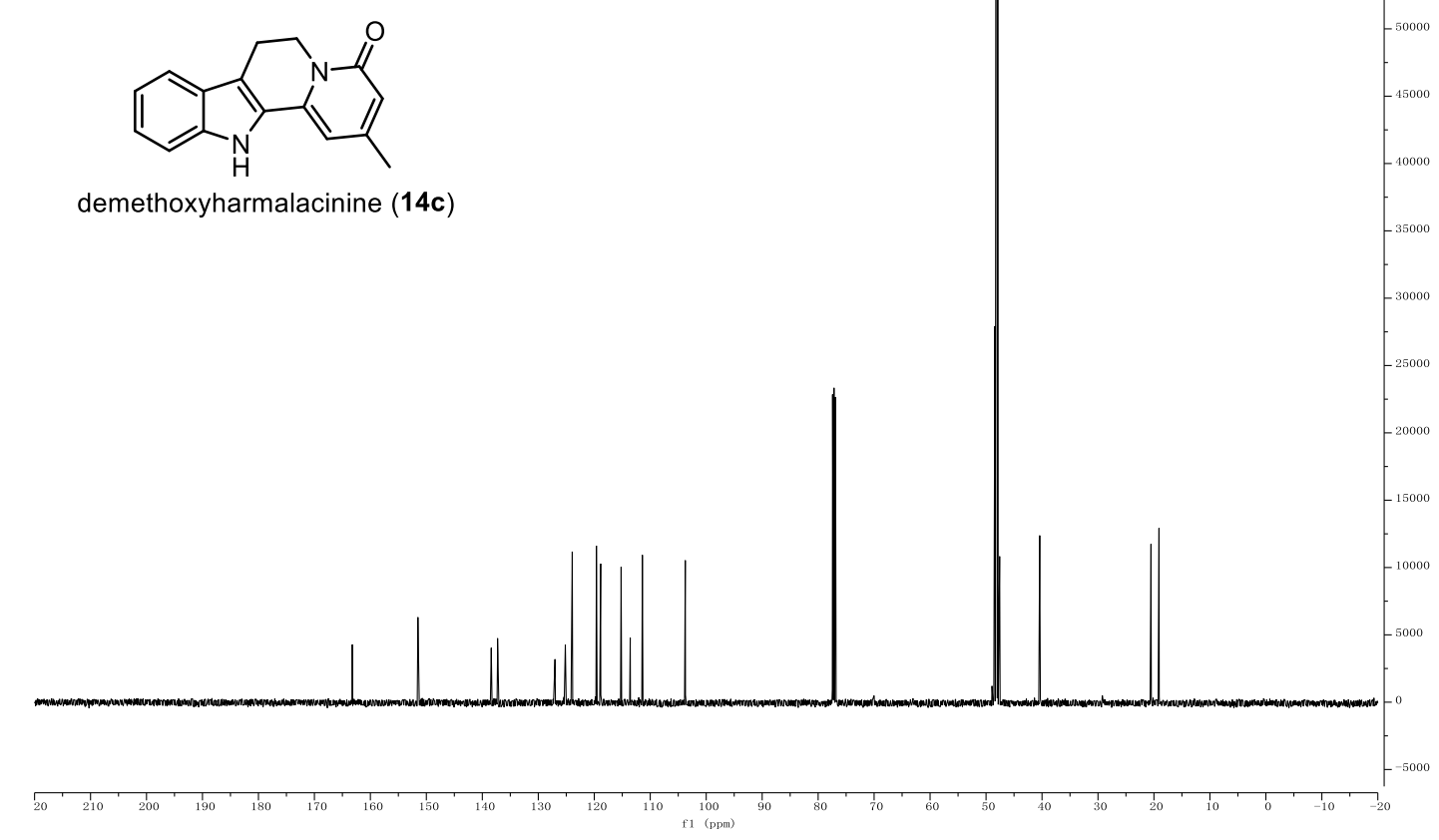

demethoxyharmalacinine (14c) 
${ }^{1} \mathbf{H}$ NMR $\left(500 \mathrm{MHz}, \mathrm{CDCl}_{3}\right)$ of $\mathbf{1 4 d .}$
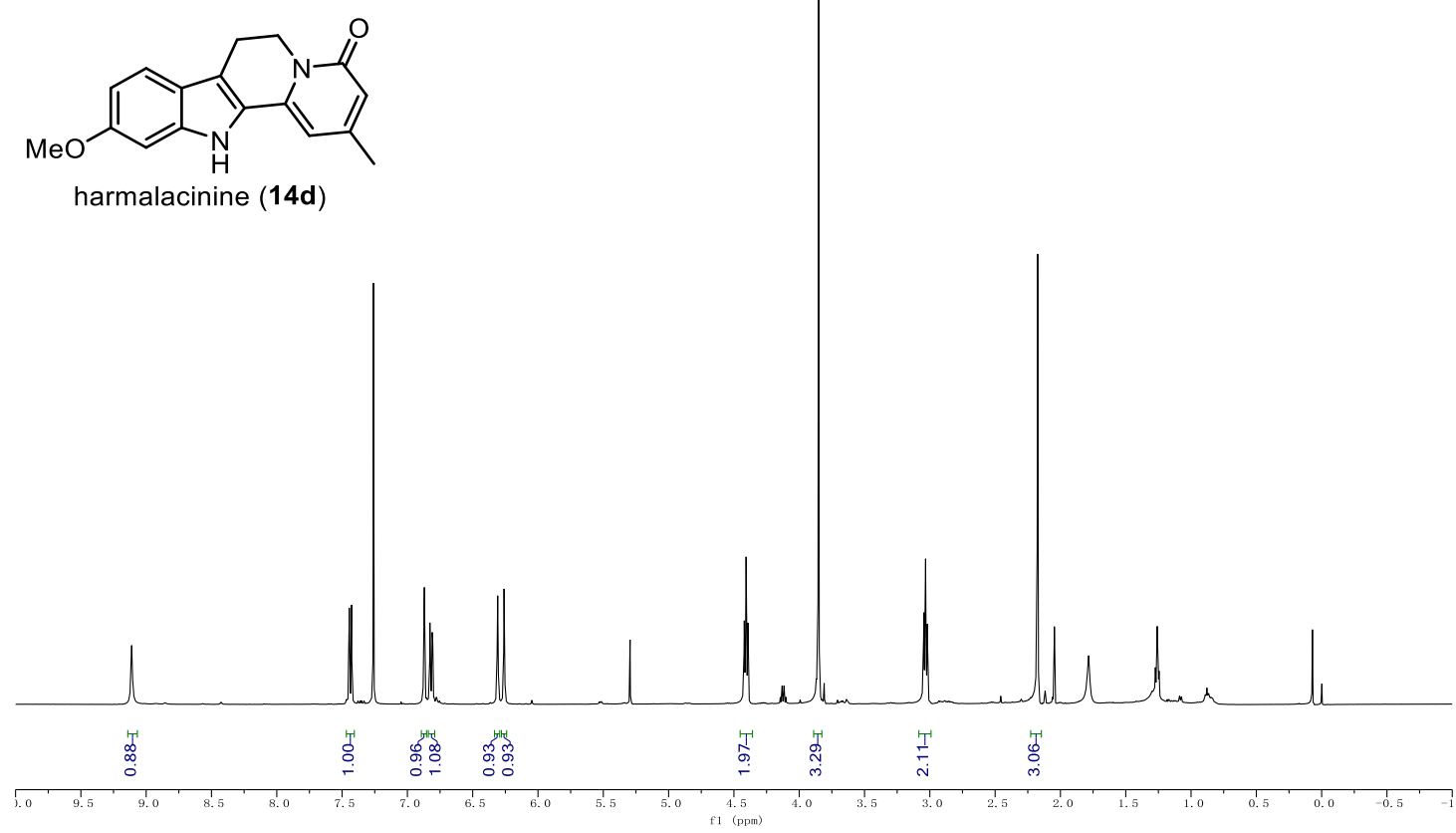

${ }^{13} \mathbf{C ~ N M R}\left(126 \mathrm{MHz}, \mathrm{CDCl}_{3}\right)$ of $\mathbf{1 4 d}$.

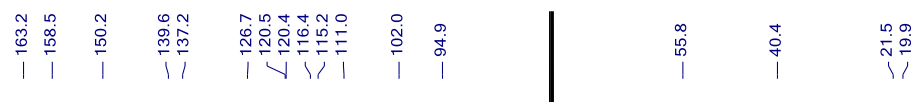<smiles>COc1ccc2c3c([nH]c2c1)-c1cc(C)cc(=O)n1CC3</smiles>

harmalacinine (14d)

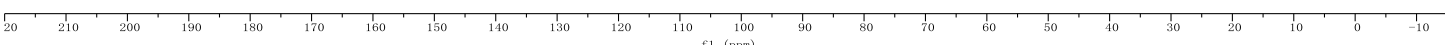


${ }^{1} \mathbf{H}$ NMR $\left(500 \mathrm{MHz}, \mathrm{CDCl}_{3}\right)$ of 15.

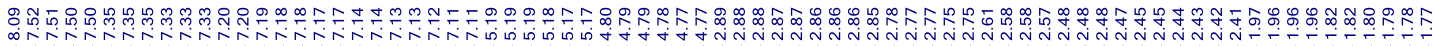

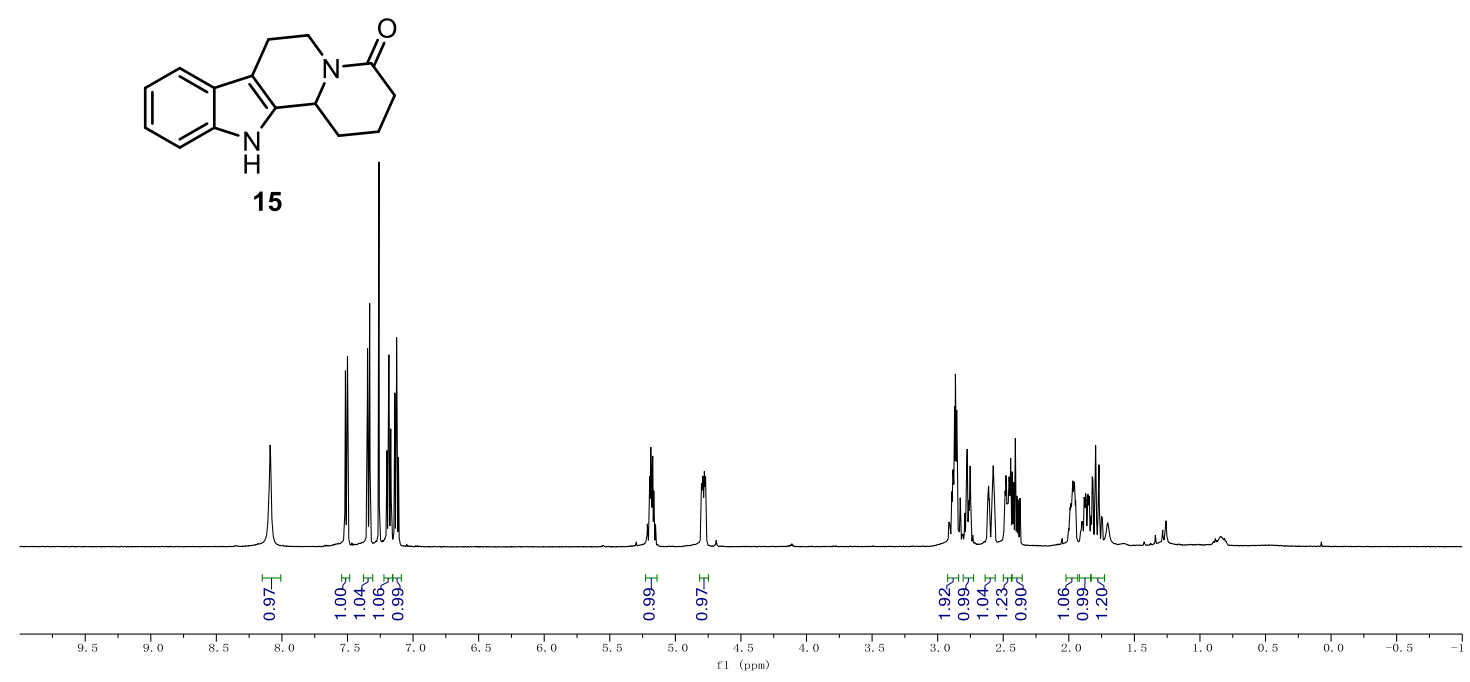

${ }^{13} \mathbf{C}$ NMR $\left(126 \mathrm{MHz}, \mathrm{CDCl}_{3}\right)$ of $\mathbf{1 5}$.

然

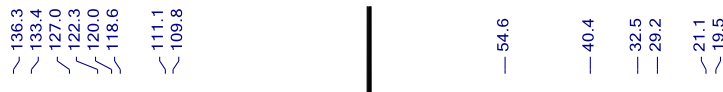

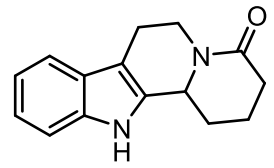

15
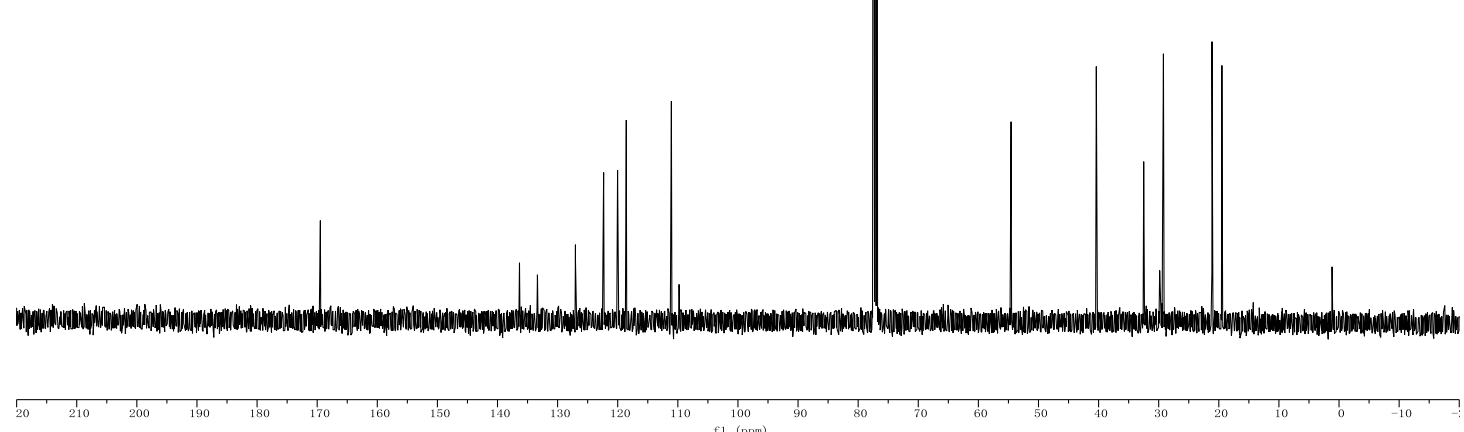
${ }^{1}$ H NMR (500 MHz, d6-DMSO) of 16.

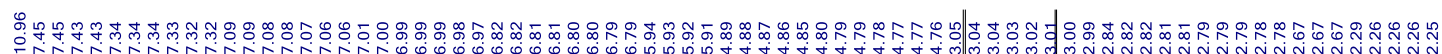<smiles>O=C1C=CCC2c3[nH]c4ccccc4c3CCN12</smiles>

16

${ }^{13}$ C NMR (126 MHz, d6-DMSO) of 16.

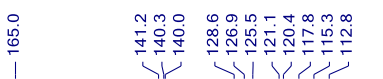<smiles>O=C1C=CCC2c3[nH]c4ccccc4c3CCN12</smiles>

16
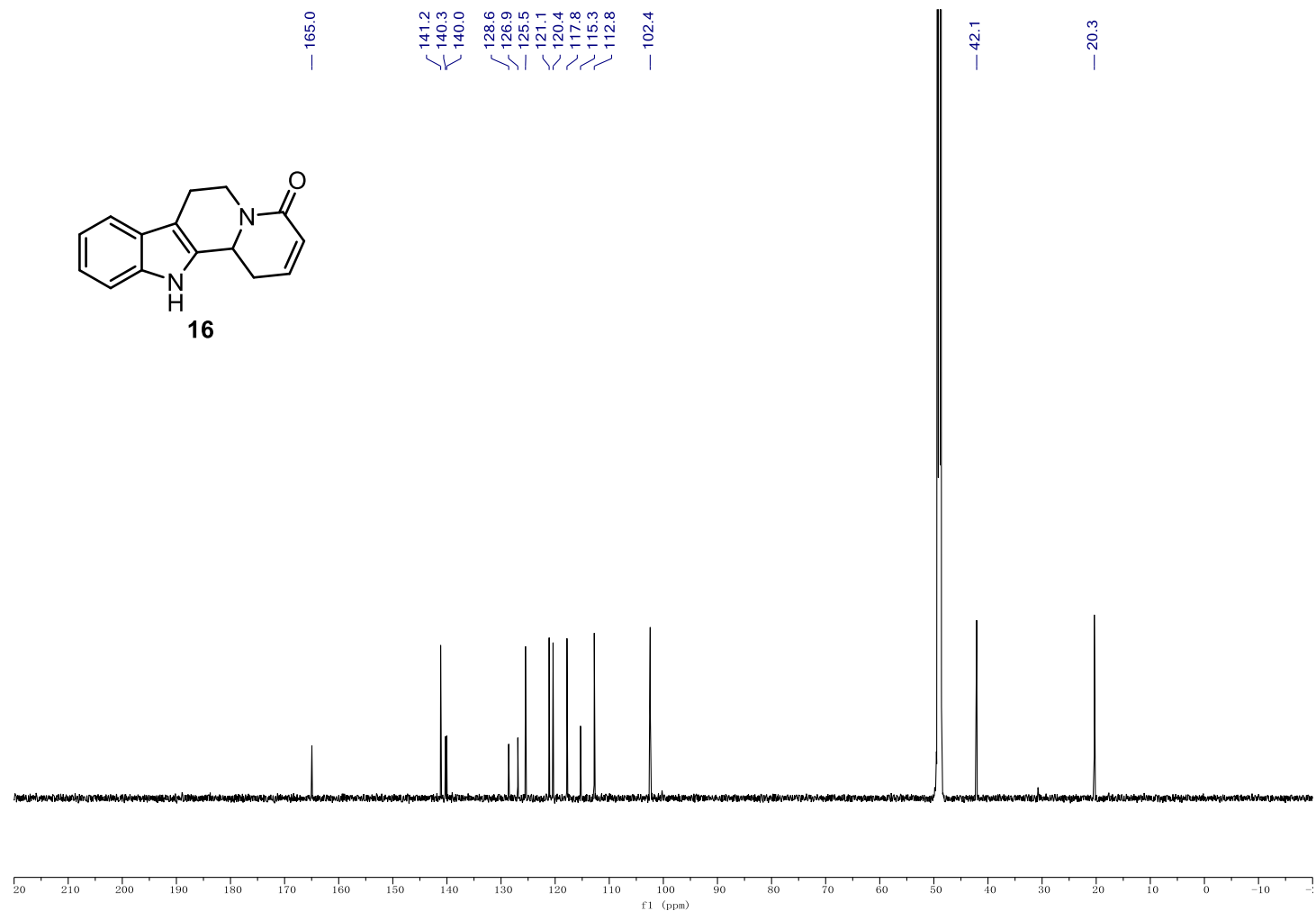
${ }^{1} \mathbf{H}$ NMR $\left(500 \mathrm{MHz}, \mathrm{CD}_{2} \mathrm{Cl}_{2}\right)$ of 24.

1

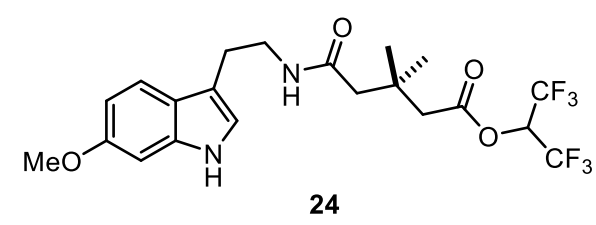

${ }^{13}$ C NMR (126 MHz, $\left.\mathrm{CD}_{2} \mathrm{Cl}_{2}\right)$ of 24.

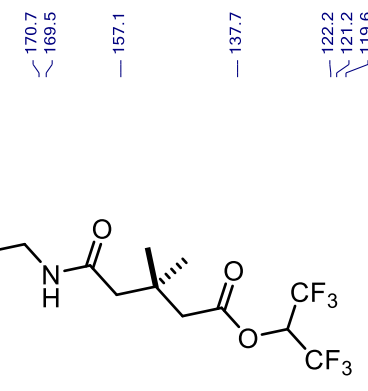

24

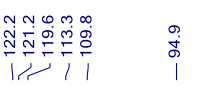

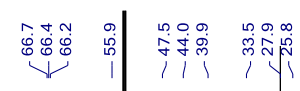<smiles>COc1ccc2c(CCNC(=O)CC(C)(C)CC(=O)OC(C(F)(F)F)C(F)(F)F)c[nH]c2c1</smiles>
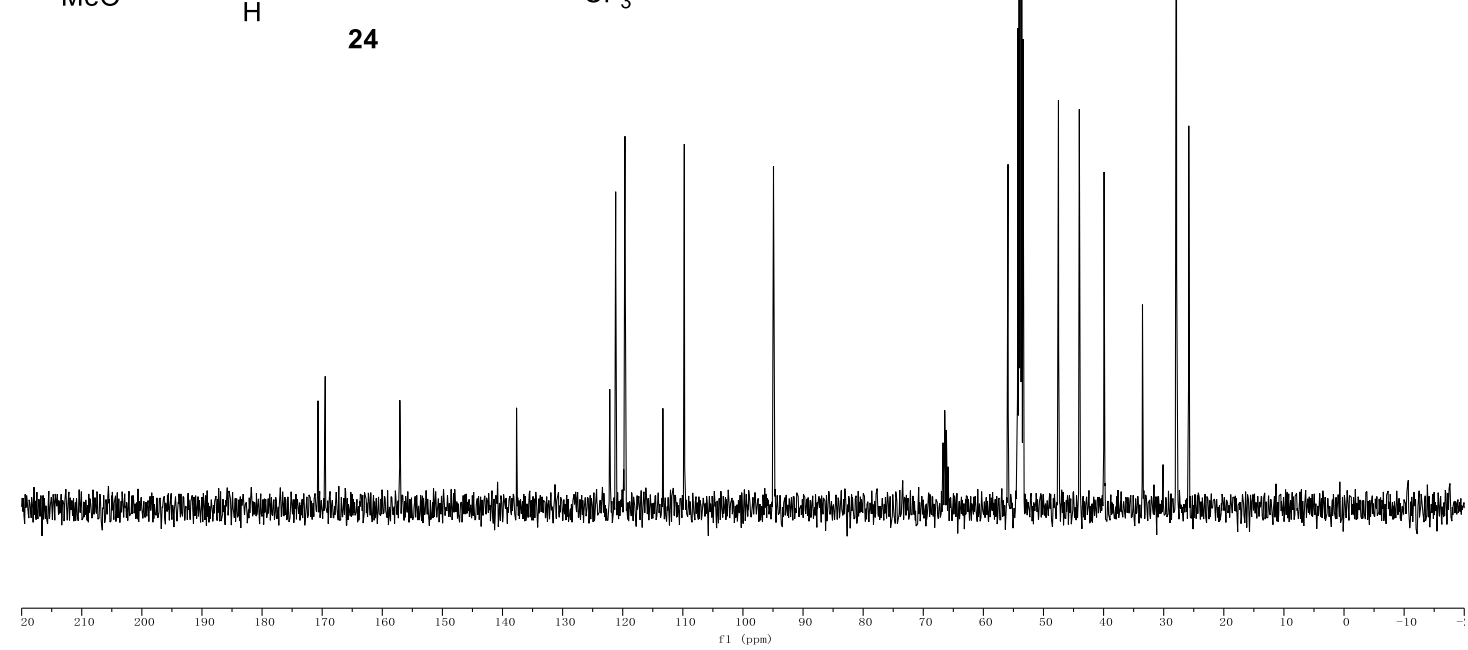
${ }^{19}$ F NMR (471 MHz, $\left.\mathrm{CD}_{2} \mathrm{Cl}_{2}\right)$ of 24.

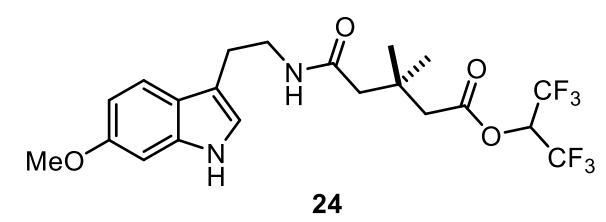

${ }^{\mathbf{1}} \mathbf{H}$ NMR $\left(700 \mathrm{MHz}, \mathrm{CDCl}_{3}\right)$ of 25.

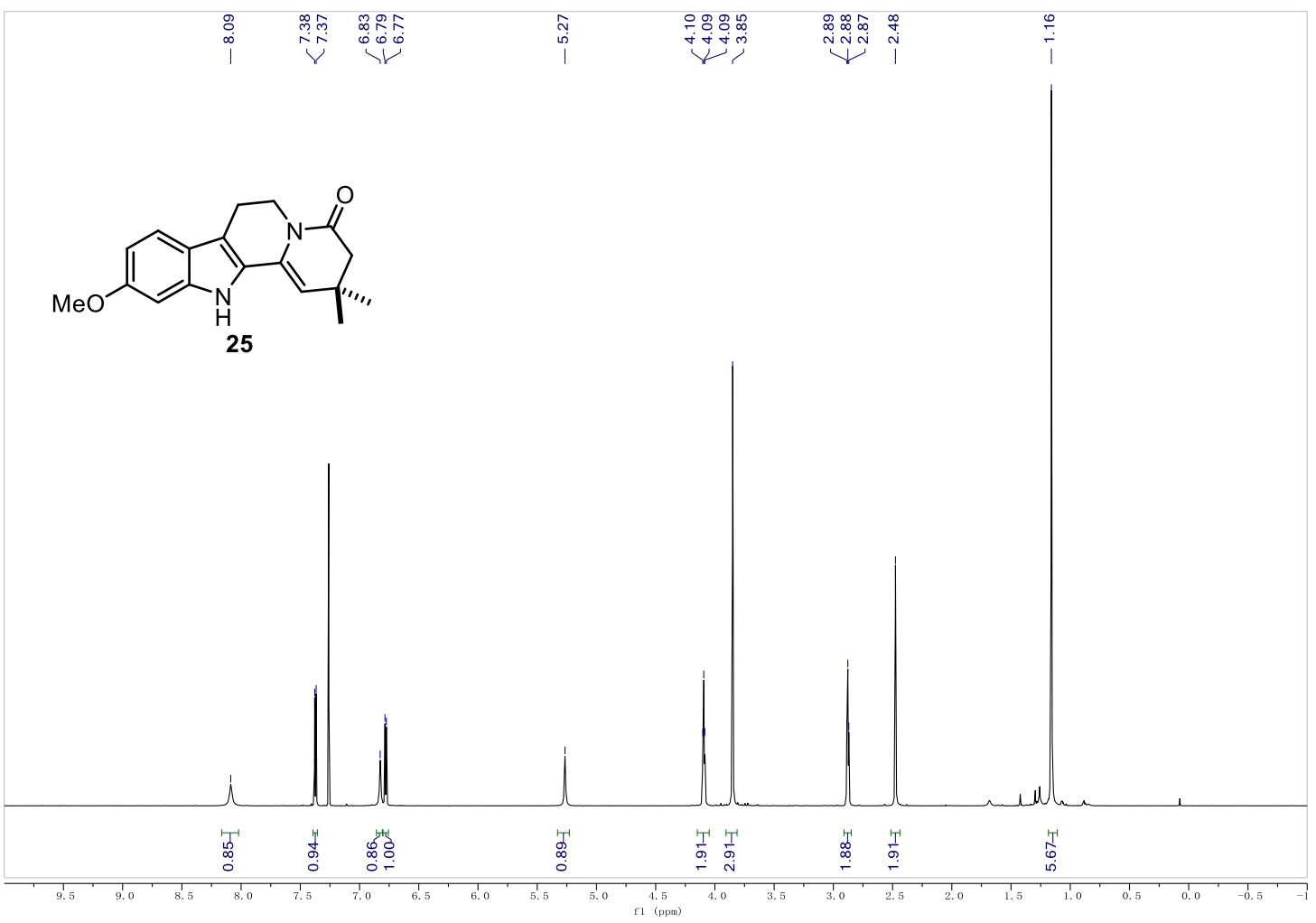


${ }^{13} \mathrm{C}$ NMR $\left(176 \mathrm{MHz}, \mathrm{CDCl}_{3}\right)$ of 25.

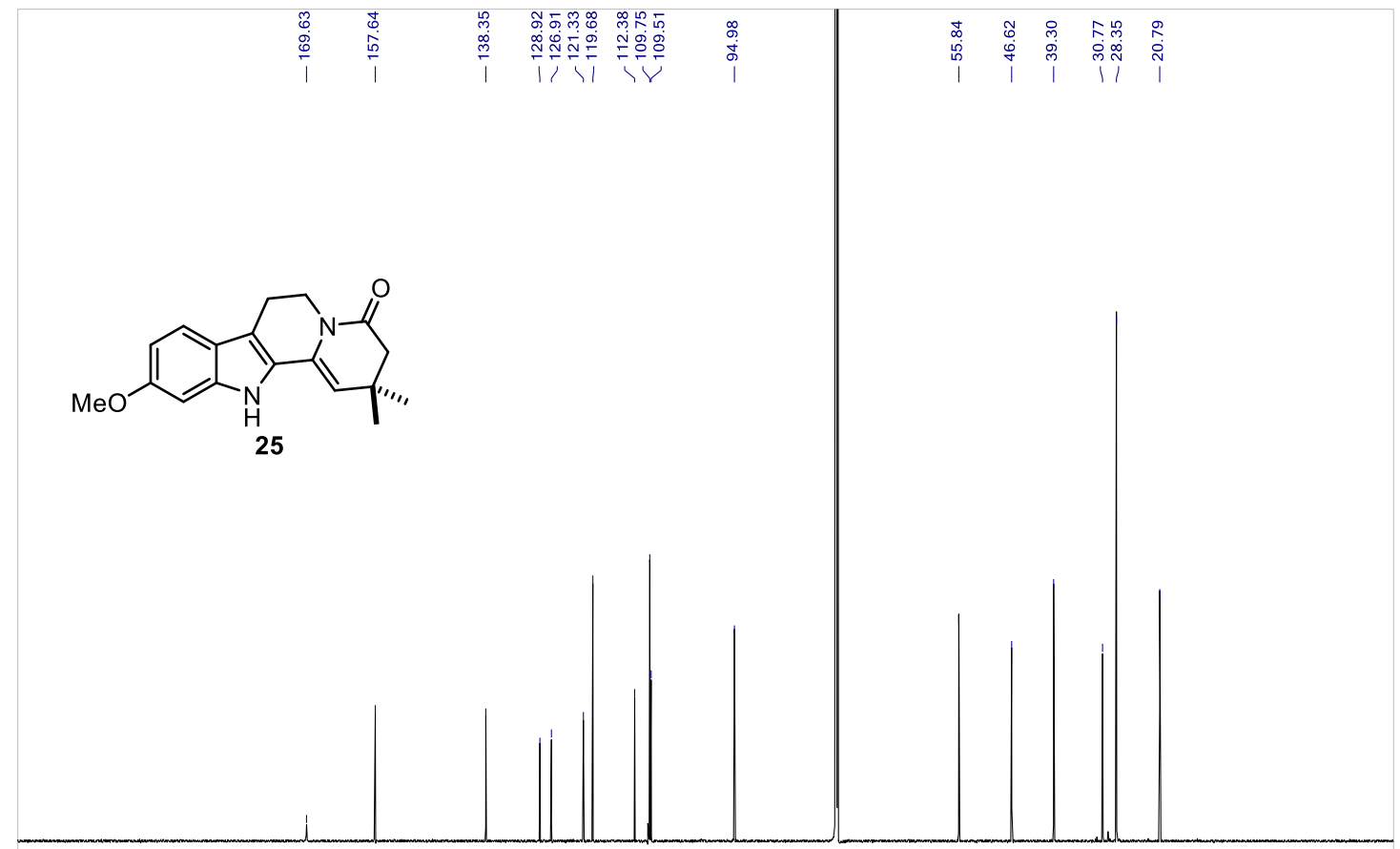

${ }^{\mathbf{1}} \mathbf{H}$ NMR $\left(500 \mathrm{MHz}, \mathrm{CDCl}_{3}\right)$ of $\mathbf{S 1 5}$.

尊<smiles>COc1ccc2c3c([nH]c2c1)C1=CC(C)(C)C(O)C(=O)N1CC3</smiles> 
${ }^{13} \mathbf{C ~ N M R}\left(126 \mathrm{MHz}, \mathrm{CDCl}_{3}\right)$ of $\mathbf{S 1 5}$.

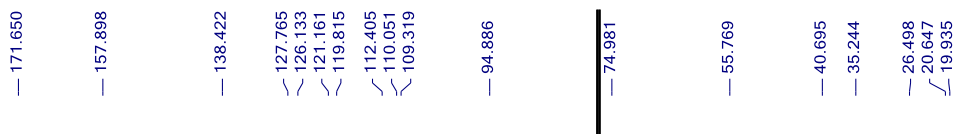<smiles>COc1ccc2c3c([nH]c2c1)C1=CC(C)(C)C(O)C(=O)N1CC3</smiles>

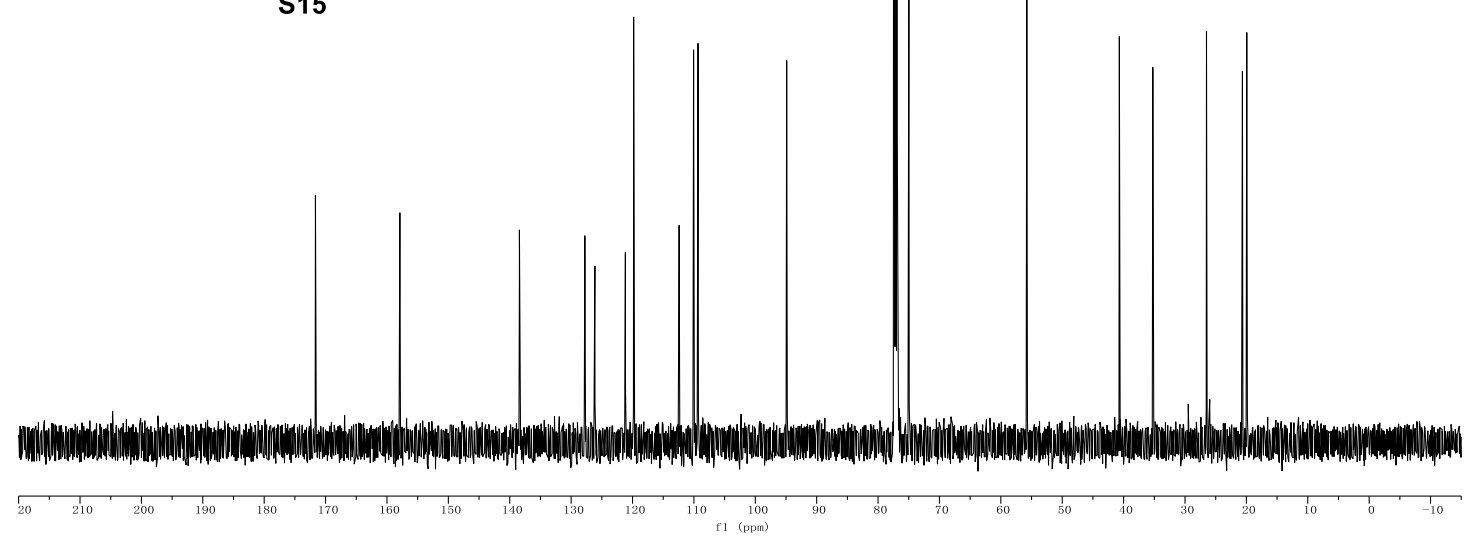

${ }^{1} \mathbf{H}$ NMR $\left(700 \mathrm{MHz}, \mathrm{CDCl}_{3}\right)$ of 26.

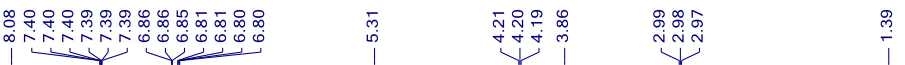

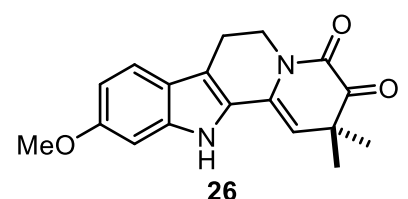


${ }^{13}$ C NMR $\left(176 \mathrm{MHz}, \mathrm{CDCl}_{3}\right)$ of 26.

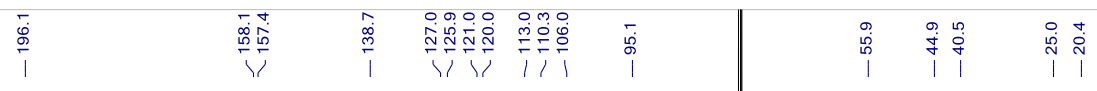<smiles>COc1ccc2c3c([nH]c2c1)C1=CC(C)(C)C(=O)C(=O)N1CC3</smiles>
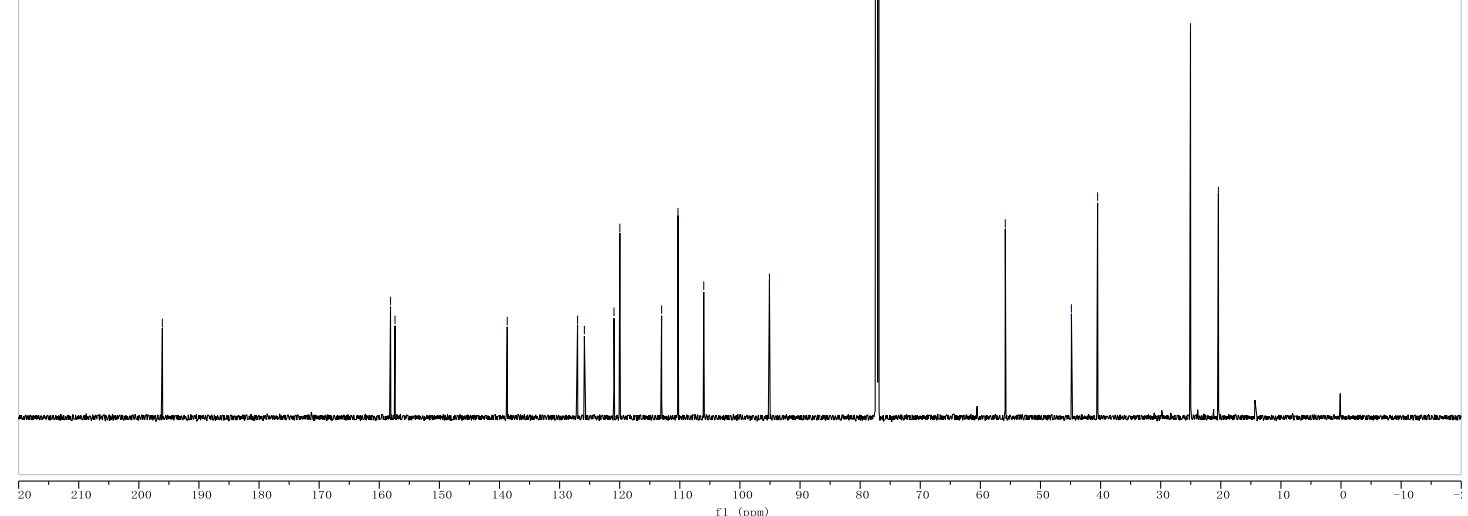

${ }^{1} \mathbf{H}$ NMR (700 MHz, $\mathbf{C D}_{\mathbf{3}}$ OD) of peganumine A (6).

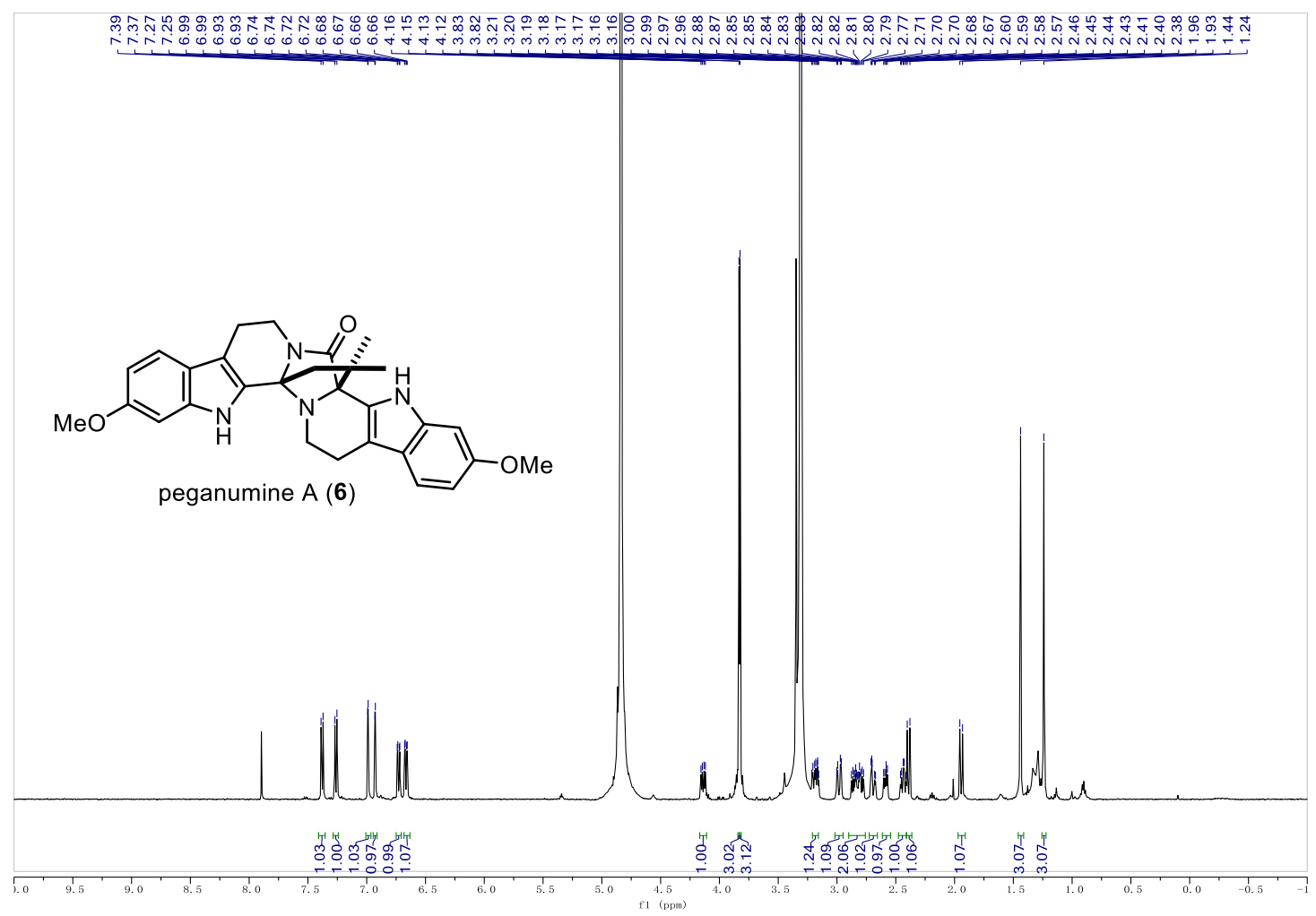


${ }^{13} \mathrm{C}$ NMR (176 MHz, CD3OD) of peganumine A (6).

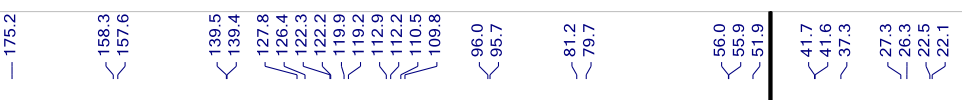

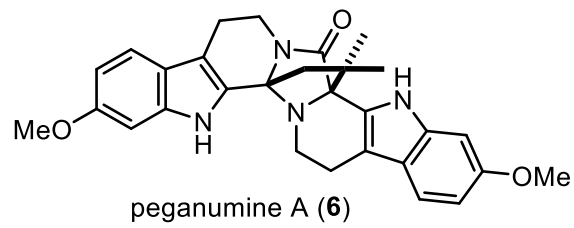

ganumine A (6)

${ }^{1}$ H NMR (500 MHz, $\boldsymbol{d}_{\mathbf{6}}$-DMSO) of peganumine A (6).

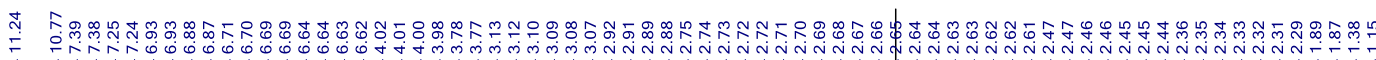
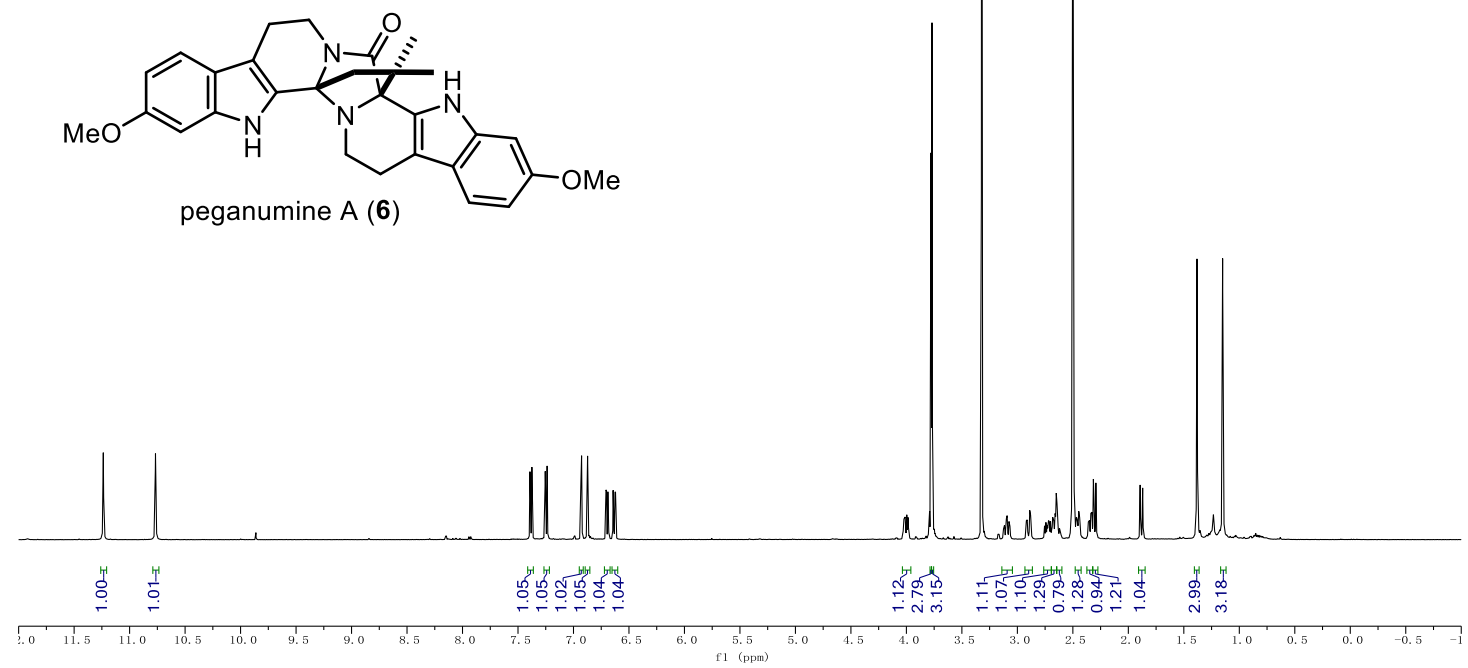
${ }^{13}$ C NMR (126 MHz, $\boldsymbol{d}_{6}$-DMSO) of peganumine A (6).

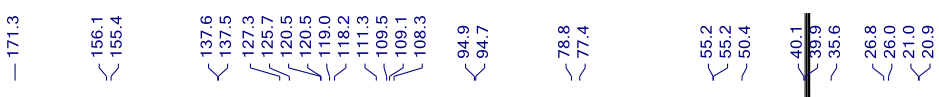

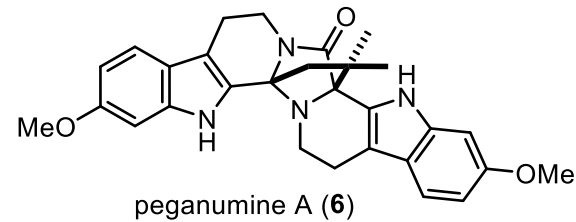

peganumine $A(6)$
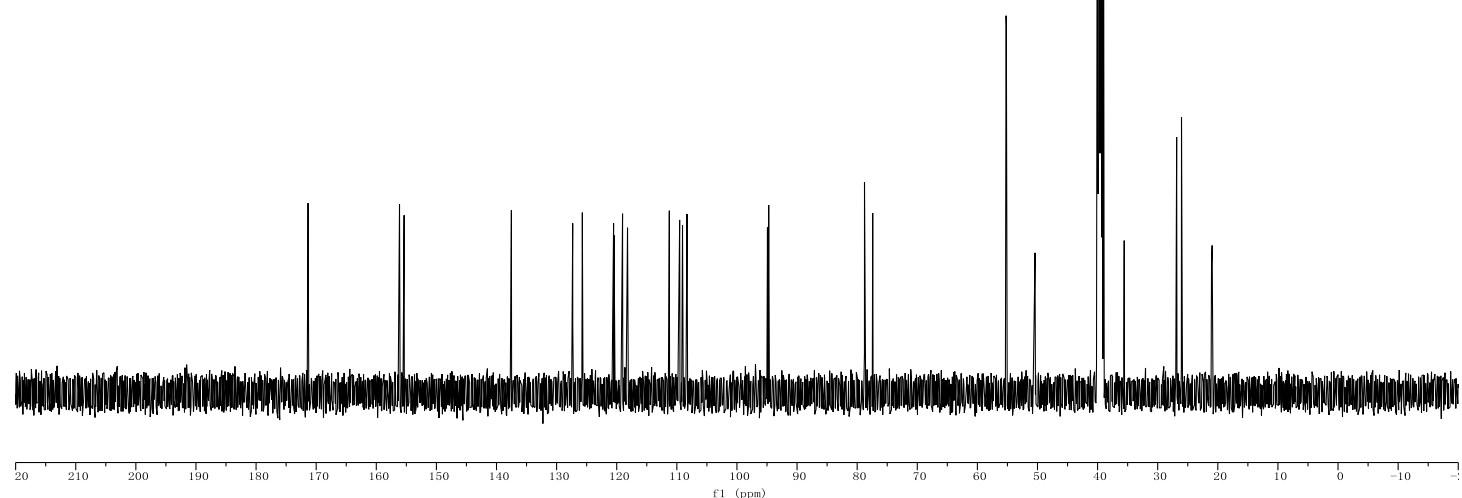

${ }^{1} \mathbf{H}$ NMR $\left(500 \mathrm{MHz}, \mathrm{CDCl}_{3}\right)$ of $\mathbf{S 2 1}$.

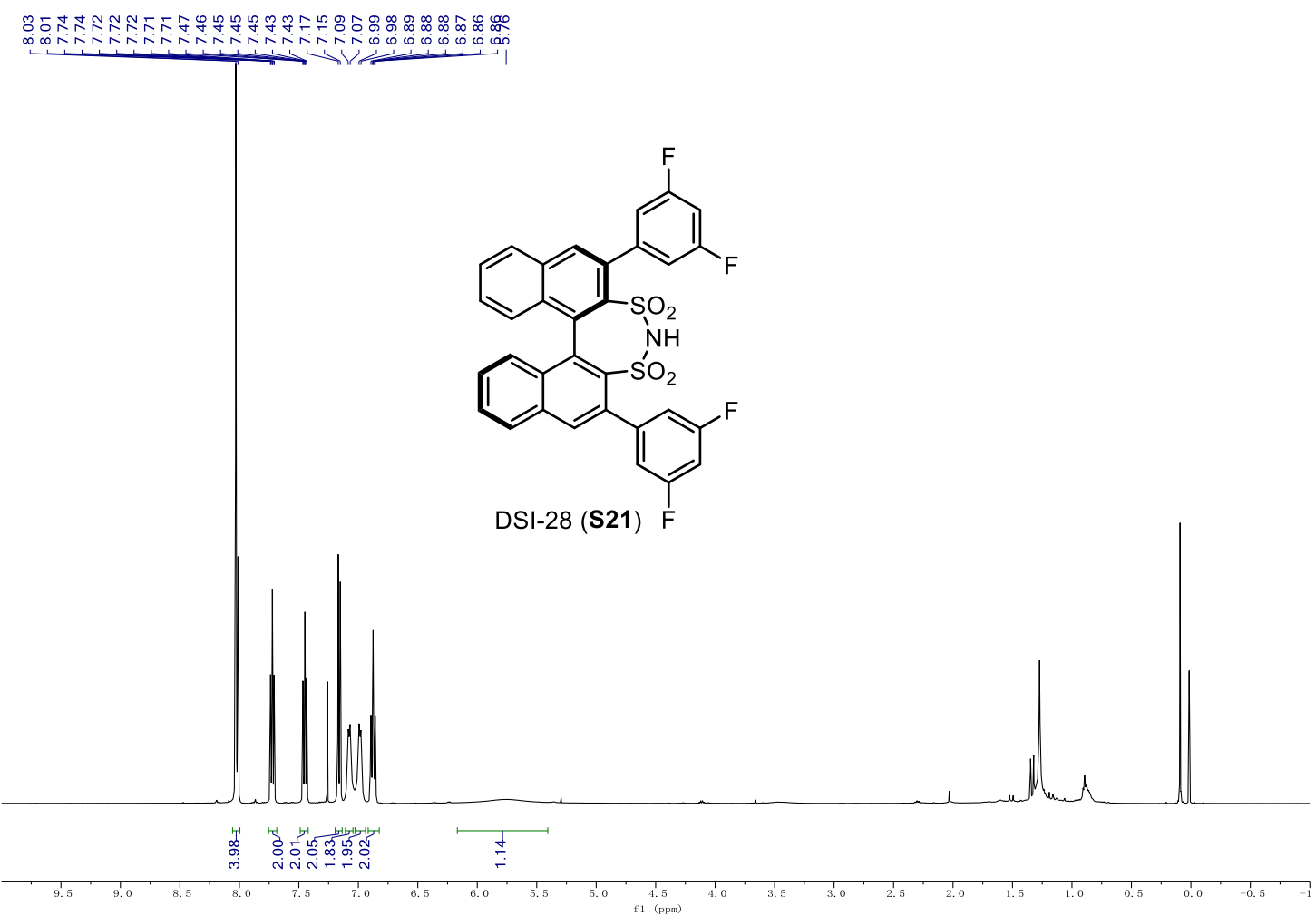


${ }^{13} \mathbf{C ~ N M R}\left(126 \mathrm{MHz}, \mathrm{CDCl}_{3}\right)$ of $\mathbf{S 2 1 .}$

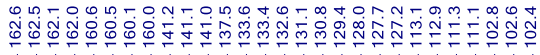
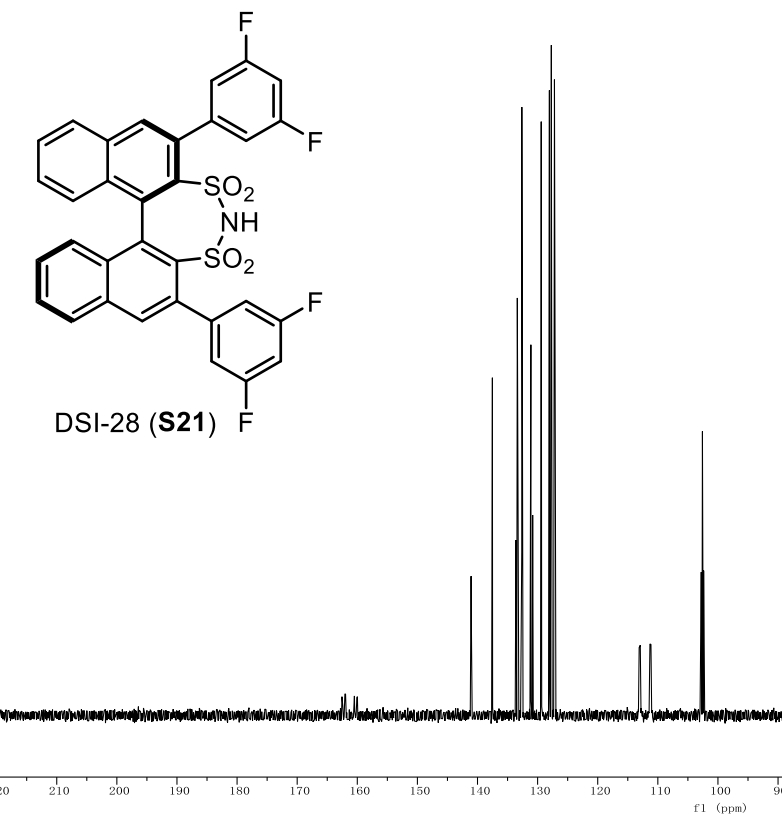

${ }^{19} \mathbf{F ~ N M R}\left(471 \mathrm{MHz}, \mathrm{CDCl}_{3}\right)$ of $\mathbf{S 2 1 .}$

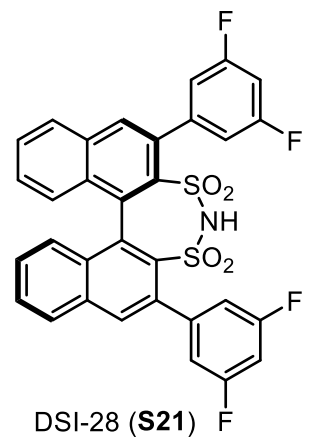


${ }^{1} \mathbf{H}$ NMR (500 MHz, $\left.\mathrm{CDCl}_{3}\right)$ of $\mathbf{S 2 2}$.

等

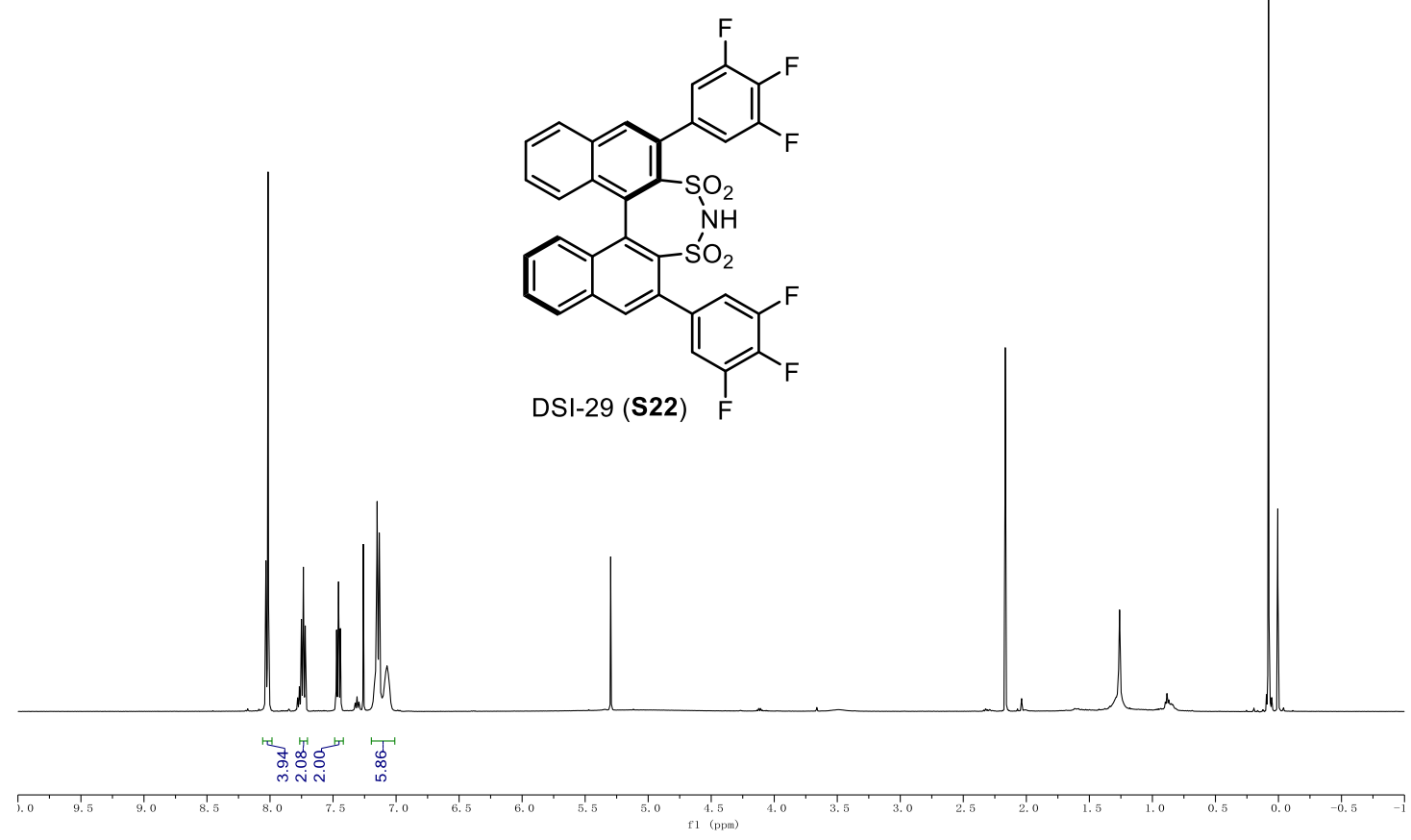

${ }^{13} \mathbf{C ~ N M R}\left(126 \mathrm{MHz}, \mathrm{CDCl}_{3}\right)$ of $\mathbf{S 2 2}$.

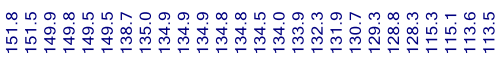

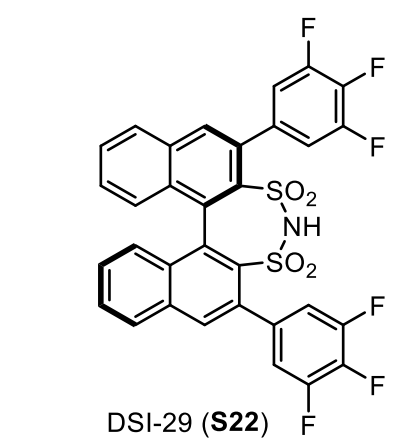


${ }^{19}$ F NMR (471 MHz, $\left.\mathrm{CDCl}_{3}\right)$ of $\mathbf{S 2 2}$.

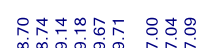

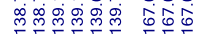

نَنَ

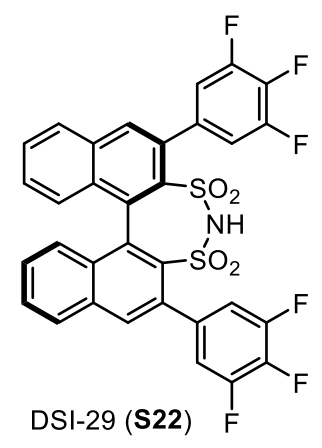

${ }^{1} \mathbf{H}$ NMR $\left(500 \mathrm{MHz}, \mathrm{CD}_{2} \mathrm{Cl}_{2}\right)$ of $\mathbf{S 2 3}$.

$\underbrace{0}$
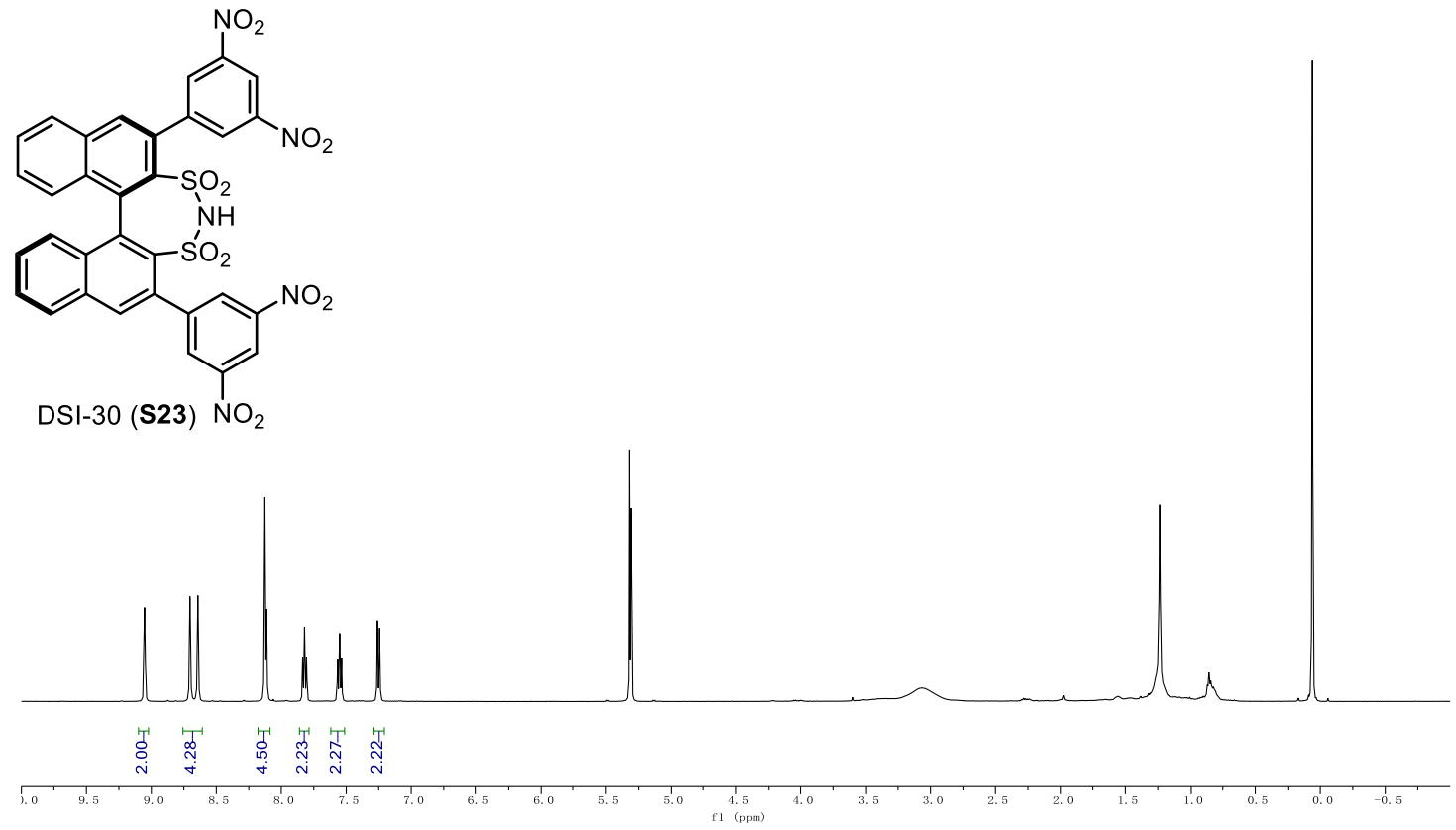
${ }^{13} \mathbf{C}$ NMR (126 MHz, $\left.\mathrm{CD}_{2} \mathrm{Cl}_{2}\right)$ of $\mathbf{S 2 3}$.

等

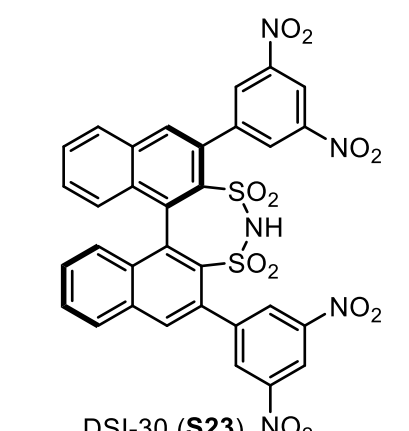

DSI-30 (S23) $\mathrm{NO}_{2}$

${ }^{1} \mathbf{H}$ NMR $\left(500 \mathrm{MHz}, \mathrm{CDCl}_{3}\right)$ of $\mathbf{3 2}$.

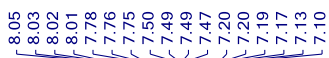

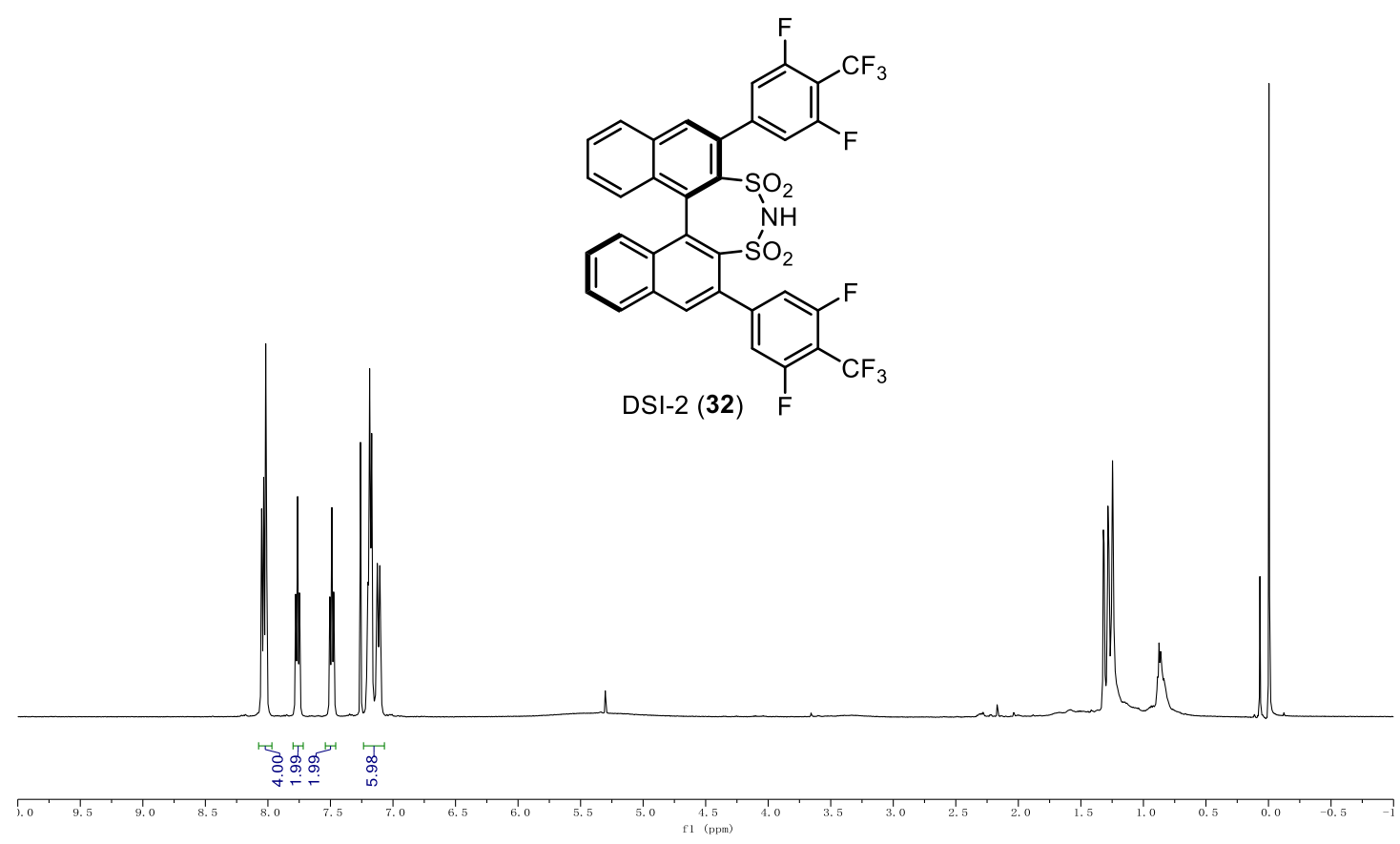


${ }^{13}$ C NMR $\left(126 \mathrm{MHz}, \mathrm{CDCl}_{3}\right)$ of 32.

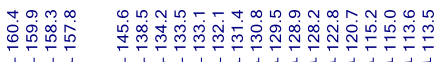

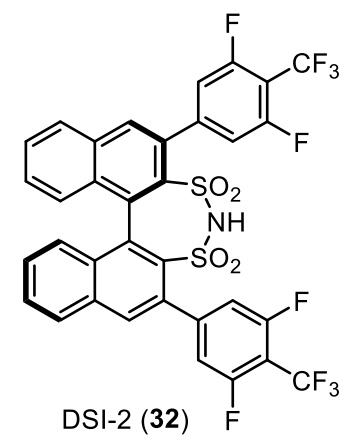

${ }^{19} \mathbf{F}$ NMR $\left(471 \mathrm{MHz}, \mathrm{CDCl}_{3}\right)$ of 32.

$\underbrace{=0.9}$<smiles></smiles>

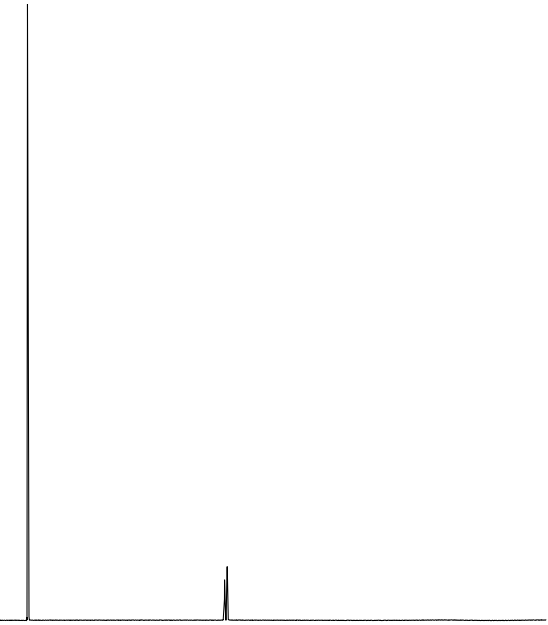


${ }^{1} \mathbf{H}$ NMR $\left(700 \mathrm{MHz}, \mathrm{CDCl}_{3}\right)$ of 35.

$\underbrace{\text { P. }}$

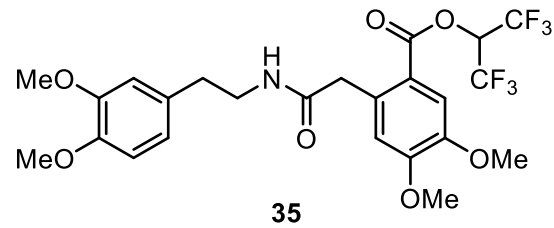

35

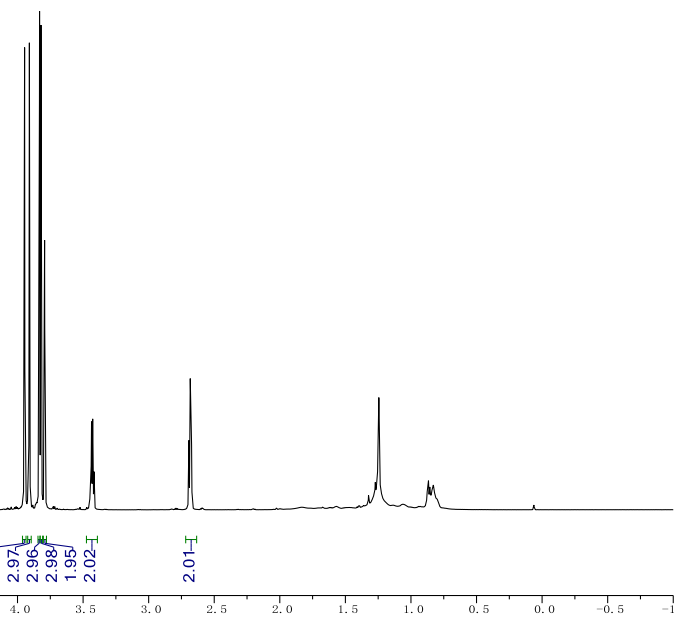

${ }^{13} \mathbf{C ~ N M R}\left(176 \mathrm{MHz}, \mathrm{CDCl}_{3}\right)$ of $\mathbf{3 5}$.

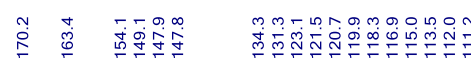
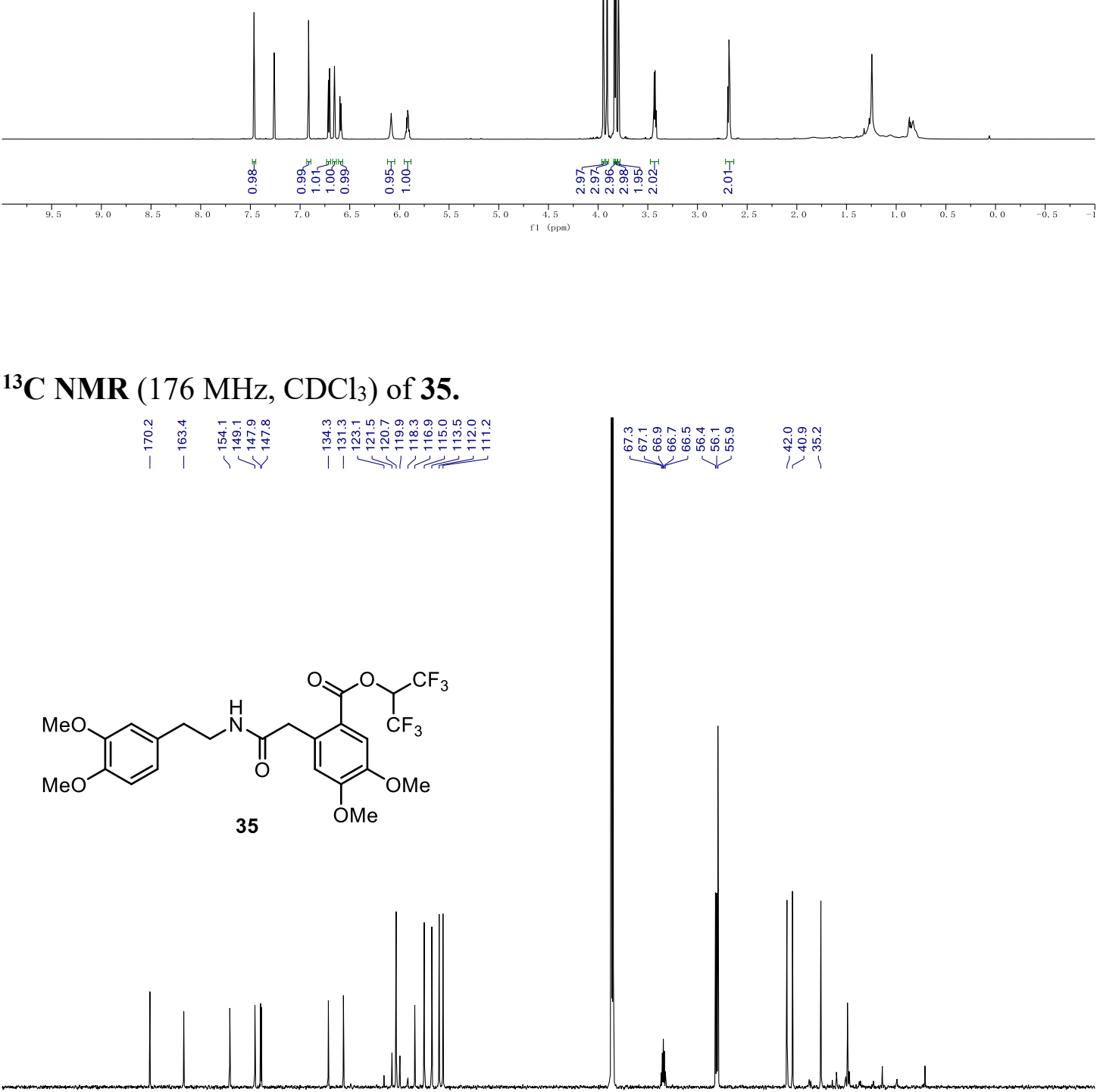
${ }^{19} \mathbf{F}$ NMR $\left(565 \mathrm{MHz}, \mathrm{CDCl}_{3}\right)$ of 35.

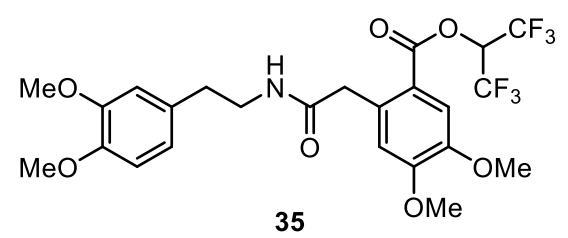

$\mid$

${ }^{1} \mathbf{H}$ NMR $\left(700 \mathrm{MHz}, \mathrm{CDCl}_{3}\right)$ of $\mathbf{3 6}$.

i

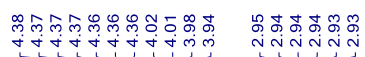

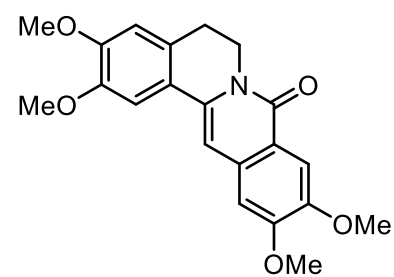

8-oxopseudopalmatine (36)

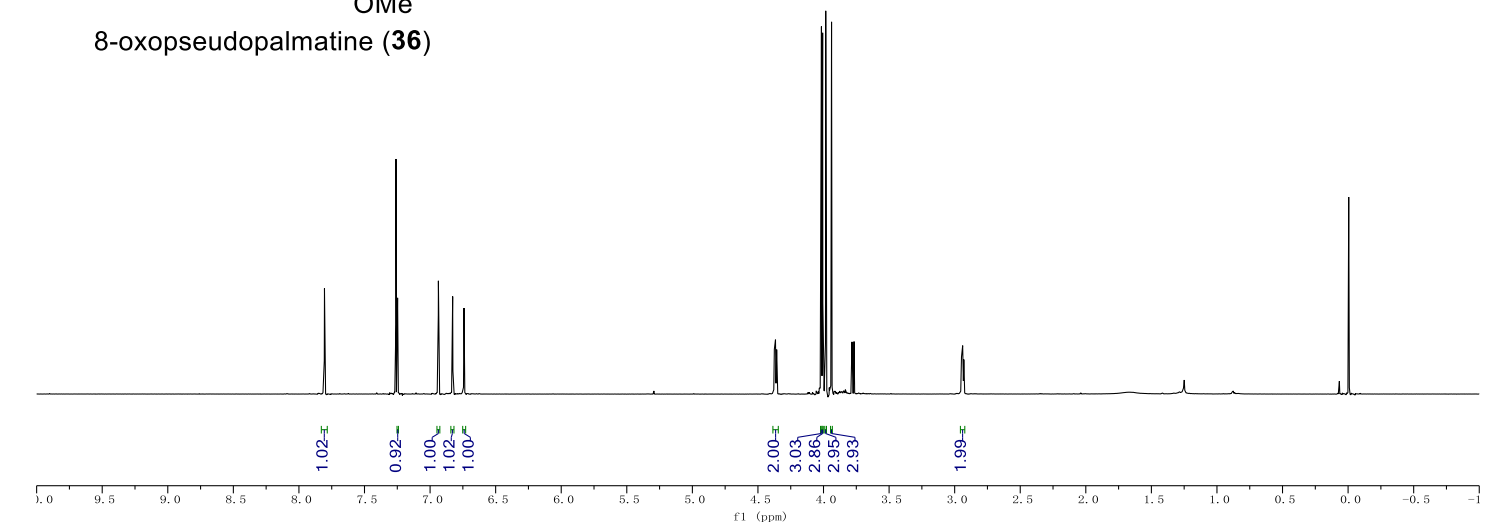


${ }^{13} \mathbf{C}$ NMR $\left(176 \mathrm{MHz}, \mathrm{CDCl}_{3}\right)$ of $\mathbf{3 6}$.

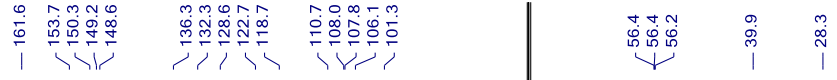

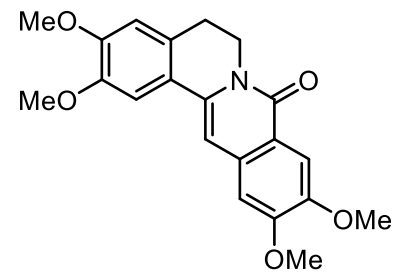

8-oxopseudopalmatine (36)

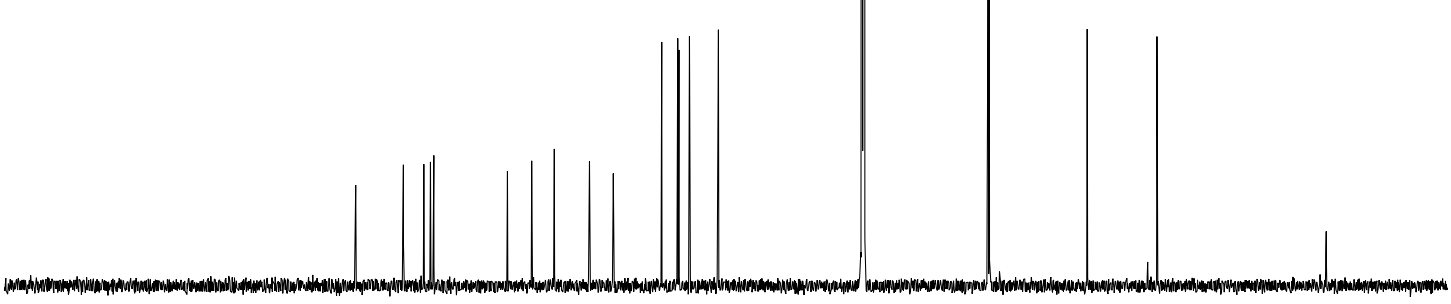

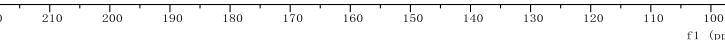

${ }^{1} \mathbf{H}$ NMR (700 MHz, $\left.\mathrm{CDCl}_{3}\right)$ of ilicifoline B (5).

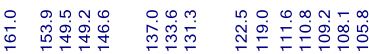

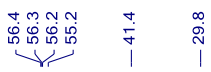

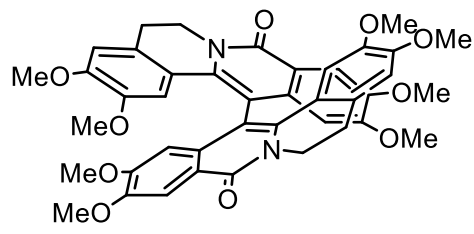

ilicifonline B (5) 
${ }^{13}$ C NMR (176 MHz, $\left.\mathrm{CDCl}_{3}\right)$ of ilicifoline B (5).
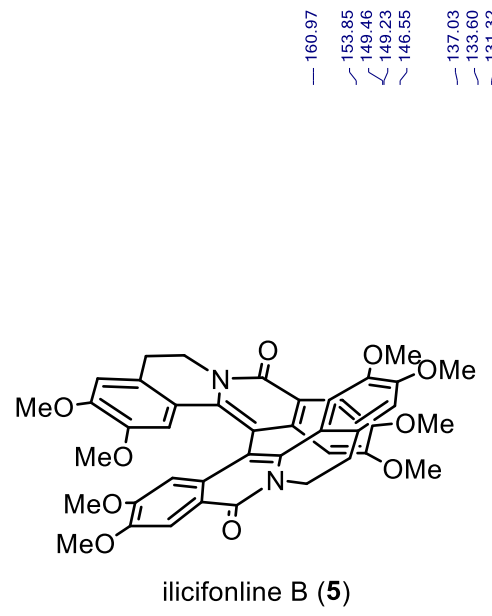

${ }^{1}$ H NMR (500 MHz, CD 3 OD) of 22.

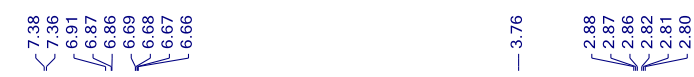<smiles>COc1ccc2c(CCN)c[nH]c2c1</smiles> 
${ }^{13} \mathrm{C}$ NMR (126 MHz, CD $\left.3 \mathrm{OD}\right)$ of 22.

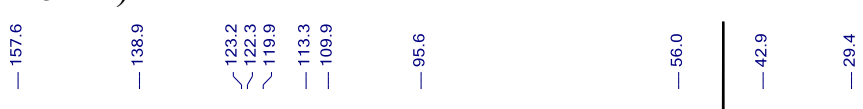<smiles>COc1ccc2c(CCN)c[nH]c2c1</smiles>

22

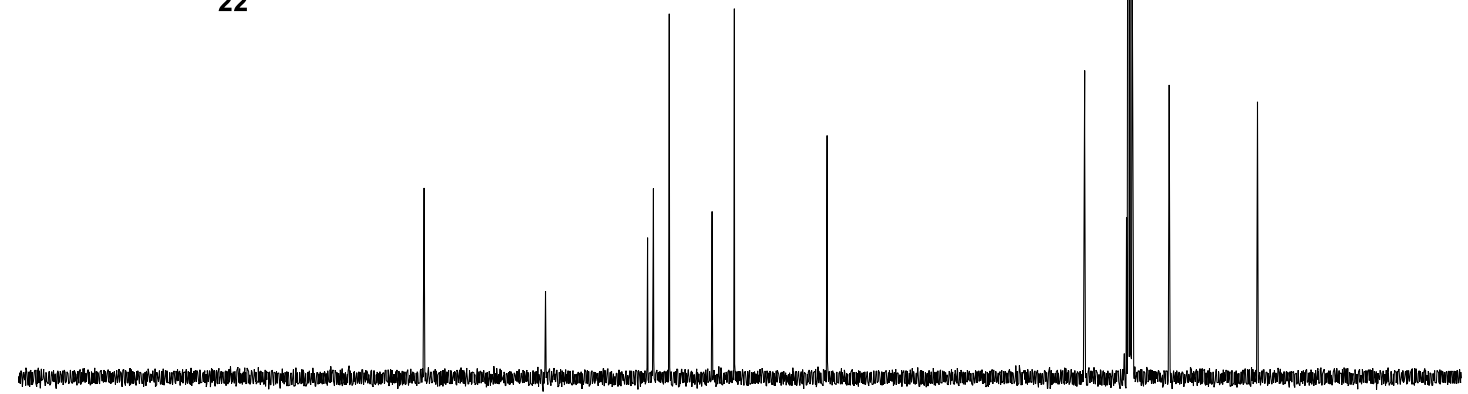

${ }^{1} \mathbf{H}$ NMR (500 MHz, CD3 OD) of S36.

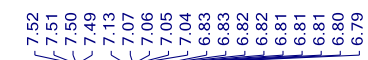

ming

$\overbrace{\substack{\mathrm{S} 36 \\ \mathrm{~S} 36}}^{\mathrm{NH}_{2}}$ 
${ }^{13} \mathbf{C}$ NMR (126 MHz, CD 3 OD) of $\mathbf{S 3 6}$.

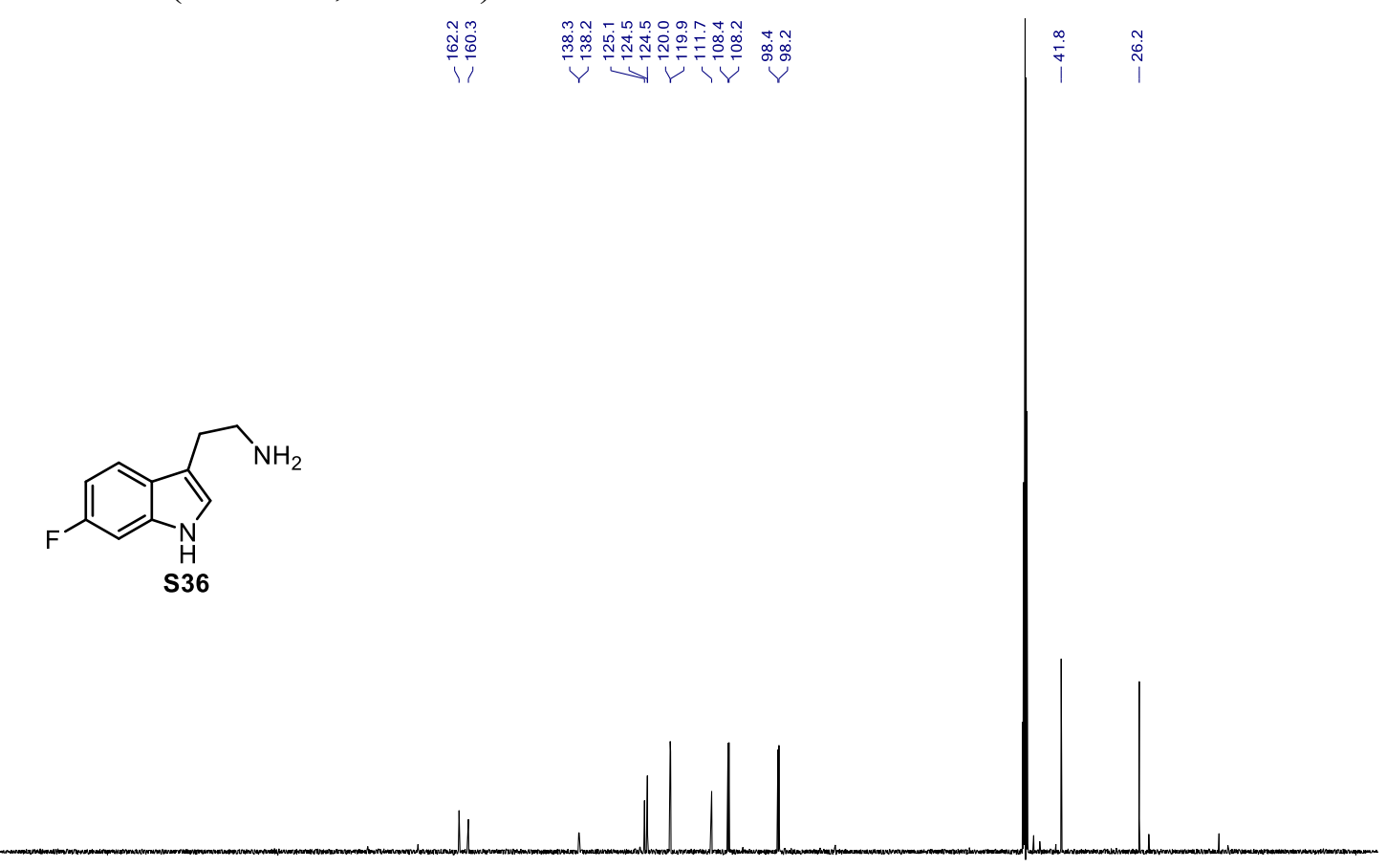

${ }^{19}$ F NMR (471 MHz, CD3 OD) of $\mathbf{S 3 6 .}$<smiles>NCCc1c[nH]c2cc(F)ccc12</smiles> 
${ }^{1} \mathbf{H}$ NMR (500 MHz, CD 3 OD) of $\mathbf{S 3 7 . ~}$
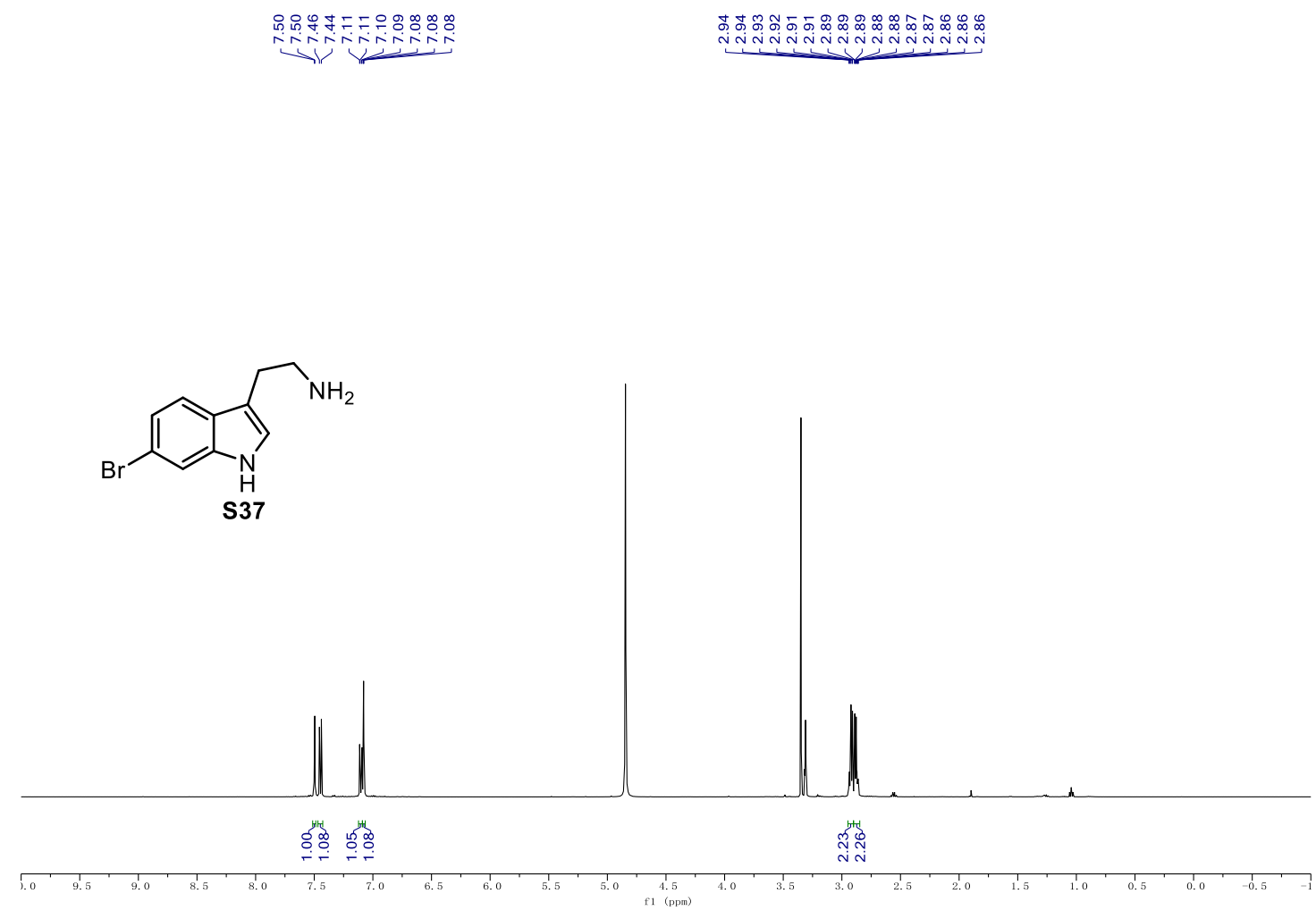

${ }^{13} \mathbf{C}$ NMR (126 MHz, CD $\left.{ }_{3} \mathrm{OD}\right)$ of $\mathbf{S 3 7 .}$
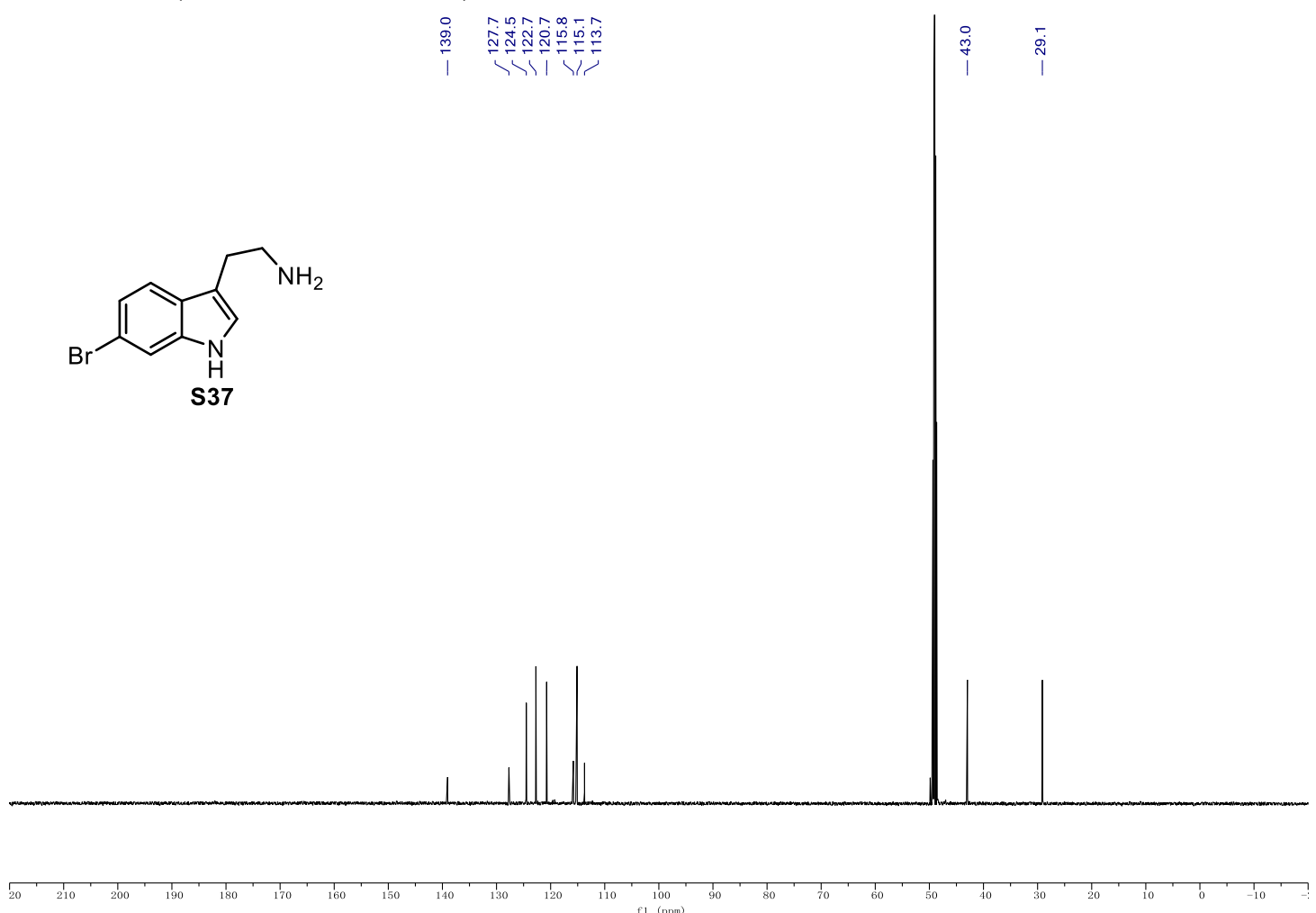
${ }^{1} \mathbf{H}$ NMR (500 MHz, CD3 OD: $\mathrm{CDCl}_{3}(\mathrm{v} / \mathrm{v}=1: 1)$ ) of $\mathbf{S 3 8}$.

管

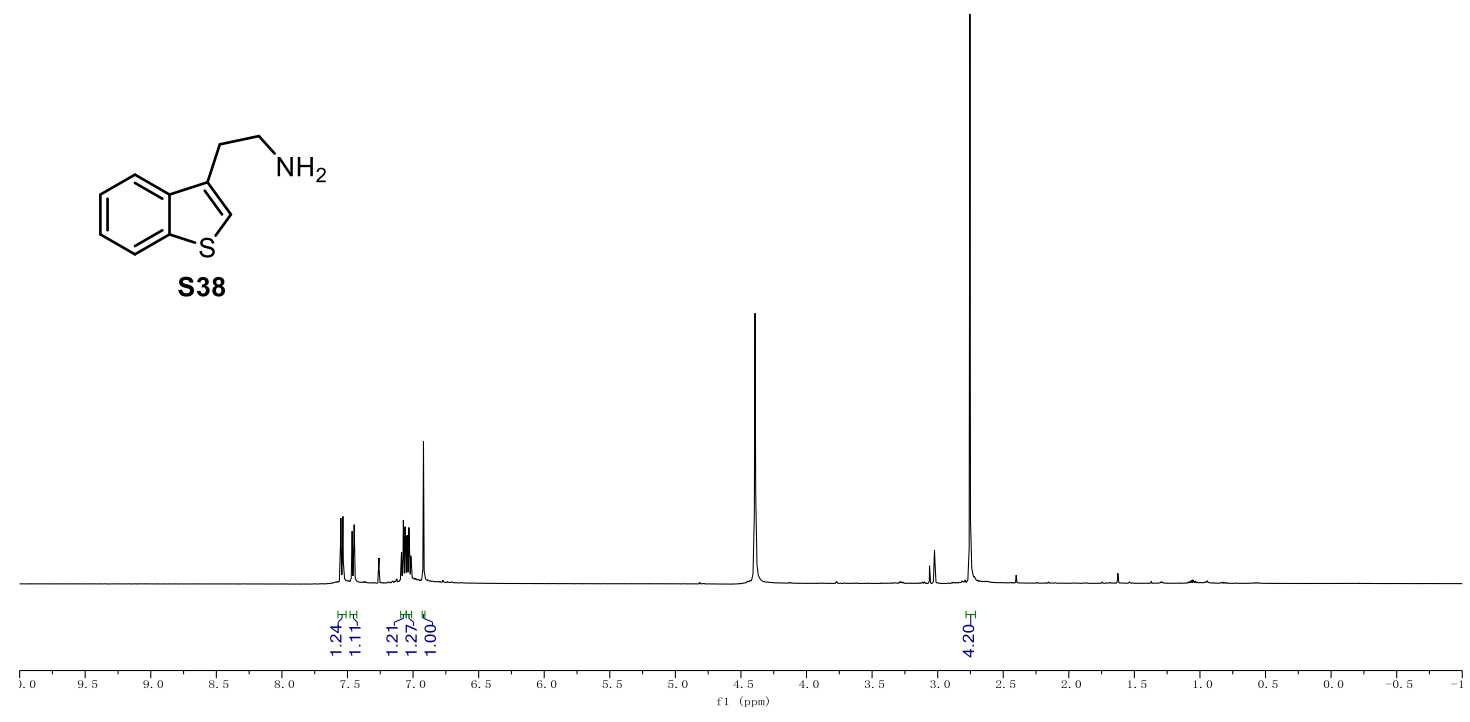

${ }^{13} \mathbf{C}$ NMR (126 MHz, CD $\left.3 \mathrm{OD}: \mathrm{CDCl}_{3}(\mathrm{v} / \mathrm{v}=1: 1)\right)$ of $\mathbf{S 3 8}$.

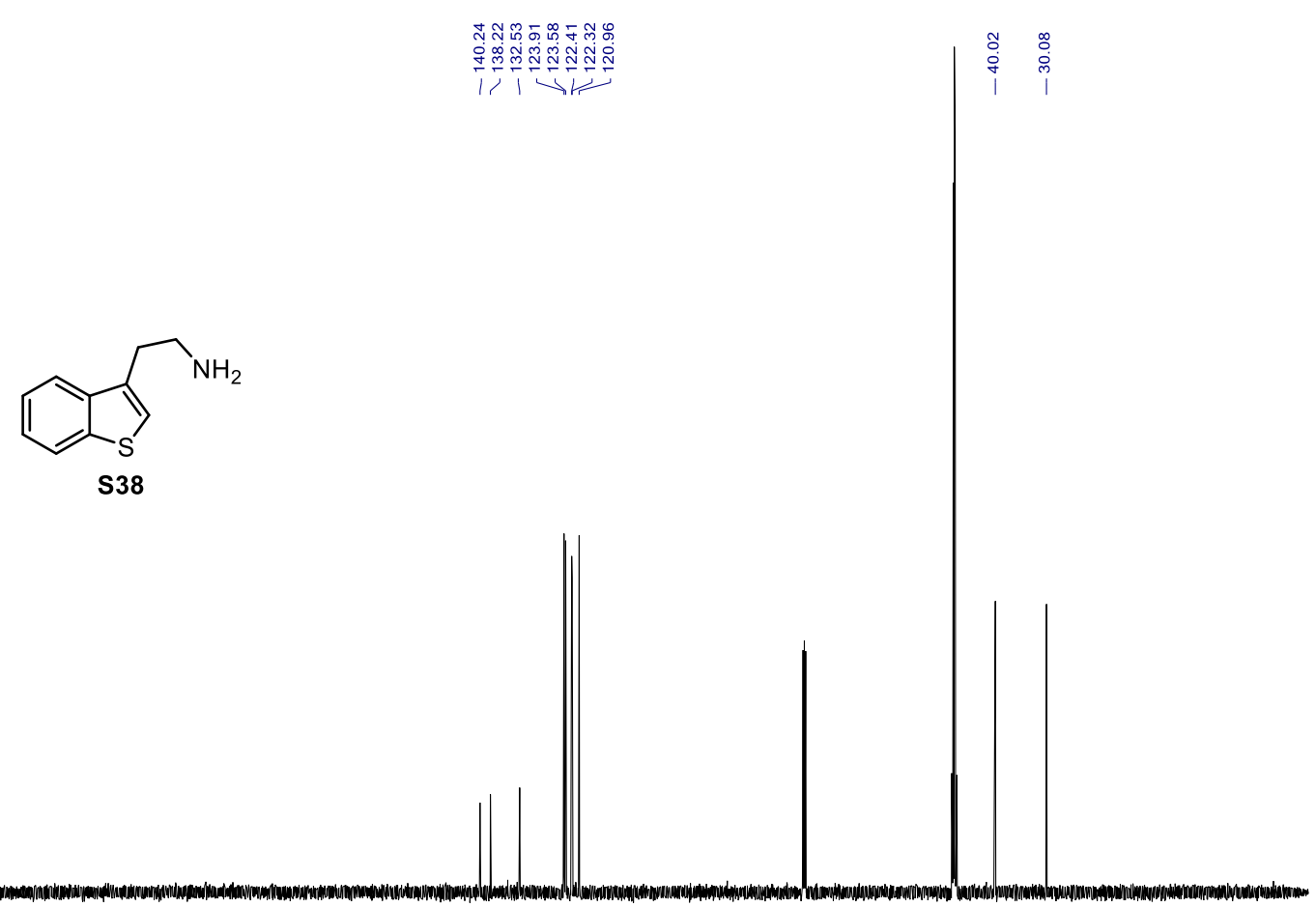

